\title{
The Agricultural Survey of Afghanistan
}
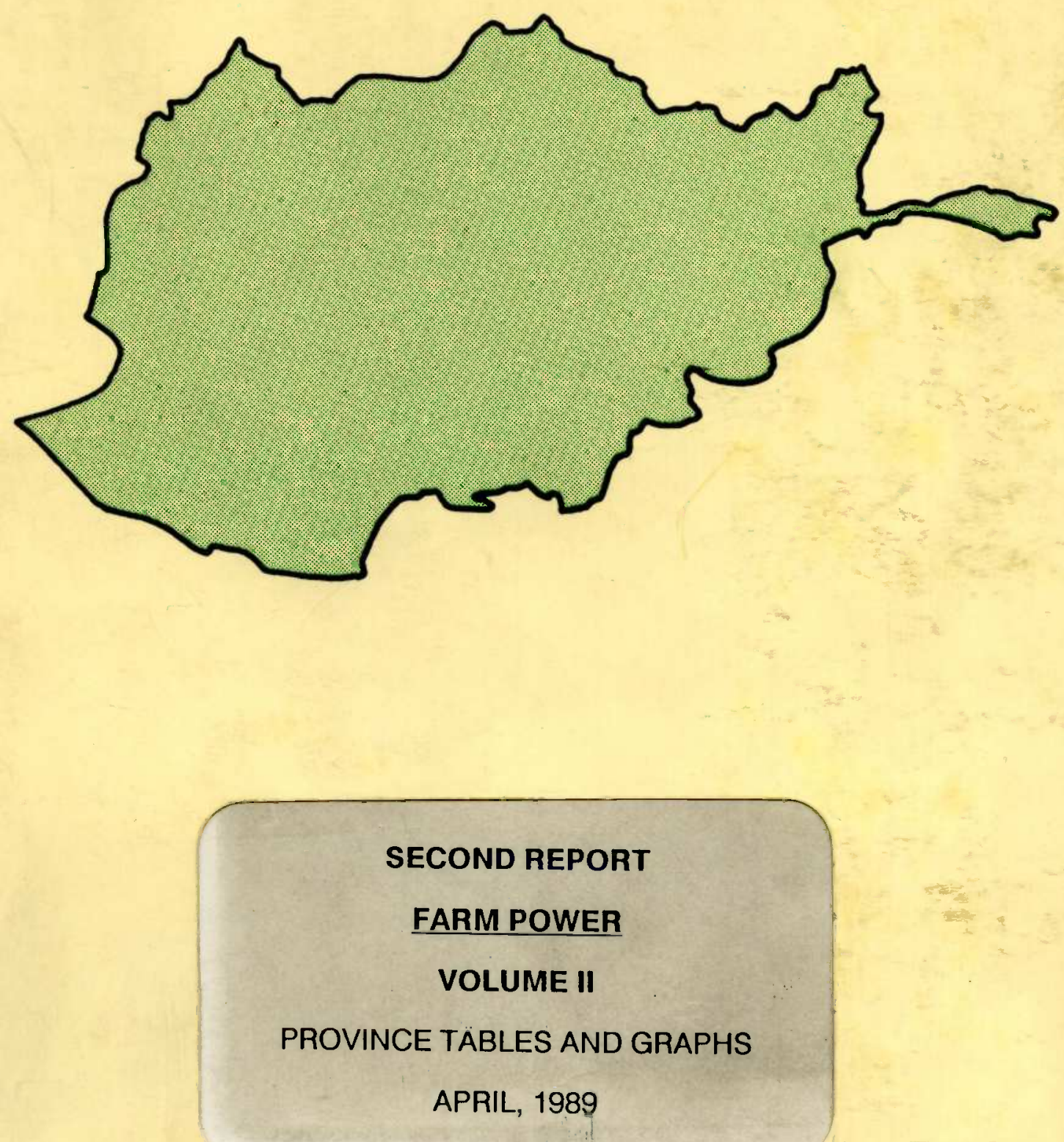

The Swedish Committee for Afghanistan 
BADAKHSHAN 


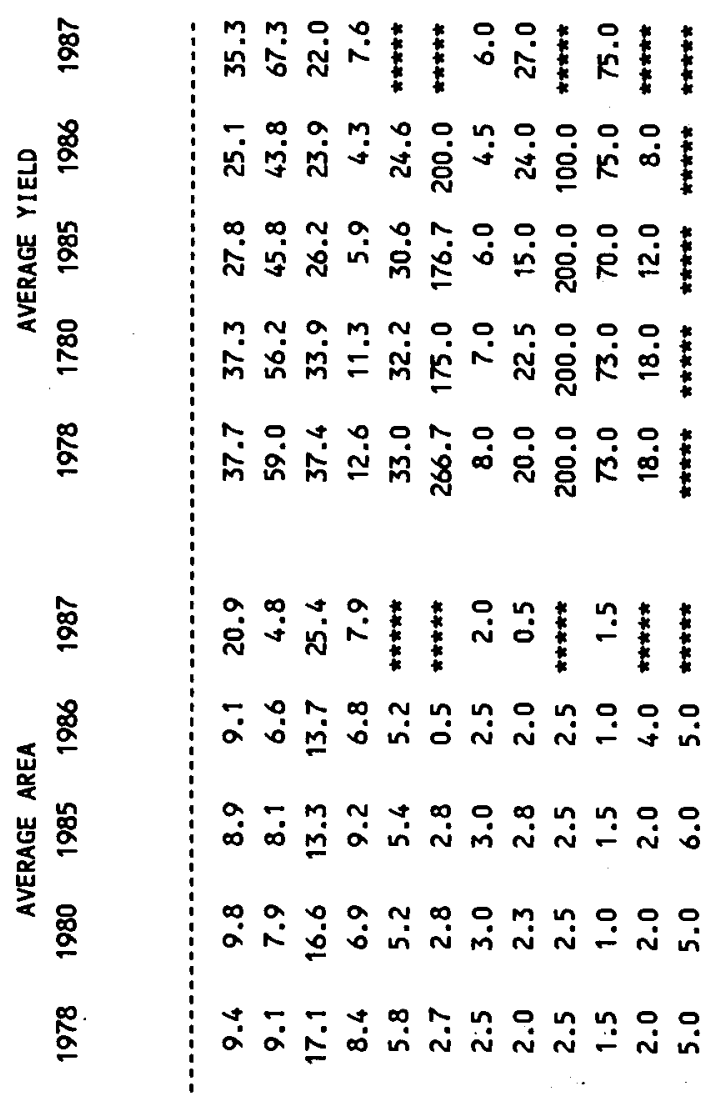

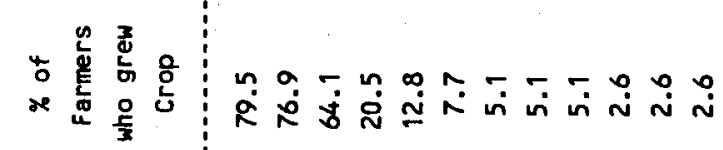

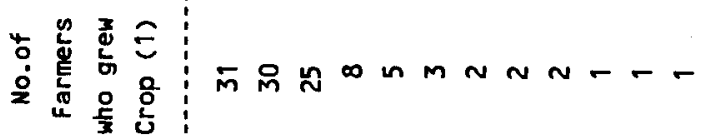
矛占

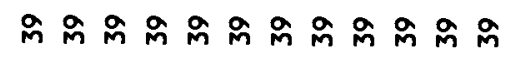


PROVINCE OF BDN

Average Number of Livestock Shot

Number of Farmers reporting

Livestock Shot

Average Number of Livestock Killed by Mines

Number of Farmers reporting Livestock killed by Mines

Number of Farmers Questioned. 12

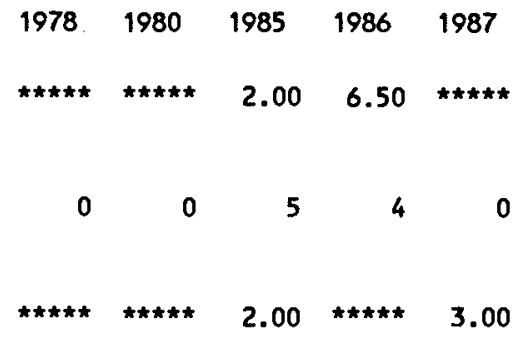

$\begin{array}{llll}0 & 1 & 0 & 1\end{array}$

IABLE 9B - DIRECT EFFECTS OF THE HAR - FARMERS IN CAMPS

PROVINCE OF BDN

Average Number of Livestock Shot

$\begin{array}{lllll}1978 & 1980 & 1985 & 1986 & 1987\end{array}$

Number of Farmers reporting

Livestock shot

$\begin{array}{lllll}0 & 4 & 12 & 5 & 0\end{array}$

Average Number of Livestock Killed by Mines

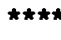

$10.80 \quad 18.80$

Number of Farmers reporting Livestock

killed by Mines

$\begin{array}{lllll}0 & 0 & 5 & 5 & 0\end{array}$

Number of Farmers Questioned 27 
IABLE 1-5 - ANALYSIS OF GREATEST FARMING PROBLEMS - COMMUNITY SURVEY

PROVINCE OF BDN

\section{PERCENTAGE OF VILLAGES GIVING FOLLOWING PRIORITY TO PROBLEM}

Rainfall

Crop Disease

Birds or Rats

Seed Availability

Fertilizer Availability

Crop Protection Chemicals Credit

Irrigation Water Availability Flooding

Labour Availabilty

Power for Land Preparation

Direct War Effects

Animal Diseases

Extension

Other Problems

\section{6}

Rainfall

Crop Disease

Birds or Rats

Seed Availability

Fertilizer Availability

Crop Protection Chemicals

Credit

Irrigation Water Availability

Flooding

Labour Availabilty

Power for Land Preparation

Direct War Effects

Animal Diseases

Extension

Other Problems

1985

Rainfall

Crop Disease

Birds or Rats

Seed Availability

Fertilizer Availability

Crop Protection Chemicals

Credit

Irrigation Water Availability

Flooding

Labour Availabilty

Power for Land Preparation

Direct War Effects

Animal Diseases

Extension

Other Problems

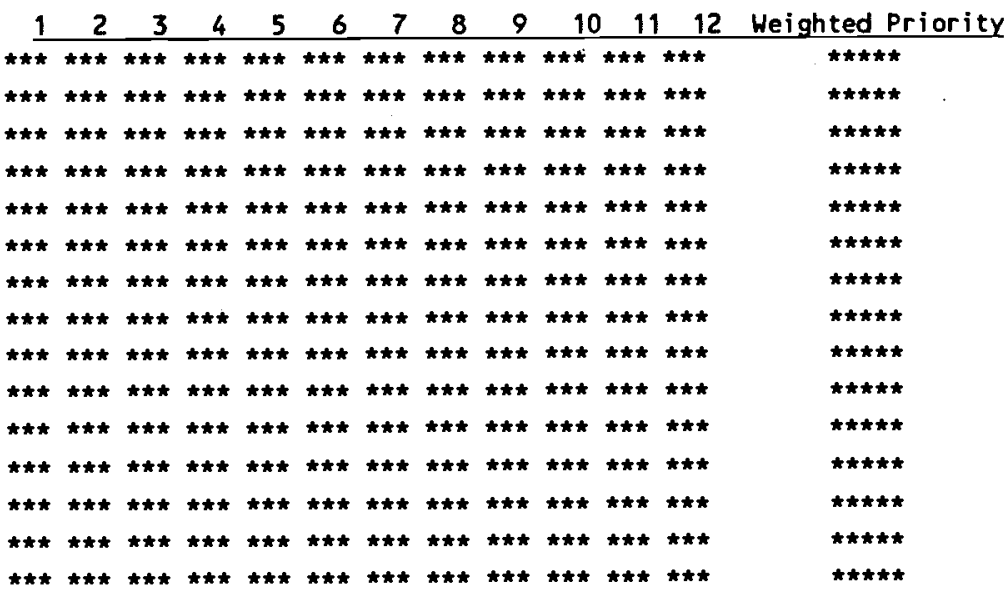

$\begin{array}{llllllllllll}1 & 2 & 3 & 4 & 5 & 6 & 7 & 8 & 9 & 10 & 11 & 12 .\end{array}$

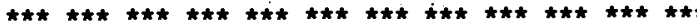

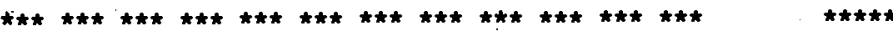

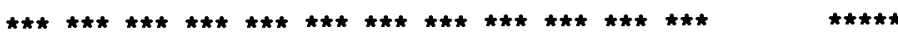

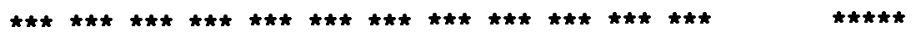

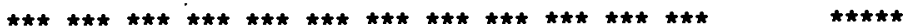

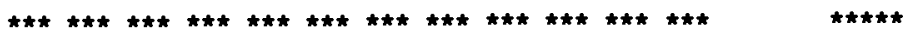

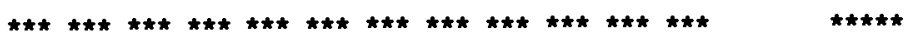

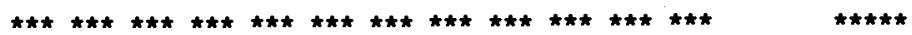

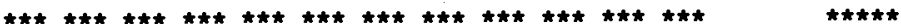

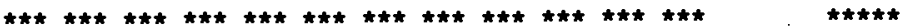

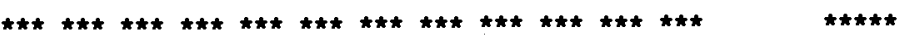

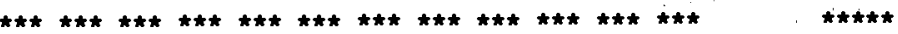

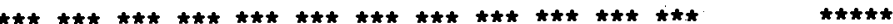

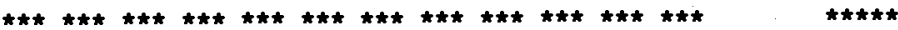

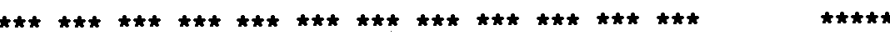

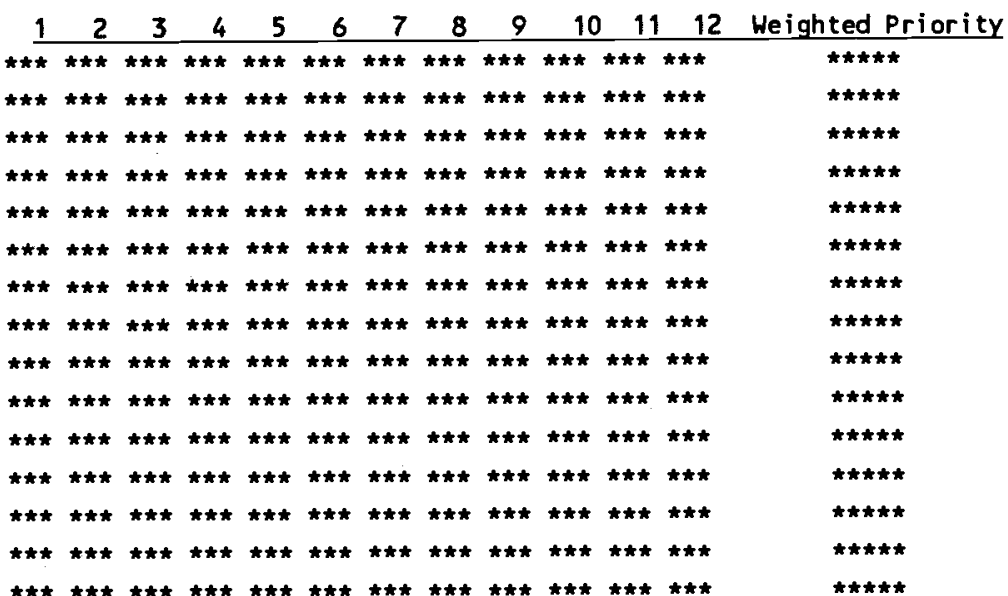


IABLE 1 -5 (contd) - ANALYSIS OF GREATEST FARMING PROBLEMS - COMMUNITY SURVEY

PROVINCE OF BON

1980 PERCENTAGE OF VILLAGES GIVING FOLLOWING PRIORITY TO PROBLEM

Rainfall

Crop Disease

Birds or Rats

Seed Availability

Fertilizer Availability

Crop Protection Chemicals

Credit

Irrigation Hater Availability

Flooding

Labour Availabilty

Power for Land Preparation

Direct War Effects

Animal Diseases

Extension

other Problems

\section{8}

Rainfall

Crop Disease

Birds or Rats

Seed Availability

Fertilizer Availability

Crop Protection Chemicals

Credit

Irrigation Water Availability

Flooding

Labour Availabilty

Power for Land Preparation

Direct War Effects

Animal Diseases

Extension

Other Problems

\begin{tabular}{|c|c|c|c|c|c|c|c|c|c|c|c|c|}
\hline 1 & 2 & 3 & 4 & 5 & 6 & 7 & 8 & 9 & 1 & 1 & 12 & Weighted Priority \\
\hline$\star \star \star$ & 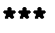 & $\star \star \star$ & $\star \star \star$ & $\star \star \star *$ & $\star \star \star *$ & $\star \star * *$ & $\star \star * *$ & $\star \star * *$ & $\star \star *$ & $\star \star * *$ & $\star \star * *$ & $\star \star \star \star \star \star$ \\
\hline$\forall \star \star *$ & $\star \star \star *$ & $\star \star \star *$ & $\star \star \star *$ & $\star * *$ & 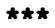 & $\star * * *$ & 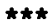 & $\star * * *$ & 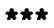 & $\hbar \star \star \star$ & $\hbar \star \star \star$ & $\hbar \star \star \star \star$ \\
\hline$\star \star \star$ & $\star \star \star \star$ & $\star \star \star \star$ & $\star \star \star \star$ & $\star \star \star \star$ & 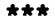 & $\star \star \star \star *$ & $\star \star \star \star$ & 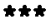 & 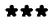 & 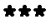 & 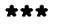 & 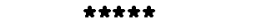 \\
\hline 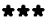 & $\star \star \star$ & $\star \star \star *$ & $\star \star \star *$ & $\star \star \star *$ & $\star \star * *$ & $\star \star \star \star *$ & $\star \star \star \star *$ & $\star \star \star *$ & $\star \star \star \star *$ & $\star \star \star *$ & $\star \star \star \star *$ & $\hbar \hbar \hbar \hbar \star$ \\
\hline$\star \star \star$ & $\star \star \star$ & $\star \star \star$ & $\star \star \star$ & $\star \star \star$ & $\star \star \star$ & $\star \star \star \star$ & *** & $\star \star * *$ & 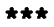 & $\hbar *$ * & 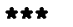 & $\star \star \star \star \star \star$ \\
\hline$\star \star \star$ & $\star \star \star$ & $\star \star \star$ & $\star \star \star \star$ & $\star \star \star$ & 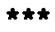 & $\star * *$ & $\star * *$ & $\star \star \star *$ & $\star * *$ & $\star \star \star *$ & $\star \star *$ & $\star \star \star \star \star \star *$ \\
\hline$\star \star \star$ & $\star \star \star *$ & $\star \star \star$ & $\star \star * *$ & $\star \star \star$ & $\star \star *$ & $\star \star \star *$ & $\star \star *$ & $\star \star \star *$ & $\star \star *$ & $\star \star \star$ & $\star \star \star$ & $\star \star \star \star \star$ \\
\hline 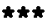 & $\star \star \star \star$ & 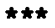 & 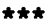 & $\star \star \star \star$ & 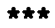 & 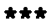 & $\star \star \star *$ & 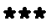 & 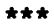 & 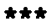 & $\star * *$ & 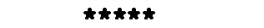 \\
\hline$\star \star \star \star$ & $\star \star \star \star$ & $\star \star \star$ & $\star \star \star *$ & $\star \star \star *$ & $\star \star \star \star *$ & $\star \star \star *$ & $\star \star * *$ & $\star * * *$ & $\star \star * *$ & $\star \star \star *$ & $\star \star * *$ & $\star \star \star * \star * *$ \\
\hline$\star \star \star \star *$ & $\star \star \star \star$ & $\star \star \star \star$ & $\star \star \star *$ & $\star \star \star \star$ & $\star \star \star \star *$ & $\star \star \star \star *$ & $\star \star \star \star$ & $\star \star \star \star$ & $\star \star \star \star$ & $\star \star \star \star$ & $\star \star \star \star$ & 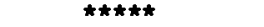 \\
\hline$\star \star \star$ & $\star \star \star$ & $\star \star \star \star$ & 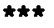 & $\star \star \star \star$ & $\star \star \star \star *$ & 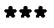 & 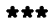 & $\star \star \star \star \hbar$ & 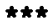 & $\star \star \star \star$ & 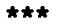 & 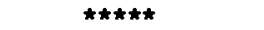 \\
\hline ** & $\star \star \star \star$ & $\star \star \star \star$ & $\star * *$ & $\neq * \star *$ & $\neq * *$ & 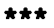 & $\star \star \star *$ & 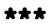 & $\star \star * *$ & $\star * * *$ & 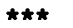 & $\hbar \star \star \star \star \star$ \\
\hline 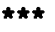 & $\star \star \star$ & $\star \star \star$ & $\star \star \star$ & $\star \star \star$ & $\star \star \star \star$ & $\star \star \star$ & $\star \star * *$ & $\star \star *$ & 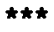 & $\star \star \star *$ & $\star \star *$ & 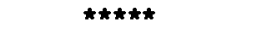 \\
\hline 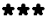 & $\star \star \star \star$ & 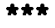 & $\star \star \star \star *$ & $\star \star * \star$ & $\star * * *$ & $\star \star \star *$ & $\star \star \star \star$ & $\star \star \star \star *$ & $\star \star \star \star *$ & $\star \star \star \star *$ & $\star \star \star \star$ & $\star \star \star \star \star \star *$ \\
\hline 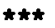 & $\star \star \star \star$ & $\star \star \star$ & 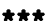 & $\star \star \star \star *$ & $\star \star \star \star$ & $\star \star \star *$ & $\star \star \star \star$ & $\hbar \star \star \star *$ & $\star \star \star \star *$ & $\star \star \star \star *$ & $\star \star \star *$ & $\star \star \star \star \star \star *$ \\
\hline
\end{tabular}

$\begin{array}{llllllllllll}1 & 2 & 3 & 4 & 5 & 6 & 7 & 8 & 9 & 10 & 11 & 12\end{array}$ Weighted Priority

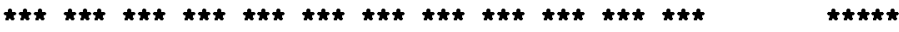

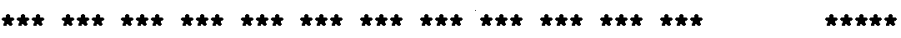

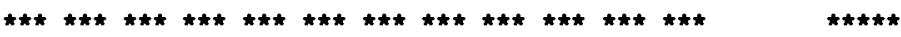

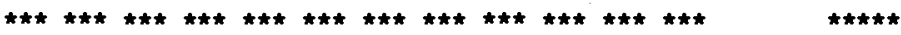

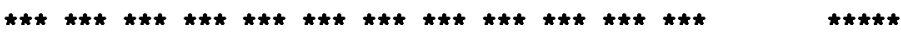

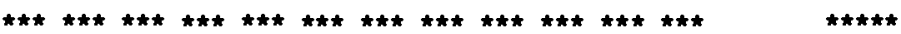

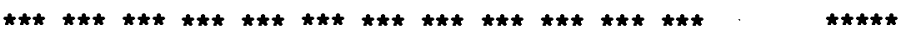

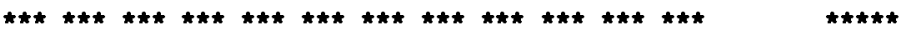

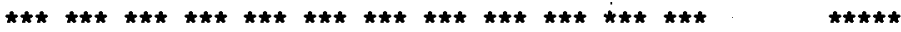

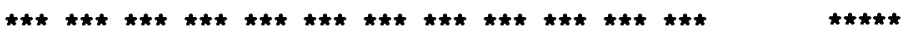

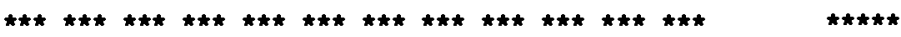

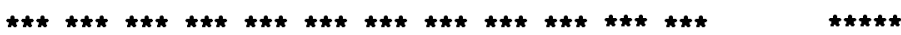

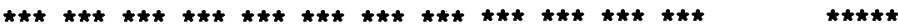

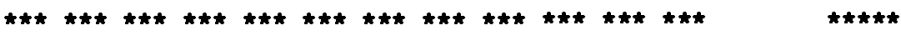

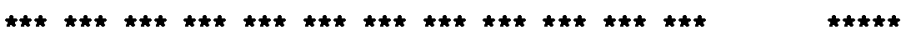




\section{TABLE OF CONTENTS}

\section{VOLUME TWO}

The Provinces are ordered alphabetically.

Volume II contains the graphs and base tables for all the 29 provinces. Each Province has the following graphs and tables are given:

1. Farm size distribution - bar chart, 1978

table, 1978

table, 1987

2. Changes in the use of farm power, 1978-87, for the province's two main crops, four bar charts. Method of land preparation: own oxen, shared oxen, hired oxen, own tractor, hired tractor, manual, and other, 1978-87, four tables.

3. Changes in oxen/family, family labour, and hired labour, 1978-87, four bar charts.

4. Number of trained oxen per family, family labour and hired labour, 1978-1987, four bar charts.

5. Number of trained oxen per family, 1978-87, two tables.

6. Family and hired labour, 1978-87, two tables.

7. Direct effects of war, two tables.

8. Percent of farmers growing different crops (1978), with average areas and yields, one table.

9. Analysis of greatest farming problems, five tables. 


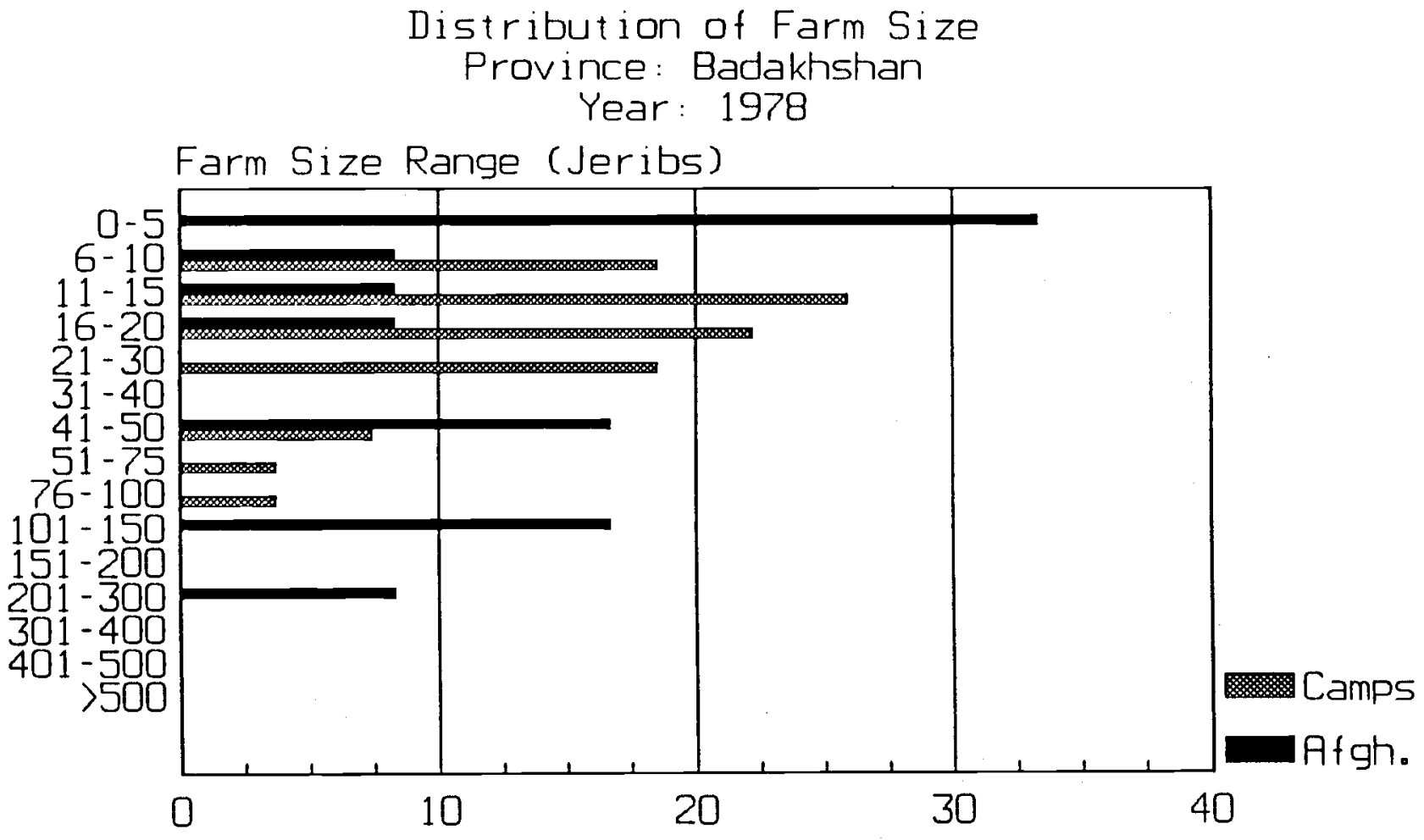

Percentage of Farmers

Farmers in Afghanistan

Total Number of Farms

Average Area

Average Area Irrigated

Average Dryland Area

Farmers who left in 1987

Total Number of Farms

Average Area

Average Area Irrigated

Average Dryland Area

\section{Average of Both Groups}

Provincial Average Area

Provincial Average Irrigated Area

Provincial Average Dryland Area
1978

12

51.25

4.63

54.23

27

23.22

16.74

12.00

31.85

15.18

50.17

2.88

29.87

52.14

(All areas in Jeribs. 5 Jeribs $=1$ Hectare) 


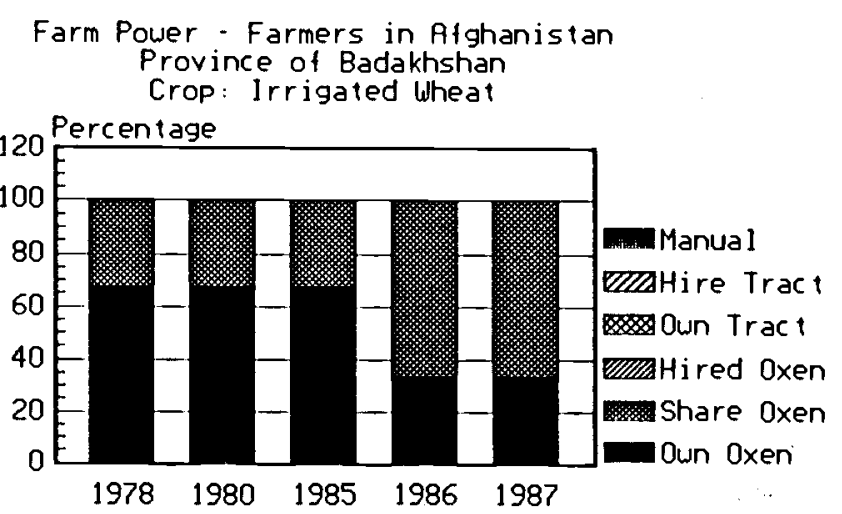

Year

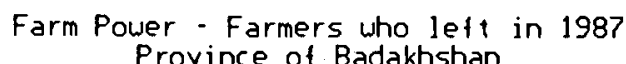

Province of Badakhshan

Crop: Irrigated wheat

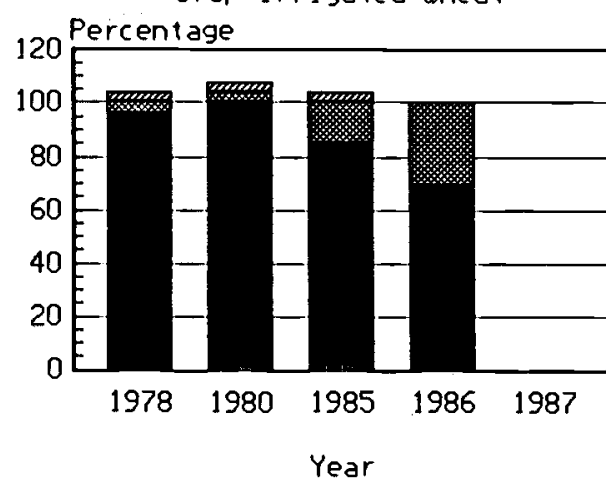

Base Figures for above graphs

Province: Badakshan

Farmers in Afghanistan

Irr. Wheat Own Oxen

Shared oxen

Hired oxen

Own Tractor

Hired Tractor

Barley

Manual Means

Own Oxen

Shared oxen

Hired oxen

Own Tractor

Hired Tractor

Manual Means

Farmers who left in 1987

$\begin{array}{ll}\text { Irr. Wheat } & \text { Own Oxen } \\ & \text { Shared Oxen } \\ & \text { Hired Oxen } \\ & \text { Own Tractor } \\ & \text { Hired Tractor } \\ & \text { Manual Means } \\ \text { Barley } & \text { Own Oxen } \\ & \text { Shared Oxen } \\ & \text { Hired Oxen } \\ & \text { Own Tractor } \\ & \text { Hired Tractor }\end{array}$
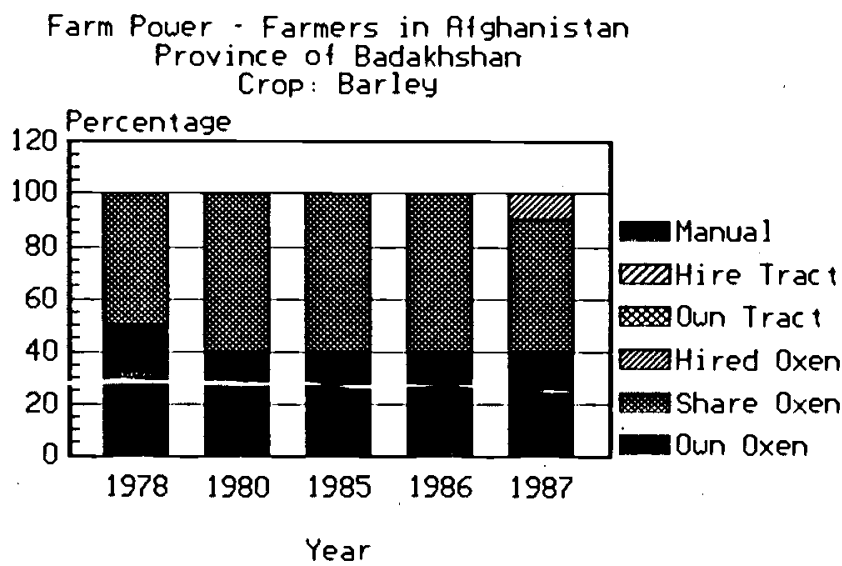

Farm Power - Farmers who lett in 1987

Province of Badakhshan Crop : Barley

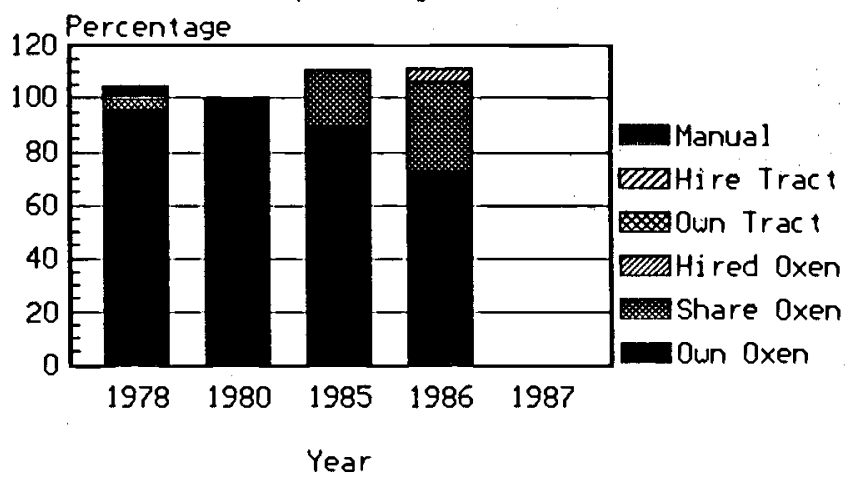

\section{Percentage of Farmers $1978 \underline{1980 \quad 1985 \quad 1986}$}

1987

$\begin{array}{ccccc}66.67 & 66.67 & 66.67 & 33.33 & 33.33 \\ 33.33 & 33.33 & 33.33 & 66.67 & 66.67 \\ - & - & - & - & - \\ - & - & - & - & - \\ - & - & - & - & - \\ - & - & - & - & - \\ 50.00 & 40.00 & 40.00 & 40.00 & 40.00 \\ 50.00 & 60.00 & 60.00 & 60.00 & 50.00 \\ - & - & - & - & - \\ - & - & - & - & - \\ - & - & - & - & - \\ - & - & - & - & -\end{array}$

$\begin{array}{ccccc}96.30 & 100.00 & 85.19 & 69.23 & - \\ 3.70 & 3.85 & 14.81 & 30.77 & - \\ 3.70 & 3.85 & 3.70 & - & - \\ - & - & - & - & - \\ - & - & - & - & - \\ - & - & - & - & - \\ 95.24 & 100.00 & 89.47 & 72.22 & - \\ 4.76 & - & 21.05 & 33.33 & - \\ - & - & - & 5.56 & - \\ - & - & - & - & - \\ - & - & - & - & -\end{array}$



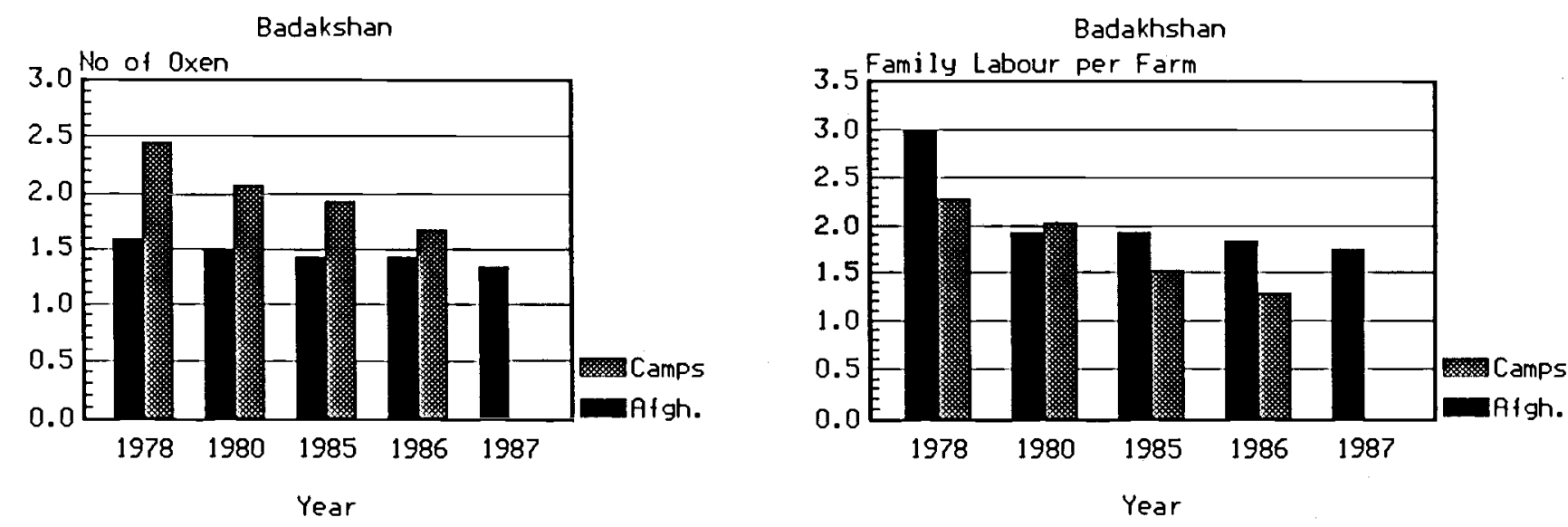

Badakhshan
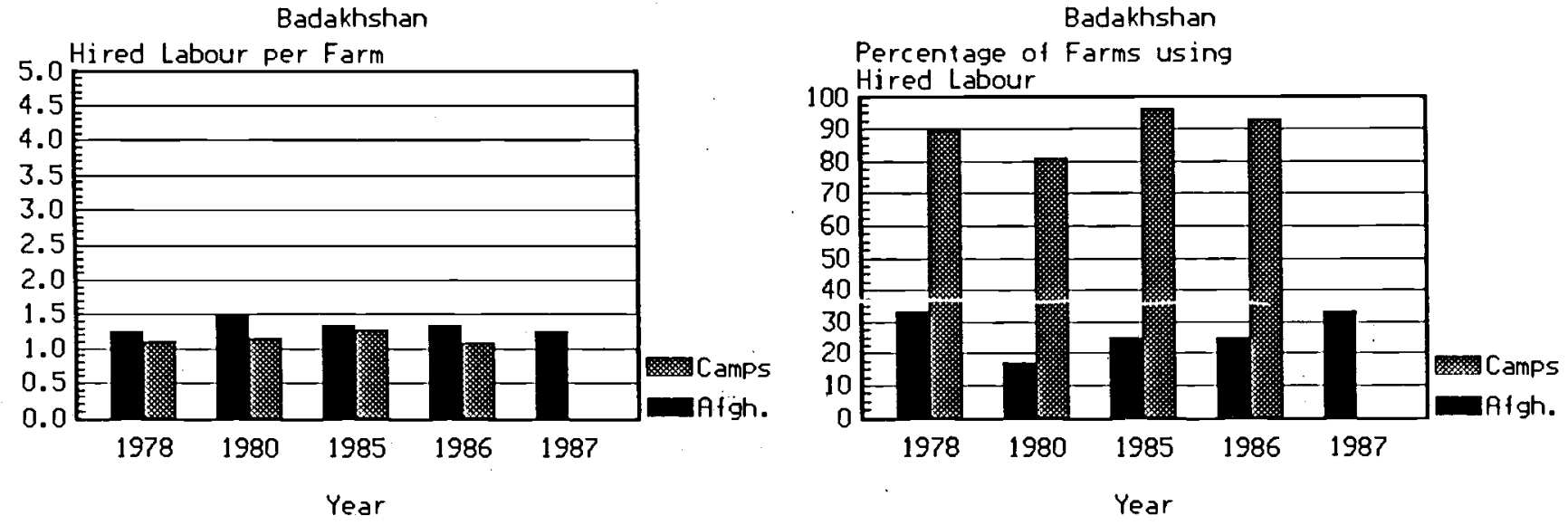
PROVINCE OF BON

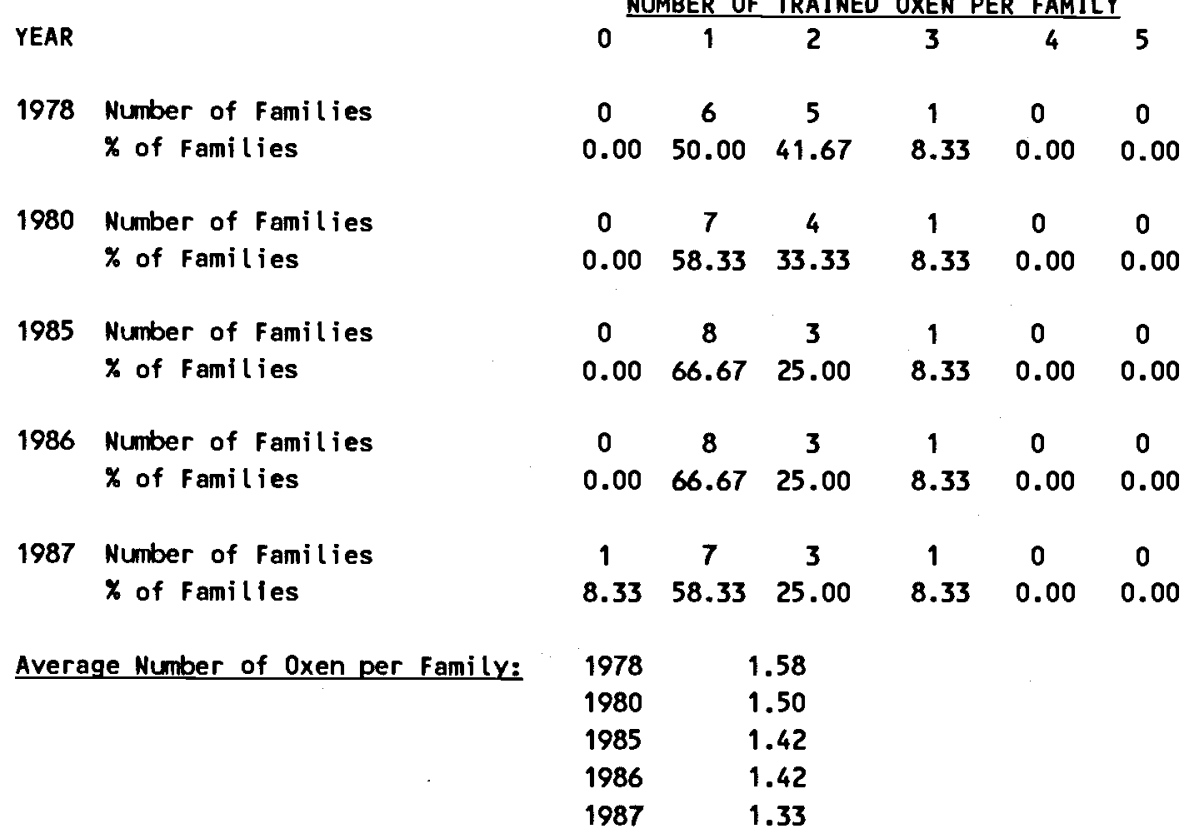

Number of Farmers Questioned 12

\section{IABLE 7B - AVERAGE NUMBER OF TRAINED OXEN FOR FARMERS IN THE CAMPS}

PROVINCE OF BDN

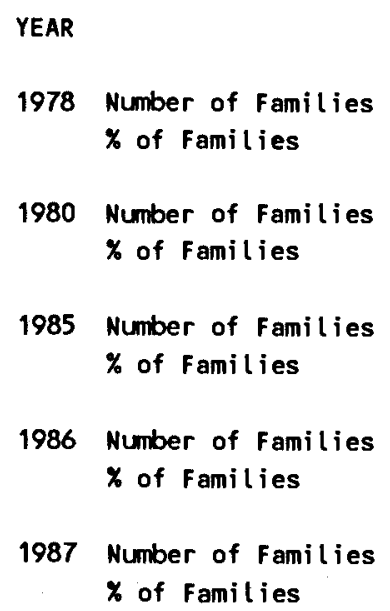


IABLE $8(A)$ - AVERAGE LABOUR (FAMILY and HIRED) FOR FARMERS IN AFGANISTAN

PROVINCE OF BDN

\begin{tabular}{|c|c|c|c|c|c|}
\hline & 1978 & 1980 & 1985 & 1986 & 1987 \\
\hline Average Family Labour per Farm & 1.92 & 1.92 & 1.92 & 1.83 & 1.75 \\
\hline $\begin{array}{l}\text { Average No. of Hired Labour per Farm } \\
\text { (for farms using hired labour) }\end{array}$ & 1.25 & 1.50 & 1.33 & 1.33 & 1.25 \\
\hline Number of Farms us ing Hired Labour & 4 & 2 & 3 & 3 & 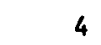 \\
\hline
\end{tabular}

IABLE $8(B)$ - AVERAGE LABOUR (FAMILY and HIRED) FOR FARMERS IN CAMPS

PROVINCE OF BDN

Average Family Labour per Farm

Average No. of Hired Labour per Farm

(for farms using hired labour)

Number of Farms using Hired Labour

Number of Farmers Questioned 27

$\begin{array}{rrrrr}1978 & 1980 & 1985 & 1986 & 1987 \\ 2.28 & 2.02 & 1.52 & 1.28 & 0.00 \\ 1.10 & 1.14 & 1.27 & 1.08 & \star \star \star \star \star \\ 24 & 22 & 26 & 25 & 0\end{array}$




\section{BALKH}




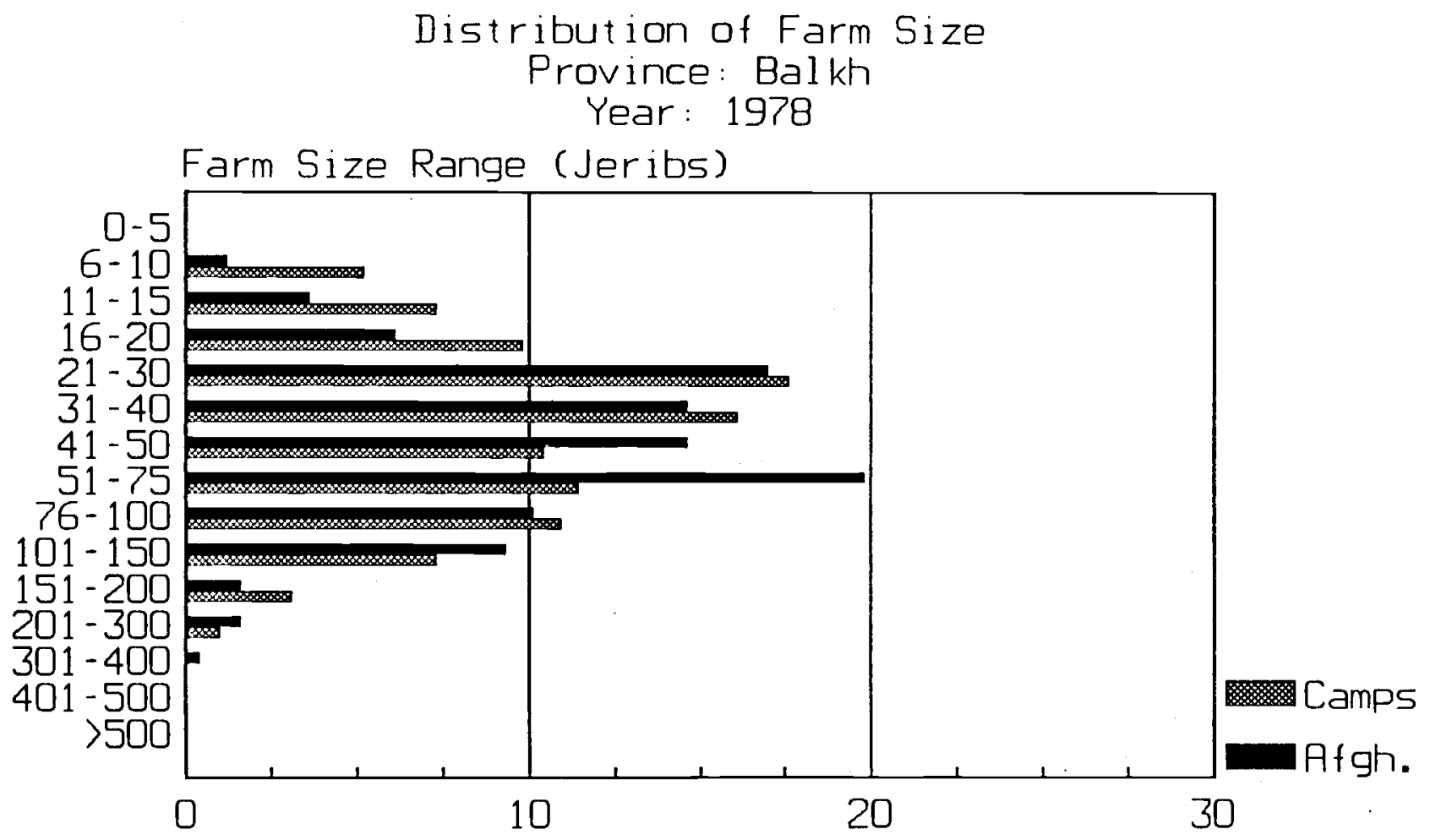

Percentage of Farmers

Farmers in Afghanistan

Total Number of Farms

Average Area

Average Area Irrigated

Average Dryland Area

Farmers who left in 1987

Total Number of Farms

Average Area

Average Area Irrigated

Average Dryland Area

\section{Average of Both Groups}

Provincial Average Area

Provincial Average Irrigated Area

Provincial Average Dryland Area
1978

247

59.21

54.92

57.20

193

53.35

43.21

54.45

56.64

49.31

38.25

56.11

27.67

43.95

(All areas in Jeribs. 5 Jeribs $=1$ Hectare) 
Farm Power - Farmers in Atghanistan Province of Balkh Crop: Irrigated wheat

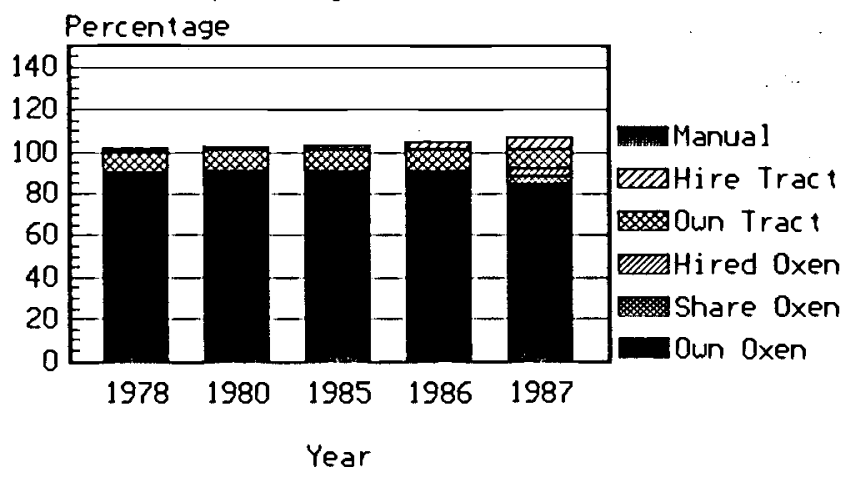

Farm Power - Farmers who lett in 1987 Province of Balkh Crop: Irrigated wheat

Percentage

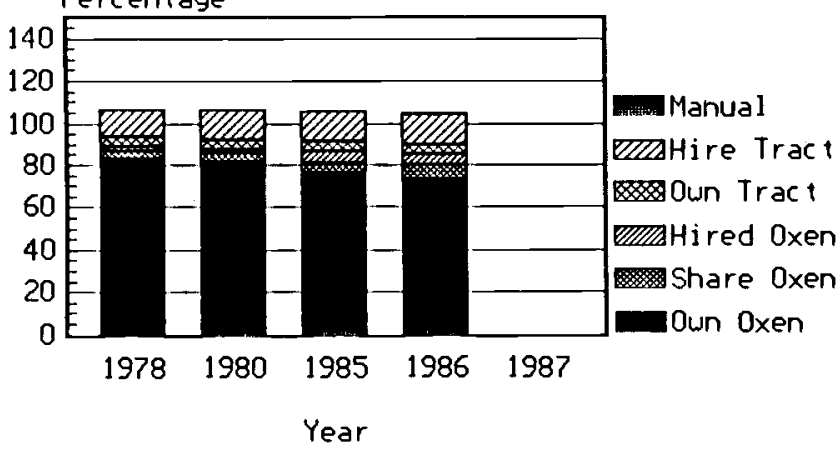

Base Figures for above graphs

Province: Balkh

Farmers in Afghanistan

Irr. Wheat own Oxen

Shared Oxen

Hired oxen

Own Tractor

Hired Tractor

Barley

Manual Means

Own Oxen

Shared oxen

Hired oxen

Own Tractor

Hired Tractor

Manual Means

Farmers who left in 1987

Irr. Wheat own oxen

Shared Oxen

Hired oxen

Own Tractor

Hired Tractor

Barley
Own Oxen

Shared oxen

Hired Oxen

Own Tractor

Hirod Tractor

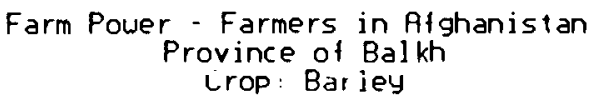

Farm Power - Farmers in Atghanistan Province of $\mathrm{Balkh}$ Lrop: Bar jey

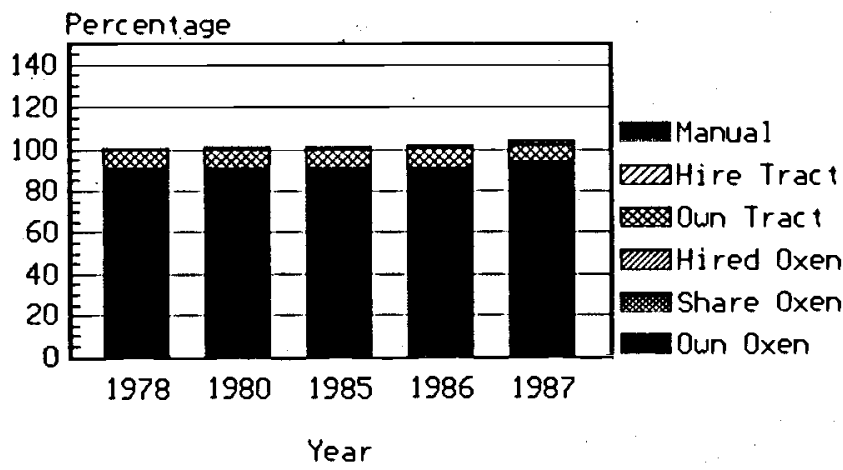

Farm Power - Farmers who left in 1987 Province of Balkh Crop: Barley

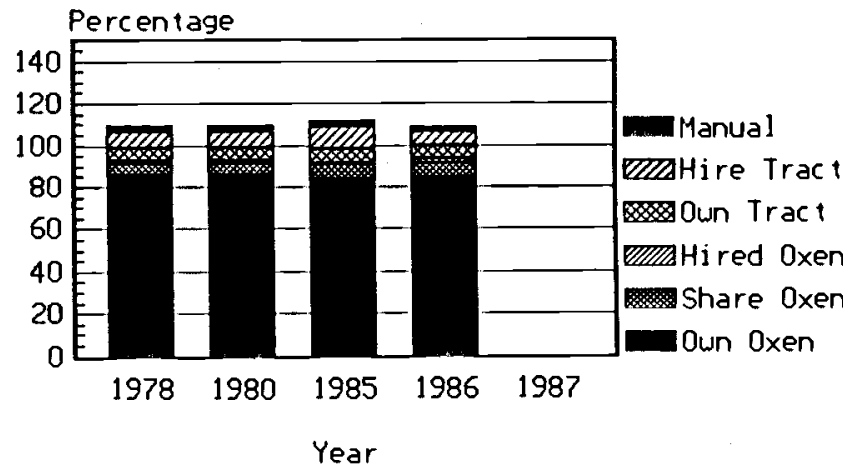

\begin{tabular}{rrrrr}
\multicolumn{5}{c}{ Percentage of Farmers } \\
1978 & $\underline{1980}$ & $\underline{1985}$ & $\underline{1986}$ & \\
& & & & \\
88.89 & 89.30 & 87.30 & 87.17 & 84.21 \\
.53 & .53 & 1.59 & 1.60 & 4.39 \\
.53 & .53 & 1.59 & 1.60 & 3.51 \\
10.05 & 10.70 & 10.58 & 10.70 & 8.77 \\
1.59 & 1.60 & 2.65 & 3.74 & 6.14 \\
- & - & - & - & - \\
89.86 & 89.16 & 89.85 & 88.66 & 90.40 \\
.97 & 1.48 & .51 & 1.55 & 1.60 \\
- & - & .51 & .52 & 1.60 \\
8.70 & 9.36 & 8.63 & 9.28 & 8.00 \\
.97 & .99 & 1.52 & 1.55 & 2.40 \\
- & - & - & - & -
\end{tabular}

$\begin{array}{rrrrr}82.82 & 81.48 & 75.78 & 73.02 & - \\ 4.29 & 4.32 & 4.97 & 7.14 & - \\ 1.84 & 1.85 & 6.21 & 4.76 & - \\ 4.29 & 4.32 & 4.35 & 4.76 & - \\ 13.50 & 14.20 & 14.29 & 14.29 & - \\ - & - & - & .79 & - \\ 86.32 & 86.17 & 84.04 & 84.81 & - \\ 5.26 & 5.32 & 7.45 & 6.33 & - \\ 1.05 & 1.06 & - & 2.53 & - \\ 6.32 & 6.38 & 6.38 & 6.33 & - \\ 7.37 & 7.15 & 10.64 & 6.33 & -\end{array}$


Balkh

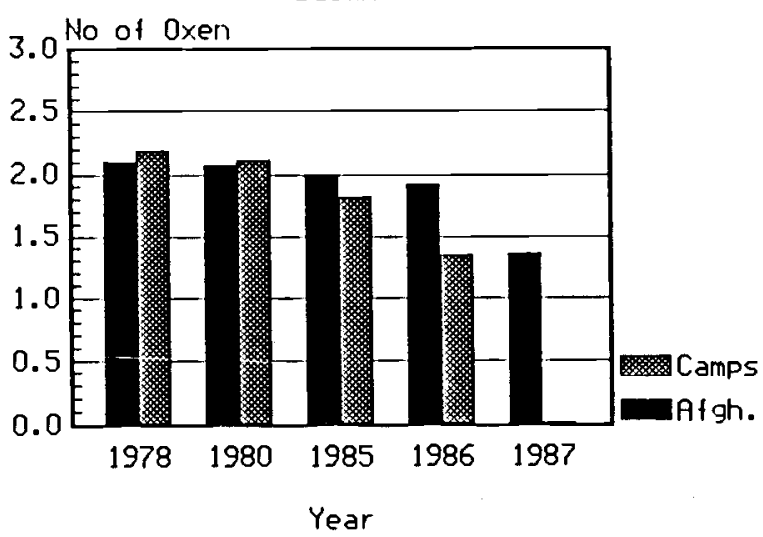

Balkh

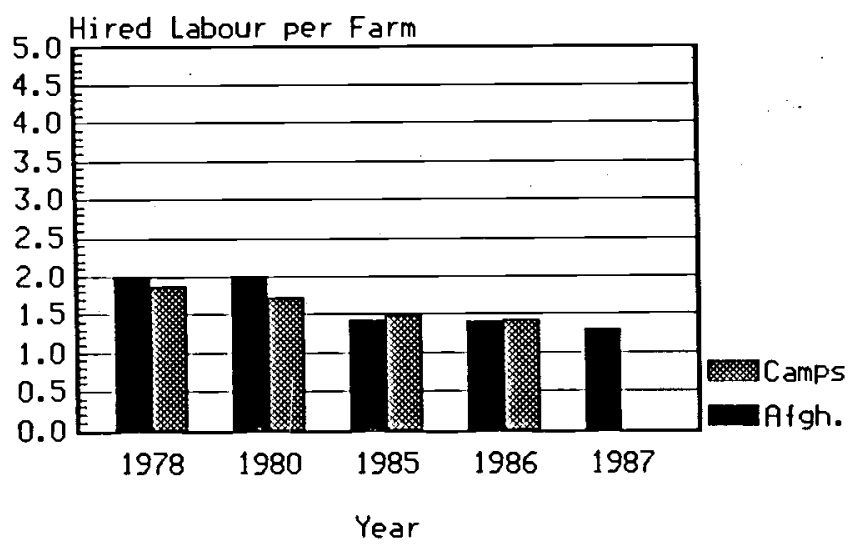

Balkh

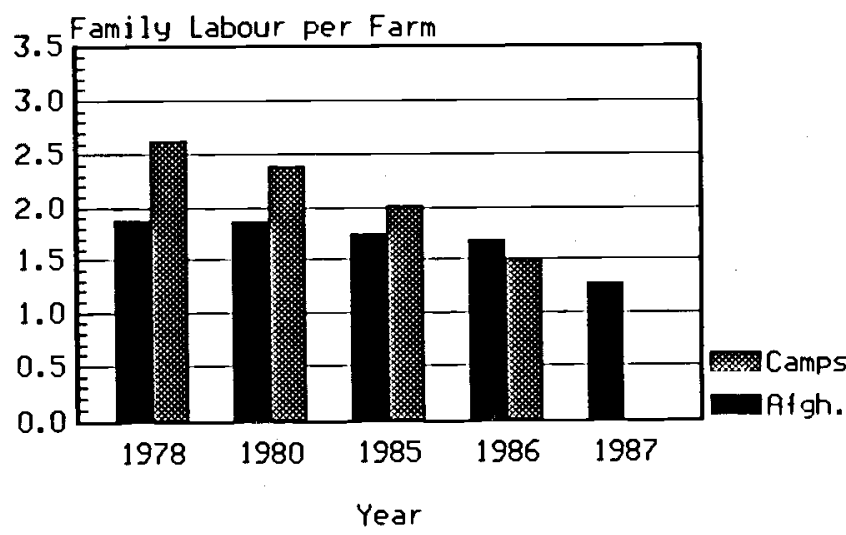

Balkh

Percentage of Farms using

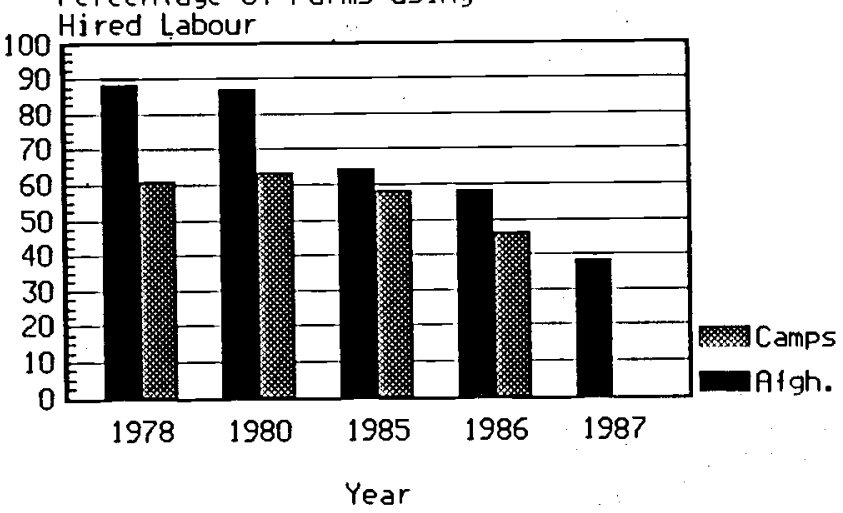


PROVINCE OF BLK

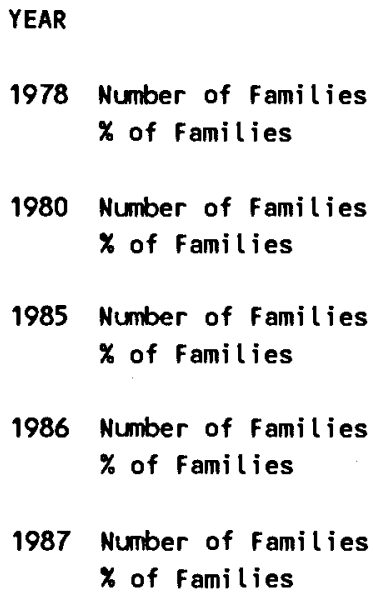

Number of Farmers Questioned 248

\section{TABLE 7B - AVERAGE NUMBER OF TRAINED OXEN FOR FARMERS IN THE CAMPS}

PROVINCE OF BLK

YEAR

1978 Number of Families

$\%$ of Families

1980 Number of Families

$\%$ of Families

1985 Number of Families $\%$ of Families

1986 Number of Families $\%$ of Families

1987 Number of Families * of Families

Average Number of Oxen per Family:

\begin{tabular}{|c|c|c|c|c|c|}
\hline \multicolumn{2}{|c|}{ NUMBER OF } & TRAINED & \multicolumn{3}{|c|}{ OXEN PER FAMILY } \\
\hline 0 & 1 & 2 & 3 & 4 & 5 \\
\hline 16 & 7 & 113 & 10 & 30 & 0 \\
\hline 9.09 & 3.98 & 64.20 & 5.68 & 17.05 & 0.00 \\
\hline 20 & 8 & 109 & 10 & 29 & 0 \\
\hline 11.36 & 4.55 & 61.93 & 5.68 & 16.48 & 0.00 \\
\hline 34 & 9 & 110 & 4 & 20 & 0 \\
\hline 19.21 & 5.08 & 62.15 & 2.26 & 11.30 & 0.00 \\
\hline 70 & 8 & 82 & 2 & 15 & 0 \\
\hline 39.55 & 4.52 & 46.33 & 1.13 & 8.47 & 0.00 \\
\hline 17 & 0 & 1 & 0 & 0 & 0 \\
\hline 99.44 & 0.00 & 0.56 & 0.00 & 0.00 & 0.00 \\
\hline
\end{tabular}

$\begin{array}{ll}1978 & 2.18 \\ 1980 & 2.11 \\ 1985 & 1.81 \\ 1986 & 1.34 \\ 1987 & 0.01\end{array}$


PROVINCE OF BLK

\begin{tabular}{|c|c|c|c|c|c|}
\hline & 1978 & 1980 & 1985 & 1986 & 1987 \\
\hline Average family Labour per Farm & 1.87 & 1.86 & 1.75 & 1.68 & 1.27 \\
\hline $\begin{array}{l}\text { Average No. of Hired Labour per farm } \\
\text { (for farms using hired labour) }\end{array}$ & 1.99 & 1.95 & 1.42 & 1.40 & 1.30 \\
\hline Number of Farms using Hired Labour & 217 & 215 & 159 & 145 & 95 \\
\hline
\end{tabular}

\section{IABLE $8(B)$ - AVERAGE LABOUR (FAMILY and HIRED) FOR FARMERS IN CAMPS}

PROVINCE OF BLK

\begin{tabular}{|c|c|c|c|c|c|}
\hline & 1978 & 1980 & 1985 & 1986 & 1987 \\
\hline Average Family Labour per farm & 2.62 & 2.39 & 2.01 & 1.50 & 0.01 \\
\hline $\begin{array}{l}\text { Average No. of Hired Labour per farm } \\
\text { (for farms using hired labour) }\end{array}$ & 1.86 & 1.73 & 1.49 & 1.42 & $\star \star \star \star \star$ \\
\hline Number of Farms using Hired Labour & 11.0 & 113 & 104 & 83 & 0 \\
\hline Number of Farmers Questioned 179 & & & & & \\
\hline
\end{tabular}


PROVINCE OF BLK

Average Number of Livestock Shot

Number of Farmers reporting

Livestock Shot

Average Number of Livestock Killed by Mines

Number of Farmers reporting Livestock

killed by Mines

Number of Farmers Questioned 247

$\begin{array}{lllll}1978 & 1980 & 1985 & 1986 & 1987 \\ \star \star \star \star \star & 34.67 & 8.70 & 3.94 & 9.90\end{array}$

$\begin{array}{lllll}0 & 3 & 20 & 16 & 10\end{array}$

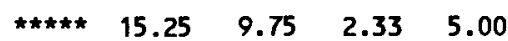

$\begin{array}{lllll}0 & 4 & 4 & 3 & 1\end{array}$

\section{IABLE 9B - DIRECT EFFECTS OF THE WAR - FARMERS IN CAMPS}

\section{PROVINCE OF BLK}

\begin{tabular}{|c|c|c|c|c|c|}
\hline & 1978 & 1980 & 1985 & 1986 & 1987 \\
\hline Average Number of Livestock Shot & 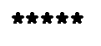 & 9.38 & 10.77 & 8.07 & 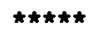 \\
\hline \multicolumn{6}{|l|}{ Number of Farmers reporting } \\
\hline Livestock Shot & 0 & 21 & 73 & 30 & 0 \\
\hline $\begin{array}{l}\text { Average Number of Livestock Killed } \\
\text { by Mines }\end{array}$ & 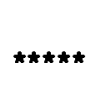 & 1.00 & 2.81 & 2.54 & 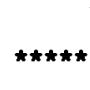 \\
\hline \multicolumn{6}{|l|}{ Number of Farmers reporting $L$ ivestock } \\
\hline killed by Mines & 0 & 1 & 21 & 13 & 0 \\
\hline
\end{tabular}




\begin{tabular}{|c|c|}
\hline$\underline{\underline{\mathbf{g}}}$ & 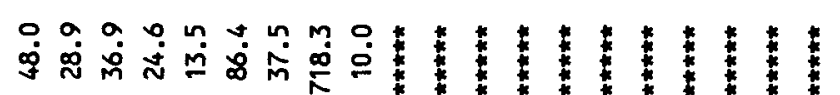 \\
\hline $0 \stackrel{8}{8}$ & 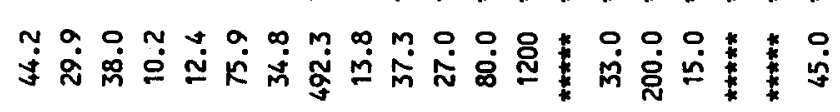 \\
\hline & 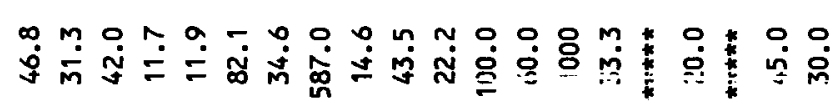 \\
\hline & 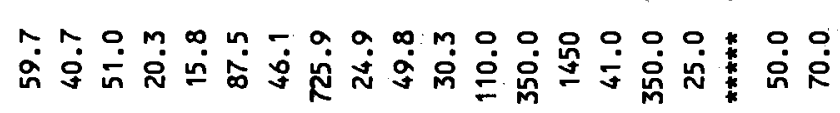 \\
\hline$\stackrel{\infty}{\varrho}$ & 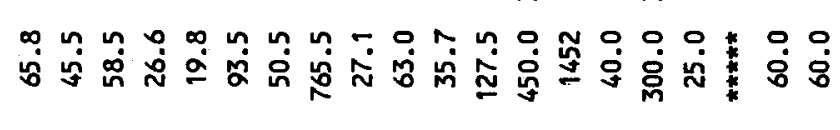 \\
\hline कీ & 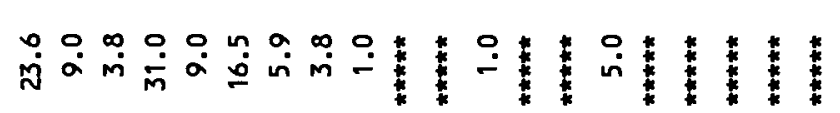 \\
\hline & 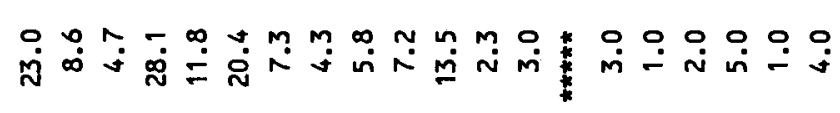 \\
\hline & 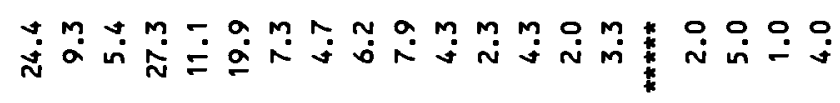 \\
\hline & 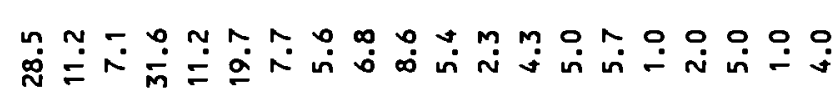 \\
\hline$\widehat{\sigma}$ & 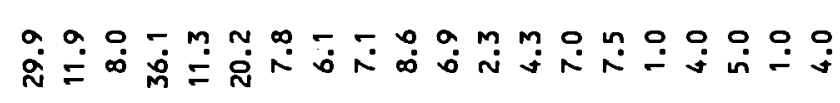 \\
\hline
\end{tabular}

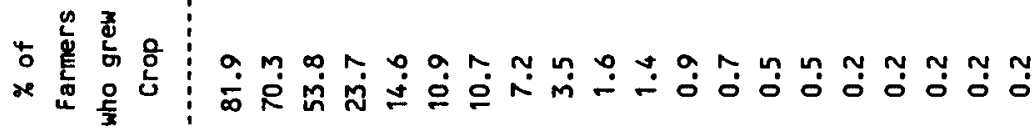

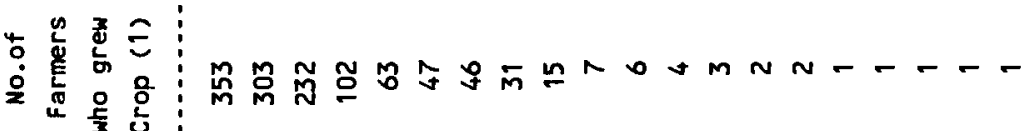

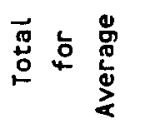

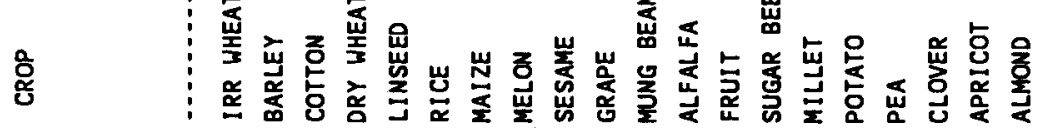


PROVINCE OF BLK

\section{PERCENTAGE OF VILLAGES GIVING FOLLOWING PRIORITY TO PROBLEM}

Rainfall

Crop Disease

Birds or Rats

Seed Availability

Fertilizer Availability

Crop Protection Chemicals

Credit

Irrigation Water Availability

Flooding

Labour Availabilty

Power for Land Preparation

Direct War Effects

Animal Diseases

Extension

Other Problems

\begin{tabular}{rrrrrrrrrrrrr}
1 & 2 & 3 & 4 & 5 & 6 & 7 & 8 & 9 & 10 & 11 & 12 & Weighted Priority \\
\hline 0 & 1 & 0 & 1 & 0 & 0 & 0 & 0 & 0 & 0 & 0 & 0 & 0.38 \\
1 & 3 & 18 & 33 & 15 & 5 & 1 & 0 & 0 & 0 & 0 & 0 & 9.42 \\
0 & 3 & 13 & 8 & 8 & 1 & 0 & 0 & 0 & 0 & 0 & 0 & 4.32 \\
0 & 1 & 21 & 14 & 10 & 1 & 1 & 0 & 0 & 0 & 0 & 0 & 5.92 \\
0 & 2 & 5 & 0 & 0 & 0 & 0 & 0 & 0 & 0 & 0 & 0 & 1.19 \\
0 & 0 & 0 & 0 & 2 & 2 & 0 & 0 & 0 & 0 & 0 & 0 & 0.37 \\
0 & 1 & 3 & 2 & 1 & 0 & 0 & 0 & 0 & 0 & 0 & 0 & 1.12 \\
8 & 25 & 1 & 0 & 1 & 0 & 0 & 0 & 0 & 0 & 0 & 0 & 9.44 \\
0 & 0 & 0 & 0 & 1 & 0 & 1 & 0 & 0 & 0 & 0 & 0 & 0.17 \\
0 & 33 & 24 & 14 & 14 & 3 & 0 & 1 & 0 & 0 & 0 & 0 & 14.02 \\
1 & 15 & 2 & 1 & 0 & 0 & 0 & 0 & 0 & 0 & 0 & 0 & 4.29 \\
89 & 10 & 0 & 0 & 0 & 0 & 0 & 0 & 0 & 0 & 0 & 0 & 41.52 \\
0 & 1 & 10 & 14 & 14 & 3 & 0 & 0 & 0 & 0 & 0 & 0 & 4.79 \\
0 & 1 & 2 & 9 & 13 & 2 & 0 & 0 & 0 & 0 & 0 & 0 & 2.90 \\
0 & 0 & 0 & 1 & 0 & 0 & 0 & 0 & 0 & 0 & 0 & 0 & 0.13
\end{tabular}

1986

Rainfall

Crop Disease

Birds or Rats

Seed Availability

Fertilizer Availability

Crop Protection Chemicals

Credit

Irrigation Water Availability

flooding

Labour Availabilty

Power for Land Preparation

Direct War Effects

Animal Diseases

Extension

other Problems

\section{5}

Rainfall

Crop Disease

Birds or Rats

Seed Availability

Fertilizer Availability

Crop Protection Chemicals

Credit

Irrigation Water Availability

Flooding

Labour Availabilty

Power for Land Preparation

Direct War Effects

Animal Diseases

Extension

Other Problems

$\begin{array}{rrrrrrrrrrrrr}1 & 2 & 3 & 4 & 5 & 6 & 7 & 8 & 9 & 10 & 11 & 12 & \text { Heighted Priority } \\ 23 & 33 & 2 & 1 & 3 & 0 & 0 & 0 & 0 & 0 & 0 & 0 & 18.88 \\ 0 & 0 & 11 & 9 & 3 & 3 & 0 & 0 & 0 & 0 & 0 & 0 & 3.37 \\ 0 & 2 & 8 & 17 & 14 & 0 & 0 & 0 & 0 & 0 & 0 & 0 & 4.97 \\ 0 & 1 & 29 & 9 & 1 & 5 & 1 & 0 & 0 & 0 & 0 & 0 & 6.21 \\ 0 & 0 & 7 & 2 & 0 & 1 & 0 & 0 & 0 & 0 & 0 & 0 & 1.40 \\ 0 & 1 & 0 & 1 & 1 & 0 & 0 & 0 & 0 & 0 & 0 & 0 & 0.50 \\ 0 & 1 & 2 & 1 & 1 & 0 & 0 & 0 & 0 & 0 & 0 & 0 & 0.85 \\ 0 & 1 & 3 & 1 & 1 & 0 & 0 & 0 & 0 & 0 & 0 & 0 & 1.02 \\ 0 & 0 & 0 & 0 & 1 & 0 & 0 & 0 & 0 & 0 & 0 & 0 & 0.10 \\ 0 & 34 & 23 & 15 & 16 & 3 & 0 & 1 & 0 & 0 & 0 & 0 & 14.86 \\ 0 & 5 & 3 & 2 & 0 & 0 & 0 & 0 & 0 & 0 & 0 & 0 & 1.84 \\ 77 & 21 & 2 & 0 & 0 & 0 & 0 & 0 & 0 & 0 & 0 & 0 & 40.20 \\ 0 & 1 & 5 & 11 & 6 & 2 & 0 & 0 & 0 & 0 & 0 & 0 & 2.97 \\ 0 & 1 & 3 & 8 & 8 & 3 & 0 & 0 & 0 & 0 & 0 & 0 & 2.70 \\ 0 & 0 & 0 & 1 & 0 & 0 & 0 & 0 & 0 & 0 & 0 & 0 & 0.13\end{array}$

$\begin{array}{rrrrrrrrrrrrr}1 & 2 & 3 & 4 & 5 & 6 & 7 & 8 & 9 & 10 & 11 & 12 & \text { Weighted Priority } \\ 21 & 33 & 2 & 3 & 6 & 0 & 0 & 0 & 0 & 0 & 0 & 0 & 18.60 \\ 0 & 1 & 11 & 9 & 7 & 2 & 0 & 0 & 0 & 0 & 0 & 0 & 3.92 \\ 0 & 0 & 7 & 18 & 8 & 1 & 1 & 0 & 0 & 0 & 0 & 0 & 4.11 \\ 0 & 1 & 31 & 7 & 5 & 0 & 1 & 0 & 0 & 0 & 0 & 0 & 6.36 \\ 0 & 1 & 2 & 3 & 0 & 0 & 0 & 0 & 0 & 0 & 0 & 0 & 1.02 \\ 0 & 1 & 0 & 0 & 0 & 0 & 0 & 0 & 0 & 0 & 0 & 0 & 0.27 \\ 0 & 1 & 2 & 2 & 0 & 1 & 0 & 0 & 0 & 0 & 0 & 0 & 0.98 \\ 1 & 2 & 3 & 0 & 1 & 1 & 0 & 0 & 0 & 0 & 0 & 0 & 1.79 \\ 0 & 0 & 0 & 0 & 1 & 0 & 0 & 0 & 0 & 0 & 0 & 0 & 0.11 \\ 0 & 34 & 23 & 15 & 7 & 3 & 0 & 1 & 0 & 0 & 0 & 0 & 14.24 \\ 0 & 3 & 6 & 1 & 0 & 0 & 0 & 0 & 0 & 0 & 0 & 0 & 1.82 \\ 78 & 21 & 1 & 0 & 0 & 0 & 0 & 0 & 0 & 0 & 0 & 0 & 41.19 \\ 0 & 0 & 8 & 10 & 1 & 1 & 0 & 0 & 0 & 0 & 0 & 0 & 2.64 \\ 0 & 0 & 1 & 7 & 16 & 5 & 0 & 0 & 0 & 0 & 0 & 0 & 2.82 \\ 0 & 0 & 0 & 1 & 0 & 0 & 0 & 0 & 0 & 0 & 0 & 0 & 0.13\end{array}$


IABLE 1 -5 (contd) - ANALYSIS OF GREATEST FARMING PROBLEMS - COMMUNITY SURVEY

PROVINCE OF BLK

$\underline{1980}$

PERCENTAGE OF VILLAGES GIVING FOLLOWING PRIORITY TO PROBLEM

Rainfall

Crop Disease

Birds or Rats

Seed Availability

Fertilizer Availability

Crop Protection Chemicals

Credit

Irrigation Water Availability

Flooding

Labour Availabilty

Power for Land Preparation

Direct War Effects

Animal Diseases

Extension

Other Problems

$\begin{array}{rrrrrrrrrrrrc}1 & 2 & 3 & 4 & 5 & 6 & 7 & 8 & 9 & 10 & 11 & 12 & \text { Weighted Priority } \\ 10 & 15 & 1 & 0 & 0 & 0 & 0 & 0 & 0 & 0 & 0 & 0 & 11.79 \\ 0 & 1 & 8 & 8 & 2 & 0 & 0 & 0 & 0 & 0 & 0 & 0 & 3.71 \\ 0 & 9 & 6 & 0 & 1 & 0 & 0 & 0 & 0 & 0 & 0 & 0 & 4.37 \\ 0 & 1 & 2 & 1 & 0 & 0 & 1 & 0 & 0 & 0 & 0 & 0 & 1.16 \\ 0 & 1 & 2 & 0 & 0 & 0 & 0 & 0 & 0 & 0 & 0 & 0 & 0.87 \\ 0 & 0 & 0 & 0 & 0 & 0 & 0 & 0 & 0 & 0 & 0 & 0 & 0.00 \\ 0 & 0 & 2 & 2 & 0 & 0 & 0 & 0 & 0 & 0 & 0 & 0 & 0.87 \\ 1 & 6 & 0 & 0 & 0 & 0 & 0 & 0 & 0 & 0 & 0 & 0 & 2.61 \\ 0 & 0 & 0 & 0 & 1 & 0 & 0 & 0 & 0 & 0 & 0 & 0 & 0.15 \\ 0 & 7 & 9 & 3 & 6 & 0 & 0 & 1 & 0 & 0 & 0 & 0 & 5.61 \\ 0 & 6 & 2 & 1 & 0 & 0 & 0 & 0 & 0 & 0 & 0 & 0 & 2.54 \\ 84 & 10 & 0 & 0 & 0 & 0 & 0 & 0 & 0 & 0 & 0 & 0 & 57.69 \\ 0 & 1 & 0 & 7 & 1 & 1 & 0 & 0 & 0 & 0 & 0 & 0 & 1.76 \\ 0 & 7 & 18 & 2 & 2 & 0 & 0 & 0 & 0 & 0 & 0 & 0 & 6.87 \\ 0 & 0 & 0 & 0 & 0 & 0 & 0 & 0 & 0 & 0 & 0 & 0 & 0.00\end{array}$

\section{$\underline{1978}$}

Rainfall

Crop Disease

Birds or Rats

Seed Availability

Fertilizer Availability

Crop Protection Chemicals

Credit

Irrigation Water Availability

Flooding

Labour Availabilty

Power for Land Preparation

Direct Har Effects

Animal Diseases

Extension

other Problems

\begin{tabular}{rrllllllllllc}
1 & 2 & 3 & 4 & 5 & 6 & 7 & 8 & 9 & 10 & 11 & 12 & Weighted Priority \\
\hline 0 & 0 & 0 & 0 & 0 & 0 & 0 & 0 & 0 & 0 & 0 & 0 & 0.00 \\
1 & 0 & 0 & 0 & 0 & 0 & 0 & 0 & 0 & 0 & 0 & 0 & 8.39 \\
0 & 0 & 1 & 0 & 0 & 0 & 0 & 0 & 0 & 0 & 0 & 0 & 2.80 \\
0 & 0 & 1 & 0 & 0 & 0 & 0 & 0 & 0 & 0 & 0 & 0 & 2.80 \\
0 & 0 & 0 & 0 & 0 & 0 & 0 & 0 & 0 & 0 & 0 & 0 & 0.00 \\
0 & 0 & 0 & 0 & 0 & 0 & 0 & 0 & 0 & 0 & 0 & 0 & 0.00 \\
1 & 1 & 0 & 1 & 0 & 0 & 0 & 0 & 0 & 0 & 0 & 0 & 14.69 \\
5 & 0 & 0 & 0 & 0 & 0 & 0 & 0 & 0 & 0 & 0 & 0 & 33.57 \\
0 & 0 & 0 & 0 & 0 & 0 & 0 & 0 & 0 & 0 & 0 & 0 & 0.00 \\
0 & 0 & 0 & 0 & 0 & 0 & 0 & 0 & 0 & 0 & 0 & 0 & 0.00 \\
0 & 0 & 0 & 0 & 0 & 0 & 0 & 0 & 0 & 0 & 0 & 0 & 0.00 \\
1 & 0 & 0 & 0 & 0 & 0 & 0 & 0 & 0 & 0 & 0 & 0 & 8.39 \\
0 & 0 & 0 & 0 & 0 & 0 & 0 & 0 & 0 & 0 & 0 & 0 & 0.00 \\
0 & 8 & 0 & 0 & 0 & 0 & 0 & 0 & 0 & 0 & 0 & 0 & 29.37 \\
0 & 0 & 0 & 0 & 0 & 0 & 0 & 0 & 0 & 0 & 0 & 0 & 0.00
\end{tabular}




\section{BADGHIS}




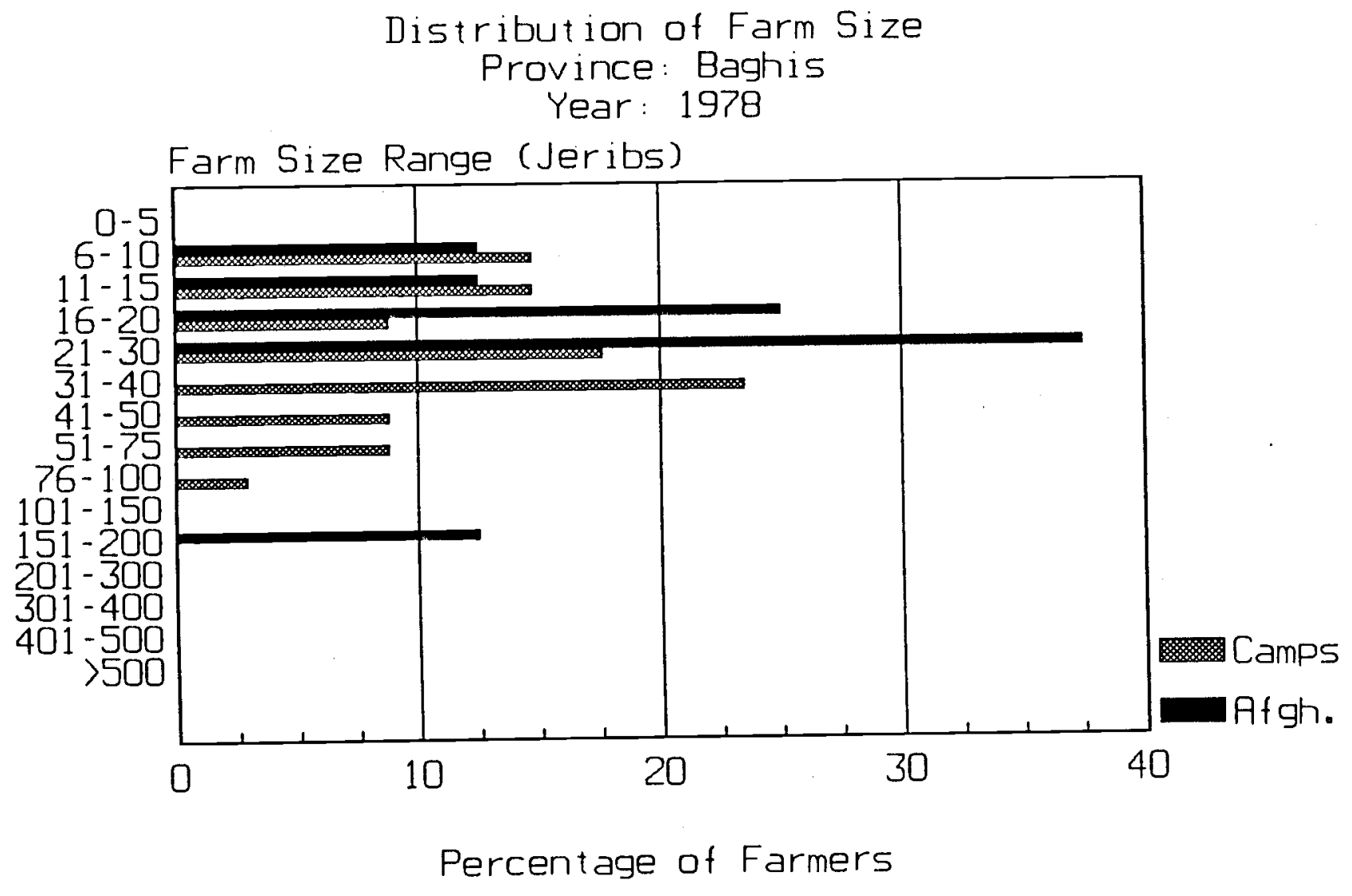

Farmers in Afghanistan

1978

1987

Total Number of Farms

8

Average Area

36.63

8

Average Area Irrigated

11.00

36.63

Average Dryland Area

30.86

10.71

24.86

Farmers who left in 1987

Total Number of Farms

Average Area

Average Area Irrigated

Average Dryland Area

34

30.99

17.98

26.70

32.06

16.45

27.67
36.63

10.71

24.86

(A11 areas in Jeribs. 5 Jeribs $=1$ Hectare) 
Farm Power - Farmers in Atghanistan Province of Baghis

Crop: Irrigated wheat

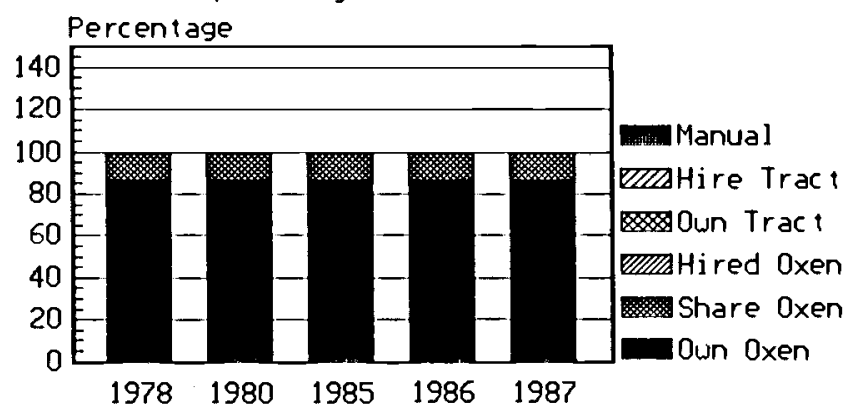

Year

Farm Power - Farmers who lett in 1987 Province of Baghis

Crop: Irrigated wheat

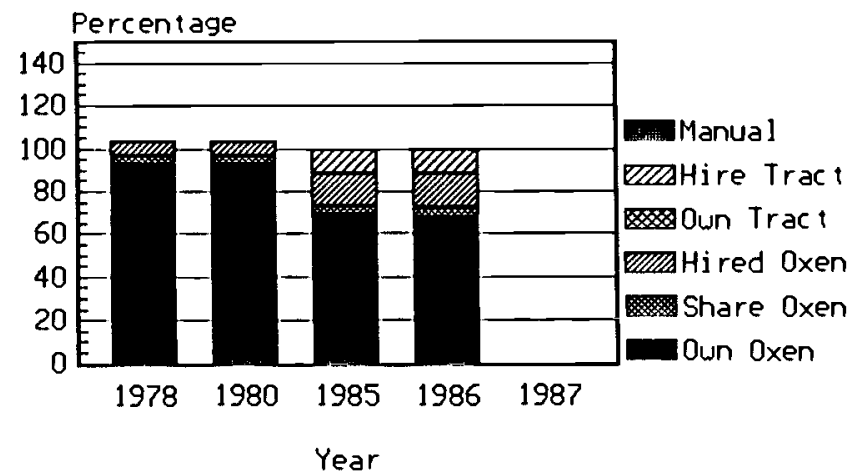

Base Fiqures for above graphs

Province: Baghis

Farmers in Afghanistan

Irr. Wheat own oxen Shared Oxen Hired Oxen Own Tractor Hired Tractor

Barley Manual Means Own Oxen Shared oxen Hired Oxen Own Tractor Hired Tractor Manual Means

Farmers who left in 1987

Irr. Wheat own oxen

Shared oxen

Hired oxen

Own Tractor

Hired Tractor

Barley

Manual Means

Own Oxen

Shared oxen

Hired oxen

Own Tractor

rimat masntav

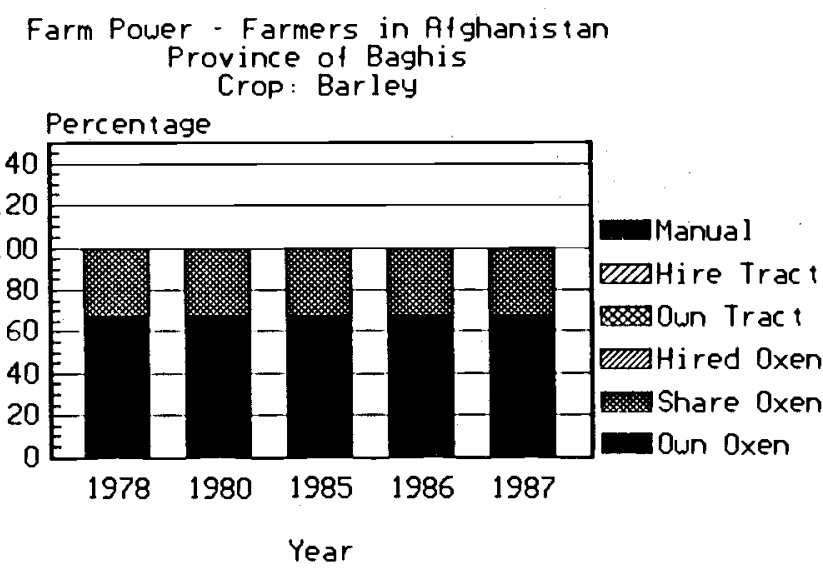

Farm Power - Farmers who lett in 1987 Province of Baghis Crop: Barley

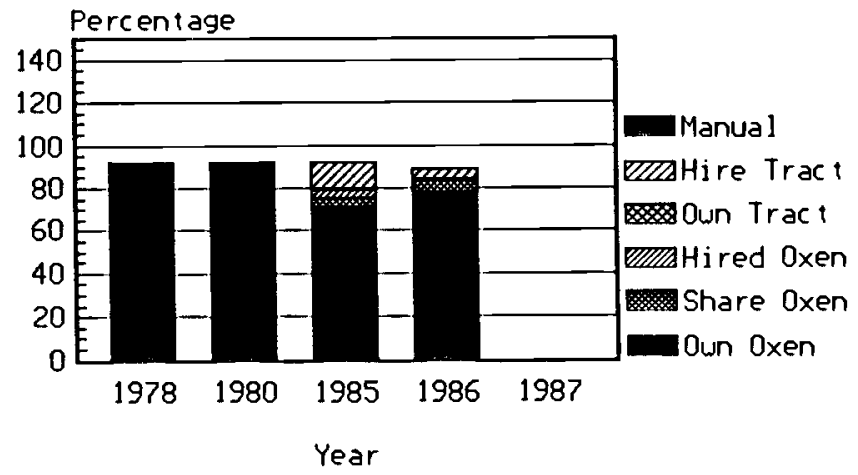

Percentage of Farmers
$1978 \underline{1980 \quad 1985 \quad \underline{1986}} \quad 1987$

$\begin{array}{ccccc}85.71 & 85.71 & 85.71 & 85.71 & 85.71 \\ 14.29 & 14.29 & 14.29 & 14.29 & 14.29 \\ - & - & - & - & - \\ - & - & - & - & - \\ - & - & - & - & - \\ 66.67 & 66.67 & 66.67 & 66.67 & 66.67 \\ 33.33 & 33.33 & 33.33 & 33.33 & 33.33 \\ - & - & - & - & - \\ - & - & - & - & - \\ - & - & - & - & - \\ - & - & - & - & -\end{array}$

68.00

4.00

16.00

12.00

77.78

5.56

$-$

$-$ 
Badghis

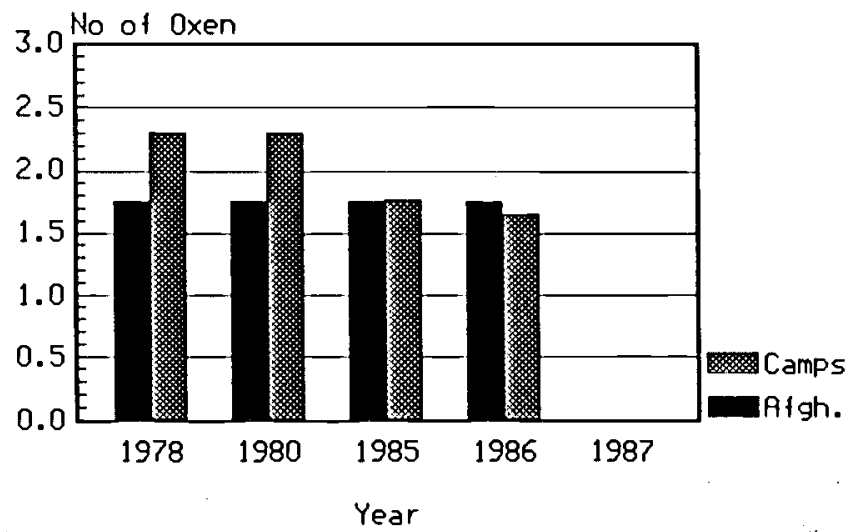

Badghis

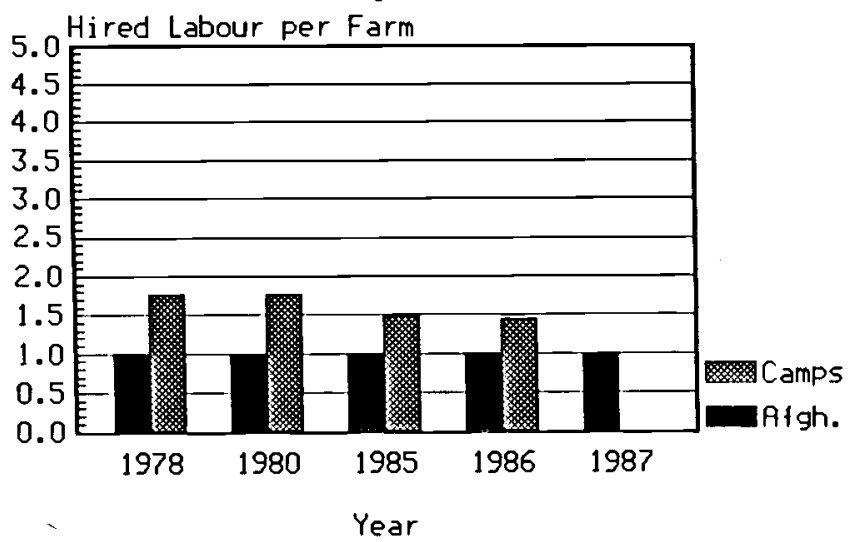

Badghis

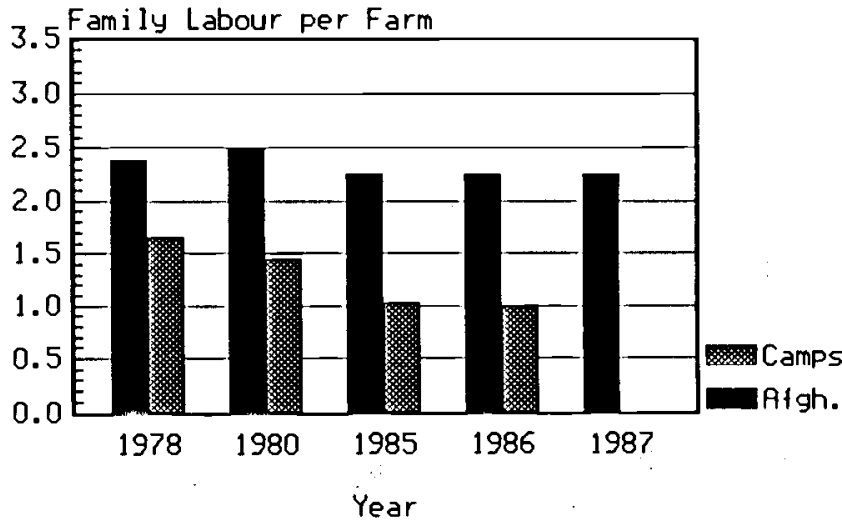

Badghis

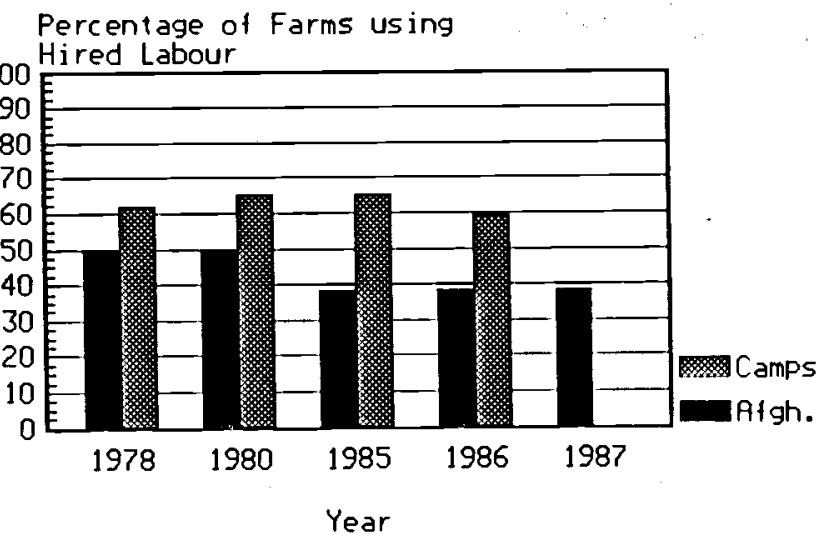


PROVINCE OF BGS

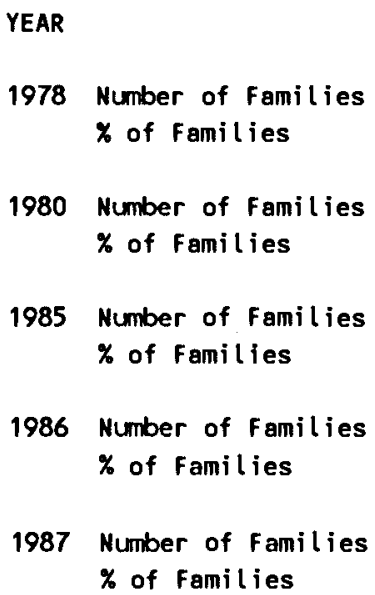

$\begin{array}{llllll}0 & 2 & 6 & 0 & 0 & 0\end{array}$

$\begin{array}{llllll}0.00 & 25.00 & 75.00 & 0.00 & 0.00 & 0.00\end{array}$

Number of Farmers Questioned 8

IABLE 7B - AVERAGE NUMBER OF TRAINED OXEN FOR FARMERS IN THE CAMPS

PROVINCE OF BGS

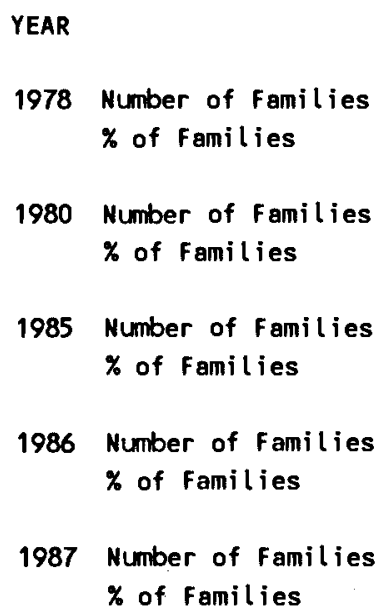


PROVINCE OF BGS

\begin{tabular}{|c|c|c|c|c|c|}
\hline & 1978 & 1980 & 1985 & 1986 & 1987 \\
\hline Average Family Labour per Farm & 2.38 & 2.50 & 2.25 & 2.25 & 2.25 \\
\hline $\begin{array}{l}\text { Average No. of Hired Labour per Farm } \\
\text { (for farms using hired labour) }\end{array}$ & 1.00 & 1.00 & 1.00 & 1.00 & 1.00 \\
\hline Number of Farms using Hired Labour. & 4 & 4 & 3 & 3 & 3 \\
\hline
\end{tabular}

IABLE $8(B)$ - AVERAGE LABOUR (FAMILY and HIRED) FOR FARMERS IN CAMPS

PROVINCE OF BGS

\begin{tabular}{|c|c|c|c|c|c|}
\hline & 1978 & 1980 & 1985 & 1986 & 1987 \\
\hline Average Family Labour per Farm & 1.65 & 1.44 & 1.03 & 1.00 & 0.00 \\
\hline $\begin{array}{l}\text { Average No. of Hired Labour per Farm } \\
\text { (for farms using hired labour) }\end{array}$ & 1.76 & 1.77 & 1.49 & 1.45 & $\star \star \star \star \star * *$ \\
\hline Number of Farms using Hired Labour & 21 & 22 & 22 & 20 & 0 \\
\hline
\end{tabular}


IABLE 9A - DIRECT EFFECTS OF THE WAR - FARMERS IN AFGANISTAN

PROVINCE OF BGS

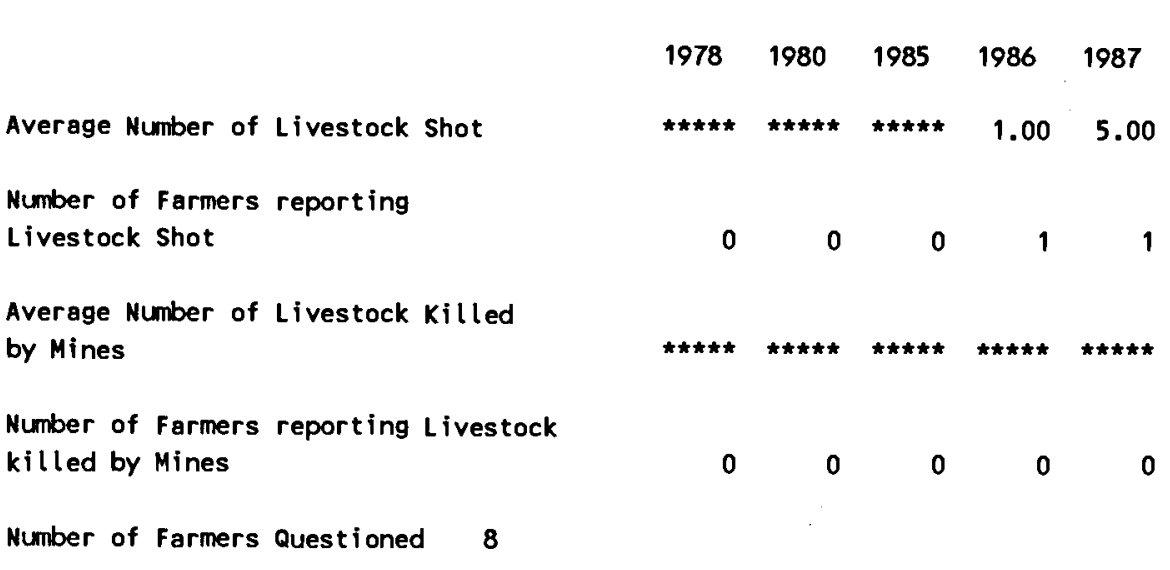

TABLE 9B - DIRECT EFFECTS OF THE WAR - FARMERS IN CAMPS

PROVINCE OF BGS

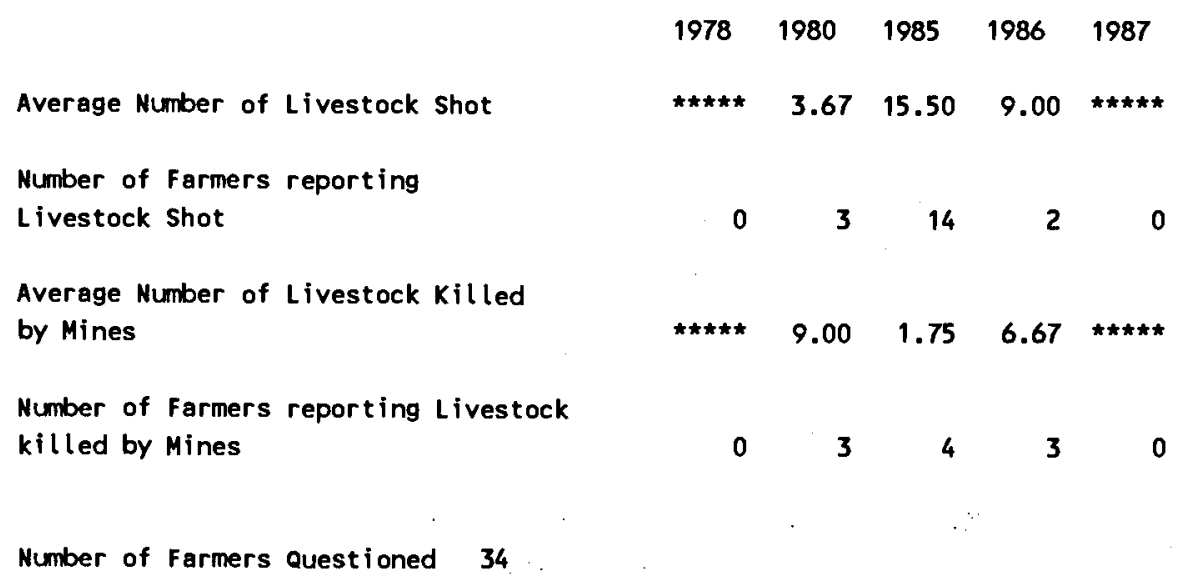




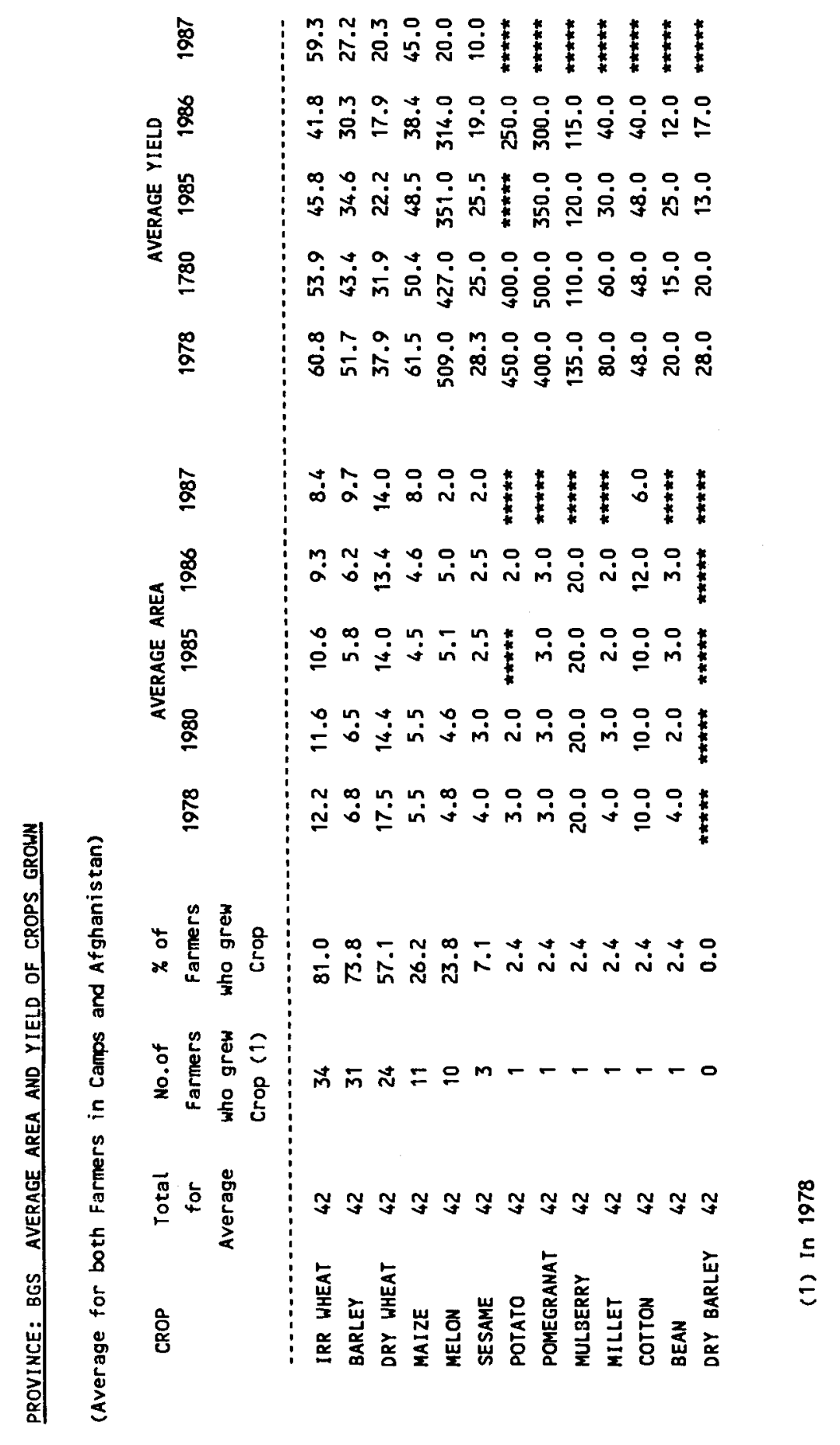


PROVINCE OF BGS

Rainfall

Crop Disease

Birds or Rats

Seed Availability

Fertilizer Availability

Crop Protection Chemicals

Credit

Irrigation Hater Availability

Flooding

Labour Availabilty

Power for Land Preparation

Direct War Effects

Animal Diseases

Extension

Other Problems

\section{6}

Rainfall

Crop Disease

Birds or Rats

Seed Availability

Fertilizer Availability

Crop Protection Chemicals

Credit

Irrigation Water Availability

Flooding

Labour Availabilty

Power for Land Preparation

Direct War Effects

Animal Diseases

Extension

Other Problems

$\underline{1985}$

\section{Rainfall}

Crop Disease

Birds or Rats

Seed Availability

Fertilizer Availability

Crop Protection Chemicals

Credit

Irrigation Water Availability

Flooding

Labour Availabilty

Power for Land Preparation

Direct Har Effects

Animal Diseases

Extension

Other Problems

\begin{tabular}{|c|c|c|c|c|c|c|c|c|c|c|c|c|}
\hline 1 & 2 & 3 & 4 & 5 & 6 & 7 & 8 & 9 & 10 & 11 & 12 & Weighted \\
\hline ** & $\star \star * *$ & $\star \star \star *$ & $\star \star \star$ & $\star \star \star$ & 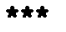 & $\star \star \star$ & $\star \star \star *$ & $\star \star \star *$ & $\star \star \star \star$ & $\star \star \star$ & $\star \star \star \star$ & $\star \star \star \star * * *$ \\
\hline 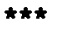 & $\star \star \star$ & $\star \star \star$ & $\star \star \star$ & $\star \star \star$ & $\star \star \star$ & $\star \star \star$ & $\star \star \star$ & $\star \star \star$ & $\star \star \star$ & $\star \star \star$ & $\star \star \star$ & $\star \star \star \star \star \star$ \\
\hline$* *$ & $\star \star \star *$ & $\star \star \star \star$ & $\star \star \star$ & $\star \star \star$ & $\star \star \star$ & $\star \star \star$ & $\star \star \star$ & $\star \star \star$ & $\star \star \star$ & $\star \star \star \star$ & $\star \star \star$ & $\star \star \star \star \star \star$ \\
\hline$* *$ & $\star \star \star$ & $\star \star \star$ & $\star \star \star$ & $\star \star \star \star$ & $\star \star \star$ & $\star \star \star$ & $\star \star \star$ & $\star \star \star$ & $\star \star \star$ & $\star \star \star \star$ & $\star \star \star$ & $\star \star \star \star \star$ \\
\hline$\star \star \star \star *$ & $\star \star \star *$ & 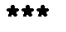 & $\star \star \star$ & $\star \star \star$ & $\star \star \star$ & $\star \star \star$ & $\star \star \star$ & $\star \star \star$ & $\star \star \star \star$ & $\star \star \star \star$ & $\star \star \star *$ & 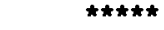 \\
\hline$* \star *$ & $\star \star \star *$ & $\star \star \star \star$ & $\star \star *$ & $\star \star \star *$ & 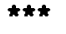 & $\star \star \star$ & $\star \star \star$ & $\star \star \star$ & $\star \star \star$ & $\star \star \star$ & $\star \star \star$ & $\star \star \star \star \star \star$ \\
\hline$\star \star \star$ & $\star \star \star \star$ & 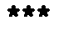 & $\star \star \star$ & $\star \star \star$ & $\star \star \star$ & $\star \star \star$ & $\star \star \star$ & $\star \star \star$ & $\star \star \star \star$ & $\star \star \star *$ & $\star \star \star$ & 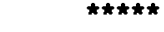 \\
\hline$* \star \star$ & $\star \star \star *$ & $\star \star \star *$ & $\star \star \star \star$ & $\star \star \star \star *$ & $\star \star \star$ & $\star \star \star$ & $\star \star \star$ & $\star \star \star$ & $\star \star \star$ & $\star \star \star$ & $\star \star \star$ & $\star \star \star \star \star \star$ \\
\hline$\star \star$ & $\star \star \star$ & $\star \star \star$ & $\star \star \star$ & $\star \star \star \star$ & $\star \star \star \star$ & $\star \star \star$ & $\star \star \star \star$ & $\star \star \star$ & $\star \star \star$ & $\star \star \star$ & $\star \star \star \star$ & $\star \star \star \star \star \star$ \\
\hline$\star \star \star \star$ & $\star \star \star *$ & $\star \star \star \star$ & 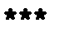 & $\star \star \star \star$ & $\star \star \star$ & $\star \star \star$ & $\star \star \star \star$ & $\star \star * *$ & $\star \star * *$ & $\star \star \star \star *$ & 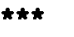 & 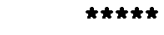 \\
\hline$\star \star \star$ & $\star \star \star$ & $\star \star \star \star$ & $\star \star \star \star$ & $\star \star \star \star$ & $\star \star \star \star$ & 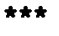 & $\star \star \star \star *$ & $\star \star \star \star$ & $\star \star \star \star$ & $\star \star \star \star$ & $\star \star \star \star$ & 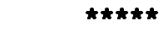 \\
\hline$* \star \star$ & $\star \star \star$ & $\star \star \star$ & 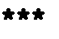 & 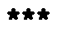 & $\star \star \star$ & $\star \star \star \star$ & $\star \star \star \star$ & $\star \star \star \star$ & $\star \star \star \star$ & $\star \star \star \star$ & $\star \star \star \star$ & 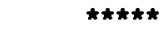 \\
\hline$\star$ & $\star \star \star *$ & $\star \star \star \star$ & $\star \star \star \star$ & $\star \star \star \star$ & $\star \star \star \star$ & 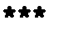 & $\star \star \star$ & $\star \star \star \star$ & $\star \star \star \star$ & 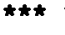 & 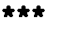 & $\star \star \star \star \star * *$ \\
\hline$r k t$ & $\star \star \star$ & $\star \star \star$ & $\star \star \star$ & $\star \star \star$ & $\star \star \star \star$ & $\star \star \star \star$ & $\star \star \star \star *$ & 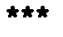 & $\star \star \star \star$ & $\star \star \star \star$ & $\star \star \star$ & $\star \star \star \star \star \star$ \\
\hline$* \star \star *$ & $\star \star \star$ & $\star \star \star$ & $\star \star \star$ & 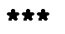 & $\star \star \star$ & $\star \star \star$ & $\star \star \star$ & $\star \star \star *$ & $\star \star \star \star$ & 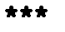 & $\star \star \star \star$ & 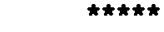 \\
\hline
\end{tabular}

$\begin{array}{llllllllllll}1 & 2 & 3 & 4 & 5 & 6 & 7 & 8 & 9 & 10 & 11 & 12\end{array}$ Weighted Priority

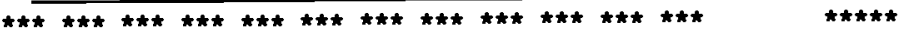

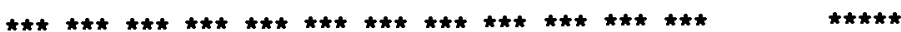

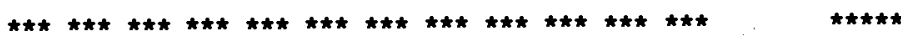

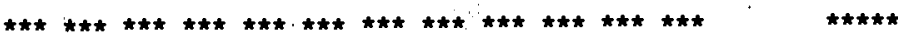

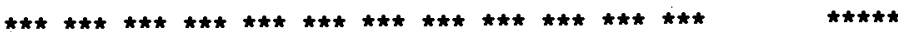

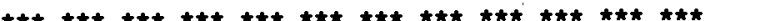

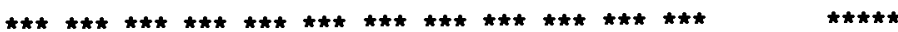

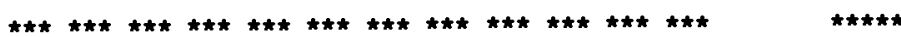

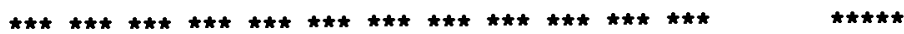

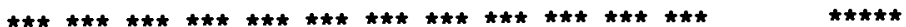

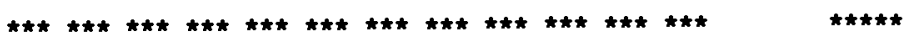

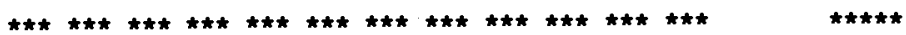

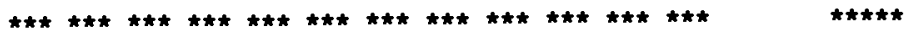

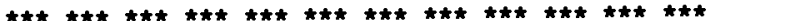

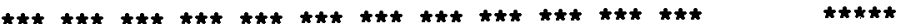

$\begin{array}{lllllllllllll}1 & 2 & 3 & 4 & 5 & 6 & 7 & 8 & 9 & 10 & 11 & 12 & \text { Heighted Priority }\end{array}$

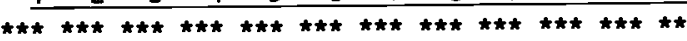

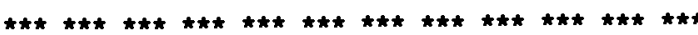

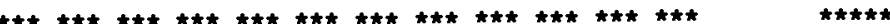

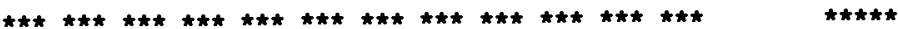

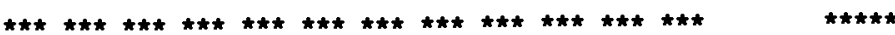

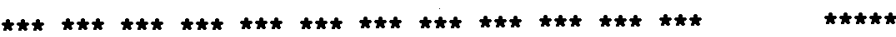

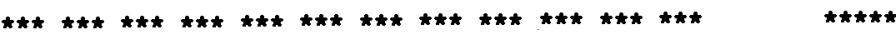

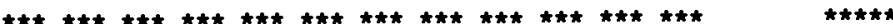

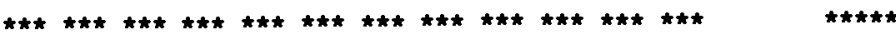

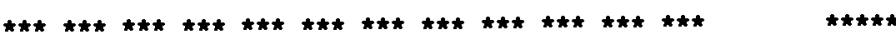

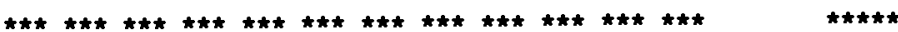

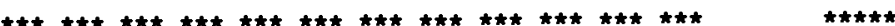

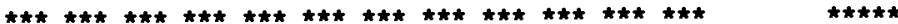

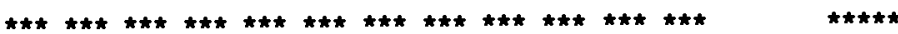

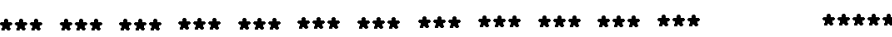


PROVINCE OF BGS

1980 PERCENTAGE OF VILLAGES GIVING FOLLOWING PRIORITY TO PROBLEM

Rainfall

Crop Oisease

Birds or Rats

Seed Availability

Fertilizer Availability

Crop Protection Chemicals

Credit

Irrigation Water Availability

Flooding

Labour Availabilty

0 irect War Effects

Animal 0 iseases

Extension

other Problems
Power for Land Preparation

\begin{tabular}{|c|c|c|c|c|c|c|c|c|c|c|c|c|}
\hline 1 & 2 & 3 & 4 & 5 & 6 & 7 & 8 & 9 & 10 & 11 & 12 & Weighted Priority \\
\hline$\star \star \star$ & $\star \star \star$ & $\star \star \star$ & $\star \star \star$ & $\star \star \star$ & $\star \star \star$ & $\star \star \star \star$ & $\star \star \star$ & $\star \star \star$ & $\star \star \star$ & $\star \star \star \star$ & $\star \star \star$ & 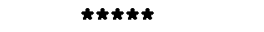 \\
\hline$\star \star \star \star$ & $\star \star \star *$ & $\star \star \star$ & $\star \star \star$ & $\star \star \star$ & $\star \star \star$ & $\star \star \star \star \Delta$ & $\star \star \star \star$ & $\star \star \star$ & $\star \star \star \star$ & $\star \star \star *$ & $\star \star \star$ & $\star \star \star \star \star *$ \\
\hline$\star \star * *$ & $\star \star \star *$ & $\star \star \star$ & $\star \star \star$ & $\star \star \star$ & $\star \star \star$ & $\star \star \star$ & $\star \star \star$ & $\star \star \star$ & $\star \star \star$ & $\star \star \star$ & $\star \star \star$ & 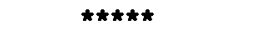 \\
\hline$\star \star \star *$ & $\star \star \star$ & $\star \star \star$ & $\star \star \star$ & $\star \star \star$ & $\star \star \star$ & $\star \star \star$ & $\star \star \star$ & $\star \star \star$ & $\star \star \star$ & $\star \star \star$ & $\star \star \star$ & 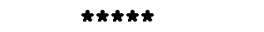 \\
\hline$* * *$ & $\star \star *$ & $\star \star \star$ & $\star \star \star$ & $\star \star \star$ & $\star \star \star$ & $\star \star \star$ & $\star \star \star$ & $\star \star \star$ & $\star \star \star$ & $\star \star \star *$ & $\star \star \star$ & $\star \star \star \star \star$ \\
\hline$\star \star \star \star$ & $\star \star \star \star$ & 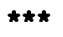 & $\star \star \star \star$ & $\star \star \star$ & $\star \star \star$ & $\star \star \star$ & $\star \star \star \star$ & $\star \star \star$ & $\star \star \star$ & $\star \star \star \star *$ & $\star \star \star \star$ & 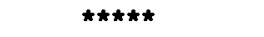 \\
\hline 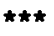 & $\hbar \hbar k$ & $\star \star \star \star *$ & $\star \star \star$ & $\star \star \star *$ & $\star \star \star$ & $\star \star \star$ & $\star \star \star \star$ & $\star \star \star$ & $\star \star \star$ & $\star \star \star$ & $\star \star \star$ & $\star \star \star \star \star$ \\
\hline$\star * *$ & $\hbar \star \star *$ & $\star \star \star$ & $\star \star \star$ & $\star \star \star$ & $\star \star \star$ & $\star \star \star$ & $\star \star \star$ & $\star \star \star$ & $\star \star \star$ & $\star \star \star *$ & $\star \star \star \star$ & $\star \star \star \star \star \star$ \\
\hline$\star \star \star *$ & $\star \star \star *$ & $\star \star \star \star$ & $\star \star \star \star$ & $\star \star \star \star$ & $\star \star \star \star$ & $\star \star \star \star$ & $\star \star \star$ & 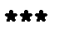 & $\star \star \star$ & $\star \star \star$ & $\star \star \star$ & $\star \star \star \star \star \star$ \\
\hline$\star \star \star \star *$ & $\star \star \star *$ & $\star \star \star *$ & $\star \star \star \star$ & $\star \star \star \star$ & $\star \star \star \star$ & $\star \star \star *$ & $\star \star \star \star$ & $\star \star \star$ & $\star \star \star *$ & $\star \star \star \star$ & $\star \star \star *$ & 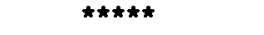 \\
\hline 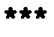 & $\star \star \star \star$ & $\star \star \star$ & $\star \star \star$ & $\star \star \star$ & $\star \star \star$ & $\star \star \star$ & $\star \star \star$ & $\star \star \star$ & $\star \star \star \star$ & $\star \star \star$ & $\star \star \star$ & 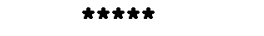 \\
\hline 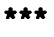 & $\star \star \star *$ & $\star \star \star *$ & $\star \star \star *$ & $\star \star \star *$ & $\star \star \star \star$ & $\star \star \star \star$ & $\star \star \star$ & $\star \star \star \star$ & $\star \star * *$ & $\star \star \star \star$ & $\star \star \star *$ & 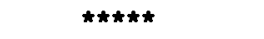 \\
\hline 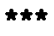 & $\star \star \star \star$ & $\star \star \star *$ & $\star \star \star$ & $\star \star \star *$ & $\star \star \star$ & 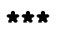 & $\star \star \star \star$ & $\star \star \star$ & $\star \star \star \star$ & $\star \star \star$ & 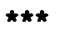 & $\star \star \star \star \star \star$ \\
\hline * & $\star \star \star$ & $\star \star \star$ & $\star \star \star \star$ & $\star \star \star$ & $\star \star \star$ & $\star \star \star \star$ & $\star \star \star \star$ & $\star \star \star$ & $\star \star \star$ & $\star \star \star \star$ & $\star \star \star \star$ & 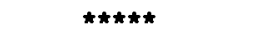 \\
\hline$* \star$ & $\star \star \star$ & $\star \star \star \star *$ & $\star \star * *$ & $\star \star \star \star$ & $\star \star \star$ & $\star \star \star \star$ & $\star \star \star$ & $\star \star \star \star *$ & $\star \star \star \star$ & $\star \star \star \star$ & $\star \star \star$ & 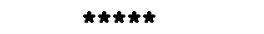 \\
\hline
\end{tabular}

1978

Rainfall

Crop 0 isease

Birds or Rats

Seed Availability

Fertilizer Availability

Crop Protection Chemicals

Credit

Irrigation Water Availability

Flooding

Labour Avai labilty

Power for Land Preparation

Oirect War Effects

Animal 0 iseases

Extension

other Problems

\begin{tabular}{|c|c|c|c|c|c|c|c|c|c|c|c|c|}
\hline 1 & 2 & 3. & 4 & 5 & 6 & 7 & 8 & 9 & 10 & 11 & 12 & Weighted Priority \\
\hline$\star \star \star *$ & $\star \star \star$ & $\star \star \star$ & $\star \star \star *$ & 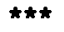 & $\star \star \star *$ & $\star \star \star \star$ & $\star \star \star *$ & $\star \star \star \star$ & $\star \star \star *$ & $\star \star \star \star$ & $\star \star \star$ & 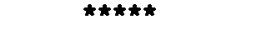 \\
\hline$\star \star \star$ & $\star \star \star$ & $\star \star \star$ & $\star \star \star$ & $\star \star \star$ & $\star \star \star$ & $\star \star \star$ & $\star \star \star *$ & $\star \star \star$ & $\star \star \star \star$ & $\star \star \star \star$ & $\star \star \star *$ & $\star \star \star \star \star \star *$ \\
\hline$\star \star \star$ & $\star \star \star$ & $\star \star \star$ & $\star \star \star$ & $\star \star \star$ & $\star \star \star$ & $\star \star \star$ & $\star \star \star$ & $\star \star \star$ & $\star \star \star$ & $\star \star \star \star$ & $\star \star * *$ & 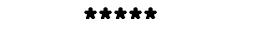 \\
\hline$\star \star \star$ & $\star \star \star$ & $\star \star \star$ & $\star \star \star$ & $\star \star \star$ & $\star \star \star$ & $\star \star \star$ & $\star \star \star$ & $\star \star \star \star$ & $\star \star \star *$ & $\star \star *$ & $\star \star \star *$ & $\star * * * *$ \\
\hline$\star \star \star$ & $\star \star *$ & $\star \star \star *$ & $\star \star \star$ & $\star \star \star *$ & $\star \star \star$ & $\star \star \star$ & $\star \star \star$ & $\star \star \star$ & 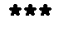 & $\star \star \star$ & 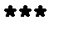 & 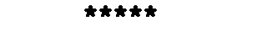 \\
\hline$\star \star \star$ & $\star \star \star$ & $\star \star \star \star$ & $\star \star \star$ & $\star \star \star \star$ & $\star \star \star$ & $\star \star \star$ & $\star \star \star$ & $\star \star \star *$ & $\star \star \star \star$ & $\star \star \star \star$ & 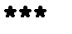 & 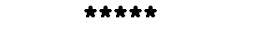 \\
\hline$\star \star \star \star$ & $\star * *$ & $\star \star \star$ & $\star \star \star$ & $\star \star \star$ & $\star \star \star$ & $\star \star \star$ & $\star \star \star$ & $\star \star \star$ & 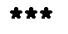 & $\star \star \star$ & $\star \star \star$ & $\star \star \star \star \star \star$ \\
\hline$\hbar \star \star \star$ & $\star \star \star \star$ & $\star \star \star$ & $\star \star \star$ & $\star \star \star$ & $\star \star \star$ & $\star \star \star$ & $\star \star \star$ & $\star \star \star \star$ & $\star \star \star$ & $\star \star \star$ & $\star * *$ & 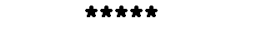 \\
\hline$\star \star \star \star$ & $* * *$ & $\star \star \star *$ & $\star \star \star$ & 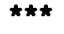 & $\star \star \star *$ & $\star \star \star$ & $\star \star \star *$ & 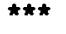 & $\star \star \star$ & $\star \star \star$ & $\star \star \star$ & $\star \star \star \star \star \star$ \\
\hline$\star \star \star \star$ & $\star \star \star$ & $\star \star \star$ & $\star \star \star \star$ & $\star \star \star$ & $\star \star \star$ & $\star \star \star$ & $\star \star \star$ & $\star \star \star$ & $\star \star \star$ & $\star \star \star$ & $\star \star \star$ & $\star \star * * * *$ \\
\hline$\star \star$ & $\star \star \star \star$ & $\star \star \star *$ & $\star \star \star *$ & $\star \star \star *$ & $\star \star \star *$ & $\star \star \star *$ & $\star \star \star *$ & $\star \star \star *$ & $\star \star \star \star$ & $\star \star \star$ & $\star \star \star \star$ & 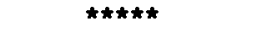 \\
\hline$\star \star \star \star$ & $\star \star * *$ & $\star \star * *$ & $\star \star * *$ & $\star \star \star$ & $\star \star \star *$ & $\star \star \star$ & $\star \star \star$ & $\star \star \star$ & $\star \star \star$ & $\star \star \star$ & $\star \star \star \star$ & 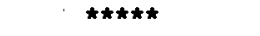 \\
\hline$\star \star$ & $\star \star \star$ & $\star \star \star \star$ & $\star \star \star *$ & $\star \star \star$ & $\star \star \star$ & $\star \star \star$ & $\star \star \star$ & $\star \star \star$ & $\star \star \star$ & $\star \star \star$ & $\star \star \star \star$ & $\star \star \star \star \star \star *$ \\
\hline kt & & & ; & t & * & * * & $*$ & $* * *$ & $\star \star \star$ & $\star \star \star \star$ & $\star \star \star *$ & $\star \star \star \star \star \star$ \\
\hline & & $x$ & * * & 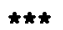 & ** & $* *$ & $\star \star \star \star$ & $\star \star \star \star$ & t* * & 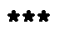 & t* $\star$ & $\star \star \star \star \star$ \\
\hline
\end{tabular}




\section{BAGHLAN}




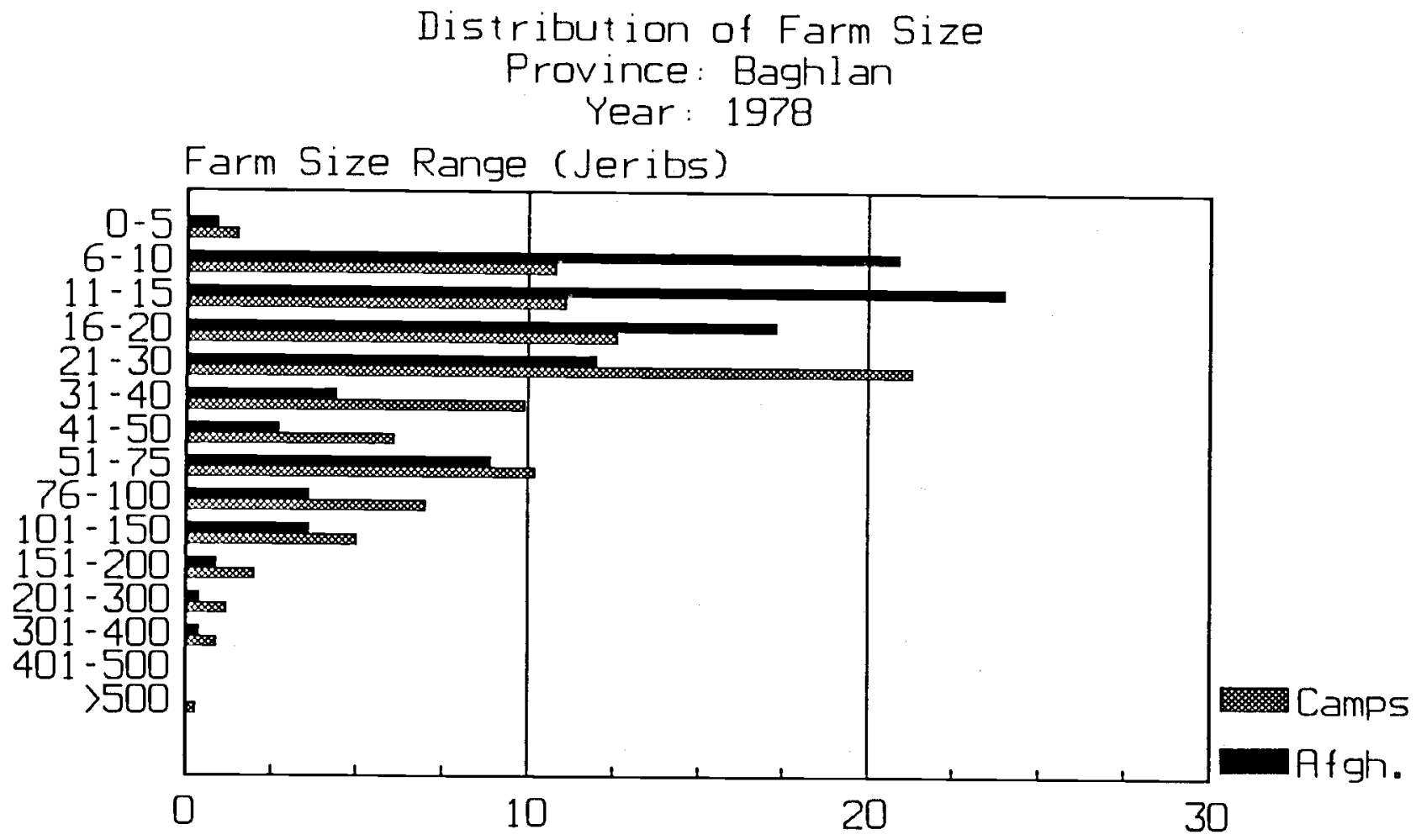

Percentage of Farmers

Farmers in Afghanistan

Total Number of Farms

Average Area

Average Area Irrigated

Average Dryland Area

$\underline{1978}$

225

32.22

16.94

73.04

342

47.88

27.06

64.55

41.67

22.75

66.84
1987

225

23.25

9.57

64.93

Farmers who left in 1987

Total Number of Farms

Average Area Irrigated

Average Dryland Area

\section{Average of Both Groups}

Provincial Average Area

Provincial Average Irrigated Area

Provincial Average Dryland Area

(All areas in Jeribs. 5 Jeribs $=1$ Hectare) 


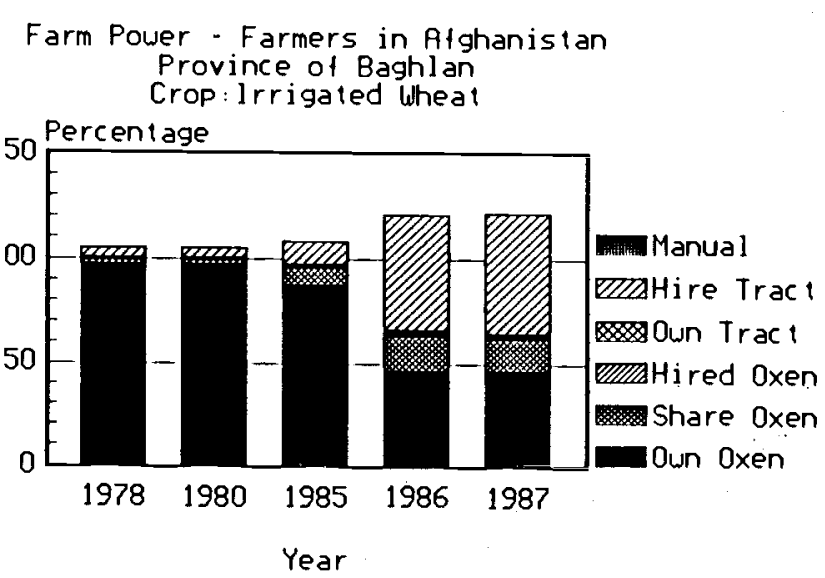

Farm Power - Farmers who lett in 1987 Province of Baghlan Crop: lrrigated wheat

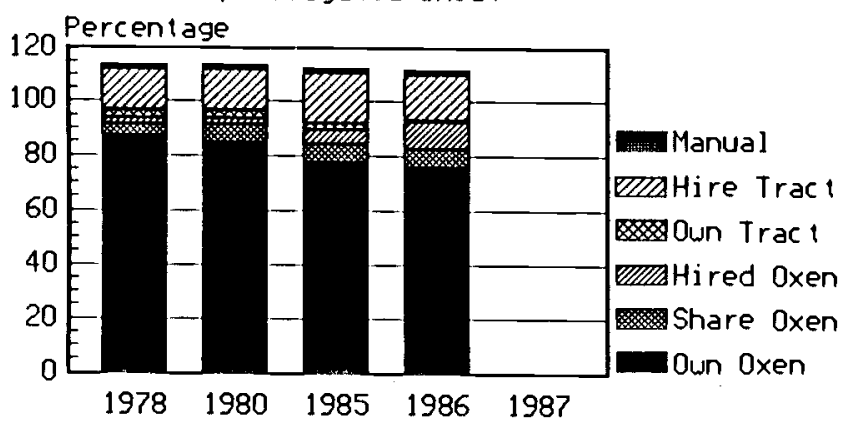

Year

Base Figures for above graphs

Province: Baghlan

Farmers in Afghanistan

Irr. Wheat Own Oxen Shared oxen

Hired oxen

Own Tractor

Hired Tractor

Rice

Manual Means

Own Oxen

Shared oxen

Hired oxen

Own Tractor

Hired Tractor

Manual Means

Farmers who left in 1987

$\begin{array}{ll}\text { Irr. Wheat } & \text { Own Oxen } \\ & \text { Shared Oxen } \\ & \text { Hired Oxen } \\ & \text { Own Tractor } \\ & \text { Hired Tractor } \\ & \text { Manual Means } \\ \text { Rice } & \text { Own Oxen } \\ & \text { Shared Oxen } \\ & \text { Hired Oxen } \\ & \text { Own Tractor }\end{array}$

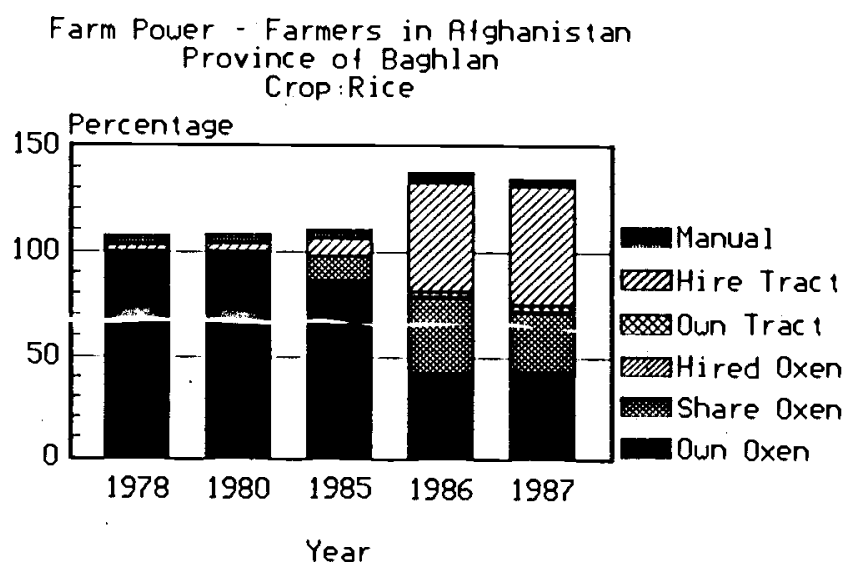

Farm Power - Farmers who lett in 1987 Province of Baghlan Crop:Rice

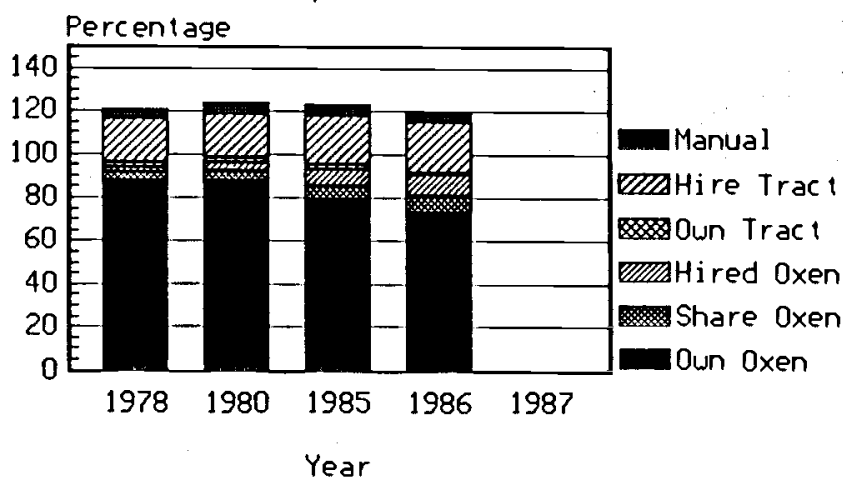

\begin{tabular}{ccrrrr} 
& \multicolumn{2}{l}{ Percentage of } & Farmers \\
\cline { 3 - 4 } & & & & \\
& & & & \\
& & & & \\
96.70 & 96.70 & 86.96 & 45.73 & 45.75 \\
2.83 & 2.83 & 9.18 & 17.59 & 16.51 \\
- & - & .48 & .50 & - \\
.47 & .47 & - & 1.51 & 1.89 \\
5.19 & 5.19 & 11.11 & 55.28 & 57.55 \\
- & - & - & - & - \\
97.93 & 97.93 & 85.82 & 42.06 & 42.76 \\
1.38 & 1.38 & 11.35 & 35.71 & 28.28 \\
- & - & - & .79 & .69 \\
- & - & - & 2.38 & 2.76 \\
3.45 & 4.14 & 8.51 & 51.59 & 55.86 \\
4.14 & 4.14 & 4.26 & 4.76 & 4.14
\end{tabular}

86.99

84.34

77.19

6.67

.456 .76

2.40

2.49

3.08

2.85

14.73

1.71

87.84

15.66

1.78

5.26

2.46

18.95

1.75

79.17

87.86

6.25

2.70

4.29
3.57

7.64

2. 08

$\begin{array}{rr}75.56 & - \\ 6.67 & - \\ 10.22 & - \\ 164 & - \\ 16.89 & - \\ 2.22 & - \\ 72.80 & - \\ 8.00 & - \\ 9.60 & - \\ .80 & -\end{array}$



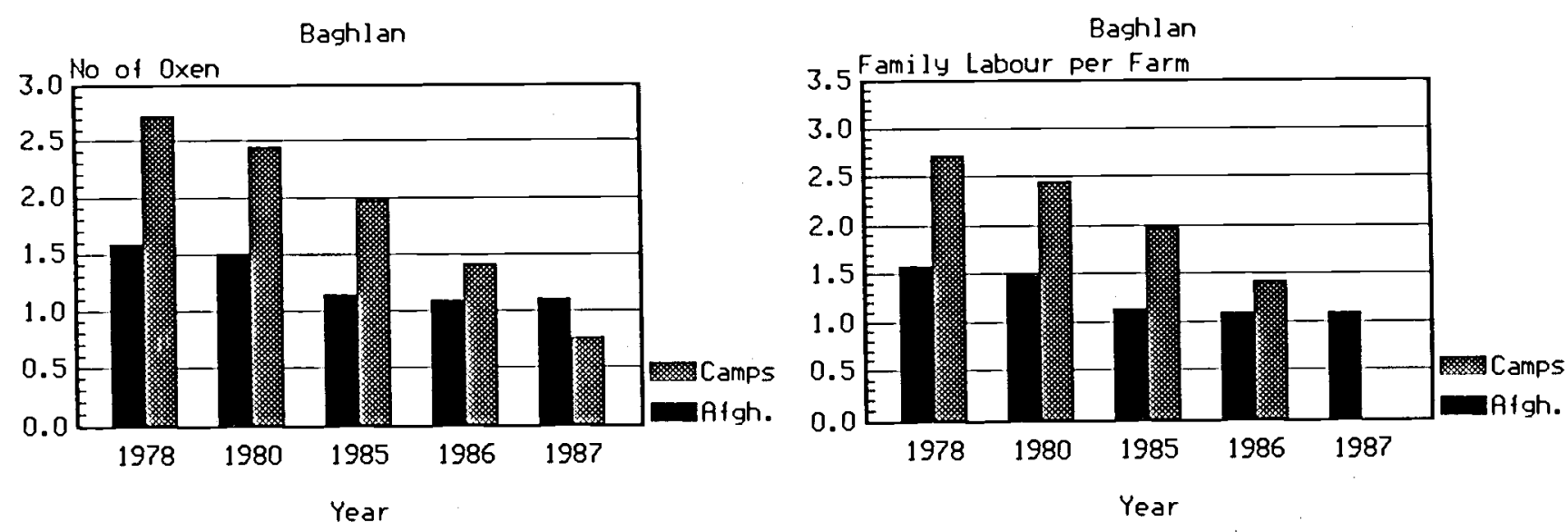

Baghlan

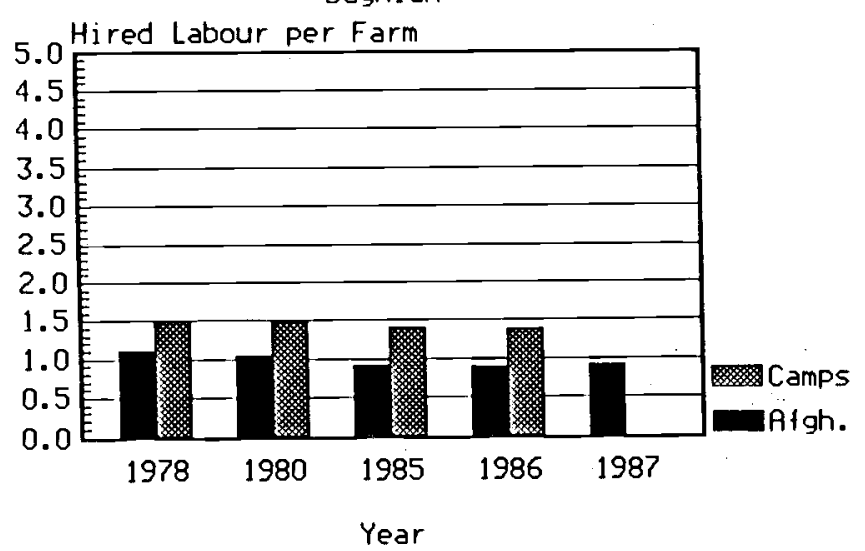

Baghlan

Percentage of Farms using

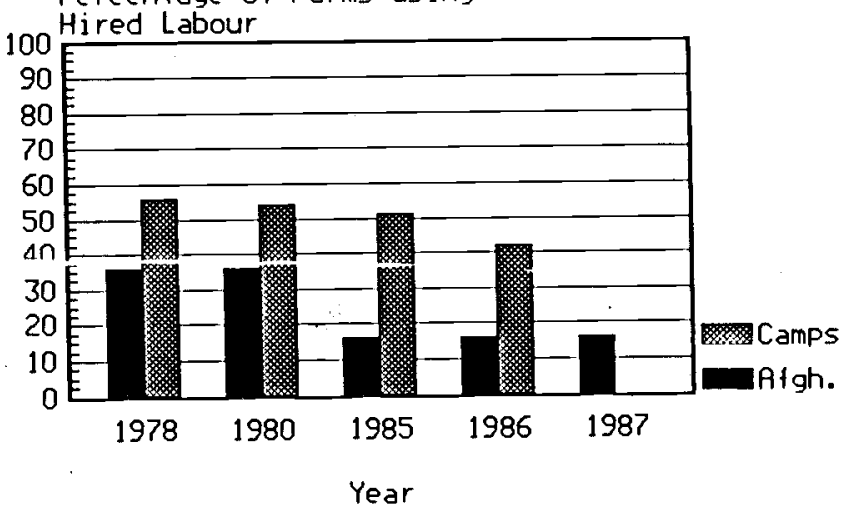


PROVINCE OF BGL

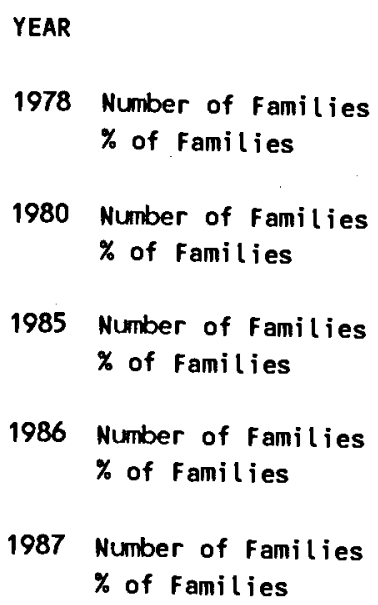

\begin{tabular}{|c|c|c|c|c|c|}
\hline 0 & 1 & 2 & 3 & 4 & 5 \\
\hline 2 & 5 & 185 & 22 & 9 & 0 \\
\hline 0.90 & 2.24 & 82.96 & 9.87 & 4.04 & 0.00 \\
\hline 2 & 5 & 186 & 22 & 8 & 0 \\
\hline 0.90 & 2.24 & 83.41 & 9.87 & 3.59 & 0.00 \\
\hline 9 & 20 & 178 & 15 & 2 & 1 \\
\hline 4.00 & 8.89 & 79.11 & 6.67 & 0.89 & 0.44 \\
\hline 46 & 70 & 93 & 13 & 2 & 1 \\
\hline 20.44 & 31.11 & 41.33 & 5.78 & 0.89 & 0.44 \\
\hline 47 & 69 & 93 & 10 & 5 & 1 \\
\hline 20.89 & 30.67 & 41.33 & 4.44 & 2.22 & 0.44 \\
\hline
\end{tabular}

2.14

2.13

1.93

1.37

1.38

Number of Farmers Questioned 225

\section{IABLE 7B - AVERAGE NUMBER OF IRAINED OXEN FOR FARMERS IN THE CAMPS} PROVINCE OF BGL

YEAR

1978 Number of Families $\%$ of Families

1980 Number of Families $\%$ of Families

1985 Number of Families $\%$ of Families

1986 Number of Families $\%$ of Families

1987 Number of Families \% of Families

Average Number of Oxen per Family:

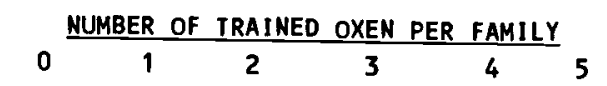

$\begin{array}{clllll}21 & 19 & 216 & 18 & 46 & 0 \\ 6.56 & 5.94 & 67.50 & 5.63 & 14.38 & 0.00 \\ 36 & 22 & 210 & 17 & 37 & 0 \\ 11.18 & 6.83 & 65.22 & 5.28 & 11.49 & 0.00 \\ & & & & & \\ 50 & 24 & 214 & 13 & 22 & 1 \\ 15.43 & 7.41 & 66.05 & 4.01 & 6.79 & 0.31 \\ 114 & 21 & 166 & 6 & 17 & 1 \\ 35.08 & 6.46 & 51.08 & 1.85 & 5.23 & 0.31 \\ 330 & 0 & 0 & 0 & 0 & 0 \\ 100.0 & 0.00 & 0.00 & 0.00 & 0.00 & 0.00\end{array}$

$\begin{array}{ll}1978 & 2.15 \\ 1980 & 1.99 \\ 1985 & 1.80 \\ 1986 & 1.37 \\ 1987 & 0.00\end{array}$

Number of Farmers Questioned 330 
PROVINCE OF BGL

$\begin{array}{lrrrrr}\text { Average Family Labour per farm } & 1.58 & 1.47 & 1.13 & 1.08 & 1.09 \\ \begin{array}{l}\text { Average No. of Hired Labour per Farm } \\ \text { (for farms using hired labour) }\end{array} & 1.10 & 1.04 & 0.90 & 0.88 & 0.91 \\ \begin{array}{l}\text { Number of Farms using Hired Labour } \\ \text { Number of Farmers Questioned } 225\end{array} & 81 & 80 & 37 & 36 & 37\end{array}$

TABLE $8(B)$ - AVERAGE LABOUR (FAMILY and HIRED) FOR FARMERS IN CAMPS

PROVINCE OF BGL

$\begin{array}{lrrrrr}\text { Average Family Labour per Farm } & 2.72 & 2.44 & 1.97 & 1.41 & 0.00 \\ \begin{array}{l}\text { Average No. of Hired Labour per Farm } \\ \text { (for farms using hired labour) }\end{array} & 1.50 & 1.49 & 1.39 & 1.38 & \star \star \star \star \star \\ \text { Number of Farms using Hired Labour } & 186 & 177 & 169 & 138 & 0\end{array}$

Number of Farmers Questioned 330 
PROVINCE OF BGL

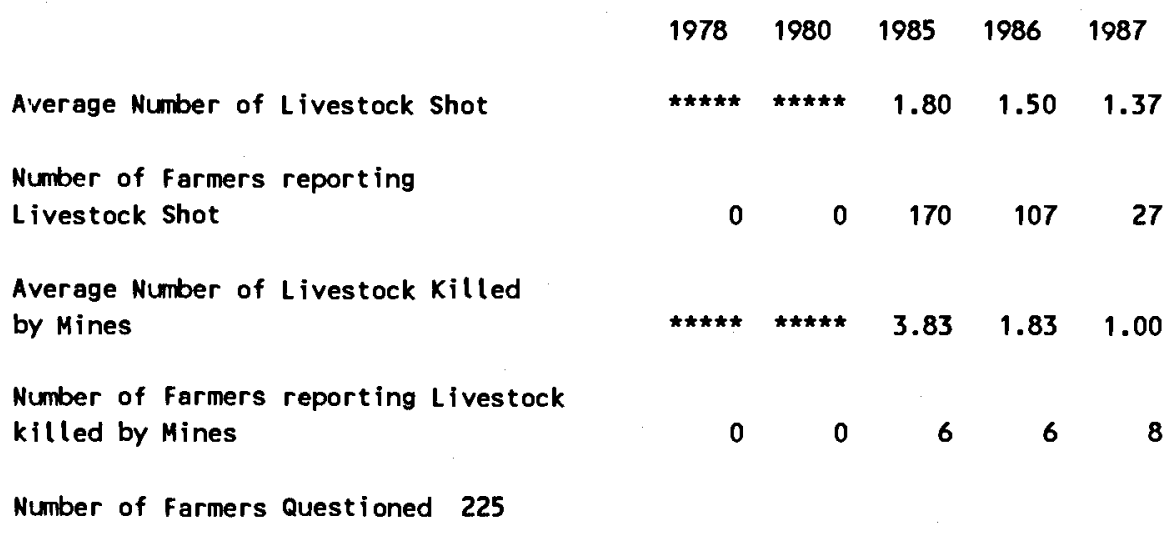

IABLE 9B - DIRECT EFFECTS OF THE WAR - FARMERS IN CAMPS

PROVINCE OF BGL

\begin{tabular}{|c|c|c|c|c|c|}
\hline & 1978 & 1980 & 1985 & 1986 & 1987 \\
\hline Average Number of Livestock Shot & 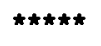 & 6.18 & 10.44 & 7.58 & $\star \star \star \star \star \star$ \\
\hline \multicolumn{6}{|l|}{ Number of Farmers reporting } \\
\hline Livestock Shot & 0 & 34 & 147 & 69 & 0 \\
\hline $\begin{array}{l}\text { Average Number of Livestock Kille } \\
\text { by Mines }\end{array}$ & $\star \star \star \star \star \star$ & 2.75 & 5.07 & 3.90 & 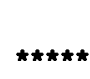 \\
\hline $\begin{array}{l}\text { Number of Farmers } r \\
\text { killed by Mines }\end{array}$ & 0 & 8 & 46 & 31 & 0 \\
\hline
\end{tabular}

Number of Farmers Questioned 330 


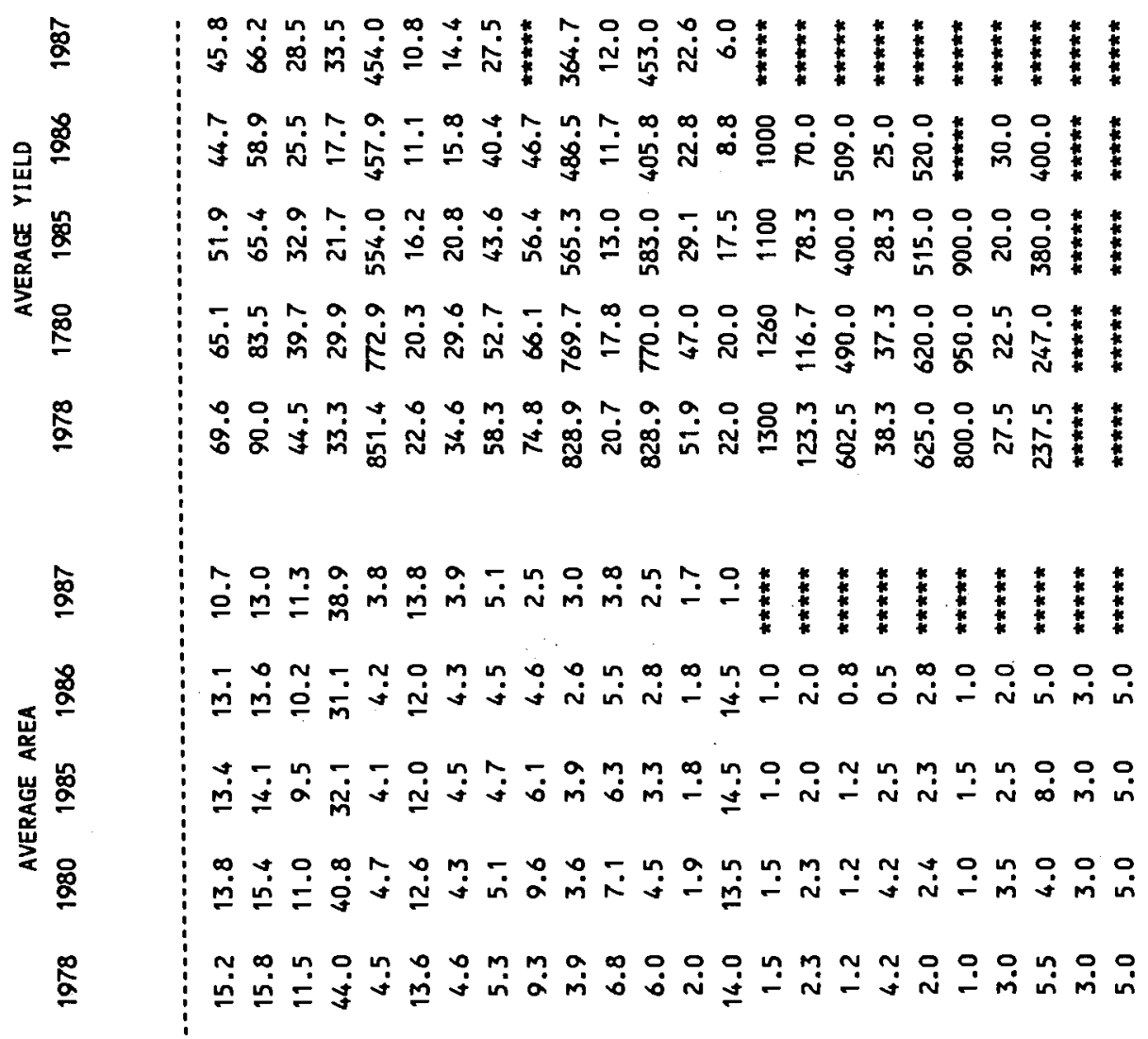

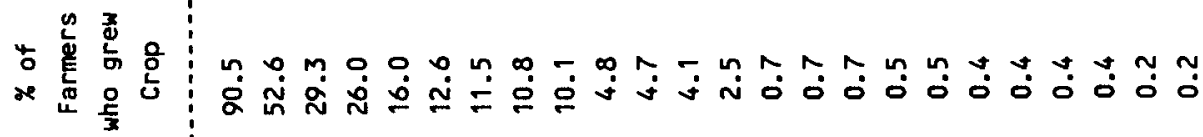

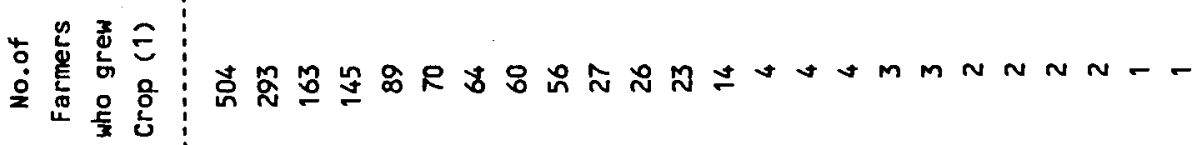
嵒敦高

:

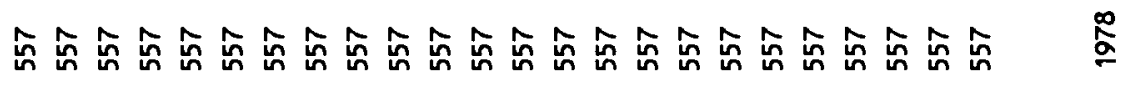

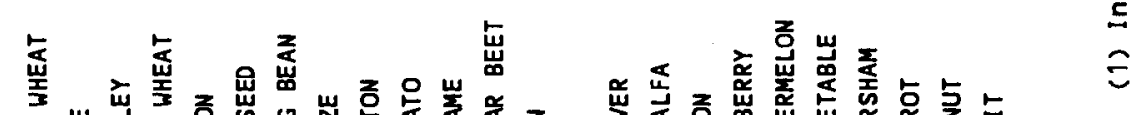


IABLE 1-5 - ANALYSIS OF GREATEST FARMING PROBLEMS - COMMUNITY SURVEY

PROVINCE OF BGL

Rainfall

Crop Disease

Birds or Rats

Seed Availability

Fertilizer Availability

Crop Protection Chemicals

Credit

Irrigation Water Availability

Flooding

Labour Availabilty

Power for Land Preparation

Direct War Effects

Animal Diseases

Extension

Other Problems

\begin{tabular}{rrrrrrrrrrrrr}
1 & 2 & 3 & 4 & 5 & 6 & 7 & 8 & 9 & 10 & 11 & 12 & Weighted Priority \\
\hline 0 & 0 & 0 & 0 & 0 & 0 & 0 & 0 & 0 & 0 & 0 & 0 & 0.00 \\
0 & 2 & 0 & 2 & 5 & 0 & 0 & 0 & 0 & 0 & 0 & 0 & 1.15 \\
0 & 5 & 25 & 30 & 25 & 7 & 2 & 0 & 0 & 0 & 0 & 0 & 10.77 \\
0 & 36 & 20 & 0 & 5 & 0 & 0 & 0 & 0 & 0 & 0 & 0 & 11.41 \\
2 & 16 & 2 & 2 & 0 & 0 & 0 & 0 & 0 & 0 & 0 & 0 & 5.09 \\
0 & 2 & 14 & 5 & 2 & 0 & 0 & 0 & 0 & 0 & 0 & 0 & 3.20 \\
0 & 2 & 0 & 2 & 0 & 5 & 0 & 0 & 0 & 0 & 0 & 0 & 1.08 \\
2 & 0 & 0 & 0 & 0 & 0 & 0 & 0 & 0 & 0 & 0 & 0 & 1.00 \\
0 & 0 & 0 & 2 & 0 & 0 & 0 & 0 & 0 & 0 & 0 & 0 & 0.25 \\
0 & 32 & 34 & 27 & 5 & 0 & 0 & 0 & 0 & 0 & 0 & 0 & 15.41 \\
0 & 0 & 5 & 0 & 0 & 0 & 0 & 0 & 0 & 0 & 0 & 0 & 0.67 \\
95 & 5 & 0 & 0 & 0 & 0 & 0 & 0 & 0 & 0 & 0 & 0 & 43.04 \\
0 & 2 & 0 & 0 & 0 & 0 & 0 & 0 & 0 & 0 & 0 & 0 & 0.50 \\
0 & 0 & 0 & 25 & 27 & 14 & 2 & 2 & 0 & 0 & 0 & 0 & 6.42 \\
0 & 0 & 0 & 0 & 0 & 0 & 0 & 0 & 0 & 0 & 0 & 0 & 0.00
\end{tabular}

1986

Rainfall

Crop Disease

Birds or Rats

Seed Availability

Fertilizer Availability

Crop Protection Chemicals

Credit

Irrigation Water Availability

flooding

Labour Availabilty

Power for Land Preparation

Direct War Effects

Animal Diseases

Extension

Other Problems

\begin{tabular}{rrrrrrrrrrrrc}
1 & 2 & 3 & 4 & 5 & 6 & 7 & 8 & 9 & 10 & 11 & 12 & Weighted Priority \\
\hline 0 & 7 & 0 & 0 & 0 & 0 & 0 & 0 & 0 & 0 & 0 & 0 & 1.52 \\
2 & 0 & 0 & 0 & 0 & 0 & 0 & 0 & 0 & 0 & 0 & 0 & 1.01 \\
0 & 2 & 11 & 43 & 25 & 9 & 0 & 0 & 0 & 0 & 0 & 0 & 9.89 \\
0 & 16 & 18 & 5 & 0 & 2 & 0 & 0 & 0 & 0 & 0 & 0 & 6.91 \\
0 & 30 & 5 & 0 & 0 & 2 & 0 & 0 & 0 & 0 & 0 & 0 & 7.41 \\
0 & 5 & 11 & 2 & 0 & 2 & 0 & 0 & 0 & 0 & 0 & 0 & 3.12 \\
0 & 16 & 7 & 2 & 2 & 0 & 0 & 0 & 0 & 0 & 0 & 0 & 5.00 \\
5 & 2 & 0 & 0 & 2 & 0 & 0 & 0 & 0 & 0 & 0 & 0 & 2.73 \\
0 & 0 & 0 & 2 & 0 & 0 & 0 & 0 & 0 & 0 & 0 & 0 & 0.25 \\
0 & 18 & 39 & 32 & 7 & 0 & 0 & 0 & 0 & 0 & 0 & 0 & 13.92 \\
0 & 0 & 2 & 0 & 0 & 0 & 0 & 0 & 0 & 0 & 0 & 0 & 0.34 \\
93 & 5 & 0 & 0 & 0 & 0 & 0 & 0 & 0 & 0 & 0 & 0 & 42.46 \\
0 & 0 & 0 & 0 & 2 & 0 & 0 & 0 & 0 & 0 & 0 & 0 & 0.20 \\
0 & 0 & 5 & 7 & 34 & 5 & 7 & 0 & 0 & 0 & 0 & 0 & 5.24 \\
0 & 0 & 0 & 0 & 0 & 0 & 0 & 0 & 0 & 0 & 0 & 0 & 0.00
\end{tabular}

1985

Rainfall

Crop Disease

Birds or Rats

Seed Availability

Fertilizer Availability

Crop Protection Chemicals

Credit

Irrigation Water Availability flooding

Labour Availabilty

Power for Land Preparation

Direct War Effects

Animal Diseases

Extension

Other Problems

\begin{tabular}{rrrrrrrrrrrrr}
1 & 2 & 3 & 4 & 5 & 6 & 7 & 8 & 9 & 10 & 11 & 12 & Heighted Priority \\
\hline 2 & 5 & 0 & 0 & 0 & 2 & 0 & 0 & 0 & 0 & 0 & 0 & 2.23 \\
2 & 5 & 0 & 7 & 0 & 7 & 0 & 0 & 0 & 0 & 0 & 0 & 3.35 \\
0 & 2 & 18 & 39 & 25 & 2 & 0 & 0 & 0 & 0 & 0 & 0 & 10.09 \\
0 & 23 & 18 & 2 & 2 & 0 & 0 & 0 & 0 & 0 & 0 & 0 & 8.37 \\
0 & 18 & 5 & 0 & 5 & 0 & 0 & 0 & 0 & 0 & 0 & 0 & 5.23 \\
0 & 7 & 4 & 0 & 0 & 0 & 0 & 0 & 0 & 0 & 0 & 0 & 2.92 \\
0 & 2 & 0 & 2 & 7 & 0 & 0 & 0 & 0 & 0 & 0 & 0 & 1.39 \\
0 & 5 & 5 & 0 & 0 & 2 & 0 & 0 & 0 & 0 & 0 & 0 & 1.89 \\
0 & 0 & 0 & 2 & 2 & 0 & 0 & 0 & 0 & 0 & 0 & 0 & 0.46 \\
0 & 27 & 34 & 23 & 2 & 0 & 0 & 0 & 0 & 0 & 0 & 0 & 14.13 \\
0 & 0 & 2 & 2 & 0 & 0 & 0 & 0 & 0 & 0 & 0 & 0 & 0.60 \\
95 & 2 & 2 & 0 & 0 & 0 & 0 & 0 & 0 & 0 & 0 & 0 & 44.18 \\
0 & 2 & 0 & 0 & 0 & 0 & 0 & 0 & 0 & 0 & 0 & 0 & 0.52 \\
0 & 0 & 2 & 14 & 23 & 7 & 2 & 0 & 0 & 0 & 0 & 0 & 4.62 \\
0 & 0 & 0 & 0 & 0 & 0 & 0 & 0 & 0 & 0 & 0 & 0 & 0.00
\end{tabular}


IABLE 1-5(contd) - ANALYSIS OF GREATEST FARMING PROBLEMS - COMMUNITY SURVEY

PROVINCE OF BGL

PERCENTAGE OF VILLAGES GIVING FOLLOWING PRIORITY TO PROBLEM

Rainfall

Crop Disease

Birds or Rats

Seed Availability

Fertilizer Availability

Crop Protection Chemicals

Credit

Irrigation Water Availability

Flooding

Labour Availabilty

Power for Land Preparation

Direct War Effects

Animal Diseases

Extension

Other Problems

\begin{tabular}{rrrrrrrrrrrrr}
1 & 2 & 3 & 4 & 5 & 6 & 7 & 8 & 9 & 10 & 11 & 12 & Weighted Priority \\
\hline 5 & 0 & 2 & 2 & 5 & 0 & 0 & 0 & 0 & 0 & 0 & 0 & 5.23 \\
5 & 5 & 0 & 5 & 0 & 0 & 0 & 0 & 0 & 0 & 0 & 0 & 6.13 \\
45 & 9 & 0 & 0 & 2 & 0 & 0 & 0 & 0 & 0 & 0 & 0 & 38.91 \\
2 & 20 & 14 & 5 & 0 & 0 & 0 & 0 & 0 & 0 & 0 & 0 & 14.02 \\
0 & 2 & 2 & 5 & 7 & 0 & 0 & 0 & 0 & 0 & 0 & 0 & 3.39 \\
0 & 7 & 5 & 0 & 0 & 0 & 0 & 0 & 0 & 0 & 0 & 0 & 3.80 \\
0 & 7 & 11 & 2 & 0 & 0 & 0 & 0 & 0 & 0 & 0 & 0 & 5.99 \\
0 & 0 & 5 & 0 & 0 & 2 & 0 & 0 & 0 & 0 & 0 & 0 & 1.46 \\
2 & 0 & 0 & 0 & 0 & 0 & 0 & 0 & 0 & 0 & 0 & 0 & 1.75 \\
9 & 0 & 0 & 2 & 2 & 0 & 0 & 0 & 0 & 0 & 0 & 0 & 7.80 \\
0 & 5 & 2 & 2 & 0 & 0 & 0 & 0 & 0 & 0 & 0 & 0 & 2.78 \\
2 & 2 & 2 & 2 & 0 & 0 & 0 & 0 & 0 & 0 & 0 & 0 & 3.65 \\
0 & 0 & 0 & 0 & 0 & 0 & 0 & 0 & 0 & 0 & 0 & 0 & 0.00 \\
0 & 11 & 0 & 0 & 5 & 0 & 0 & 0 & 0 & 0 & 0 & 0 & 5.08 \\
0 & 0 & 0 & 0 & 0 & 0 & 0 & 0 & 0 & 0 & 0 & 0 & 0.00
\end{tabular}

$\underline{1978}$

Rainfall

Crop Disease

Birds or Rats

Seed Availability

Fertilizer Availability

Crop Protection Chemicals

Credit

Irrigation Water Availability

flooding

Labour Availabilty

Power for Land Preparation

Direct Har Effects

Animal Diseases

Extension

Other Problems

\begin{tabular}{rrrrrrrrrrrrr}
1 & 2 & 3 & 4 & 5 & 6 & 7 & 8 & 9 & 10 & 11 & 12 & Weighted Priority \\
\hline 9 & 5 & 0 & 2 & 0 & 0 & 0 & 0 & 0 & 0 & 0 & 0 & 21.30 \\
2 & 0 & 0 & 0 & 2 & 0 & 0 & 0 & 0 & 0 & 0 & 0 & 4.87 \\
2 & 11 & 9 & 0 & 0 & 0 & 0 & 0 & 0 & 0 & 0 & 0 & 19.61 \\
9 & 0 & 2 & 0 & 0 & 0 & 0 & 0 & 0 & 0 & 0 & 0 & 17.58 \\
0 & 0 & 5 & 2 & 0 & 0 & 0 & 0 & 0 & 0 & 0 & 0 & 3.72 \\
2 & 0 & 0 & 0 & 0 & 0 & 0 & 0 & 0 & 0 & 0 & 0 & 4.06 \\
2 & 7 & 0 & 0 & 0 & 0 & 0 & 0 & 0 & 0 & 0 & 0 & 10.14 \\
5 & 2 & 0 & 2 & 0 & 0 & 0 & 0 & 0 & 0 & 0 & 0 & 11.16 \\
0 & 0 & 0 & 0 & 0 & 0 & 0 & 0 & 0 & 0 & 0 & 0 & 0.00 \\
0 & 0 & 0 & 2 & 0 & 0 & 0 & 0 & 0 & 0 & 0 & 0 & 1.01 \\
0 & 0 & 5 & 0 & 2 & 0 & 0 & 0 & 0 & 0 & 0 & 0 & 3.52 \\
0 & 0 & 0 & 0 & 0 & 0 & 0 & 0 & 0 & 0 & 0 & 0 & 0.00 \\
0 & 2 & 0 & 0 & 0 & 0 & 0 & 0 & 0 & 0 & 0 & 0 & 2.03 \\
0 & 0 & 0 & 2 & 0 & 0 & 0 & 0 & 0 & 0 & 0 & 0 & 1.01 \\
0 & 0 & 0 & 0 & 0 & 0 & 0 & 0 & 0 & 0 & 0 & 0 & 0.00
\end{tabular}




\section{BAMYAN}




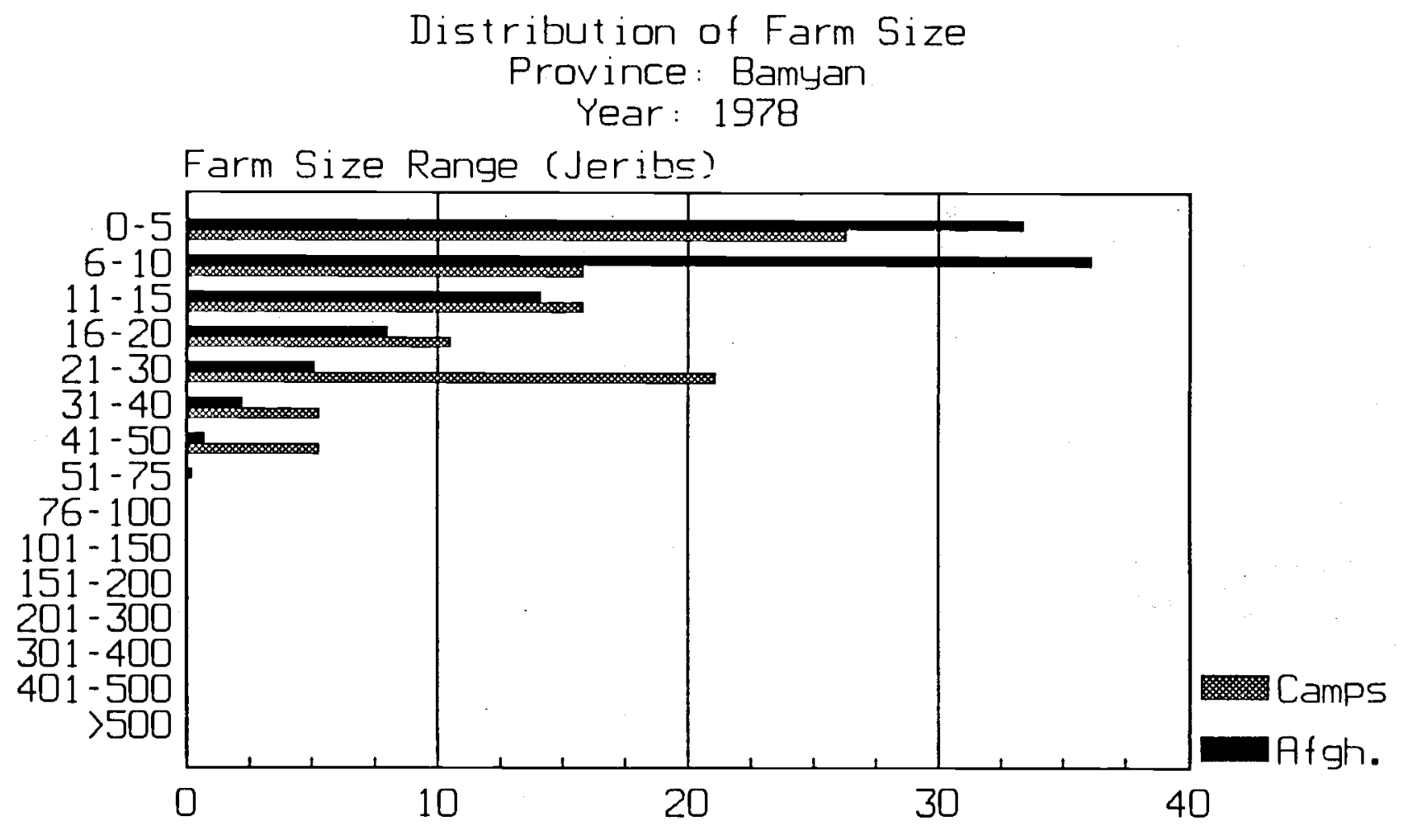

Percentage of Farmers

Farmers in Afghanistan

Total Number of Farms

Average Area

9.77

Average Area Irrigated

7.82

4.49

Average Dryland Area

5.99

4.09

Farmers who left in 1987

Total Number of Farms

Average Area

19

0

Average Area Irrigated

16.53

13.89

Average Dryland Area

11.25

Average of Both Groups

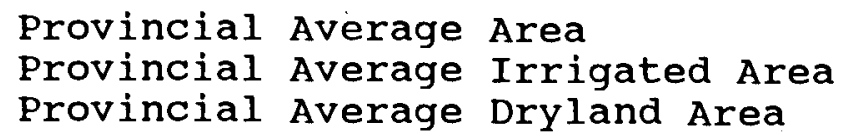

10.07

5.37

Provincial Average Irrigated Area

8.09

4.49

6.29

4.09

(All areas in Jeribs. 5 Jeribs $=1$ Hectare) 
Farm Power - Farmers in Atghanistan Province of Bamyan

Crop: Irrigated wheat

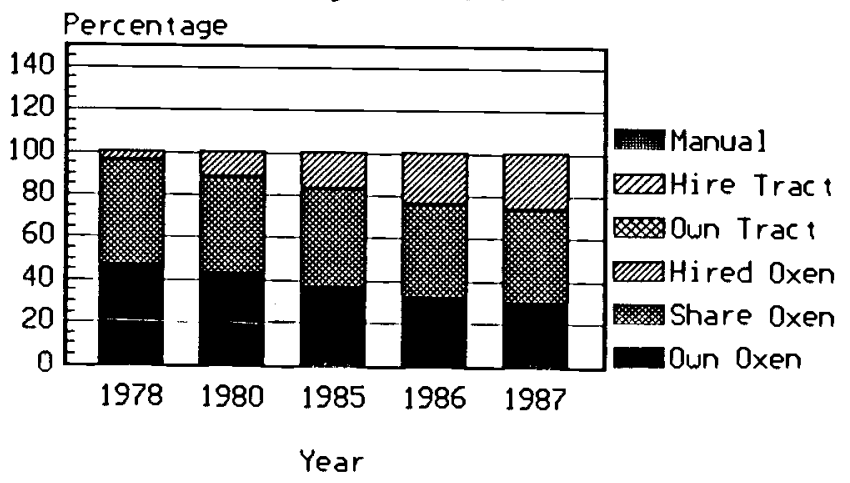

Farm Power - Farmers who left in 1987 Province of Bamyan

Crop: Irrigated wheat

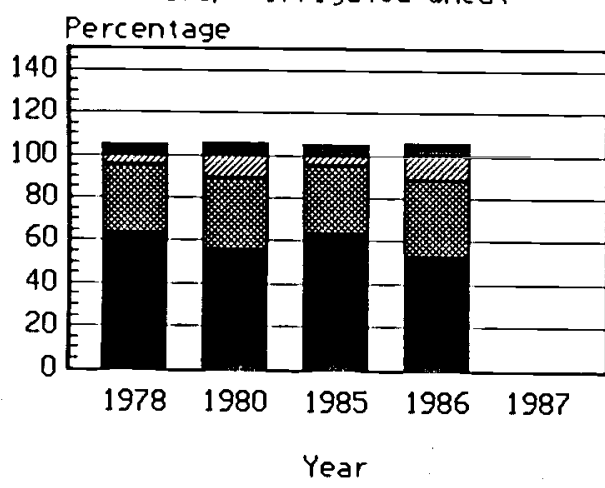

Base Figures for above graphs Oun Tract שखा Share Oxen Oun Oxen

\section{Farmers in Afghanistan}

Irr. Wheat Own oxen

Shared Oxen

Hired oxen

Own Tractor

Hired Tractor

Barley Manual Means

Own Oxen

Shared oxen

Hired oxen Own Tractor Hired Tractor Manual Means

Farmers who left in 1987

$\begin{array}{ll}\text { Irr. Wheat } & \text { Own Oxen } \\ & \text { Shared Oxen } \\ & \text { Hired Oxen } \\ & \text { Own Tractor } \\ & \text { Hired Tractor } \\ & \text { Manual Means } \\ \text { Barley } & \text { Own Oxen } \\ & \text { Shared Oxen } \\ & \text { Hired oxen } \\ & \text { Own Tractor }\end{array}$

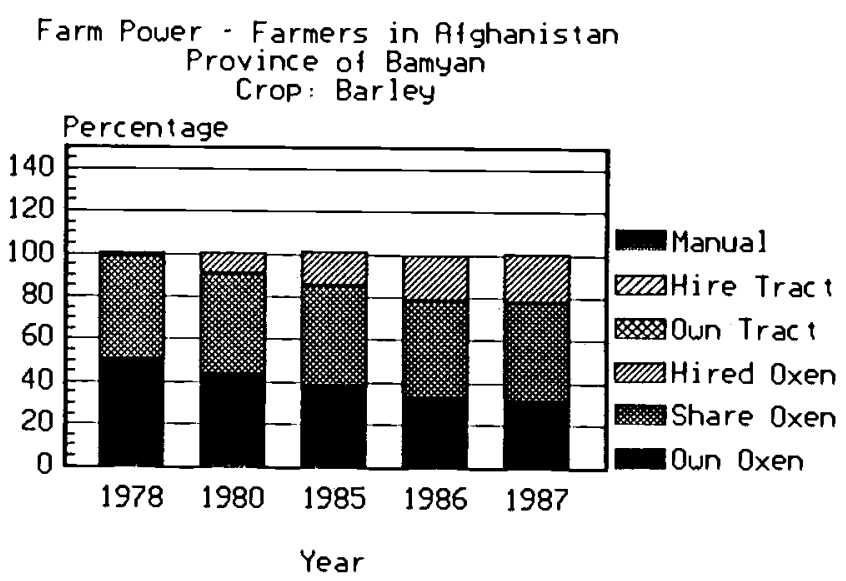
Farm Power - Farmers who lett in 1987 Province of Bamyan Crop: Barley

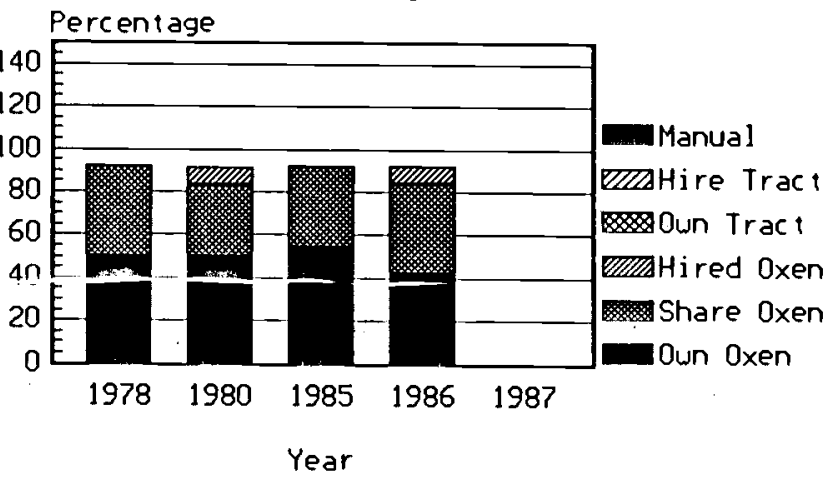

\begin{tabular}{ccccc}
\multicolumn{7}{c}{ Percentage of } & Farmers \\
1978 & $\underline{1980}$ & $\underline{1985}$ & $\underline{1986}$ & $\underline{1987}$ \\
& & & & \\
46.27 & 42.61 & 36.72 & 32.16 & 29.68 \\
49.75 & 45.86 & 46.40 & 44.22 & 44.14 \\
4.23 & 11.78 & 17.12 & 23.87 & 26.93 \\
- & - & - & - & - \\
- & - & - & - & - \\
- & - & - & - & - \\
50.21 & 43.21 & 38.33 & 32.79 & 31.44 \\
48.51 & 47.33 & 47.08 & 45.75 & 46.29 \\
1.28 & 9.47 & 15.42 & 21.46 & 22.71 \\
- & - & - & - & - \\
- & - & - & - & - \\
- & - & - & - & -
\end{tabular}



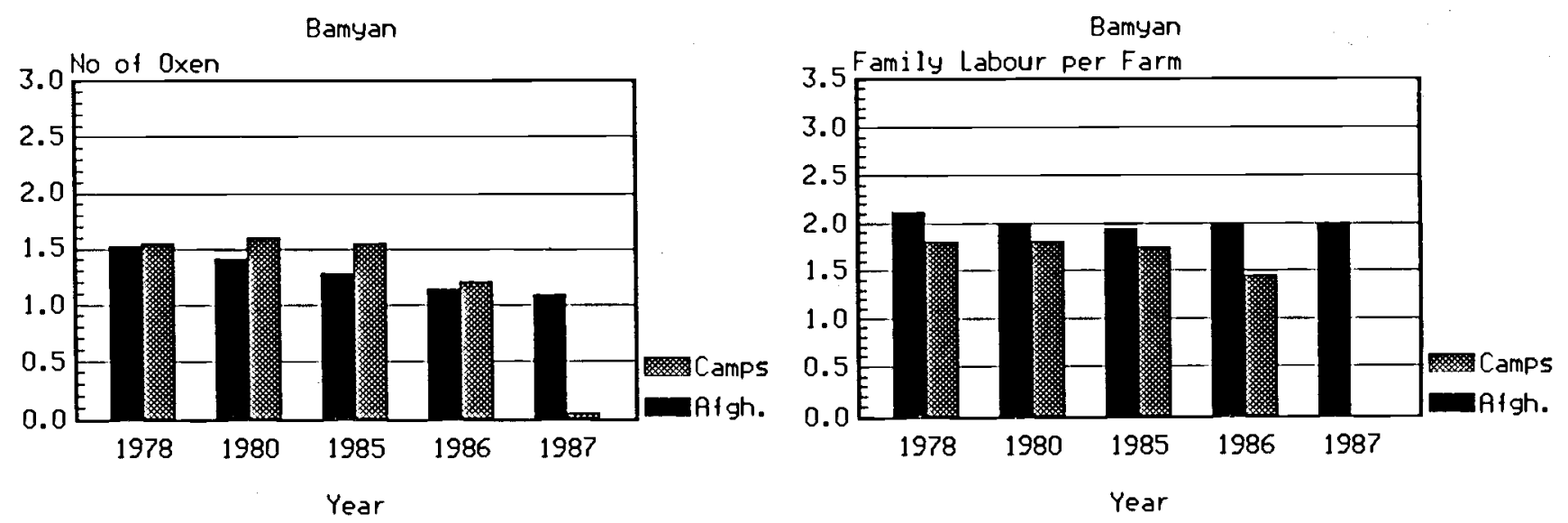

Bamyan

Bamyan
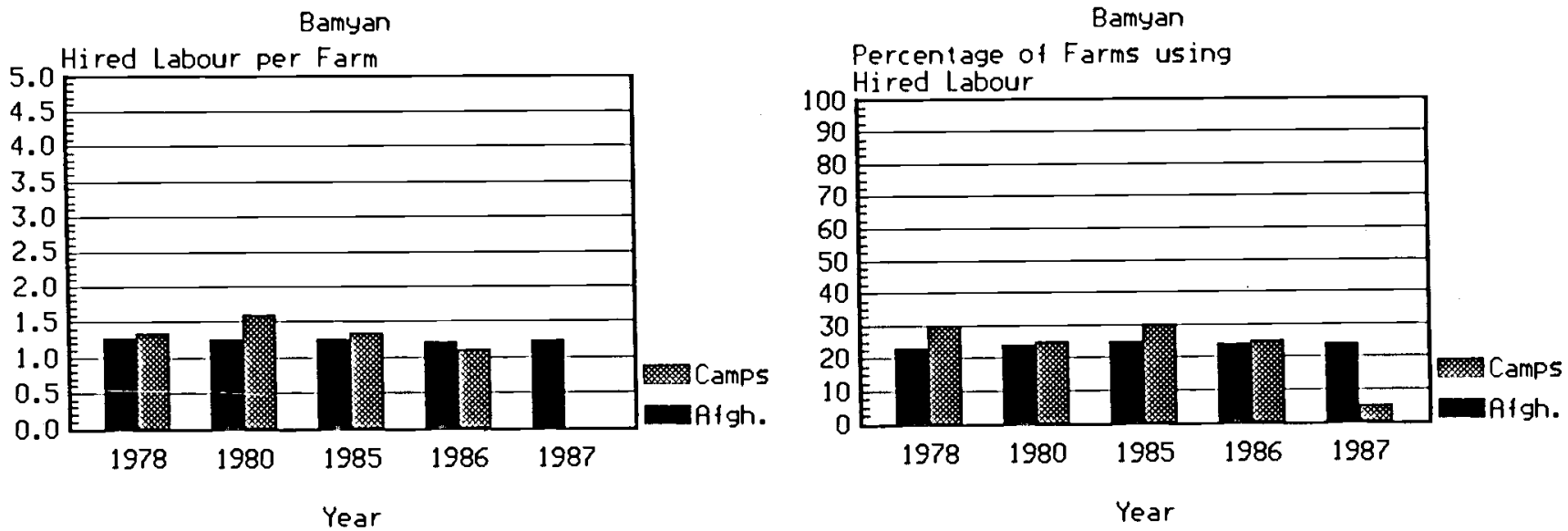
PROVINCE OF BYN

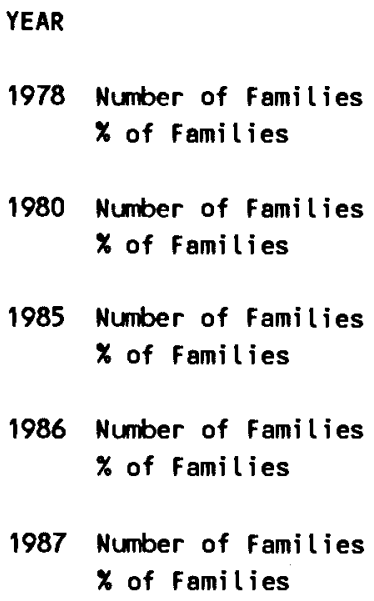

Number of Farmers Questioned 410

$\begin{array}{ll}1978 & 1.52 \\ 1980 & 1.41 \\ 1985 & 1.27 \\ 1986 & 1.13 \\ 1987 & 1.08\end{array}$

\begin{tabular}{|c|c|c|c|c|c|}
\hline \multicolumn{3}{|c|}{ NUMBER OF TRAINED } & \multicolumn{3}{|c|}{ OXEN PER FAMILY } \\
\hline 0 & 1 & 2 & 3 & 4 & 5 \\
\hline 21 & 196 & 164 & 13 & 11 & 3 \\
\hline 5.15 & 48.04 & 40.20 & 3.19 & 2.70 & 0.74 \\
\hline 48 & 187 & 145 & 16 & 9 & 3 \\
\hline 11.76 & 45.83 & 35.54 & 3.92 & 2.21 & 0.74 \\
\hline 72 & 191 & 124 & 9 & 11 & 2 \\
\hline 17.60 & 46.70 & 30.32 & 2.20 & 2.69 & 0.49 \\
\hline 97 & ליו & 113 & 7 & 7 & 1 \\
\hline 23.66 & 45.12 & 27.56 & 1.71 & 1.71 & 0.24 \\
\hline 110 & 180 & 105 & 9 & 5 & 1 \\
\hline 6.83 & 43.90 & 25.61 & 2.20 & 1.22 & 0.24 \\
\hline
\end{tabular}

\section{IABLE 7B - AVERAGE NUMBER OF TRAINED OXEN FOR FARMERS IN THE CAMPS}

PROVINCE OF BYN

\begin{tabular}{|c|c|c|c|c|c|c|c|}
\hline \multirow[b]{2}{*}{ YEAR } & & \multicolumn{2}{|c|}{ NUMBER OF } & TRAINED & \multicolumn{3}{|c|}{ OXEN PER FAMILY } \\
\hline & & 0 & 1 & 2 & 3 & 4 & 5 \\
\hline \multirow[t]{2}{*}{1978} & Number of Families & 2 & 7 & 10 & 0 & 1 & 0 \\
\hline & \% of Families & 10.00 & 35.00 & 50.00 & 0.00 & 5.00 & 0.00 \\
\hline \multirow[t]{2}{*}{1980} & Number of Families & 2 & 7 & 9 & 1 & 1 & 0 \\
\hline & \% of Fanilies & 10.00 & 35.00 & 45.00 & 5.00 & 5.00 & 0.00 \\
\hline \multirow[t]{2}{*}{1985} & Number of Families & 2 & 7 & 10 & 0 & 1 & 0 \\
\hline & \% of Families & 10.00 & 35.00 & 50.00 & 0.00 & 5.00 & 0.00 \\
\hline \multirow[t]{2}{*}{1986} & Number of Families & 5 & 8 & 6 & 0 & 1 & 0 \\
\hline & $\%$ of Families & 25.00 & 40.00 & 30.00 & 0.00 & 5.00 & 0.00 \\
\hline \multirow[t]{2}{*}{1987} & Number of Families & 19 & 1 & 0 & 0 & 0 & 0 \\
\hline & $x$ of Families & 95.00 & 5.00 & 0.00 & 0.00 & 0.00 & 0.00 \\
\hline \multirow{5}{*}{\multicolumn{2}{|c|}{ Average Number of Oxen per Family: }} & 1978 & \multicolumn{2}{|c|}{1.55} & & & \\
\hline & & 1980 & \multicolumn{2}{|c|}{1.60} & & & \\
\hline & & 1985 & \multicolumn{2}{|c|}{1.55} & & & \\
\hline & & 1986 & \multicolumn{2}{|c|}{1.20} & & & \\
\hline & & 1987 & \multicolumn{2}{|c|}{0.05} & & & \\
\hline
\end{tabular}

Number of Farmers Questioned 20 
TABLE $8(A)$ - AVERAGE LABOUR (FAMILY and HIRED) FOR FARMERS IN AFGANISTAN

PROVINCE OF BYN

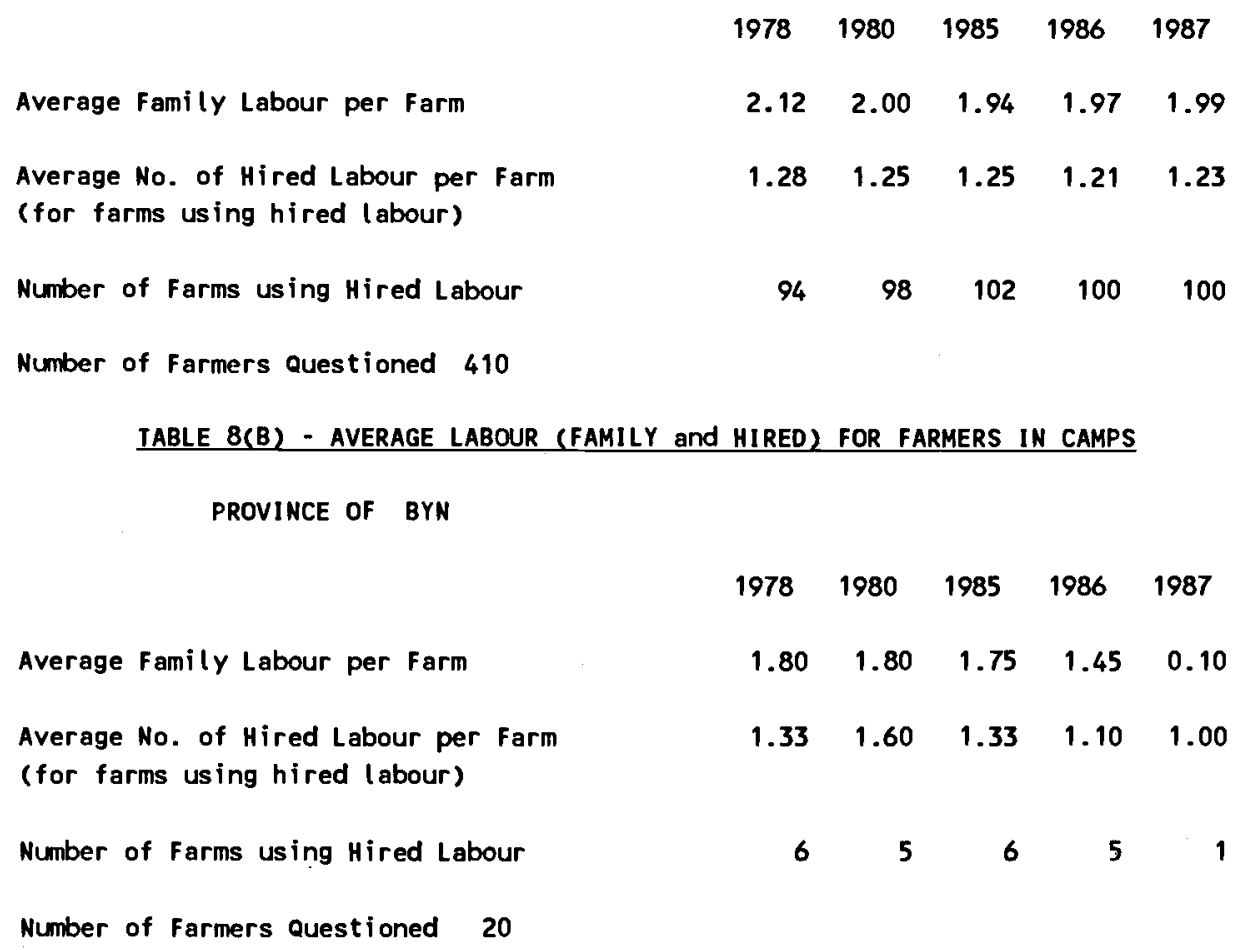


PROVINCE OF BYN

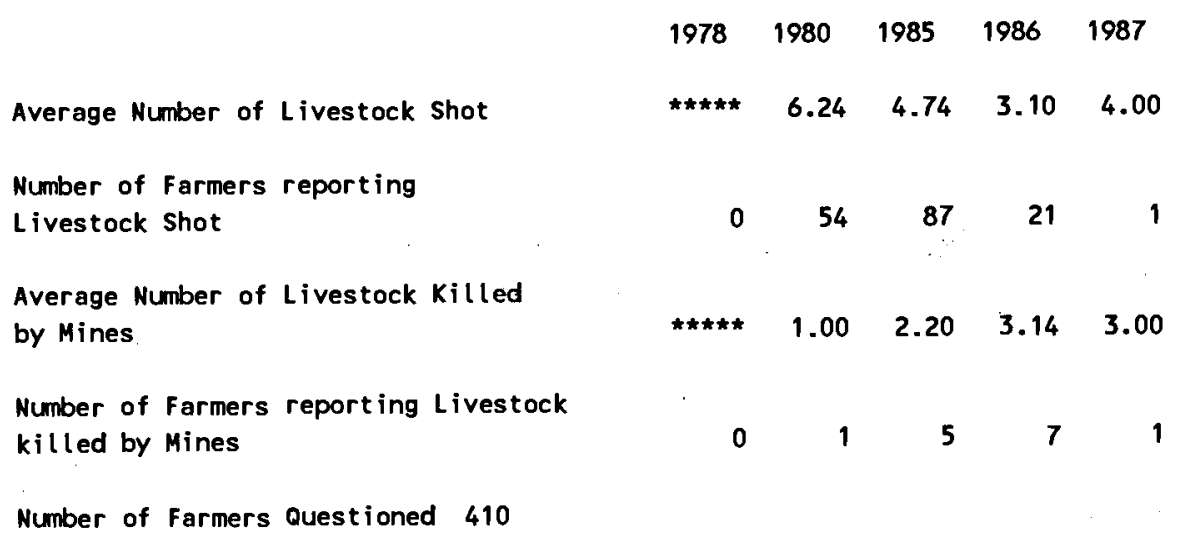

IABLE 9B - DIRECT EFFECTS OF THE WAR - FARMERS IN CAMPS

PROVINCE OF BYN

Average Number of Livestock Shot

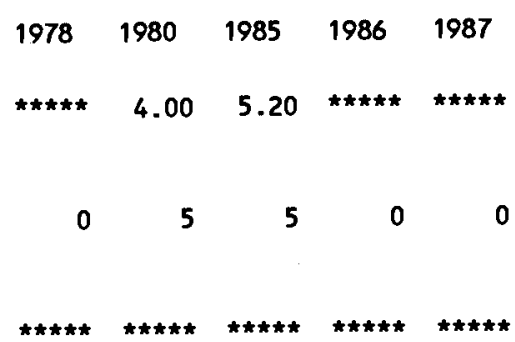

Average Number of Livestock Killed by Mines

Number of Farmers reporting Livestock killed by Mines

$\begin{array}{lllll}0 & 0 & 0 & 0 & 0\end{array}$

Number of Farmers Questioned 20 


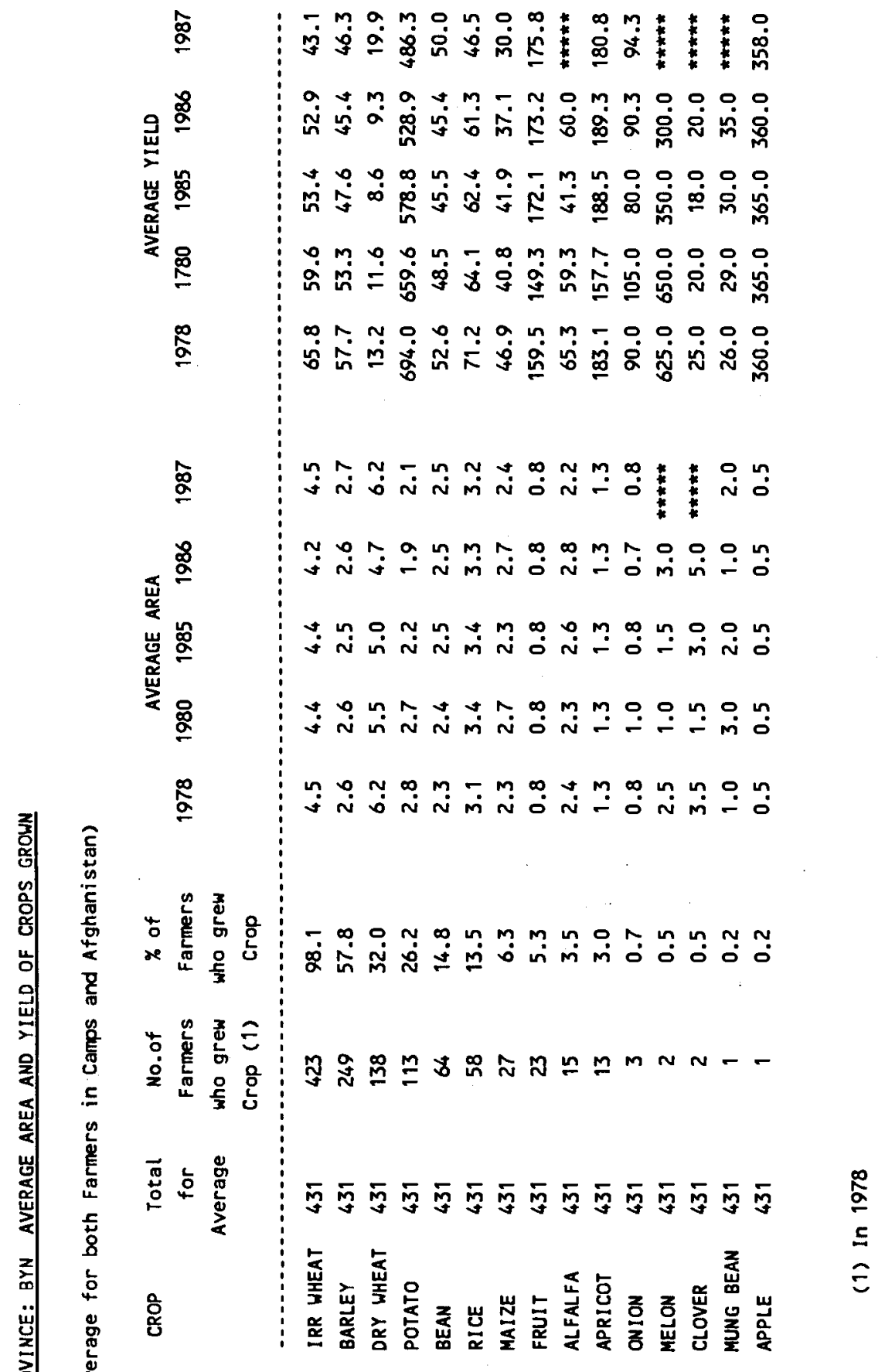


IABLE 1-5 - ANALYSIS OF GREATEST FARMING PROBLEMS - COMMUNITY SURVEY

PROVINCE OF BYN

\section{PERCENTAGE OF VILLAGES GIVING FOLLOWING PRIORITY TO PROBLEM}

Rainfall

Crop Disease

Birds or Rats

Seed Availability

Fertilizer Availability

Crop Protection Chemicals

Credit

Irrigation Water Availability

Flooding

Labour Availabilty

Power for Land Preparation

Direct War Effects

Animal Diseases

Extension

Other Problems

$\begin{array}{rrrrrrrrrrrrr}1 & 2 & 3 & 4 & 5 & 6 & 7 & 8 & 9 & 10 & 11 & 12 & \text { Weighted Priority } \\ 1 & 0 & 0 & 0 & 0 & 0 & 0 & 0 & 0 & 0 & 0 & 0 & 0.45 \\ 82 & 11 & 1 & 0 & 0 & 0 & 0 & 0 & 0 & 0 & 0 & 0 & 38.58 \\ 1 & 5 & 2 & 8 & 19 & 13 & 5 & 5 & 1 & 0 & 0 & 0 & 6.01 \\ 0 & 0 & 0 & 0 & 0 & 0 & 0 & 1 & 0 & 0 & 0 & 0 & 0.06 \\ 0 & 2 & 16 & 10 & 2 & 0 & 0 & 0 & 0 & 0 & 0 & 0 & 4.05 \\ 1 & 11 & 8 & 22 & 16 & 0 & 1 & 0 & 0 & 0 & 0 & 0 & 7.99 \\ 0 & 0 & 0 & 0 & 0 & 0 & 0 & 0 & 0 & 0 & 0 & 0 & 0.00 \\ 0 & 2 & 5 & 2 & 1 & 0 & 0 & 0 & 0 & 0 & 0 & 0 & 1.53 \\ 7 & 18 & 14 & 7 & 3 & 2 & 1 & 0 & 1 & 0 & 0 & 0 & 10.36 \\ 0 & 3 & 0 & 0 & 1 & 0 & 0 & 0 & 0 & 0 & 0 & 0 & 0.77 \\ 0 & 17 & 38 & 16 & 2 & 1 & 0 & 0 & 0 & 0 & 0 & 0 & 11.06 \\ 2 & 3 & 3 & 1 & 0 & 0 & 0 & 0 & 0 & 0 & 0 & 0 & 2.16 \\ 2 & 9 & 3 & 5 & 6 & 5 & 1 & 0 & 0 & 0 & 0 & 0 & 4.97 \\ 2 & 2 & 0 & 3 & 4 & 2 & 3 & 0 & 0 & 0 & 0 & 0 & 2.42 \\ 1 & 15 & 13 & 19 & 13 & 9 & 5 & 0 & 0 & 0 & 0 & 0 & 9.60\end{array}$

$\underline{1986}$

Rainfall

Crop Disease

Birds or Rats

Seed Availability

Fertilizer Availability

Crop Protection Chemicals

Credit

Irrigation Water Availability

Flooding

Labour Availabilty

Power for Land Preparation

Direct War Effects

Animal Diseases

Extension

other Problems

\begin{tabular}{rrrrrrrrrrrrr}
1 & 2 & 3 & 4 & 5 & 6 & 7 & 8 & 9 & 10 & 11 & 12 & Weighted Priority \\
\hline 5 & 25 & 13 & 2 & 0 & 0 & 0 & 0 & 0 & 0 & 0 & 0 & 10.04 \\
5 & 7 & 4 & 6 & 10 & 7 & 1 & 0 & 0 & 0 & 0 & 0 & 6.84 \\
0 & 7 & 7 & 15 & 5 & 4 & 11 & 2 & 0 & 0 & 0 & 0 & 5.99 \\
0 & 0 & 0 & 2 & 0 & 0 & 0 & 0 & 0 & 0 & 0 & 0 & 0.23 \\
0 & 3 & 7 & 8 & 3 & 1 & 0 & 0 & 0 & 0 & 0 & 0 & 3.08 \\
0 & 4 & 6 & 20 & 17 & 13 & 0 & 0 & 0 & 0 & 0 & 0 & 6.51 \\
0 & 0 & 0 & 0 & 0 & 0 & 0 & 0 & 0 & 0 & 0 & 0 & 0.00 \\
8 & 17 & 15 & 1 & 2 & 0 & 0 & 0 & 0 & 0 & 0 & 0 & 9.95 \\
0 & 0 & 0 & 4 & 0 & 0 & 0 & 1 & 0 & 0 & 0 & 0 & 0.53 \\
2 & 0 & 0 & 0 & 1 & 0 & 0 & 0 & 0 & 0 & 0 & 0 & 1.03 \\
0 & 2 & 29 & 28 & 5 & 0 & 0 & 0 & 0 & 0 & 0 & 0 & 8.44 \\
9 & 4 & 3 & 0 & 1 & 0 & 0 & 0 & 0 & 0 & 0 & 0 & 5.70 \\
24 & 16 & 7 & 4 & 1 & 1 & 0 & 0 & 0 & 0 & 0 & 0 & 15.97 \\
7 & 1 & 0 & 1 & 1 & 3 & 3 & 2 & 0 & 0 & 0 & 0 & 4.26 \\
35 & 13 & 7 & 4 & 6 & 4 & 4 & 1 & 0 & 0 & 0 & 0 & 21.43
\end{tabular}

1985

Rainfall

Crop Disease

Birds or Rats

Seed Availability

Fertilizer Availability

Crop Protection Chemicals

Credit

Irrigation Water Availability

Flooding

Labour Availabilty

Power for Land Preparation

Direct War Effects

Animal Diseases

Extension

other Problems

$\begin{array}{rrrrrrrrrrrrr}1 & 2 & 3 & 4 & 5 & 6 & 7 & 8 & 9 & 10 & 11 & 12 & \text { Weighted Priority } \\ 16 & 13 & 16 & 0 & 1 & 0 & 0 & 0 & 0 & 0 & 0 & 0 & 12.78 \\ 6 & 11 & 16 & 10 & 7 & 3 & 1 & 0 & 0 & 0 & 0 & 0 & 10.27 \\ 2 & 3 & 4 & 14 & 7 & 4 & 5 & 3 & 0 & 0 & 0 & 0 & 5.48 \\ 0 & 0 & 1 & 0 & 0 & 0 & 1 & 0 & 0 & 0 & 0 & 0 & 0.23 \\ 0 & 2 & 3 & 2 & 1 & 0 & 0 & 0 & 0 & 0 & 0 & 0 & 1.32 \\ 0 & 2 & 11 & 6 & 10 & 6 & 2 & 0 & 0 & 0 & 0 & 0 & 4.61 \\ 0 & 0 & 0 & 0 & 0 & 0 & 0 & 0 & 0 & 0 & 0 & 0 & 0.00 \\ 6 & 10 & 10 & 14 & 0 & 0 & 0 & 0 & 0 & 0 & 0 & 0 & 8.58 \\ 1 & 2 & 1 & 1 & 0 & 0 & 1 & 0 & 0 & 0 & 0 & 0 & 1.33 \\ 0 & 0 & 0 & 1 & 0 & 1 & 0 & 0 & 0 & 0 & 0 & 0 & 0.20 \\ 0 & 2 & 7 & 10 & 3 & 1 & 0 & 1 & 0 & 0 & 0 & 0 & 3.28 \\ 32 & 7 & 11 & 1 & 0 & 1 & 0 & 0 & 0 & 0 & 0 & 0 & 18.82 \\ 16 & 14 & 5 & 2 & 0 & 3 & 1 & 1 & 1 & 0 & 0 & 0 & 11.97 \\ 1 & 1 & 3 & 4 & 1 & 1 & 3 & 0 & 1 & 0 & 0 & 0 & 2.15 \\ 20 & 29 & 5 & 7 & 3 & 8 & 2 & 2 & 0 & 0 & 0 & 0 & 18.97\end{array}$


PROVINCE OF BYN

Rainfall

Crop Disease

Birds or Rats

Seed Availability

Fertilizer Availability

Crop Protection Chemicals

Credit

Irrigation Water Availability

Flooding

Direct War Effects

Animal Diseases

Extension
Labour Availabilty

Power for Land Preparation

other Problems

\begin{tabular}{rrrrrrrrrrrrc}
1 & 2 & 3 & 4 & 5 & 6 & 7 & 8 & 9 & 10 & 11 & 12 & Heighted Priority \\
\hline 0 & 6 & 8 & 4 & 1 & 0 & 0 & 0 & 0 & 0 & 0 & 0 & 3.73 \\
9 & 7 & 1 & 3 & 10 & 4 & 2 & 0 & 0 & 0 & 0 & 0 & 8.99 \\
5 & 11 & 8 & 1 & 3 & 6 & 4 & 6 & 0 & 0 & 0 & 0 & 8.88 \\
0 & 0 & 0 & 0 & 0 & 0 & 0 & 0 & 0 & 0 & 0 & 0 & 0.00 \\
0 & 1 & 0 & 1 & 0 & 1 & 0 & 0 & 0 & 0 & 0 & 0 & 0.50 \\
0 & 5 & 20 & 10 & 5 & 5 & 0 & 0 & 0 & 0 & 0 & 0 & 7.16 \\
0 & 0 & 0 & 0 & 0 & 0 & 0 & 0 & 0 & 0 & 0 & 0 & 0.00 \\
0 & 3 & 6 & 2 & 0 & 0 & 0 & 0 & 0 & 0 & 0 & 0 & 2.17 \\
7 & 6 & 3 & 1 & 1 & 2 & 0 & 1 & 0 & 0 & 0 & 0 & 6.47 \\
1 & 1 & 1 & 1 & 0 & 3 & 0 & 0 & 0 & 0 & 0 & 0 & 1.40 \\
0 & 0 & 0 & 1 & 2 & 0 & 0 & 0 & 0 & 0 & 0 & 0 & 0.35 \\
19 & 18 & 5 & 3 & 0 & 1 & 0 & 0 & 0 & 0 & 0 & 0 & 15.81 \\
21 & 20 & 1 & 1 & 1 & 0 & 3 & 0 & 0 & 0 & 0 & 0 & 16.70 \\
0 & 0 & 4 & 1 & 3 & 3 & 4 & 0 & 1 & 0 & 0 & 0 & 1.83 \\
39 & 16 & 6 & 3 & 2 & 1 & 0 & 0 & 0 & 0 & 0 & 0 & 26.00
\end{tabular}

1978

Rainfall

Crop Disease

Birds or Rats

Seed Availability

Fertilizer Availability

Crop Protection Chemicals

Credit

Irrigation Water Availability

flooding

Labour Availabilty

Power for Land Preparation

Direct War Effects

Animal Diseases

Extension

Other Problems

\begin{tabular}{rrrrrrrrrrrrr}
1 & 2 & 3 & 4 & 5 & 6 & 7 & 8 & 9 & 10 & 11 & 12 & Weighted Priority \\
\hline 0 & 3 & 1 & 3 & 0 & 0 & 0 & 0 & 0 & 0 & 0 & 0 & 2.36 \\
10 & 9 & 6 & 2 & 0 & 1 & 0 & 0 & 0 & 0 & 0 & 0 & 15.69 \\
32 & 4 & 7 & 7 & 10 & 2 & 0 & 0 & 0 & 0 & 0 & 0 & 36.47 \\
0 & 0 & 0 & 0 & 0 & 0 & 0 & 0 & 0 & 0 & 0 & 0 & 0.00 \\
0 & 0 & 0 & 0 & 1 & 0 & 0 & 0 & 0 & 0 & 0 & 0 & 0.18 \\
0 & 14 & 6 & 6 & 1 & 0 & 0 & 0 & 0 & 0 & 0 & 0 & 9.32 \\
0 & 0 & 0 & 0 & 0 & 0 & 0 & 0 & 0 & 0 & 0 & 0 & 0.00 \\
0 & 2 & 3 & 2 & 0 & 0 & 0 & 0 & 0 & 0 & 0 & 0 & 2.28 \\
2 & 1 & 0 & 0 & 0 & 0 & 0 & 0 & 0 & 0 & 0 & 0 & 2.28 \\
0 & 0 & 1 & 0 & 0 & 0 & 0 & 0 & 0 & 0 & 0 & 0 & 0.30 \\
0 & 0 & 0 & 0 & 0 & 0 & 0 & 0 & 0 & 0 & 0 & 0 & 0.00 \\
0 & 0 & 0 & 1 & 0 & 0 & 0 & 0 & 0 & 0 & 0 & 0 & 0.23 \\
9 & 3 & 0 & 2 & 0 & 0 & 1 & 0 & 0 & 0 & 0 & 0 & 10.18 \\
10 & 2 & 4 & 1 & 0 & 0 & 0 & 0 & 0 & 0 & 0 & 0 & 11.50 \\
7 & 3 & 3 & 0 & 2 & 1 & 0 & 0 & 0 & 0 & 0 & 0 & 9.20
\end{tabular}


FARAH 


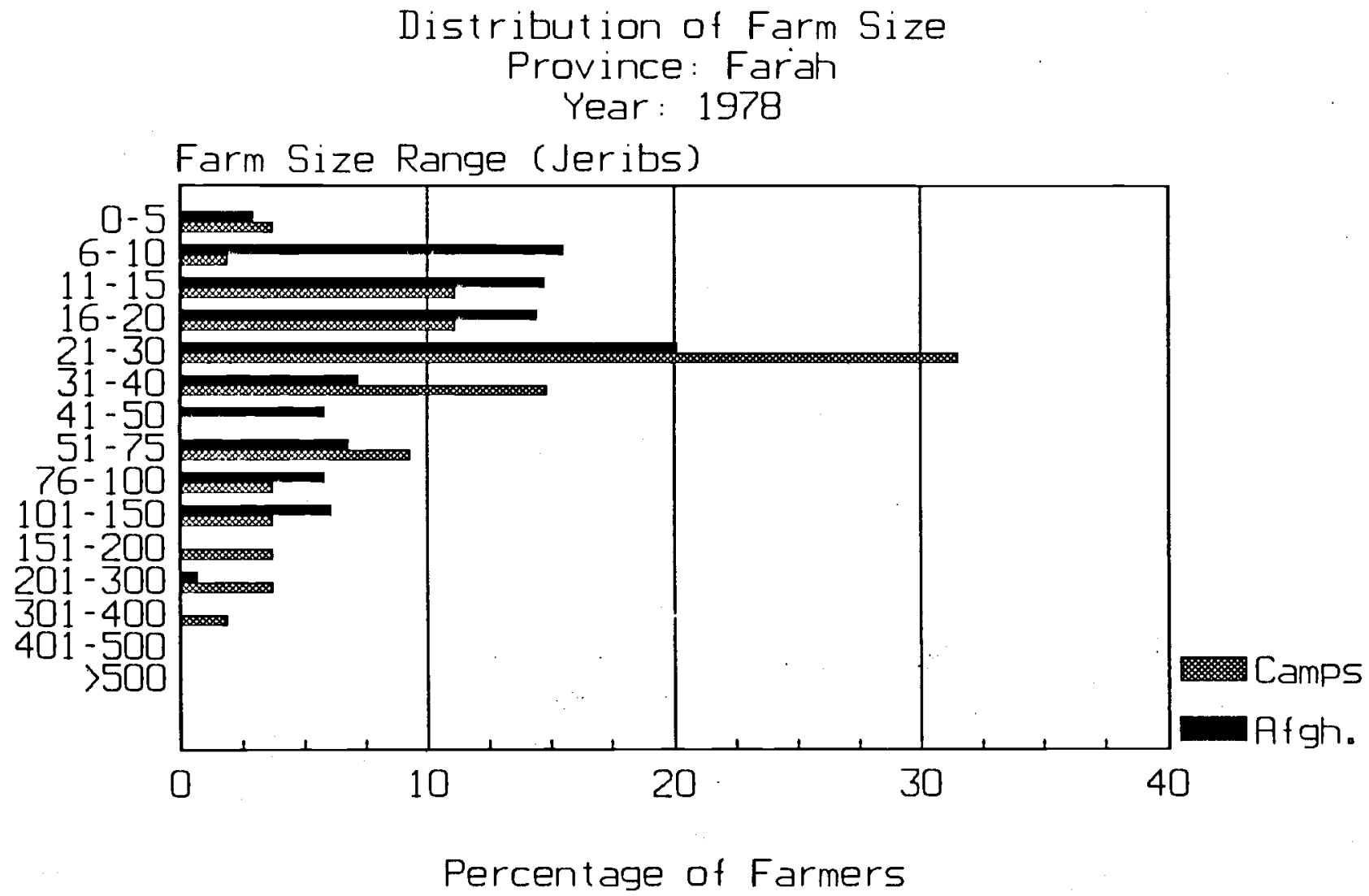

Farmers in Afghanistan

1978

1987

Total Number of Farms

278

Average Area

35.48

17.81

Average Area Irrigated

35.28

17.79

Average Dryland Area

11.00

9.20

Farmers who left in 1987

Total Number of Farms

Average Area

Average Area Irrigated

Average Dryland Area

$\begin{array}{rl}54 & 0 \\ 51.43 & - \\ 50.98 & - \\ 8.00 & -\end{array}$

Average of Both Groups

Provincial Average Area
Provincial Average Irrigated Area
Provincial Average Dryland Area

38.07

17.81

37.83

17.79

9.88

9.20

(All areas in Jeribs. 5 Jeribs $=1$ Hectare) 
Farm Power - Farmers in Alghanistan Province of Farah Crop: Irrigated wheat

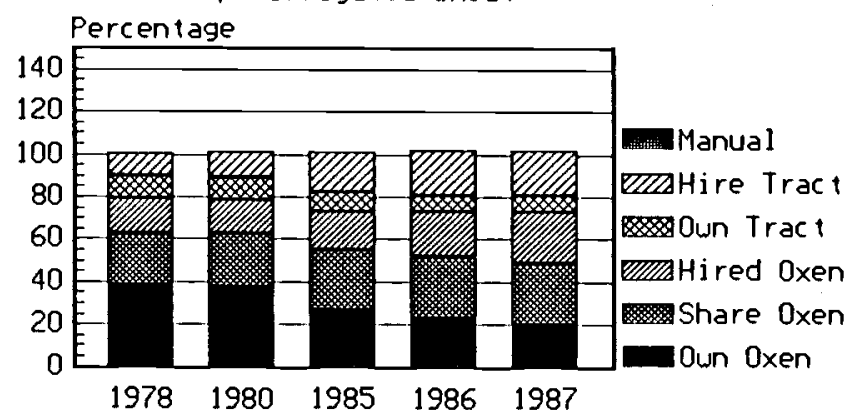

Year

Farm Power - Farmers who left in 1987 Province of Farah Crop: Irrigated wheat

Percentage

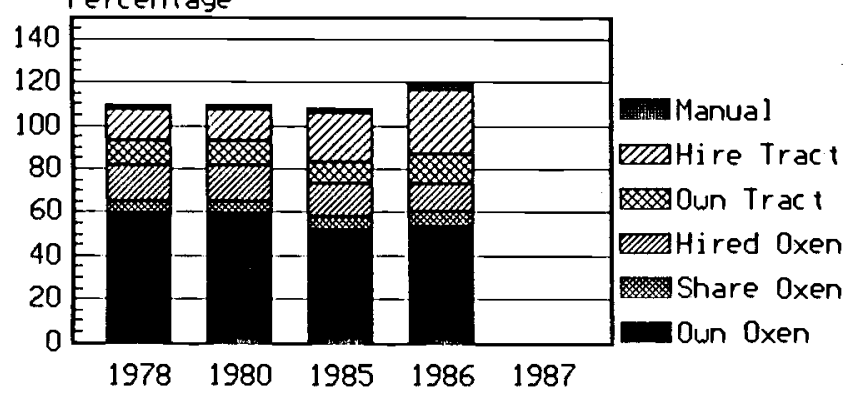

Year

Base Figures for above graphs

Province: Farah

\section{Farmers in Afghanistan}

Irr. Wheat own oxen

Shared oxen

Hired oxen

Own Tractor

Hired Tractor

Maize

Manual Means

Own Oxen

Shared oxen

Hired oxen

Own Tractor

Hired Tractor

Manual Means

Farmers who left in 1987
Irr. Wheat own oxen

Shared oxen

Hired oxen

Own Tractor

Hired Tractor

Maize
Manual Means

Own Oxen

Shared oxen

Hired oxen

own Tractor
Farm Power - Farmers in Atghanistan

Province of Farah Crop: Maize

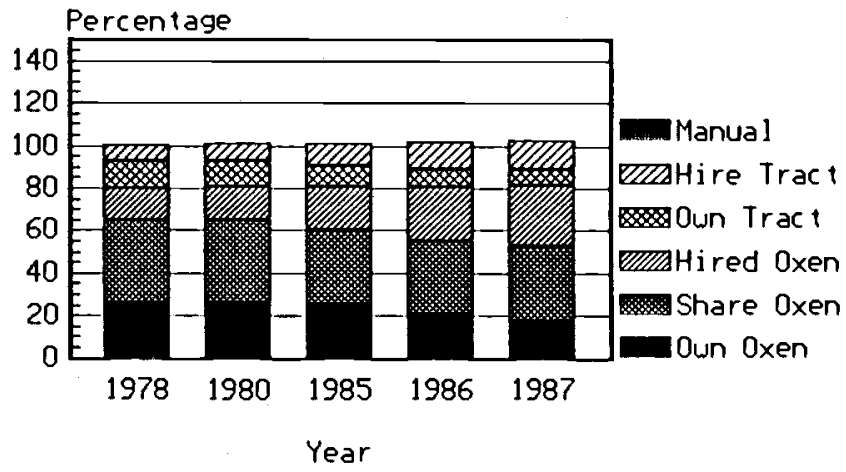

Farm Power - Farmers who lett in 1987 Province of Farah Crop: Maize

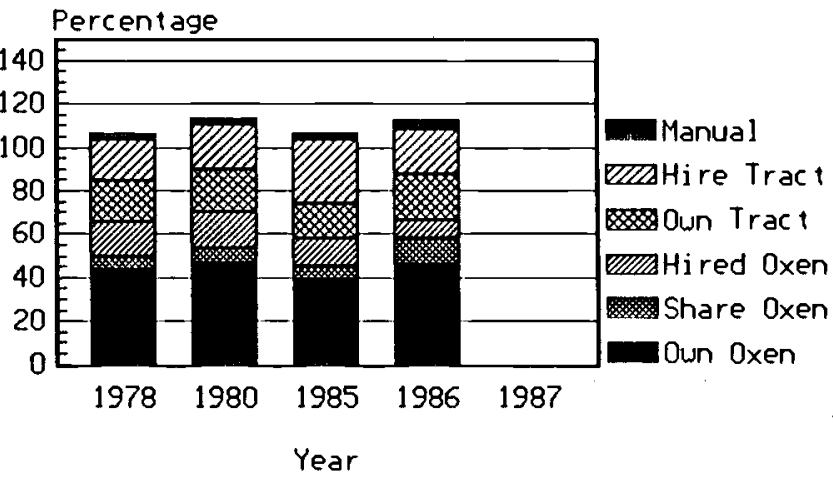

Percentage of Farmers

$1978 \quad \underline{1980} \quad \underline{1985} \quad \underline{1986} \quad \underline{1987}$

$\begin{array}{ccccc}38.41 & 37.73 & 26.94 & 23.15 & 20.00 \\ 24.28 & 24.54 & 27.85 & 28.70 & 29.23 \\ 16.30 & 16.48 & 18.26 & 21.30 & 24.10 \\ 10.51 & 10.26 & 9.13 & 7.41 & 7.18 \\ 11.23 & 12.09 & 19.18 & 21.30 & 21.03 \\ - & - & - & - & - \\ 26.32 & 26.32 & 25.33 & 21.09 & 17.56 \\ 38.16 & 38.16 & 34.67 & 34.01 & 35.11 \\ 15.75 & 16.45 & 20.67 & 25.85 & 29.01 \\ 12.50 & 11.84 & 10.00 & 8.16 & 7.63 \\ 7.89 & 8.55 & 10.67 & 12.93 & 12.98 \\ - & - & - & - & -\end{array}$

59.26

59.26

51.92

53.33

5.56

5.56

5.77

6.67

16.67

16.67

$11 \cdot 11$

11.11

15.38

13.33

14.81

1.85

14.81

1.85

9.62

13.33

$23.08 \quad 30.00$

1.92

3.33

43.75

46.67

6.25

6.67

38.71

45.83

6.45

12.50

15.63

16.67

12.90

20.00

16.13

8.33

20.83 
Farah

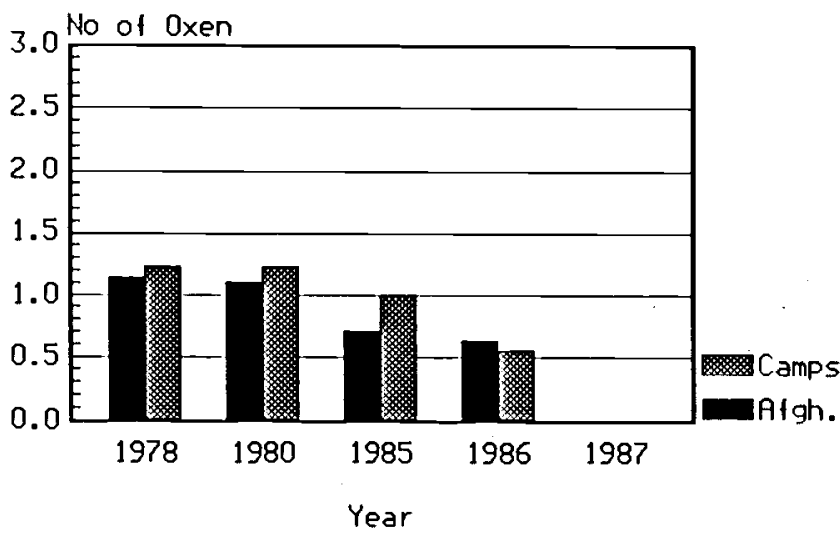

Farah

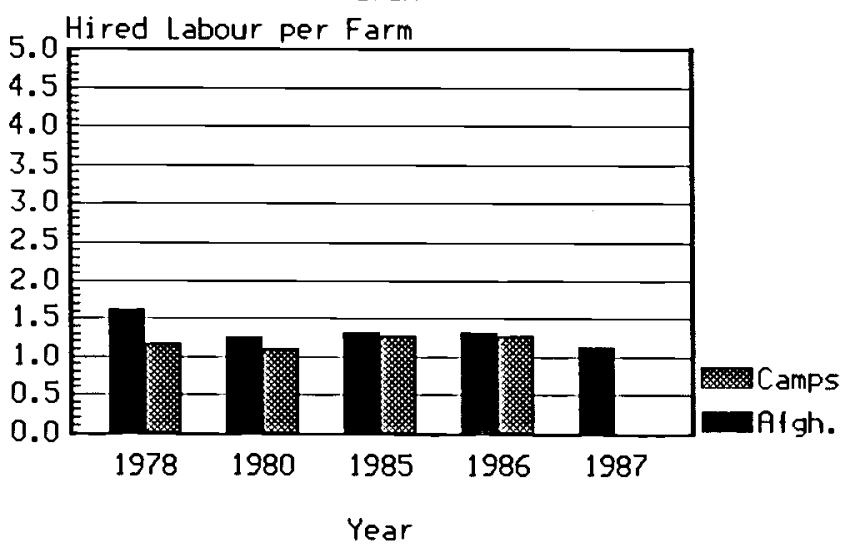

Farah

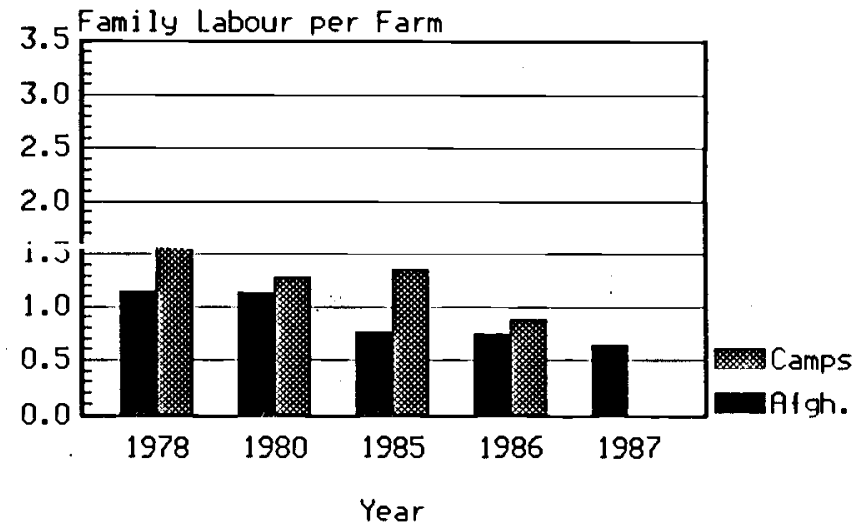

Farah

Percentage of Farms using

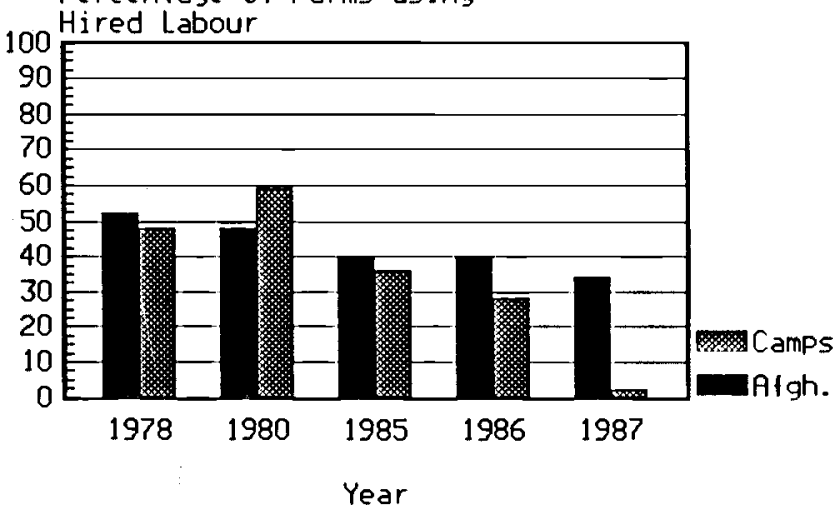


PROVINCE OF FRA

YEAR

1978 Number of Families

\% of Families

1980 Number of Families

$\%$ of Families

1985 Number of Families

$\%$ of Families

1986 Number of Families

$\%$ of Families

1987 Number of Fanilies

\% of Families

Average Number of oxen per Family:

1978

1980

1985

1986

1987
NUMBER OF TRAINED OXEN PER FAMILY

$\begin{array}{llllll}99 & 71 & 78 & 4 & 15 & 1\end{array}$

$\begin{array}{llllll}36.94 & 26.49 & 29.10 & 1.49 & 5.60 & 0.37\end{array}$

$\begin{array}{lllll}103 & 70 & 75 & 5 & 13\end{array}$

$\begin{array}{llllll}38.58 & 26.22 & 28.09 & 1.87 & 4.87 & 0.37\end{array}$

$\begin{array}{llllll}158 & 60 & 43 & 5 & 6 & 1\end{array}$

$\begin{array}{lllllll}57.88 & 21.98 & 15.75 & 1.83 & 2.20 & 0.37\end{array}$

$\begin{array}{llllll}165 & 62 & 36 & 5 & 4 & 1\end{array}$

$\begin{array}{llllll}60.44 & 22.71 & 13.19 & 1.83 & 1.47 & 0.37\end{array}$

$\begin{array}{llllll}182 & 56 & 26 & 5 & 4 & 2\end{array}$

$\begin{array}{llllll}66.18 & 20.36 & 9.45 & 1.82 & 1.45 & 0.73\end{array}$

$\begin{array}{ll}978 & 1.13 \\ 980 & 1.09 \\ 985 & 0.70 \\ 986 & 0.62 \\ 987 & 0.54\end{array}$

Number of Farmers Questioned 277

\section{TABLE 7B - AVERAGE MUMBER OF TRAINED OXEN FOR FARMERS IN THE CAMPS}

PROVINCE OF FRA

YEAR

1978 Number of Families

\% of Families

1980 Number of Families

$\%$ of Families

1985 Number of Families

$\%$ of Families

1986 Number of Fanilies

$\%$ of Families

1987 Number of Families

$\%$ of Families

Average Number of Oxen per Family:

$\begin{array}{ll}1978 & 1.23 \\ 1980 & 1.23 \\ 1985 & 1.00 \\ 1986 & 0.54 \\ 1987 & 0.02\end{array}$

Number of Farmers Questioned 58

\begin{tabular}{|c|c|c|c|c|c|}
\hline \multicolumn{2}{|c|}{ NUMBER OF } & \multirow{2}{*}{$\frac{\text { IRAINED }}{2}$} & \multirow{2}{*}{$\frac{\text { OXEN }}{3}$} & \multicolumn{2}{|c|}{ FAMILY } \\
\hline 0 & 1 & & & 4 & 5 \\
\hline 20 & 6 & 28 & 1 & 1 & 0 \\
\hline 35.71 & 10.71 & 50.00 & 1.79 & 1.79 & 0.00 \\
\hline 20 & 6 & 28 & 1 & 1 & 0 \\
\hline 35.71 & 10.71 & 50.00 & 1.79 & 1.79 & 0.00 \\
\hline 26 & 6 & 23 & 0 & 1 & 0 \\
\hline 46.43 & 10.71 & 41.07 & 0.00 & 1.79 & 0.00 \\
\hline 40 & 3 & 12 & 1 & 0 & 0 \\
\hline 71.43 & 5.36 & 21.43 & 1.79 & 0.00 & 0.00 \\
\hline 57 & 1 & 0 & 0 & 0 & 0 \\
\hline 8.28 & 1.72 & 0.00 & 0.00 & 0.00 & 0.00 \\
\hline
\end{tabular}




\section{IABLE $8(A)$ - AVERAGE LABOUR (FAMILY and HIRED) FOR FARMERS IN AFGANISTAN}

PROVINCE OF FRA

\begin{tabular}{|c|c|c|c|c|}
\hline & 1978 & 1980 & 1985 & 1986 \\
\hline Average Family Labour per Farm & 1.14 & 1.13 & 0.75 & 0.74 \\
\hline $\begin{array}{l}\text { Average No. of Hired Labour per Farm. } \\
\text { (for farms using hired labour) }\end{array}$ & 1.61 & 1.24 & 1.32 & 1.31 \\
\hline Number of Farms using Hired Labour & 143 & 132 & 111 & 110 \\
\hline
\end{tabular}

IABLE 8(B) - AVERAGE LABOUR (FAMILY and HIRED) FOR FARMERS IN CAMPS

PROVINCE OF FRA

\begin{tabular}{|c|c|c|c|c|c|}
\hline & 1978 & 1980 & 1985 & 1986 & 1987 \\
\hline Average Family Labour per Farm & 1.58 & 1.28 & 1.35 & 0.88 & 0.03 \\
\hline $\begin{array}{l}\text { Average No. of Hired Labour per Farm } \\
\text { (for farms using hired labour) }\end{array}$ & 1.17 & 1.11 & 1.27 & 1.28 & 1.50 \\
\hline Number of Farms using Hired Labour & 28 & 34 & 21 & 16 & 1 \\
\hline
\end{tabular}

Number of Farmers Questioned $\mathbf{5 8}$ 


\section{IABLE 9A - DIRECT EFFECTS OF THE WAR - FARMERS IN AFGANISTAN}

PROVINCE OF FRA

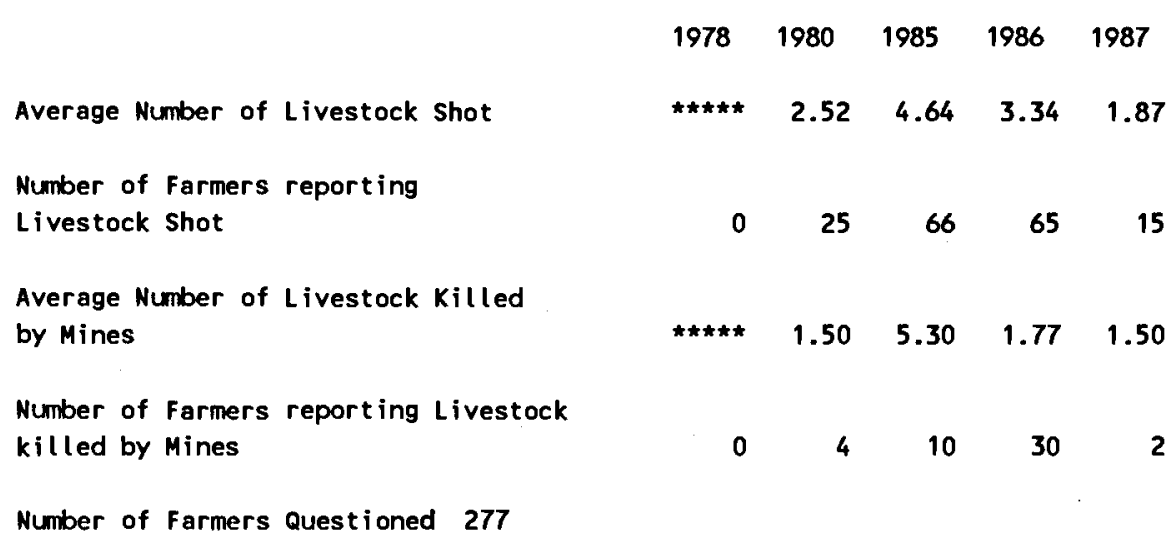

\section{IABLE 9B - DIRECT EFFECTS OF THE WAR - FARMERS IN CAMPS}

PROVINCE OF FRA

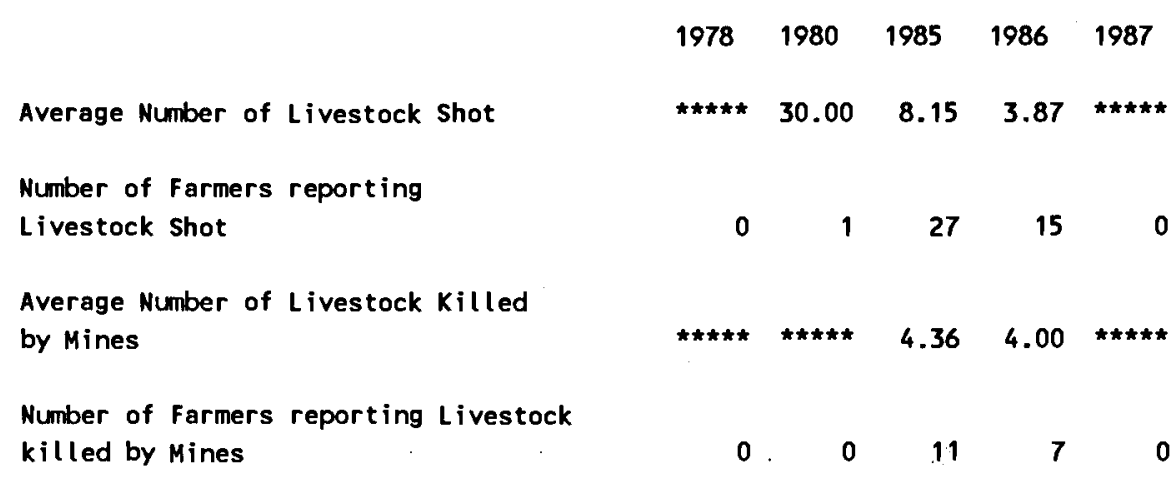

Number of Farmers Questioned 58 


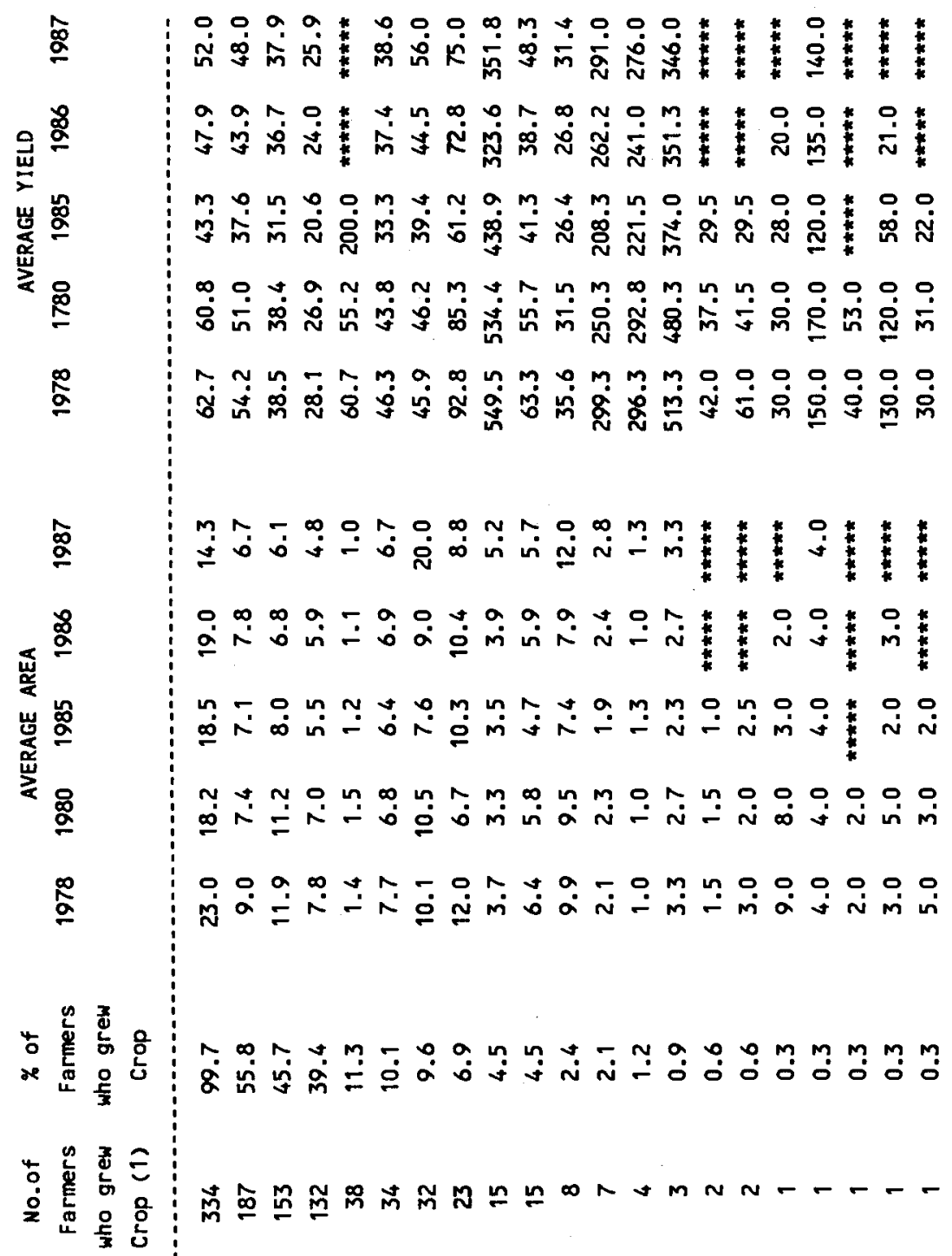
空京兽

웅

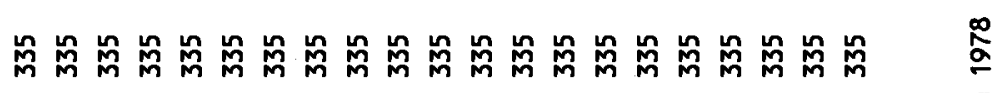

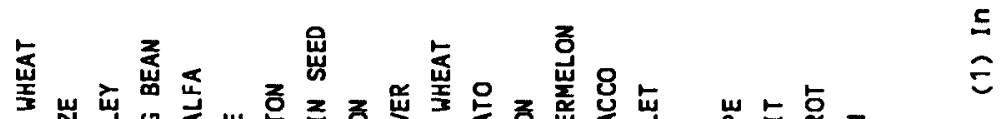


IABLE 1-5 - ANALYSIS OF GREATEST FARMING PROBLEMS - COMMUNITY SURVEY

PROVINCE OF FRA

1987 PERCENTAGE OF VILLAGES GIVING FOLLOWING PRIORITY TO PROBLEM

Rainfall

Crop Disease

Birds or Rats

Seed Availability

Fertilizer Availability

Crop Protection Chemicals

Credit

Irrigation Water Availability

flooding

Labour Availabilty

Power for Land Preparation

Direct War Effects

Animal Diseases

Extension

other Problems

\begin{tabular}{rrrrrrrrrrrrr}
1 & 2 & 3 & 4 & 5 & 6 & 7 & 8 & 9 & 10 & 11 & 12 & Weighted Priority \\
\hline 0 & 0 & 0 & 0 & 0 & 0 & 0 & 0 & 0 & 0 & 0 & 0 & 0.00 \\
0 & 0 & 0 & 4 & 0 & 0 & 0 & 0 & 0 & 0 & 0 & 0 & 0.45 \\
4 & 6 & 19 & 31 & 0 & 0 & 0 & 0 & 0 & 0 & 0 & 0 & 10.29 \\
0 & 2 & 4 & 2 & 1 & 0 & 0 & 0 & 0 & 0 & 0 & 0 & 1.60 \\
34 & 13 & 1 & 0 & 0 & 0 & 0 & 0 & 0 & 0 & 0 & 0 & 20.08 \\
0 & 4 & 8 & 0 & 0 & 0 & 0 & 0 & 0 & 0 & 0 & 0 & 2.28 \\
2 & 18 & 19 & 2 & 4 & 0 & 0 & 0 & 0 & 0 & 0 & 0 & 9.46 \\
1 & 10 & 10 & 16 & 5 & 0 & 0 & 0 & 0 & 0 & 0 & 0 & 6.95 \\
0 & 0 & 0 & 0 & 0 & 0 & 0 & 0 & 0 & 0 & 0 & 0 & 0.00 \\
4 & 8 & 23 & 7 & 2 & 0 & 0 & 0 & 0 & 0 & 0 & 0 & 8.74 \\
2 & 14 & 6 & 2 & 1 & 0 & 0 & 0 & 0 & 0 & 0 & 0 & 6.15 \\
53 & 23 & 5 & 1 & 0 & 0 & 0 & 0 & 0 & 0 & 0 & 0 & 32.69 \\
0 & 0 & 0 & 0 & 1 & 0 & 0 & 0 & 0 & 0 & 0 & 0 & 0.12 \\
0 & 0 & 4 & 5 & 0 & 0 & 0 & 0 & 0 & 0 & 0 & 0 & 1.19 \\
0 & 0 & 0 & 0 & 0 & 0 & 0 & 0 & 0 & 0 & 0 & 0 & 0.00
\end{tabular}

1986

Rainfall

Crop Disease

Birds or Rats

Seed Availability

Fertilizer Availability

Crop Protection Chemicals

Credit

Irrigation Hater Avai lability

flooding

Labour Availabilty

Power for Land Preparation

Direct War Effects

Animal Diseases

Extension

Other Problems

\begin{tabular}{rrrrrrrrrrrrr}
1 & 2 & 3 & 4 & 5 & 6 & 7 & 8 & 9 & 10 & 11 & 12 & Weighted Priority \\
\hline 0 & 1 & 0 & 0 & 0 & 0 & 0 & 0 & 0 & 0 & 0 & 0 & 0.31 \\
0 & 0 & 4 & 0 & 0 & 0 & 0 & 0 & 0 & 0 & 0 & 0 & 0.62 \\
20 & 11 & 24 & 7 & 0 & 0 & 0 & 0 & 0 & 0 & 0 & 0 & 18.36 \\
4 & 0 & 1 & 0 & 0 & 0 & 0 & 0 & 0 & 0 & 0 & 0 & 2.06 \\
5 & 8 & 13 & 5 & 0 & 0 & 0 & 0 & 0 & 0 & 0 & 0 & 7.53 \\
13 & 4 & 1 & 0 & 0 & 0 & 0 & 0 & 0 & 0 & 0 & 0 & 7.94 \\
11 & 1 & 0 & 1 & 0 & 0 & 0 & 0 & 0 & 0 & 0 & 0 & 6.04 \\
2 & 13 & 4 & 1 & 2 & 0 & 0 & 0 & 0 & 0 & 0 & 0 & 5.66 \\
1 & 0 & 2 & 0 & 0 & 0 & 0 & 0 & 0 & 0 & 0 & 0 & 1.03 \\
13 & 1 & 5 & 4 & 0 & 0 & 0 & 0 & 0 & 0 & 0 & 0 & 8.41 \\
5 & 1 & 7 & 5 & 1 & 0 & 0 & 0 & 0 & 0 & 0 & 0 & 4.77 \\
19 & 47 & 12 & 1 & 1 & 0 & 0 & 0 & 0 & 0 & 0 & 0 & 24.32 \\
0 & 0 & 0 & 0 & 0 & 0 & 0 & 0 & 0 & 0 & 0 & 0 & 0.00 \\
6 & 13 & 24 & 16 & 0 & 0 & 0 & 0 & 0 & 0 & 0 & 0 & 1.2 .64 \\
0 & 0 & 0 & 2 & 0 & 0 & 0 & 0 & 0 & 0 & 0 & 0 & 0.31
\end{tabular}

1985

Rainfall

Crop Disease

Birds or Rats

Seed Availability

Fertilizer Availability

Crop Protection Chemicals

Credit

Irrigation Water Availability

Flooding

Labour Availabilty

Power for Land Preparation

Direct War Effects

Animal Diseases

Extension

Other Problems

\begin{tabular}{rrrrrrrrrrrrr}
1 & 2 & 3 & 4 & 5 & 6 & 7 & 8 & 9 & 10 & 11 & 12 & Heighted Priority \\
\hline 6 & 36 & 7 & 0 & 0 & 0 & 0 & 0 & 0 & 0 & 0 & 0 & 13.84 \\
2 & 0 & 0 & 1 & 0 & 0 & 0 & 0 & 0 & 0 & 0 & 0 & 1.42 \\
4 & 13 & 10 & 16 & 0 & 0 & 0 & 0 & 0 & 0 & 0 & 0 & 9.07 \\
0 & 1 & 0 & 0 & 0 & 0 & 0 & 0 & 0 & 0 & 0 & 0 & 0.31 \\
5 & 5 & 14 & 2 & 0 & 0 & 0 & 0 & 0 & 0 & 0 & 0 & 6.61 \\
1 & 1 & 0 & 0 & 0 & 0 & 0 & 0 & 0 & 0 & 0 & 0 & 0.94 \\
0 & 4 & 1 & 0 & 0 & 0 & 0 & 0 & 0 & 0 & 0 & 0 & 1.15 \\
73 & 22 & 2 & 0 & 0 & 0 & 0 & 0 & 0 & 0 & 0 & 0 & 44.47 \\
0 & 0 & 0 & 0 & 0 & 0 & 0 & 0 & 0 & 0 & 0 & 0 & 0.00 \\
0 & 0 & 4 & 1 & 0 & 0 & 0 & 0 & 0 & 0 & 0 & 0 & 0.79 \\
0 & 1 & 0 & 1 & 0 & 0 & 0 & 0 & 0 & 0 & 0 & 0 & 0.47 \\
7 & 11 & 43 & 8 & 0 & 0 & 0 & 0 & 0 & 0 & 0 & 0 & 15.26 \\
1 & 0 & 0 & 2 & 0 & 0 & 0 & 0 & 0 & 0 & 0 & 0 & 0.94 \\
0 & 5 & 12 & 6 & 0 & 0 & 0 & 0 & 0 & 0 & 0 & 0 & 4.14 \\
0 & 0 & 2 & 1 & 0 & 0 & 0 & 0 & 0 & 0 & 0 & 0 & 0.58
\end{tabular}


PROVINCE OF FRA

\section{PERCENTAGE OF VILLAGES GIVING FOLLOUING PRIORITY TO PROBLEM}

Rainfall

Crop 0 isease

Birds or Rats

Seed Availability

Fertilizer Availability

Crop Protection Chemicals Credit

Irrigation Water Avail ability

Flooding

Labour Avail labilty

Power for Land Preparation

Direct War Effects

Animal Oiseases

Extension

Other Problems

\begin{tabular}{rrrrrrrrrrrrr}
1 & 2 & 3 & 4 & 5 & 6 & 7 & 8 & 9 & 10 & 11 & 12 & Heighted Priority \\
\hline 0 & 0 & 0 & 0 & 0 & 0 & 0 & 0 & 0 & 0 & 0 & 0 & 0.00 \\
0 & 0 & 1 & 0 & 0 & 0 & 0 & 0 & 0 & 0 & 0 & 0 & 0.31 \\
14 & 13 & 4 & 1 & 0 & 0 & 0 & 0 & 0 & 0 & 0 & 0 & 17.58 \\
0 & 1 & 0 & 0 & 0 & 0 & 0 & 0 & 0 & 0 & 0 & 0 & 0.47 \\
0 & 7 & 4 & 0 & 0 & 0 & 0 & 0 & 0 & 0 & 0 & 0 & 3.76 \\
0 & 2 & 0 & 0 & 0 & 0 & 0 & 0 & 0 & 0 & 0 & 0 & 0.94 \\
19 & 5 & 1 & 0 & 0 & 0 & 0 & 0 & 0 & 0 & 0 & 0 & 17.19 \\
0 & 2 & 1 & 0 & 0 & 0 & 0 & 0 & 0 & 0 & 0 & 0 & 1.26 \\
24 & 6 & 4 & 1 & 0 & 0 & 0 & 0 & 0 & 0 & 0 & 0 & 22.27 \\
1 & 1 & 1 & 0 & 0 & 0 & 0 & 0 & 0 & 0 & 0 & 0 & 1.72 \\
0 & 0 & 0 & 0 & 0 & 0 & 0 & 0 & 0 & 0 & 0 & 0 & 0.00 \\
17 & 27 & 2 & 4 & 0 & 0 & 0 & 0 & 0 & 0 & 0 & 0 & 24.77 \\
0 & 0 & 0 & 0 & 0 & 0 & 0 & 0 & 0 & 0 & 0 & 0 & 0.00 \\
6 & 8 & 6 & 1 & 0 & 0 & 0 & 0 & 0 & 0 & 0 & 0 & 9.77 \\
0 & 0 & 0 & 0 & 0 & 0 & 0 & 0 & 0 & 0 & 0 & 0 & 0.00
\end{tabular}

1978

Rainfall

Crop 0 iseese

Birds or Rats

Seed Availability

Fertilizer Availability

Crop Protection Chemicals

Credit

Irrigation Water Availability

Flooding

Labour Availabilty

Power for Land Preparation

Oirect War Effects

Animal 0 iseases

Extension

other Problems

\begin{tabular}{rrrlllllllllr}
1 & 2 & 3 & 4 & 5 & 6 & 7 & 8 & 0 & 10 & 11 & 12 & Weighted Priority \\
\hline 0 & 0 & 0 & 0 & 0 & 0 & 0 & 0 & 0 & 0 & 0 & 0 & 0.00 \\
0 & 1 & 0 & 0 & 0 & 0 & 0 & 0 & 0 & 0 & 0 & 0 & 2.64 \\
6 & 2 & 0 & 0 & 0 & 0 & 0 & 0 & 0 & 0 & 0 & 0 & 31.72 \\
1 & 0 & 0 & 0 & 0 & 0 & 0 & 0 & 0 & 0 & 0 & 0 & 5.29 \\
1 & 0 & 1 & 0 & 0 & 0 & 0 & 0 & 0 & 0 & 0 & 0 & 7.05 \\
0 & 0 & 0 & 0 & 0 & 0 & 0 & 0 & 0 & 0 & 0 & 0 & 0.00 \\
2 & 2 & 0 & 0 & 0 & 0 & 0 & 0 & 0 & 0 & 0 & 0 & 15.86 \\
0 & 0 & 0 & 1 & 0 & 0 & 0 & 0 & 0 & 0 & 0 & 0 & 1.32 \\
1 & 0 & 0 & 0 & 0 & 0 & 0 & 0 & 0 & 0 & 0 & 0 & 5.29 \\
0 & 0 & 0 & 0 & 0 & 0 & 0 & 0 & 0 & 0 & 0 & 0 & 0.00 \\
0 & 0 & 0 & 0 & 0 & 0 & 0 & 0 & 0 & 0 & 0 & 0 & 0.00 \\
0 & 0 & 0 & 0 & 0 & 0 & 0 & 0 & 0 & 0 & 0 & 0 & 0.00 \\
0 & 1 & 0 & 0 & 0 & 0 & 0 & 0 & 0 & 0 & 0 & 0 & 2.64 \\
5 & 0 & 1 & 0 & 0 & 0 & 0 & 0 & 0 & 0 & 0 & 0 & 22.91 \\
1 & 0 & 0 & 0 & 0 & 0 & 0 & 0 & 0 & 0 & 0 & 0 & 5.29
\end{tabular}


FARYAB 


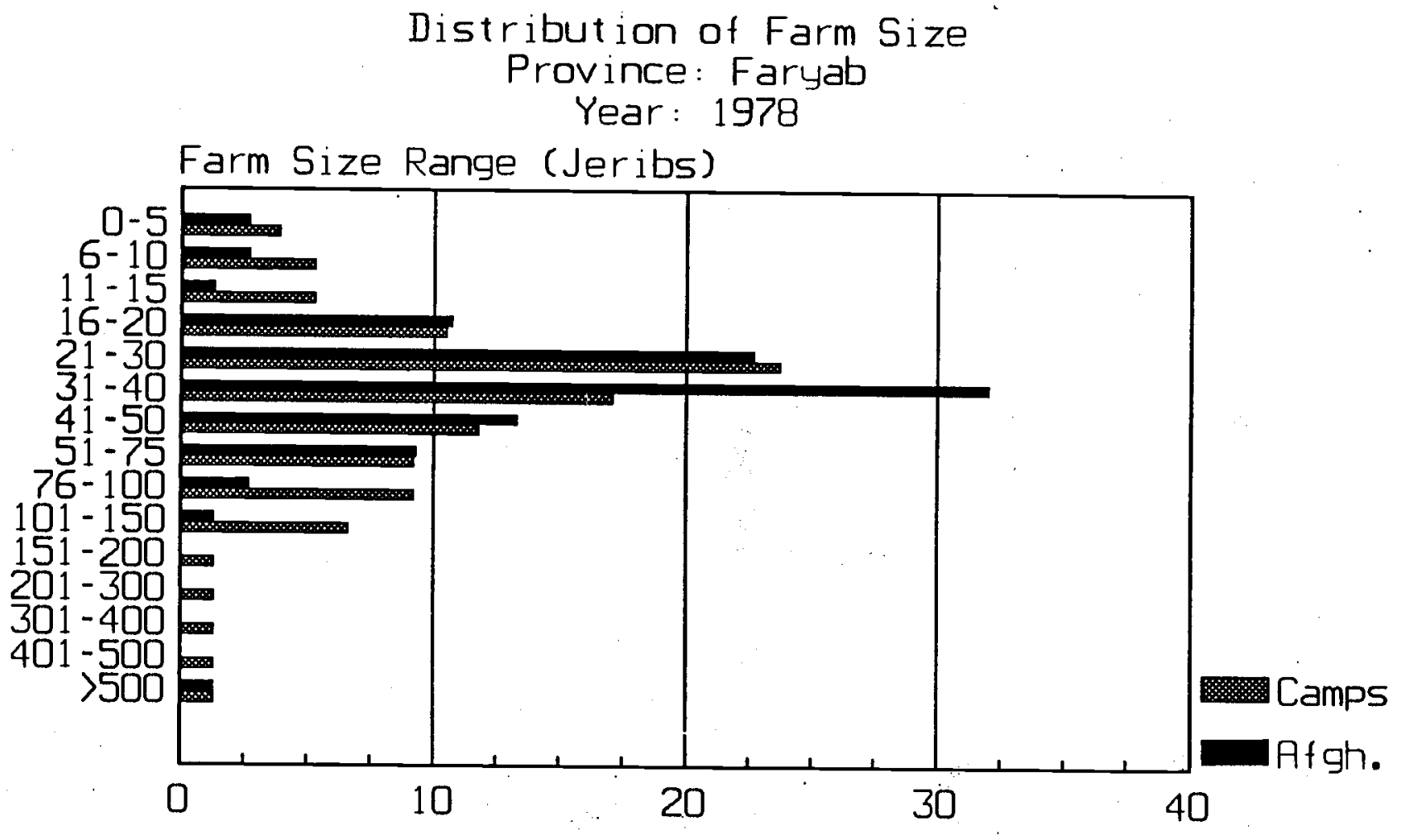

Percentage of. Farmers

Farmers in Afghanistan

Total Number of Farms

Average Area

Average Area Irrigated

Average Dryland Area

Farmers who left in 1987

Total Number of Farms

Average Area

Average Area Irrigated

Average Dryland Area

Average of Both Groups

Provincial Average Area

Provincial Average Irrigated Area

Provincial Average Dryland Area
1978

75

42.77

12.57

42.03

76

51.28

32.75

45.70

47.05

25.82

43.68

30.47

8.79

28.49

(All areas in Jeribs. 5 Jeribs $=1$ Hectare) 
Farm Power - Farmers in Atghanistan Province of Faryab Crop: Dry wheat

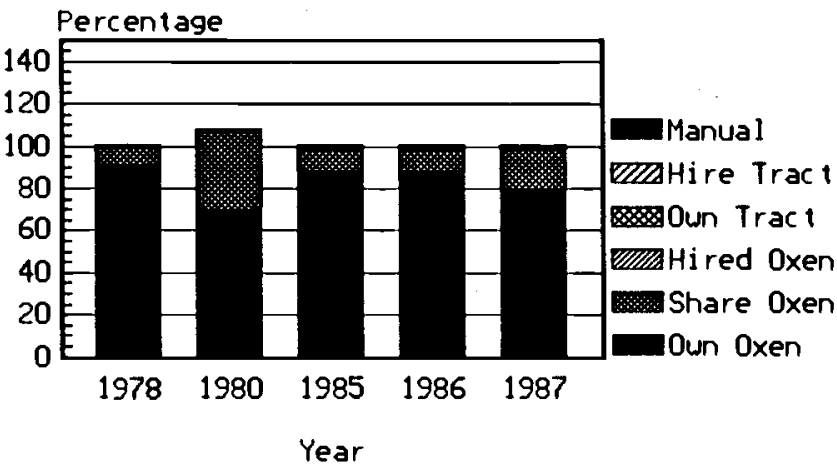

Farm Power - Farmers who lett in 1987 Province of Faryab Crop: Dry wheat

Percentage

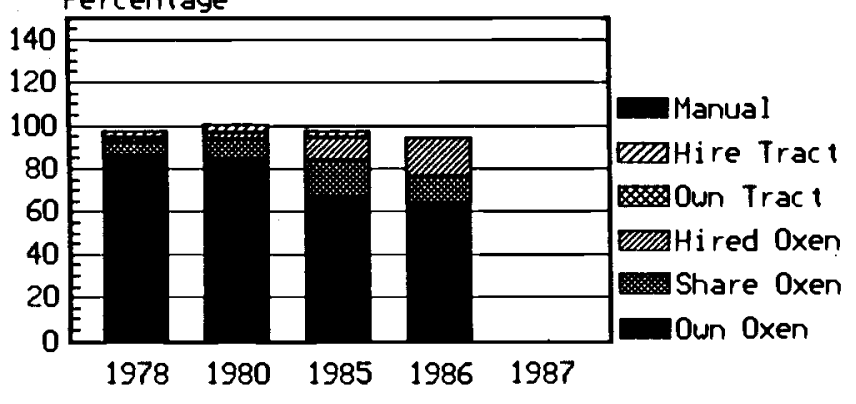

Year

Base Figures for above graphs

Province: Faryab

Farmers in Afghanistan

Dry wheat own oxen

Shared oxen

Hired oxen

Own Tractor

Hired Tractor

Barley

Manual Means

Own oxen

shared oxen

Hired Oxen

Own Tractor

Hired Tractor

Manual Means

Farmers who left in 1987

Dry wheat Own oxen

Shared oxen

Hired oxen

own Tractor

Hired Tractor

Manual Means

Barley

Own oxen

Shared oxen

Hired oxen

Own Tractor

Hired Tractor
Farm Power - Farmers in Atghanistan Province of Faryab

Crop: Barley

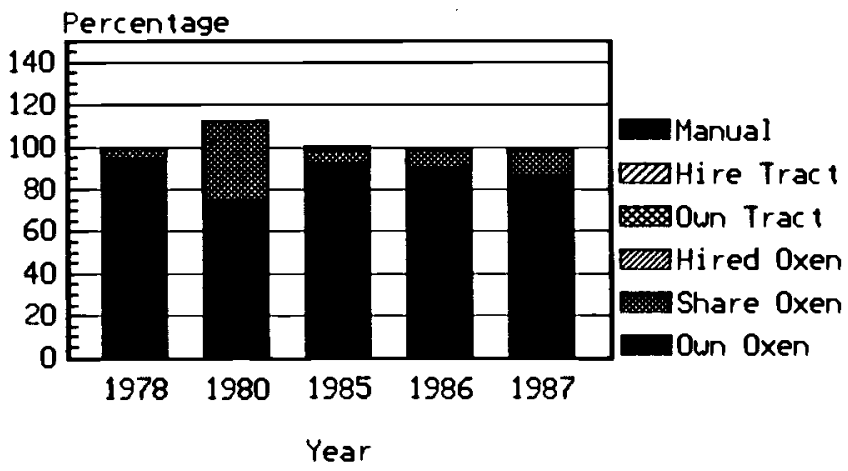

Farm Power - Farmers who lett in 1987

Province of Faryab Crop: Barley

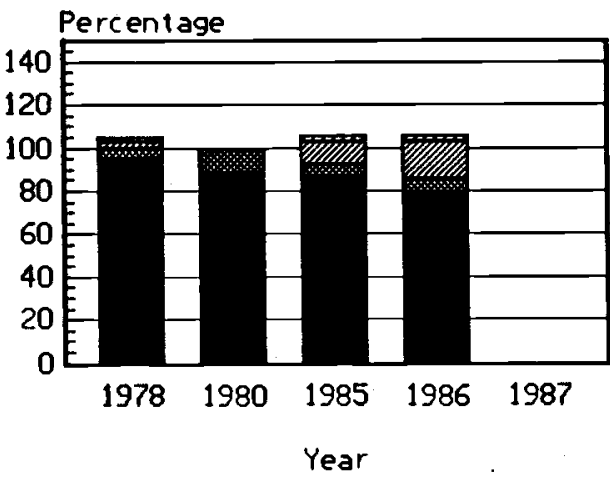

Manual Eathire Tract Own Tract שTIGHired Oxen Share Oxen uown Oxen

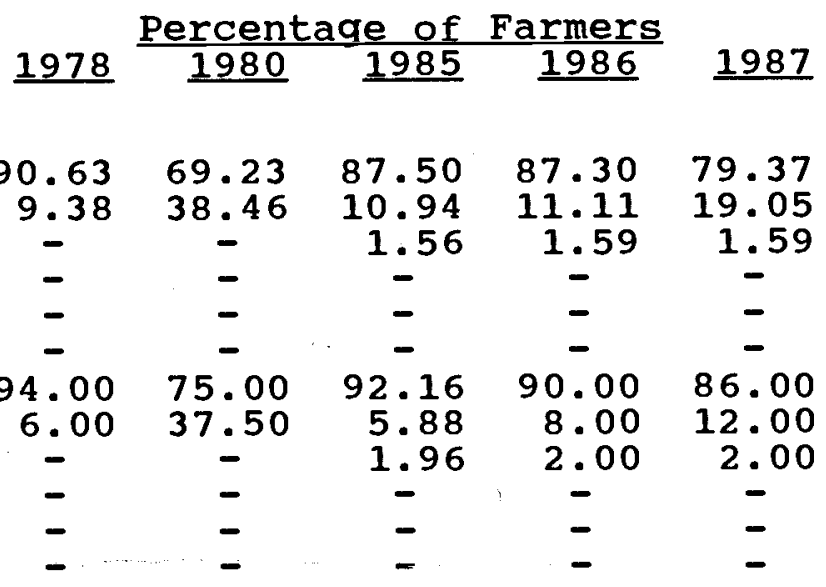

$\begin{array}{rrrcc}86.11 & 84.38 & 66.67 & 63.64 & - \\ 5.56 & 9.38 & 16.67 & 12.12 & - \\ 2.78 & 3.13 & 11.11 & 18.18 & - \\ - & - & - & - & = \\ 2.78 & 3.13 & 2.78 & - & - \\ - & - & - & - & - \\ 94.74 & 87.88 & 86.49 & 79.41 & 100.00 \\ 5.26 & 12.12 & 5.41 & 5.88 & - \\ 2.63 & - & 10.81 & 17.65 & - \\ - & - & - & - & - \\ 2.63 & - & 2.70 & 2.94 & -\end{array}$


Faryab

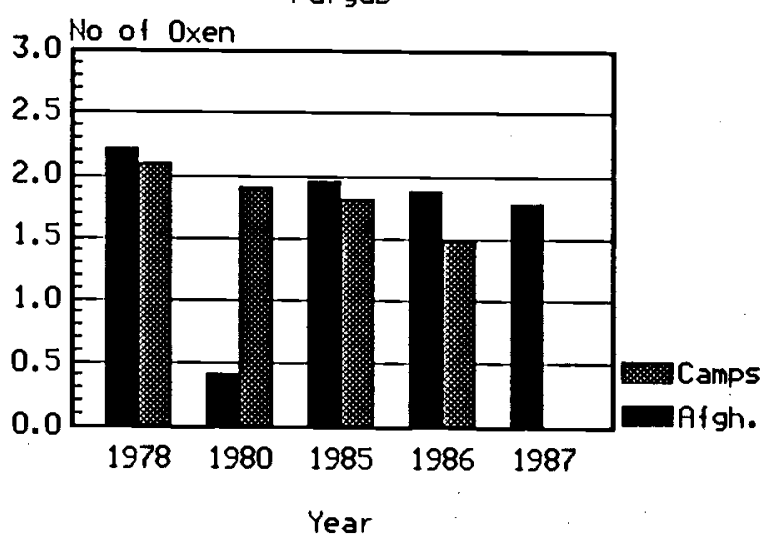

Faryab

5.0 Hired Labour per Farm

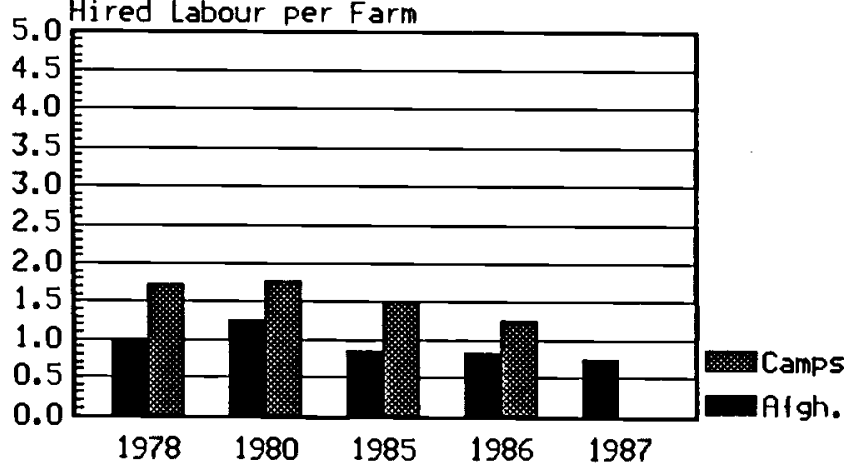

Year
Faryab

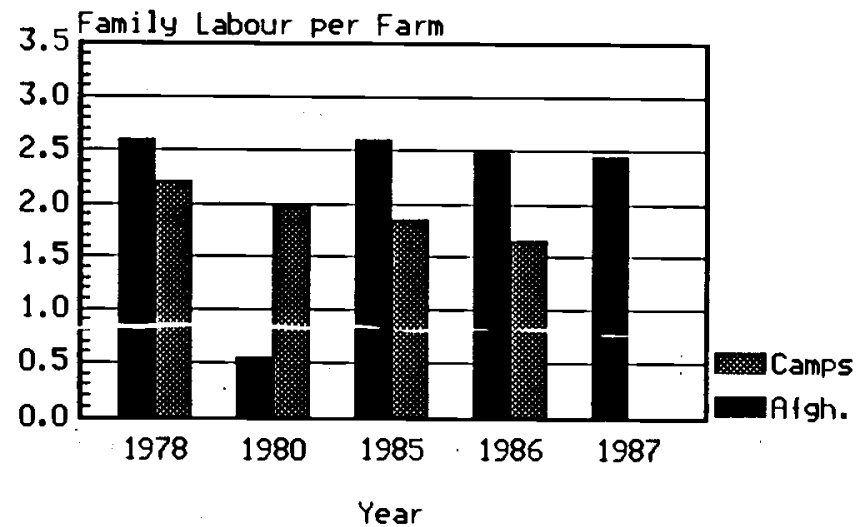

Faryab

Percentage of Farms using

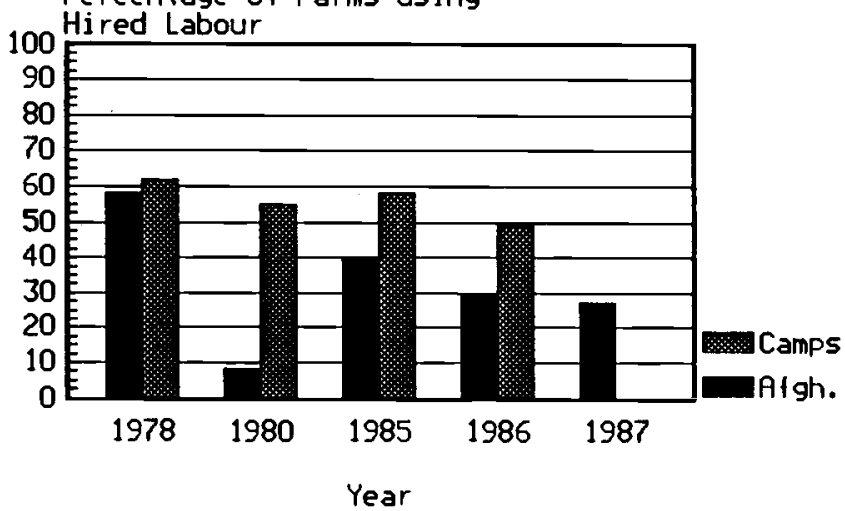


PROVINCE OF FRY

\begin{tabular}{|c|c|c|c|c|c|c|c|}
\hline \multirow[b]{2}{*}{ YEAR } & & & & & & & \\
\hline & & $\mathbf{0}$ & 1 & 2 & 3 & 4 & 5 \\
\hline 1978 & $\begin{array}{l}\text { Number of Families } \\
x \text { of Families }\end{array}$ & 1 & $\begin{array}{l}7 \\
9.72\end{array}$ & $\begin{array}{l}46 \\
63.89\end{array}$ & $\begin{array}{c}13 \\
18.06\end{array}$ & ${ }^{4} .56$ & $\begin{array}{l}1 \\
1.39\end{array}$ \\
\hline 1980 & $\begin{array}{l}\text { Number of Families } \\
x \text { of Familles }\end{array}$ & $\begin{array}{l}58 \\
79.45\end{array}$ & $\begin{array}{l}5 \\
6.85\end{array}$ & $\begin{array}{c}6 \\
8.22\end{array}$ & ${ }_{4.11}^{3}$ & $\begin{array}{c}1 \\
1.37\end{array}$ & $\begin{array}{c}0 \\
0.00\end{array}$ \\
\hline 1985 & $\begin{array}{l}\text { Number of Families } \\
x \text { of Families }\end{array}$ & $\begin{array}{l}3 \\
4.11\end{array}$ & $\begin{array}{c}8 \\
10.96\end{array}$ & $\begin{array}{l}54 \\
73.97\end{array}$ & $\begin{array}{c}6 \\
8.22\end{array}$ & 2.74 & $\begin{array}{c}0 \\
0.00\end{array}$ \\
\hline 1986 & $\begin{array}{l}\text { Number of Families } \\
\chi \text { of Families }\end{array}$ & ${ }^{4}$ & $\begin{array}{c}9 \\
12.33\end{array}$ & $\begin{array}{l}54 \\
73.97\end{array}$ & $5^{4}$ & $2^{2} .74$ & $\begin{array}{c}0 \\
0.00\end{array}$ \\
\hline 1987 & $\begin{array}{l}\text { Number of Families } \\
x \text { of Families }\end{array}$ & $\begin{array}{l}4 \\
5.48\end{array}$ & $\begin{array}{l}14 \\
19.18\end{array}$ & $\begin{array}{c}51 \\
69.86\end{array}$ & 2.74 & $2^{2} .74$ & $\begin{array}{c}0 \\
0.00\end{array}$ \\
\hline Avere & ge Number of Oxen per Family: & $\begin{array}{l}1978 \\
1980 \\
1985 \\
1986 \\
1987\end{array}$ & & $\begin{array}{l}2.21 \\
0.41 \\
1.95 \\
1.88 \\
1.78\end{array}$ & & & \\
\hline
\end{tabular}

Number of Farmers Questioned 73

IABLE 7B - AVERAGE NUMBER OF TRAINED OXEN FOR FARMERS IN THE CAMPS

PROVINCE OF FRY

YEAR

1978 Number of Families

$x$ of Families

1980 Number of Families

* of Families

1985 Number of Families

$x$ of Families

1986 Number of Families

$x$ of Families

1987 Number of Families

$x$ of Families

Average Number of Oxen per Family:

1978

1980

1985

1986

1987
$0 \begin{array}{ccccc}\text { NUMBER OF } & \text { TRAINED } & \text { OXEN PER } & \text { FAMILY } \\ 0 & 1 & 2 & 3 & 4\end{array}$

$\begin{array}{cccccc}4 & 6 & 46 & 4 & 7 & 1 \\ 5.88 & 8.82 & 67.65 & 5.88 & 10.29 & 1.47\end{array}$

$\begin{array}{llllll}9 & 8 & 40 & 3 & 7 & 1\end{array}$

$\begin{array}{llllll}13.24 & 11.76 & 58.82 & 4.41 & 10.29 & 1.47\end{array}$

$\begin{array}{cccccc}9 & 9 & 43 & 3 & 4 & 1 \\ 13.04 & 13.04 & 62.32 & 4.35 & 5.80 & 1.45\end{array}$

$\begin{array}{llllll}17 & 8 & 39 & 4 & 1 & 0\end{array}$

$\begin{array}{llllll}24.64 & 11.59 & 56.52 & 5.80 & 1.45 & 0.00\end{array}$

$\begin{array}{llllll}69 & 0 & 0 & 0 & 0 & 0\end{array}$

$\begin{array}{cccccc}100.0 & 0.00 & 0.00 & 0.00 & 0.00 & 0.00\end{array}$

$\begin{array}{ll}1978 & 2.10 \\ 1980 & 1.91 \\ 1985 & 1.81 \\ 1986 & 1.48 \\ 1987 & 0.00\end{array}$

Number of Farmers Questioned 69 
IABLE 8(A) - AVERAGE LABOUR (FAMILY and HIRED) FOR FARMERS IN AFGANISTAN

PROVINCE OF FRY

\begin{tabular}{|c|c|c|c|c|c|}
\hline & 1978 & 1980 & 1985 & 1986 & 1987 \\
\hline Average Family Labour per Farm & 2.60 & 0.55 & 2.59 & 2.48 & 2.45 \\
\hline $\begin{array}{l}\text { Average No. of Hired Labour per Farm } \\
\text { (for farms using hired (abour) }\end{array}$ & 0.45 & $1: 2 \overline{3}$ & 0.84 & 0.82 & 0.73 \\
\hline Number of Farms using Hired Labour " ". & 42 & 6 & 29 & 22 & 20 \\
\hline
\end{tabular}

IABLE 8(B) - AVERAGE LABOUR (FAMILY and HIRED) FOR FARMERS IN CAMPS

PROVINCE OF FRY

\begin{tabular}{|c|c|c|c|c|c|}
\hline & 1978 & 1980 & 1985 & 1986 & 1987 \\
\hline Average Family Labour per Farm & 2.20 & 1.96 & 1.85 & 1.65 & 0.00 \\
\hline $\begin{array}{l}\text { Average No. of Hired Labour per Farm } \\
\text { (for farms using hired (abour) }\end{array}$ & 1.71 & 1.76 & 1.51 & 1.24 & $\star \star \star \star \star$ \\
\hline Number of Farms using Hired Labour & 43 & 38 & 40 & 34 & 0 \\
\hline
\end{tabular}


PROVINCE OF FRY

Average Number of Livestock Shot

\begin{tabular}{|c|c|c|c|c|}
\hline 1978 & 1980 & 1985 & 1986 & 1987 \\
\hline$\hbar \hbar \hbar \hbar \hbar$ & 1.00 & 8.46 & 4.69 & 2.56 \\
\hline 0 & 1 & 24 & 13 & \\
\hline 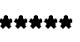 & 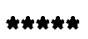 & 1.00 & 3.00 & \\
\hline
\end{tabular}

Number of Farmers reporting Livestock

killed by Mines

0

0

2

1

Number of Farmers Questioned 75

\section{IABLE 9B - DIRECT EFFECTS OF THE WAR - FARMERS IN CAMPS}

PROVINCE OF FRY

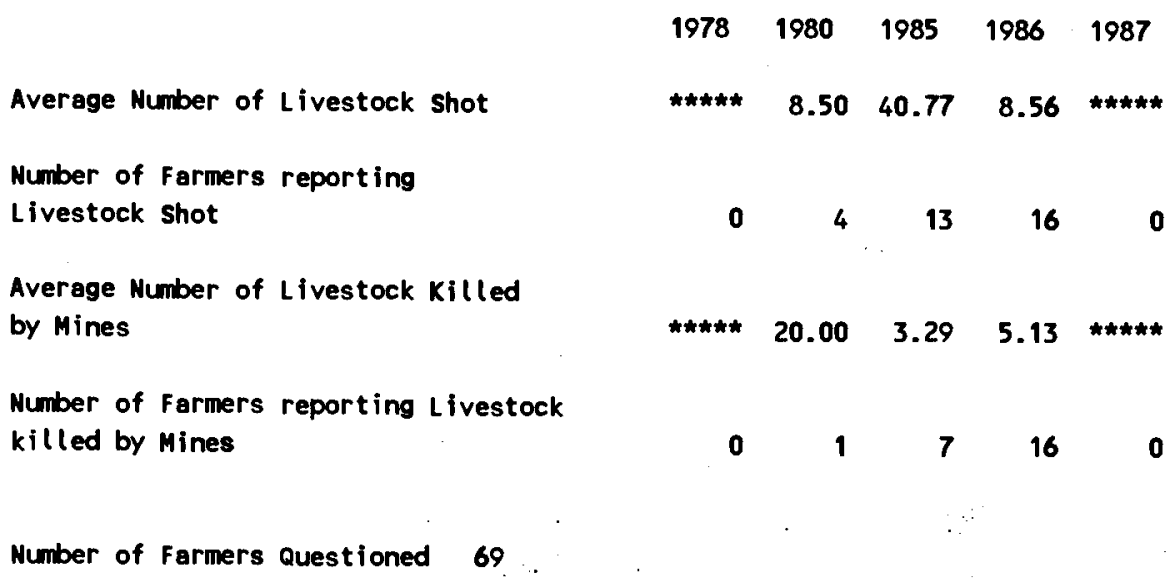




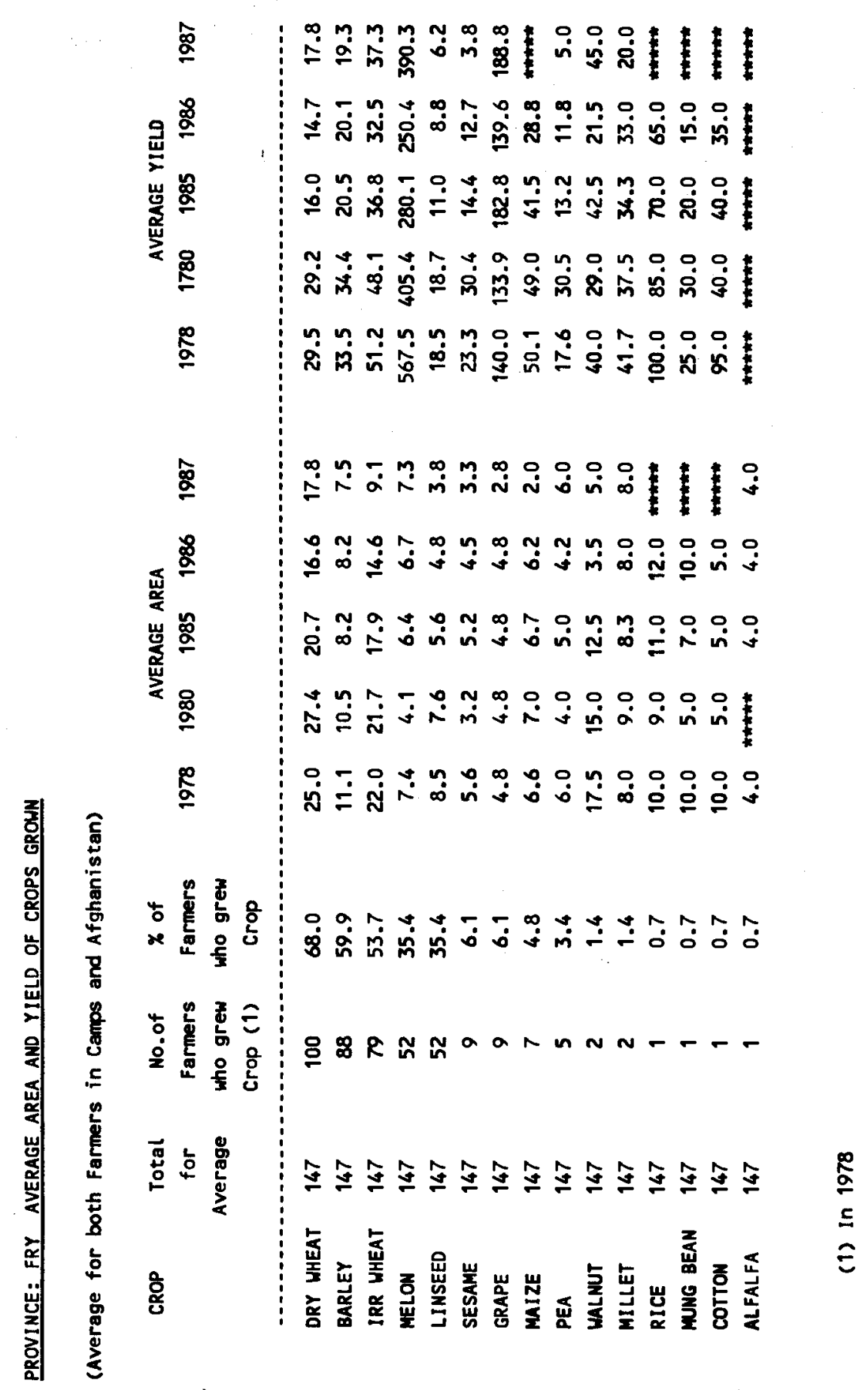


PROVINCE OF FRY

Rainfall

Crop 0 isease

8 irds or Rats

Seed Avail ability

Fertilizer Availability

Credit

Irrigation Water Availability

Flooding

Labour Availabilty

Power for Land Preparation

Oirect War Effects

Animal Diseases

Extension

Other Problems
Crop Protection Chemicals

\begin{tabular}{rrrrrrrrrrrrr}
1 & 2 & 3 & 4 & 5 & 6 & 7 & 8 & 9 & 10 & 11 & 12 & Weighted Priority \\
\hline 8 & 8 & 0 & 0 & 0 & 0 & 0 & 0 & 0 & 0 & 0 & 0 & 5.44 \\
5 & 8 & 26 & 15 & 13 & 10 & 0 & 0 & 0 & 0 & 0 & 0 & 12.09 \\
0 & 8 & 0 & 5 & 3 & 0 & 3 & 0 & 0 & 0 & 0 & 0 & 2.83 \\
0 & 13 & 13 & 13 & 0 & 0 & 0 & 0 & 0 & 0 & 0 & 0 & 6.55 \\
13 & 13 & 8 & 0 & 0 & 0 & 0 & 0 & 0 & 0 & 0 & 0 & 10.27 \\
0 & 0 & 3 & 3 & 3 & 0 & 0 & 0 & 0 & 0 & 0 & 0 & 0.95 \\
0 & 0 & 0 & 0 & 0 & 0 & 0 & 0 & 0 & 0 & 0 & 0 & 0.00 \\
5 & 0 & 0 & 0 & 0 & 0 & 0 & 0 & 0 & 0 & 0 & 0 & 2.42 \\
0 & 0 & 0 & 0 & 3 & 0 & 0 & 0 & 0 & 0 & 0 & 0 & 0.24 \\
8 & 21 & 13 & 3 & 0 & 0 & 0 & 0 & 0 & 0 & 0 & 0 & 10.78 \\
0 & 5 & 0 & 0 & 0 & 0 & 0 & 0 & 0 & 0 & 0 & 0 & 1.21 \\
62 & 23 & 8 & 3 & 0 & 0 & 0 & 0 & 0 & 0 & 0 & 0 & 35.96 \\
0 & 0 & 8 & 0 & 0 & 0 & 0 & 0 & 0 & 0 & 0 & 0 & 1.21 \\
0 & 0 & 3 & 0 & 0 & 0 & 0 & 0 & 0 & 0 & 0 & 0 & 0.40 \\
0 & 3 & 13 & 33 & 23 & 8 & 3 & 3 & 0 & 0 & 0 & 0 & 9.65
\end{tabular}

1986

Rainfall

Crop 0 isease

Birds or Rats

Seed Avai labllity

Fertilizer Availability

Crop Protection Chemicals

Credit

Irrigation Water Availability

Flooding

Labour Avail labilty

Power for Land Preparation

0 irect War Effects

Animal 0 iseases

Extension

Other Problems

$\begin{array}{lllllllllllll}1 & 2 & 3 & 4 & 5 & 6 & 7 & 8 & 9 & 10 & 11 & 12 & \text { Weighted Priority }\end{array}$

$\begin{array}{lllllllllllll}36 & 33 & 3 & 0 & 3 & 0 & 0 & 0 & 0 & 0 & 0 & 0 & 27.28\end{array}$

$\begin{array}{lllllllllllll}5 & 3 & 26 & 10 & 8 & 8 & 0 & 0 & 0 & 0 & 0 & 0 & 10.29\end{array}$

$\begin{array}{lllllllllllll}0 & 5 & 3 & 2 & z & n & 3 & 0 & 0 & 0 & 0 & 0 & 3.15\end{array}$

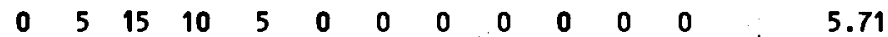

$\begin{array}{llllllllllllll}3 & 13 & 15 & 0 & 0 & 0 & 0 & 0 & 0 & 0 & 0 & 0 & & 7.13\end{array}$

$\begin{array}{lllllllllllll}0 & 0 & 0 & 5 & 5 & 0 & 0 & 0 & 0 & 0 & 0 & 0 & 1.17\end{array}$

$\begin{array}{lllllllllllll}0 & 0 & 0 & 0 & 0 & 0 & 0 & 0 & 0 & 0 & 0 & 0 & 0.00\end{array}$

$\begin{array}{rllllllllllll}23 & 0 & 0 & 0 & 0 & 0 & 0 & 0 & 0 & 0 & 0 & 0 & 11.67\end{array}$

$\begin{array}{lllllllllllll}0 & 0 & 0 & 0 & 3 & 0 & 0 & 0 & 0 & 0 & 0 & 0 & 0.26\end{array}$

$\begin{array}{lllllllllllll}0 & 5 & 3 & 8 & 0 & 0 & 0 & 0 & 0 & 0 & 0 & 0 & 2.70\end{array}$

$\begin{array}{lllllllllllll}3 & 0 & 0 & 0 & 0 & 0 & 0 & 0 & 0 & 0 & 0 & 0 & 1.30\end{array}$

$\begin{array}{lllllllllllll}31 & 33 & 18 & 8 & 0 & 0 & 0 & 0 & 0 & 0 & 0 & 0 & \\ & 0 & 3 & 0 & 3 & 0 & 0 & 0 & 0 & 0 & 0 & 0 & \end{array}$

$\begin{array}{lllllllllllll}0 & 0 & 3 & 0 & 3 & 0 & 0 & 0 & 0 & 0 & 0 & 0 & 0.69\end{array}$

$\begin{array}{lllllllllllll}0 & 0 & 0 & 0 & 0 & 0 & 0 & 0 & 0 & 0 & 0 & 0 & 0.00\end{array}$

$\begin{array}{lllllllllllll}0 & 3 & 0 & 0 & 0 & 0 & 0 & 0 & 0 & 0 & 0 & 0 & \\ \end{array}$
Rainfall

Crop 0 isease

Birds or Rets

Seed Avai lability

Fertilizer Availability

Crop Protection Chemicals

Credit

Irrigation Water Availability

Flooding

Labour Avai labilty

Power for Land Preparation

0 irect War Effects

Animal 0 iseases

Extension

Other Problems

\begin{tabular}{rrrrrrrrrrrrr}
1 & 2 & 3 & 4 & 5 & 6 & 7 & 8 & 9 & 10 & 11 & 12 & Weighted Priority \\
\hline 33 & 36 & 0 & 0 & 3 & 0 & 0 & 0 & 0 & 0 & 0 & 0 & 26.65 \\
5 & 5 & 26 & 8 & 8 & 5 & 0 & 0 & 0 & 0 & 0 & 0 & 10.58 \\
0 & 10 & 5 & 5 & 5 & 5 & 0 & 0 & 0 & 0 & 0 & 0 & 5.15 \\
0 & 5 & 13 & 10 & 5 & 0 & 0 & 0 & 0 & 0 & 0 & 0 & 5.37 \\
3 & 13 & 10 & 0 & 0 & 0 & 0 & 0 & 0 & 0 & 0 & 0 & 6.38 \\
0 & 0 & 0 & 5 & 3 & 0 & 0 & 0 & 0 & 0 & 0 & 0 & 0.92 \\
0 & 0 & 0 & 0 & 0 & 0 & 0 & 0 & 0 & 0 & 0 & 0 & 0.00 \\
23 & 0 & 0 & 0 & 0 & 0 & 0 & 0 & 0 & 0 & 0 & 0 & 11.88 \\
0 & 0 & 0 & 3 & 3 & 0 & 0 & 0 & 0 & 0 & 0 & 0 & 0.59 \\
0 & 0 & 0 & 8 & 0 & 0 & 0 & 0 & 0 & 0 & 0 & 0 & 0.99 \\
0 & 3 & 0 & 0 & 0 & 0 & 0 & 0 & 0 & 0 & 0 & 0 & 0.66 \\
36 & 23 & 26 & 5 & 0 & 0 & 0 & 0 & 0 & 0 & 0 & 0 & 29.47 \\
0 & 3 & 0 & 0 & 3 & 0 & 0 & 0 & 0 & 0 & 0 & 0 & 0.92 \\
0 & 0 & 0 & 0 & 0 & 0 & 0 & 0 & 0 & 0 & 0 & 0 & 0.00 \\
0 & 0 & 3 & 0 & 0 & 0 & 0 & 0 & 0 & 0 & 0 & 0 & 0.44
\end{tabular}


PROVINCE OF FRY

\section{Rainfall}

Crop 0 isease

Birds or Rats

Fertilizer Availability

Crop Protection Chemicals

Credit

Irrigation Water Availability

Flooding

Labour Availabilty

Power for Land Preparation

Direct Wer Effects

Animal Diseases

Extension

other Problems
Seed Availability

\begin{tabular}{ccccccccccccc}
1 & 2 & 3 & 4 & 5 & 6 & 7 & 8 & 0 & 10 & 11 & 12 & Weighted Priority \\
\hline 0 & 0 & 0 & 0 & 0 & 0 & 0 & 0 & 0 & 0 & 0 & 0 & 0.00 \\
0 & 0 & 0 & 0 & 0 & 0 & 0 & 0 & 0 & 0 & 0 & 0 & 0.00 \\
3 & 0 & 0 & 0 & 0 & 0 & 0 & 0 & 0 & 0 & 0 & 0 & 100.0 \\
0 & 0 & 0 & 0 & 0 & 0 & 0 & 0 & 0 & 0 & 0 & 0 & 0.00 \\
0 & 0 & 0 & 0 & 0 & 0 & 0 & 0 & 0 & 0 & 0 & 0 & 0.00 \\
0 & 0 & 0 & 0 & 0 & 0 & 0 & 0 & 0 & 0 & 0 & 0 & 0.00 \\
0 & 0 & 0 & 0 & 0 & 0 & 0 & 0 & 0 & 0 & 0 & 0 & 0.00 \\
0 & 0 & 0 & 0 & 0 & 0 & 0 & 0 & 0 & 0 & 0 & 0 & 0.00 \\
0 & 0 & 0 & 0 & 0 & 0 & 0 & 0 & 0 & 0 & 0 & 0 & 0.00 \\
0 & 0 & 0 & 0 & 0 & 0 & 0 & 0 & 0 & 0 & 0 & 0 & 0.00 \\
0 & 0 & 0 & 0 & 0 & 0 & 0 & 0 & 0 & 0 & 0 & 0 & 0.00 \\
0 & 0 & 0 & 0 & 0 & 0 & 0 & 0 & 0 & 0 & 0 & 0 & 0.00 \\
0 & 0 & 0 & 0 & 0 & 0 & 0 & 0 & 0 & 0 & 0 & 0 & 0.00 \\
0 & 0 & 0 & 0 & 0 & 0 & 0 & 0 & 0 & 0 & 0 & 0 & 0.00 \\
0 & 0 & 0 & 0 & 0 & 0 & 0 & 0 & 0 & 0 & 0 & 0 & 0.00
\end{tabular}

1978

Rainfall

Crop Oisease

Birds or Rats

Seed Availability

Fertilizer Availability

Crop Protection chemicals

Credit

Irrigation Water Availability

Flooding

Labour Availabilty

Power for Land Preparation

Direct War Effects

Animal Diseases

Extension

Other Problems

\begin{tabular}{rrrrrrrrrrrrr}
1 & 2 & 3 & 4 & 5 & 6 & 7 & 8 & 9 & 10 & 11 & 12 & Weighted Priority \\
\hline 0 & 0 & 0 & 0 & 0 & 0 & 0 & 0 & 0 & 0 & 0 & 0 & 0.00 \\
0 & 0 & 0 & 0 & 0 & 0 & 0 & 0 & 0 & 0 & 0 & 0 & 0.00 \\
0 & 0 & 3 & 0 & 0 & 0 & 0 & 0 & 0 & 0 & 0 & 0 & 2.60 \\
0 & 8 & 0 & 0 & 0 & 0 & 0 & 0 & 0 & 0 & 0 & 0 & 11.26 \\
18 & 0 & 0 & 0 & 0 & 0 & 0 & 0 & 0 & 0 & 0 & 0 & 52.50 \\
0 & 3 & 0 & 0 & 0 & 0 & 0 & 0 & 0 & 0 & 0 & 0 & 3.76 \\
0 & 0 & 0 & 0 & 0 & 0 & 0 & 0 & 0 & 0 & 0 & 0 & 0.00 \\
10 & 0 & 0 & 0 & 0 & 0 & 0 & 0 & 0 & 0 & 0 & 0 & 30.00 \\
0 & 0 & 0 & 0 & 0 & 0 & 0 & 0 & 0 & 0 & 0 & 0 & 0.00 \\
0 & 0 & 0 & 0 & 0 & 0 & 0 & 0 & 0 & 0 & 0 & 0 & 0.00 \\
0 & 0 & 0 & 0 & 0 & 0 & 0 & 0 & 0 & 0 & 0 & 0 & 0.00 \\
0 & 0 & 0 & 0 & 0 & 0 & 0 & 0 & 0 & 0 & 0 & 0 & 0.00 \\
0 & 0 & 0 & 0 & 0 & 0 & 0 & 0 & 0 & 0 & 0 & 0 & 0.00 \\
0 & 0 & 0 & 0 & 0 & 0 & 0 & 0 & 0 & 0 & 0 & 0 & 0.00 \\
0 & 0 & 0 & 0 & 0 & 0 & 0 & 0 & 0 & 0 & 0 & 0 & 0.00
\end{tabular}




\section{GHOR}




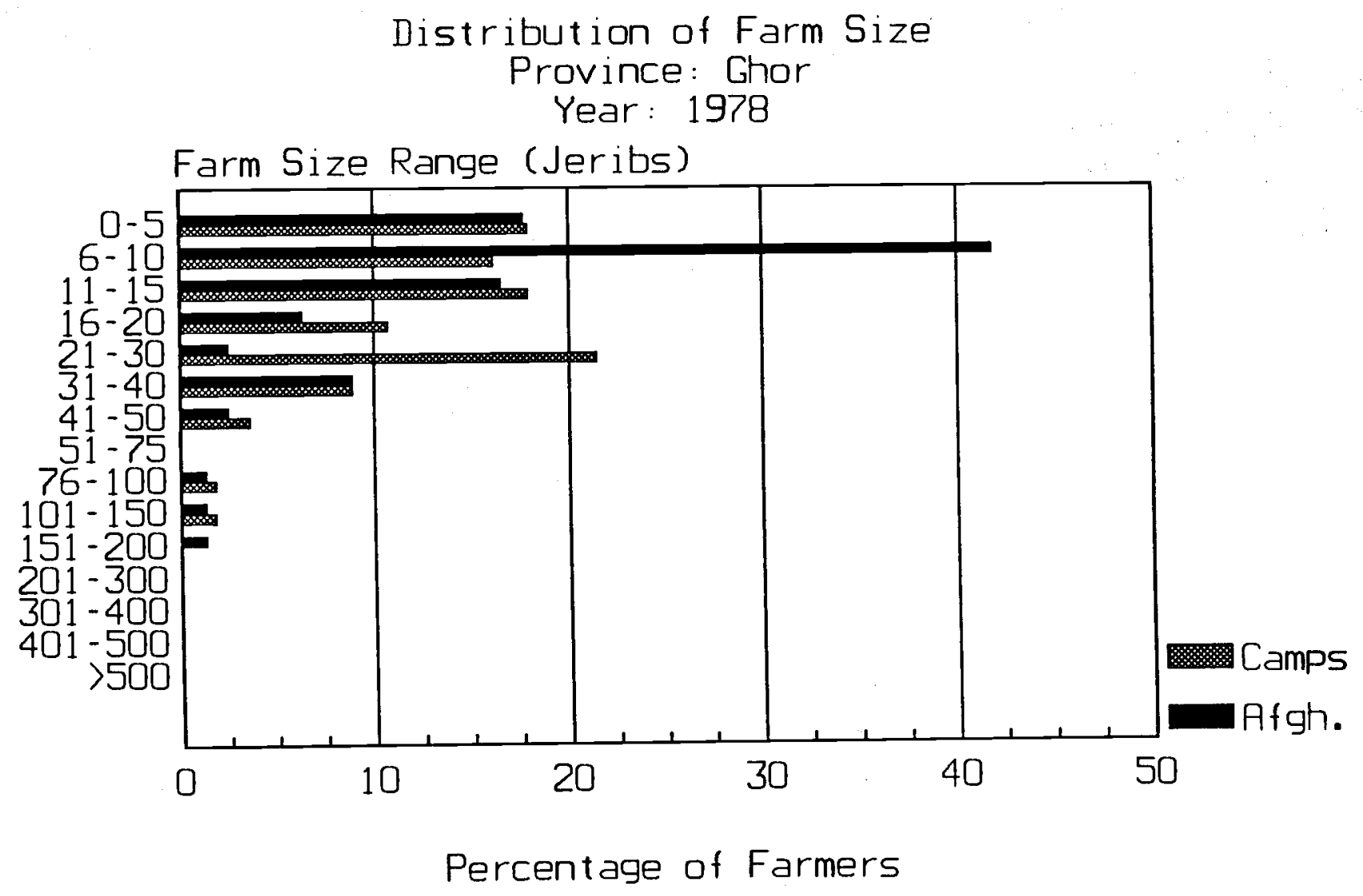

Province: Ghor

Farmers in Afghanistan

1978

$\underline{1987}$

Total Number of Farms

79

79

Average Area

16.78

8.99

Average Area Irrigated

6.09

4.63

Average Dryland Area

14.86

9.62

Farmers who left in 1987

Total Number of Farms

Average Area

Average Area Irrigated

Average Dryland Area

56
20.96
9.26
16.26

\section{Average of Both Groups}

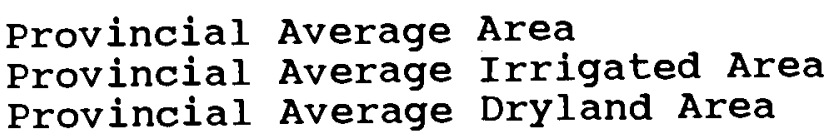

(A1l areas in Jeribs. 5 Jeribs $=1$ Hectare)

$\begin{array}{rr}18.51 & 8.99 \\ 7.48 & 4.63 \\ 15.43 & 9.62\end{array}$



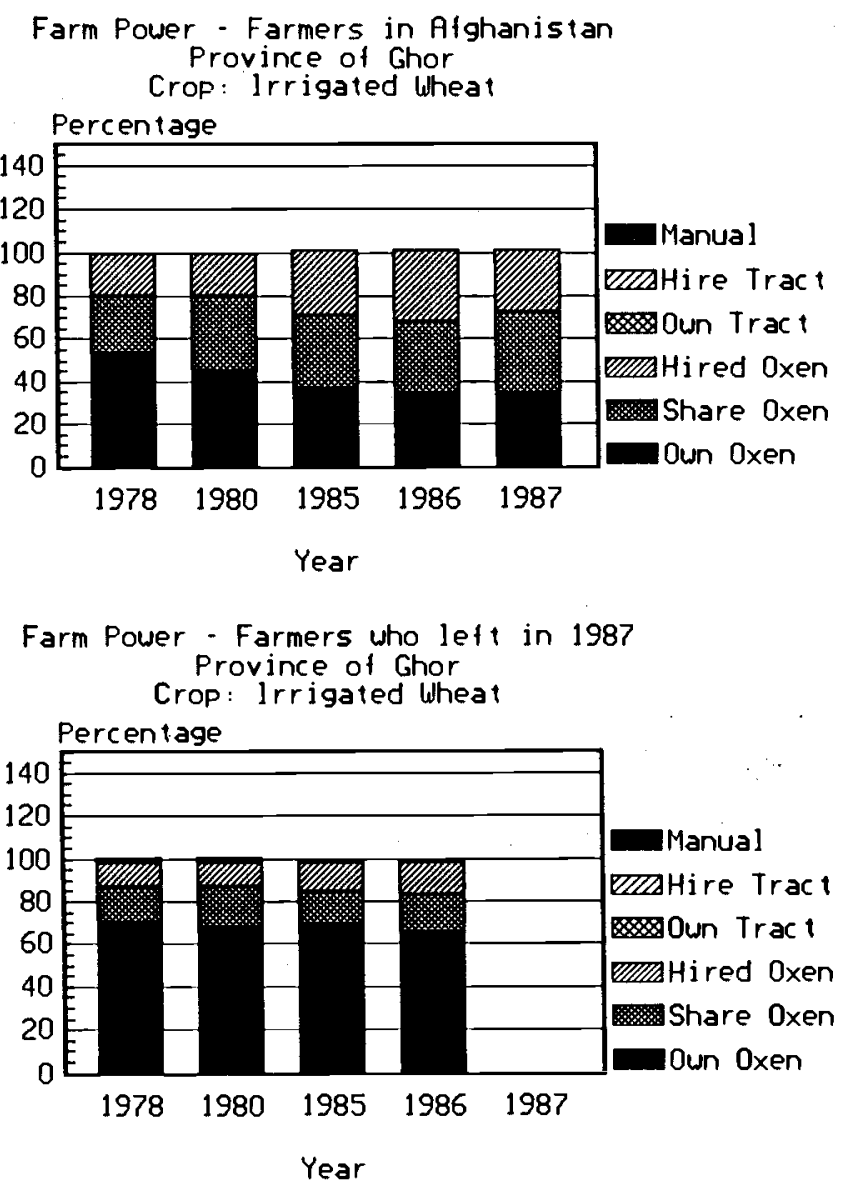

Base Figures for above graphs

\section{Province: Ghor}

\section{Farmers in Afghanistan}

$\begin{array}{cl}\text { Irr. Wheat } & \text { Own Oxen } \\ \text { Shared Oxen } & \text { Hired Oxen } \\ \text { Own Tractor } \\ \text { Hired Tractor } \\ \text { Manual Means } \\ \text { Dry Wheat } & \text { Own Oxen } \\ & \text { Shared Oxen } \\ & \text { Hired Oxen } \\ \text { Own Tractor } \\ \text { Hired Tractor } \\ \text { Manual Means }\end{array}$

Farmers who left in 1987

$\begin{array}{cl}\text { Irr. Wheat } & \text { Own Oxen } \\ & \text { Shared Oxen } \\ & \text { Hired oxen } \\ & \text { Own Tractor } \\ & \text { Hired Tractor } \\ & \text { Manual Means } \\ \text { Dry wheat } & \text { Own Oxen } \\ & \text { Shared Oxen } \\ & \text { Hired Oxen } \\ \text { Own Tractor } & \end{array}$

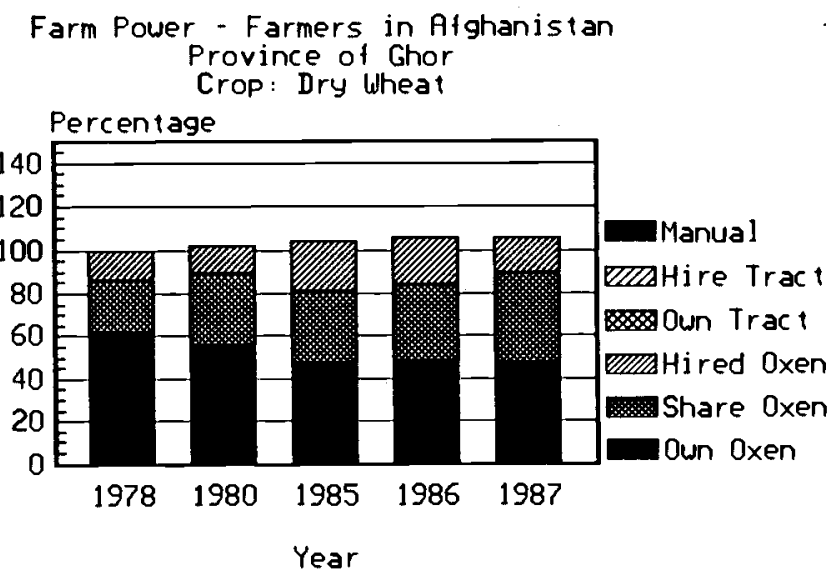
Farm Power - Farmers who left in 1987 Piovince of Chor Crop: Dry wheat

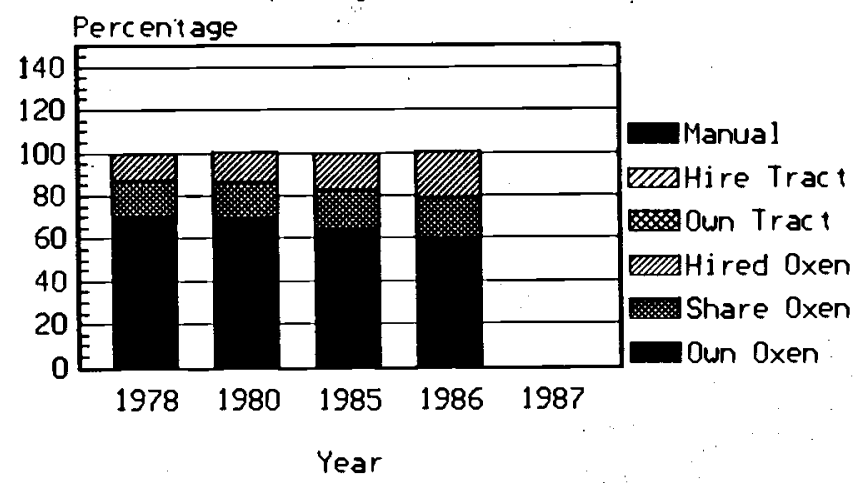

\begin{tabular}{ccccc} 
& \multicolumn{9}{c}{ Percentage of } & Farmers & \\
1978 & \multicolumn{1}{l}{1980} & $\underline{1985}$ & $\underline{1986}$ & 1987 \\
& & & & \\
53.62 & 44.93 & 36.76 & 34.78 & 34.78 \\
26.09 & 34.78 & 33.82 & 33.33 & 37.68 \\
20.29 & 20.29 & 30.88 & 33.33 & 28.99 \\
- & - & - & - & - \\
- & - & - & - & - \\
- & - & - & - & - \\
62.07 & 55.36 & 47.06 & 48.15 & 47.27 \\
24.14 & 33.93 & 33.33 & 35.19 & 41.82 \\
13.79 & 12.50 & 23.53 & 22.22 & 16.36 \\
- & - & - & - & - \\
- & - & - & - & - \\
- & - & - & - & -
\end{tabular}

$\begin{array}{ccccc}69.81 & 67.92 & 69.23 & 65.38 & - \\ 16.98 & 18.87 & 15.38 & 17.31 & - \\ 11.32 & 11.32 & 13.46 & 15.38 & - \\ - & - & - & - & - \\ - & - & - & - & - \\ 1.89 & 1.89 & 1.92 & 1.92 & - \\ 70.27 & 69.44 & 63.64 & 59.38 & - \\ 16.22 & 16.67 & 18.18 & 18.75 & - \\ 13.51 & 13.89 & 18.18 & 21.88 & - \\ - & - & - & - & -\end{array}$


PROVINCE OF GOR

\begin{tabular}{|c|c|c|c|c|c|c|c|}
\hline \multirow[b]{2}{*}{ YEAR } & & & & & & & \\
\hline & & 0 & 1 & 2 & 3 & 4 & 5 \\
\hline \multirow[t]{2}{*}{1978} & Number of Families & 13 & 23 & 33 & 6 & 2 & 1 \\
\hline & $X$ of Families & 16.67 & 29.49 & 42.31 & 7.69 & 2.56 & 1.28 \\
\hline \multirow[t]{2}{*}{1980} & Number of Families & 14 & 27 & 30 & 6 & 1 & 1 \\
\hline & x of Families & 17.72 & 34.18 & 37.97 & 7.59 & 1.27 & 1.27 \\
\hline \multirow[t]{2}{*}{1985} & Number of Families & 23 & 25 & 28 & 2 & 1 & 0 \\
\hline & $x$ of Families & 29.11 & 31.65 & 35.44 & 2.53 & 1.27 & 0.00 \\
\hline \multirow[t]{2}{*}{1986} & Number of Families & 23 & 25 & 28 & 2 & 1 & 0 \\
\hline & \% of Families & 29.11 & 31.65 & 35.44 & 2.53 & 1.27 & 0.00 \\
\hline \multirow[t]{2}{*}{1987} & Number of Families & 19 & 29 & 28 & 2 & 1 & 0 \\
\hline & $X$ of Families & 24.05 & 36.71 & 35.44 & 2.53 & 1.27 & 0.00 \\
\hline \multirow{5}{*}{\multicolumn{2}{|c|}{ Average Number of Oxen per Family: }} & 1978 & \multicolumn{2}{|r|}{1.54} & & & \\
\hline & & 1980 & \multicolumn{2}{|r|}{1.44} & & & \\
\hline & & 1985 & \multicolumn{2}{|r|}{1.15} & & & \\
\hline & & 1986 & \multicolumn{2}{|r|}{1.15} & & & \\
\hline & & 1987 & \multicolumn{2}{|r|}{1.20} & & & \\
\hline
\end{tabular}

Number of Farmers Questioned 79

IABLE 78 - AVERAGE NUMBER OF TRAINED OXEN FOR FARMERS IN THE CAMPS

PROVINCE OF GOR

YEAR

1978 Number of Families

$x$ of Families

1980 Number of Families

$X$ of Families

1985 Number of Families

\% of Families

1986 Number of Families

$\%$ of Families

1987 Number of Families

\% of Families

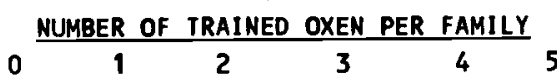

$\begin{array}{llllll}7 & 9 & 34 & 3 & 2 & 0\end{array}$

$\begin{array}{llllll}12.73 & 16.36 & 61.82 & 5.45 & 3.64 & 0.00\end{array}$

$\begin{array}{llllll}7 & 10 & 35 & 2 & 1 & 0\end{array}$

$\begin{array}{llllll}12.73 & 18.18 & 63.64 & 3.64 & 1.82 & 0.00\end{array}$

$\begin{array}{llllll}7 & 10 & 37 & 0 & 1 & 1\end{array}$

$\begin{array}{llllll}12.50 & 17.86 & 66.07 & 0.00 & 1.79 & 1.79\end{array}$

$\begin{array}{llllll}10 & 12 & 32 & 0 & 2 & 0\end{array}$

$\begin{array}{llllll}17.86 & 21.43 & 57.14 & 0.00 & 3.57 & 0.00\end{array}$

$\begin{array}{llllll}56 & 0 & 0 & 0 & 0 & 0\end{array}$

$\begin{array}{llllll}100.0 & 0.00 & 0.00 & 0.00 & 0.00 & 0.00\end{array}$

Number of Farmers Questioned 56 
IABLE 8(A) - AVERAGE LABOUR (FAMILY and HIRED) FOR FARMERS IN AFGANISTAN

PROVINCE OF GOR

$\begin{array}{rrrrr}1978 & 1980 & 1985 & 1986 & 1987 \\ 1.56 & 1.53 & 1.41 & 1.39 & 1.49 \\ 1.11 & 1.00 & 0.92 & 1.04 & 1.00 \\ & & & & \\ 14 & 14 & 12 & 12 & 13\end{array}$

Number of Farmers Questioned 79

TABLE $8(B)$ - AVERAGE LABOUR (FAMILY and HIRED) FOR FARMERS IN CAMPS

PROVINCE OF GOR

\begin{tabular}{|c|c|c|c|c|c|}
\hline & 1978 & 1980 & 1985 & 1986 & 1987 \\
\hline Average Family Labour per Farm & 1.77 & 1.69 & 1.46 & 1.52 & 0.00 \\
\hline $\begin{array}{l}\text { Average No. of Hired Labour per Farm } \\
\text { (for farms using hired (abour) }\end{array}$ & 1.17 & 1.17 & 1.11 & 1.12 & 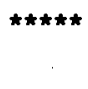 \\
\hline Number of Farms & 23 & 23 & 21 & 21 & 0 \\
\hline
\end{tabular}

Number of Farmers Questioned 56 
PROVINCE OF GOR

Average Number of Livestock Shot

Number of Farmers reporting

Livestock Shot

Average Number of Livestock Killed by Mines

Number of Farmers reporting Livestock killed by Mines

Number of Farmers Questioned 79 $\begin{array}{lllll}1978 & 1980 & 1985 & 1986 & 1987\end{array}$

$\begin{array}{lllll}\star \star \star * * & 3.67 & 1.50 & 3.25 & 3.50\end{array}$

$0.12 \quad 6 \quad 4 \quad 4$

$\begin{array}{llll}\star \star \star \star * & 5.00 & 12.00 & 1.00 \quad \star \star \star \star \star\end{array}$

$\begin{array}{lllll}0 & 1 & 2 & 1 & 0\end{array}$

IABLE 9B - DIRECT EFFECTS OF THE WAR - FARMERS IN CAMPS

PROVINCE OF GOR

Average Number of Livestock Shot

Number of Farmers reporting

Livestock Shot

Average Number of Livestock Killed by Mines

Number of Farmers reporting Livestock killed by Mines

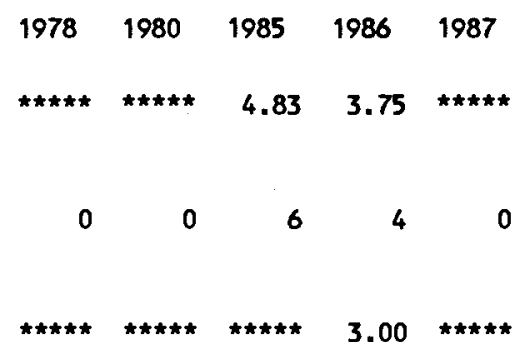

$\begin{array}{lllll}0 & 0 & 0 & 8 & 0\end{array}$

Number of Farmers Questioned 56 


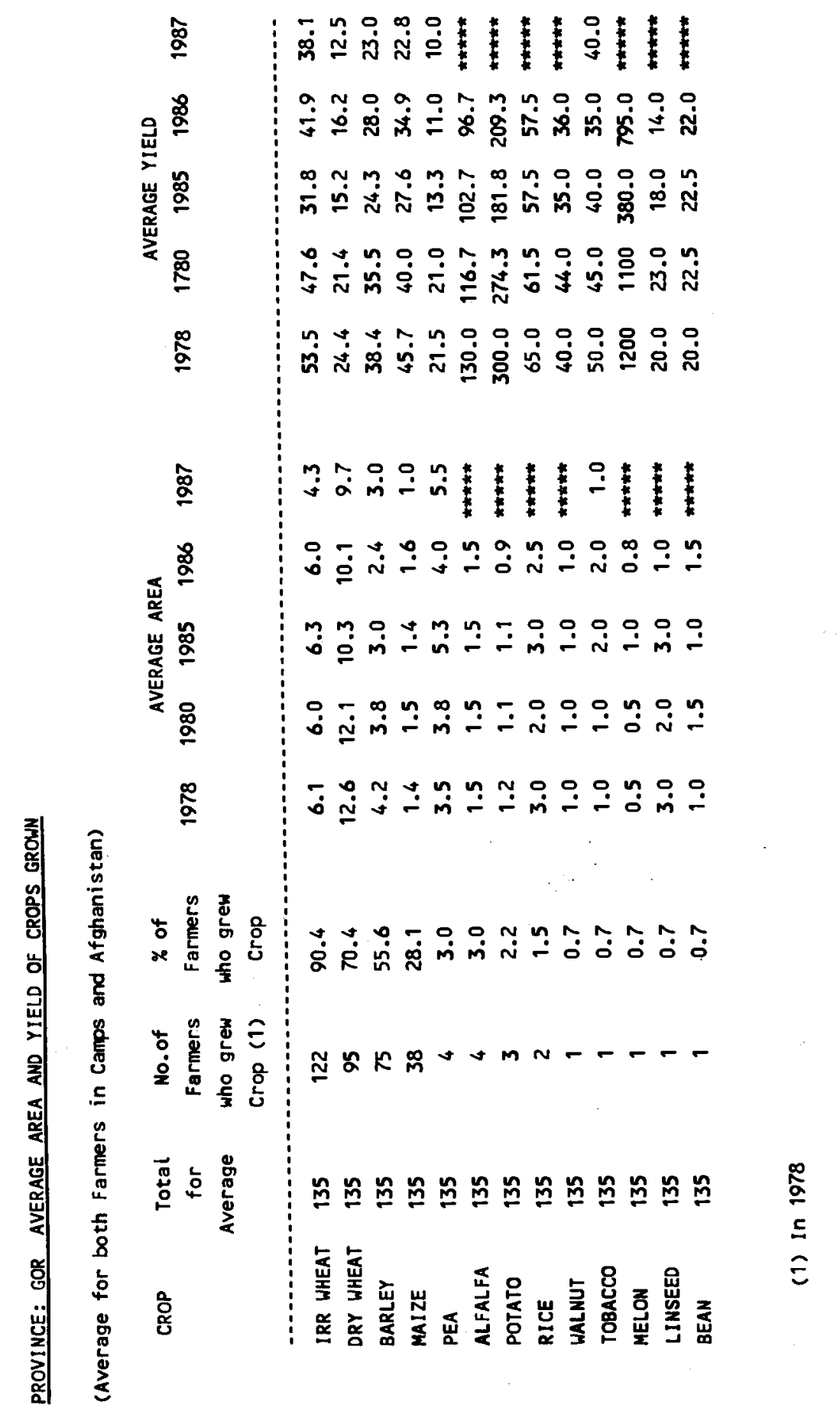


PROVINCE OF GOR

Rainfall

Crop 0 isease

Birds or Rats

Seed Availability

Fertilizer Availability

Crop Protection Chemicals

Credit

Irrigation Water Availability

Flooding

Labour Availabilty

Power for Land Preparation

0 irect War Effects

Animal 0 iseases

Extension

Other Problems

\begin{tabular}{rrrrrrrrrrrrr}
1 & 2 & 3 & 4 & 5 & 6 & 7 & 8 & 9 & 10 & 11 & 12 & Weighted Priority \\
\hline 37 & 37 & 16 & 0 & 0 & 0 & 0 & 0 & 0 & 0 & 0 & 0 & 22.63 \\
0 & 5 & 11 & 5 & 32 & 11 & 16 & 5 & 0 & 0 & 0 & 0 & 6.90 \\
0 & 0 & 11 & 11 & 5 & 16 & 26 & 5 & 11 & 0 & 0 & 0 & 5.76 \\
0 & 0 & 0 & 0 & 0 & 0 & 0 & 0 & 0 & 0 & 0 & 0 & 0.00 \\
0 & 0 & 16 & 21 & 5 & 0 & 0 & 0 & 0 & 0 & 0 & 0 & 4.33 \\
0 & 0 & 0 & 0 & 0 & 0 & 0 & 0 & 0 & 0 & 0 & 0 & 0.00 \\
0 & 0 & 0 & 0 & 0 & 0 & 0 & 0 & 0 & 0 & 0 & 0 & 0.00 \\
0 & 53 & 26 & 11 & 0 & 0 & 0 & 0 & 0 & 0 & 0 & 0 & 14.10 \\
47 & 5 & 0 & 0 & 5 & 0 & 5 & 0 & 0 & 0 & 0 & 0 & 19.37 \\
0 & 0 & 0 & 0 & 0 & 0 & 0 & 0 & 0 & 0 & 0 & 0 & 0.00 \\
0 & 0 & 5 & 11 & 5 & 0 & 0 & 0 & 0 & 0 & 0 & 0 & 2.03 \\
16 & 0 & 16 & 26 & 26 & 0 & 0 & 0 & 0 & 0 & 0 & 0 & 12.30 \\
0 & 0 & 0 & 0 & 5 & 21 & 5 & 5 & 0 & 0 & 0 & 0 & 2.23 \\
0 & 0 & 0 & 0 & 5 & 21 & 21 & 32 & 5 & 11 & 0 & 0 & 4.92 \\
0 & 0 & 5 & 16 & 16 & 26 & 0 & 5 & 5 & 0 & 0 & 0 & 5.42
\end{tabular}

1986

\section{Rainfall}

Crop Oisease

Birds or Rats

Seed Avai lability

Fertilizer Availability

Crop Protection Chemicals

Credit

Irrigation Water Availability

Flooding

Labour Availabilty

Power for Land Preparation

Oirect War Effects

Animal 0 iseases

Extension

Other Problems

1985

Rainfall

Crop Oisease

Birds or Rats

Seed Availability

Fertilizer Availability

Crop Protection Chemicals

Credit

Irrigation Water Availability

Flooding

Labour Availabilty

Power for Land Preparation

Oirect War Effects

Animal 0 iseases

Extension

Other Problems

$\begin{array}{rrrrrrrrrrrrr}1 & 2 & 3 & 4 & 5 & 6 & 7 & 8 & 9 & 10 & 11 & 12 & \text { Heighted Priority } \\ 11 & 0 & 0 & 0 & 0 & 0 & 0 & 0 & 0 & 0 & 0 & 0 & 4.65 \\ 0 & 5 & 37 & 11 & 5 & 11 & 0 & 0 & 0 & 0 & 0 & 0 & 8.98 \\ 0 & 5 & 16 & 32 & 11 & 0 & 0 & 0 & 0 & 0 & 0 & 0 & 7.90 \\ 0 & 5 & 0 & 0 & 0 & 5 & 0 & 0 & 0 & 0 & 0 & 0 & 1.55 \\ 5 & 26 & 11 & 5 & 0 & 0 & 0 & 0 & 0 & 0 & 0 & 0 & 10.26 \\ 0 & 0 & 0 & 0 & 0 & 0 & 0 & 0 & 0 & 0 & 0 & 0 & 0.00 \\ 0 & 0 & 0 & 0 & 0 & 0 & 0 & 0 & 0 & 0 & 0 & 0 & 0.00 \\ 0 & 47 & 5 & 0 & 0 & 0 & 0 & 0 & 0 & 0 & 0 & 0 & 11.23 \\ 0 & 5 & 0 & 0 & 0 & 0 & 0 & 0 & 0 & 0 & 0 & 0 & 1.16 \\ 0 & 0 & 0 & 0 & 0 & 0 & 0 & 0 & 0 & 0 & 0 & 0 & 0.00 \\ 5 & 0 & 5 & 5 & 5 & 0 & 0 & 0 & 0 & 0 & 0 & 0 & 4.14 \\ 84 & 5 & 5 & 0 & 5 & 0 & 0 & 0 & 0 & 0 & 0 & 0 & 39.58 \\ 0 & 0 & 0 & 0 & 0 & 0 & 0 & 0 & 0 & 0 & 0 & 0 & 0.00 \\ 0 & 0 & 16 & 32 & 26 & 16 & 11 & 0 & 0 & 0 & 0 & 0 & 9.96 \\ 0 & 0 & 0 & 5 & 0 & 0 & 0 & 0 & 0 & 0 & 0 & 0 & 0.58\end{array}$

$\begin{array}{rrrrrrrrrrrrr}1 & 2 & 3 & 4 & 5 & 6 & 7 & 8 & 9 & 10 & 11 & 12 & \text { Weighted Priority } \\ 95 & 0 & 0 & 0 & 0 & 0 & 0 & 0 & 0 & 0 & 0 & 0 & 36.92 \\ 0 & 0 & 0 & 11 & 26 & 26 & 5 & 0 & 0 & 0 & 0 & 0 & 5.08 \\ 0 & 0 & 0 & 5 & 5 & 26 & 11 & 11 & 0 & 0 & 0 & 0 & 3.73 \\ 0 & 0 & 32 & 11 & 26 & 5 & 0 & 11 & 0 & 0 & 0 & 0 & 8.03 \\ 0 & 0 & 16 & 16 & 0 & 0 & 0 & 0 & 0 & 0 & 0 & 0 & 3.59 \\ 0 & 0 & 5 & 0 & 0 & 0 & 0 & 0 & 0 & 0 & 0 & 0 & 0.68 \\ 0 & 0 & 0 & 0 & 0 & 0 & 0 & 0 & 0 & 0 & 0 & 0 & 0.00 \\ 0 & 100 & 0 & 0 & 0 & 0 & 0 & 0 & 0 & 0 & 0 & 0 & 19.49 \\ 0 & 0 & 0 & 0 & 0 & 0 & 0 & 0 & 0 & 0 & 0 & 0 & 0.00 \\ 0 & 0 & 0 & 0 & 0 & 0 & 0 & 0 & 0 & 0 & 0 & 0 & 0.00 \\ 0 & 0 & 26 & 26 & 11 & 0 & 0 & 0 & 0 & 0 & 0 & 0 & 6.80 \\ 0 & 0 & 16 & 21 & 11 & 5 & 0 & 0 & 0 & 0 & 0 & 0 & 5.27 \\ 0 & 0 & 5 & 11 & 11 & 16 & 16 & 5 & 0 & 0 & 0 & 0 & 4.69 \\ 0 & 0 & 0 & 0 & 11 & 16 & 47 & 16 & 11 & 0 & 0 & 0 & 5.71 \\ 0 & 0 & 0 & 0 & 0 & 0 & 0 & 0 & 0 & 0 & 0 & 0 & 0.00\end{array}$


PROVINCE OF GOR

\section{PERCENTAGE OF VILLAGES GIVING FOLLOWING PRIORITY TO PROBLEM}

Rainfall

Crop 0 isease

Birds or Rats

Seed Availability

Fertilizer Availability

Crop Protection Chemicals

Credit

Irrigation Water Availability

Flooding

Labour Availabilty

Power for Land Preparation

Oirect War Effects

Animal 0 iseases

Extension

Other Problems

\begin{tabular}{rrrrrrrrrrrrr}
1 & 2 & 3 & 4 & 5 & 6 & 7 & 8 & 9 & 10 & 11 & 12 & Heighted Priority \\
\hline 0 & 0 & 5 & 0 & 0 & 0 & 0 & 0 & 0 & 0 & 0 & 0 & 0.79 \\
0 & 11 & 37 & 26 & 5 & 5 & 0 & 0 & 0 & 0 & 0 & 0 & 11.79 \\
0 & 0 & 0 & 32 & 32 & 11 & 5 & 0 & 0 & 0 & 0 & 0 & 7.57 \\
0 & 0 & 0 & 0 & 0 & 0 & 0 & 0 & 0 & 0 & 0 & 0 & 0.00 \\
0 & 11 & 21 & 0 & 0 & 0 & 0 & 0 & 0 & 0 & 0 & 0 & 5.56 \\
0 & 0 & 0 & 5 & 0 & 0 & 0 & 0 & 0 & 0 & 0 & 0 & 0.60 \\
5 & 0 & 0 & 0 & 0 & 0 & 0 & 0 & 0 & 0 & 0 & 0 & 2.38 \\
5 & 53 & 0 & 0 & 0 & 0 & 0 & 0 & 0 & 0 & 0 & 0 & 14.30 \\
0 & 5 & 11 & 5 & 0 & 0 & 0 & 0 & 0 & 0 & 0 & 0 & 3.38 \\
0 & 5 & 5 & 0 & 5 & 0 & 0 & 0 & 0 & 0 & 0 & 0 & 2.46 \\
0 & 0 & 0 & 0 & 5 & 0 & 0 & 0 & 0 & 0 & 0 & 0 & 0.48 \\
84 & 5 & 5 & 0 & 0 & 0 & 0 & 0 & 0 & 0 & 0 & 0 & 40.11 \\
0 & 0 & 0 & 0 & 5 & 0 & 0 & 0 & 0 & 0 & 0 & 0 & 0.48 \\
0 & 0 & 5 & 16 & 21 & 37 & 11 & 5 & 0 & 0 & 0 & 0 & 8.25 \\
0 & 0 & 5 & 5 & 5 & 0 & 0 & 0 & 0 & 0 & 0 & 0 & 1.87
\end{tabular}

$\underline{1978}$

Rainfall

Crop 0 isease

Birds or Rats

Seed Availability

Fertilizer Availability

Crop Protection Chemicals

Credit

Irrigation Water Avai lability

Flooding

Labour Availabilty

Power for Land Preparation

Direct War Effects

Animal 0 iseases

Extension

other Problems

\begin{tabular}{llllllllllllc}
1 & 2 & 3 & 4 & 5 & 6 & 7 & 8 & 9 & 10 & 11 & 12 & Heighted Priority \\
\hline 0 & 0 & 0 & 0 & 0 & 0 & 0 & 0 & 0 & 0 & 0 & 0 & 0.00 \\
0 & 0 & 0 & 0 & 0 & 0 & 0 & 0 & 0 & 0 & 0 & 0 & 0.00 \\
0 & 0 & 0 & 0 & 0 & 0 & 0 & 0 & 0 & 0 & 0 & 0 & 0.00 \\
0 & 0 & 0 & 0 & 0 & 0 & 0 & 0 & 0 & 0 & 0 & 0 & 0.00 \\
0 & 0 & 0 & 0 & 0 & 0 & 0 & 0 & 0 & 0 & 0 & 0 & 0.00 \\
0 & 0 & 0 & 0 & 0 & 0 & 0 & 0 & 0 & 0 & 0 & 0 & 0.00 \\
0 & 0 & 0 & 0 & 0 & 0 & 0 & 0 & 0 & 0 & 0 & 0 & 0.00 \\
0 & 0 & 0 & 0 & 0 & 0 & 0 & 0 & 0 & 0 & 0 & 0 & 0.00 \\
0 & 0 & 0 & 0 & 0 & 0 & 0 & 0 & 0 & 0 & 0 & 0 & 0.00 \\
0 & 0 & 0 & 0 & 0 & 0 & 0 & 0 & 0 & 0 & 0 & 0 & 0.00 \\
0 & 0 & 0 & 0 & 0 & 0 & 0 & 0 & 0 & 0 & 0 & 0 & 0.00 \\
0 & 0 & 0 & 0 & 0 & 0 & 0 & 0 & 0 & 0 & 0 & 0 & 0.00 \\
0 & 0 & 0 & 0 & 0 & 0 & 0 & 0 & 0 & 0 & 0 & 0 & 0.00 \\
0 & 0 & 0 & 0 & 0 & 0 & 0 & 0 & 0 & 0 & 0 & 0 & 0.00 \\
0 & 0 & 0 & 0 & 0 & 0 & 0 & 0 & 0 & 0 & 0 & 0 & 0.00
\end{tabular}




\section{GHAZNI}




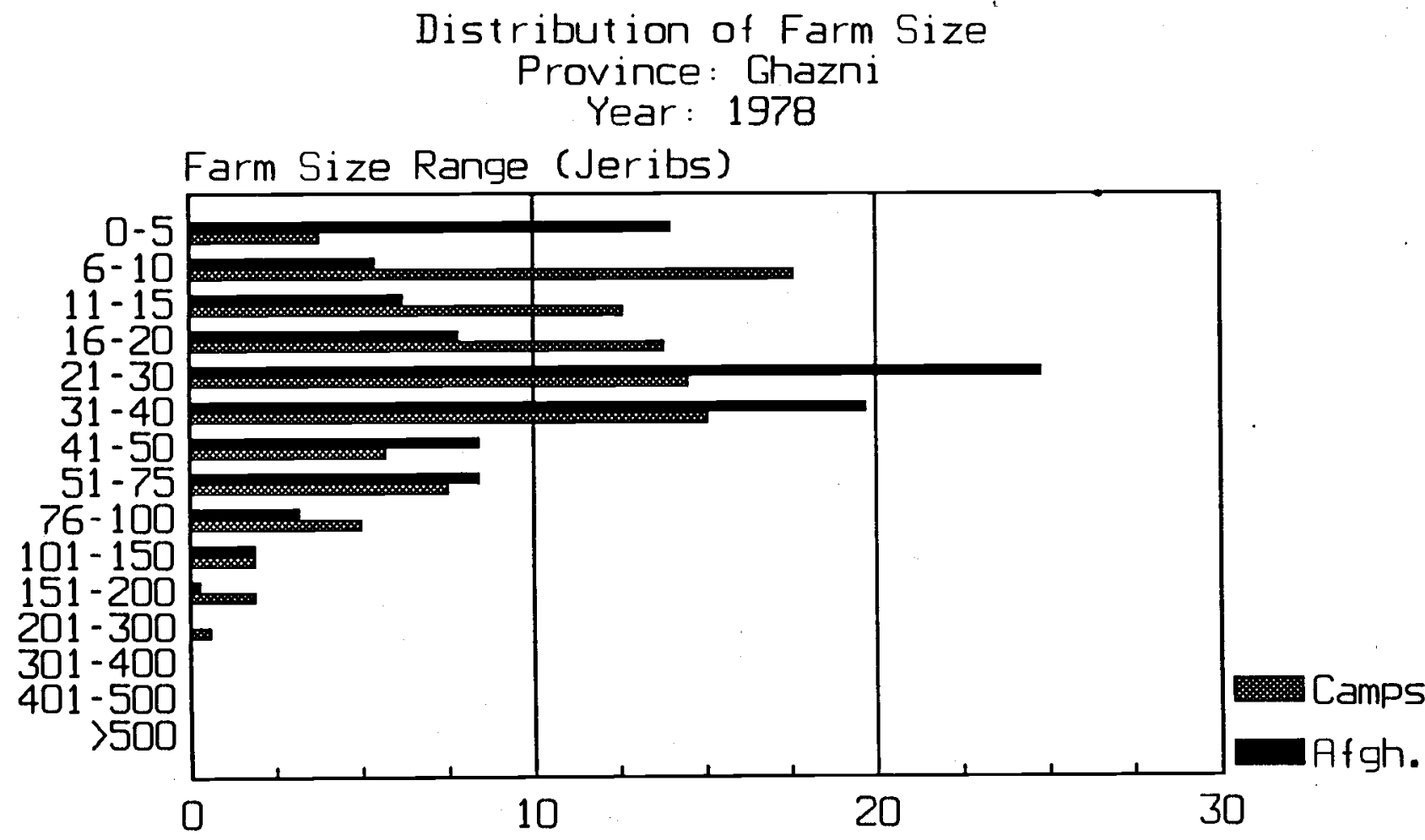

Percentage of Farmers

Farmers in Afghanistan

1978

1987

Total Number of Farms

371

366

Average Area

Average Area Irrigated

31.08

19.46

Average Dryland Area

29.75

10.14

18.27

3.47

Farmers who left in 1987

Total Number of Farms

159

1

Average Area

33.90

8.00

Average Area Irrigated

28.00

8.00

Average Dryland Area

20.44

Average of Both Groups

Provincial Average Area

Provincial Average Irrigated Area

Provincial Average Dryland Area
31.93

29.22

14.78
19.43

18.24

3.47

(All areas in Jeribs. 5 Jeribs $=1$ Hectare) 


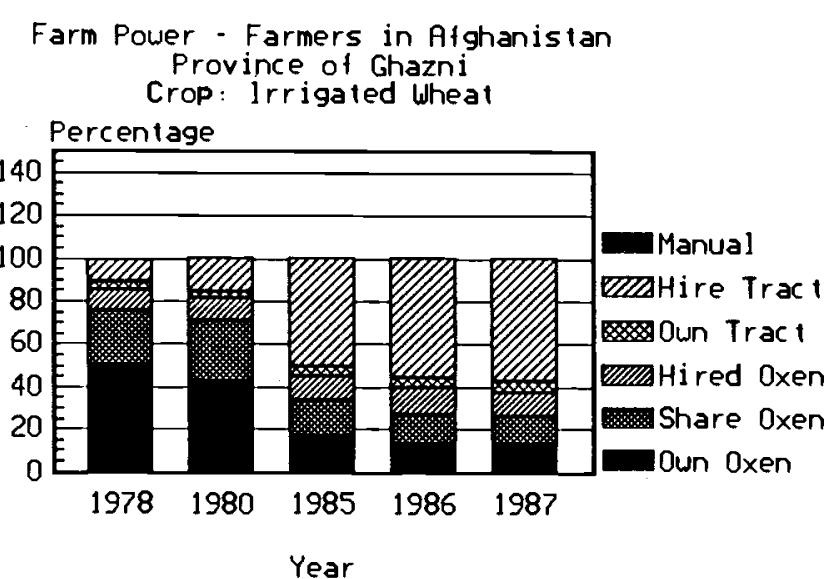

Farm Power - Farmers who left in 1987 Province of Ghazni Crop: Irrigated wheat

Percentage

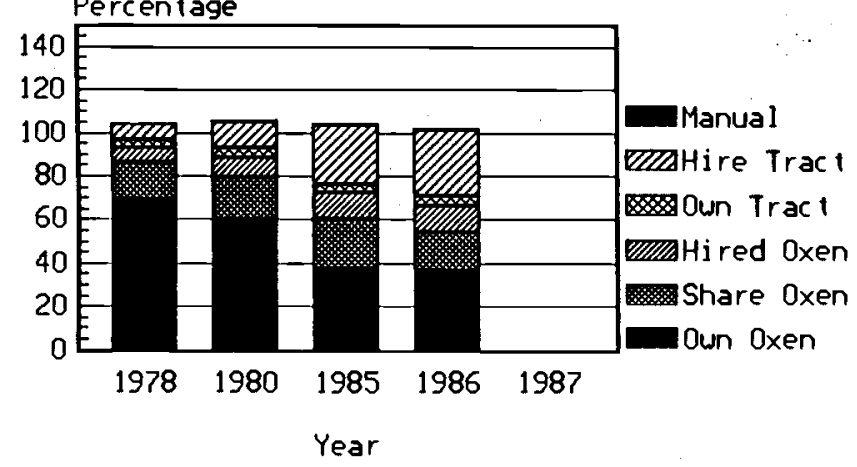

Base Fiqures for above graphs

Province: Ghazni

Farmers in Afghanistan

Irr. Wheat Own oxen

shared oxen

Hired oxen

Own Tractor

Hired Tractor

Barley

Manual Means

Own Oxen

Shared oxen

Hired oxen

Own Tractor

Hired Tractor

Manual Means

Farmers who left in 1987

Irr. Wheat own oxen

Shared oxen

Hired oxen

Own Tractor

Hired Tractor

Barley
Manual Means

Own oxen

Shared oxen

Hired oxen

Own Tractor

Hired Tractor
Farm Power - Farmers in Atghanistan Province of Ghazni

Crop: Barley

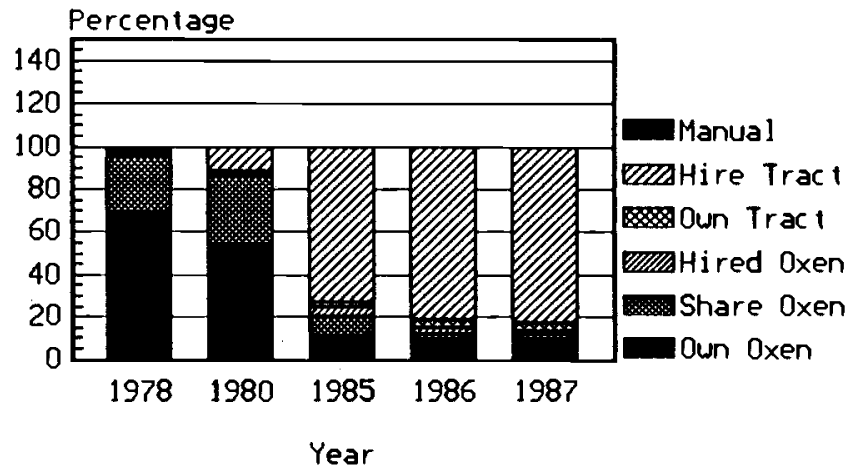

Farm Power - Farmers who lett in 1987 Fiuriice of Ghazni Crop: Barley

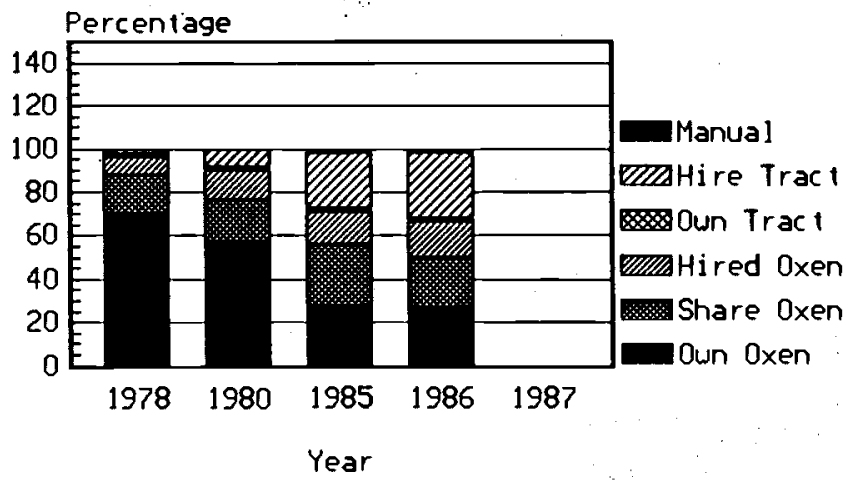

Percentage of Farmers

$1978 \quad \underline{1980 \quad 1985 \quad 1986} \quad 1987$

50.56

43.06

16.71

13.37

13.03

24.72

28.06

16.99

10.56

11.70

13.65

13.31

3.33

3.06

4.18

12.53

4.74

11.11

15.56

50.97

55.99

69.48

53.90

11.28

31.82

9.02

9.17

11.05

5.10

58.07

25.32

1.95

1.95

4.51

2.75

9.52

2.75

2.26

3.67

1.90

1.30

11.04

72.93

81.65

1.90

3. 81

82.86

(1)

$\begin{array}{rrrrr}69.38 & 60.38 & 37.50 & 36.76 & - \\ 16.88 & 18.87 & 22.50 & 17.65 & - \\ 6.25 & 8.81 & 12.50 & 11.76 & - \\ 4.38 & 4.40 & 3.75 & 4.41 & - \\ 7.50 & 12.58 & 26.88 & 30.88 & - \\ - & .63 & .63 & .74 & - \\ 70.00 & 57.35 & 27.69 & 26.79 & - \\ 17.14 & 19.12 & 27.69 & 23.21 & - \\ 8.57 & 13.24 & 15.38 & 16.07 & - \\ 1.43 & 1.47 & 1.54 & 1.79 & - \\ 2.86 & 8.82 & 26.15 & 30.36 & -\end{array}$


Ghazni

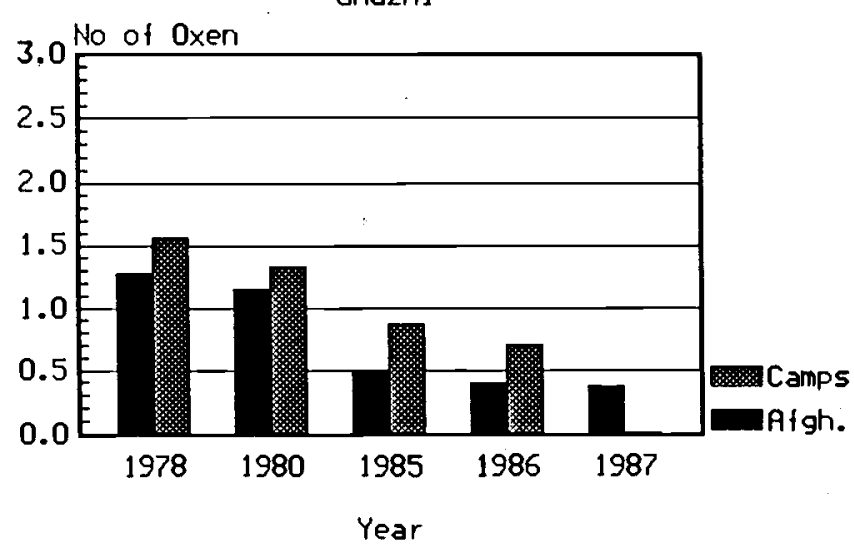

Ghazni

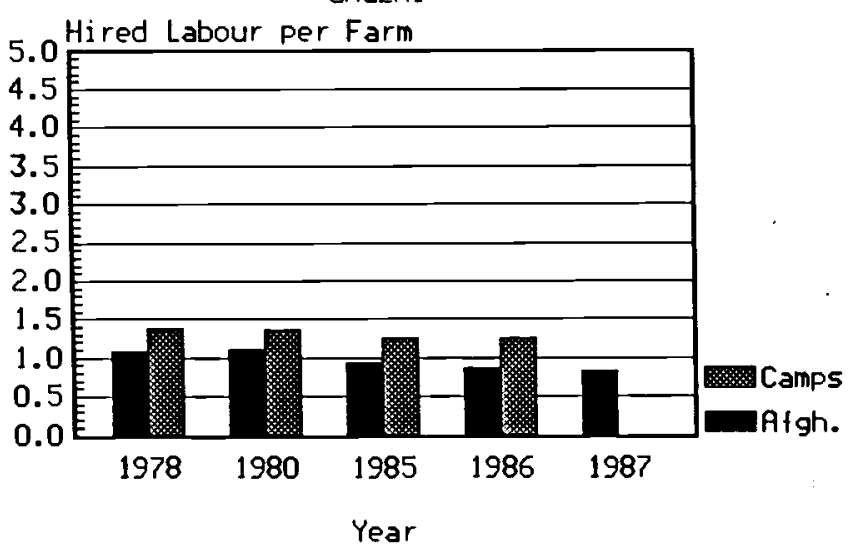

Ghazni

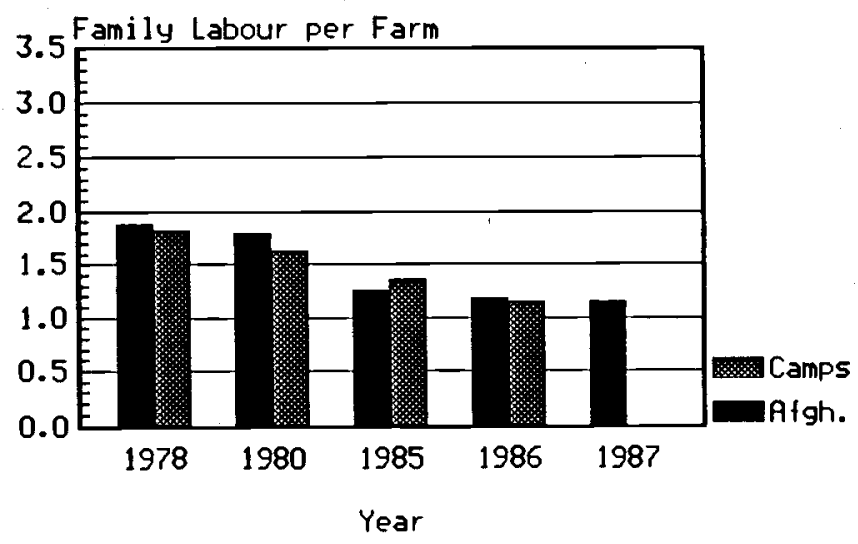

Ghazni

Percentage of Farms using

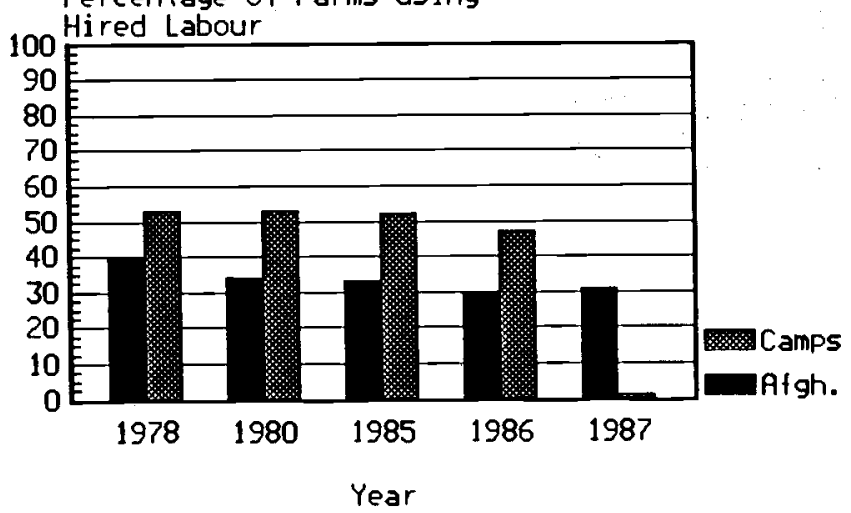


PROVINCE OF GZN

YEAR

1978 Number of Fanilies

x of Families

1980 Number of Families

$\boldsymbol{X}$ of Families

1985 Number of Families

$x$ of Families

1986 Number of Families

$x$ of Families

1987 Number of Families

$x$ of Families

Average Number of Oxen per Family:

\section{TABLE 7B - AVERAGE NU}

YEAR

1978 Number of Families

$x$ of Families

1980 Number of Families

$\boldsymbol{X}$ of Families

1985 Number of Families

$X$ of Families

1986 Number of Familles

\% of Families

1987 Number of Families

x of Families

Average Number of Oxen per Family:

$\begin{array}{ll}1978 & 1.56 \\ 1980 & 1.33 \\ 1985 & 0.87 \\ 1986 & 0.70 \\ 1987 & 0.01\end{array}$

Number of Farmers Questioned 371
MUMBER OF IRAINED OXEN PER FAMILY

$\begin{array}{llllll}100 & 91 & 167 & 7 & 6 & 0\end{array}$

$\begin{array}{llllll}26.95 & 24.53 & 45.01 & 1.89 & 1.62 & 0.00\end{array}$

$\begin{array}{llllll}115 & 104 & 140 & 7 & 5 & 0\end{array}$

$\begin{array}{llllll}31.00 & 28.03 & 37.74 & 1.89 & 1.35 & 0.00\end{array}$

$\begin{array}{llllll}254 & 57 & 58 & 2 & 0 & 0\end{array}$

$\begin{array}{llllll}68.46 & 15.36 & 15.63 & 0.54 & 0.00 & 0.00\end{array}$

$\begin{array}{llllll}277 & 45 & 48 & 1 & 0 & 0\end{array}$

$\begin{array}{llllll}74.66 & 12.13 & 12.94 & 0.27 & 0.00 & 0.00\end{array}$

$\begin{array}{llllll}283 & 43 & 44 & 1 & 0 & 0\end{array}$

$\begin{array}{llllll}76.28 & 11.59 & 11.86 & 0.27 & 0.00 & 0.00\end{array}$

$\begin{array}{ll}1978 & 1.27 \\ 1980 & 1.15 \\ 1985 & 0.48 \\ 1986 & 0.39 \\ 1987 & 0.36\end{array}$

1.27

39

0.36

TRAINED OXEN FOR FARMERS IN THE CAMPS

MUMBER OF TRAINED OXEN PER FAMILY

$0 \begin{array}{lllll} & 1 & 2 & 3 & 4\end{array}$

$\begin{array}{llllll}27 & 26 & 100 & 2 & 4 & 0\end{array}$

$\begin{array}{llllll}16.98 & 16.35 & 62.89 & 1.26 & 2.52 & 0.00\end{array}$

$\begin{array}{llllll}43 & 28 & 84 & 1 & 3 & 0\end{array}$

$\begin{array}{llllll}27.04 & 17.61 & 52.83 & 0.63 & 1.89 & 0.00\end{array}$

$\begin{array}{llllll}75 & 32 & 51 & 0 & 1 & 0\end{array}$

$\begin{array}{llllll}47.17 & 20.13 & 32.08 & 0.00 & 0.63 & 0.00\end{array}$

$\begin{array}{llllll}94 & 21 & 43 & 0 & 1 & 0\end{array}$

$\begin{array}{llllll}59.12 & 13.21 & 27.04 & 0.00 & 0.63 & 0.00\end{array}$

$\begin{array}{llllll}159 & 1 & 0 & 0 & 0 & 0\end{array}$

$\begin{array}{llllll}99.38 & 0.63 & 0.00 & 0.00 & 0.00 & 0.00\end{array}$

Number of Farmers Questioned 160 
PROVINCE OF GZN

\begin{tabular}{|c|c|c|c|c|c|}
\hline & 1978 & 1980 & 1985 & 1986 & 1987 \\
\hline Average Family Labour per Farm & 1.88 & 1.79 & 1.25 & 1.18 & 1.14 \\
\hline $\begin{array}{l}\text { Average No. of Hired Labour per Farm } \\
\text { (for farms using hired (abour) }\end{array}$ & 1.08 & 1.10 & 0.93 & 0.86 & 0.83 \\
\hline Number of Farms using Hired Labour & 148 & 126 & 123 & 113 & 114 \\
\hline
\end{tabular}

\section{IABLE 8(B) - AVERAGE LABOUR (FAMILY and HIRED) FOR FARMERS IN CAMPS}

PROVINCE OF GZN

\begin{tabular}{|c|c|c|c|c|c|}
\hline & 1978 & 1980 & 1985 & 1986 & 1987 \\
\hline Average Family Labour per Farm & 1.81 & 1.63 & 1.35 & 1.14 & 0.02 \\
\hline $\begin{array}{l}\text { Average No. of Hired Labour per Farm } \\
\text { (for farms using hired (abour) }\end{array}$ & 1.37 & 1.35 & 1.25 & 1.24 & 0.30 \\
\hline Number of Farms using Hired Labour & 85 & 84 & 83 & 75 & 1 \\
\hline
\end{tabular}


PROVINCE OF GZN

Average Number of Livestock Shot

$\begin{array}{lllll}1978 & 1980 & 1985 & 1986 & 1987\end{array}$

Number of Farmers reporting

Livestock Shot

$\star \star \star \star \star \quad 4.56 \quad 4.00 \quad 4.36 \quad 2.62$

Average Number of Livestock Killed

by Mines

$\begin{array}{lllll}\star \star \star \star * & 3.82 & 4.75 & 2.38 & 2.60\end{array}$

Number of Farmers reporting Livestock

killed by Mines

$\begin{array}{lllll}0 & 11 & 8 & 24 & 10\end{array}$

Number of Farmers Questioned 371

IABLE 9B - DIRECT EFFECTS OF THE HAR - FARMERS IN CAMPS

PROVINCE OF GZN

Average Number of Livestock Shot

$\begin{array}{lllll}1978 & 1980 & 1985 & 1986 & 1987\end{array}$

Number of Farmers reporting

Livestock shot

$0 \quad 9 \quad 51 \quad 15 \quad 0$

Average Number of Livestock Killed

by Mines

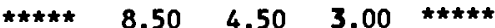

Number of Farmers reporting Livestock

killed by Mines

$\begin{array}{lllll}0 & 2 & 12 & 15 & 0\end{array}$

Number of Farmers Questioned 160 


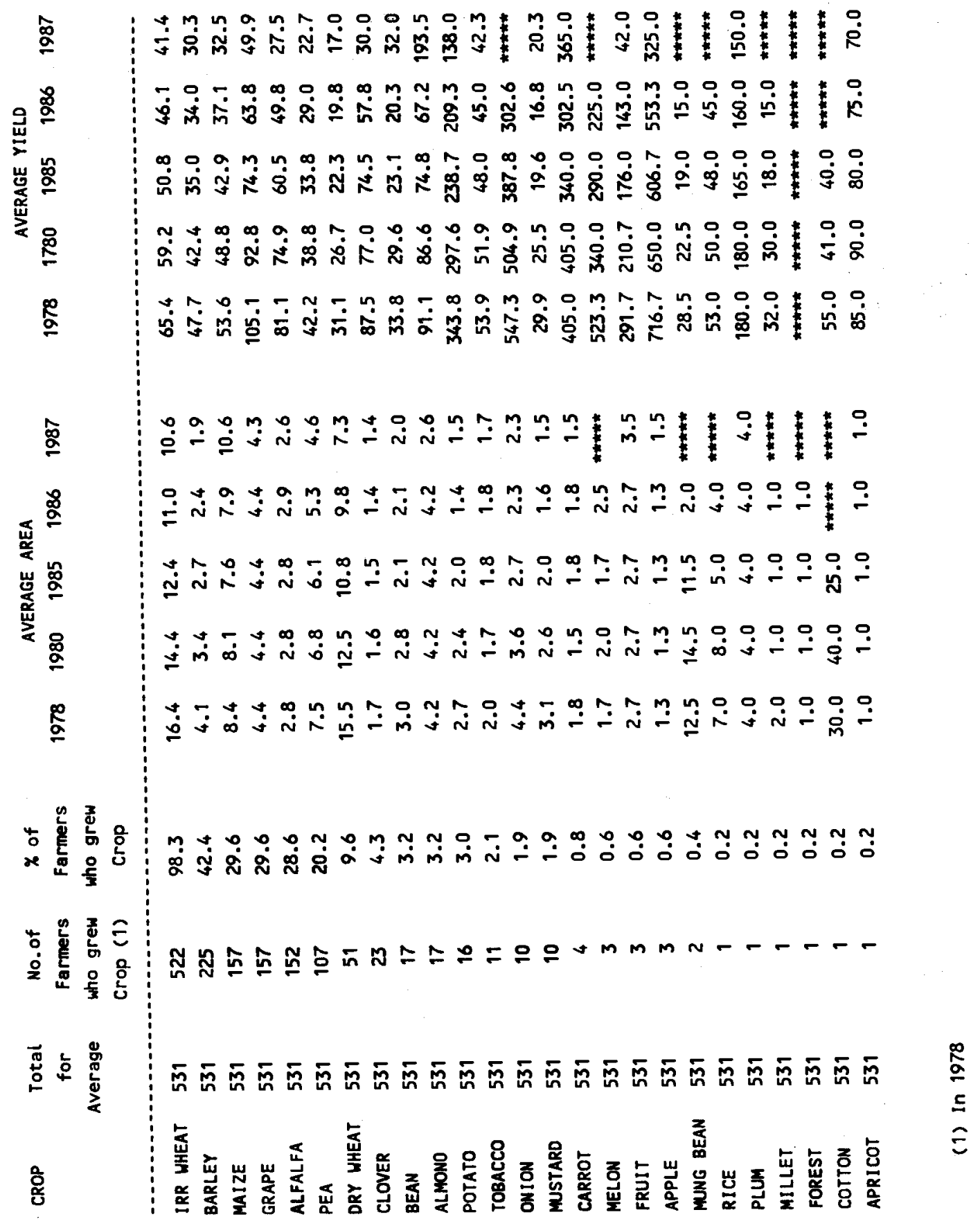


PROVINCE OF GZN

1987

\section{PERCENTAGE OF VILLAGES GIVING FOLLONING PRIORITY TO PROBLEM}

Rainfall

Crop Disease

Birds or Rats

Seed Availability

Fertilizer Availability

Crop Protection Chemicals

Credit

Irrigation Water Availability

Flooding

Labour Availabilty

Power for Land Preparation

Direct War Effects

Animal Diseases

Extension

Other Problems

\begin{tabular}{rrrrrrrrrrrrr}
1 & 2 & 3 & 4 & 5 & 6 & 7 & 8 & 9 & 10 & 11 & 12 & Weighted Priority \\
\hline 6 & 46 & 12 & 3 & 3 & 0 & 3 & 0 & 0 & 0 & 0 & 0 & 13.12 \\
1 & 1 & 3 & 8 & 8 & 18 & 22 & 12 & 2 & 0 & 0 & 0 & 5.27 \\
0 & 0 & 5 & 4 & 2 & 1 & 3 & 3 & 2 & 0 & 0 & 0 & 1.59 \\
0 & 2 & 0 & 3 & 3 & 4 & 6 & 5 & 3 & 2 & 0 & 0 & 1.90 \\
1 & 7 & 5 & 10 & 7 & 12 & 3 & 3 & 1 & 0 & 0 & 0 & 4.80 \\
0 & 1 & 0 & 1 & 3 & 7 & 21 & 18 & 5 & 1 & 0 & 0 & 3.16 \\
0 & 0 & 0 & 4 & 9 & 20 & 8 & 2 & 3 & 1 & 0 & 0 & 3.06 \\
62 & 17 & 8 & 2 & 1 & 1 & 0 & 0 & 0 & 0 & 0 & 0 & 28.16 \\
0 & 0 & 1 & 2 & 0 & 1 & 0 & 1 & 0 & 0 & 0 & 0 & 0.36 \\
1 & 4 & 11 & 17 & 39 & 14 & 3 & 1 & 1 & 0 & 0 & 0 & 8.29 \\
1 & 8 & 18 & 28 & 12 & 3 & 3 & 4 & 2 & 0 & 0 & 0 & 8.20 \\
28 & 15 & 37 & 15 & 3 & 2 & 0 & 0 & 0 & 0 & 0 & 0 & 19.97 \\
0 & 0 & 0 & 0 & 3 & 3 & 1 & 2 & 3 & 3 & 0 & 0 & 0.71 \\
1 & 0 & 1 & 3 & 1 & 0 & 5 & 4 & 2 & 2 & 2 & 0 & 1.40 \\
0 & 0 & 0 & 0 & 0 & 0 & 0 & 0 & 0 & 0 & 0 & 0 & 0.00
\end{tabular}

1986

Rainfall

Crop Disease

Birds or Rats

Seed Availability

Fertilizer Availability

Crop Protection Chemicals

Credit

Irrigation Water Availability

Flooding

Labour Availabilty

Power for Land Preparation

Direct War Effects

Animal Diseases

Extension

other Problems

\begin{tabular}{rrrrrrrrrrrrr}
1 & 2 & 3 & 4 & 5 & 6 & 7 & 8 & 9 & 10 & 11 & 12 & Heighted Priority \\
\hline 0 & 4 & 0 & 0 & 1 & 0 & 0 & 0 & 0 & 0 & 0 & 0 & 0.91 \\
4 & 5 & 6 & 8 & 18 & 23 & 11 & 1 & 1 & 0 & 0 & 0 & 7.88 \\
0 & 0 & 3 & 1 & 1 & 2 & 7 & 4 & 3 & 0 & 0 & 0 & 1.42 \\
0 & 2 & 1 & 2 & 4 & 7 & 6 & 7 & 2 & 0 & 0 & 0 & 2.15 \\
0 & 6 & 6 & 3 & 3 & 1 & 2 & 0 & 0 & 0 & 0 & 0 & 2.57 \\
2 & 4 & 3 & 4 & 10 & 18 & 20 & 6 & 0 & 0 & 0 & 0 & 5.89 \\
13 & 6 & 3 & 7 & 17 & 5 & 9 & 2 & 0 & 0 & 0 & 0 & 9.55 \\
34 & 32 & 17 & 7 & 0 & 0 & 0 & 0 & 0 & 0 & 0 & 0 & 23.16 \\
1 & 0 & 2 & 1 & 3 & 1 & 0 & 0 & 0 & 0 & 0 & 0 & 0.90 \\
5 & 3 & 20 & 32 & 18 & 7 & 3 & 0 & 0 & 0 & 0 & 0 & 10.61 \\
6 & 13 & 14 & 15 & 7 & 12 & 5 & 1 & 0 & 0 & 0 & 0 & 9.98 \\
35 & 26 & 21 & 13 & 3 & 2 & 0 & 0 & 0 & 0 & 0 & 0 & 23.85 \\
0 & 0 & 1 & 1 & 0 & 1 & 0 & 1 & 0 & 0 & 0 & 0 & 0.30 \\
0 & 0 & 2 & 2 & 1 & 2 & 2 & 3 & 0 & 1 & 0 & 0 & 0.83 \\
0 & 0 & 0 & 0 & 0 & 0 & 0 & 0 & 0 & 0 & 0 & 0 & 0.00
\end{tabular}

$\underline{1985}$

Rainfall

Crop Disease

Birds or Rats

Seed Availability

Fertilizer Availability

Crop Protection Chemicals

Credit

Irrigation Hater Availability

Flooding

Labour Availabilty

Power for Land Preparation

Direct War Effects

Animal Diseases

Extension

other Problems

\begin{tabular}{rrrrrrrrrrrrr}
1 & 2 & 3 & 4 & 5 & 6 & 7 & 8 & 9 & 10 & 11 & 12 & Weighted Priority \\
\hline 2 & 2 & 0 & 1 & 2 & 0 & 0 & 0 & 0 & 0 & 0 & 0 & 1.34 \\
1 & 1 & 2 & 0 & 2 & 5 & 3 & 1 & 1 & 0 & 0 & 0 & 1.60 \\
0 & 0 & 1 & 1 & 3 & 2 & 3 & 0 & 0 & 0 & 0 & 0 & 0.79 \\
1 & 1 & 2 & 3 & 8 & 17 & 3 & 1 & 0 & 0 & 0 & 0 & 3.31 \\
1 & 8 & 3 & 2 & 0 & 0 & 1 & 0 & 0 & 0 & 0 & 0 & 2.61 \\
0 & 1 & 0 & 1 & 3 & 1 & 2 & 2 & 0 & 0 & 0 & 0 & 0.75 \\
3 & 10 & 10 & 10 & 19 & 13 & 4 & 0 & 1 & 0 & 0 & 0 & 9.18 \\
21 & 37 & 15 & 9 & 2 & 0 & 0 & 0 & 0 & 0 & 0 & 0 & 20.55 \\
6 & 1 & 3 & 1 & 3 & 3 & 0 & 0 & 0 & 0 & 0 & 0 & 3.73 \\
3 & 7 & 23 & 29 & 16 & 1 & 2 & 0 & 0 & 0 & 0 & 0 & 10.74 \\
1 & 5 & 19 & 21 & 15 & 7 & 3 & 0 & 0 & 0 & 0 & 0 & 8.57 \\
58 & 23 & 12 & 4 & 0 & 0 & 0 & 0 & 0 & 0 & 0 & 0 & 32.36 \\
1 & 1 & 1 & 3 & 8 & 16 & 12 & 2 & 0 & 0 & 0 & 0 & 3.59 \\
0 & 0 & 1 & 2 & 2 & 1 & 3 & 2 & 2 & 0 & 0 & 0 & 0.89 \\
0 & 0 & 0 & 0 & 0 & 0 & 0 & 0 & 0 & 0 & 0 & 0 & 0.00
\end{tabular}


IABLE $1-5$ (contd) - ANALYSIS OF GREATEST FARMING PROBLEMS - COMMUNITY SURVEY

PROVINCE OF GZN

Rainfall

Crop Disease

Birds or Rats

Seed Availability

Fertilizer Availability

Crop Protection Chemicals

Credit

Irrigation Water Availability

Flooding

Labour Availabilty

Power for Land Preparation

Direct War Effects

Animal Diseases

Extension

Other Problems

\begin{tabular}{rrrrrrrrrrrrr}
1 & 2 & 3 & 4 & 5 & 6 & 7 & 8 & 9 & 10 & 11 & 12 & Weighted Priority \\
\hline 0 & 3 & 0 & 0 & 0 & 0 & 0 & 0 & 0 & 0 & 0 & 0 & 0.79 \\
0 & 1 & 3 & 2 & 0 & 1 & 0 & 0 & 0 & 0 & 0 & 0 & 1.14 \\
0 & 2 & 0 & 1 & 1 & 0 & 0 & 0 & 0 & 0 & 0 & 0 & 0.76 \\
4 & 27 & 18 & 10 & 3 & 0 & 0 & 0 & 0 & 0 & 0 & 0 & 16.90 \\
2 & 2 & 2 & 0 & 1 & 0 & 0 & 0 & 0 & 0 & 0 & 0 & 2.03 \\
0 & 0 & 3 & 1 & 0 & 0 & 0 & 0 & 0 & 0 & 0 & 0 & 0.66 \\
24 & 28 & 15 & 3 & 0 & 0 & 0 & 0 & 0 & 0 & 0 & 0 & 27.74 \\
3 & 13 & 8 & 9 & 0 & 0 & 0 & 0 & 0 & 0 & 0 & 0 & 9.07 \\
8 & 3 & 0 & 1 & 0 & 0 & 0 & 0 & 0 & 0 & 0 & 0 & 5.65 \\
0 & 2 & 3 & 1 & 1 & 0 & 0 & 0 & 0 & 0 & 0 & 0 & 1.29 \\
0 & 0 & 0 & 0 & 0 & 0 & 0 & 0 & 0 & 0 & 0 & 0 & 0.00 \\
35 & 5 & 26 & 8 & 0 & 0 & 0 & 0 & 0 & 0 & 0 & 0 & 30.42 \\
2 & 0 & 2 & 1 & 0 & 0 & 0 & 0 & 0 & 0 & 0 & 0 & 1.53 \\
0 & 0 & 0 & 1 & 2 & 1 & 0 & 0 & 0 & 0 & 0 & 0 & 0.43 \\
3 & 0 & 0 & 0 & 0 & 0 & 0 & 0 & 0 & 0 & 0 & 0 & 1.58
\end{tabular}

1978

Rainfall

Crop Disease

Birds or Rats

Seed Avai lability

Fertilizer Availability

Crop Protection Chemicals

Credit

Irrigation Water Availability

Flooding

Labour Availabilty

Power for Land Preparation

Direct War Effects

Animal Diseases

Extension

Other Problems

\begin{tabular}{rrrlllllllllc}
1 & 2 & 3 & 4 & 5 & 6 & 7 & 8 & 9 & 10 & 11 & 12 & Heighted Priority \\
\hline 0 & 0 & 0 & 0 & 0 & 0 & 0 & 0 & 0 & 0 & 0 & 0 & 0.00 \\
0 & 0 & 1 & 0 & 0 & 0 & 0 & 0 & 0 & 0 & 0 & 0 & 0.67 \\
2 & 0 & 0 & 0 & 0 & 0 & 0 & 0 & 0 & 0 & 0 & 0 & 4.01 \\
4 & 3 & 0 & 0 & 0 & 0 & 0 & 0 & 0 & 0 & 0 & 0 & 13.02 \\
2 & 3 & 0 & 0 & 0 & 0 & 0 & 0 & 0 & 0 & 0 & 0 & 7.01 \\
0 & 0 & 0 & 0 & 0 & 0 & 0 & 0 & 0 & 0 & 0 & 0 & 0.00 \\
1 & 1 & 1 & 0 & 0 & 0 & 0 & 0 & 0 & 0 & 0 & 0 & 3.67 \\
7 & 0 & 0 & 0 & 0 & 0 & 0 & 0 & 0 & 0 & 0 & 0 & 16.03 \\
18 & 3 & 0 & 0 & 0 & 0 & 0 & 0 & 0 & 0 & 0 & 0 & 46.08 \\
0 & 0 & 0 & 0 & 0 & 0 & 0 & 0 & 0 & 0 & 0 & 0 & 0.00 \\
0 & 1 & 0 & 0 & 0 & 0 & 0 & 0 & 0 & 0 & 0 & 0 & 1.00 \\
0 & 0 & 0 & 0 & 0 & 0 & 0 & 0 & 0 & 0 & 0 & 0 & 0.00 \\
0 & 1 & 0 & 0 & 0 & 0 & 0 & 0 & 0 & 0 & 0 & 0 & 1.00 \\
0 & 0 & 0 & 1 & 0 & 0 & 0 & 0 & 0 & 0 & 0 & 0 & 0.50 \\
3 & 1 & 0 & 0 & 0 & 0 & 0 & 0 & 0 & 0 & 0 & 0 & 7.01
\end{tabular}




\section{HELMAND}




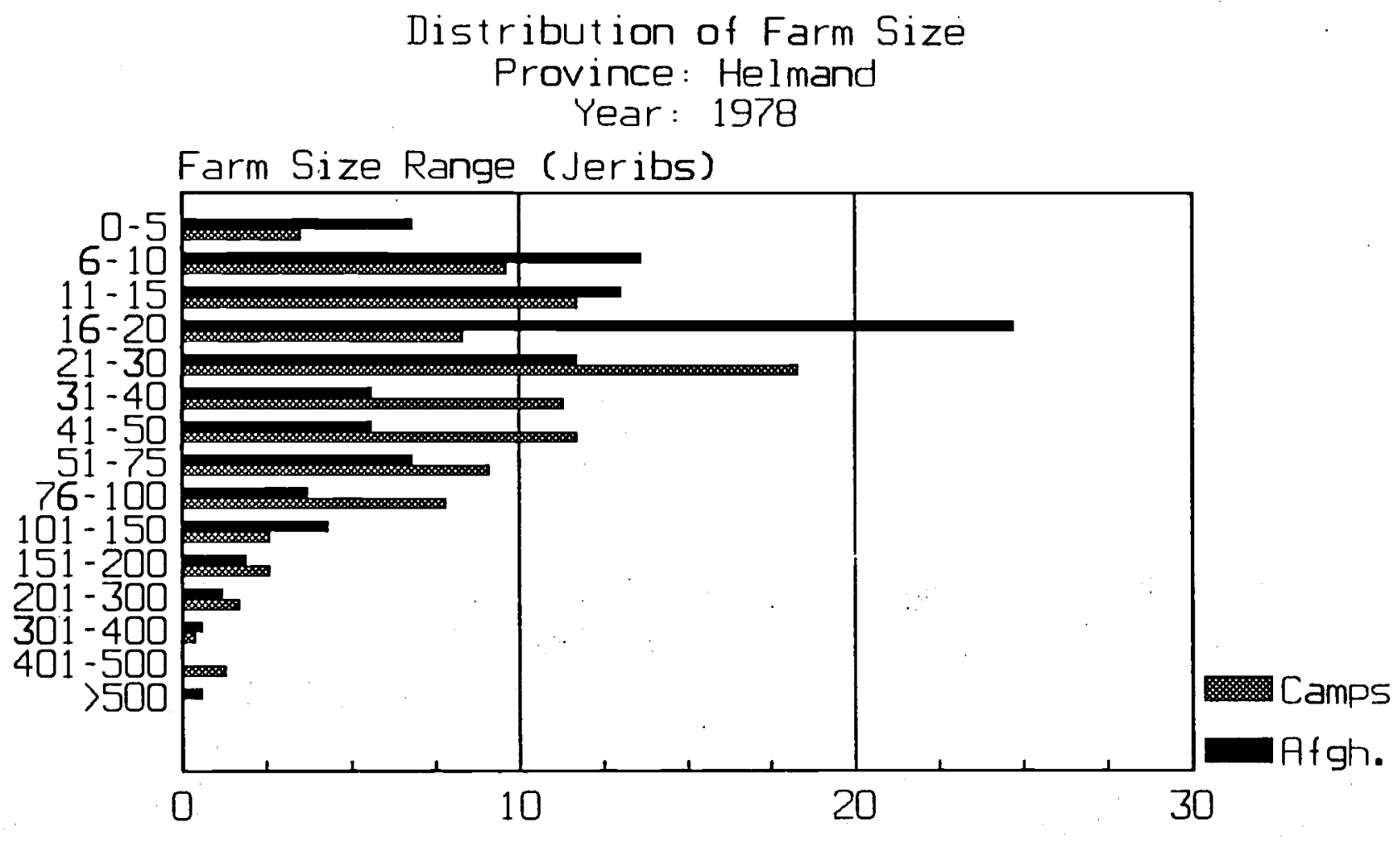

Percentage of Farmers

Farmers in Afghanistan

1978 1987

Total Number of Farms

Average Area

41.20

13.25

Average Area Irrigated

27.51

10.12

Average Dryland Area

Farmers who left in 1987

Total Number of Farms

Average Area

Average Area Irrigated

Average Dryland Area

\section{Average of Both Groups}

Provincial Average Area

Provincial Average Irrigated Area

47.32

13.25

Provincial Average Dryland Area

41.62

10.12

77.41

29.64

(All areas in Jeribs. 5 Jeribs $=1$ Hectare) 
Farm Power - Farmers in Atghanistan Province of Helmand

Crop: Irrigated wheat

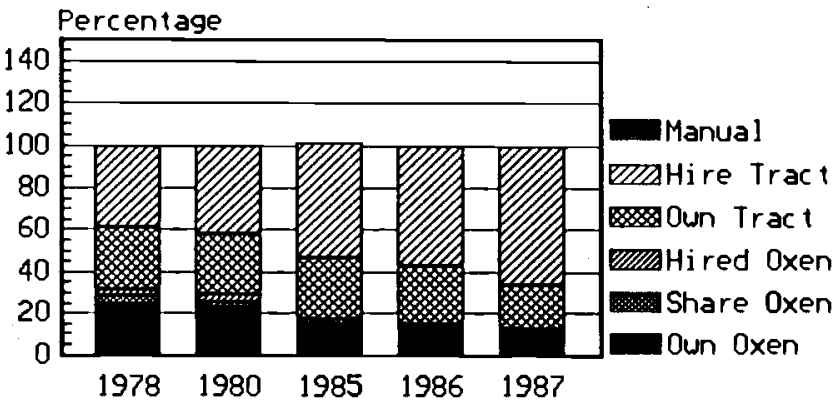

$\begin{array}{lllll}1978 & 1980 & 1985 & 1986 & 1987\end{array}$

Year

Farm Power - Farmers who lett in 1987

Province of Helmand

Crop: Irrigated wheat

Percentage

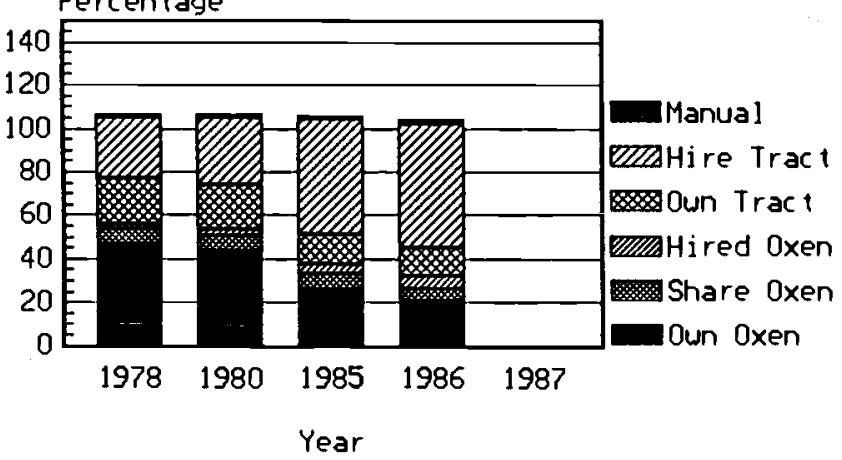

Base Figures for above graphs

Province: Helmand

Farmers in Afghanistan

Irr. Wheat own oxen

Shared oxen

Hired oxen

own Tractor

Hired Tractor

Maize

Manual Means

Own Oxen

Shared Oxen

Hired oxen

Own Tractor

Hired Tractor

Manual Means

Farmers who left in 1987

Irr. Wheat own oxen

Shared oxen

Hired oxen

Own Tractor

Hired Tractor

Maize

Manual Means

Own Oxen
$1978 \frac{\text { Percentage of Farmers }}{1980} 1985 \quad 1986$

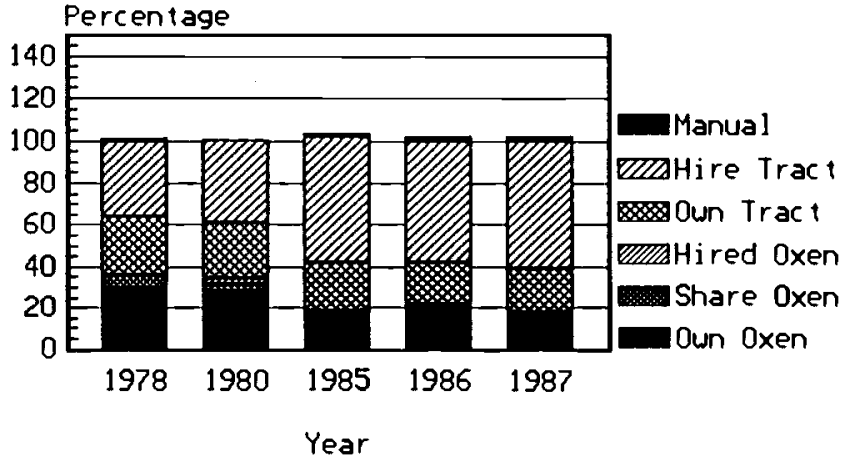

Farm Power - Farmers who lett in 1987

Province of Helmand Crop: Maize

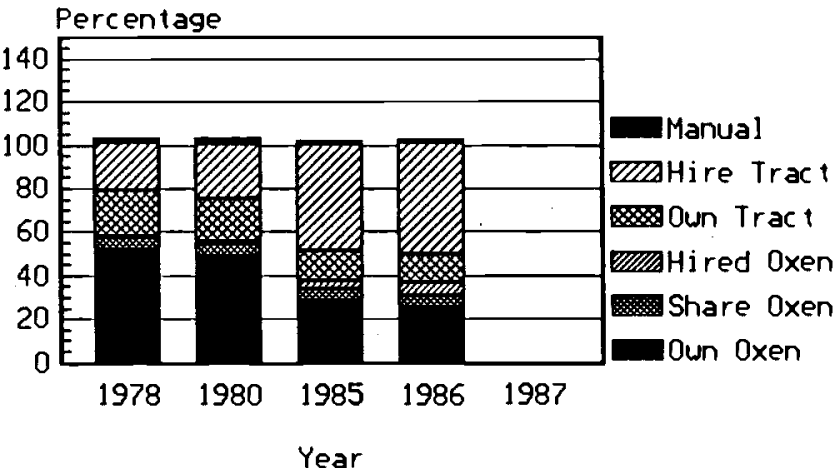

Shared oxen

Hired oxen

own Tractor

$\begin{array}{rr}46.96 & 43.48 \\ 6.52 & 6.96 \\ 2.17 & 2.61 \\ 20.87 & 20.87 \\ 28.70 & 30.87 \\ 1.30 & 1.30 \\ 52.12 & 48.78 \\ 5.45 & 5.49 \\ .61 & 1.22 \\ 20.61 & \text { ग0 1? }\end{array}$

$$
\begin{aligned}
& 1 \\
& 2 \\
& 5 \\
& 1
\end{aligned}
$$

3.91
.87
2.61

2.61

53.51

$-$

18.64

$-$

23.73

59.32

1.69

12.39

.88

1.77
27

27.43

57.52

1 - 8

21.82

$-$

20.00

58.18

1.82
10.71

1987

1.79

21.43

66.07

17.65

-

21.57

60.78

1.96
-

\begin{abstract}
1.69
\end{abstract}
26.03
6. 85
4.57
13.70
52.97
1.37
28.21
5.77
3.85
13.46


PROVINCE OF HLM

YEAR

1978 Number of Families

$\chi$ of Families

1980 Number of Families

$X$ of Families

1985 Number of Families

$x$ of Families

1986 Number of Families

x of Families

1987 Number of Families

$\chi$ of Families

Average Number of Oxen per Family:

1978

1980

1985

1986

1987 \begin{tabular}{ccccc} 
NUMBER & OF & TRAINED & OXEN & PER FAMILY \\
\hline 1 & 2 & 3 & 4
\end{tabular}

$\begin{array}{llllll}108 & 7 & 38 & 5 & 3 & 0\end{array}$

$\begin{array}{llllll}67.08 & 4.35 & 23.60 & 3.11 & 1.86 & 0.00\end{array}$

$\begin{array}{llllll}114 & 5 & 39 & 2 & 1 & 0\end{array}$

$\begin{array}{llllll}70.81 & 3.11 & 24.22 & 1.24 & 0.62 & 0.00\end{array}$

$\begin{array}{llllll}137 & 2 & 21 & 0 & 2 & 0\end{array}$

$\begin{array}{llllll}84.57 & 1.23 & 12.96 & 0.00 & 1.23 & 0.00\end{array}$

$\begin{array}{llllll}140 & 0 & 21 & 1 & 0 & 0\end{array}$

$\begin{array}{llllll}86.42 & 0.00 & 12.96 & 0.62 & 0.00 & 0.00\end{array}$

$\begin{array}{llllll}143 & 2 & 17 & 0 & 0 & 0\end{array}$

$\begin{array}{llllll}88.27 & 1.23 & 10.49 & 0.00 & 0.00 & 0.00\end{array}$

0.68

0.58

0.32

0.28

0.22

Number of Farmers Questioned 162

\section{IABLE 7B - AVERAGE NUMBER OF TRAINED OXEN FOR FARMERS IN THE CAMPS}

PROVINCE OF HLM

YEAR

1978 Number of Families

$\boldsymbol{X}$ of Families

1980 Number of Families

$x$ of Families

1985 Number of Families

\% of Families

1986 Number of Families

\% of Families

1987 Number of Families

\% of Families
NUMBER OF TRAINEO OXEN PER FAMILY

$\begin{array}{llllll}0 & 1 & 2 & 3 & 4 & 5\end{array}$

$\begin{array}{llllll}108 & 15 & 91 & 10 & 6 & 0\end{array}$

$\begin{array}{llllll}46.96 & 6.52 & 39.57 & 4.35 & 2.61 & 0.00\end{array}$

$\begin{array}{llllll}117 & 15 & 86 & 8 & 4 & 0\end{array}$

$\begin{array}{llllll}50.87 & 6.52 & 37.39 & 3.48 & 1.74 & 0.00\end{array}$

163 ic ju $\quad$ is $\quad \overline{3} \quad$ U

$\begin{array}{llllll}70.26 & 5.17 & 21.55 & 1.72 & 1.29 & 0.00\end{array}$

$\begin{array}{llllll}188 & 7 & 35 & 2 & 0 & 0\end{array}$

$\begin{array}{llllll}81.03 & 3.02 & 15.09 & 0.86 & 0.00 & 0.00\end{array}$

$\begin{array}{llllll}232 & 0 & 0 & 0 & 0 & 0\end{array}$

$\begin{array}{llllll}100.0 & 0.00 & 0.00 & 0.00 & 0.00 & 0.00\end{array}$

Average Number of Oxen per Family:

$\begin{array}{ll}1978 & 1.09 \\ 1980 & 0.99 \\ 1985 & 0.59 \\ 1986 & 0.36 \\ 1987 & 0.00\end{array}$

Number of Farmers Questioned 232 
PROVINCE OF HLM

\begin{tabular}{|c|c|c|c|c|c|}
\hline & 1978 & 1980 & 1985 & 1986 & 1987 \\
\hline Average Family Labour per Farm & 1.37 & 1.23 & 1.10 & 1.01 & 1.00 \\
\hline $\begin{array}{l}\text { Average No. of Hired Labour per Farm } \\
\text { (for farms using hired labour) }\end{array}$ & 1.87 & 1.75 & 1.64 & 1.64 & 1.61 \\
\hline Number of Farms Using Hired Labour & 91 & 80 & 50 & 49 & 49 \\
\hline \multicolumn{6}{|l|}{ Number of Farmers Questioned 162} \\
\hline IABLE $8(B)$ - AVERAGE LABOUR (FAMILY and & HIRED) & FOR FAR & RMERS IN & CAMPS & \\
\hline PROVINCE OF HLM & & & & & \\
\hline & 1978 & 1980 & 1985 & 1986 & 1987 \\
\hline Average Family Labour per Farm & 1.74 & 1.57 & 1.23 & 0.93 & 0.00 \\
\hline $\begin{array}{l}\text { Average No. of Hired Labour per Farm } \\
\text { (for farms using hired (abour) }\end{array}$ & 2.28 & 2.35 & 1.59 & 1.43 & 1.00 \\
\hline Number of Farms using Hired Labour & 153 & 159 & 135 & 113 & 1 \\
\hline Vumber of Farmers Questioned 232 & & & & & \\
\hline
\end{tabular}




\section{TABLE 9A - DIRECT EFFECTS OF THE HAR - FARMERS IN AFGANISTAN}

PROVINCE OF HLM

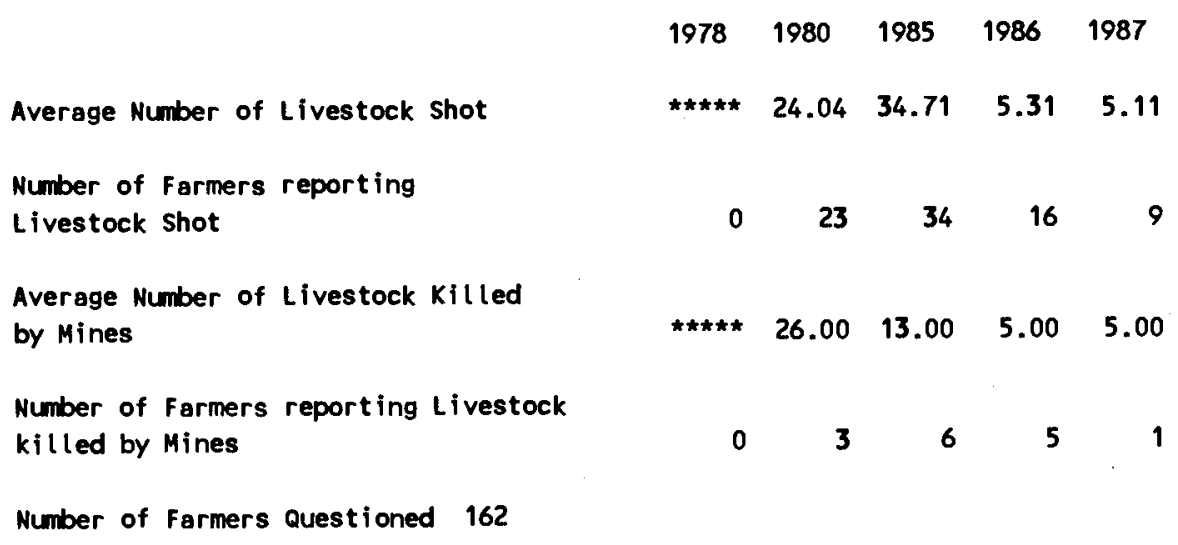

\section{TABLE 9B - DIRECT EFFECTS OF THE WAR - FARMERS IN CAMPS}

PROVINCE OF HLM

\begin{tabular}{l|rrrrr} 
& 1978 & 1980 & 1985 & 1986 & 1987 \\
$\begin{array}{l}\text { Average Number of Livestock Shot } \\
\text { Number of Farmers reporting }\end{array}$ & $\star \star \star \star \star$ & 2.71 & 3.75 & 3.56 & $\star \star \star \star \star$
\end{tabular}

Number of Farmers Questioned 232 


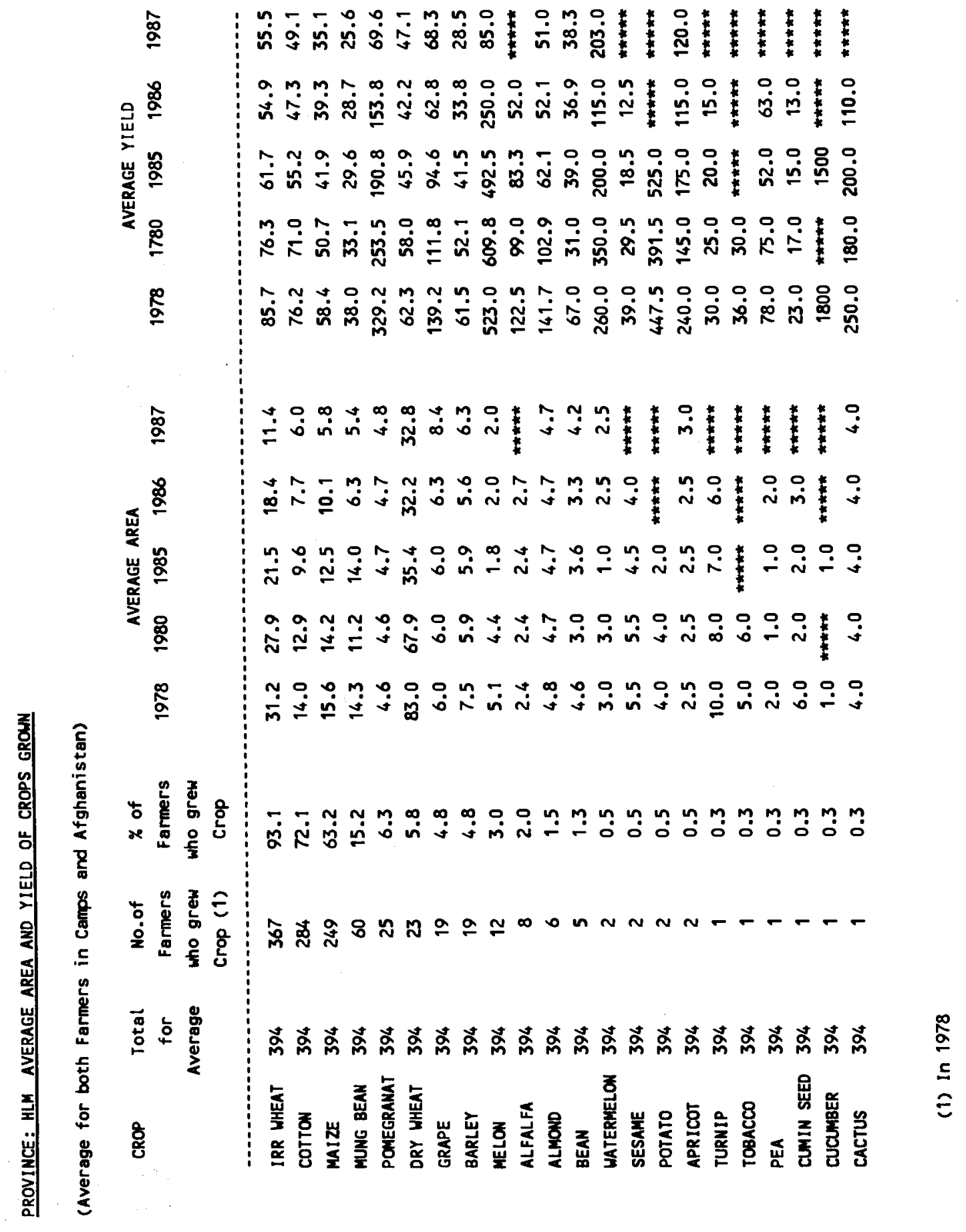


PROVINCE OF HLM

Rainfall

Crop Disease

Birds or Rats

Seed Availability

Fertilizer Availability

Crop Protection Chemicals

Credit

Irrigation Hater Availability

Flooding

Labour Availabilty

Power for Land Preparation

Direct Har Effects

Animal Diseases

Extension

Other Problems

\begin{tabular}{rrrrrrrrrrrrc}
1 & 2 & 3 & 4 & 5 & 6 & 7 & 8 & 9 & 10 & 11 & 12 & Heighted Priority \\
\hline 0 & 4 & 11 & 4 & 0 & 0 & 0 & 0 & 0 & 0 & 0 & 0 & 3.03 \\
0 & 0 & 0 & 4 & 43 & 26 & 4 & 0 & 0 & 0 & 0 & 0 & 6.49 \\
0 & 2 & 0 & 0 & 2 & 0 & 2 & 2 & 0 & 0 & 0 & 0 & 0.93 \\
0 & 0 & 0 & 0 & 9 & 22 & 4 & 0 & 0 & 0 & 0 & 0 & 2.63 \\
2 & 17 & 24 & 26 & 2 & 0 & 0 & 0 & 0 & 0 & 0 & 0 & 11.35 \\
0 & 0 & 0 & 0 & 0 & 0 & 0 & 0 & 0 & 0 & 0 & 0 & 0.00 \\
0 & 0 & 0 & 0 & 0 & 0 & 2 & 0 & 0 & 0 & 0 & 0 & 0.14 \\
7 & 17 & 37 & 17 & 0 & 0 & 0 & 0 & 0 & 0 & 0 & 0 & 14.03 \\
0 & 0 & 0 & 0 & 0 & 0 & 0 & 0 & 0 & 0 & 0 & 0 & 0.00 \\
0 & 0 & 0 & 4 & 15 & 9 & 15 & 0 & 0 & 0 & 0 & 0 & 3.41 \\
11 & 30 & 17 & 30 & 0 & 0 & 0 & 0 & 0 & 0 & 0 & 0 & 17.38 \\
74 & 17 & 2 & 0 & 0 & 0 & 0 & 0 & 0 & 0 & 0 & 0 & 36.66 \\
0 & 0 & 0 & 2 & 0 & 13 & 17 & 4 & 2 & 0 & 0 & 0 & 2.63 \\
0 & 0 & 0 & 0 & 9 & 4 & 0 & 4 & 0 & 0 & 0 & 0 & 1.32 \\
0 & 0 & 0 & 0 & 0 & 0 & 0 & 0 & 0 & 0 & 0 & 0 & 0.00
\end{tabular}

$\underline{1986}$

Rainfall

Crop Disease

Birds or Rats

Seed Availability

Fertilizer Avai lability

Crop Protection Chemicals

Credit

Irrigation Water Avail lability

Flooding

Labour Availabilty

Power for Land Preparation

Direct War Effects

Animal Diseases

Extension

other Problems

\section{5}

\begin{tabular}{rrrrrrrrrrrrr}
1 & 2 & 3 & 4 & 5 & 6 & 7 & 8 & 9 & 10 & 11 & 12 & Weighted Priority \\
\hline 0 & 7 & 4 & 7 & 2 & 0 & 0 & 0 & 0 & 0 & 0 & 0 & 2.84 \\
0 & 0 & 0 & 4 & 28 & 33 & 7 & 0 & 0 & 0 & 0 & 0 & 5.50 \\
0 & 0 & 0 & 0 & 2 & 7 & 11 & 2 & 0 & 0 & 0 & 0 & 1.40 \\
0 & 0 & 0 & 0 & 15 & 9 & 7 & 4 & 0 & 0 & 0 & 0 & 2.51 \\
2 & 11 & 22 & 41 & 0 & 0 & 0 & 0 & 0 & 0 & 0 & 0 & 10.57 \\
0 & 0 & 0 & 0 & 0 & 0 & 2 & 2 & 0 & 0 & 0 & 0 & 0.24 \\
0 & 0 & 0 & 0 & 0 & 2 & 0 & 0 & 0 & 0 & 0 & 0 & 0.15 \\
7 & 37 & 33 & 13 & 0 & 0 & 0 & 0 & 0 & 0 & 0 & 0 & 16.43 \\
0 & 0 & 0 & 0 & 0 & 0 & 0 & 0 & 0 & 0 & 0 & 0 & 0.00 \\
0 & 0 & 0 & 2 & 9 & 15 & 15 & 2 & 0 & 0 & 0 & 0 & 3.05 \\
7 & 28 & 35 & 24 & 0 & 0 & 0 & 0 & 0 & 0 & 0 & 0 & 16.05 \\
83 & 11 & 2 & 2 & 0 & 0 & 0 & 0 & 0 & 0 & 0 & 0 & 37.49 \\
0 & 0 & 0 & 0 & 20 & 7 & 7 & 2 & 0 & 0 & 0 & 0 & 2.60 \\
0 & 0 & 0 & 0 & 7 & 2 & 4 & 0 & 4 & 0 & 0 & 0 & 1.16 \\
0 & 0 & 0 & 0 & 0 & 0 & 0 & 0 & 0 & 0 & 0 & 0 & 0.00
\end{tabular}

Rainfall

Crop Disease

Birds or Rats

Seed Availability

Fertilizer Availability

Crop Protection Chemicals

Credit

Irrigation Water Availability

Flooding

Labour Availabilty

Power for Land Preparation

Direct War Effects

Animal Diseases

Extension

Other Problems

\begin{tabular}{rrrrrrrrrrrrr}
1 & 2 & 3 & 4 & 5 & 6 & 7 & 8 & 9 & 10 & 11 & 12 & Weighted Priority \\
\hline 0 & 11 & 2 & 4 & 2 & 0 & 0 & 0 & 0 & 0 & 0 & 0 & 3.18 \\
0 & 0 & 0 & 7 & 28 & 24 & 13 & 2 & 0 & 0 & 0 & 0 & 5.55 \\
0 & 0 & 0 & 2 & 4 & 7 & 9 & 2 & 0 & 0 & 0 & 0 & 1.66 \\
0 & 0 & 0 & 0 & 11 & 4 & 7 & 2 & 0 & 0 & 0 & 0 & 1.70 \\
0 & 7 & 43 & 26 & 2 & 0 & 0 & 0 & 0 & 0 & 0 & 0 & 10.23 \\
0 & 0 & 0 & 0 & 0 & 2 & 2 & 0 & 0 & 0 & 0 & 0 & 0.28 \\
0 & 0 & 0 & 0 & 2 & 2 & 0 & 0 & 0 & 0 & 0 & 0 & 0.33 \\
9 & 46 & 24 & 13 & 4 & 0 & 0 & 0 & 0 & 0 & 0 & 0 & 18.05 \\
0 & 0 & 0 & 0 & 0 & 0 & 0 & 0 & 0 & 0 & 0 & 0 & 0.00 \\
0 & 0 & 0 & 2 & 11 & 15 & 4 & 2 & 2 & 0 & 0 & 0 & 2.64 \\
0 & 26 & 26 & 43 & 2 & 0 & 0 & 0 & 0 & 0 & 0 & 0 & 13.68 \\
89 & 9 & 0 & 0 & 0 & 0 & 0 & 0 & 0 & 0 & 0 & 0 & 38.69 \\
0 & 0 & 0 & 2 & 11 & 20 & 7 & 2 & 0 & 0 & 0 & 0 & 2.97 \\
0 & 0 & 0 & 0 & 4 & 7 & 2 & 2 & 0 & 0 & 0 & 0 & 1.05 \\
0 & 0 & 0 & 0 & 0 & 0 & 0 & 0 & 0 & 0 & 0 & 0 & 0.00
\end{tabular}


PROVINCE OF HLM

\section{PERCENTAGE OF VILLAGES GIVING FOLLONING PRIORITY TO PROBLEM}

Rainfall

Crop Disease

Birds or Rats

Seed Availability

Fertilizer Availability

Crop Protection Chemicals

Credit

Irrigation Water Availability

Flooding

Labour Avai labilty

Power for Land Preparation

Direct War Effects

Animal Diseases

Extension

other Problems

\begin{tabular}{ccccccccccccc}
1 & 2 & 3 & 4 & 5 & 6 & 7 & 8 & 9 & 10 & 11 & 12 & Weighted Priority \\
\hline 0 & 7 & 7 & 4 & 0 & 0 & 0 & 0 & 0 & 0 & 0 & 0 & 2.79
\end{tabular}

$\begin{array}{lllllllllllll}0 & 0 & 4 & 2 & 17 & 35 & 9 & 0 & 0 & 0 & 0 & 0 & 5.36\end{array}$

$\begin{array}{lllllllllllll}0 & 2 & 0 & 0 & 7 & 4 & 13 & 0 & 0 & 0 & 0 & 0 & 2.13\end{array}$

$\begin{array}{lllllllllllll}0 & 0 & 0 & 0 & 9 & 7 & 0 & 2 & 0 & 0 & 0 & 0 & 1.33\end{array}$

$\begin{array}{lllllllllllll}0 & 9 & 39 & 28 & 0 & 0 & 0 & 0 & 0 & 0 & 0 & 0 & 10.47\end{array}$

$\begin{array}{lllllllllllll}0 & 0 & 0 & 0 & 2 & 0 & 0 & 0 & 0 & 0 & 0 & 0 & 0.19\end{array}$

$\begin{array}{lllllllllllll}0 & 0 & 0 & 0 & 0 & 0 & 2 & 0 & 0 & 0 & 0 & 0 & 0.13\end{array}$

$\begin{array}{lllllllllllll}22 & 43 & 11 & 13 & 2 & 0 & 0 & 0 & 0 & 0 & 0 & 0 & 21.75\end{array}$

$\begin{array}{lllllllllllll}0 & 0 & 0 & 0 & 0 & 0 & 0 & 0 & 0 & 0 & 0 & 0 & 0.00\end{array}$

$\begin{array}{lllllllllllll}0 & 0 & 0 & 2 & 11 & 4 & 7 & 2 & 0 & 0 & 0 & 0 & 1.99\end{array}$

$\begin{array}{lllllllllllll}0 & 15 & 35 & 41 & 4 & 0 & 0 & 0 & 0 & 0 & 0 & 0 & 13.01\end{array}$

$\begin{array}{lllllllllllll}76 & 22 & 0 & 0 & 0 & 0 & 0 & 0 & 0 & 0 & 0 & 0 & 37.22\end{array}$

$\begin{array}{lllllllllllll}0 & 0 & 0 & 4 & 17 & 9 & 4 & 0 & 0 & 0 & 0 & 0 & 2.84\end{array}$

$\begin{array}{lllllllllllll}0 & 0 & 0 & 0 & 7 & 0 & 2 & 0 & 2 & 0 & 0 & 0 & 0.79\end{array}$

$\begin{array}{lllllllllllll}0 & 0 & 0 & 0 & 0 & 0 & 0 & 0 & 0 & 0 & 0 & 0 & 0.00\end{array}$

\section{8}

Rainfall

Crop Disease

Birds or Rats

Seed Availability

Fertilizer Availability

Crop Protection Chemicals

Credit

Irrigation Water Availability

Flooding

Labour Availabilty

Power for Land Preparation

Direct War Effects

Animal Diseases

Extension

Other Problems

\begin{tabular}{ccccccccccccc}
1 & 2 & 3 & 4 & 5 & 6 & 7 & 8 & 9 & 10 & 11 & 12 & Heighted Priority \\
\hline 0 & 0 & 0 & 0 & 0 & 0 & 0 & 0 & 0 & 0 & 0 & 0 & 0.00 \\
9 & 2 & 2 & 0 & 0 & 0 & 0 & 0 & 0 & 0 & 0 & 0 & 36.62 \\
2 & 2 & 4 & 2 & 2 & 0 & 0 & 0 & 0 & 0 & 0 & 0 & 19.82 \\
0 & 0 & 0 & 0 & 0 & 0 & 0 & 0 & 0 & 0 & 0 & 0 & 0.00 \\
0 & 0 & 0 & 0 & 0 & 0 & 0 & 0 & 0 & 0 & 0 & 0 & 0.00 \\
0 & 0 & 0 & 0 & 0 & 0 & 0 & 0 & 0 & 0 & 0 & 0 & 0.00 \\
0 & 0 & 0 & 0 & 0 & 0 & 0 & 0 & 0 & 0 & 0 & 0 & 0.00 \\
0 & 4 & 0 & 0 & 0 & 0 & 0 & 0 & 0 & 0 & 0 & 0 & 7.58 \\
0 & 0 & 0 & 0 & 0 & 0 & 0 & 0 & 0 & 0 & 0 & 0 & 0.00 \\
0 & 0 & 0 & 0 & 0 & 0 & 0 & 0 & 0 & 0 & 0 & 0 & 0.00 \\
0 & 4 & 2 & 2 & 0 & 0 & 0 & 0 & 0 & 0 & 0 & 0 & 11.99 \\
2 & 0 & 0 & 0 & 0 & 0 & 0 & 0 & 0 & 0 & 0 & 0 & 7.58 \\
0 & 4 & 4 & 2 & 0 & 0 & 0 & 0 & 0 & 0 & 0 & 0 & 14.52 \\
0 & 0 & 0 & 2 & 0 & 0 & 0 & 0 & 0 & 0 & 0 & 0 & 1.89 \\
0 & 0 & 0 & 0 & 0 & 0 & 0 & 0 & 0 & 0 & 0 & 0 & 0.00
\end{tabular}




\section{HERAT}




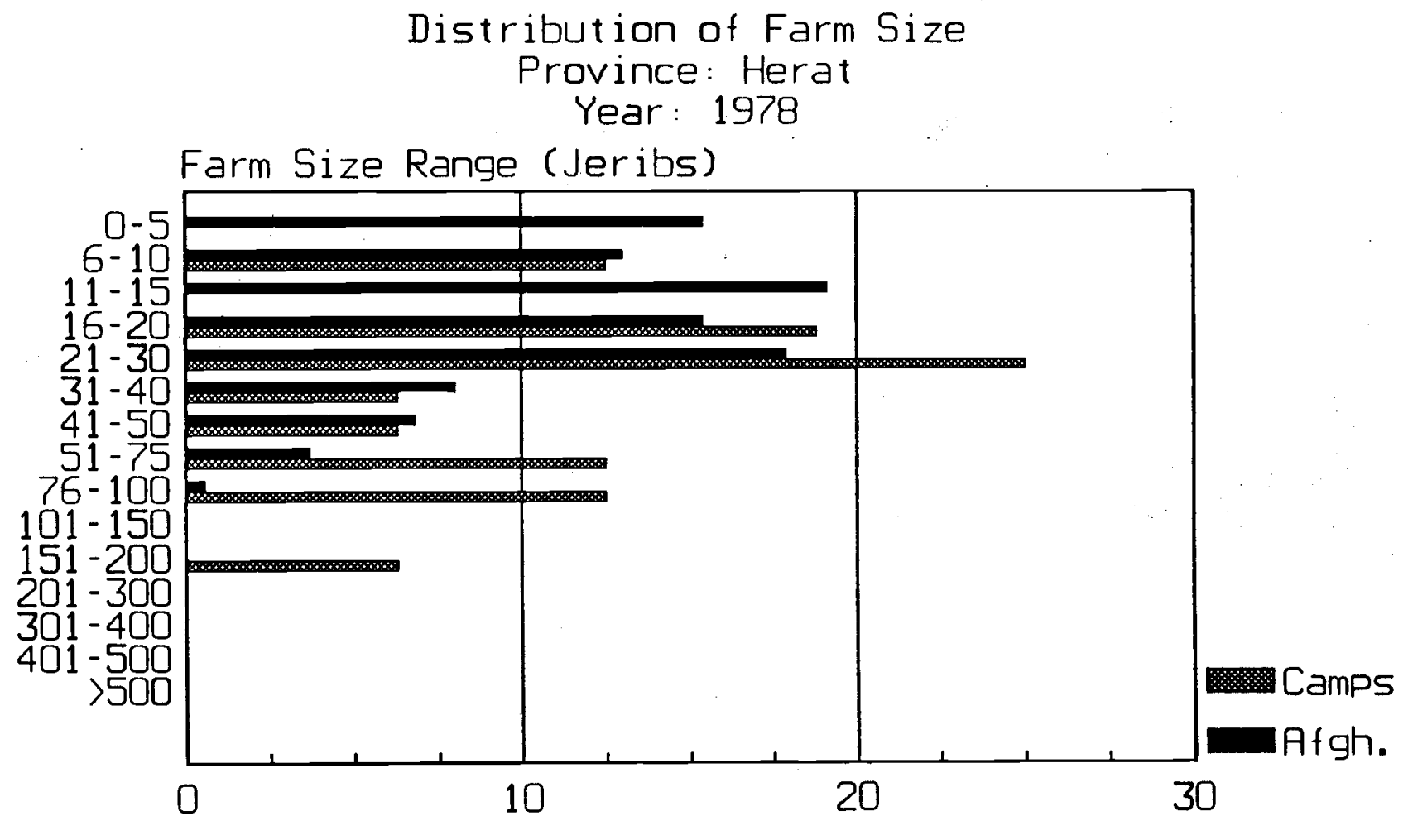

Percentage of Farmers

Farmers in Afghanistan

1978

1987

Total Number of Farms

Average Area

20.94

11.35

Average Area Irrigated

19.86

10.42

Average Dryland Area

11.11

2.96

Farmers who left in 1987

Total Number of Farms

Average Area

16

45.75

39.13

Average Area Irrigated

35.33

\section{Average of Both Groups}

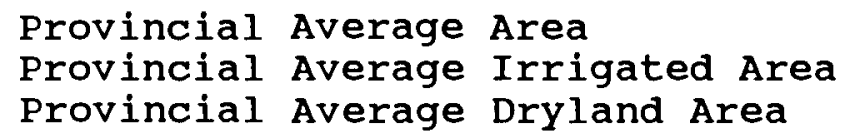

23.17

11.35

21.65

10.42

13.53

2.96

(All areas in Jeribs. 5 Jeribs $=1$ Hectare) $\cdot$ pa 


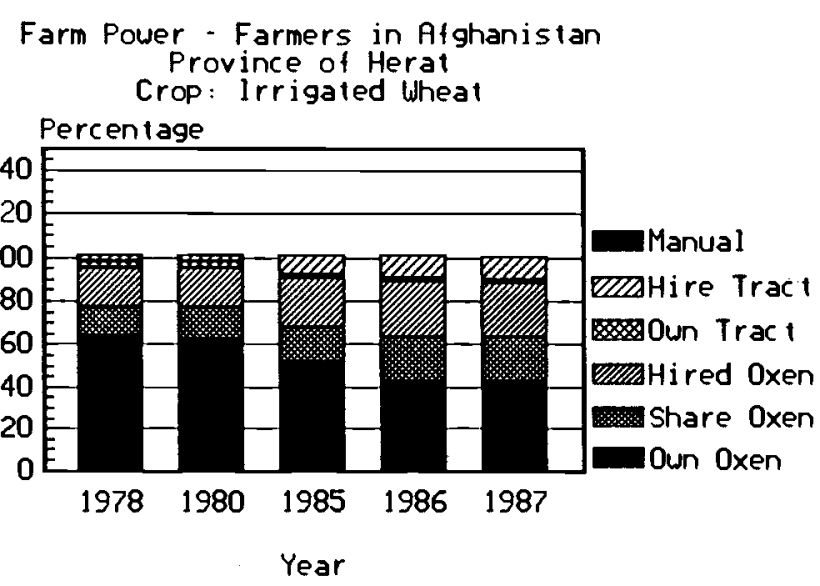

Farm Power - Farmers who lett in 1987 Province of Herat Crop: Irrigated wheat

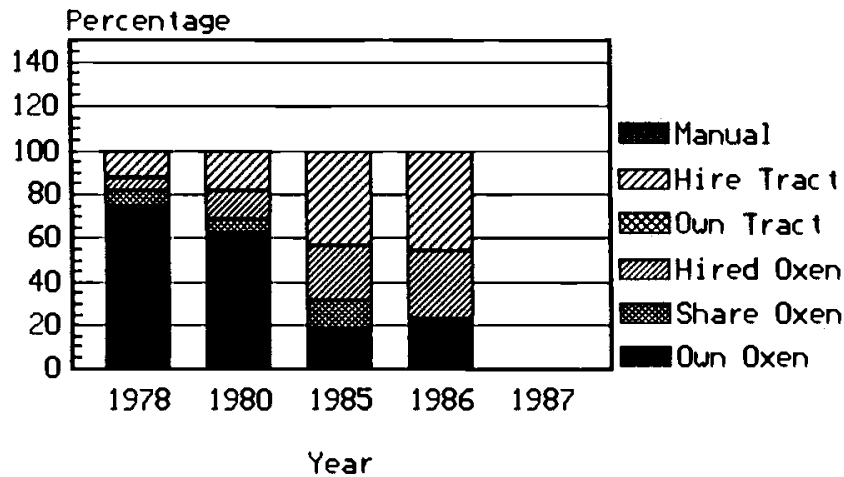

Base Figures for above graphs

Province: Herat

\section{Farmers in Afghanistan}

Irr. Wheat Own Oxen Shared oxen

Hired oxen

Own Tractor

Hired Tractor

Barley

Manual Means

Own Oxen

Shared oxen

Hired oxen

Own Tractor

Hired Tractor

Manual Means
Farm Power - Farmers in Atghanistan Province of Herat

Crop: Barley

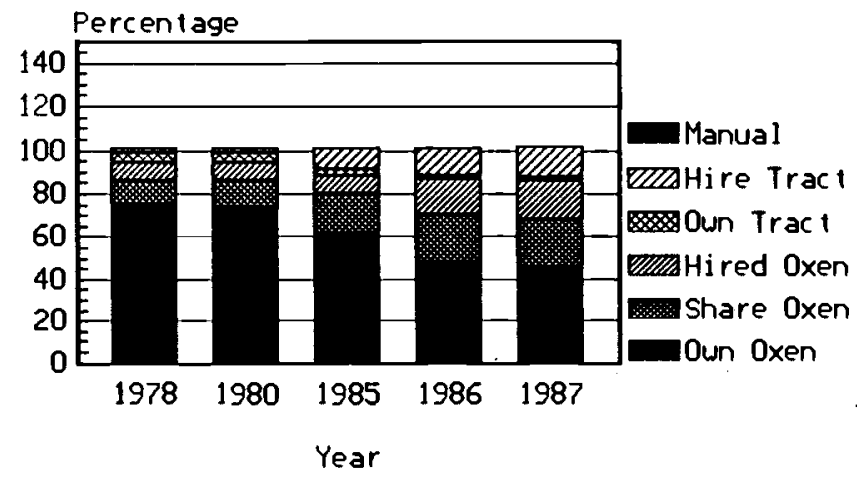

Farm Power - Farmers who lett in 1987 Province of Herat Crop: Barley

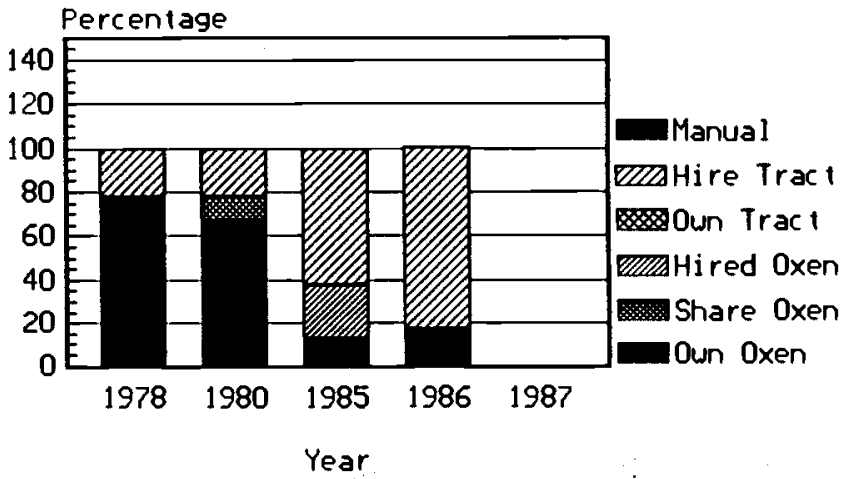

\begin{tabular}{rrrrrr} 
& \multicolumn{2}{l}{ Percentage of } & Farmers & \\
\cline { 3 - 4 } 1978 & & 1980 & $\underline{1985}$ & $\underline{1986}$ & 1987 \\
& & & & \\
63.89 & 62.50 & 51.75 & 43.17 & 42.96 \\
13.19 & 14.58 & 16.08 & 20.14 & 20.00 \\
18.06 & 18.06 & 22.38 & 25.90 & 25.19 \\
2.78 & 2.78 & 2.10 & 1.44 & 1.48 \\
3.47 & 3.47 & 9.09 & 10.79 & 11.11 \\
- & - & - & - & - \\
75.34 & 73.97 & 61.43 & 47.76 & 46.15 \\
10.96 & 12.33 & 18.57 & 22.39 & 21.54 \\
8.22 & 8.22 & 8.57 & 16.42 & 18.46 \\
4.11 & 4.11 & 2.86 & 1.49 & 1.54 \\
2.74 & 2.74 & 10.00 & 13.43 & 13.85 \\
- & - & - & - & -
\end{tabular}

Farmers who left in 1987

$\begin{array}{ll}\text { Irr. Wheat } & \text { Own Oxen } \\ & \text { Shared Oxen } \\ \text { Hired oxen } & \text { Own Tractor } \\ & \text { Hired Tractor } \\ & \text { Manual Means } \\ & \text { Own Oxen } \\ \text { Shared Oxen } \\ \text { Hired oxen } \\ \text { Own Tractor } \\ \text { Ow }\end{array}$

$\begin{array}{rcccc}75.00 & 62.50 & 18.75 & 23.08 & - \\ 6.25 & 6.25 & 12.50 & - & - \\ 6.25 & 12.50 & 25.00 & 30.77 & - \\ - & - & - & - & - \\ 12.50 & 18.75 & 43.75 & 46.15 & - \\ - & - & - & - & - \\ 77.78 & 66.67 & 12.50 & 16.67 & - \\ - & 11.11 & - & - & - \\ - & - & 25.00 & - & - \\ - & - & - & - & -\end{array}$



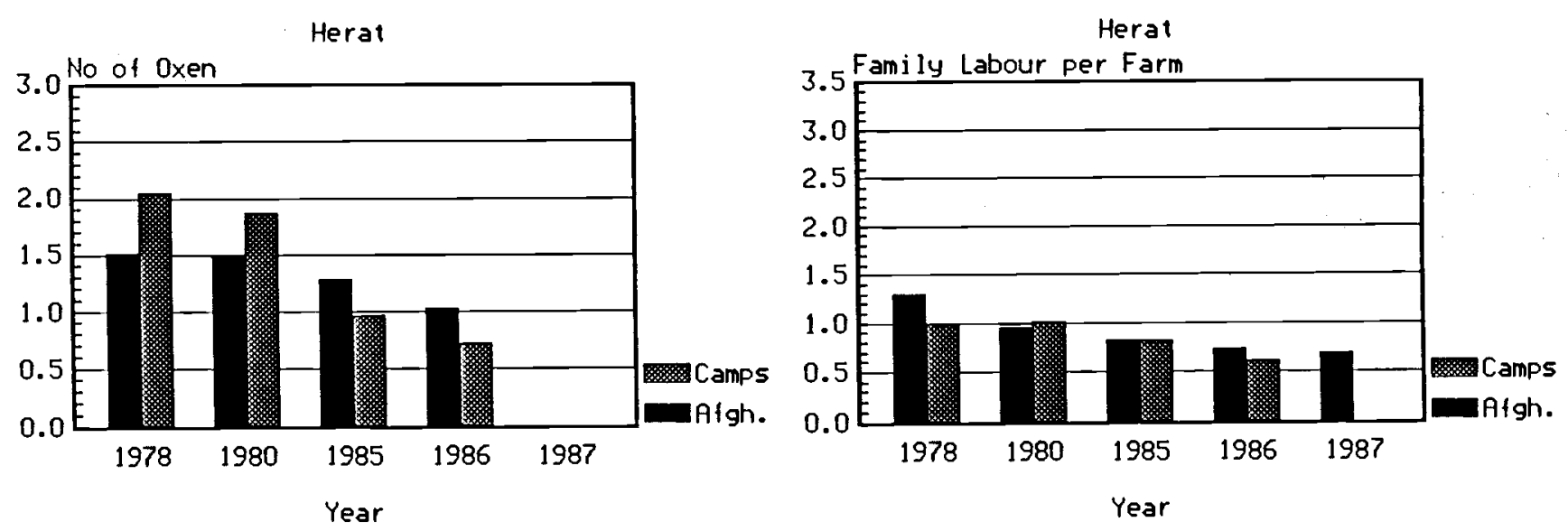

Herat

Herat
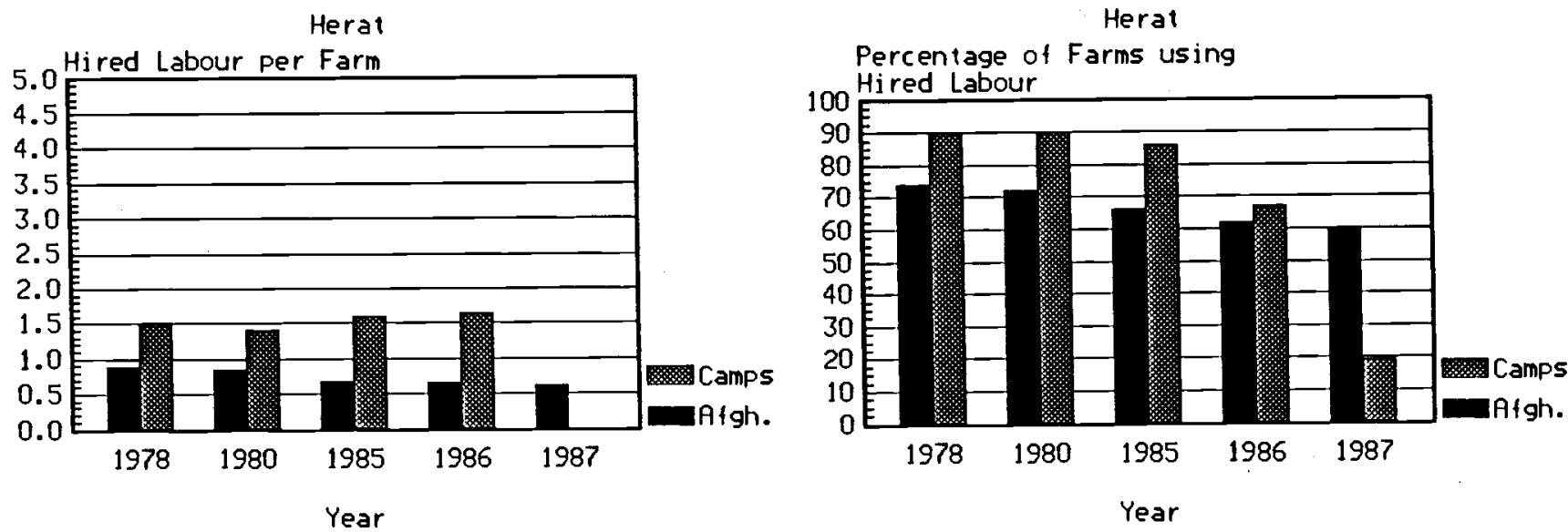
PROVINCE OF HRT

YEAR

1978 Number of Families

\% of Families

1980 Number of Families

$x$ of Families

1985 Number of Families

$x$ of Families

1986 Number of Fanilies

$\%$ of Families

1987 Number of Families

$x$ of Families

Average Number of Oxen per Family: \begin{tabular}{lcccc} 
NUMBER OF & TRAINED & OXEN PER FAMILY \\
\hline & 1 & 2 & 3 & 4
\end{tabular}

$\begin{array}{llllll}44 & 25 & 73 & 4 & 15 & 0\end{array}$

$\begin{array}{llllll}27.33 & 15.53 & 45.34 & 2.48 & 9.32 & 0.00\end{array}$

$\begin{array}{llllll}44 & 27 & 73 & 5 & 13 & 0\end{array}$

$\begin{array}{llllll}27.16 & 16.67 & 45.06 & 3.09 & 8.02 & 0.00\end{array}$

$\begin{array}{llllll}58 & 29 & 61 & 4 & 11 & 0\end{array}$

$\begin{array}{llllll}35.58 & 17.79 & 37.42 & 2.45 & 6.75 & 0.00\end{array}$

$\begin{array}{llllll}68 & 33 & 56 & 2 & 4 & 0\end{array}$

$\begin{array}{llllll}41.72 & 20.25 & 34.36 & 1.23 & 2.45 & 0.00\end{array}$

$\begin{array}{llllll}71 & 32 & 54 & 2 & 4 & 0\end{array}$

$\begin{array}{llllll}43.56 & 19.63 & 33.13 & 1.23 & 2.45 & 0.00\end{array}$

Number of Farmers Questioned 163

IABLE 7B - AVERAGE NUMBER OF TRAINED OXEN FOR FARMERS. IN THE CAMPS

PROVINCE OF HRT

YEAR

1978 Number of Families

$\%$ of Families

1980 Number of Families

$x$ of Families

1985 Number of Families

$x$ of Families

1986 Number of Families

$x$ of Families

1987 Number of Families

$x$ of Families

Average Number of Oxen per Family:

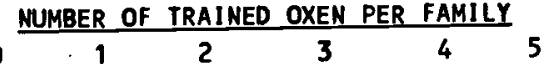

$\begin{array}{llllll}4 & 1 & 10 & 2 & 4 & 0\end{array}$

$\begin{array}{llllll}19.05 & 4.76 & 47.62 & 9.52 & 19.05 & 0.00\end{array}$

$\begin{array}{cccccc}6 & 1 & 10 & 1 & 3 & 0 \\ 28.57 & 4.76 & 47.62 & 4.76 & 14.29 & 0.00\end{array}$

$\begin{array}{llllll}12 & 2 & 5 & 0 & 2 & 0\end{array}$

$\begin{array}{llllll}57.14 & 9.52 & 23.81 & 0.00 & 9.52 & 0.00\end{array}$

$\begin{array}{llllll}15 & 1 & 3 & 0 & 2 & 0\end{array}$

$\begin{array}{llllll}71.43 & 4.76 & 14.29 & 0.00 & 9.52 & 0.00\end{array}$

$\begin{array}{llllll}19 & 0 & 2 & 0 & 0 & 0\end{array}$

$\begin{array}{llllll}90.48 & 0.00 & 9.52 & 0.00 & 0.00 & 0.00\end{array}$

$\begin{array}{ll}1978 & 2.05 \\ 1980 & 1.71 \\ 1985 & 0.95 \\ 1986 & 0.71 \\ 1987 & 0.19\end{array}$

Number of Farmers Questioned 21 
PROVINCE OF HRT

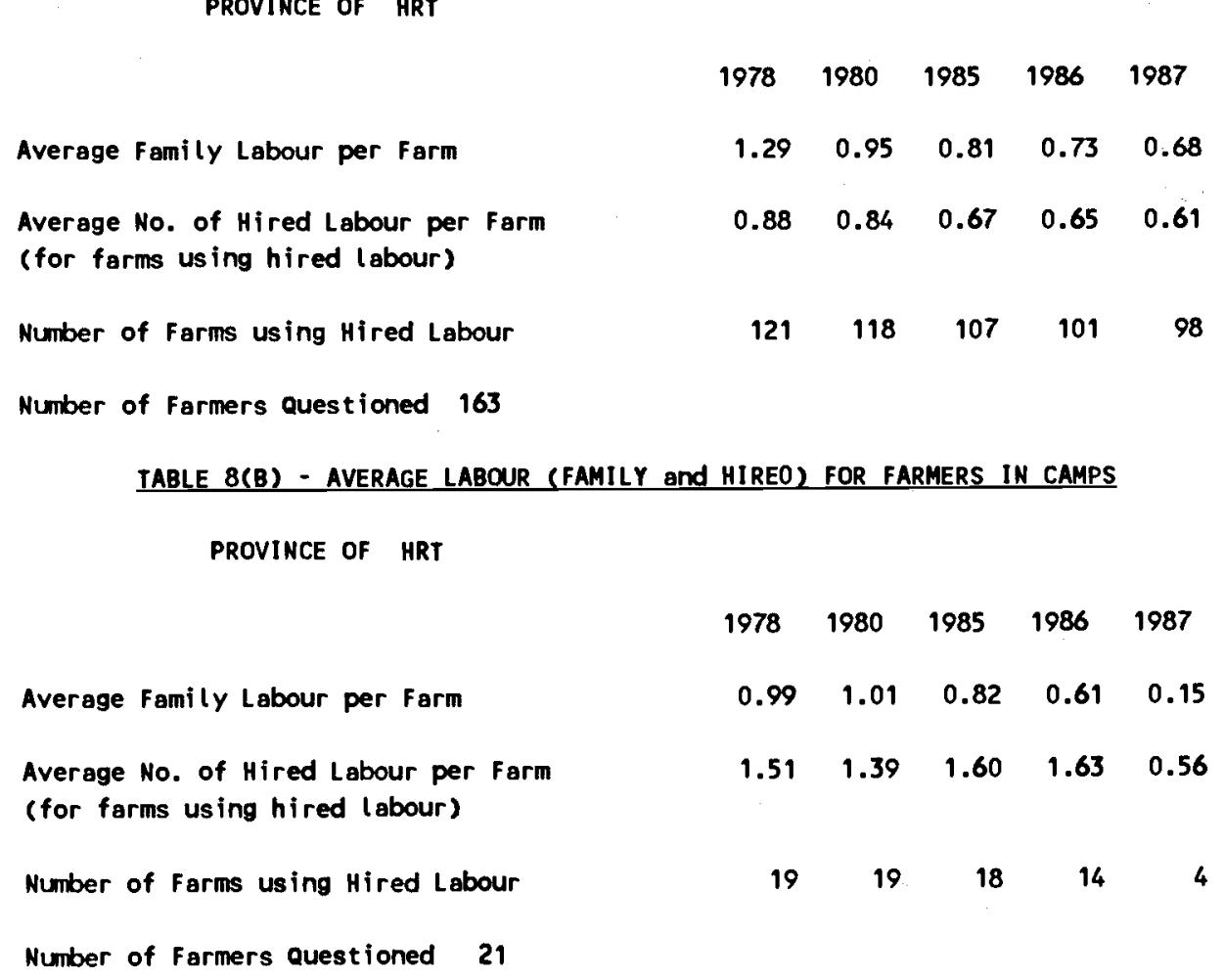


PROVINCE OF HRT

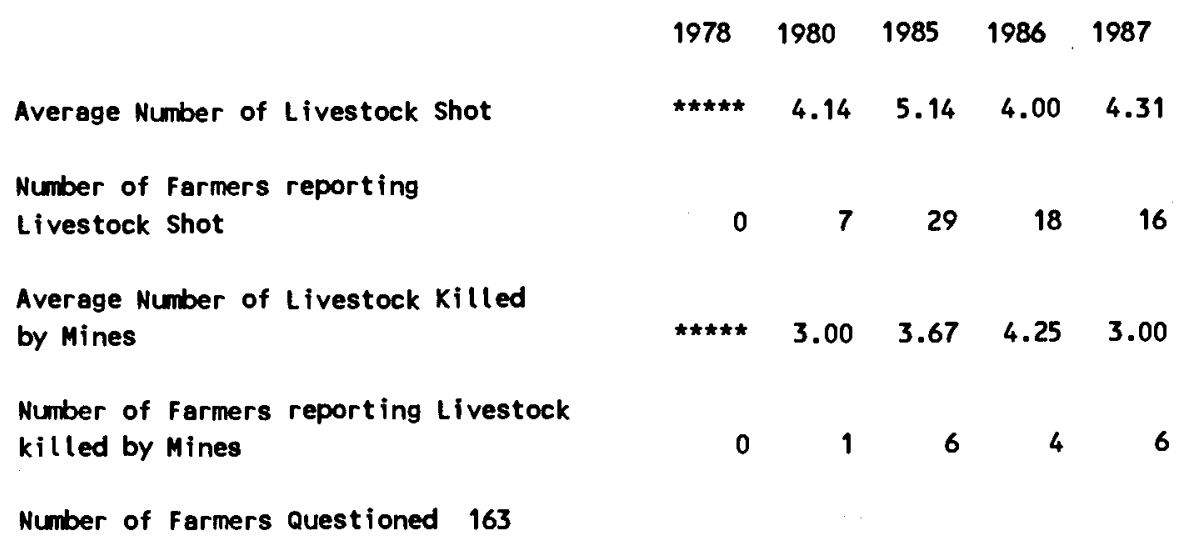

\section{TABLE 9B - DIRECT EFFECTS OF THE HAR - FARMERS IN CAMPS}

PROVINCE OF HRT

Average Number of Livestock Shot

Number of Farmers reporting

Livestock Shot

Average Number of Livestock Killed by Mines

Number of Farmers reporting Livestock killed by Mines

Number of Farmers Questioned

21

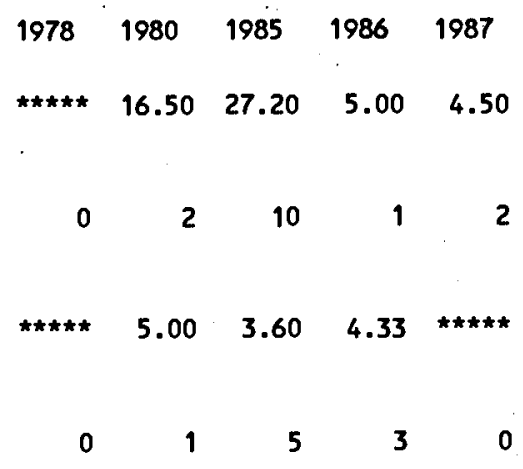




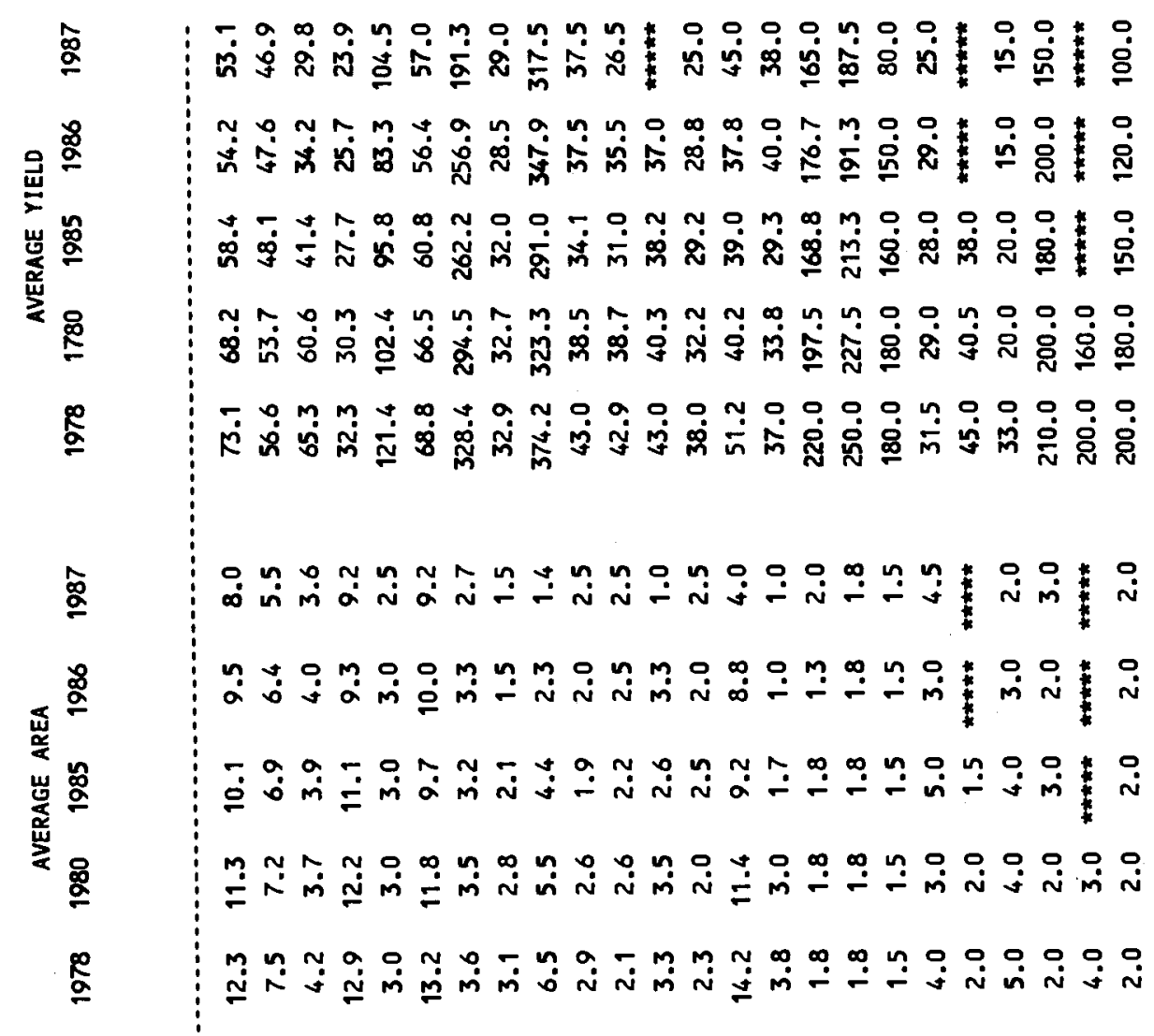

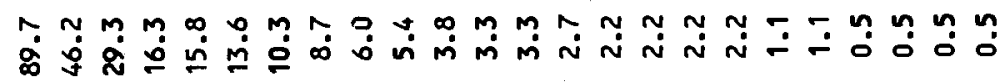

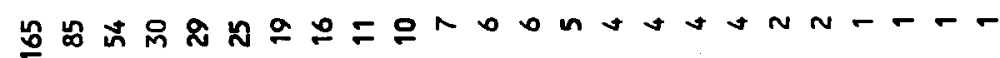
蹗高喜

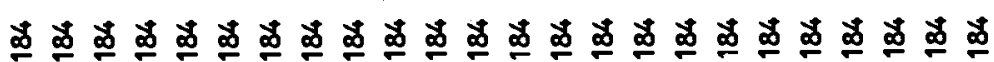

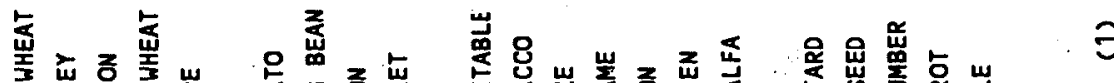


IABLE 1-5 - ANALYSIS OF GREATEST FARMING PROBLEMS - COMMUNITY SURVEY

PROVINCE OF HRT

PERCENTAGE OF VILLAGES GIVING FOLLONING PRIORITY TO PROBLEM

Rainfall

Crop Disease

Birds or Rats

Seed Availability

Fertilizer Availability

Crop Protection Chemicals

Credit

Irrigation Water Availability

Flooding

Labour Availabilty

Power for Land Preparation

Direct War Effects

Animal Diseases

Extension

Other Problems

\begin{tabular}{rrrrrrrrrrrrr}
1 & 2 & 3 & 4 & 5 & 6 & 7 & 8 & 9 & 10 & 11 & 12 & Heighted Priority \\
\hline 0 & 0 & 0 & 0 & 0 & 0 & 0 & 0 & 0 & 0 & 0 & 0 & 0.00 \\
0 & 3 & 19 & 22 & 22 & 10 & 2 & 0 & 0 & 0 & 0 & 0 & 8.29 \\
0 & 2 & 6 & 2 & 11 & 14 & 3 & 0 & 0 & 0 & 0 & 0 & 3.51 \\
0 & 8 & 22 & 2 & 13 & 16 & 0 & 0 & 0 & 0 & 0 & 0 & 7.12 \\
27 & 5 & 0 & 6 & 3 & 3 & 0 & 0 & 0 & 0 & 0 & 0 & 13.48 \\
0 & 3 & 6 & 10 & 14 & 5 & 0 & 0 & 0 & 0 & 0 & 0 & 4.09 \\
0 & 0 & 2 & 5 & 3 & 14 & 3 & 0 & 0 & 0 & 0 & 0 & 2.18 \\
3 & 0 & 8 & 0 & 0 & 0 & 0 & 0 & 0 & 0 & 0 & 0 & 2.44 \\
0 & 0 & 0 & 0 & 2 & 0 & 0 & 0 & 0 & 0 & 0 & 0 & 0.13 \\
0 & 21 & 17 & 30 & 6 & 11 & 0 & 0 & 0 & 0 & 0 & 0 & 11.25 \\
0 & 2 & 0 & 2 & 5 & 2 & 0 & 0 & 0 & 0 & $n$ & 0 & 1.01 \\
73 & 27 & 0 & 0 & 0 & 0 & 0 & 0 & 0 & 0 & 0 & 0 & 36.32 \\
0 & 0 & 0 & 0 & 2 & 3 & 0 & 0 & 0 & 0 & 0 & 0 & 0.36 \\
0 & 27 & 13 & 10 & 5 & 3 & 13 & 0 & 0 & 0 & 0 & 0 & 9.82 \\
0 & 0 & 0 & 0 & 0 & 0 & 0 & 0 & 0 & 0 & 0 & 0 & 0.00
\end{tabular}

\section{6}

Rainfall

Crop Disease

Birds or Rats

Seed Availability

Fertilizer Availability

Crop Protection Chemicals

Credit

Irrigation Water Availability

flooding

Labour Avail labilty

Power for Land Preparation

Direct Har Effects

Animal Diseases

Extension

Other Problems

\begin{tabular}{rrrrrrrrrrrrr}
1 & 2 & 3 & 4 & 5 & 6 & 7 & 8 & 9 & 10 & 11 & 12 & Weighted Priority \\
\hline 0 & 0 & 0 & 0 & 0 & 0 & 2 & 0 & 0 & 0 & 0 & 0 & 0.10 \\
0 & 10 & 10 & 8 & 3 & 13 & 0 & 0 & 0 & 0 & 0 & 0 & 5.45 \\
0 & 0 & 0 & 0 & 2 & 0 & 0 & 0 & 0 & 0 & 0 & 0 & 0.14 \\
0 & 8 & 16 & 30 & 8 & 19 & 0 & 0 & 0 & 0 & 0 & 0 & 9.28 \\
0 & 24 & 11 & 0 & 5 & 2 & 2 & 0 & 0 & 0 & 0 & 0 & 7.34 \\
0 & 5 & 5 & 8 & 8 & 3 & 0 & 0 & 0 & 0 & 0 & 0 & 3.47 \\
2 & 6 & 6 & 5 & 13 & 13 & 3 & 0 & 0 & 0 & 0 & 0 & 5.67 \\
24 & 11 & 8 & 2 & 0 & 0 & 0 & 0 & 0 & 0 & 0 & 0 & 13.94 \\
0 & 0 & 0 & 0 & 0 & 0 & 0 & 0 & 0 & 0 & 0 & 0 & 0.00 \\
0 & 8 & 17 & 10 & 16 & 2 & 2 & 0 & 0 & 0 & 0 & 0 & 6.81 \\
0 & 0 & 2 & 3 & 2 & 0 & 0 & 0 & 0 & 0 & 0 & 0 & 0.71 \\
71 & 10 & 14 & 5 & 0 & 0 & 0 & 0 & 0 & 0 & 0 & 0 & 35.34 \\
0 & 0 & 0 & 2 & 0 & 0 & 0 & 0 & 0 & 0 & 0 & 0 & 0.17 \\
0 & 17 & 13 & 30 & 27 & 3 & 0 & 0 & 0 & 0 & 0 & 0 & 11.37 \\
0 & 0 & 2 & 0 & 0 & 0 & 0 & 0 & 0 & 0 & 0 & 0 & 0.23
\end{tabular}

$\underline{1985}$

Rainfall

Crop Disease

Birds or Rats

Seed Availability

Fertilizer Availability

Crop Protection Chemicals

Credit

Irrigation Water Availability

Flooding

Labour Avai labilty

Power for Land Preparation

Direct War Effects

Animal Diseases

Extension

Other Problems

\begin{tabular}{rrrrrrrrrrrrr}
1 & 2 & 3 & 4 & 5 & 6 & 7 & 8 & 9 & 10 & 11 & 12 & Weighted Priority \\
\hline 0 & 0 & 0 & 0 & 0 & 0 & 0 & 0 & 0 & 0 & 0 & 0 & 0.00 \\
0 & 8 & 10 & 19 & 16 & 8 & 0 & 0 & 0 & 0 & 0 & 0 & 6.76 \\
0 & 0 & 0 & 8 & 17 & 3 & 0 & 0 & 0 & 0 & 0 & 0 & 2.48 \\
0 & 2 & 10 & 30 & 27 & 16 & 0 & 0 & 0 & 0 & 0 & 0 & 8.06 \\
0 & 0 & 0 & 0 & 0 & 0 & 0 & 0 & 0 & 0 & 0 & 0 & 0.00 \\
0 & 2 & 8 & 14 & 8 & 8 & 0 & 0 & 0 & 0 & 0 & 0 & 4.09 \\
0 & 0 & 2 & 6 & 6 & 17 & 0 & 0 & 0 & 0 & 0 & 0 & 2.60 \\
8 & 13 & 25 & 0 & 0 & 0 & 0 & 0 & 0 & 0 & 0 & 0 & 9.38 \\
0 & 0 & 0 & 0 & 0 & 0 & 0 & 0 & 0 & 0 & 0 & 0 & 0.00 \\
0 & 35 & 2 & 6 & 8 & 3 & 0 & 0 & 0 & 0 & 0 & 0 & 8.94 \\
2 & 29 & 2 & 2 & 0 & 2 & 0 & 0 & 0 & 0 & 0 & 0 & 7.03 \\
90 & 10 & 0 & 0 & 0 & 0 & 0 & 0 & 0 & 0 & 0 & 0 & 39.26 \\
0 & 0 & 0 & 0 & 3 & 2 & 0 & 0 & 0 & 0 & 0 & 0 & 0.37 \\
0 & 6 & 40 & 14 & 11 & 22 & 0 & 0 & 0 & 0 & 0 & 0 & 10.68 \\
0 & 0 & 0 & 0 & 2 & 3 & 0 & 0 & 0 & 0 & 0 & 0 & 0.35
\end{tabular}


PROVINCE OF HRT

Rainfall

Crop Disease

Birds or Rats

Seed Availability

Fertilizer Availability

Crop Protection Chemicals

Credit

Irrigation Water Availability

Flooding

Labour Availabilty

Power for Land Preparation

Direct War Effects

Animal Diseases

Extension

Other Problems

\begin{tabular}{rrrrrrrrrrrrr}
1 & 2 & 3 & 4 & 5 & 6 & 7 & 8 & 9 & 10 & 11 & 12 & Weighted Priority \\
\hline 0 & 0 & 0 & 0 & 0 & 2 & 0 & 0 & 0 & 0 & 0 & 0 & 0.11 \\
8 & 3 & 14 & 16 & 10 & 14 & 0 & 0 & 0 & 0 & 0 & 0 & 9.60 \\
3 & 0 & 27 & 11 & 2 & 0 & 0 & 0 & 0 & 0 & 0 & 0 & 6.50 \\
0 & 3 & 8 & 16 & 11 & 16 & 0 & 0 & 0 & 0 & 0 & 0 & 5.56 \\
24 & 13 & 0 & 2 & 6 & 0 & 0 & 0 & 0 & 0 & 0 & 0 & 13.55 \\
0 & 2 & 8 & 6 & 10 & 2 & 0 & 0 & 0 & 0 & 0 & 0 & 3.06 \\
0 & 0 & 0 & 10 & 16 & 22 & 2 & 0 & 0 & 0 & 0 & 0 & 4.04 \\
16 & 25 & 0 & 0 & 3 & 0 & 0 & 0 & 0 & 0 & 0 & 0 & 12.43 \\
0 & 0 & 0 & 0 & 0 & 0 & 0 & 0 & 0 & 0 & 0 & 0 & 0.00 \\
0 & 2 & 22 & 10 & 10 & 3 & 0 & 0 & 0 & 0 & 0 & 0 & 5.54 \\
0 & 0 & 0 & 0 & 0 & 2 & 0 & 0 & 0 & 0 & 0 & 0 & 0.11 \\
54 & 2 & 0 & 0 & 0 & 0 & 0 & 0 & 0 & 0 & 0 & 0 & 23.31 \\
0 & 0 & 5 & 0 & 0 & 0 & 0 & 0 & 0 & 0 & 0 & 0 & 0.68 \\
0 & 48 & 16 & 27 & 2 & 0 & 0 & 0 & 0 & 0 & 0 & 0 & 15.39 \\
0 & 0 & 0 & 0 & 0 & 2 & 0 & 0 & 0 & 0 & 0 & 0 & 0.11
\end{tabular}

1978

Rainfall

Crop Disease

Birds or Rats

Seed Avail lability

Fertilizer Avail lability

crop Protection chemicals

Credit

Irrigation Water Availability

Flooding

Labour Availabilty

Power for Land Preparation

Direct War Effects

Animal Diseases

Extension

other Problems

\begin{tabular}{rrrrrrrrrrrrr}
1 & 2 & 3 & 4 & 5 & 6 & 7 & 8 & 9 & 10 & 11 & 12 & Weighted Priority \\
\hline 0 & 0 & 0 & 0 & 0 & 0 & 0 & 0 & 0 & 0 & 0 & 0 & 0.00 \\
3 & 6 & 22 & 11 & 2 & 0 & 0 & 0 & 0 & 0 & 0 & 0 & 9.69 \\
11 & 17 & 10 & 16 & 13 & 2 & 0 & 0 & 0 & 0 & 0 & 0 & 17.13 \\
2 & 2 & 5 & 3 & 2 & 0 & 0 & 0 & 0 & 0 & 0 & 0 & 2.92 \\
8 & 32 & 2 & 2 & 0 & 0 & 0 & 0 & 0 & 0 & 0 & 0 & 14.22 \\
0 & 5 & 0 & 0 & 0 & 0 & 0 & 0 & 0 & 0 & 0 & 0 & 1.37 \\
6 & 5 & 0 & 0 & 0 & 0 & 0 & 0 & 0 & 0 & 0 & 0 & 5.02 \\
32 & 10 & 0 & 0 & 0 & 0 & 0 & 0 & 0 & 0 & 0 & 0 & 20.99 \\
14 & 5 & 2 & 0 & 0 & 0 & 0 & 0 & 0 & 0 & 0 & 0 & 9.89 \\
0 & 0 & 2 & 2 & 0 & 0 & 0 & 0 & 0 & 0 & 0 & 0 & 0.53 \\
0 & 0 & 0 & 0 & 0 & 0 & 0 & 0 & 0 & 0 & 0 & 0 & 0.00 \\
0 & 0 & 0 & 0 & 0 & 0 & 0 & 0 & 0 & 0 & 0 & 0 & 0.00 \\
16 & 13 & 2 & 3 & 2 & 0 & 0 & 0 & 0 & 0 & 0 & 0 & 13.72 \\
0 & 0 & 3 & 8 & 16 & 8 & 0 & 0 & 0 & 0 & 0 & 0 & 4.34 \\
0 & 0 & 0 & 0 & 2 & 0 & 0 & 0 & 0 & 0 & 0 & 0 & 0.18
\end{tabular}


JOWZJAN 


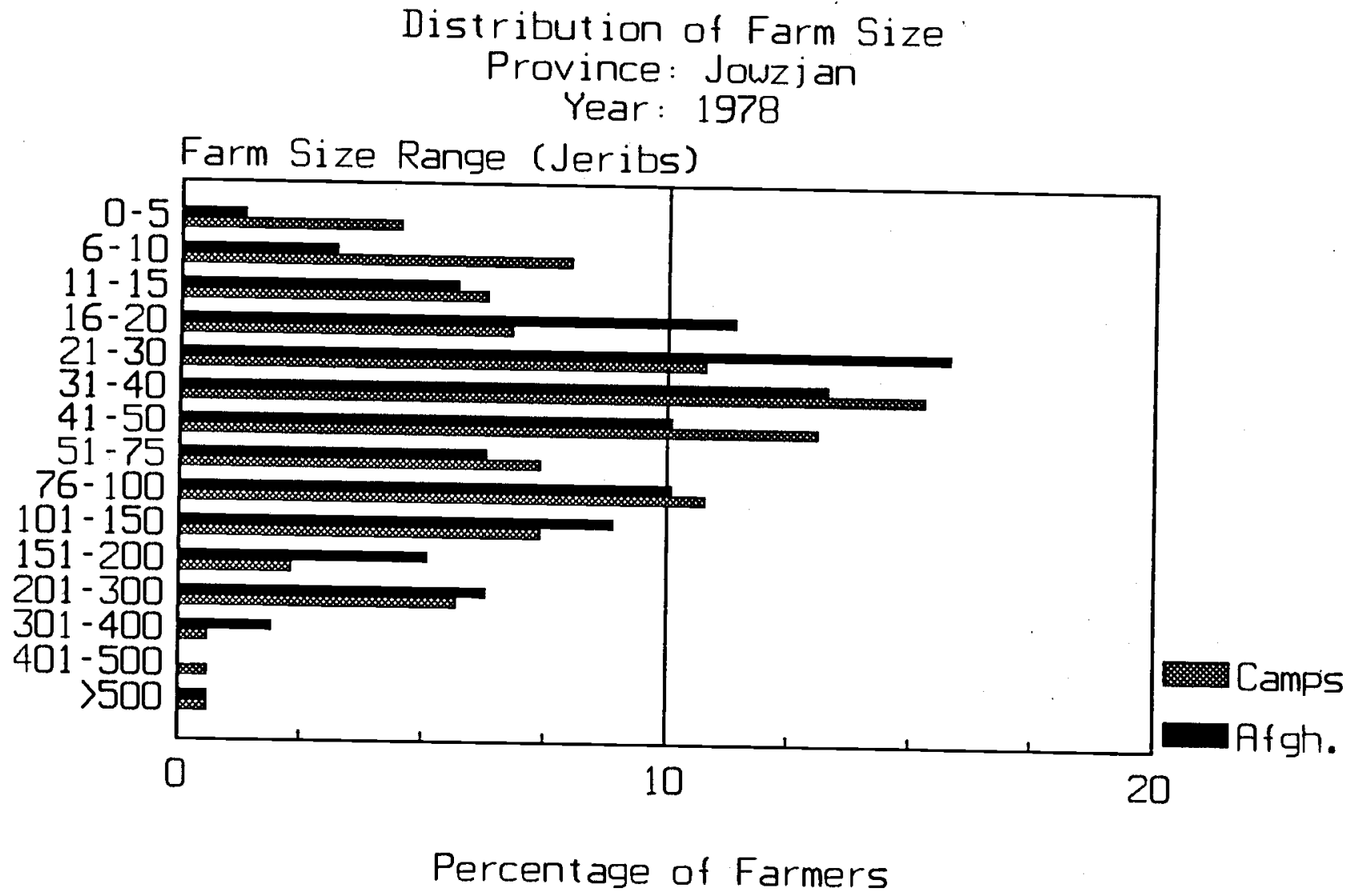

Farmers in Afghanistan 1978 1987

Total Number of Farms

Average Area

158

156

Average Area Irrigated

Average Dryland Area

81.17

47.62

14.75

8.31

70.35

45.56

Farmers who left in 1987

Total Number of Farms

Average Area

Average Area Irrigated

Average Dryland Area

176

71.55

33.66

86.20

Average of Both Groups

Provincial Average Area

Provincial Average Irrigated Area

Provincial Average Dryland Area

76.10

47.62

24.39

8.31

81.58

45.56

(All areas in Jeribs. 5 Jeribs $=1$ Hectare) 
Farm Power - Farmers in Atghanistan Province of Jowzian Crop: Barley

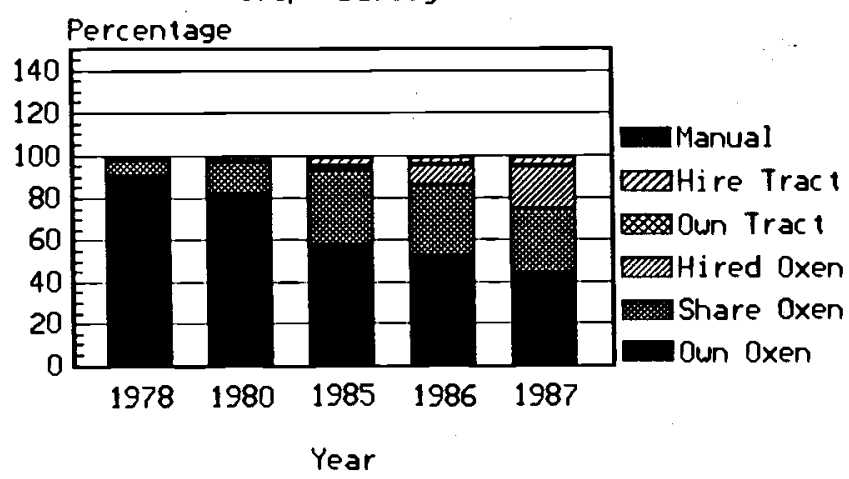

Farm Power - Farmers who lett in 1987 Province of Jowzjan Crop: Barley

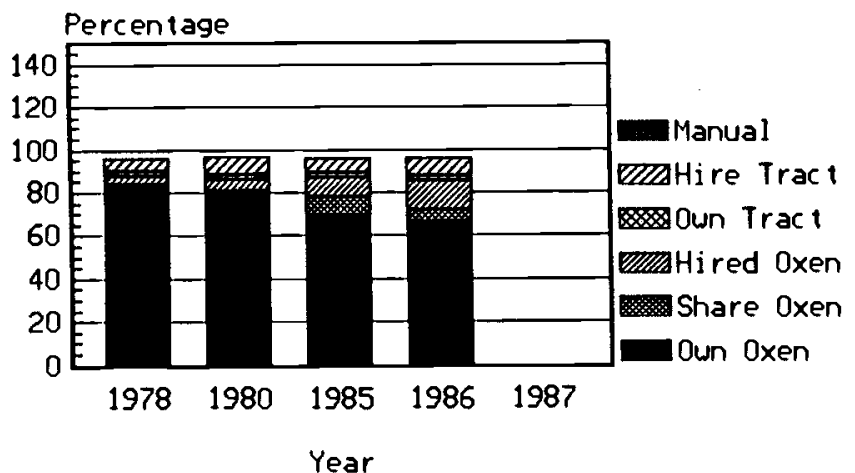

Base Figures for above araphs

Province: Jowzjan

Farmers in Afghanistan

Dry Wheat own oxen

Shared oxen

Hired oxen

Own Tractor

Hired Tractor

Barley

Manual Means

Own Oxen

Shared oxen

Hired oxen

Own Tractor

Hired Tractor

Manual Means

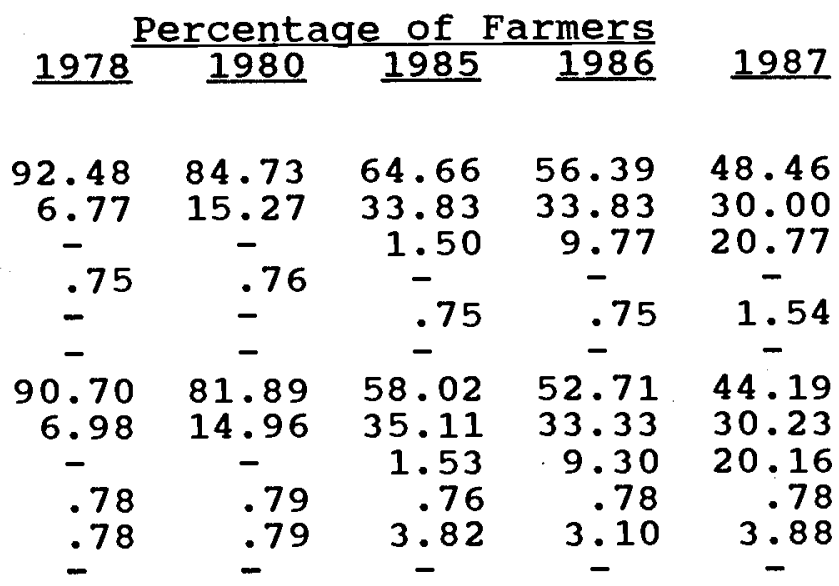

90.67

88.24

75.34

72.41

5.33

8.82

16.44

12.07

Hired oxen

own Tractor

Hired Tractor

Manual Means

Barley

Own Oxen

shared oxen

Hired oxen

Own Tractor
4.00

4.41

5.33

$-$

5.88

82.05

78.90

1. 71

1.83

3.42

5.50

1.83
9.59

4. 11

12.07

68.97

3. 45

8.62

66.29

9. 48

1. 72

5.62

13.48

2.25
Manual שäHire Tract Oun Tract שmHired oxen Share Oxen own Oxen 

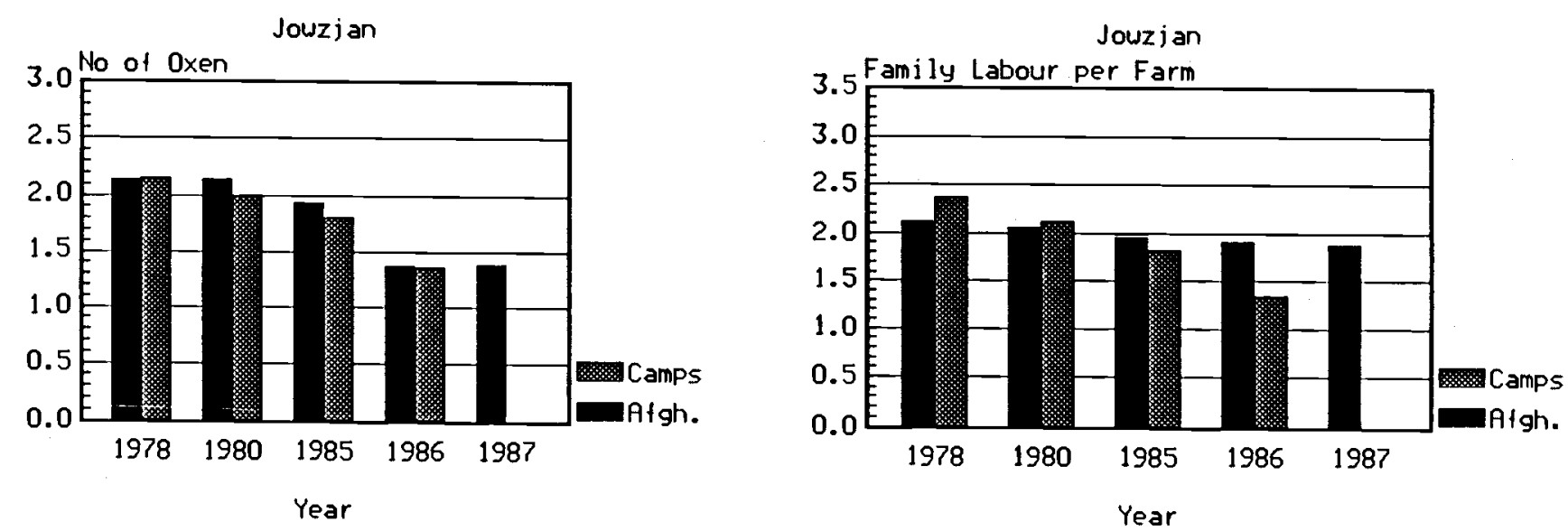

Jowzjan
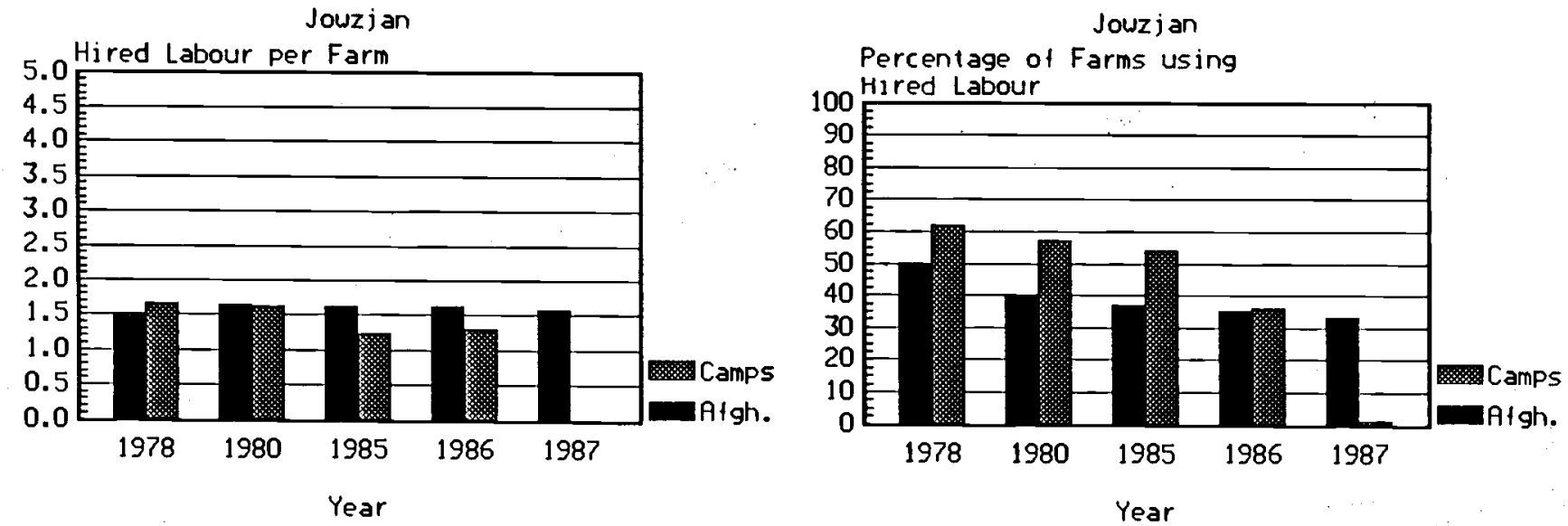
PROVINCE OF JZJ

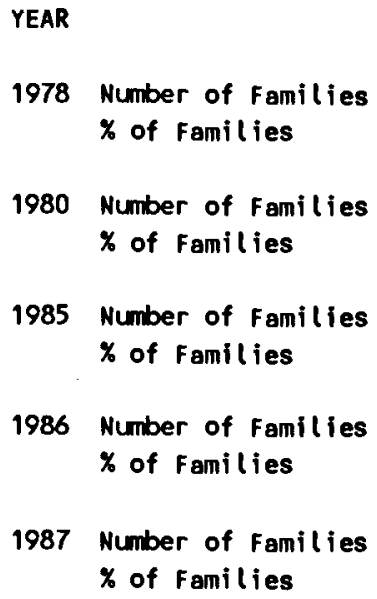

\begin{tabular}{|c|c|c|c|c|c|}
\hline \multicolumn{2}{|c|}{ NUMBER OF } & TRAINED & \multicolumn{3}{|c|}{ OXEN PER FAMIL } \\
\hline 0 & 1 & 2 & 3 & 4 & 5 \\
\hline 5 & 9 & 118 & 2 & 19 & 1 \\
\hline 3.25 & 5.84 & 76.62 & 1.30 & 12.34 & 0.65 \\
\hline 7 & 24 & 102 & 1 & 21 & 0 \\
\hline 4.52 & 15.48 & 65.81 & 0.65 & 13.55 & 0.00 \\
\hline 17 & 49 & 70 & 6 & 13 & 0 \\
\hline 10.97 & 31.61 & 45.16 & 3.87 & 8.39 & 0.00 \\
\hline 29 & 49 & 66 & 6 & 5 & $\mathbf{0}$ \\
\hline 18.71 & 31.61 & 42.58 & 3.87 & 3.23 & 0.00 \\
\hline 4 & 43 & 62 & 1 & 2 & 0 \\
\hline 30.3 & 27.74 & 40.00 & 0.65 & 1.29 & 0.0 \\
\hline
\end{tabular}

$\begin{array}{ll}1978 & 2.16 \\ 1980 & 2.03 \\ 1985 & 1.67 \\ 1986 & 1.41 \\ 1987 & 1.15\end{array}$

Number of Farmers Questioned 156

NUMBER OF TRAINED OXEN PER FAMILY

$\begin{array}{cccccc}0 & 1 & 2 & 3 & 4 & 5 \\ 28 & 10 & 93 & 9 & 24 & 3 \\ 16.77 & 5.99 & 55.69 & 5.39 & 14.37 & 1.80 \\ 39 & 11 & 91 & 7 & 17 & 1 \\ 23.49 & 6.63 & 54.82 & 4.22 & 10.24 & 0.60 \\ 42 & 19 & 86 & 5 & 16 & 2 \\ 24.71 & 11.18 & 50.59 & 2.94 & 9.41 & 1.18 \\ 78 & 12 & 66 & 2 & 11 & 2 \\ 45.61 & 7.02 & 38.60 & 1.17 & 6.43 & 1.17 \\ 169 & 0 & 2 & 0 & 0 & 0 \\ 98.83 & 0.00 & 1.17 & 0.00 & 0.00 & 0.00\end{array}$

$\begin{array}{ll}1978 & 2.00 \\ 1980 & 1.73 \\ 1985 & 1.65 \\ 1986 & 1.19 \\ 1987 & 0.02\end{array}$

Number of Farmers Questioned 171 
TABLE 8(A) - AVERAGE LABOUR (FAMILY and HIRED) FOR FARMERS IN AFGANISTAN

PROVINCE OF JZJ

$\begin{array}{lrrrrr} & 1978 & 1980 & 1985 & 1986 & 1987 \\ & & & & & \\ \text { Average Family Labour per Farm } & 2.11 & 2.05 & 1.95 & 1.90 & 1.87 \\ \begin{array}{l}\text { Average No. of Hired Labour per Farm } \\ \text { (for farms using hired labour) }\end{array} & 1.47 & 1.64 & 1.61 & 1.61 & 1.56 \\ \text { Number of Farms using Hired Labour } & 76 & 63 & 57 & 54 & 52 \\ \text { Number of Farmers Questioned 156 } & & & & & \\ \qquad \begin{array}{l}\text { IABLE 8(B) - AVERAGE LABOUR (FAMILY and HIRED) FOR FARMERS IN CAMPS }\end{array}\end{array}$

PROVINCE OF JZJ

Average Family Labour per Farm

Average No. of $\mathrm{Hi}$ red Labour per Farm

(for farms using hired labour)

Number of Farms using Hired Labour

Number of Farmers Questioned 171

$\begin{array}{lllll}1978 & 1980 & 1985 & 1986 & 1987 \\ 2.37 & 2.12 & 1.82 & 1.34 & 0.05 \\ 1.65 & 1.61 & 1.23 & 1.30 & 2.00\end{array}$

$\begin{array}{lllll}106 & 97 & 93 & 61 & 2\end{array}$


PROVINCE OF JZJ

Number of Families $x$ of Families

Number of Families \% of Families

Number of Fanilies

x of Families

Number of Families

$X$ of Families

Number of Families

$X$ of Families

age Number of Oxen per Family:

NUMBER OF TRAINED OXEN PER FAMILY

0

$\begin{array}{llllll}1 & 2 & 3 & 4 & 5 & \\ 47 & 43 & 62 & 1 & 2 & 0\end{array}$

$\begin{array}{llllll}30.32 & 27.74 & 40.00 & 0.65 & 1.29 & 0.00\end{array}$

$\begin{array}{llllll}29 & 49 & 66 & 6 & 5 & 0\end{array}$

$\begin{array}{llllll}18.71 & 31.61 & 42.58 & 3.87 & 3.23 & 0.00\end{array}$

$\begin{array}{llllll}17 & 49 & 70 & 6 & 13 & 0\end{array}$

$\begin{array}{llllll}10.97 & 31.61 & 45.16 & 3.87 & 8.39 & 0.00\end{array}$

$\begin{array}{llllll}7 & 24 & 102 & 1 & 21 & 0\end{array}$

$\begin{array}{llllll}4.52 & 15.48 & 65.81 & 0.65 & 13.55 & 0.00\end{array}$

$\begin{array}{llllll}5 & 9 & 118 & 2 & 19 & 1\end{array}$

$\begin{array}{llllll}3.25 & 5.84 & 76.62 & 1.30 & 12.34 & 0.65\end{array}$

$\begin{array}{ll}1987 & 1.15 \\ 1986 & 1.41 \\ 1985 & 1.67 \\ 1980 & 2.03 \\ 1978 & 2.16\end{array}$

ser of Farmers Questioned 156

IABLE 7B - AVERAGE NUMBER OF TRAINED OXEN FOR FARMERS IN THE CAMPS

PROVINCE OF JZJ

NUMBER OF TRAINED OXEN PER FAMILY

Number of Families

$x$ of Families

Number of Families

$x$ of Families

Number of Families

$x$ of Families

Number of Families

$x$ of Families

Number of Families

$X$ of Families

$\begin{array}{cccccc} & & & & \\ 169 & 1 & 2 & 3 & 4 & 5 \\ 98.83 & 0.00 & 1.17 & 0.00 & 0.00 & 0.00 \\ & & & & & \\ 78 & 12 & 66 & 2 & 11 & 2 \\ 45.61 & 7.02 & 38.60 & 1.17 & 6.43 & 1.17 \\ & & & & & \\ 42 & 19 & 86 & 5 & 16 & 2 \\ 24.71 & 11.18 & 50.59 & 2.94 & 9.41 & 1.18 \\ & & & & & \\ 39 & 11 & 91 & 7 & 17 & 1 \\ 23.49 & 6.63 & 54.82 & 4.22 & 10.24 & 0.60 \\ & & & & & \\ 28 & 10 & 93 & 9 & 24 & 3 \\ 16.77 & 5.99 & 55.69 & 5.39 & 14.37 & 1.80 \\ 1987 & & 0.02 & & & \\ 1986 & & 1.19 & & & \\ 1985 & & 1.65 & & & \\ 1980 & & 1.73 & & & \\ 1978 & & 2.00 & . & & \end{array}$

ber of Farmers Questioned 171 


\begin{tabular}{|c|c|}
\hline 产 & 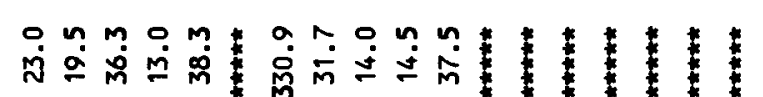 \\
\hline$\stackrel{2}{\stackrel{8}{2}}$ & 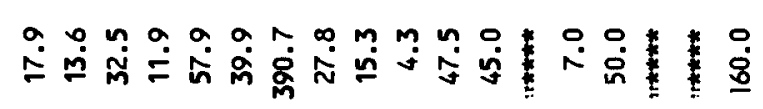 \\
\hline & 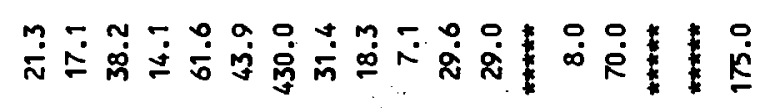 \\
\hline & 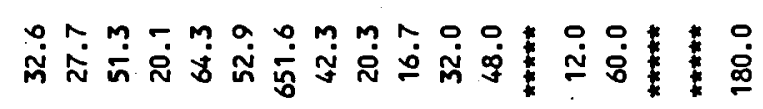 \\
\hline$g$ & 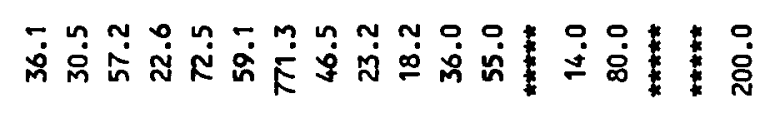 \\
\hline 台 & 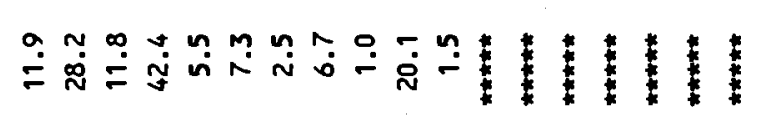 \\
\hline & 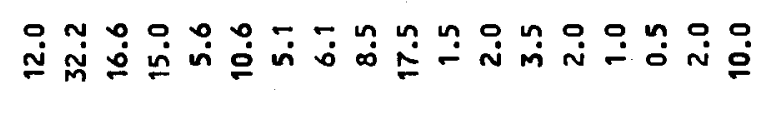 \\
\hline 兽 & 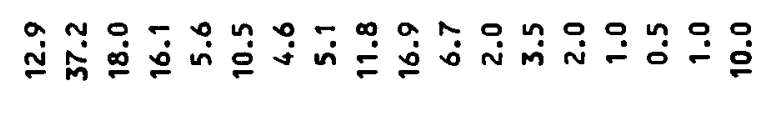 \\
\hline 8 & 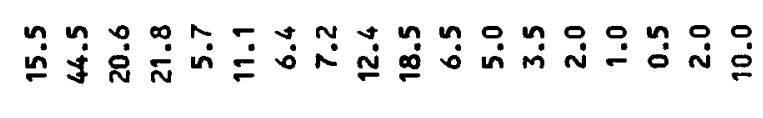 \\
\hline$\stackrel{\infty}{\underline{\alpha}}$ & 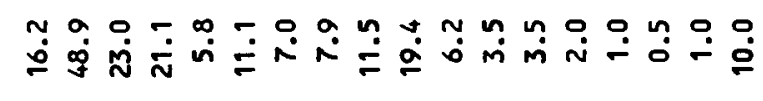 \\
\hline
\end{tabular}

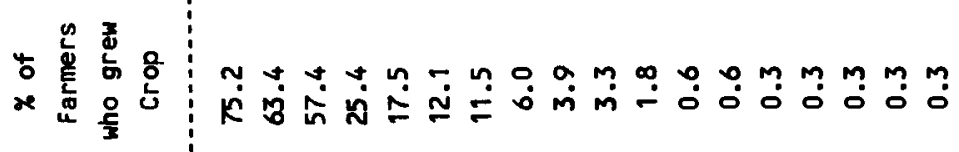

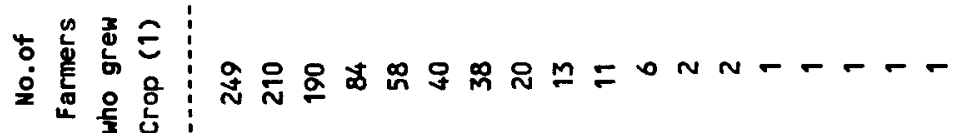


PROVINCE OF JZJ

\section{PERCENTAGE OF VILLAGES GIVING FOLLOWING PRIORITY TO PROBLEM}

Rainfall

Crop Disease

Birds or Rats

Seed Availabil ity

Fertilizer Availability

Crop Protection Chemicals

Credit

Irrigation Hater Availability

Flooding

Labour Availabilty

Power for Land Preparation

Direct War Effects

Animal Diseases

Extension

other Problems

$\begin{array}{ccccccccccccc}1 & 2 & 3 & 4 & 5 & 6 & 7 & 8 & 9 & 10 & 11 & 12 & \text { Weighted Priority } \\ 2 & 0 & 2 & 0 & 0 & 0 & 0 & 0 & 0 & 0 & 0 & 0 & 1.10\end{array}$

$\begin{array}{lllllllllllll}0 & 6 & 11 & 17 & 13 & 6 & 0 & 0 & 0 & 0 & 0 & 0 & 7.72\end{array}$

$\begin{array}{lllllllllllll}9 & 2 & 2 & 5 & 2 & 0 & 0 & 0 & 0 & 0 & 0 & 0 & 6.42\end{array}$

$\begin{array}{lllllllllllll}0 & 3 & 2 & 3 & 8 & 0 & 0 & 0 & 0 & 0 & 0 & 0 & 2.34\end{array}$

$\begin{array}{lllllllllllll}0 & 2 & 8 & 6 & 0 & 0 & 0 & 0 & 0 & 0 & 0 & 0 & 2.61\end{array}$

$\begin{array}{lllllllllllll}0 & 0 & 2 & 9 & 6 & 3 & 0 & 0 & 0 & 0 & 0 & 0 & 2.45\end{array}$

$\begin{array}{lllllllllllll}0 & 8 & 23 & 13 & 3 & 3 & 0 & 0 & 0 & 0 & 0 & 0 & 8.45\end{array}$

$\begin{array}{lllllllllllll}44 & 16 & 0 & 0 & 0 & 0 & 0 & 0 & 0 & 0 & 0 & 0 & 27.23\end{array}$

$\begin{array}{lllllllllllll}5 & 0 & 2 & 3 & 2 & 0 & 0 & 0 & 0 & 0 & 0 & 0 & 3.33\end{array}$

$\begin{array}{lllllllllllll}2 & 9 & 6 & 5 & 5 & 2 & 0 & 0 & 0 & 0 & 0 & 0 & \\ 0 & 0 & 0 & 0 & 0 & 0 & 0 & 0 & 0 & 0 & 0 & 0 & 5.65\end{array}$

$\begin{array}{lllllllllllll}0 & 0 & 0 & 0 & 0 & 0 & 0 & 0 & 0 & 0 & 0 & 0 & 0.00\end{array}$

$\begin{array}{lllllllllllll}33 & 33 & 11 & 2 & 0 & 0 & 0 & 0 & 0 & 0 & 0 & 0 & 28.13\end{array}$

$\begin{array}{lllllllllllll}0 & 2 & 2 & 8 & 14 & 8 & 3 & 0 & 0 & 0 & 0 & 0 & 4.13\end{array}$

$\begin{array}{lllllllllllll}0 & 0 & 2 & 0 & 2 & 0 & 0 & 0 & 0 & 0 & 0 & 0 & 0.44\end{array}$

$\begin{array}{lllllllllllll}0 & 0 & 0 & 0 & 0 & 0 & 0 & 0 & 0 & 0 & 0 & 0 & 0.00\end{array}$

1978

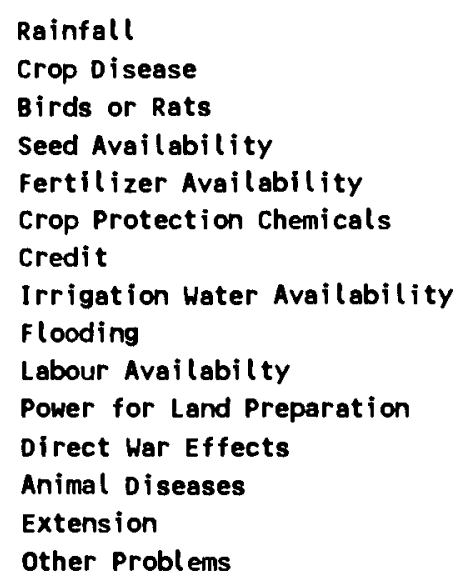

\begin{tabular}{ccccccccccccc}
1 & 2 & 3 & 4 & 5 & 6 & 7 & 8 & 0 & 10 & 11 & 12 & Heighted Priority \\
\hline 0 & 0 & 0 & 0 & 0 & 0 & 0 & 0 & 0 & 0 & 0 & 0 & 0.00 \\
2 & 0 & 0 & 0 & 0 & 0 & 0 & 0 & 0 & 0 & 0 & 0 & 16.67 \\
3 & 0 & 0 & 0 & 0 & 0 & 0 & 0 & 0 & 0 & 0 & 0 & 33.33 \\
0 & 0 & 0 & 0 & 0 & 0 & 0 & 0 & 0 & 0 & 0 & 0 & 0.00 \\
0 & 0 & 0 & 0 & 0 & 0 & 0 & 0 & 0 & 0 & 0 & 0 & 0.00 \\
0 & 0 & 0 & 0 & 0 & 0 & 0 & 0 & 0 & 0 & 0 & 0 & 0.00 \\
0 & 0 & 0 & 0 & 0 & 0 & 0 & 0 & 0 & 0 & 0 & 0 & 0.00 \\
0 & 0 & 0 & 0 & 0 & 0 & 0 & 0 & 0 & 0 & 0 & 0 & 0.00 \\
3 & 0 & 0 & 0 & 0 & 0 & 0 & 0 & 0 & 0 & 0 & 0 & 33.33 \\
2 & 0 & 0 & 0 & 0 & 0 & 0 & 0 & 0 & 0 & 0 & 0 & 16.67 \\
0 & 0 & 0 & 0 & 0 & 0 & 0 & 0 & 0 & 0 & 0 & 0 & 0.00 \\
0 & 0 & 0 & 0 & 0 & 0 & 0 & 0 & 0 & 0 & 0 & 0 & 0.00 \\
0 & 0 & 0 & 0 & 0 & 0 & 0 & 0 & 0 & 0 & 0 & 0 & 0.00 \\
0 & 0 & 0 & 0 & 0 & 0 & 0 & 0 & 0 & 0 & 0 & 0 & 0.00 \\
0 & 0 & 0 & 0 & 0 & 0 & 0 & 0 & 0 & 0 & 0 & 0 & 0.00
\end{tabular}


KABUL 


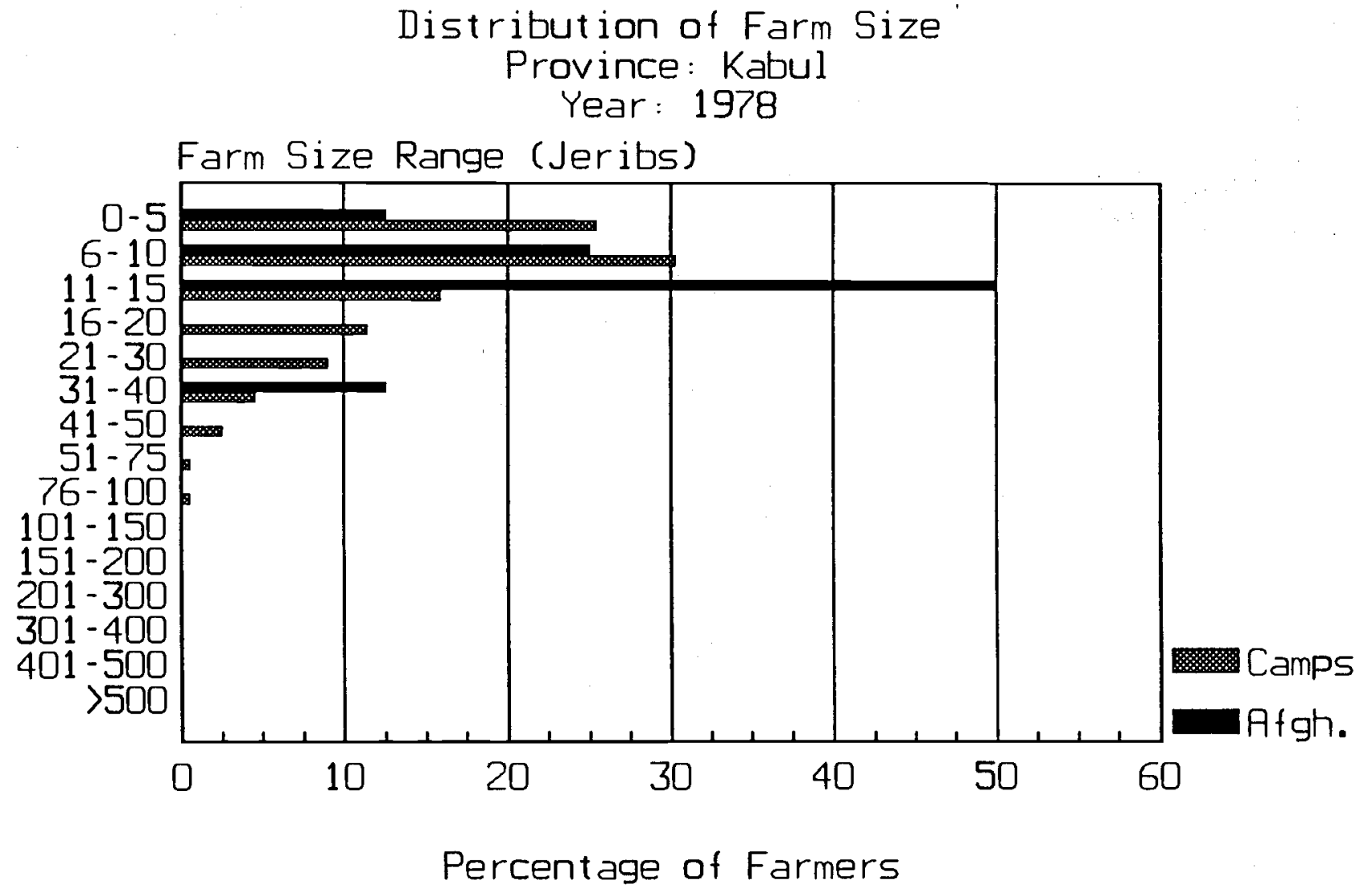

Farmers in Afghanistan

1978

1987

Total Number of Farms

Average Area

Average Area Irrigated

Average Dryland Area

$\begin{array}{cc}8 & 8 \\ 12.50 & 2.06 \\ 10.00 & 2.06 \\ 20.00 & -\end{array}$

Farmers who left in 1987

Total Number of Farms

201

3

Average Area

13.59

3.67

Average Area Irrigated

12.45

3.67

Average Dryland Area

12.08

5.00

Average of Both Groups

Provincial Average Area

Provincial Average Irrigated Area

13.55

2.50

Provincial Average Dryland Area

12.35

2.50

12.45

5.00

(All areas in Jeribs. 5 Jeribs $=1$ Hectare) 
Farm Power - Farmers in Afghanistan Province of Kabul

Crop: Irrigated wheat

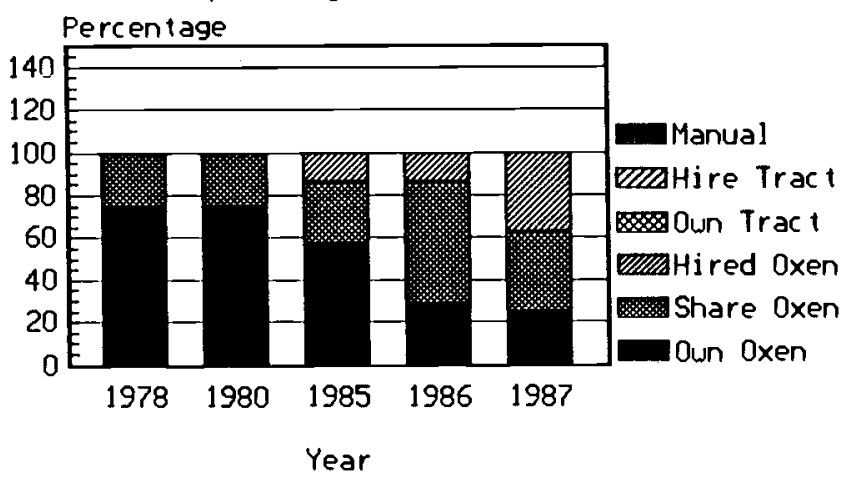

Farm Power - Farmers who left in 1987 Province of Kabul Crop: Irrigated wheat

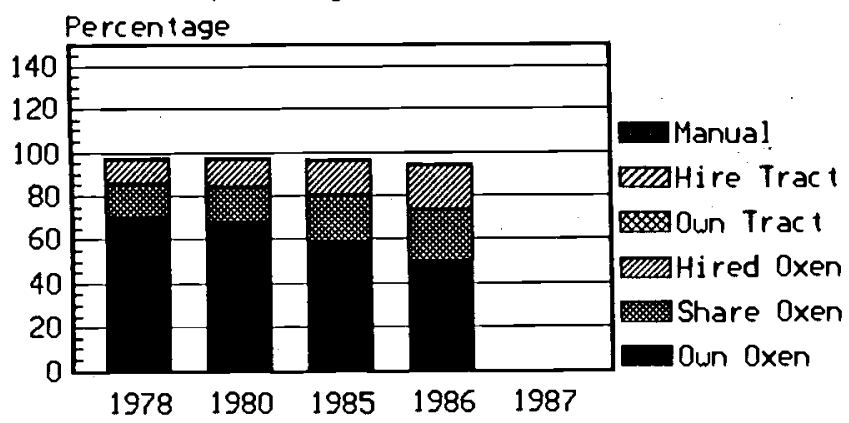

Year

Base Figures for above graphs

Province: Kabul

Farmers in Afqhanistan

Irr. Wheat own oxen Shared oxen

Hired oxen

Own Tractor

Hired Tractor

Manual Means

Maize

Own oxen

Shared oxen

Hired oxen

Own Tractor

Hired Tractor

Manual Means

Farmers who left in 1987

$\begin{array}{ll}\text { Irr. Wheat } & \text { Own Oxen } \\ & \text { Shared Oxen } \\ & \text { Hired Oxen } \\ & \text { Own Tractor } \\ & \text { Hired Tractor } \\ & \text { Manual Means } \\ \text { Maize } & \text { Own Oxen } \\ & \text { Shared oxen } \\ & \text { Hired Oxen } \\ & \text { Own Tractor }\end{array}$
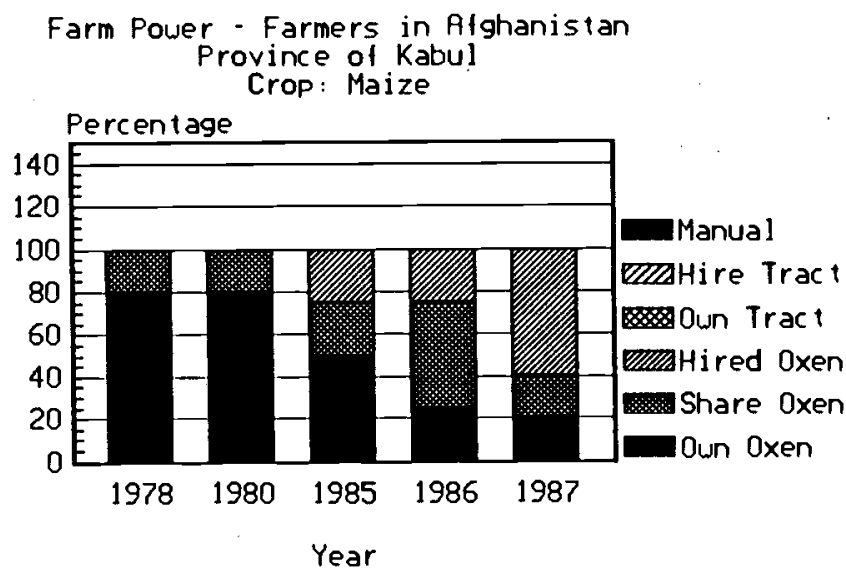

Farm Power - Farmers who lett in 1987 Province of Kabul Crop: Maize

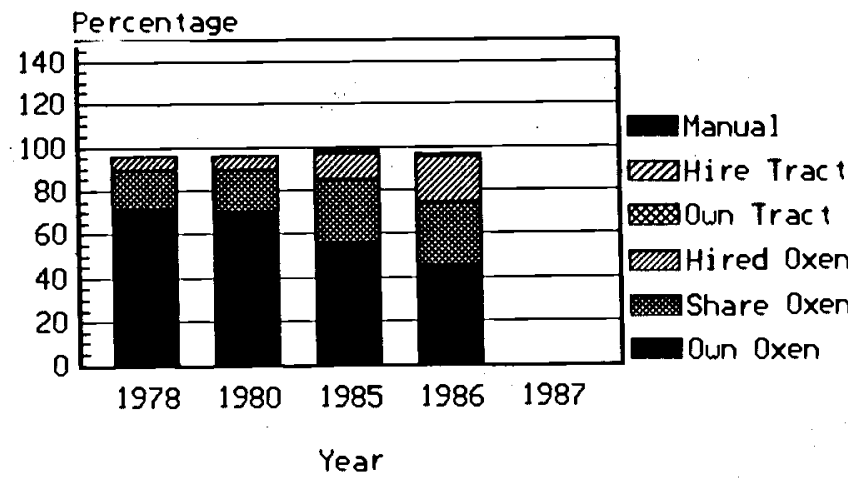

Percentage of Farmers $1978 \quad \underline{1980 \quad 1985} \quad \underline{1986}$

1987

$\begin{array}{ccccc}75.00 & 75.00 & 57.14 & 28.57 & 25.00 \\ 25.00 & 25.00 & 28.57 & 57.14 & 37.50 \\ - & - & 14.29 & 14.29 & 37.50 \\ - & - & - & - & - \\ - & - & - & - & - \\ - & - & - & - & - \\ 80.00 & 80.00 & 50.00 & 25.00 & 20.00 \\ 20.00 & 20.00 & 25.00 & 50.00 & 20.00 \\ - & - & 25.00 & 25.00 & 60.00 \\ - & - & - & - & - \\ - & - & - & - & - \\ - & - & - & - & -\end{array}$

69.89

15.05

11.29

$-$

.54

1.72

71.72
17.17

7.07
68.13

15.38

13.19

.55

$$
-
$$

70.30

18.81

6.93
58.62

21.26

16.09

.57

$-$

56.04

12.09
28.57
49.55

23.42

20.72

.90

$-$

44.83

$29.31 \quad 100.00$

20.69100 .00

66.67

33.33

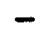

-
- 
Kabul

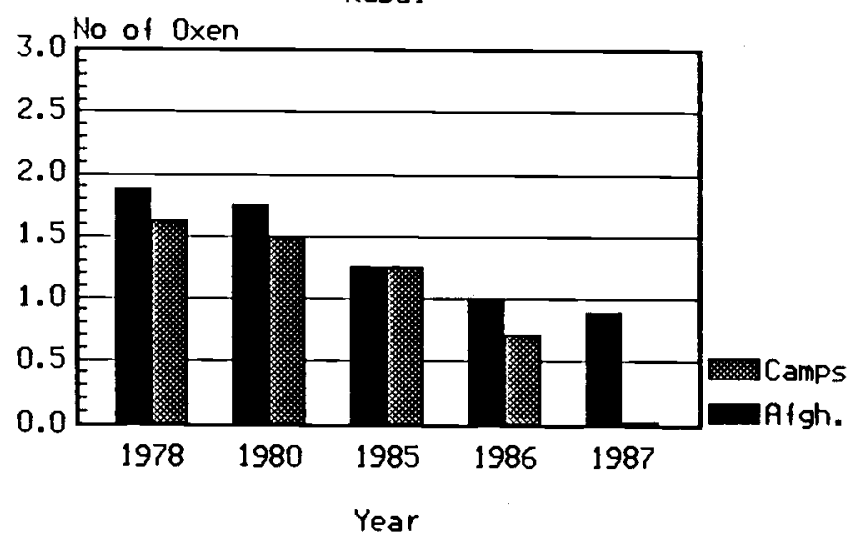

Kabul

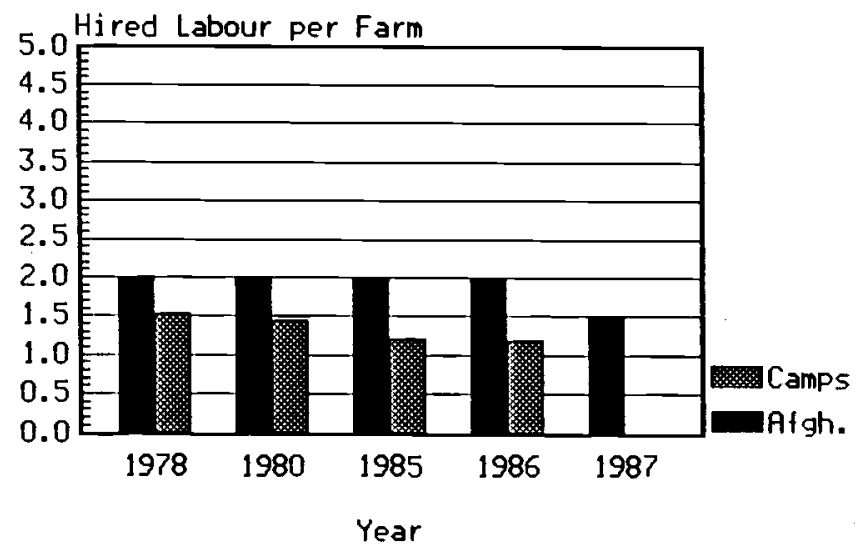

Kabul

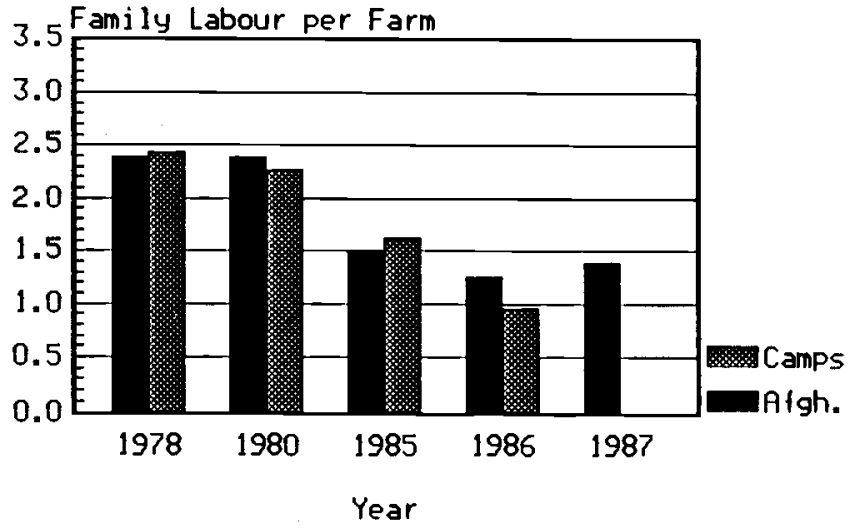

Kabul

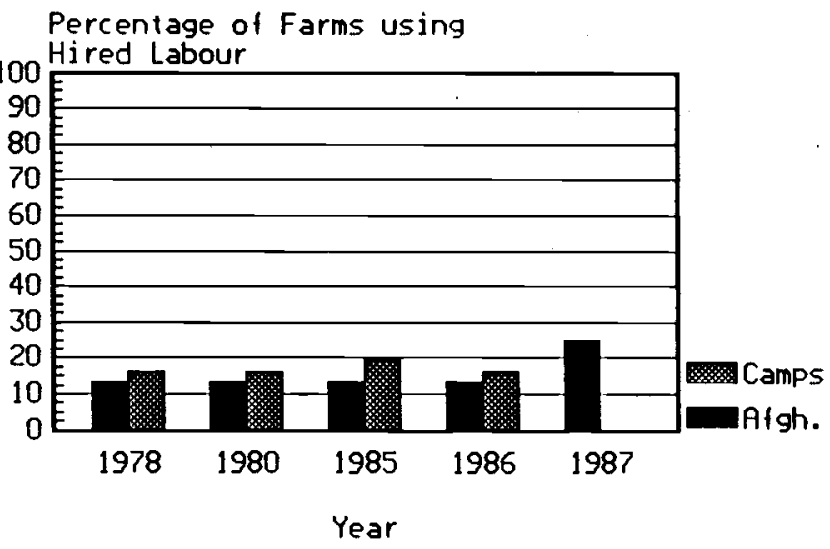


PROVINCE OF KBL

\begin{tabular}{|c|c|c|c|c|c|c|c|}
\hline YEAR & & 0 & 1 & 2 & 3 & 4 & 5 \\
\hline 1978 & $\begin{array}{l}\text { Number of Families } \\
x \text { of Families }\end{array}$ & $\begin{array}{c}0 \\
0.00\end{array}$ & $\begin{array}{c}1 \\
12.50\end{array}$ & $\begin{array}{c}7 \\
87.50\end{array}$ & $\begin{array}{c}0 \\
0.00\end{array}$ & $\begin{array}{c}0 \\
0.00\end{array}$ & $\begin{array}{c}0 \\
0.00\end{array}$ \\
\hline 1980 & $\begin{array}{l}\text { Number of Families } \\
x \text { of Families }\end{array}$ & $\begin{array}{c}0 \\
0.00\end{array}$ & $\begin{array}{c}2 \\
25.00\end{array}$ & $\begin{array}{c}6 \\
75.00\end{array}$ & $\begin{array}{c}0 \\
0.00\end{array}$ & $\begin{array}{c}0 \\
0.00\end{array}$ & $\begin{array}{c}0 \\
0.00\end{array}$ \\
\hline 1985 & $\begin{array}{l}\text { Number of Families } \\
x \text { of Families }\end{array}$ & $\begin{array}{c}2 \\
25.00\end{array}$ & $\begin{array}{c}2 \\
25.00\end{array}$ & $\begin{array}{c}4 \\
50.00\end{array}$ & $\begin{array}{c}0 \\
0.00\end{array}$ & $\begin{array}{c}0 \\
0.00\end{array}$ & $\begin{array}{c}0 \\
0.00\end{array}$ \\
\hline 1986 & $\begin{array}{l}\text { Number of Families } \\
x \text { of Families }\end{array}$ & $\begin{array}{c}2 \\
25 . n n\end{array}$ & $\begin{array}{c}4 \\
5 n .0 n\end{array}$ & $\begin{array}{c}2 \\
25 . \mathrm{nn}\end{array}$ & $\begin{array}{c}0 \\
0.00\end{array}$ & $\begin{array}{c}0 \\
0.00\end{array}$ & $\begin{array}{c}0 \\
0.00\end{array}$ \\
\hline 1987 & $\begin{array}{l}\text { Number of Families } \\
X \text { of Families }\end{array}$ & $\begin{array}{c}3 \\
37.50\end{array}$ & $\begin{array}{c}3 \\
37.50\end{array}$ & $\begin{array}{c}2 \\
25.00\end{array}$ & $\begin{array}{c}0 \\
0.00\end{array}$ & $\begin{array}{c}0 \\
0.00\end{array}$ & $\begin{array}{c}0 \\
0.00\end{array}$ \\
\hline Avera & ge Number of Oxen per Family: & $\begin{array}{l}1978 \\
1980 \\
1985 \\
1986 \\
1987\end{array}$ & $\begin{array}{l}1 \\
1 \\
1\end{array}$ & $\begin{array}{l}1.88 \\
1.75 \\
1.25 \\
1.00 \\
0.88\end{array}$ & & & \\
\hline
\end{tabular}

Number of Farmers Questioned 8

IABLE 7B - AVERAGE NUMBER OF TRAINED OXEN FOR FARMERS IN THE CAMPS

PROVINCE OF KBL

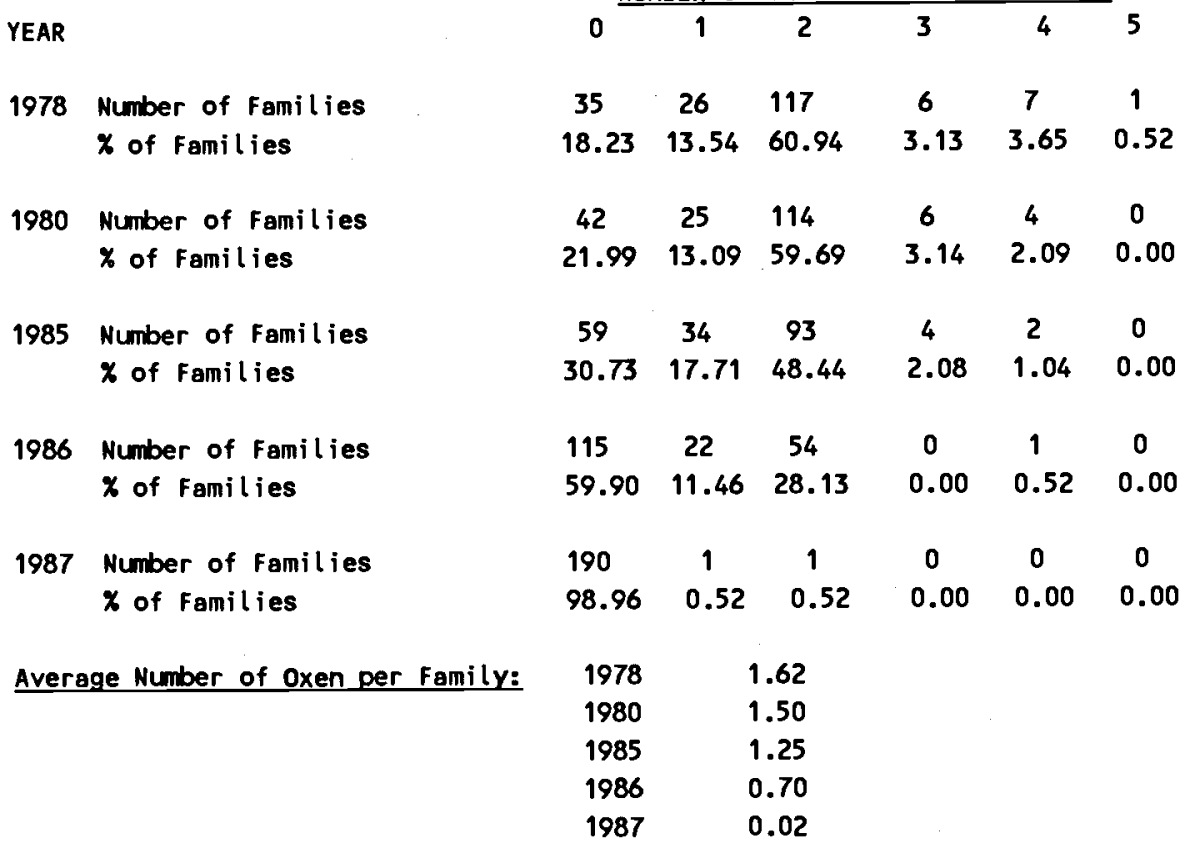

Number of Farmers Questioned 192 
PROVINCE OF KBL

\begin{tabular}{|c|c|c|c|c|c|}
\hline & 1978 & 1980 & 1985 & 1986 & 1987 \\
\hline Average Family Labour per Farm & 2.38 & 2.38 & 1.50 & 1.25 & 1.38 \\
\hline $\begin{array}{l}\text { Average No. of Hired Labour per Farm } \\
\text { (for farms using hired labour) }\end{array}$ & 2.00 & 2.00 & 2.00 & 2.00 & 1.50 \\
\hline Number of Farms using Hired Labour & 1 & 1 & 1 & 1 & 2 \\
\hline \multicolumn{6}{|l|}{ Number of Farmers Questioned 8} \\
\hline \multicolumn{6}{|c|}{ IABLE $8(B)$ - AVERAGE LABOUR (FAMILY and HIRED) FOR FARMERS IN CAMPS } \\
\hline \multirow[t]{2}{*}{ PROVINCE OF KBL } & & & & & \\
\hline & 1978 & 1980 & 1985 & 1986 & 1987 \\
\hline Average Family Labour per Farm & 2.43 & 2.27 & 1.62 & 0.95 & 0.01 \\
\hline $\begin{array}{l}\text { Average No. of Hired Labour per Farm } \\
\text { (for farms using hired (abour) }\end{array}$ & 1.52 & 1.44 & 1.20 & 1.18 & 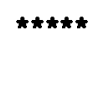 \\
\hline Number of Farms using Hired Labour & 31 & 31 & 37 & 31 & 0 \\
\hline Number of Farmers Questioned 192 & & & & & \\
\hline
\end{tabular}




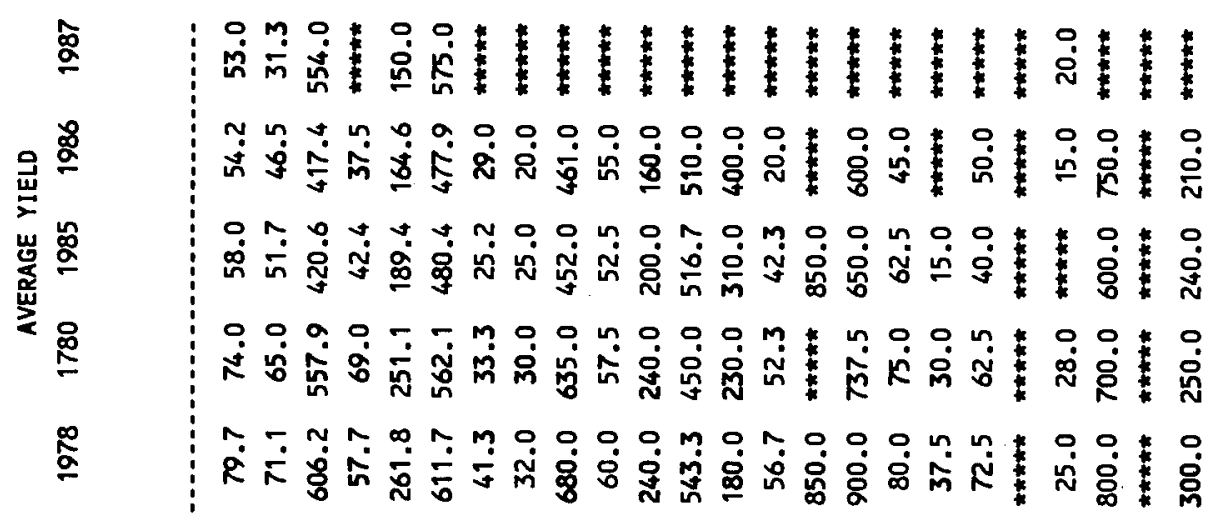

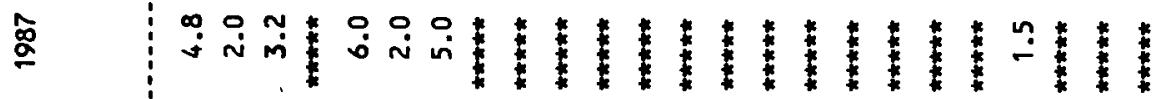

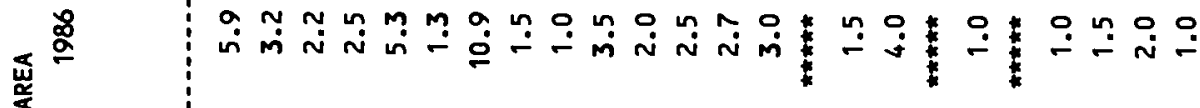
岀总

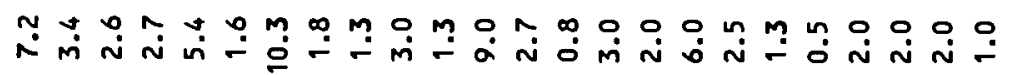

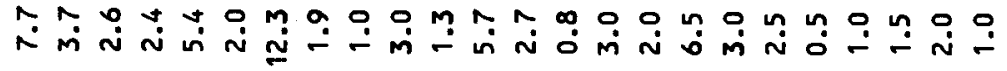

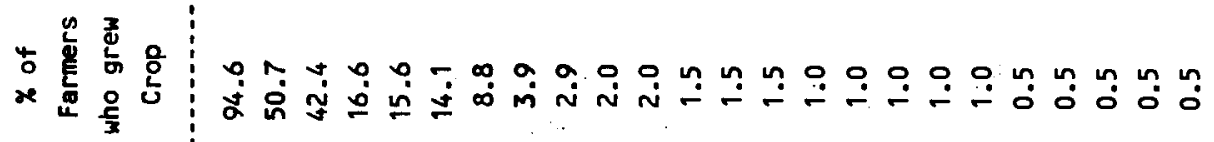

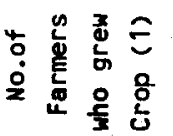

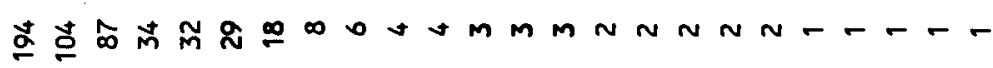
矛部

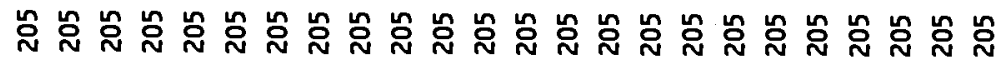

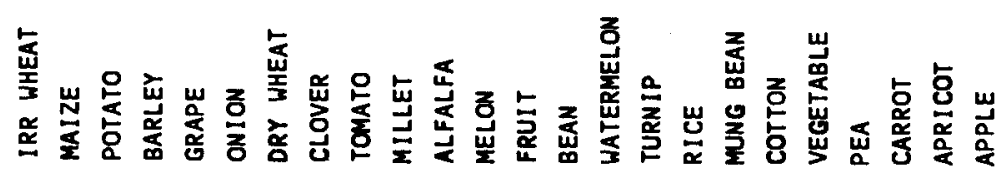


PROVINCE OF KBL

Rainfall

Crop Disease

Birds or Rats

Seed Avail lability

Fertilizer Availability

Crop Protection Chemicals

Credit

Irrigation Water Availability

Flooding

Labour Availabilty

Power for Land Preparation

Direct War Effects

Animal Diseases

Extension

other Problems

\begin{tabular}{rrrrrrrrrrrrr}
1 & 2 & 3 & 4 & 5 & 6 & 7 & 8 & 9 & 10 & 11 & 12 & Weighted Priority \\
\hline 0 & 0 & 0 & 0 & 0 & 0 & 0 & 0 & 0 & 0 & 0 & 0 & 0.00 \\
0 & 0 & 0 & 0 & 0 & 0 & 0 & 0 & 0 & 0 & 0 & 0 & 0.00 \\
0 & 0 & 0 & 0 & 0 & 0 & 0 & 0 & 0 & 0 & 0 & 0 & 0.00 \\
0 & 0 & 0 & 0 & 0 & 0 & 0 & 0 & 0 & 0 & 0 & 0 & 0.00 \\
0 & 100 & 0 & 0 & 0 & 0 & 0 & 0 & 0 & 0 & 0 & 0 & 27.27 \\
0 & 0 & 100 & 0 & 0 & 0 & 0 & 0 & 0 & 0 & 0 & 0 & 18.18 \\
0 & 0 & 0 & 0 & 0 & 0 & 0 & 0 & 0 & 0 & 0 & 0 & 0.00 \\
0 & 0 & 0 & 0 & 0 & 0 & 0 & 0 & 0 & 0 & 0 & 0 & 0.00 \\
0 & 0 & 0 & 0 & 0 & 0 & 0 & 0 & 0 & 0 & 0 & 0 & 0.00 \\
0 & 0 & 0 & 0 & 0 & 0 & 0 & 0 & 0 & 0 & 0 & 0 & 0.00 \\
0 & 0 & 0 & 0 & 0 & 0 & 0 & 0 & 0 & 0 & 0 & 0 & 0.00 \\
100 & 0 & 0 & 0 & 0 & 0 & 0 & 0 & 0 & 0 & 0 & 0 & 54.55 \\
0 & 0 & 0 & 0 & 0 & 0 & 0 & 0 & 0 & 0 & 0 & 0 & 0.00 \\
0 & 0 & 0 & 0 & 0 & 0 & 0 & 0 & 0 & 0 & 0 & 0 & 0.00 \\
0 & 0 & 0 & 0 & 0 & 0 & 0 & 0 & 0 & 0 & 0 & 0 & 0.00
\end{tabular}

1986

Rainfall

Crop Disease

Birds or Rats

Seed Availabil ity

Fertilizer Availability

Crop Protection Chemicals

Credit

Irrigation Water Availability

flooding

Labour Availabilty

Power for Land Preparation

Direct Har Effects

Animal Diseases

Extension

other Problems

\begin{tabular}{rrrrrrrrrrrrr}
1 & 2 & 3 & 4 & 5 & 6 & 7 & 8 & 9 & 10 & 11 & 12 & Weighted Priority \\
\hline 0 & 0 & 0 & 0 & 0 & 0 & 0 & 0 & 0 & 0 & 0 & 0 & 0.00 \\
0 & 100 & 0 & 0 & 0 & 0 & 0 & 0 & 0 & 0 & 0 & 0 & 24.00 \\
0 & 0 & 0 & 0 & 0 & 0 & 0 & 0 & 0 & 0 & 0 & 0 & 0.00 \\
0 & 0 & 0 & 0 & 0 & 0 & 0 & 0 & 0 & 0 & 0 & 0 & 0.00 \\
0 & 0 & 100 & 0 & 0 & 0 & 0 & 0 & 0 & 0 & 0 & 0 & 16.97 \\
0 & 0 & 0 & 100 & 0 & 0 & 0 & 0 & 0 & 0 & 0 & 0 & 12.00 \\
0 & 0 & 0 & 0 & 0 & 0 & 0 & 0 & 0 & 0 & 0 & 0 & 0.00 \\
100 & 0 & 0 & 0 & 0 & 0 & 0 & 0 & 0 & 0 & 0 & 0 & 48.00 \\
0 & 0 & 0 & 0 & 0 & 0 & 0 & 0 & 0 & 0 & 0 & 0 & 0.00 \\
0 & 0 & 0 & 0 & 0 & 0 & 0 & 0 & 0 & 0 & 0 & 0 & 0.00 \\
0 & 0 & 0 & 0 & 0 & 0 & 0 & 0 & 0 & 0 & 0 & 0 & 0.00 \\
0 & 0 & 0 & 0 & 0 & 0 & 0 & 0 & 0 & 0 & 0 & 0 & 0.00 \\
0 & 0 & 0 & 0 & 0 & 0 & 0 & 0 & 0 & 0 & 0 & 0 & 0.00 \\
0 & 0 & 0 & 0 & 0 & 0 & 0 & 0 & 0 & 0 & 0 & 0 & 0.00 \\
0 & 0 & 0 & 0 & 0 & 0 & 0 & 0 & 0 & 0 & 0 & 0 & 0.00
\end{tabular}

Rainfall

Crop Disease

Birds or Rats

Seed Availability

Fertilizer Availability

Crop Protection Chemicals

Credit

Irrigation Water Availabil ity

Flooding

Labour Availabilty

Power for Land Preparation

Direct War Effects

Animal Diseases

Extension

other Problems

\begin{tabular}{rrrrrrrrrrrrr}
1 & 2 & 3 & 4 & 5 & 6 & 7 & 8 & 0 & 10 & 11 & 12 & Weighted Priority \\
\hline 0 & 0 & 0 & 0 & 0 & 0 & 0 & 0 & 0 & 0 & 0 & 0 & 0.00 \\
0 & 0 & 0 & 0 & 0 & 0 & 0 & 0 & 0 & 0 & 0 & 0 & 0.00 \\
0 & 0 & 0 & 0 & 0 & 0 & 0 & 0 & 0 & 0 & 0 & 0 & 0.00 \\
0 & 0 & 0 & 0 & 0 & 0 & 0 & 0 & 0 & 0 & 0 & 0 & 0.00 \\
0 & 0 & 0 & 0 & 0 & 0 & 0 & 0 & 0 & 0 & 0 & 0 & 0.00 \\
0 & 0 & 0 & 0 & 0 & 0 & 0 & 0 & 0 & 0 & 0 & 0 & 0.00 \\
0 & 0 & 0 & 0 & 0 & 0 & 0 & 0 & 0 & 0 & 0 & 0 & 0.00 \\
0 & 0 & 0 & 0 & 0 & 0 & 0 & 0 & 0 & 0 & 0 & 0 & 0.00 \\
0 & 0 & 0 & 0 & 0 & 0 & 0 & 0 & 0 & 0 & 0 & 0 & 0.00 \\
0 & 0 & 0 & 0 & 0 & 0 & 0 & 0 & 0 & 0 & 0 & 0 & 0.00 \\
0 & 0 & 0 & 0 & 0 & 0 & 0 & 0 & 0 & 0 & 0 & 0 & 0.00 \\
100 & 0 & 0 & 0 & 0 & 0 & 0 & 0 & 0 & 0 & 0 & 0 & 100.0 \\
0 & 0 & 0 & 0 & 0 & 0 & 0 & 0 & 0 & 0 & 0 & 0 & 0.00 \\
0 & 0 & 0 & 0 & 0 & 0 & 0 & 0 & 0 & 0 & 0 & 0 & 0.00 \\
0 & 0 & 0 & 0 & 0 & 0 & 0 & 0 & 0 & 0 & 0 & 0 & 0.00
\end{tabular}


PROVINCE OF KBL

Rainfall

Crop Disease

Birds or Rats

Seed Availability

Fertilizer Availability

Crop Protection Chemicals

Credit

Irrigation Water Availability

Flooding

Labour Availabilty

Power for Land Preparation

Direct War Effects

Animal Diseases

Extension

other Problems

\begin{tabular}{rrrlllllllllc}
1 & 2 & 3 & 4 & 5 & 6 & 7 & 8 & 9 & 10 & 11 & 12 & Weighted Priority \\
\hline 0 & 0 & 0 & 0 & 0 & 0 & 0 & 0 & 0 & 0 & 0 & 0 & 0.00 \\
0 & 0 & 0 & 0 & 0 & 0 & 0 & 0 & 0 & 0 & 0 & 0 & 0.00 \\
100 & 0 & 0 & 0 & 0 & 0 & 0 & 0 & 0 & 0 & 0 & 0 & 100.0 \\
0 & 0 & 0 & 0 & 0 & 0 & 0 & 0 & 0 & 0 & 0 & 0 & 0.00 \\
0 & 0 & 0 & 0 & 0 & 0 & 0 & 0 & 0 & 0 & 0 & 0 & 0.00 \\
0 & 0 & 0 & 0 & 0 & 0 & 0 & 0 & 0 & 0 & 0 & 0 & 0.00 \\
0 & 0 & 0 & 0 & 0 & 0 & 0 & 0 & 0 & 0 & 0 & 0 & 0.00 \\
0 & 0 & 0 & 0 & 0 & 0 & 0 & 0 & 0 & 0 & 0 & 0 & 0.00 \\
0 & 0 & 0 & 0 & 0 & 0 & 0 & 0 & 0 & 0 & 0 & 0 & 0.00 \\
0 & 0 & 0 & 0 & 0 & 0 & 0 & 0 & 0 & 0 & 0 & 0 & 0.00 \\
0 & 0 & 0 & 0 & 0 & 0 & 0 & 0 & 0 & 0 & 0 & 0 & 0.00 \\
0 & 0 & 0 & 0 & 0 & 0 & 0 & 0 & 0 & 0 & 0 & 0 & 0.00 \\
0 & 0 & 0 & 0 & 0 & 0 & 0 & 0 & 0 & 0 & 0 & 0 & 0.00 \\
0 & 0 & 0 & 0 & 0 & 0 & 0 & 0 & 0 & 0 & 0 & 0 & 0.00 \\
0 & 0 & 0 & 0 & 0 & 0 & 0 & 0 & 0 & 0 & 0 & 0 & 0.00
\end{tabular}

\section{$\underline{1978}$}

Rainfall

Crop Disease

Birds or Rats

Seed Availability

Fertilizer Availability

Crop Protection Chemicals

Credit

Irrigation Water Availability

Flooding

Labour Avai labilty

Power for Land Preparation

Direct War Effects

Animal Diseases

Extension

other Problems

\begin{tabular}{|c|c|c|c|c|c|c|c|c|c|c|c|c|}
\hline 1 & 2 & 3 & 4 & 5 & 6 & 7 & 8 & 9 & 10 & 11 & 12 & Weighted $P$ \\
\hline 0 & 0 & 0 & 0 & 0 & 0 & 0 & 0 & 0 & 0 & 0 & 0 & $\star \star \star \star \star \star$ \\
\hline 0 & 0 & 0 & 0 & 0 & 0 & 0 & 0 & 0 & 0 & 0 & 0 & 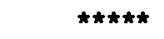 \\
\hline 0 & 0 & 0 & 0 & 0 & 0 & 0 & 0 & 0 & 0 & 0 & 0 & 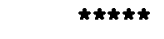 \\
\hline 0 & 0 & 0 & 0 & 0 & 0 & 0 & 0 & 0 & 0 & 0 & 0 & 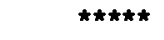 \\
\hline 0 & 0 & 0 & 0 & 0 & 0 & 0 & 0 & 0 & 0 & 0 & 0 & 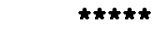 \\
\hline 0 & 0 & 0 & 0 & 0 & 0 & 0 & 0 & 0 & 0 & 0 & 0 & 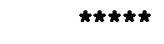 \\
\hline 0 & 0 & 0 & 0 & 0 & 0 & 0 & 0 & 0 & 0 & 0 & 0 & 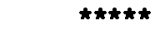 \\
\hline 0 & 0 & 0 & $\hat{\varepsilon}$ & $\hat{0}$ & 0 & $\hat{0}$ & 0 & 0 & 0 & $n$ & 0 & 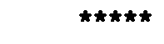 \\
\hline 0 & 0 & 0 & 0 & 0 & 0 & 0 & 0 & 0 & 0 & 0 & 0 & $\star \star \star \star \star \star$ \\
\hline 0 & 0 & 0 & 0 & 0 & 0 & 0 & 0 & 0 & 0 & 0 & 0 & 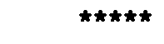 \\
\hline 0 & 0 & 0 & 0 & 0 & 0 & 0 & 0 & 0 & 0 & 0 & 0 & $\star \star \star \star \star \star$ \\
\hline 0 & 0 & 0 & 0 & 0 & 0 & 0 & 0 & 0 & 0 & 0 & 0 & $\star \star \star \star \star \star$ \\
\hline 0 & 0 & 0 . & 0 & 0 & 0 & 0 & 0 & 0 & 0 & 0 & 0 & 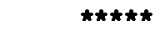 \\
\hline 0 & 0 & 0 & 0 & 0 & 0 & 0 & 0 & 0 & 0 & 0 & 0 & 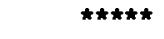 \\
\hline 0 & 0 & 0 & 0 & 0 & 0 & 0 & 0 & 0 & 0 & 0 & 0 & 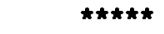 \\
\hline
\end{tabular}


KANDAHAR 


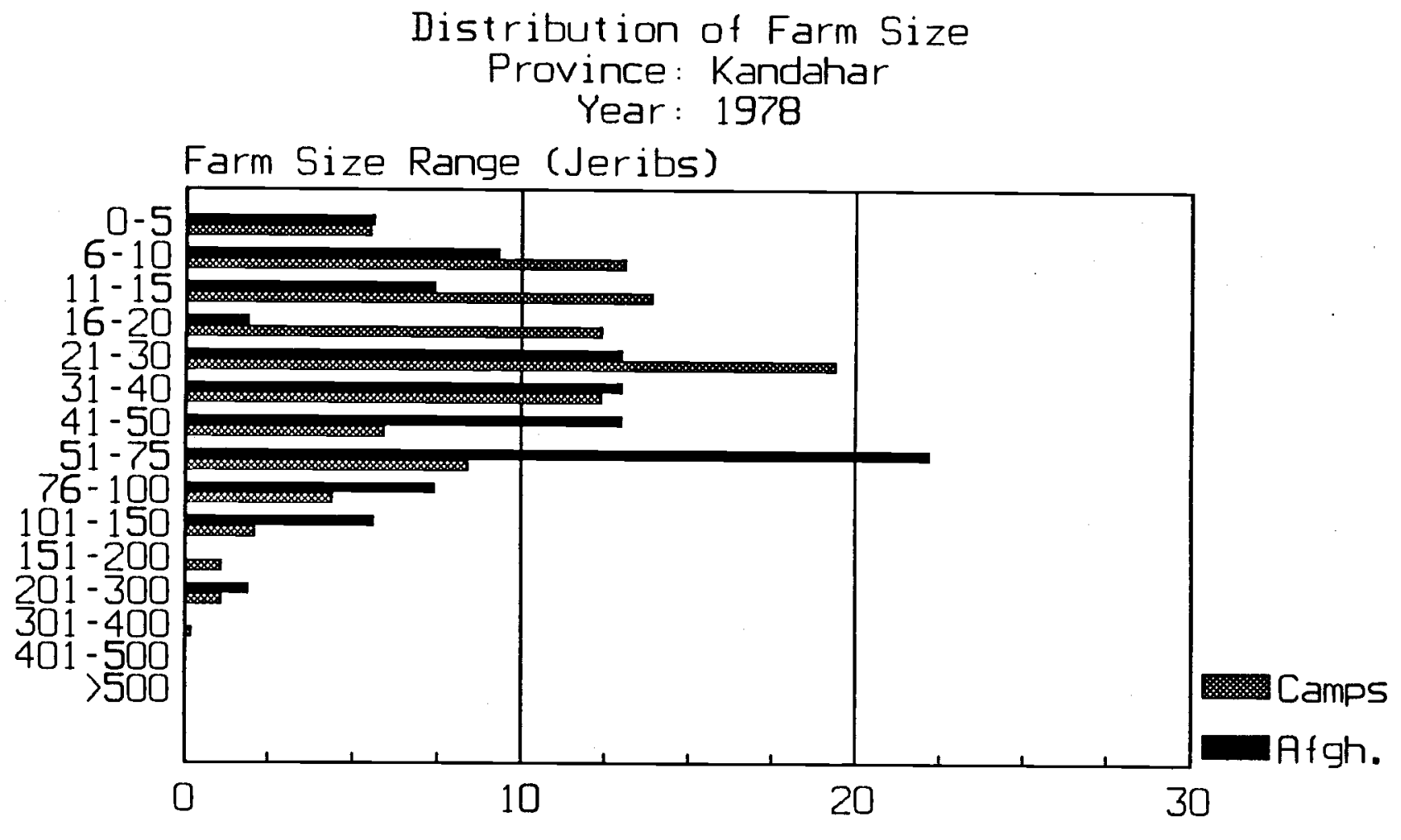

Percentage of Farmers

Farmers in Afqhanistan

1978

1987

Total Number of Farms

Average Area

Average Area Irrigated

Average Dryland Area

Total Number of Farms

Average Area

Average Area Irrigated

Average Dryland Area

54

46.22

46.00

7.75

474

34.85

33.95

25.47
54

27.11

26.17

4.29

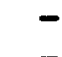

18.33

50.00

\section{Average of Both Groups}

Provincial Average Area

Provincial Average Irrigated Area

36.02

27.11

Provincial Average Dryland Area

(All areas in Jeribs. 5 Jeribs $=1$ Hectare) 
Farm Power - Farmers in Atghanistan Province of Kandahar Crop: Irrigated wheat

Percentage

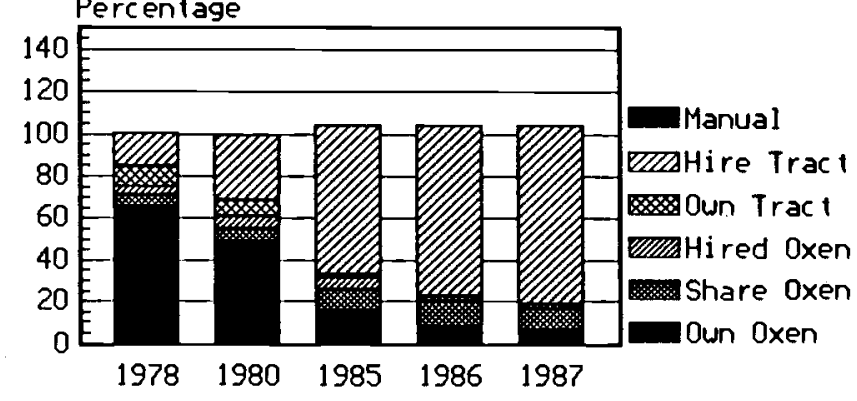

Year

Farm Power - Farmers who left in 1987 Province of Kandahar

Crop: Irrigated wheat

\section{Percentage}

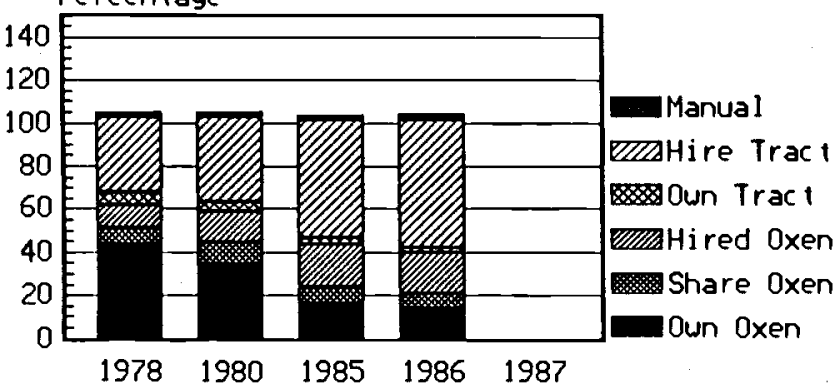

Year

Base Figures for above graphs

Province: Kandahar

Farmers in Afghanistan

Irr. Wheat own oxen

Shared oxen

Hired oxen

Own Tractor

Hired Tractor

Maize

Manual Means

Own Oxen

Shared Oxen

Hired oxen

Own Tractor

Hired Tractor

Manual Means

Farmers who left in 1987

Irr. Wheat own oxen

Maize
Shared oxen

Hired oxen

Own Tractor

Hired Tractor

Manual Means

Own Oxen

Shared oxen

Hired oxen
43.60

7.35

11.14

5.69

35.07

1.90

34.16

10.15

14.36

4.46

39.85

1.98

59.86

9.52

47.95

13.01

13.70
16.03

8.15

19.02

3.53

54.62

2.17

23.85

10.00

17.69
14.12

6.92

19.02

2.31

58.79

2.88

23.77

7.38

20.49
Manual EzaHire Trac 1 Own Tract שIISHired Oxen Share 0xen oun oxen
6.12

10.20

2.04

$-$

85.71

14.29

28.57

$-$

71.43 

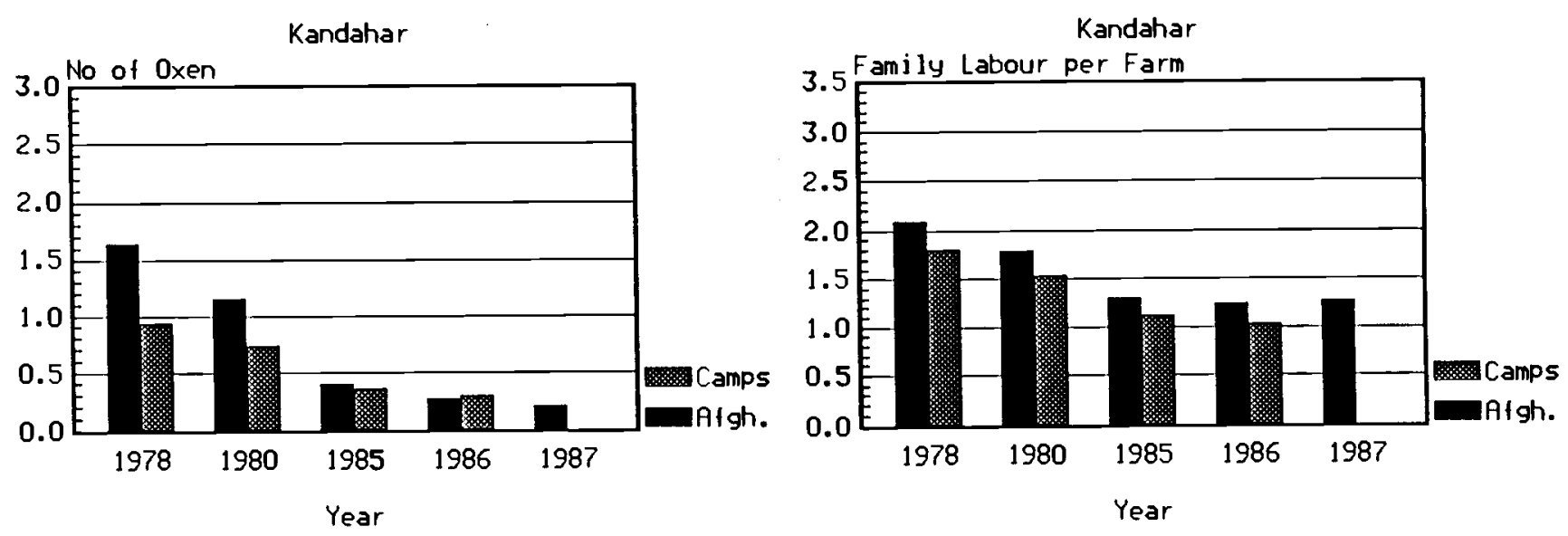

Kandahar
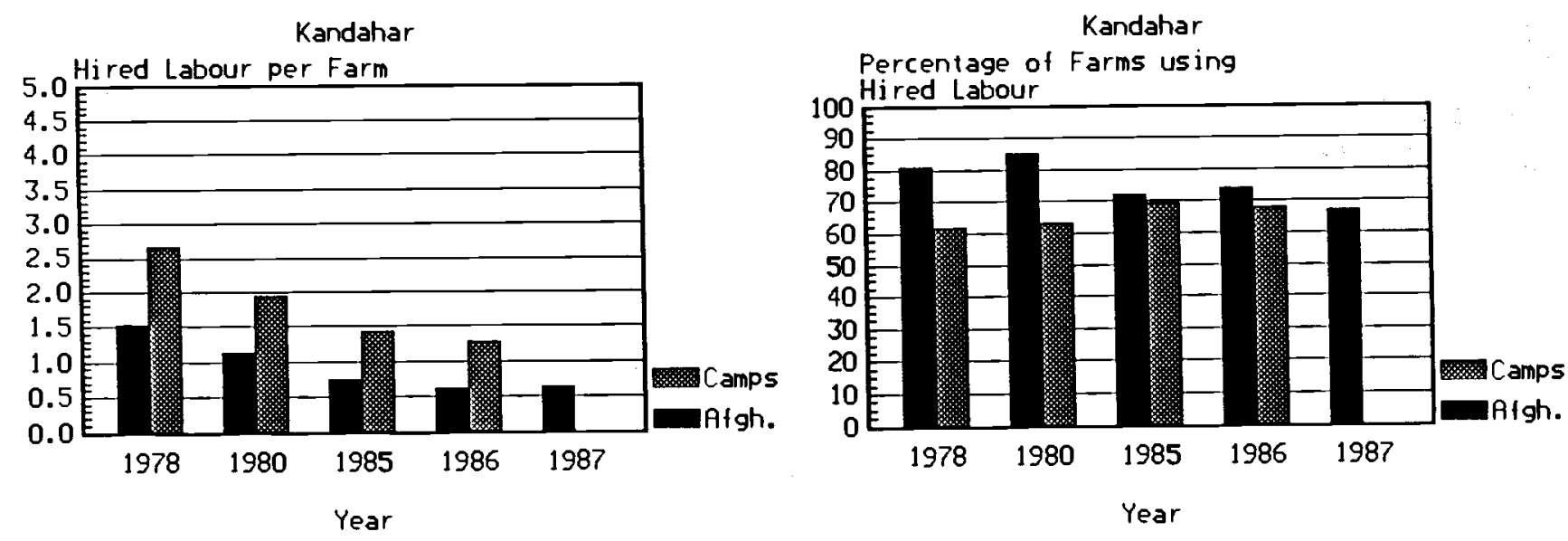
PROVINCE OF KDR

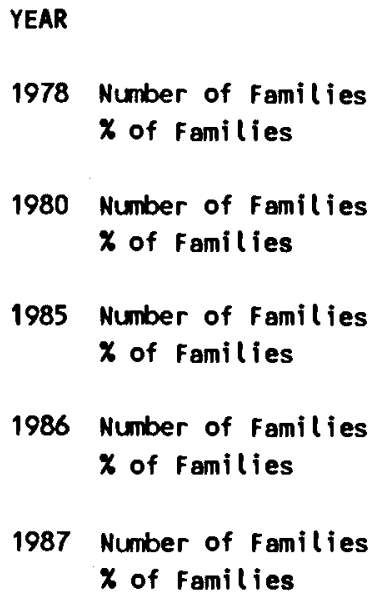

$\begin{array}{ll}1978 & 1.64 \\ 1980 & 1.15 \\ 1985 & 0.39 \\ 1986 & 0.26 \\ 1987 & 0.20\end{array}$

Number of Farmers Questioned 54

\section{IABLE 7B - AVERAGE NUMBER OF TRAINED OXEN FOR FARMERS IN THE CAMPS}

PROVINCE OF KDR

\begin{tabular}{|c|c|c|c|c|c|c|c|}
\hline \multirow{2}{*}{\multicolumn{2}{|c|}{ YEAR }} & \\
\hline & & 0 & 1 & 2 & 3 & 4 & 5 \\
\hline 1978 & $\begin{array}{l}\text { Number of Families } \\
\% \text { of Families }\end{array}$ & $\begin{array}{l}257 \\
54.68\end{array}$ & $\begin{array}{l}41 \\
8.72\end{array}$ & $\begin{array}{l}141 \\
30.00\end{array}$ & $\begin{array}{l}15 \\
3.19\end{array}$ & $\begin{array}{l}13 \\
2.77\end{array}$ & $\begin{array}{c}3 \\
0.64\end{array}$ \\
\hline 1980 & $\begin{array}{l}\text { Number of Families } \\
\% \text { of Families }\end{array}$ & $\begin{array}{l}297 \\
63.06\end{array}$ & $\begin{array}{l}39 \\
8.28\end{array}$ & $\begin{array}{l}114 \\
24.20\end{array}$ & $\begin{array}{l}9 \\
1.91\end{array}$ & $\begin{array}{l}11 \\
2.34\end{array}$ & $\begin{array}{c}1 \\
0.21\end{array}$ \\
\hline 1985 & $\begin{array}{l}\text { Number of Families } \\
x \text { of Families }\end{array}$ & $\begin{array}{l}383 \\
81.14\end{array}$ & $\begin{array}{l}29 \\
6.14\end{array}$ & $\begin{array}{c}48 \\
10.17\end{array}$ & $\begin{array}{c}7 \\
1.48\end{array}$ & $\begin{array}{c}4 \\
0.85\end{array}$ & $\begin{array}{c}1 \\
0.21\end{array}$ \\
\hline 1986 & $\begin{array}{l}\text { Number of Families } \\
\% \text { of Families }\end{array}$ & $\begin{array}{l}400 \\
84.39\end{array}$ & $\begin{array}{l}23 \\
4.85\end{array}$ & $\begin{array}{l}42 \\
8.86\end{array}$ & $\begin{array}{c}5 \\
1.05\end{array}$ & $\begin{array}{c}3 \\
0.63\end{array}$ & $\begin{array}{c}1 \\
0.21\end{array}$ \\
\hline 1987 & $\begin{array}{l}\text { Number of Families } \\
\% \text { of Families }\end{array}$ & $\begin{array}{l}474 \\
100.0\end{array}$ & $\begin{array}{c}0 \\
0.00\end{array}$ & $\begin{array}{c}0 \\
0.00\end{array}$ & $\begin{array}{c}0 \\
0.00\end{array}$ & $\begin{array}{c}0 \\
0.00\end{array}$ & $\begin{array}{c}0 \\
0.00\end{array}$ \\
\hline Avers & ge Number of Oxen per Family: & $\begin{array}{l}1978 \\
1980 \\
1985 \\
1986 \\
1987\end{array}$ & & $\begin{array}{l}0.93 \\
0.73 \\
0.35 \\
0.29 \\
0.00\end{array}$ & & & \\
\hline
\end{tabular}

Number of Farmers Questioned 474 
PROVINCE OF KDR

\begin{tabular}{|c|c|c|c|c|c|}
\hline & 1978 & 1980 & 1985 & 1986 & 1987 \\
\hline Average Family Labour per Farm & 2.09 & 1.78 & 1.30 & 1.23 & 1.26 \\
\hline $\begin{array}{l}\text { Average No. of Hired Labour per Farm } \\
\text { (for farms using hired (abour) }\end{array}$ & 1.52 & 1.13 & 0.73 & 0.61 & 0.64 \\
\hline Number of Farms using Hired Labour & 44 & 46 & 39 & 40 & 36 \\
\hline \multicolumn{6}{|l|}{ Number of Farmers Questioned 54} \\
\hline PROVINCE OF KDR & $\cdot$ & & & & \\
\hline & 1978 & 1980 & 1985 & 1986 & 1987 \\
\hline Average Family Labour per Farm & 1.80 & 1.54 & 1.12 & 1.03 & 0.00 \\
\hline $\begin{array}{l}\text { Average No. of Hired Labour per Farm } \\
\text { (for farms using hired labour) }\end{array}$ & 2.65 & 1.93 & 1.43 & 1.28 & $\star \star \star \star \star \star$ \\
\hline Number of Farms using Hired Labour & 295 & 298 & 327 & 321 & 0 \\
\hline Number of Farmers Questioned 474 & & & & & \\
\hline
\end{tabular}


PROVINCE OF KDR

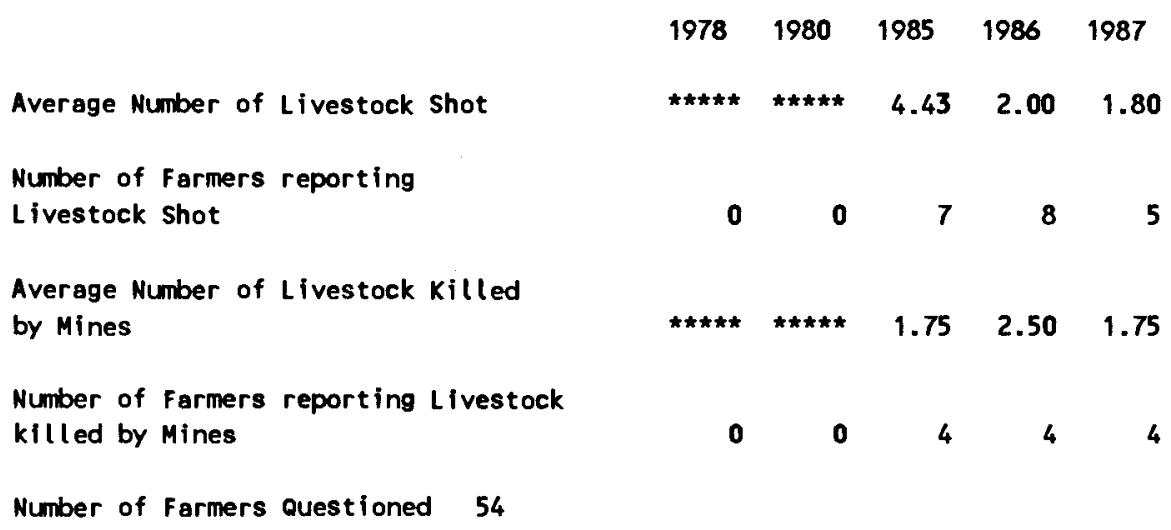

\section{TABLE 9B - DIRECT EFFECTS OF THE WAR - FARMERS IN CAMPS}

PROVINCE OF KDR

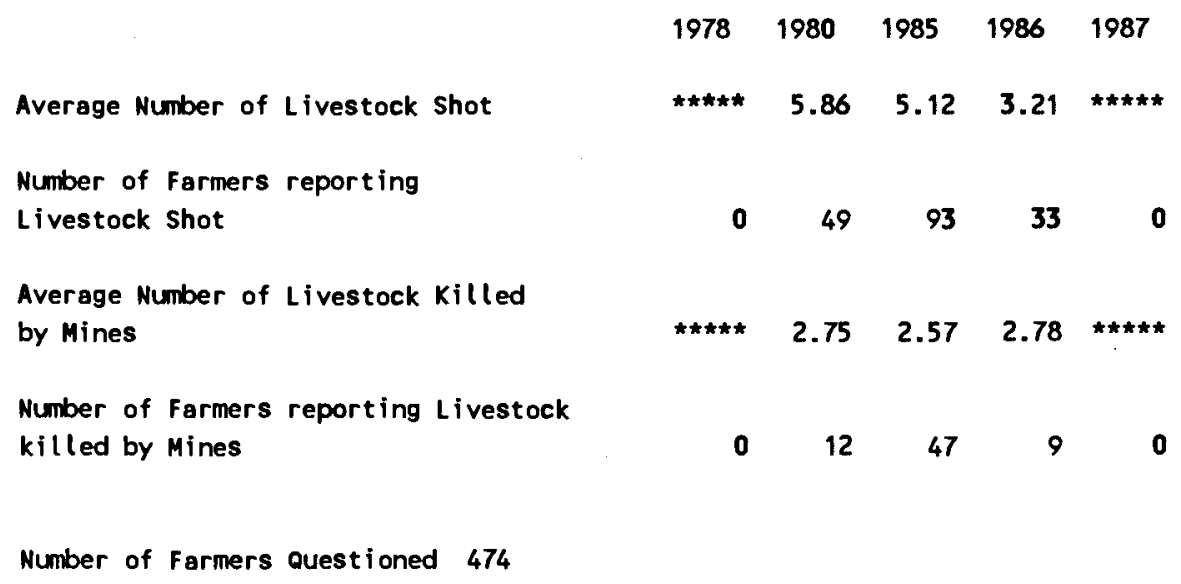




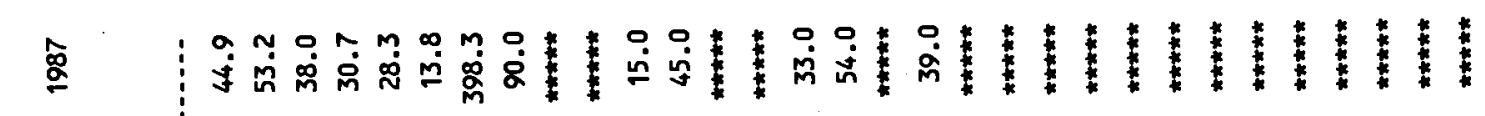
胥

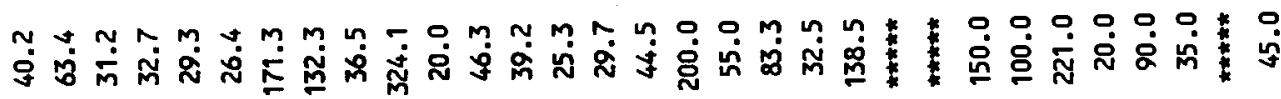
mâ

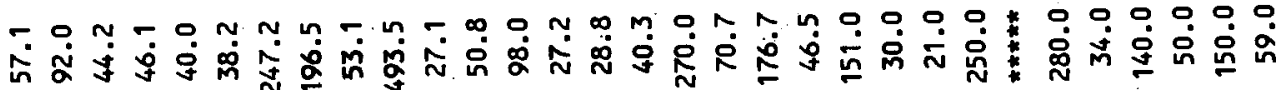

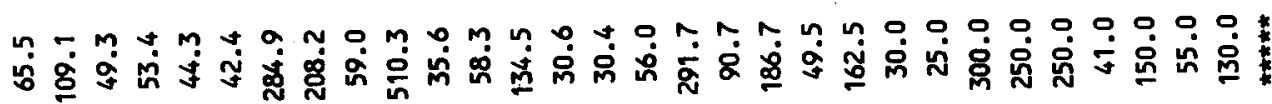

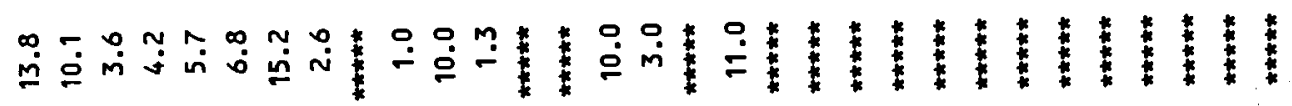

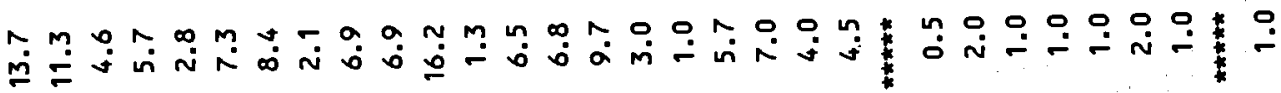

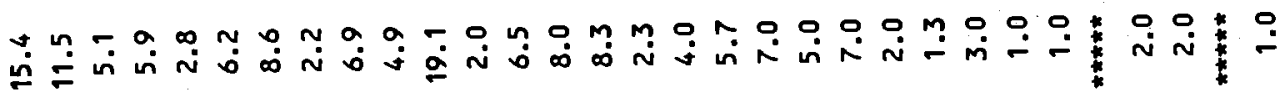

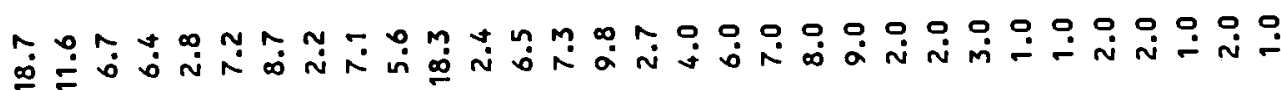

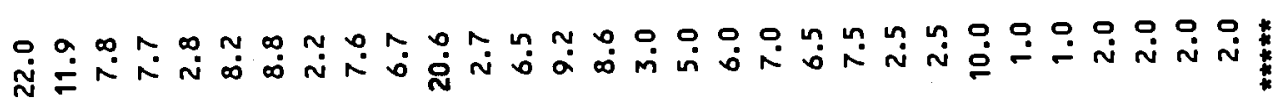
吕

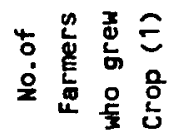

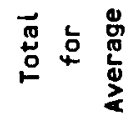

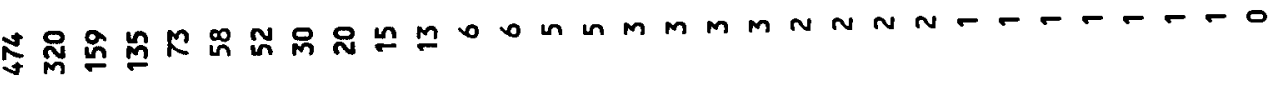

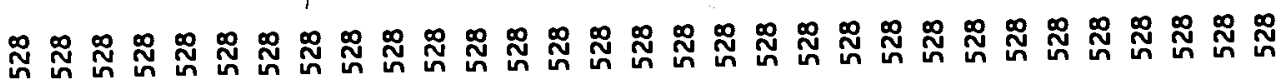

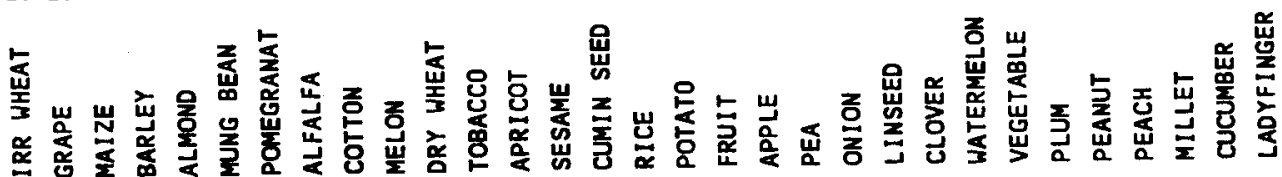


PROVINCE OF KDR

Rainfall

Crop Disease

Birds or Rats

Seed Avail lability

Fertilizer Availability

Crop Protection Chemicals

Credit

Irrigation Water Availability

Flooding

Labour Availabilty

Power for Land Preparation

Direct War Effects

Animal Diseases

Extension

Other Problems

1986

Rainfall

Crop Disease

Birds or Rats

Seed Availability

Fertilizer Availability

Crop Protection Chemicals

Credit

Irrigation Hater Availability

Flooding

Labour Availabilty

Power for Land Preparation

Direct War Effects

Animal Diseases

Extension

Other Problems

$\underline{1985}$

Rainfall

Crop Disease

Birds or Rats

Seed Availability

Fertilizer Availability

Crop Protection Chemicals

Credit

Irrigation Water Availability

Flooding

Labour Avai labilty

Power for Land Preparation

Direct War Effects

Animal Diseases

Extension

Other Problems

\begin{tabular}{|c|c|c|c|c|c|c|c|c|c|c|c|c|}
\hline 1 & 2 & 3 & 4 & 5 & 6 & 7 & 8 & 9 & 10 & 11 & 12 & Weighted \\
\hline$* * *$ & $\star \star \star \star$ & $\star \star * *$ & 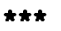 & 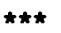 & $\star \star \star \star$ & 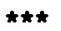 & 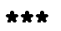 & 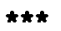 & 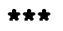 & 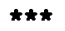 & $\star \star \star$ & 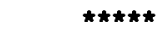 \\
\hline k* & $\hbar \hbar \hbar$ & $\star \star \star *$ & $\star \star \star \star$ & $\star \star \star$ & $\star \star \star$ & $\star \star \star \star$ & $\star \star \star \star$ & $\star \star \star \star$ & 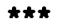 & $\star \star \star *$ & $\star \star \star *$ & $\star \star \star \star \star \star *$ \\
\hline *** & $\star \star * *$ & $\star * *$ & $\star * *$ & $\star \star \star *$ & $* * *$ & $\star \star \star \star$ & $\star \star \star \star$ & $\star \star \star \star$ & $\star \star \star$ & $\star \star \star$ & $\star \star \star \star$ & $\star \star \star \star \star \star$ \\
\hline t** & $\hbar \star \star \star$ & 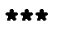 & 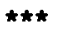 & $\star \star \star \star$ & 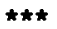 & $\star \star \star \star$ & $\star \star \star *$ & $\star \star \star \star$ & $\star * *$ & $\star * *$ & $\star \star \star \star *$ & 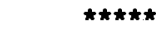 \\
\hline$\star \star$ & 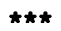 & $\star \star \star \star$ & $\star \star \star \star$ & $\star \star \star *$ & $\star \star \star *$ & $\star \star \star \star$ & $\star \star \star \star$ & 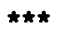 & $\star \star \star \star \star$ & 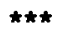 & $t \hbar \hbar$ & $\star \star \star \star \star \star$ \\
\hline$\star \star \star \star$ & $\star \star \star \star$ & $\star \star \star$ & $\star \star \star$ & $\star \star \star \star$ & $\star * *$ & $\star \star \star \star *$ & $\star \star * *$ & $\star \star \star \star *$ & $* * *$ & $* * *$ & 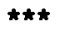 & 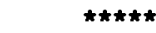 \\
\hline$\star \star \star \star$ & $\star \star \star \star$ & $\star \star \star \star *$ & $\star \star \star *$ & $\star \star \star *$ & $\star \star \star \star *$ & $\star \star \star \star$ & 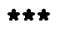 & 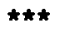 & $\star \star \star *$ & $\star \star \star \star$ & $\hbar \star \star$ & $\star \star * * * *$ \\
\hline$\star \star \star$ & $\star \star \star$ & $\star \star \star$ & $\star \star \star \star$ & $\star \star \star \star *$ & $\star \star \star *$ & $\star \star \star \star$ & $\star \star \star *$ & $\star \star \star \star$ & $\star \star \star \star$ & $\star \star \star \star$ & $\star \star \star \star$ & $\star \star \star \star \star \star$ \\
\hline$\star \star \star$ & 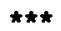 & 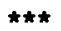 & $\star \star \star$ & $\star \star \star \star$ & $\star \star \star \star$ & $\star \star \star \star$ & $\star \star \star *$ & $\star \star \star \star$ & $* * *$ & $\star \star \star *$ & 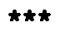 & 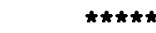 \\
\hline 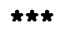 & \#* * & 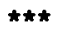 & $\star \star \star$ & $\star \star \star$ & $\star \star \star$ & $\star \star \star$ & $\star \star \star$ & $\star \star \star$ & $\star \star * *$ & $\star * *$ & $\star \star \star \star$ & 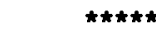 \\
\hline$\star \star \star \star$ & 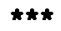 & $\star \star \star$ & $\star \star \star \star$ & $\star \star \star$ & 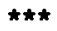 & $\star \star \star$ & $\star \star \star$ & $\star \star \star$ & $\star \star \star$ & $\star \star \star \star$ & $\star \star \star \star$ & $\star \star \star \star \star \star \star *$ \\
\hline 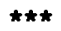 & $\hbar \star \star$ & $\hbar \star \star$ & $\star \star \star$ & $\star \star \star$ & $\star \star \star$ & $\star \star \star$ & $\star \star \star$ & $\star \star \star \star$ & 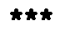 & $\star \star \star *$ & 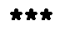 & 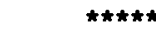 \\
\hline$\star$ & $\star \star \star \star$ & $\star \star \star \star$ & $\star \star \star \star$ & $\star \star \star \star$ & 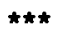 & $\star \star \star \star$ & 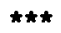 & 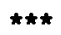 & $\star \star \star \star$ & $\star \star \star \star$ & 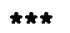 & 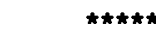 \\
\hline * & $\hbar \hbar \hbar$ & $\hbar \star \hbar$ & $\star \star \star$ & $\star \star \star$ & $\star \star \star \star$ & $\star \star \star \star$ & $\star \star \star \star$ & $\star \star \star \star$ & $\star \star \star \star *$ & 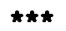 & 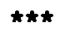 & 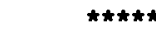 \\
\hline & $\star * *$ & $\star \star$ & $\star \star \star \star *$ & $\star \star \star \star$ & 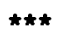 & $\star \star \star \star$ & 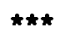 & $\star \star \star \star$ & $\star \star \star \star$ & $\star \star \star$ & $\star \star \star \star$ & 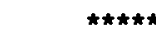 \\
\hline
\end{tabular}

$\begin{array}{llllllllllll}1 & 2 & 3 & 4 & 5 & 6 & 7 & 8 & 9 & 10 & 11 & 12\end{array}$ Weighted Priority

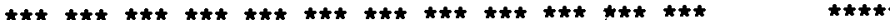

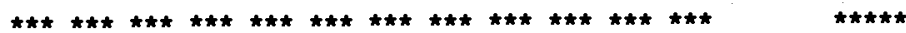

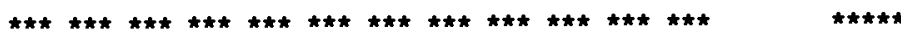

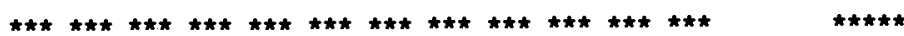

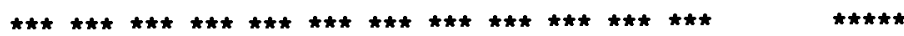

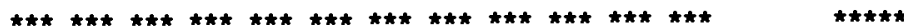

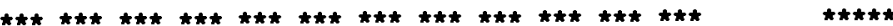

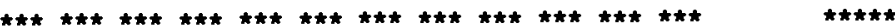

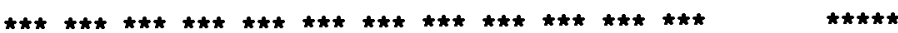

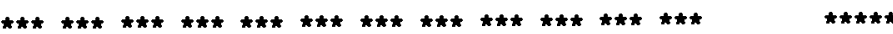

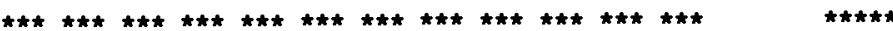

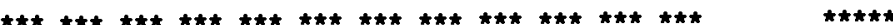

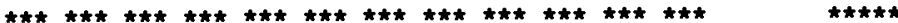

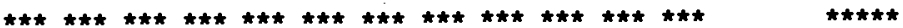

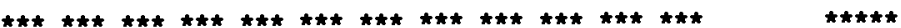

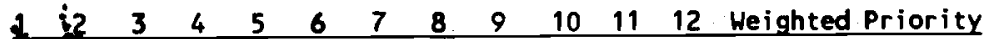

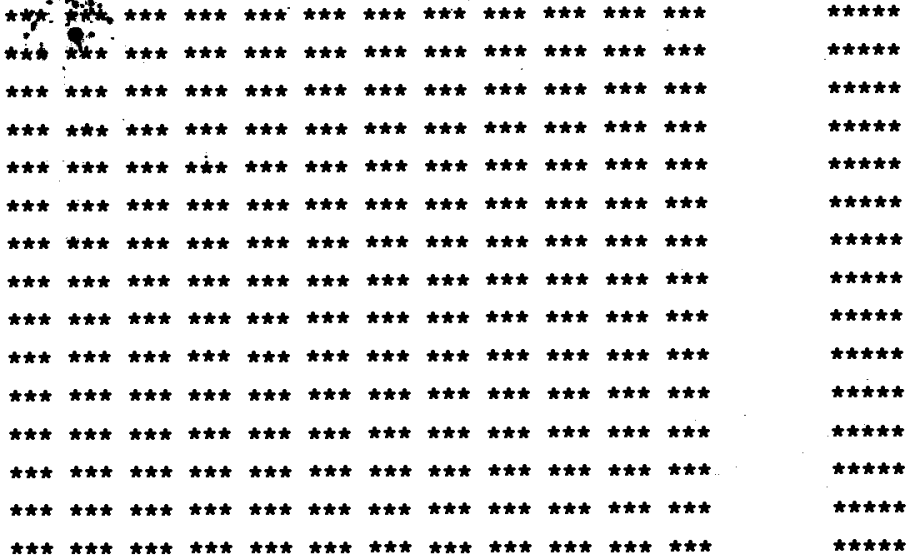




\section{TABLE 1-5(contd) - ANALYSIS OF GREATEST FARMING PROBLEMS - COMMUNITY SURVEY}

PROVINCE OF KDR

\section{PERCENTAGE OF VILLAGES GIVING FOLLONING PRIORITY IO PROBLEM}

\section{Rainfall}

Crop Disease

Birds or Rats

Seed Avai lability

Fertilizer Availability

Crop Protection Chemicals

Credit

Irrigation Water Availability

Flooding

Labour Availabilty

Power for Land Preparation

Direct War Effects

Animal Diseases

Extension

Other Problems

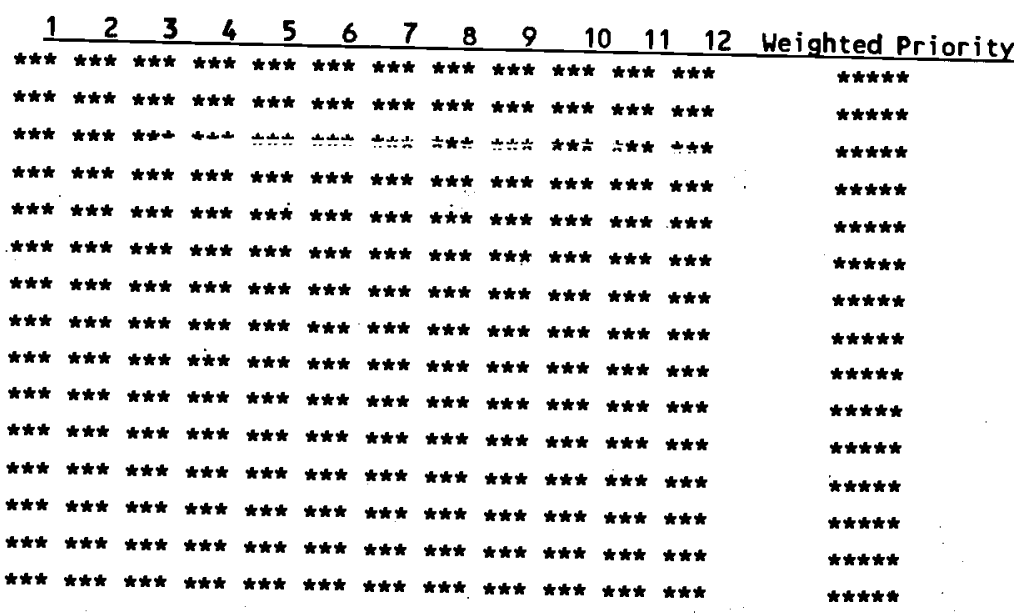

$\underline{1978}$

Rainfall

Crop Disease

Birds or Rats

Seed Availability

Fertilizer Availability

Crop Protection Chemicals

Credit

Irrigation Water Availability

Flooding

Labour Availabilty

Power for Land Preparation

Direct War Effects

Animal $D$ iseases

Extension

Other Problems

\begin{tabular}{|c|c|c|c|c|c|c|c|c|c|c|c|c|}
\hline 1 & 2 & 3 & 4 & 5 & 6 & 7 & 8 & 9 & 10 & 11 & 12 & Weighted \\
\hline$* *$ & $\star \star \star \star$ & $\star * *$ & $\star \star$ & $\star *$ & * & $\star \star$ & $\star \star *$ & $* * *$ & $\star \star \star *$ & $\star * *$ & $\star \star \star$ & $\star \star \star * * *$ \\
\hline$\star * \star$ & $\star \star \star$ & $\star \star * *$ & $\star \star \star *$ & $\star * *$ & $\star \star \star *$ & $\star \star \star *$ & $\star \star * *$ & $\star \star \star *$ & $\star \star * *$ & $\star \star \star \star$ & $* * *$ & 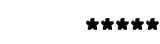 \\
\hline$\star *$ & $\star \star \star *$ & $\star \star * *$ & $\star \star \star$ & $* * *$ & $\star * *$ & $\star * *$ & $\star \star \star$ & $* * *$ & $\star \star \star \star$ & $\star \star \star$ & $\star \star \star \star$ & $\star * \star * * *$ \\
\hline$* \star \star$ & $\star * *$ & $* * *$ & 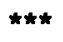 & $\star \star * *$ & $\star \star * t$ & $\star \star \star \star$ & $\star * \star *$ & $* * *$ & $\star \star * \star$ & $\star \star \star \star$ & $\star * *$ & $\star * * * *$ \\
\hline$\star * *$ & $\star \star \star *$ & $\star * *$ & $\star * *$ & $\star \star \star \star$ & $* * *$ & $* * *$ & $* * *$ & $\star * *$ & 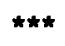 & $\star * *$ & $* * *$ & $\star \star \star \star \star \star *$ \\
\hline$* \star$ & $\star \star \star$ & $* * *$ & $* * *$ & $\star \star \star *$ & $\hbar \hbar \hbar$ & $\star \star \star \star$ & $\star * *$ & $\hbar \star \star *$ & $\star \star \star \star$ & $\star \star *$ & $\star \star * *$ & $\star * \star * *$ \\
\hline$\star \star * \star *$ & $* * *$ & $* * *$ & $\star * *$ & $\star * *$ & $\star \star *$ & $\star \star \star \star$ & $\star * *$ & $\star \star *$ & $\star \star \star *$ & $\star \star \star \star$ & $\star \star *$ & $\star * * * *$ \\
\hline$* * *$ & $\star * *$ & $\star * *$ & $\star \star *$ & $\star \star \star \star$ & $\star * *$ & $\star \star * *$ & $\star \star \star$ & $\star \star \star$ & $\star \star \star$ & $\star \star \star$ & $\star \star \star *$ & $\star \star \star * \star *$ \\
\hline$\star \star \star$ & $\star \star \star *$ & $\star \star \star \star$ & $* * *$ & $* * *$ & $* * *$ & $\star * *$ & $\star \star \star *$ & $* * *$ & $\star \star \star *$ & $\star \star \star \star$ & $\star \star \star \star$ & $\star \star \star * \star *$ \\
\hline & $\star * *$ & $\star \star$ & $\star \star \star *$ & $* * *$ & $* * *$ & $\star * *$ & $\star * *$ & $\star \star \star *$ & $\star \star \star \star$ & $* * *$ & $\star \star * \star$ & $\star \star \star \star * \star$ \\
\hline * & $\star \star \star *$ & $\star \star * t$ & $\star \star \star$ & $\star * *$ & $* * *$ & $\star \star \star$ & $* * *$ & $* * *$ & $\star * *$ & $\star \star \star \star$ & $\star * *$ & $\star \star \star \star \star \star *$ \\
\hline & $\star * *$ & $* * *$ & $\star \star \star *$ & $\star \star \star *$ & $\star * * *$ & $* * *$ & $\star \star \star *$ & $\star * *$ & $\star * \star$ & $\star \star \star \star$ & $\star \star * \star$ & $\star \star \star \star * \star *$ \\
\hline & $\star \star \star$ & $\star \star \star \star$ & $\star \star *$ & $\star \star \star *$ & $\star \star \star \star$ & $* * *$ & $\star * *$ & $\star \star \star \star$ & $\star * *$ & $\star * \star *$ & $\star * *$ & $* * * * *$ \\
\hline & $\star \star \star$ & $\star * *$ & $* * *$ & $* * *$ & $\star * *$ & $\star \star \star \star$ & $\star * *$ & $\star \star * *$ & $\star * *$ & $\star * *$ & $\star \star \star *$ & $\star \star \star \star \star * *$ \\
\hline & $\star t$ & $* *$ & $* \star \star *$ & ** & $* *$ & $* * *$ & $* *$ & $\star * \star *$ & ** & $r *$ & $\star * \star$ & $\hbar \star \hbar \star \hbar$ \\
\hline
\end{tabular}


KUNAR 


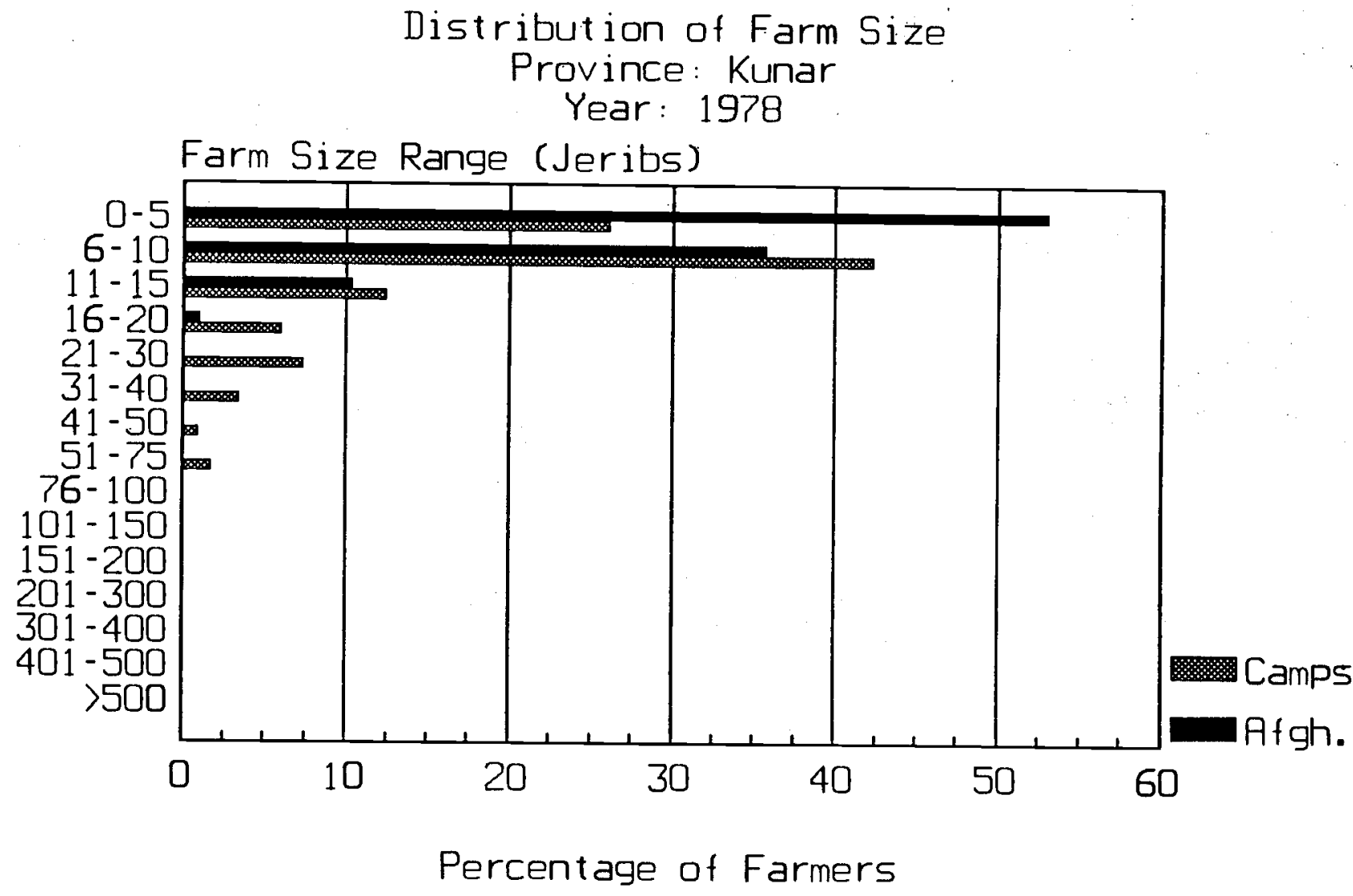

Farmers in Afghanistan 1978 1987

Total Number of Farms

Average Area

Average Area Irrigated

Average Dryland Area

$\begin{array}{ll}300 & 296 \\ 5.95 & 1.66 \\ 4.97 & 1.45 \\ 4.27 & 2.66\end{array}$

Farmers who left in 1987

Total Number of Farms

Average Area

Average Area Irrigated

Average Dryland Area

234

16

11.51

6.81

7.86

4.38

8.41

9.00

\section{Average of Both Groups}

Provincial Average Area

Provincial Average Irrigated Area

8.39

1.93

Provincial Average Dryland Area

6.31

1.61

6.30

2.94

(All areas in Jeribs. 5 Jeribs $=1$ Hectare) 
Farm Power - Farmers in Atghanistan Province of Kunar Crop: Maize

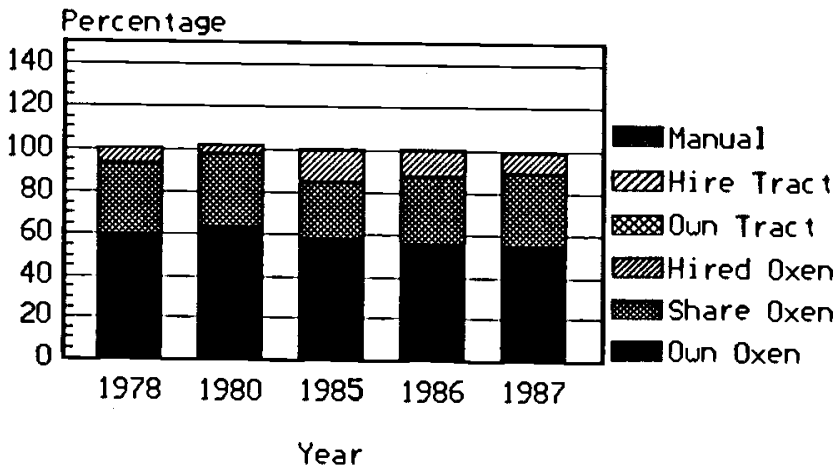

Farm Power - Farmers who lett in 1987 Province of Kunar Crop : Maize

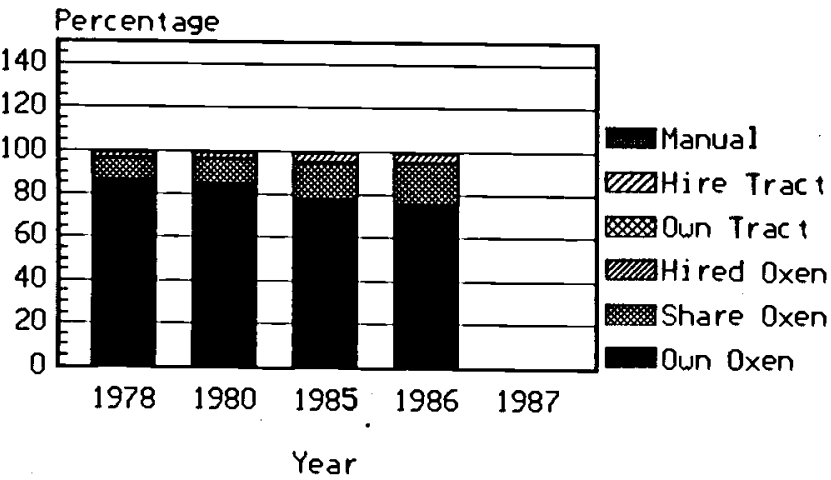

Base Figures for above graphs

Province: Kunar

Farmers in Afghanistan

Irr. Wheat own oxen

Shared oxen

Hired oxen

Own Tractor

Hired Tractor

Maize Own oxen

Shared oxen

Hired oxen

Own Tractor

Hired Tractor

Manual Means

Farmers who left in 1987

Irr. Wheat own oxen

Shared oxen

Hired oxen

Own Tractor

Hired Tractor

Maize

\section{8}

60.93

34.42

3.72

$-$

-

$-$

59.18

33.71

7.12

-

$-$
Farm Power - Farmers in Atghanistan

Province of Kunar

Crop: Irrigated wheat

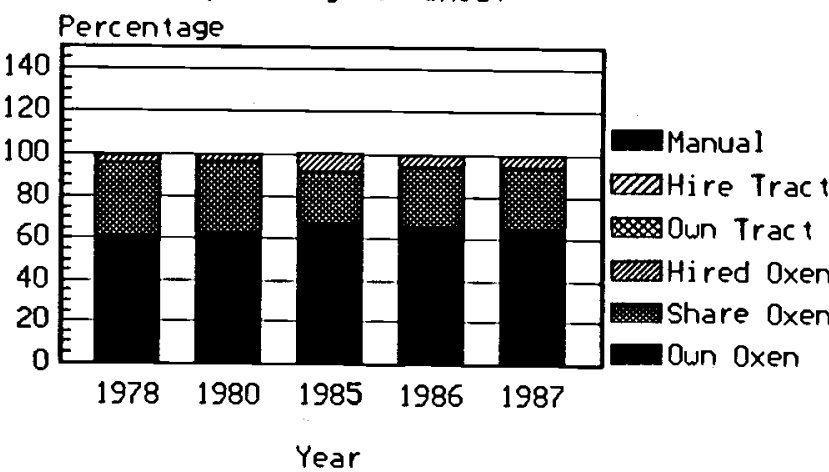

Farm Power - Farmers who lett in 1987 Province of Kunar

Crop: Irrigated wheat

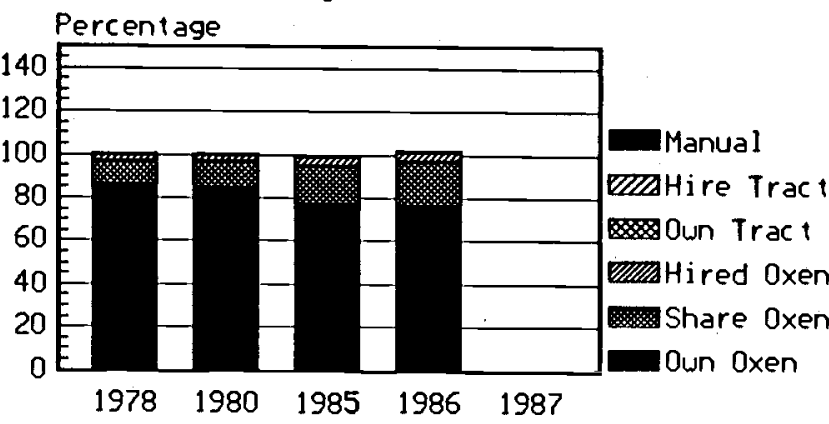

Year
Percentage of Farmers $1980 \quad \underline{1985} \quad \underline{1986}$

62.81
33.17
3.52
-
-

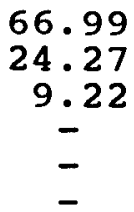

63.45

34.01

4. 06

58.23

26.10

15.66

$-$

.40

65.73

27.70

6.10

$-$

-

56.02

31.58

11.65

$-$

$-38$

.38
.38

1987

64.62

29.25

5. 66

$-$

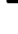

55.09

33.58

10.57

$-$

$$
\begin{array}{rcccc}
86.21 & 84.35 & 76.58 & 76.10 & 75.00 \\
10.34 & 12.17 & 17.57 & 20.13 & 18.75 \\
3.02 & 3.04 & 4.95 & 5.03 & - \\
- & - & - & - & - \\
- & - & - & - & - \\
.43 & .43 & .45 & .63 & - \\
86.10 & 84.16 & 77.78 & 75.66 & - \\
9.42 & 11.31 & 16.43 & 18.42 & - \\
3.59 & 3.62 & 4.35 & 4.61 & -
\end{array}
$$

Shared oxen

Hired Oxen

Own Tractor

Hired Tractor 

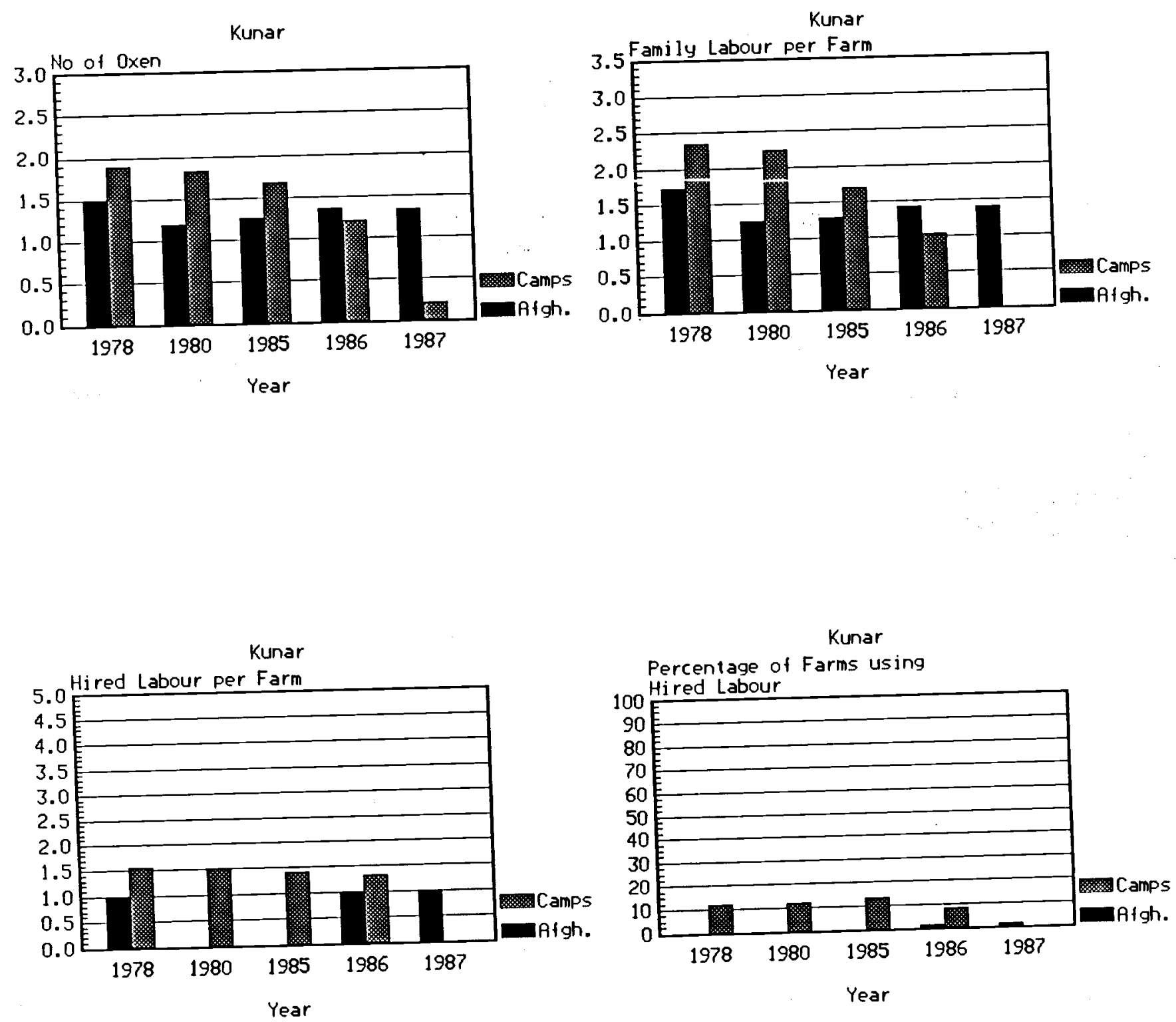
PROVINCE OF KNR

YEAR

1978 Number of Families

X of Families

1980 Number of Families

* of Fanilies

1985 Number of Families

\% of Families

1986 Number of Families

\% of Fanilies

1987 Number of Families

\% of Families

Average Number of Oxen per Family:

1980

1985

1986

1987
NUMBER OF TRAINED OXEN PER FAMILY

$\begin{array}{llllll}32 & 96 & 171 & 0 & 3 & 0\end{array}$

$\begin{array}{llllll}10.60 & 31.79 & 56.62 & 0.00 & 0.99 & 0.00\end{array}$

$\begin{array}{llllll}86 & 73 & 142 & 0 & 0 & 0\end{array}$

$\begin{array}{llllll}28.57 & 24.25 & 47.18 & 0.00 & 0.00 & 0.00\end{array}$

$\begin{array}{llllll}78 & 74 & 149 & 0 & 0 & 1\end{array}$

$\begin{array}{llllll}25.83 & 24.50 & 49.34 & 0.00 & 0.00 & 0.33\end{array}$

$\begin{array}{llllll}48 & 101 & 153 & 0 & 0 & 0\end{array}$

$\begin{array}{llllll}15.89 & 33.44 & 50.66 & 0.00 & 0.00 & 0.00\end{array}$

$\begin{array}{llllll}48 & 105 & 149 & 0 & 0 & 0\end{array}$

$\begin{array}{llllll}15.89 & 34.77 & 49.34 & 0.00 & 0.00 & 0.00\end{array}$

$\begin{array}{ll}1978 & 1.49 \\ 1980 & 1.19 \\ 1985 & 1.25 \\ 1986 & 1.35 \\ 1987 & 1.33\end{array}$

Number of Farmers Quest ioned 302

\section{TABLE 7B - AVERAGE NUMBER OF TRAINED OXEN FOR FARMERS IN THE CAMPS}

PROVINCE OF KNR

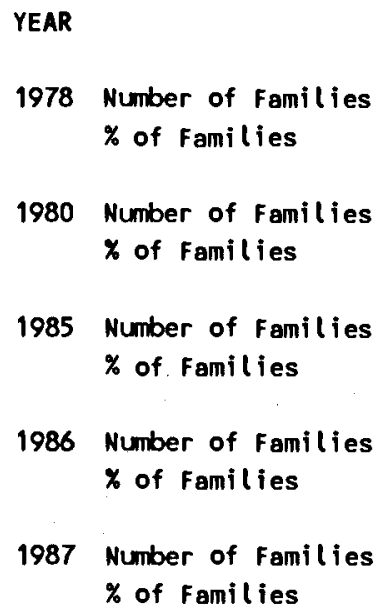

$\begin{array}{ll}1978 & 1.89 \\ 1980 & 1.83 \\ 1985 & 1.67 \\ 1986 & 1.20 \\ 1987 & 0.20\end{array}$


PROVINCE OF KNR

Average Family Labour per Farm

Average No. of Hired Labour per Farm

(for farms using hired (abour)

Number of Farms using Hired Labour

Number of Farmers Questioned 302

$\begin{array}{lllll}1978 & 1980 & 1985 & 1986 & 1987 \\ 1.73 & 1.25 & 1.28 & 1.41 & 1.40 \\ 1.00 & \star \star \star \star \star & \star \star \star \star \star & 1.00 & 1.00\end{array}$

$\begin{array}{llll}1 & 0 & 0 & 2\end{array}$

\section{IABLE $8(B)$ - AVERAGE LABOUR (FAMILY and HIRED) FOR FARMERS IN CAMPS}

PROVINCE OF KNR

Average Family Labour per Farm

Average No. of Hired Labour per Farm

(for farms using hired (abour)

Number of Farms using Hired Labour

Number of Farmers Questioned 244

$\begin{array}{lllll}1978 & 1980 & 1985 & 1986 & 1987 \\ 2.34 & 2.23 & 1.70 & 1.02 & 0.14 \\ 1.55 & 1.50 & 1.39 & 1.32 & \star \star \star \star \star\end{array}$

$\begin{array}{lllll}29 & 30 & 31 & 19 & 0\end{array}$ 
PROVINCE OF KNR

\begin{tabular}{|c|c|c|c|c|c|}
\hline & 1978 & 1980 & 1985 & 1986 & 1987 \\
\hline Average Number of Livestock Shot & 30.00 & 3.57 & 7.33 & 3.30 & 2.50 \\
\hline $\begin{array}{l}\text { Number of Farmers reporting } \\
\text { Livestock Shot }\end{array}$ & 2 & 35 & 63 & 20 & 12 \\
\hline $\begin{array}{l}\text { Average Number of Livestock Killed } \\
\text { by Mines }\end{array}$ & 3.33 & 4.94 & 3.88 & 6.13 & 4.00 \\
\hline $\begin{array}{l}\text { Number of Farmers reporting Livestock } \\
\text { killed by Mines }\end{array}$ & 3 & 17 & 16 & 8 & 1 \\
\hline
\end{tabular}

IABLE 9B - DIRECT EFFECTS OF THE WAR - FARMERS IN CAMPS

PROVINCE OF KNR

Average Number of Livestock Shot

\begin{tabular}{|c|c|c|c|}
\hline 978 & 1980 & 1985 & 1986 \\
\hline & & 4 & 2.14 \\
\hline
\end{tabular}

Number of Farmers reporting

Livestock Shot

$\begin{array}{lllll}0 & 23 & 79 & 21 & 1\end{array}$

Average Number of Livestock Killed by Mines

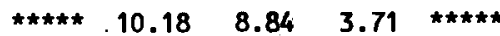

Number of Farmers reporting Livestock

killed by Mines

$\begin{array}{lllll}0 & 11 & 19 & 7 & 0\end{array}$

Number of Farmers Questioned 244 
PROVINCE OF KNR

$\begin{array}{lrrrrr} & 1978 & 1980 & 1985 & 1986 & 1987 \\ & 30.00 & 3.57 & 7.33 & 3.30 & 2.50 \\ \begin{array}{l}\text { Average Number of Livestock Shot } \\ \begin{array}{l}\text { Number of Farmers reporting } \\ \text { Livestock Shot }\end{array}\end{array} & 2 & 35 & 63 & 20 & 12 \\ \begin{array}{l}\text { Average Number of Livestock Killed } \\ \text { by Mines }\end{array} & 3.33 & 4.94 & 3.88 & 6.13 & 4.00 \\ \begin{array}{l}\text { Number of Farmers reporting Livestock } \\ \text { killed by Mines }\end{array} & 3 & 17 & 16 & 8 & 1 \\ \text { Number of Farmers Questioned 302 } & & & & & \\ \end{array}$

\section{TABLE 9B - DIRECT EFFECTS OF THE WAR - FARMERS IN CAMPS}

PROVINCE OF KNR

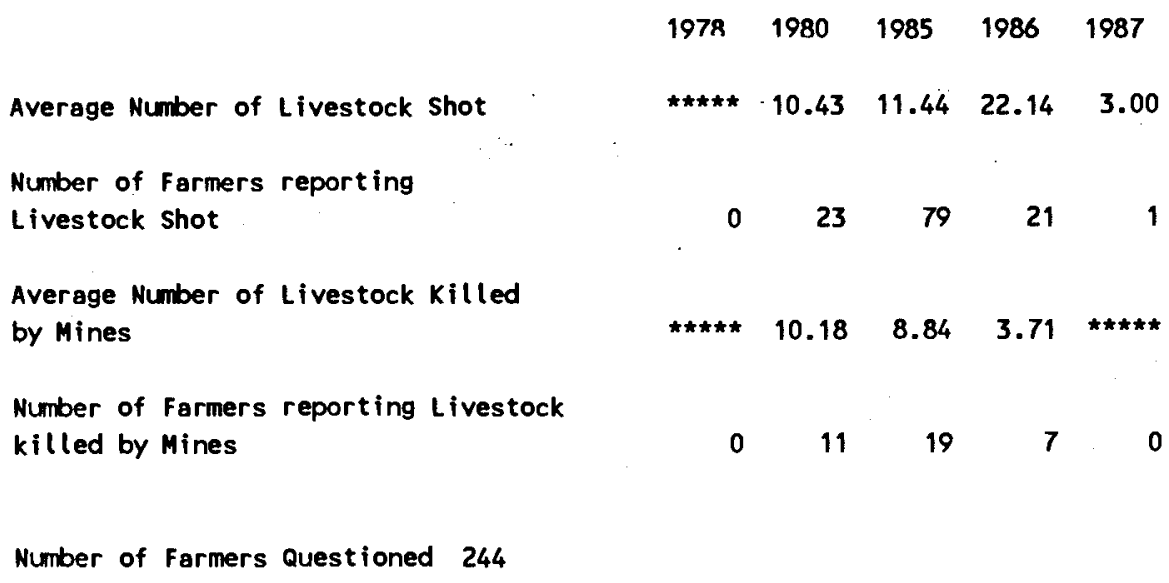




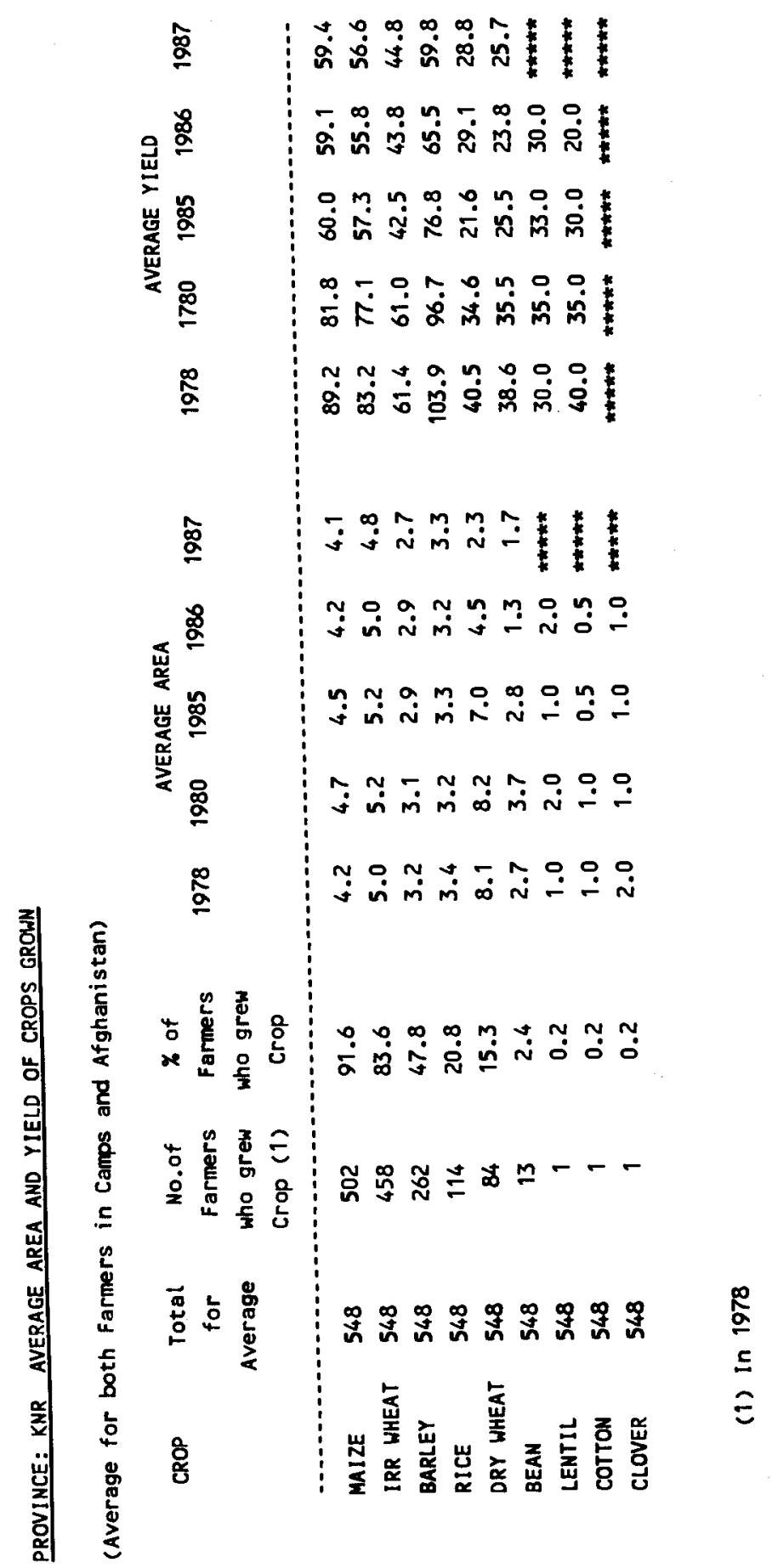


PROVINCE OF KNR

Rainfall

Crop Disease

Fertilizer Availability

Crop Protection Chemicals

Credit

Irrigation Water Availability

Flooding

Labour Availabilty

Power for Land Preparation

Direct War Effects

Animal Diseases

Extension

other Problems
Birds or Rats

Seed Availability

\begin{tabular}{rrrrrrrrrrrrr}
1 & 2 & 3 & 4 & 5 & 6 & 7 & 8 & 9 & 10 & 11 & 12 & Heighted Priority \\
\hline 0 & 0 & 8 & 6 & 2 & 0 & 2 & 0 & 0 & 0 & 0 & 0 & 2.05 \\
3 & 19 & 16 & 13 & 6 & 2 & 3 & 2 & 0 & 0 & 0 & 0 & 10.02 \\
0 & 3 & 27 & 16 & 13 & 6 & 0 & 0 & 0 & 0 & 0 & 0 & 7.79 \\
0 & 5 & 6 & 8 & 13 & 11 & 5 & 5 & 2 & 0 & 0 & 0 & 5.29 \\
16 & 15 & 2 & 2 & 0 & 0 & 0 & 0 & 0 & 0 & 0 & 0 & 10.27 \\
0 & 0 & 2 & 0 & 6 & 15 & 10 & 0 & 0 & 0 & 0 & 0 & 2.38 \\
0 & 5 & 0 & 0 & 3 & 2 & 2 & 0 & 0 & 0 & 0 & 0 & 1.51 \\
11 & 15 & 10 & 3 & 3 & 0 & 0 & 0 & 0 & 0 & 0 & 0 & 9.81 \\
0 & 2 & 3 & 3 & 3 & 0 & 0 & 0 & 0 & 0 & 0 & 0 & 1.41 \\
0 & 0 & 5 & 8 & 8 & 8 & 0 & 0 & 0 & 0 & 0 & 0 & 2.78 \\
2 & 5 & 0 & 5 & 2 & 2 & 0 & 0 & 0 & 0 & 0 & 0 & 2.46 \\
58 & 3 & 6 & 11 & 13 & 3 & 0 & 0 & 0 & 0 & 0 & 0 & 28.62 \\
0 & 3 & 3 & 5 & 3 & 6 & 6 & 0 & 2 & 0 & 0 & 0 & 2.84 \\
0 & 2 & 3 & 8 & 0 & 2 & 0 & 0 & 0 & 0 & 0 & 0 & 1.76 \\
8 & 23 & 8 & 6 & 10 & 2 & 2 & 0 & 0 & 0 & 0 & 0 & 11.02
\end{tabular}

1986

Rainfall

Crop Disease

Birds or Rats

Seed Availability

Fertilizer Availability

Crop Protection Chemicals

Credit

Irrigation Water Availability

Flooding

Labour Avai labilty

Power for Land Preparation

Direct War Effects

Animal Diseases

Extension

other Problems

1985

Rainfall

Crop Disease

Birds or Rats

Seed Avai lability

Fertilizer Availability

Crop Protection Chemicals

Credit

Irrigation Hater Availability

Flooding

Labour Avai labilty

Power for Land Preparation

Direct War Effects

Animal Diseases

Extension

other Problems

\begin{tabular}{rrrrrrrrrrrrr}
1 & 2 & 3 & 4 & 5 & 6 & 7 & 8 & 9 & 10 & 11 & 12 & Weighted Priority \\
\hline 0 & 2 & 5 & 3 & 3 & 0 & 5 & 2 & 0 & 0 & 0 & 0 & 2.00 \\
0 & 23 & 18 & 11 & 10 & 5 & 0 & 0 & 0 & 0 & 0 & 0 & 9.55 \\
0 & 5 & 16 & 19 & 13 & 3 & 0 & 0 & 0 & 0 & 0 & 0 & 6.61 \\
0 & 0 & 0 & 15 & 11 & 10 & 8 & 6 & 2 & 0 & 0 & 0 & 4.04 \\
13 & 35 & 0 & 0 & 2 & 0 & 2 & 0 & 0 & 0 & 0 & 0 & 13.08 \\
0 & 0 & 0 & 5 & 3 & 13 & 5 & 3 & 0 & 0 & 0 & 0 & 2.14 \\
0 & 5 & 2 & 0 & 2 & 5 & 0 & 0 & 0 & 0 & 0 & 0 & 1.71 \\
13 & 10 & 15 & 10 & 0 & 0 & 0 & 2 & 0 & 0 & 0 & 0 & 10.57 \\
0 & 3 & 3 & 3 & 3 & 5 & 0 & 0 & 0 & 0 & 0 & 0 & 2.07 \\
0 & 2 & 5 & 8 & 6 & 6 & 8 & 0 & 0 & 0 & 0 & 0 & 3.34 \\
0 & 2 & 8 & 2 & 3 & 2 & 2 & 0 & 0 & 0 & 0 & 0 & 2.11 \\
66 & 8 & 8 & 10 & 3 & 2 & 0 & 0 & 0 & 0 & 0 & 0 & 31.95 \\
0 & 0 & 2 & 0 & 6 & 3 & 8 & 2 & 0 & 0 & 0 & 0 & 1.56 \\
0 & 2 & 2 & 2 & 3 & 11 & 0 & 0 & 0 & 0 & 0 & 0 & 1.79 \\
6 & 3 & 16 & 5 & 11 & 2 & 3 & 2 & 0 & 0 & 0 & 0 & 7.48
\end{tabular}

$\begin{array}{rrrrrrrrrrrrr}1 & 2 & 3 & 4 & 5 & 6 & 7 & 8 & 9 & 10 & 11 & 12 & \text { Heighted Priority } \\ 19 & 15 & 6 & 15 & 0 & 0 & 0 & 0 & 5 & 0 & 0 & 0 & 14.15 \\ 0 & 6 & 26 & 16 & 6 & 2 & 2 & 0 & 2 & 0 & 0 & 0 & 7.66 \\ 0 & 0 & 3 & 15 & 11 & 3 & 0 & 2 & 0 & 0 & 0 & 0 & 3.31 \\ 0 & 0 & 0 & 0 & 6 & 11 & 11 & 0 & 0 & 3 & 0 & 0 & 2.19 \\ 5 & 44 & 3 & 6 & 5 & 0 & 0 & 0 & 0 & 0 & 0 & 0 & 13.01 \\ 0 & 0 & 0 & 2 & 3 & 6 & 0 & 2 & 0 & 0 & 0 & 0 & 1.00 \\ 0 & 0 & 6 & 0 & 2 & 2 & 2 & 2 & 0 & 0 & 2 & 0 & 1.43 \\ 6 & 23 & 21 & 3 & 0 & 2 & 0 & 0 & 0 & 0 & 0 & 0 & 11.09 \\ 0 & 0 & 2 & 0 & 0 & 5 & 0 & 0 & 2 & 2 & 0 & 0 & 0.72 \\ 0 & 0 & 2 & 3 & 3 & 8 & 5 & 5 & 5 & 0 & 0 & 0 & 2.22 \\ 6 & 2 & 3 & 5 & 5 & 2 & 0 & 0 & 2 & 0 & 0 & 0 & 4.71 \\ 60 & 8 & 2 & 13 & 5 & 5 & 0 & 3 & 2 & 0 & 0 & 0 & 30.00 \\ 0 & 2 & 3 & 6 & 2 & 2 & 0 & 0 & 2 & 0 & 0 & 0 & 1.83 \\ 0 & 0 & 0 & 0 & 8 & 0 & 11 & 8 & 2 & 3 & 0 & 0 & 2.03 \\ 2 & 2 & 16 & 2 & 5 & 3 & 5 & 3 & 0 & 0 & 0 & 0 & 4.64\end{array}$


PROVINCE OF KNR

Rainfall

Crop Disease

Birds or Rats

Seed Availability

Fertilizer Availability

Crop Protection Chemicals

Credit

Irrigation Water Avai lability

Flooding

Labour Availabilty

Power for Land Preparation

Direct War Effects

Animal Diseases

Extension

Other Problems

\begin{tabular}{ccccccccccccc}
1 & 2 & 3 & 4 & 5 & 6 & 7 & 8 & 9 & 10 & 11 & 12 & Heighted Priority \\
\hline 0 & 3 & 0 & 0 & 0 & 0 & 0 & 0 & 0 & 0 & 0 & 0 & 1.61 \\
0 & 0 & 0 & 0 & 0 & 0 & 0 & 0 & 0 & 0 & 0 & 0 & 0.00 \\
0 & 0 & 0 & 0 & 0 & 0 & 0 & 0 & 0 & 0 & 0 & 0 & 0.00 \\
0 & 0 & 0 & 3 & 5 & 0 & 0 & 0 & 0 & 0 & 0 & 0 & 1.77 \\
3 & 3 & 2 & 3 & 0 & 0 & 0 & 0 & 0 & 0 & 0 & 0 & 6.17 \\
0 & 0 & 0 & 0 & 0 & 0 & 0 & 0 & 0 & 0 & 0 & 0 & 0.00 \\
0 & 3 & 3 & 0 & 0 & 3 & 0 & 0 & 0 & 0 & 0 & 0 & 3.22 \\
5 & 6 & 8 & 2 & 0 & 0 & 0 & 0 & 0 & 0 & 0 & 0 & 11.13 \\
0 & 2 & 2 & 0 & 0 & 0 & 0 & 0 & 0 & 0 & 0 & 0 & 1.34 \\
0 & 2 & 0 & 2 & 0 & 2 & 0 & 0 & 0 & 0 & 0 & 0 & 1.48 \\
0 & 2 & 0 & 0 & 0 & 0 & 0 & 0 & 0 & 0 & 0 & 0 & 0.80 \\
65 & 6 & 0 & 0 & 0 & 0 & 0 & 0 & 0 & 0 & 0 & 0 & 67.59 \\
0 & 0 & 0 & 0 & 2 & 0 & 0 & 0 & 0 & 0 & 0 & 0 & 0.32 \\
0 & 0 & 2 & 2 & 2 & 0 & 2 & 0 & 0 & 0 & 0 & 0 & 1.49 \\
0 & 3 & 3 & 2 & 0 & 0 & 0 & 0 & 0 & 0 & 0 & 0 & 3.08
\end{tabular}

\section{8}

Rainfall

Crop Disease

Birds or Rats

Seed Avail ability

Fertilizer Availability

Crop Protection Chemicals

Credit

Irrigation Water Availability

Flooding

Labour Availabilty

Power for Land Preparation

Direct War Effects

Animal Diseases

Extension

Other Problems

\begin{tabular}{rrllllllllllc}
1 & 2 & 3 & 4 & 5 & 6 & 7 & 8 & 9 & 10 & 11 & 12 & Weighted Priority \\
\hline 2 & 0 & 0 & 0 & 0 & 0 & 0 & 0 & 0 & 0 & 0 & 0 & 5.94 \\
0 & 0 & 0 & 0 & 0 & 0 & 0 & 0 & 0 & 0 & 0 & 0 & 0.00 \\
0 & 0 & 0 & 0 & 0 & 0 & 0 & 0 & 0 & 0 & 0 & 0 & 0.00 \\
0 & 0 & 5 & 2 & 0 & 0 & 0 & 0 & 0 & 0 & 0 & 0 & 7.43 \\
0 & 0 & 0 & 0 & 0 & 0 & 0 & 0 & 0 & 0 & 0 & 0 & 0.00 \\
0 & 0 & 0 & 0 & 0 & 0 & 0 & 0 & 0 & 0 & 0 & 0 & 0.00 \\
0 & 2 & 0 & 2 & 0 & 0 & 0 & 0 & 0 & 0 & 0 & 0 & 4.46 \\
15 & 3 & 0 & 0 & 0 & 0 & 0 & 0 & 0 & 0 & 0 & 0 & 59.41 \\
0 & 0 & 0 & 0 & 0 & 0 & 0 & 0 & 0 & 0 & 0 & 0 & 0.00 \\
0 & 2 & 0 & 0 & 0 & 0 & 0 & 0 & 0 & 0 & 0 & 0 & 2.97 \\
0 & 0 & 0 & 0 & 0 & 0 & 0 & 0 & 0 & 0 & 0 & 0 & 0.00 \\
2 & 3 & 2 & 0 & 0 & 0 & 0 & 0 & 0 & 0 & 0 & 0 & 13.86 \\
0 & 0 & 0 & 0 & 0 & 0 & 0 & 0 & 0 & 0 & 0 & 0 & 0.00 \\
0 & 0 & 0 & 0 & 0 & 0 & 0 & 0 & 0 & 0 & 0 & 0 & 0.00 \\
2 & 0 & 0 & 0 & 0 & 0 & 0 & 0 & 0 & 0 & 0 & 0 & 5.94
\end{tabular}


KUNDUZ 


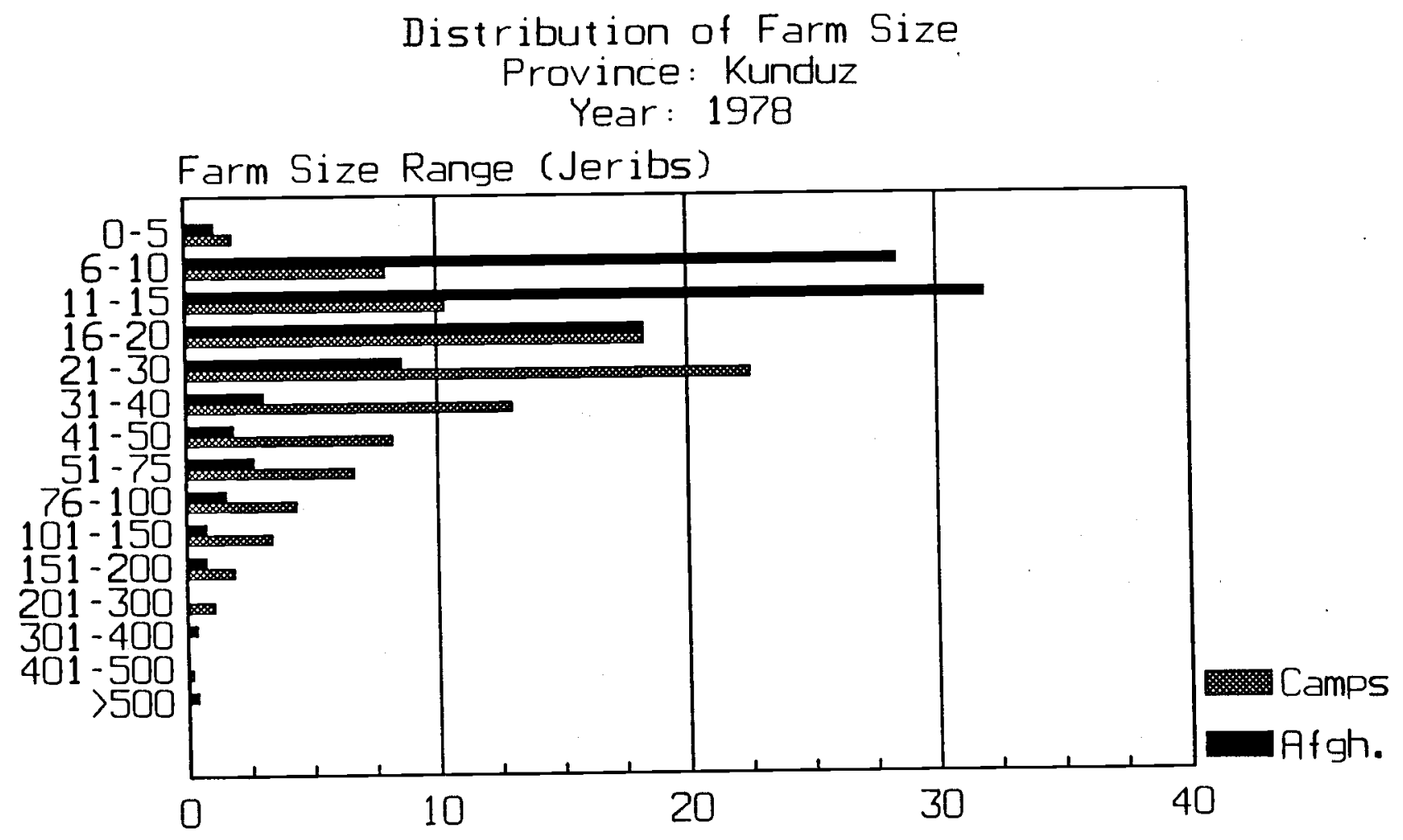

Percentage of Farmers

Farmers in Afghanistan

1978

1987

Total Number of Farms

257

238

Average Area

24.07

8.09

Average Area Irrigated

22.69

7.07

Average Dryland Area

21.05

10.95

Farmers who left in 1987

Total Number of Farms

Average Area

Average Area Irrigated

Average Dryland Area

524

1

40.04

28.83

30.00

81.21

30.00

\section{Average of Both Groups}

Provincial Average Area

Provincial Average Irrigated Area

Provincial Average Dryland Area

34.78

8.18

26.71

7.17

70.42

10.95

(All areas in Jeribs. 5 Jeribs $=1$ Hectare) 
Farm Power - Farmers in Alghanistan Province of Kunduz

Crop: Irrigated wheat

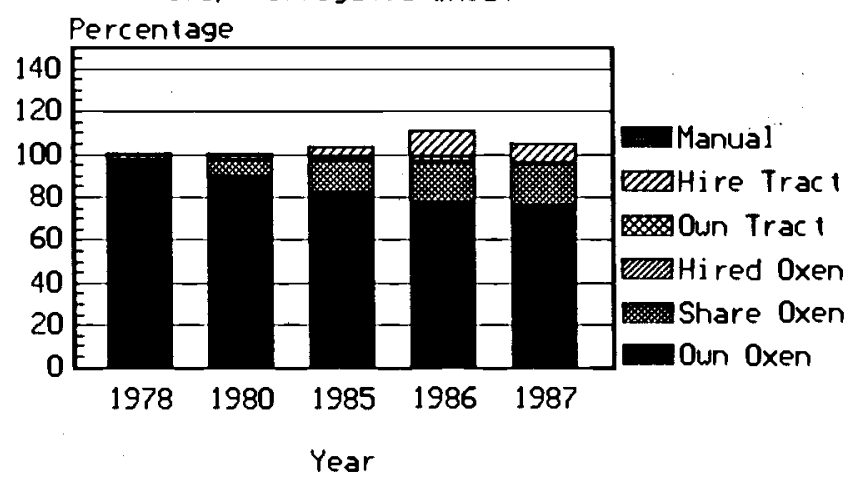

Farm Power - Farmers who left in 1987 Province of Kunduz Crop: Irrigated wheat

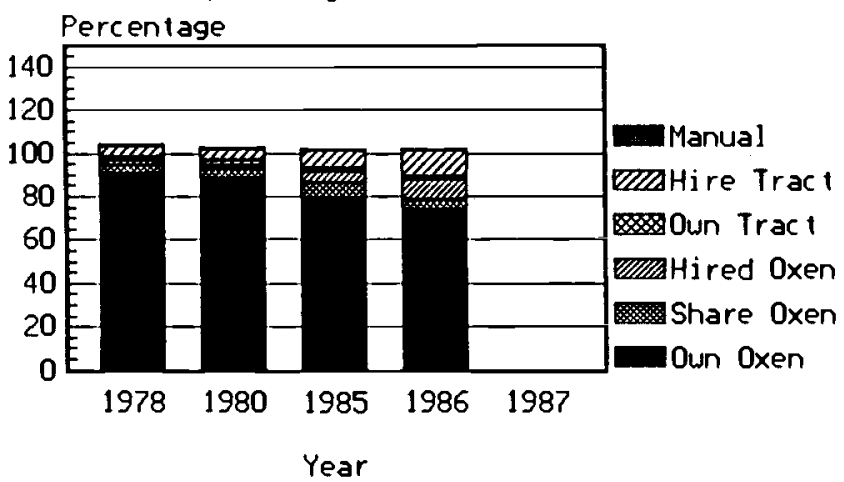

Base Figures for above graphs

Province: Kunduz

Farmers in Afghanistan

Irr. Wheat own oxen

Shared oxen

Hired oxen

Own Tractor

Hired Tractor

Rice

Manual Means

Own Oxen

Shared Oxen

Hired oxen

Own Tractor

Hired Tractor

Manual Means

Farmers who left in 1987

Irr. Wheat own oxen

Shared oxen

Hired oxen

Own Tractor

Hired Tractor

Rice

Manual Means

Own Oxen

Shared oxen

Hired oxen
Farm Power - Farmers in Atghanistan Province of Kunduz

Crop: Rice

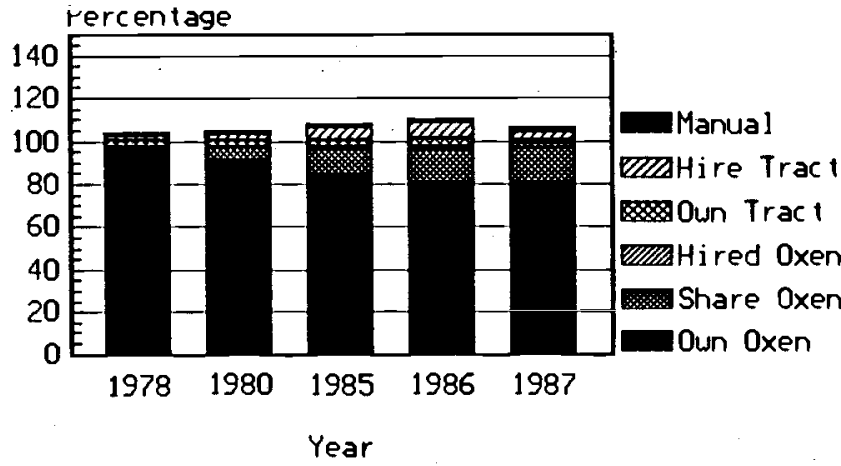

Farm Power - Farmers who lett in 1987 Province of Kunduz

Crop: Rice

Percentage

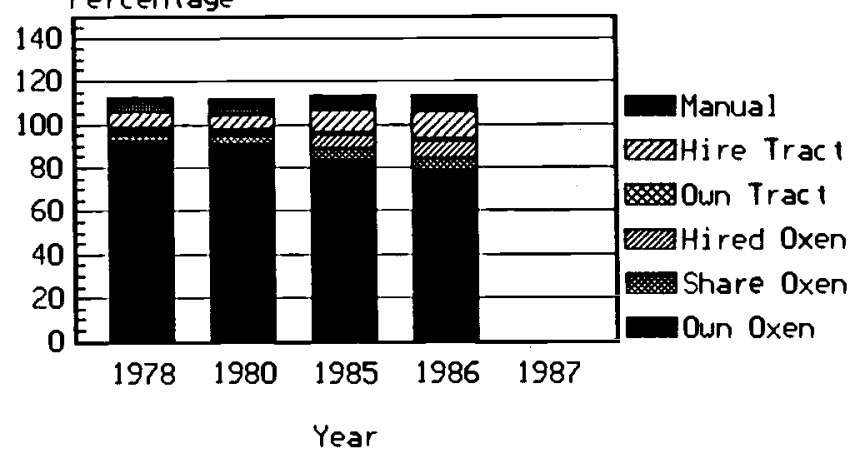

Percentage of Farmers

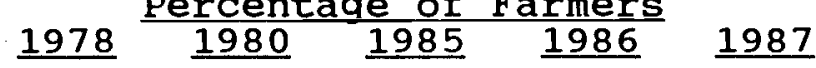

$\begin{array}{rrrrc}96.00 & 89.56 & 82.00 & 77.51 & 76.19 \\ 1.60 & 8.03 & 14.40 & 18.47 & 18.61 \\ - & - & .80 & .80 & .87 \\ 2.00 & 2.01 & 2.00 & 2.01 & - \\ .80 & .80 & 4.40 & 12.45 & 9.52 \\ - & - & - & - & - \\ 96.13 & 91.16 & 84.44 & 80.56 & 80.63 \\ 1.10 & 6.08 & 11.67 & 15.00 & 16.25 \\ - & - & 1.11 & 1.67 & 1.88 \\ 3.31 & 3.31 & 3.33 & 3.89 & 1.25 \\ 2.21 & 2.76 & 5.56 & 7.22 & 4.38 \\ 1.66 & 1.66 & 1.67 & 1.67 & 1.88\end{array}$

$\begin{array}{rrrrc}90.79 & 88.58 & 79.32 & 73.67 & 100.00 \\ 3.35 & 4.31 & 6.33 & 4.59 & - \\ 2.09 & 1.72 & 5.70 & 8.94 & 100.00 \\ 2.09 & 2.16 & 1.48 & 1.45 & - \\ 5.23 & 5.17 & 8.65 & 12.56 & - \\ .42 & .43 & .42 & .24 & - \\ 92.06 & 90.69 & 82.86 & 78.65 & - \\ 2.80 & 3.43 & 5.24 & 4.69 & - \\ 1.87 & 1.96 & 6.67 & 8.85 & -\end{array}$


PROVINCE OF KDZ

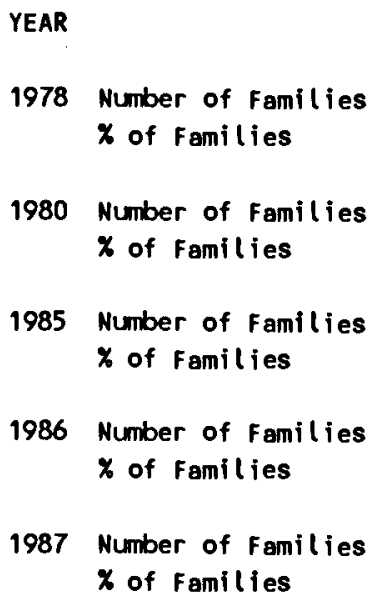

Number of Farmers Questioned 257
NUMBER OF TRAINED OXEN PER FAMILY

$\begin{array}{llllll}0 & 1 & 2 & 3 & 4 & 5\end{array}$

$\begin{array}{llllll}6 & 5 & 215 & 19 & 6 & 3\end{array}$

$\begin{array}{llllll}2.36 & 1.97 & 84.65 & 7.48 & 2.36 & 1.18\end{array}$

$\begin{array}{llllll}20 & 10 & 199 & 14 & 9 & 2\end{array}$

$\begin{array}{llllll}7.87 & 3.94 & 78.35 & 5.51 & 3.54 & 0.79\end{array}$

$\begin{array}{llllll}35 & 14 & 191 & 7 & 6 & 1\end{array}$

$\begin{array}{llllll}13.78 & 5.51 & 75.20 & 2.76 & 2.36 & 0.39\end{array}$

$\begin{array}{llllll}47 & 16 & 178 & 7 & 6 & 0\end{array}$

$\begin{array}{llllll}18.50 & 6.30 & 70.08 & 2.76 & 2.36 & 0.00\end{array}$

$\begin{array}{llllll}66 & 17 & 167 & 4 & 3 & 0\end{array}$

$\begin{array}{llllll}25.68 & 6.61 & 64.98 & 1.56 & 1.17 & 0.00\end{array}$

$\begin{array}{ll}1978 & 2.09 \\ 1980 & 1.95 \\ 1985 & 1.76 \\ 1986 & 1.64 \\ 1987 & 1.46\end{array}$
09
.95
1.64

1.46

\begin{tabular}{|c|c|c|c|c|c|}
\hline \multicolumn{2}{|c|}{ NUMBER OF } & \multicolumn{2}{|c|}{ TRAINED OXEN } & \multicolumn{2}{|c|}{ ER FAMILY } \\
\hline 0 & 1 & 2 & 3 & 4 & 5 \\
\hline 25 & 25 & 326 & 49 & 71 & 4 \\
\hline 5.00 & 5.00 & 65.20 & 9.80 & 14.20 & 0.80 \\
\hline 48 & 29 & 329 & 39 & 55 & 2 \\
\hline 9.56 & 5.78 & 65.54 & 7.77 & 10.96 & 0.40 \\
\hline 80 & 36 & 323 & 24 & 41 & 2 \\
\hline 15.81 & 7.11 & 63.83 & 4.74 & 8.10 & 0.40 \\
\hline 159 & 30 & 276 & 15 & 26 & 1 \\
\hline 31.36 & 5.92 & 54.44 & 2.96 & 5.13 & 0.20 \\
\hline 508 & 0 & 0 & 1 & 0 & 0 \\
\hline 9.80 & 0.00 & 0.00 & 0.20 & 0.00 & 0.00 \\
\hline
\end{tabular}

$\begin{array}{ll}1978 & 2.26 \\ 1980 & 2.06 \\ 1985 & 1.83 \\ 1986 & 1.45 \\ 1987 & 0.01\end{array}$

Number of Farmers Questioned 509 
PROVINCE OF KDZ

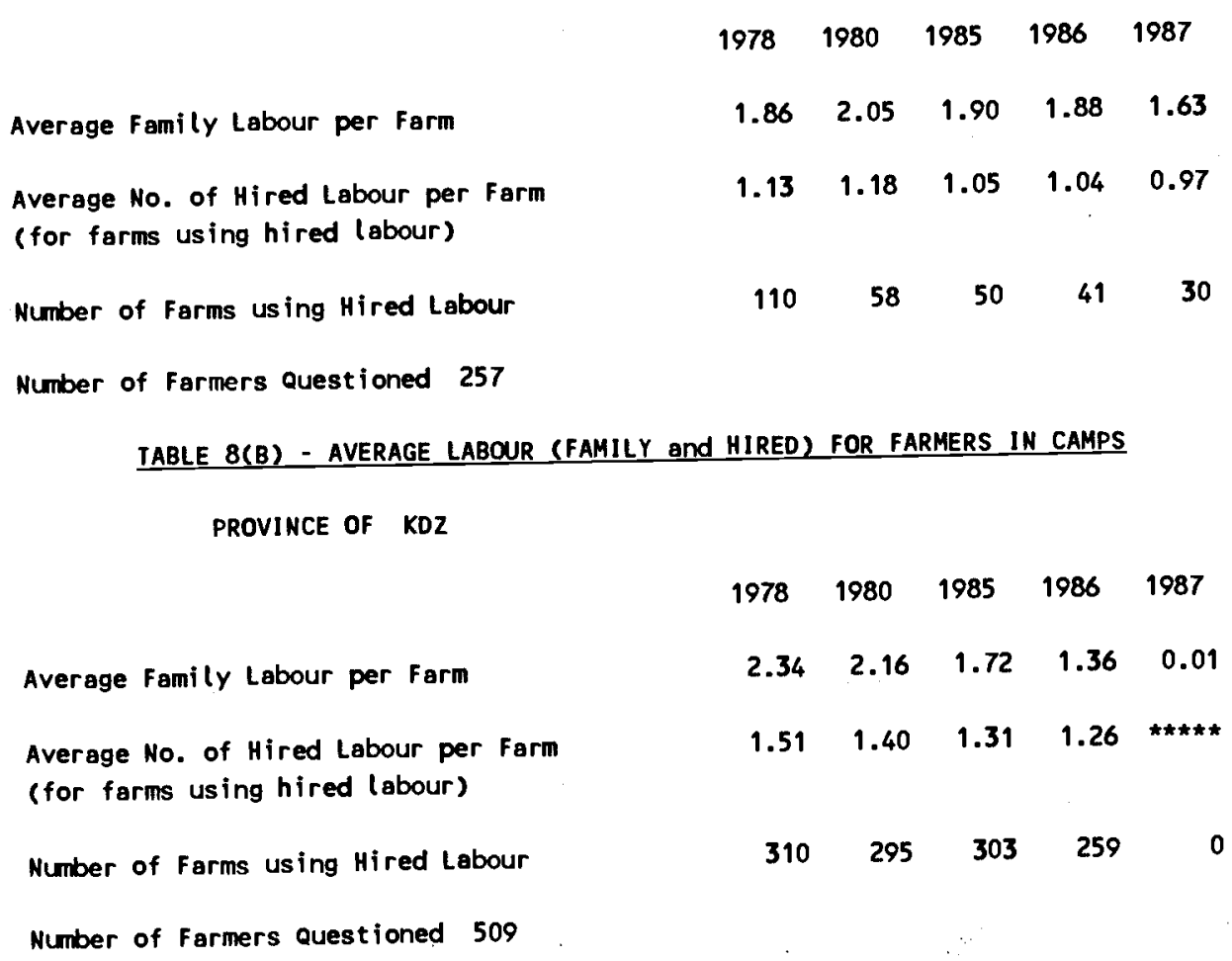




\section{IABLE 9A - DIRECT EFFECTS OF THE WAR - FARMERS IN AFGANISTAN}

PROVINCE OF KOZ

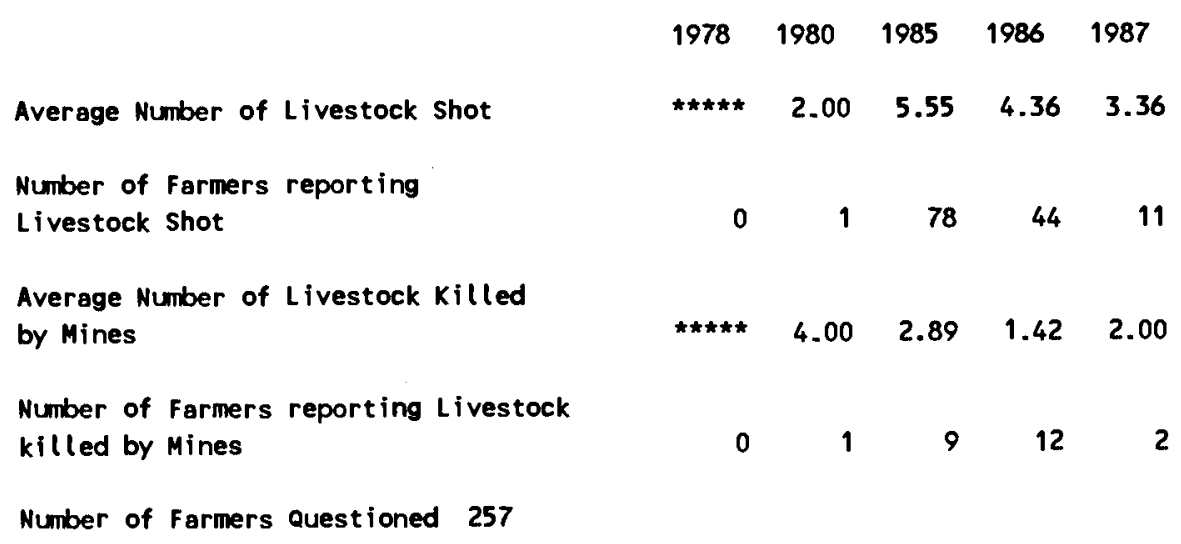

\section{IABLE 9B - DIRECT EFFECTS OF THE HAR - FARMERS IN CAMPS}

PROVINCE OF KOZ

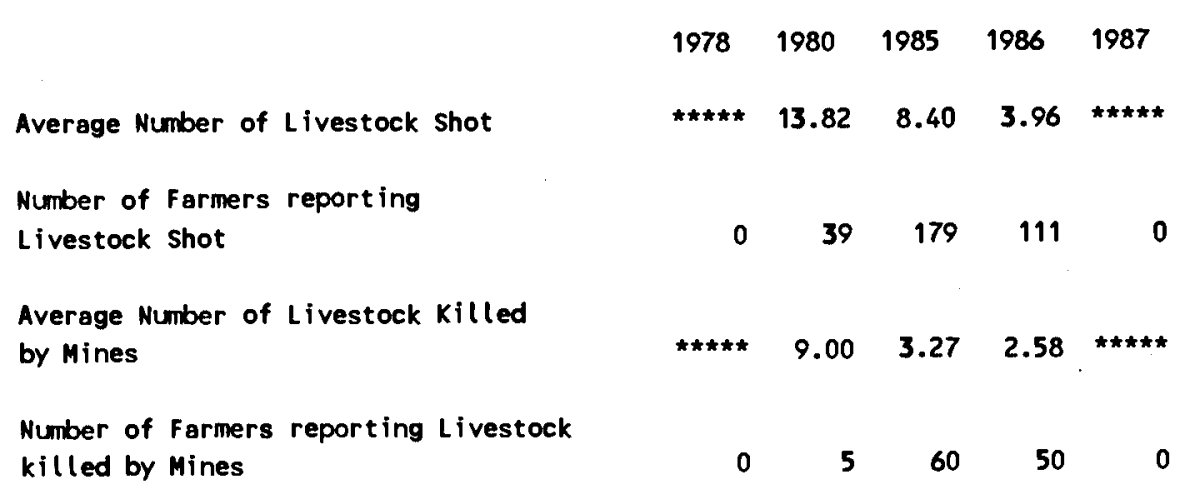

Number of Farmers Questioned 509 


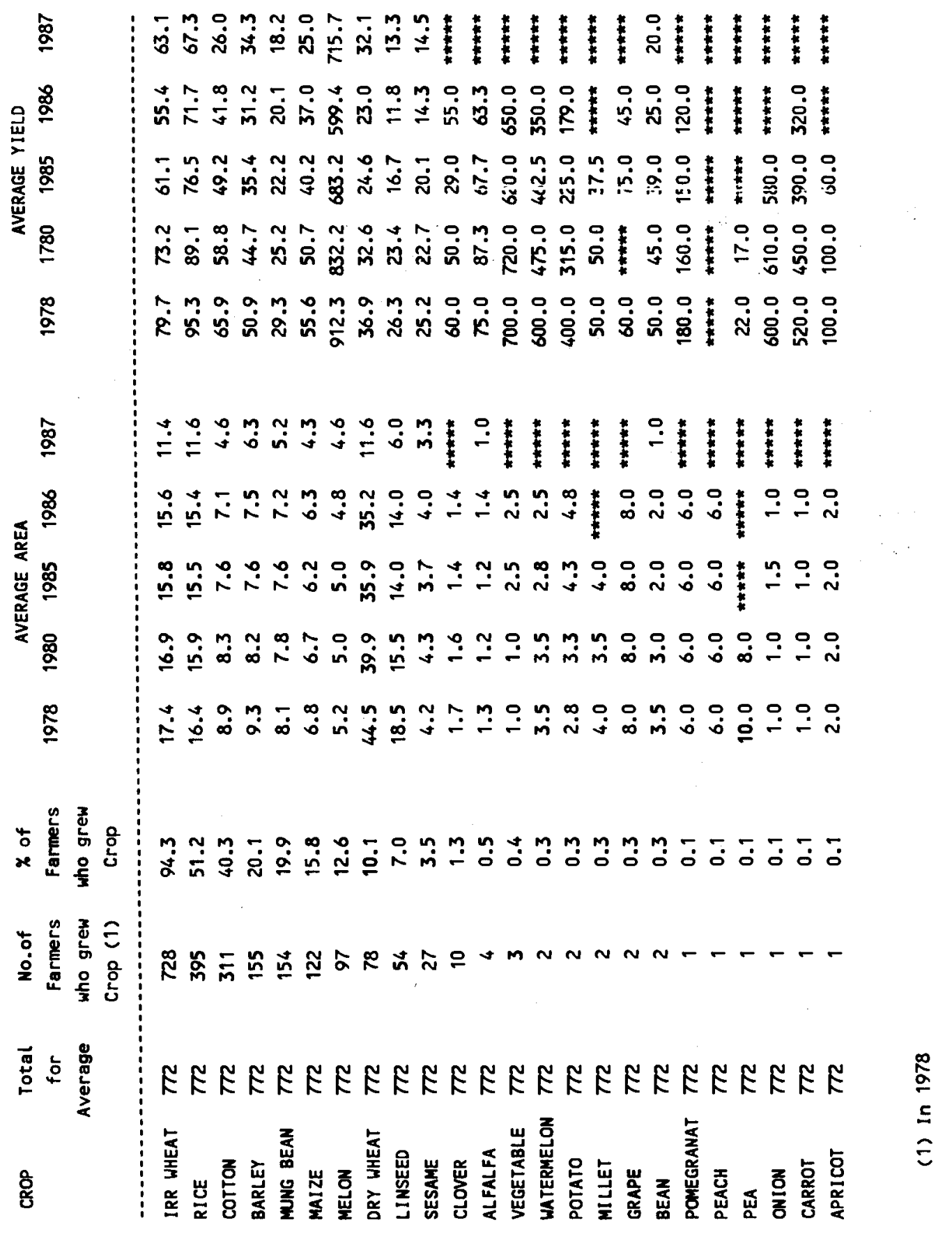


PROVINCE OF KDZ

\section{PERCENTAGE OF VILLAGES GIVING FOLLOWING PRIORITY TO PROBLEM}

Rainfall

Crop Disease

Birds or Rats

Seed Availability

Fertilizer Availability

Crop Protection Chemicals

Credit

Irrigation Water Availability

flooding

Labour Availabilty

Power for Land Preparation

Direct War Effects

Animal Diseases

Extension

Other Problems

\begin{tabular}{rrrrrrrrrrrrr}
1 & 2 & 3 & 4 & 5 & 6 & 7 & 8 & 9 & 10 & 11 & 12 & Weighted Priority \\
\hline 0 & 0 & 0 & 0 & 0 & 0 & 0 & 0 & 0 & 0 & 0 & 0 & 0.00 \\
0 & 0 & 2 & 4 & 13 & 9 & 2 & 0 & 0 & 0 & 0 & 0 & 2.63 \\
0 & 0 & 38 & 34 & 11 & 2 & 0 & 0 & 0 & 0 & 0 & 0 & 10.33 \\
0 & 0 & 6 & 2 & 4 & 2 & 2 & 0 & 0 & 0 & 0 & 0 & 1.81 \\
0 & 2 & 0 & 4 & 2 & 0 & 0 & 0 & 0 & 0 & 0 & 0 & 1.11 \\
0 & 0 & 0 & 4 & 2 & 4 & 0 & 0 & 0 & 0 & 0 & 0 & 0.96 \\
0 & 6 & 28 & 11 & 6 & 0 & 0 & 0 & 0 & 0 & 0 & 0 & 7.11 \\
6 & 30 & 4 & 0 & 0 & 2 & 0 & 0 & 0 & 0 & 0 & 0 & 10.02 \\
0 & 0 & 2 & 0 & 0 & 0 & 0 & 0 & 0 & 0 & 0 & 0 & 0.31 \\
2 & 49 & 15 & 2 & 2 & 2 & 0 & 0 & 0 & 0 & 0 & 0 & 14.29 \\
2 & 4 & 4 & 0 & 4 & 0 & 0 & 0 & 0 & 0 & 0 & 0 & 2.84 \\
89 & 9 & 2 & 0 & 0 & 0 & 0 & 0 & 0 & 0 & 0 & 0 & 41.01 \\
0 & 0 & 0 & 0 & 0 & 0 & 0 & 0 & 0 & 0 & 0 & 0 & 0.00 \\
0 & 0 & 0 & 38 & 34 & 6 & 0 & 0 & 0 & 0 & 0 & 0 & 7.59 \\
0 & 0 & 0 & 0 & 0 & 0 & 0 & 0 & 0 & 0 & 0 & 0 & 0.00
\end{tabular}

$\underline{1986}$

Rainfall

Crop Disease

Birds or Rats

Seed Availability

Fertilizer Availability

Crop Protection Chemicals

Credit

Irrigation Hater Availability

Flooding

Labour Availabilty

Power for Land Preparation

Direct War Effects

Animal Diseases

Extension

Other Problems

\begin{tabular}{rrrrrrrrrrrrr}
1 & 2 & 3 & 4 & 5 & 6 & 7 & 8 & 9 & 10 & 11 & 12 & Weighted Priority \\
\hline 0 & 9 & 2 & 0 & 0 & 0 & 0 & 0 & 0 & 0 & 0 & 0 & 2.10 \\
0 & 2 & 4 & 6 & 6 & 0 & 4 & 0 & 0 & 0 & 0 & 0 & 2.52 \\
0 & 0 & 13 & 13 & 23 & 17 & 2 & 4 & 0 & 0 & 0 & 0 & 6.67 \\
0 & 0 & 2 & 11 & 4 & 0 & 0 & 2 & 0 & 0 & 0 & 0 & 1.89 \\
0 & 0 & 9 & 9 & 2 & 0 & 0 & 0 & 0 & 0 & 0 & 0 & 2.28 \\
0 & 0 & 0 & 0 & 0 & 0 & 0 & 0 & 0 & 0 & 0 & 0 & 0.00 \\
0 & 9 & 26 & 15 & 13 & 0 & 2 & 0 & 0 & 0 & 0 & 0 & 8.17 \\
15 & 9 & 2 & 0 & 0 & 0 & 0 & 0 & 0 & 0 & 0 & 0 & 8.39 \\
0 & 0 & 0 & 0 & 2 & 0 & 0 & 0 & 0 & 0 & 0 & 0 & 0.18 \\
9 & 38 & 17 & 15 & 6 & 2 & 2 & 0 & 0 & 0 & 0 & 0 & 16.47 \\
0 & 9 & 21 & 15 & 2 & 2 & 2 & 0 & 0 & 0 & 0 & 0 & 6.82 \\
77 & 23 & 0 & 0 & 0 & 0 & 0 & 0 & 0 & 0 & 0 & 0 & 37.30 \\
0 & 0 & 0 & 0 & 0 & 2 & 0 & 0 & 0 & 0 & 0 & 0 & 0.15 \\
0 & 0 & 2 & 17 & 34 & 28 & 2 & 0 & 0 & 0 & 0 & 0 & 7.05 \\
0 & 0 & 0 & 0 & 0 & 0 & 0 & 0 & 0 & 0 & 0 & 0 & 0.00
\end{tabular}

1985

Rainfall

Crop Disease

Birds or Rats

Seed Avai lability

Fertilizer Availability

Crop Protection Chemicals

Credit

Irrigation Water Availability

flooding

Labour Availabilty

Power for Land Preparation

Direct War Effects

Animal Diseases

Extension

Other Problems

\begin{tabular}{rrrrrrrrrrrrr}
1 & 2 & 3 & 4 & 5 & 6 & 7 & 8 & 9 & 10 & 11 & 12 & Weighted Priority \\
\hline 4 & 9 & 0 & 0 & 0 & 4 & 0 & 0 & 0 & 0 & 0 & 0 & 4.01 \\
0 & 0 & 2 & 0 & 4 & 0 & 2 & 0 & 0 & 0 & 0 & 0 & 0.81 \\
0 & 2 & 28 & 34 & 15 & 4 & 0 & 0 & 0 & 0 & 0 & 0 & 9.78 \\
0 & 0 & 2 & 2 & 2 & 2 & 2 & 0 & 0 & 0 & 0 & 0 & 1.01 \\
0 & 0 & 2 & 4 & 2 & 2 & 0 & 0 & 0 & 0 & 0 & 0 & 1.11 \\
0 & 0 & 0 & 0 & 0 & 0 & 0 & 0 & 0 & 0 & 0 & 0 & 0.00 \\
0 & 13 & 23 & 21 & 4 & 4 & 0 & 0 & 0 & 0 & 0 & 0 & 9.17 \\
9 & 6 & 4 & 0 & 2 & 0 & 0 & 0 & 0 & 0 & 0 & 0 & 5.89 \\
0 & 0 & 0 & 0 & 0 & 0 & 0 & 0 & 0 & 0 & 0 & 0 & 0.00 \\
6 & 49 & 32 & 0 & 2 & 0 & 0 & 0 & 0 & 0 & 0 & 0 & 18.24 \\
0 & 2 & 6 & 4 & 4 & 4 & 0 & 0 & 0 & 0 & 0 & 0 & 2.53 \\
81 & 19 & 0 & 0 & 0 & 0 & 0 & 0 & 0 & 0 & 0 & 0 & 39.34 \\
0 & 0 & 0 & 0 & 0 & 2 & 0 & 0 & 0 & 0 & 0 & 0 & 0.15 \\
0 & 0 & 0 & 32 & 43 & 9 & 0 & 0 & 0 & 0 & 0 & 0 & 7.79 \\
0 & 0 & 0 & 0 & 0 & 2 & 0 & 0 & 0 & 0 & 0 & 0 & 0.15
\end{tabular}


PROVINCE OF KDZ

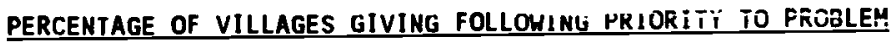

Rainfall

Crop Disease

Birds or Rats

Seed Avai lability

Fertilizer Availability

Crop Protection Chemicals

Credit

Irrigation Water Availability

Flooding

Labour Availabilty

Power for Land Preparation

Direct War Effects

Animal Diseases

Extension

Other Problems

\begin{tabular}{rrrrrrrrrrrrr}
1 & 2 & 3 & 4 & 5 & 6 & 7 & 8 & 9 & 10 & 11 & 12 & Weighted Priority \\
\hline 0 & 0 & 2 & 0 & 0 & 0 & 0 & 0 & 0 & 0 & 0 & 0 & 0.51 \\
0 & 2 & 0 & 0 & 2 & 0 & 0 & 0 & 0 & 0 & 0 & 0 & 1.07 \\
74 & 0 & 6 & 0 & 0 & 2 & 0 & 0 & 0 & 0 & 0 & 0 & 55.05 \\
0 & 0 & 6 & 2 & 0 & 0 & 0 & 0 & 0 & 0 & 0 & 0 & 1.90 \\
2 & 0 & 0 & 4 & 6 & 0 & 0 & 0 & 0 & 0 & 0 & 0 & 3.20 \\
0 & 0 & 0 & 0 & 0 & 0 & 0 & 0 & 0 & 0 & 0 & 0 & 0.00 \\
0 & 30 & 0 & 0 & 0 & 0 & 0 & 0 & 0 & 0 & 0 & 0 & 10.65 \\
4 & 2 & 0 & 0 & 4 & 0 & 0 & 0 & 0 & 0 & 0 & 0 & 4.41 \\
0 & 0 & 0 & 0 & 0 & 0 & 0 & 0 & 0 & 0 & 0 & 0 & 0.00 \\
4 & 21 & 4 & 4 & 0 & 0 & 0 & 0 & 0 & 0 & 0 & 0 & 12.43 \\
0 & 4 & 4 & 2 & 0 & 0 & 0 & 0 & 0 & 0 & 0 & 0 & 2.92 \\
9 & 0 & 4 & 0 & 0 & 0 & 0 & 0 & 0 & 0 & 0 & 0 & 7.10 \\
0 & 0 & 0 & 0 & 0 & 0 & 0 & 0 & 0 & 0 & 0 & 0 & 0.00 \\
0 & 0 & 0 & 0 & 0 & 0 & 0 & 0 & 0 & 0 & 0 & 0 & 0.00 \\
0 & 2 & 0 & 0 & 0 & 0 & 0 & 0 & 0 & 0 & 0 & 0 & 0.76
\end{tabular}

1978

Rainfall

Crop Disease

Birds or Rats

Seed Availability

Fertilizer Availability

Crop Protection Chemicals

Credit

Irrigation Water Availability

Flooding

Labour Availabilty

Power for Land Preparation

Direct War Effects

Animal Diseases

Extension

Other Problems

\begin{tabular}{rrrrrrrrrrrrr}
1 & 2 & 3 & 4 & 5 & 6 & 7 & 8 & 9 & 10 & 11 & 12 & Weighted Priority \\
\hline 0 & 0 & 0 & 0 & 0 & 0 & 0 & 0 & 0 & 0 & 0 & 0 & 0.00 \\
2 & 4 & 2 & 0 & 2 & 0 & 0 & 0 & 0 & 0 & 0 & 0 & 16.17 \\
4 & 0 & 0 & 0 & 2 & 0 & 0 & 0 & 0 & 0 & 0 & 0 & 14.04 \\
0 & 0 & 4 & 2 & 2 & 0 & 0 & 0 & 0 & 0 & 0 & 0 & 7.13 \\
2 & 0 & 0 & 4 & 2 & 0 & 0 & 0 & 0 & 0 & 0 & 0 & 10.85 \\
0 & 0 & 4 & 0 & 0 & 0 & 0 & 0 & 0 & 0 & 0 & 0 & 4.26 \\
4 & 6 & 0 & 2 & 0 & 0 & 0 & 0 & 0 & 0 & 0 & 0 & 23.94 \\
0 & 0 & 0 & 2 & 0 & 2 & 0 & 0 & 0 & 0 & 0 & 0 & 2.66 \\
0 & 0 & 0 & 0 & 0 & 0 & 0 & 0 & 0 & 0 & 0 & 0 & 0.00 \\
0 & 0 & 0 & 0 & 0 & 0 & 0 & 0 & 0 & 0 & 0 & 0 & 0.00 \\
4 & 2 & 0 & 0 & 0 & 0 & 0 & 0 & 0 & 0 & 0 & 0 & 15.96 \\
0 & 0 & 0 & 0 & 0 & 0 & 0 & 0 & 0 & 0 & 0 & 0 & 0.00 \\
0 & 0 & 2 & 0 & 0 & 0 & 0 & 0 & 0 & 0 & 0 & 0 & 2.13 \\
0 & 0 & 0 & 0 & 2 & 0 & 0 & 0 & 0 & 0 & 0 & 0 & 1.28 \\
0 & 0 & 0 & 2 & 0 & 0 & 0 & 0 & 0 & 0 & 0 & 0 & 1.60
\end{tabular}


KAPISA 


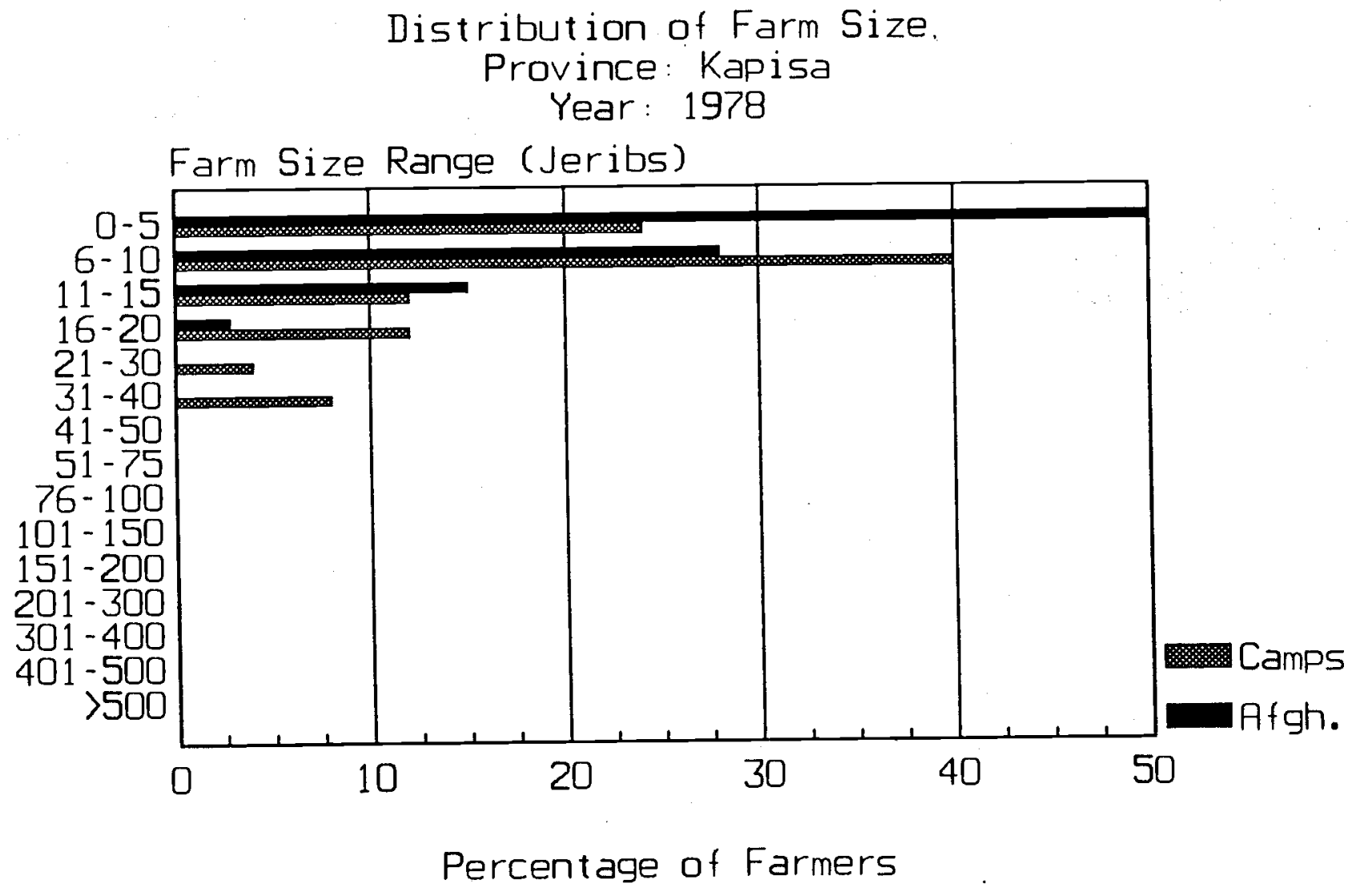

Farmers in Afghanistan

1978

1987

Total Number of Farms

Average Area

Average Area Irrigated

Average Dryland Area

107

6.35

6.26

10.00

Farmers who left in 1987

Total Number of Farms

Average Area

Average Area Irrigated

Average Dryland Area

\section{Average of Both Groups}

Provincial Average Area

Provincial Average Irrigated Area

Provincial Average Dryland Area
25

11.76

11.76

$-$

$\begin{array}{rr}7.38 & 2.45 \\ 7.30 & 2.49 \\ 10.00 & 9.00\end{array}$

1 16.00 16.00

(All areas in Jeribs. 5 Jeribs $=1$ Hectare) 
Farm Power - Farmers in Atghanistan Province of Kapisa Crop: Irrigated wheat

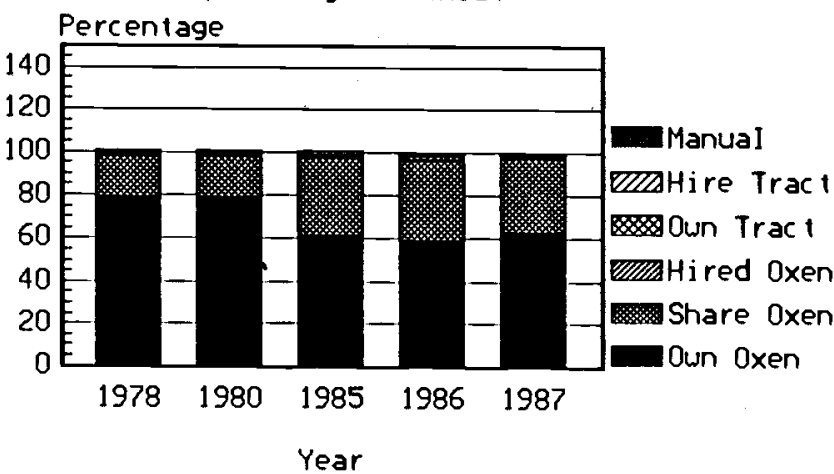

Farm Power - Farmers who lett in 1987 Province of Kapisa Crop: Irrigated wheat

Percentage

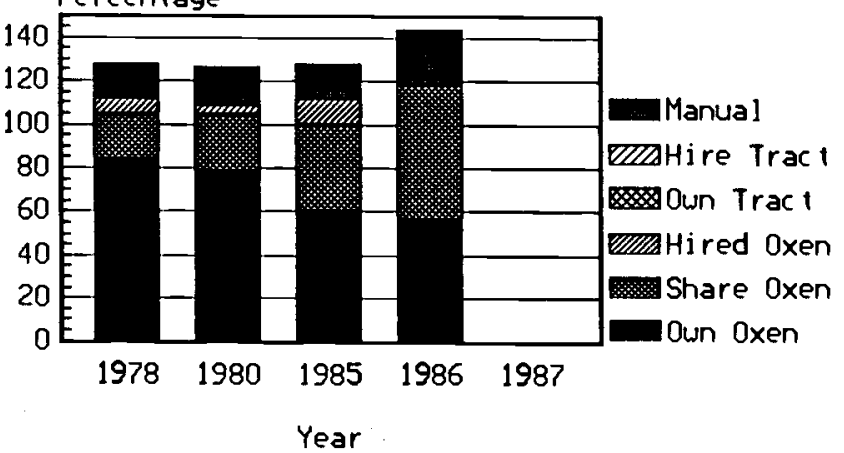

Farm Power - Farmers in Atghanistan Province of Kapisa Crop: Maize

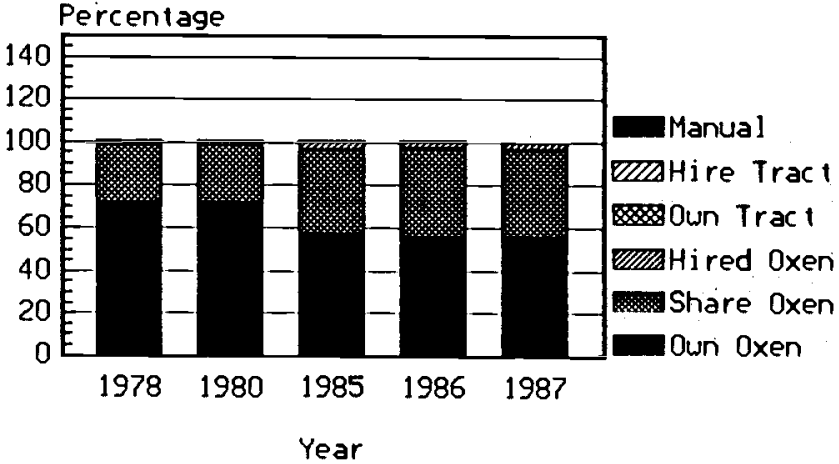

Farm Power - Farmers who lett in 1987 Province of Kapisa Crop: Maize

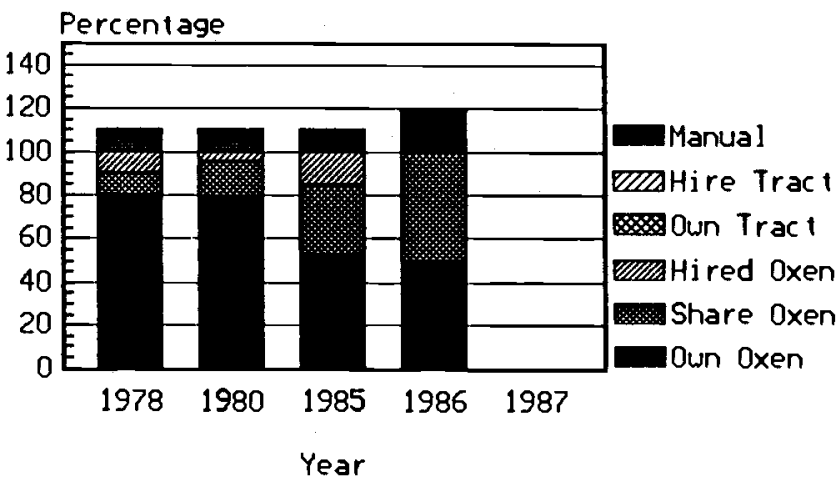

Base Figures for above graphs

Province: Kapisa

Farmers in Afghanistan

Irr. Wheat Own Oxen Shared oxen Hired oxen Own Tractor Hired Tractor

Maize Manual Means own Oxen Shared oxen Hired oxen Own Tractor Hired Tractor Manual Means

Farmers who left in 1987

$\begin{array}{ll}\text { Irr. Wheat } & \text { Own Oxen } \\ & \text { Shared Oxen } \\ & \text { Hired Oxen } \\ & \text { Own Tractor } \\ & \text { Hired Tractor } \\ & \text { Manual Means } \\ \text { Maize } & \text { Own Oxen } \\ & \text { Shared Oxen } \\ & \text { Hired Oxen } \\ & \text { Own Tractor } \\ & \text { Hired Tractor } \\ & \text { Manial Mnans }\end{array}$

84.00

20.00

8.00

$-$

$-$

16.00

80.00

10.00

10.00

(17

Percentage of Farmers $1980 \quad \underline{1985} \quad \underline{1986}$

1987
78.30

19.81

$-$

$-$

1.89

1.70

26.42

1.89

$-$

-
60.61

36.36

1.01

2.02

$-$

56.86

39.22

3.92

$-$

$-$
58.42

37.62

1.98

$-$

1.98

55.56

40.74

3.70

3.70
-

$-$
62.14

34.95

.97

1.94

$-$

56.00

40.00

4.00

$-$
78.26

26.09

4.35

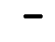

17.39

78.95

15.79

5.26

60.00

40.00

$12-$

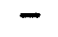

16.00

52.63

31.58

15.79$$
56.25
$$$$
62.50
$$$$
62.50
$$$$
\text { - }
$$$$
25.00
$$

50.00

50.00

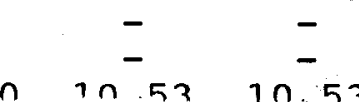

\section{(}

列 

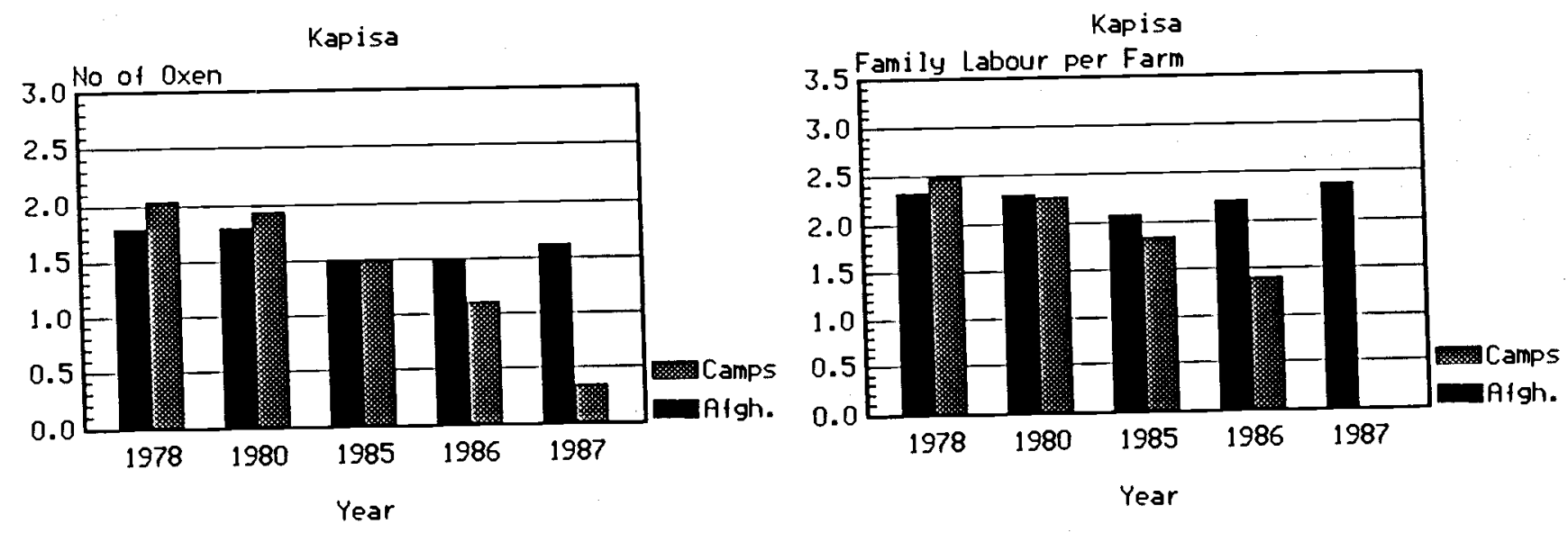

Kapisa

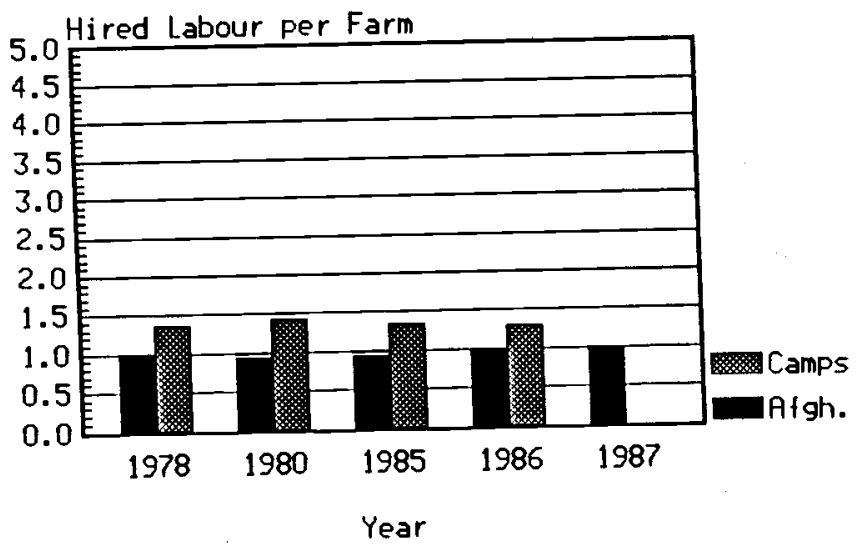

Kapisa

Percentage of Farms using

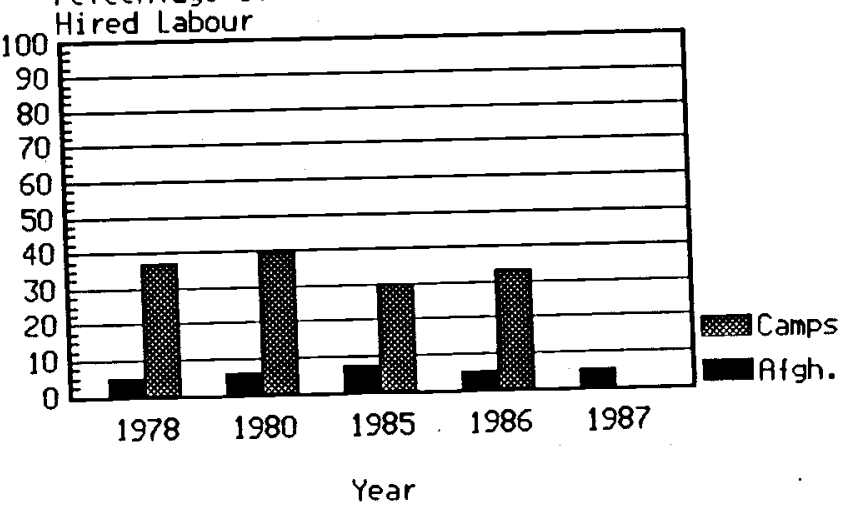


PROVINCE OF KPS

\begin{tabular}{|c|c|c|c|c|c|c|c|}
\hline \multirow{2}{*}{\multicolumn{2}{|c|}{ YEAR }} & \multicolumn{2}{|c|}{ NUMBER of } & TRAINED & \multicolumn{3}{|c|}{ OXEN PER FAMILY } \\
\hline & & 0 & 1 & 2 & 3 & 4 & 5 \\
\hline 1978 & $\begin{array}{l}\text { Number of Families } \\
\text { \% of Families }\end{array}$ & $\begin{array}{l}3 \\
2.80\end{array}$ & $\begin{array}{c}22 \\
20.56\end{array}$ & $\begin{array}{l}77 \\
71.96\end{array}$ & $\begin{array}{l}5 \\
4.67\end{array}$ & $\begin{array}{c}0 \\
0.00\end{array}$ & $\begin{array}{c}0 \\
0.00\end{array}$ \\
\hline 1980 & $\begin{array}{l}\text { Number of Families } \\
\% \text { of Families }\end{array}$ & $\begin{array}{l}3 \\
2.80\end{array}$ & $\begin{array}{c}22 \\
20.56\end{array}$ & $\begin{array}{l}77 \\
71.96\end{array}$ & $\begin{array}{l}5 \\
4.67\end{array}$ & $\begin{array}{l}0 \\
0.00\end{array}$ & $\begin{array}{l}0 \\
0.00\end{array}$ \\
\hline 1985 & $\begin{array}{l}\text { Number of Families } \\
\text { \% of Families }\end{array}$ & $\begin{array}{l}10 \\
9.35\end{array}$ & $\begin{array}{c}39 \\
36.45\end{array}$ & $\begin{array}{c}55 \\
51.40\end{array}$ & 2.80 & $\begin{array}{c}0 \\
0.00\end{array}$ & $\begin{array}{c}0 \\
0.00\end{array}$ \\
\hline 1986 & $\begin{array}{l}\text { Number of Families } \\
\% \text { of Families }\end{array}$ & $\begin{array}{c}11 \\
10.28\end{array}$ & $\begin{array}{c}37 \\
34.58\end{array}$ & $\begin{array}{c}56 \\
52.34\end{array}$ & $\begin{array}{l}3 \\
2.80\end{array}$ & $\begin{array}{l}0 \\
0.00\end{array}$ & $\begin{array}{c}0 \\
0.00\end{array}$ \\
\hline 1987 & $\begin{array}{l}\text { Number of Families } \\
\% \text { of Families }\end{array}$ & $\begin{array}{l}5 \\
4.67\end{array}$ & $\begin{array}{c}36 \\
33.64\end{array}$ & $\begin{array}{c}62 \\
57.94\end{array}$ & $\begin{array}{c}4 \\
3.74\end{array}$ & $\begin{array}{l}0 \\
0.00\end{array}$ & $\begin{array}{l}0 \\
0.00\end{array}$ \\
\hline era & ge Number of Oxen per Family: & $\begin{array}{l}1978 \\
1980 \\
1985 \\
1986 \\
1987\end{array}$ & & $\begin{array}{l}1.79 \\
1.79 \\
1.48 \\
1.48 \\
1.61\end{array}$ & & & \\
\hline
\end{tabular}

Number of Farmers Questioned 107

IABLE 7B - AVERAGE NUMBER OF TRAINED OXEN FOR FARMERS IN THE CAMPS

PROVINCE OF KPS

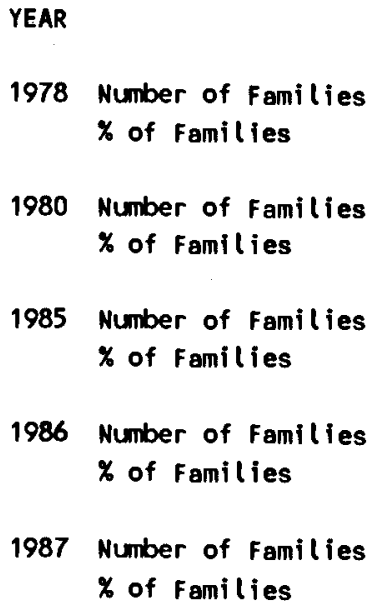

$\begin{array}{ll}1978 & 2.03 \\ 1980 & 1.93 \\ 1985 & 1.50 \\ 1986 & 1.10 \\ 1987 & 0.33\end{array}$

Number of Farmers Questioned 30 
PROVINCE OF KPS

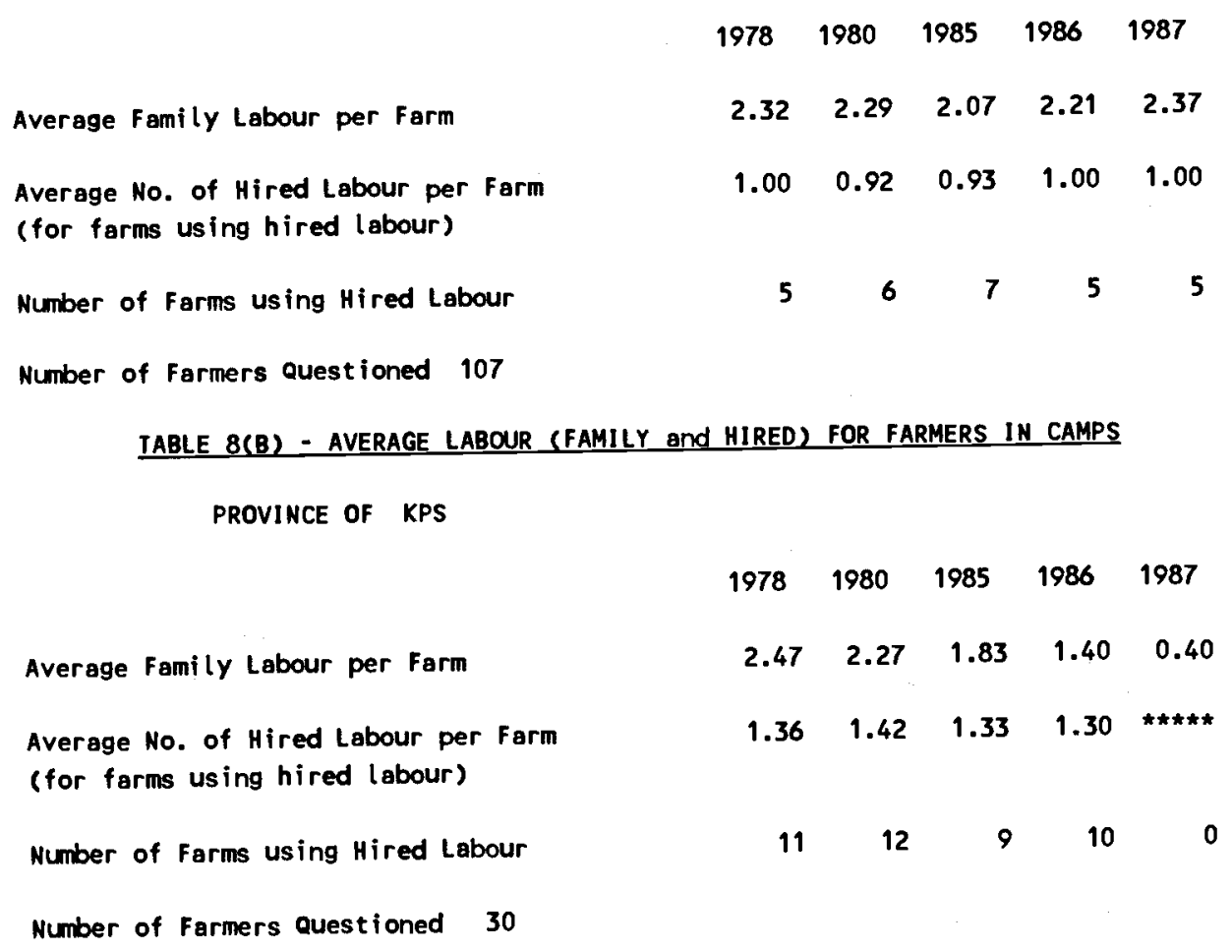


PROVINCE OF KPS

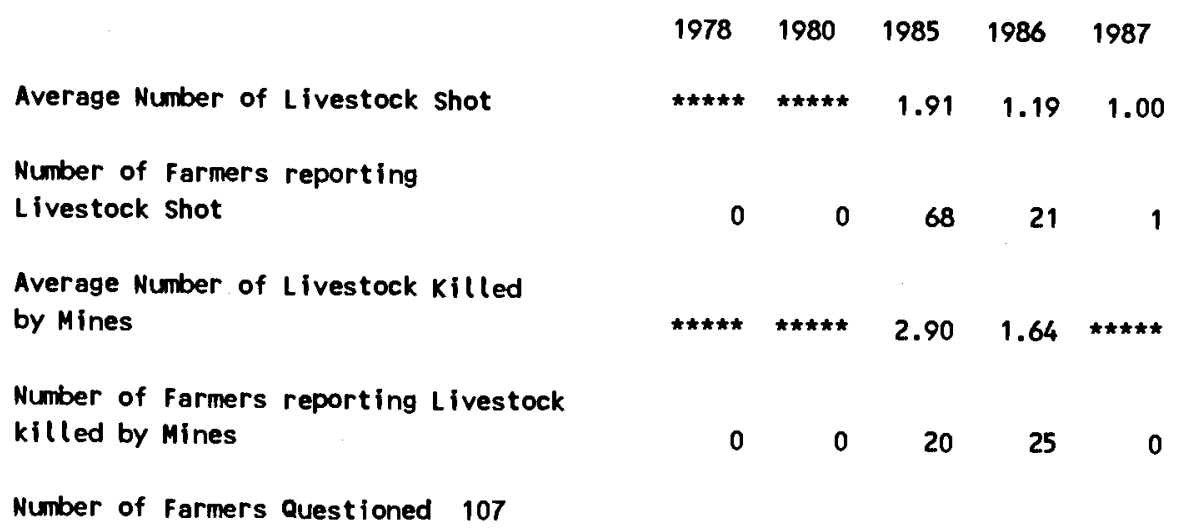

\section{IABLE 9B - DIRECT EFFECTS OF THE WAR - FARMERS IN CAMPS}

PROVINCE OF KPS

Average Number of Livestock Shot

$\begin{array}{rrrrrr}1978 & 1980 & 1985 & 1986 & 1987 \\ \star \star \star \star \star \star & 13.40 & 2.75 & 2.20 & \star \star \star \star \star \\ 0 & 5 & 12 & 10 & 0 \\ & & & & \\ \star \star \star \star \star & 2.00 & 2.40 & 1.67 & \star \star \star \star \star\end{array}$

Number of Farmers reporting Livestock

killed by Mines

$\begin{array}{lllll}0 & 1 & 5 & 3 & 0\end{array}$

Number of Farmers Questioned 30 


\begin{tabular}{|c|c|}
\hline$\underline{\underline{Q}}$ & moำ \\
\hline & 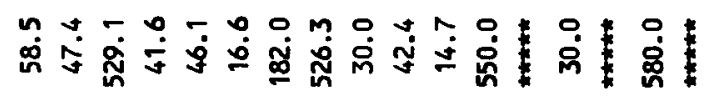 \\
\hline & 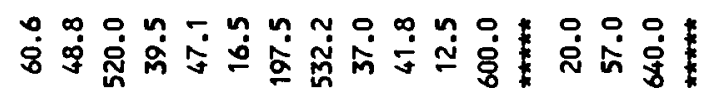 \\
\hline & 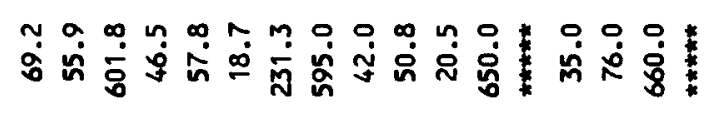 \\
\hline$\stackrel{\infty}{\sigma}$ & 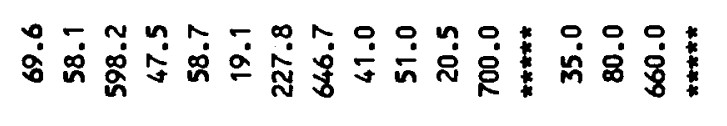 \\
\hline$\stackrel{\underline{\Phi}}{\underline{E}}$ & 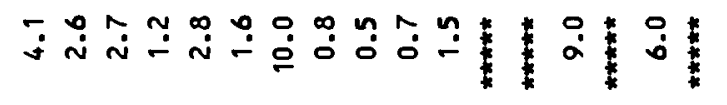 \\
\hline & 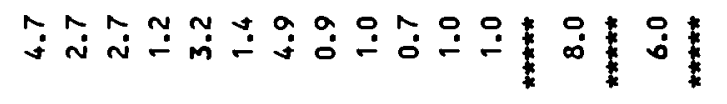 \\
\hline 蓆哀 & 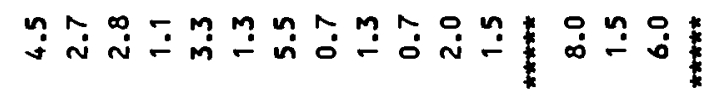 \\
\hline 票 & 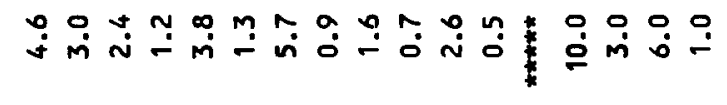 \\
\hline$\stackrel{\infty}{\alpha}$ & 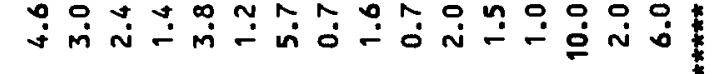 \\
\hline
\end{tabular}

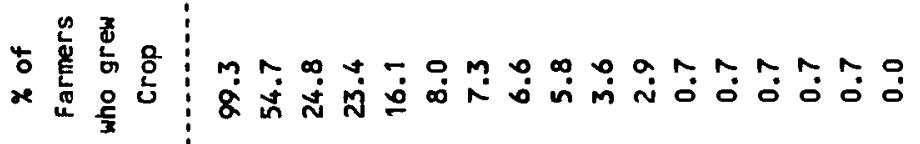

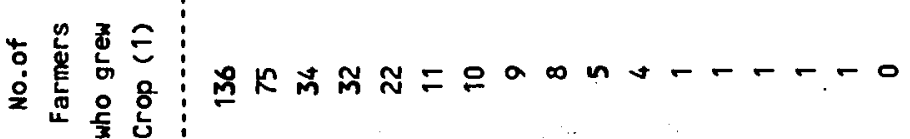

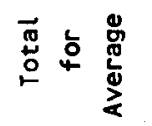

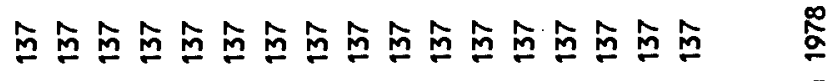

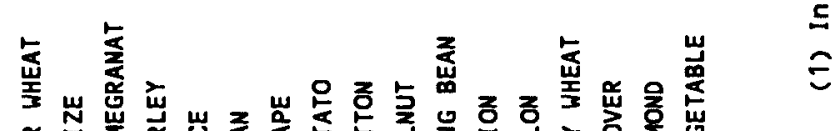

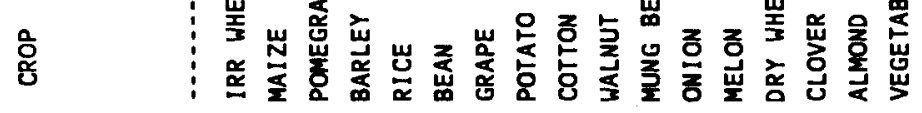


PROVINCE OF KPS

Rainfall

Crop Disease

Birds or Rats

Fertilizer Availability

Crop Protection Chemicals

Credit

Irrigation Water Availability Flooding

Labour Avai labilty

Power for Land Preparation

Animal Diseases

Extension

Other Problems
Seed Availability

Direct War Effects

$\begin{array}{rrrrrrrrrrrrr}1 & 2 & 3 & 4 & 5 & 6 & 7 & 8 & 9 & 10 & 11 & 12 & \text { Weighted Priority } \\ 0 & 3 & 0 & 1 & 0 & 0 & 1 & 0 & 0 & 0 & 0 & 0 & 0.88 \\ 10 & 4 & 12 & 29 & 32 & 9 & 0 & 0 & 0 & 0 & 0 & 0 & 13.56 \\ 0 & 0 & 0 & 1 & 1 & 2 & 10 & 0 & 0 & 0 & 0 & 0 & 1.79 \\ 33 & 7 & 20 & 23 & 9 & 0 & 0 & 0 & 0 & 0 & 0 & 0 & 22.24 \\ 36 & 12 & 17 & 9 & 1 & 1 & 0 & 0 & 0 & 0 & 0 & 0 & 21.93 \\ 0 & 1 & 1 & 10 & 16 & 19 & 3 & 1 & 0 & 0 & 0 & 0 & 4.63 \\ 0 & 0 & 0 & 0 & 0 & 0 & 0 & 0 & 0 & 0 & 0 & 0 & 0.00 \\ 9 & 9 & 10 & 1 & 1 & 0 & 0 & 0 & 0 & 0 & 0 & 0 & 7.41 \\ 0 & 0 & 6 & 6 & 9 & 7 & 3 & 0 & 0 & 0 & 0 & 0 & 2.93 \\ 0 & 0 & 1 & 1 & 1 & 4 & 0 & 0 & 0 & 0 & 0 & 0 & 0.81 \\ 0 & 4 & 14 & 6 & 1 & 3 & 0 & 0 & 0 & 0 & 0 & 0 & 4.00 \\ 7 & 57 & 13 & 6 & 0 & 1 & 0 & 0 & 0 & 0 & 0 & 0 & 18.03 \\ 1 & 0 & 0 & 1 & 7 & 0 & 3 & 0 & 0 & 0 & 0 & 0 & 1.59 \\ 0 & 0 & 0 & 0 & 0 & 0 & 0 & 0 & 0 & 0 & 0 & 0 & 0.00 \\ 0 & 0 & 1 & 0 & 0 & 0 & 0 & 0 & 0 & 0 & 0 & 0 & 0.21\end{array}$

1986

Rainfall

Crop Disease

Birds or Rats

Seed Availability

Fertilizer Availability

Crop Protection Chemicals

Credit

Irrigation Water Availability

Flooding

Labour Availabilty

Power for Land Preparation

Direct Har Effects

Animal Diseases

Extension

other Problems

\begin{tabular}{rrrrrrrrrrrrr}
1 & 2 & 3 & 4 & 5 & 6 & 7 & 8 & 9 & 10 & 11 & 12 & Weighted Priority \\
\hline 0 & 0 & 0 & 1 & 0 & 0 & 0 & 0 & 0 & 0 & 0 & 0 & 0.16 \\
9 & 6 & 10 & 26 & 26 & 7 & 0 & 0 & 0 & 0 & 0 & 0 & 12.57 \\
0 & 0 & 0 & 0 & 3 & 7 & 7 & 1 & 0 & 0 & 0 & 0 & 1.35 \\
28 & 10 & 20 & 13 & 7 & 1 & 0 & 0 & 0 & 0 & 0 & 0 & 19.96 \\
32 & 10 & 19 & 9 & 1 & 0 & 1 & 0 & 0 & 0 & 0 & 0 & 20.67 \\
0 & 1 & 0 & 10 & 9 & 13 & 3 & 0 & 0 & 0 & 0 & 0 & 3.42 \\
0 & 0 & 0 & 0 & 0 & 0 & 0 & 0 & 0 & 0 & 0 & 0 & 0.00 \\
12 & 9 & 10 & 4 & 1 & 0 & 0 & 0 & 0 & 0 & 0 & 0 & 9.32 \\
0 & 0 & 6 & 7 & 6 & 3 & 1 & 0 & 0 & 0 & 0 & 0 & 2.52 \\
0 & 0 & 1 & 0 & 1 & 0 & 0 & 0 & 0 & 0 & 0 & 0 & 0.35 \\
0 & 6 & 20 & 10 & 1 & 1 & 0 & 0 & 0 & 0 & 0 & 0 & 5.73 \\
17 & 55 & 7 & 4 & 1 & 0 & 0 & 0 & 0 & 0 & 0 & 0 & 21.94 \\
0 & 0 & 0 & 3 & 9 & 3 & 6 & 1 & 0 & 0 & 0 & 0 & 1.78 \\
0 & 0 & 0 & 0 & 0 & 0 & 0 & 0 & 0 & 0 & 0 & 0 & 0.00 \\
0 & 0 & 1 & 0 & 0 & 0 & 0 & 0 & 0 & 0 & 0 & 0 & 0.22
\end{tabular}

1985

Rainfall

Crop Disease

Birds or Rats

Seed Availability

Fertilizer Availability

Crop Protection Chemicals

Credit

Irrigation Water Availability

Flooding

Labour Availabilty

Power for Land Preparation

Direct War Effects

Animal Diseases

Extension

Other Problems

\begin{tabular}{rrrrrrrrrrrrc}
1 & 2 & 3 & 4 & 5 & 6 & 7 & 8 & 9 & 10 & 11 & 12 & Heighted Priority \\
\hline 0 & 0 & 0 & 0 & 0 & 0 & 0 & 0 & 0 & 0 & 0 & 0 & 0.00 \\
0 & 7 & 6 & 10 & 7 & 7 & 0 & 0 & 0 & 0 & 0 & 0 & 5.36 \\
0 & 0 & 0 & 0 & 0 & 3 & 1 & 0 & 0 & 0 & 0 & 0 & 0.34 \\
9 & 20 & 16 & 7 & 4 & 0 & 0 & 0 & 0 & 0 & 0 & 0 & 13.38 \\
7 & 16 & 23 & 9 & 3 & 0 & 0 & 0 & 0 & 0 & 0 & 0 & 12.82 \\
0 & 0 & 0 & 4 & 3 & 0 & 1 & 0 & 0 & 0 & 0 & 0 & 0.93 \\
0 & 0 & 3 & 0 & 0 & 0 & 0 & 0 & 0 & 0 & 0 & 0 & 0.48 \\
7 & 23 & 6 & 4 & 3 & 0 & 0 & 0 & 0 & 0 & 0 & 0 & 11.19 \\
0 & 1 & 1 & 1 & 3 & 1 & 0 & 0 & 0 & 0 & 0 & 0 & 1.19 \\
0 & 0 & 4 & 0 & 1 & 0 & 0 & 0 & 0 & 0 & 0 & 0 & 0.87 \\
0 & 14 & 25 & 26 & 3 & 0 & 0 & 0 & 0 & 0 & 0 & 0 & 11.25 \\
74 & 12 & 7 & 1 & 0 & 0 & 0 & 0 & 0 & 0 & 0 & 0 & 41.13 \\
0 & 1 & 0 & 1 & 1 & 0 & 0 & 0 & 0 & 0 & 0 & 0 & 0.69 \\
0 & 0 & 0 & 0 & 0 & 0 & 0 & 0 & 0 & 0 & 0 & 0 & 0.00 \\
0 & 1 & 0 & 0 & 0 & 0 & 0 & 0 & 0 & 0 & 0 & 0 & 0.36
\end{tabular}


PROVINCE OF KPS

Rainfall

Crop Disease

Birds or Rats

Seed Availability

Fertilizer Availability

Crop Protection Chemicals

Credit

Irrigation Water Availability

Flooding

Labour Availabilty

Power for Land Preparation

Direct War Effects

Animal Diseases

Extension

other Problems

\begin{tabular}{ccccccccccccc}
1 & 2 & 3 & 4 & 5 & 6 & 7 & 8 & 9 & 10 & 11 & 12 & Weighted Priority \\
\hline 0 & 0 & 0 & 0 & 0 & 0 & 0 & 0 & 0 & 0 & 0 & 0 & 0.00 \\
0 & 0 & 0 & 0 & 0 & 0 & 0 & 0 & 0 & 0 & 0 & 0 & 0.00 \\
0 & 0 & 0 & 0 & 0 & 0 & 0 & 0 & 0 & 0 & 0 & 0 & 0.00 \\
0 & 0 & 0 & 0 & 0 & 0 & 0 & 0 & 0 & 0 & 0 & 0 & 0.00 \\
0 & 0 & 0 & 0 & 0 & 0 & 0 & 0 & 0 & 0 & 0 & 0 & 0.00 \\
0 & 0 & 0 & 0 & 0 & 0 & 0 & 0 & 0 & 0 & 0 & 0 & 0.00 \\
0 & 0 & 0 & 0 & 0 & 0 & 0 & 0 & 0 & 0 & 0 & 0 & 0.00 \\
9 & 0 & 1 & 0 & 0 & 0 & 0 & 0 & 0 & 0 & 0 & 0 & 53.52 \\
3 & 0 & 0 & 0 & 0 & 0 & 0 & 0 & 0 & 0 & 0 & 0 & 16.90 \\
0 & 0 & 0 & 0 & 0 & 0 & 0 & 0 & 0 & 0 & 0 & 0 & 0.00 \\
1 & 1 & 0 & 0 & 0 & 0 & 0 & 0 & 0 & 0 & 0 & 0 & 12.68 \\
3 & 0 & 0 & 0 & 0 & 0 & 0 & 0 & 0 & 0 & 0 & 0 & 16.90 \\
0 & 0 & 0 & 0 & 0 & 0 & 0 & 0 & 0 & 0 & 0 & 0 & 0.00 \\
0 & 0 & 0 & 0 & 0 & 0 & 0 & 0 & 0 & 0 & 0 & 0 & 0.00 \\
0 & 0 & 0 & 0 & 0 & 0 & 0 & 0 & 0 & 0 & 0 & 0 & 0.00
\end{tabular}

1978

Rainfall

Crop Disease

Birds or Rats

Seed Availability

Fertilizer Availability

Crop Protection Chemicals

Credit

Irrigation Water Availability

Flooding

Labour Avai labilty

Power for Land Preparation

Direct War Effects

Animal Diseases

Extension

other Problems

\begin{tabular}{ccccccccccccc}
1 & 2 & 3 & 4 & 5 & 6 & 7 & 8 & 0 & 10 & 11 & 12 & Weighted Priority \\
\hline 0 & 0 & 0 & 0 & 0 & 0 & 0 & 0 & 0 & 0 & 0 & 0 & 0.00 \\
0 & 0 & 0 & 0 & 0 & 0 & 0 & 0 & 0 & 0 & 0 & 0 & 0.00 \\
0 & 0 & 0 & 0 & 0 & 0 & 0 & 0 & 0 & 0 & 0 & 0 & 0.00 \\
0 & 0 & 0 & 0 & 0 & 0 & 0 & 0 & 0 & 0 & 0 & 0 & 0.00 \\
0 & 0 & 0 & 0 & 0 & 0 & 0 & 0 & 0 & 0 & 0 & 0 & 0.00 \\
0 & 0 & 0 & 0 & 0 & 0 & 0 & 0 & 0 & 0 & 0 & 0 & 0.00 \\
0 & 0 & 0 & 0 & 0 & 0 & 0 & 0 & 0 & 0 & 0 & 0 & 0.00 \\
7 & 0 & 0 & 0 & 0 & 0 & 0 & 0 & 0 & 0 & 0 & 0 & 83.33 \\
0 & 0 & 0 & 0 & 0 & 0 & 0 & 0 & 0 & 0 & 0 & 0 & 0.00 \\
0 & 0 & 0 & 0 & 0 & 0 & 0 & 0 & 0 & 0 & 0 & 0 & 0.00 \\
1 & 0 & 0 & 0 & 0 & 0 & 0 & 0 & 0 & 0 & 0 & 0 & 16.67 \\
0 & 0 & 0 & 0 & 0 & 0 & 0 & 0 & 0 & 0 & 0 & 0 & 0.00 \\
0 & 0 & 0 & 0 & 0 & 0 & 0 & 0 & 0 & 0 & 0 & 0 & 0.00 \\
0 & 0 & 0 & 0 & 0 & 0 & 0 & 0 & 0 & 0 & 0 & 0 & 0.00 \\
0 & 0 & 0 & 0 & 0 & 0 & 0 & 0 & 0 & 0 & 0 & 0 & 0.00
\end{tabular}




\section{LOWGAR}




\section{Distribution of Farm Size \\ Province: Lowgar \\ Year: 1978}

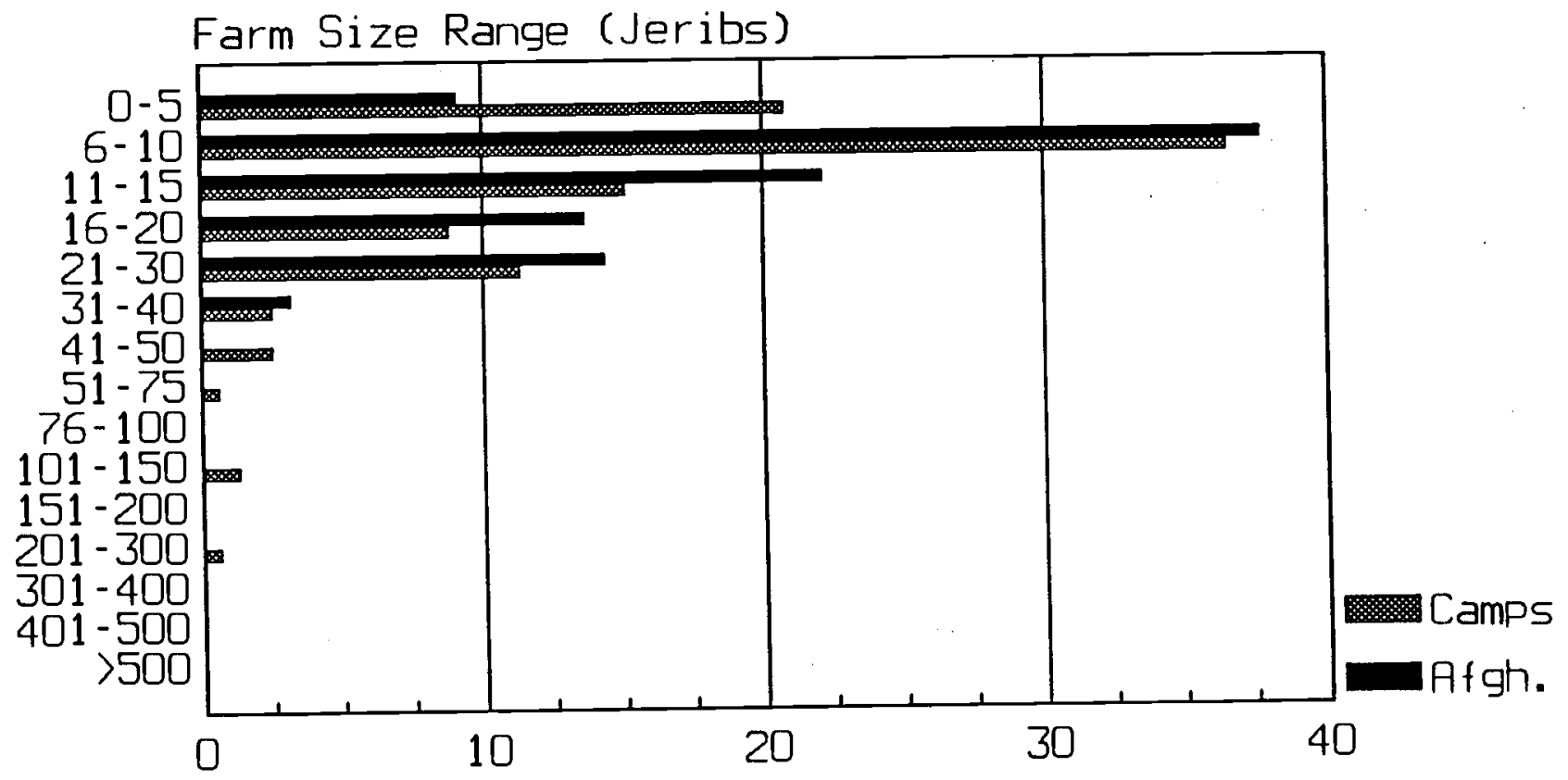

Percentage of Farmers

Farmers in Afghanistan

Total Number of Farms Average Area

Average Area Irrigated

Average Dryland Area

Farmers who left in 1987

Total Number of Farms

Average Area

Average Area Irrigated

Average Dryland Area

\section{Average of both Groups}

Provincial Average Area

Provincial Average Irrigated Area

Provincial Average Dryland Area

(All areas in Jeribs. 5 Jeribs $=1$ Hectare)
1978

1987

$\begin{array}{rl}154 & 154 \\ 13.35 & 4.42 \\ 13.29 & 4.32 \\ 2.50 & 2.00\end{array}$

159

3

15.50

13.25

12.77

7.00

7.00

14.44

4.46

13.27

4.37

10.35

2. 00 
PROVINCE OF LGR

YEAR

1978 Number of Families

\% of Families

1980 Number of Families

\% of Families

1985 Number of Families

$x$ of Fanilies

1986 Number of Families

$x$ of Families

1987 Number of Families

$x$ of Families

Average Number of Oxen per Family:

\section{PROVINCE OF LGR}

IABLE 7B - AVERAGE NUMBER OF TRAINEO OXEN FOR FARMERS IN THE CAMPS

YEAR

1978 Number of Families

$x$ of Families

1980 Number of Families

\% of Families

1985 Number of Families

$\%$ of Families

1986 Number of Families

X of Families

1987 Number of Families

$x$ of Families

Average Number of Oxen per Family:

$\begin{array}{ll}1978 & 1.46 \\ 1980 & 1.34 \\ 1985 & 1.12 \\ 1986 & 0.70 \\ 1987 & 0.01\end{array}$

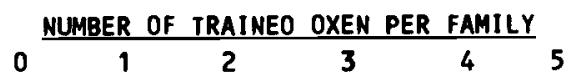

$\begin{array}{cccccc}6 & 48 & 84 & 0 & 1 & 0 \\ 4.32 & 34.53 & 60.43 & 0.00 & 0.72 & 0.00\end{array}$

$\begin{array}{llllll}8 & 51 & 79 & 0 & 1 & 0\end{array}$

$\begin{array}{llllll}5.76 & 36.69 & 56.83 & 0.00 & 0.72 & 0.00\end{array}$

$\begin{array}{llllll}11 & 68 & 59 & 0 & 1 & 0\end{array}$

$\begin{array}{llllll}7.91 & 48.92 & 42.45 & 0.00 & 0.72 & 0.00\end{array}$

$\begin{array}{llllll}20 & 74 & 44 & 0 & 1 & 0\end{array}$

$\begin{array}{llllll}14.39 & 53.24 & 31.65 & 0.00 & 0.72 & 0.00\end{array}$

$\begin{array}{llllll}33 & 69 & 36 & 0 & 1 & 0\end{array}$

$\begin{array}{llllll}23.74 & 49.64 & 25.90 & 0.00 & 0.72 & 0.00\end{array}$

$\begin{array}{ll}1978 & 1.58 \\ 1980 & 1.53 \\ 1985 & 1.37 \\ 1986 & 1.19 \\ 1987 & 1.04\end{array}$

139 
PROVINCE OF LGR

\begin{abstract}
Average Family Labour per Farm
Average No. of Hired Labour per Farm

(for farms using hired labour)
\end{abstract}

Number of Farms using Hired Labour

Number of Farmers Questioned 139

$\begin{array}{lllll}1978 & 1980 & 1985 & 1986 & 1987 \\ 2.29 & 2.16 & 1.83 & 1.83 & 1.72 \\ 2.25 & 2.25 & 1.56 & 1.40 & 1.86\end{array}$

$4 \quad 4 \quad 9 \quad 10 \quad 7$

IABLE 8(B) - AVERAGE LABOUR (FAMILY and HIRED) FOR FARMERS IN CAMPS

PROVINCE OF LGR

Average Family Labour per Farm

Average No. of Hired Labour per Farm

(for farms using hired labour)

Number of Farms using Hired Labour

Number of Farmers Questioned 155

$\begin{array}{rrrrr}1978 & 1980 & 1985 & 1986 & 1987 \\ 2.19 & 2.04 & 1.63 & 1.09 & 0.02 \\ 1.54 & 1.40 & 1.15 & 1.16 & \star \star \star \star \star \\ 26 & 25 & 27 & 16 & 0\end{array}$


PROVINCE OF LGR

Average Number of Livestock Shot

Number of Farmers reporting

Livestock Shot

Average Number of Livestock Killed by Mines

Number of Farmers reporting Livestock

killed by Mines

Number of Farmers Questioned 139

$\begin{array}{lllll}1978 & 1980 & 1985 & 1986 & 1987\end{array}$

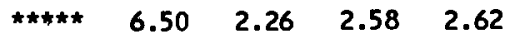

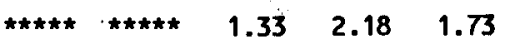

0

$0 \quad 3 \quad 17$

11

IABLE 9B - DIRECT EFFECTS OF THE WAR - FARMERS IN CAMPS

PROVINCE OF LGR

$\begin{array}{lrrrrr} & 1978 & 1980 & 1985 & 1986 & 1987 \\ \begin{array}{l}\text { Average Number of Livestock Shot } \\ \text { Number of Farmers reporting }\end{array} & \star \star \star \star \star & 4.25 & 11.83 & 3.90 & \star \star \star \star \star\end{array}$




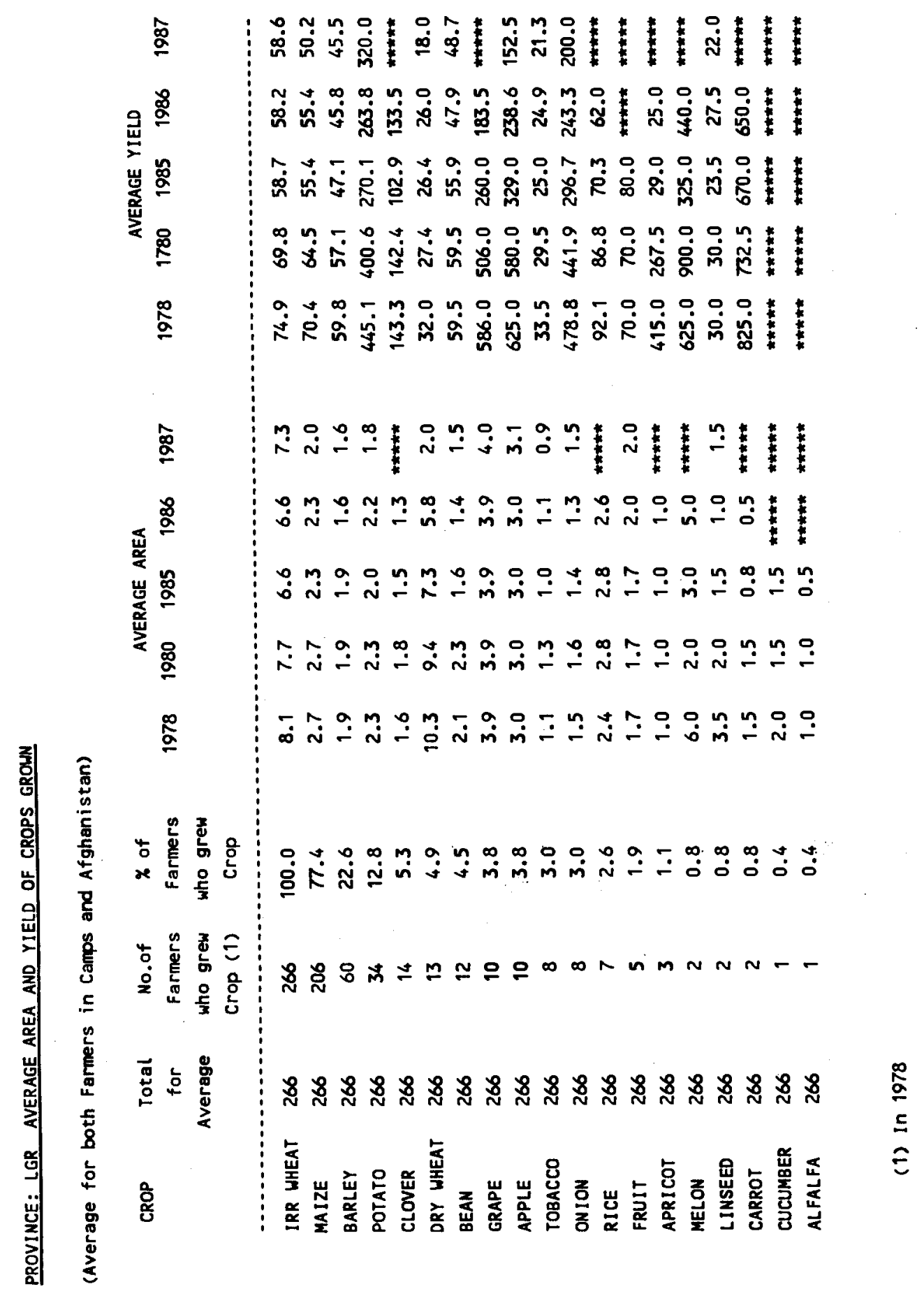


PROVINCE OF LGR

Rainfall

Crop Disease

Birds or Rats

Seed Availability

Fertilizer Availability

Crop Protection Chemicals

Credit

Irrigation Water Availability

flooding

Labour Availabilty

Power for Land Preparation

Direct War Effects

Animal Diseases

Extension

Other Problems

\begin{tabular}{rrrrrrrrrrrrr}
1 & 2 & 3 & 4 & 5 & 6 & 7 & 8 & 9 & 10 & 11 & 12 & Weighted Priority \\
\hline 0 & 0 & 0 & 0 & 0 & 0 & 3 & 0 & 0 & 0 & 0 & 0 & 0.17 \\
0 & 0 & 3 & 43 & 5 & 14 & 3 & 0 & 0 & 0 & 0 & 0 & 6.70 \\
0 & 0 & 0 & 5 & 11 & 30 & 5 & 8 & 0 & 0 & 0 & 0 & 4.45 \\
0 & 3 & 0 & 5 & 11 & 3 & 0 & 5 & 0 & 0 & 0 & 0 & 2.60 \\
3 & 49 & 24 & 0 & 0 & 0 & 3 & 0 & 0 & 0 & 0 & 0 & 15.43 \\
0 & 0 & 0 & 3 & 0 & 0 & 0 & 0 & 0 & 0 & 0 & 0 & 0.29 \\
0 & 0 & 0 & 0 & 0 & 0 & 0 & 0 & 0 & 0 & 0 & 0 & 0.00 \\
0 & 8 & 19 & 5 & 0 & 0 & 0 & 0 & 0 & 0 & 0 & 0 & 5.09 \\
0 & 0 & 0 & 0 & 0 & 3 & 0 & 0 & 0 & 0 & 0 & 0 & 0.20 \\
5 & 5 & 5 & 16 & 11 & 0 & 0 & 0 & 0 & 0 & 0 & 0 & 7.00 \\
11 & 27 & 32 & 3 & 0 & 0 & 0 & 0 & 0 & 0 & 0 & 0 & 15.55 \\
78 & 8 & 0 & 0 & 0 & 0 & 0 & 0 & 0 & 0 & 0 & 0 & 35.80 \\
0 & 0 & 5 & 0 & 8 & 11 & 11 & 0 & 0 & 0 & 0 & 0 & 2.94 \\
0 & 0 & 0 & 0 & 0 & 0 & 0 & 0 & 0 & 0 & 0 & 0 & 0.00 \\
0 & 0 & 0 & 5 & 32 & 3 & 3 & 0 & 0 & 0 & 0 & 0 & 3.77
\end{tabular}

1986

Rainfall

Crop Disease

Birds or Rats

Seed Avail ability

Fertilizer Availability

Crop Protection Chemicals

Credit

Irrigation Water Availability

Flooding

Labour Availabilty

Power for Land Preparation

Direct War Effects

Animal Diseases

Extension

other Problems

\begin{tabular}{rrrrrrrrrrrrr}
1 & 2 & 3 & 4 & 5 & 6 & 7 & 8 & 9 & 10 & 11 & 12 & Weighted Priority \\
\hline 0 & 0 & 5 & 3 & 0 & 0 & 0 & 0 & 0 & 0 & 0 & 0 & 1.07 \\
3 & 0 & 11 & 27 & 8 & 5 & 3 & 0 & 0 & 0 & 0 & 0 & 6.88 \\
0 & 5 & 3 & 3 & 19 & 19 & 11 & 0 & 0 & 0 & 0 & 0 & 5.50 \\
0 & 3 & 8 & 5 & 5 & 3 & 0 & 0 & 0 & 0 & 0 & 0 & 2.99 \\
0 & 8 & 3 & 3 & 0 & 3 & 0 & 0 & 0 & 0 & 0 & 0 & 2.62 \\
0 & 0 & 0 & 3 & 3 & 0 & 0 & 0 & 0 & 0 & 0 & 0 & 0.52 \\
0 & 0 & 0 & 0 & 0 & 0 & 0 & 0 & 0 & 0 & 0 & 0 & 0.00 \\
0 & 14 & 27 & 8 & 3 & 0 & 0 & 0 & 0 & 0 & 0 & 0 & 7.90 \\
0 & 3 & 3 & 0 & 0 & 3 & 0 & 0 & 0 & 0 & 0 & 0 & 1.16 \\
11 & 5 & 11 & 11 & 11 & 0 & 0 & 0 & 0 & 0 & 0 & 0 & 9.47 \\
8 & 46 & 14 & 11 & 0 & 0 & 0 & 0 & 0 & 0 & 0 & 0 & 16.50 \\
81 & 8 & 3 & 0 & 0 & 0 & 0 & 0 & 0 & 0 & 0 & 0 & 37.08 \\
0 & 3 & 11 & 8 & 5 & 5 & 0 & 0 & 0 & 0 & 0 & 0 & 3.86 \\
0 & 0 & 0 & 0 & 0 & 0 & 0 & 0 & 0 & 0 & 0 & 0 & 0.00 \\
0 & 0 & 0 & 8 & 22 & 19 & 5 & 0 & 0 & 0 & 0 & 0 & 4.43
\end{tabular}

1985

Rainfall

Crop Disease

Birds or Rats

Seed Availability

Fertilizer Availability

Crop Protection Chemicals

Credit

Irrigation Water Availability

Flooding

Labour Avai labilty

Power for Land Preparation

Direct War Effects

Animal Diseases

Extension

other Problems

\begin{tabular}{rrrrrrrrrrrrr}
1 & 2 & 3 & 4 & 5 & 6 & 7 & 8 & 9 & 10 & 11 & 12 & Weighted Priority \\
\hline 0 & 5 & 0 & 0 & 0 & 0 & 0 & 0 & 0 & 0 & 0 & 0 & 1.18 \\
0 & 0 & 11 & 35 & 14 & 5 & 0 & 0 & 0 & 0 & 0 & 0 & 6.97 \\
0 & 5 & 0 & 5 & 8 & 35 & 5 & 5 & 0 & 0 & 0 & 0 & 5.66 \\
0 & 0 & 0 & 8 & 3 & 8 & 0 & 3 & 0 & 0 & 0 & 0 & 1.86 \\
3 & 32 & 8 & 3 & 0 & 0 & 0 & 0 & 0 & 0 & 0 & 0 & 9.72 \\
0 & 0 & 0 & 3 & 0 & 0 & 0 & 0 & 0 & 0 & 0 & 0 & 0.29 \\
0 & 0 & 0 & 0 & 0 & 0 & 0 & 0 & 0 & 0 & 0 & 0 & 0.00 \\
5 & 24 & 11 & 19 & 0 & 0 & 0 & 0 & 0 & 0 & 0 & 0 & 10.80 \\
0 & 0 & 0 & 0 & 0 & 3 & 0 & 0 & 0 & 0 & 0 & 0 & 0.20 \\
0 & 0 & 16 & 11 & 16 & 0 & 0 & 0 & 0 & 0 & 0 & 0 & 4.95 \\
5 & 22 & 30 & 3 & 3 & 0 & 0 & 0 & 0 & 0 & 0 & 0 & 11.92 \\
86 & 3 & 3 & 0 & 0 & 0 & 0 & 0 & 0 & 0 & 0 & 0 & 38.68 \\
0 & 0 & 14 & 5 & 5 & 3 & 11 & 0 & 0 & 0 & 0 & 0 & 3.89 \\
0 & 0 & 0 & 0 & 0 & 0 & 0 & 0 & 0 & 0 & 0 & 0 & 0.00 \\
0 & 0 & 0 & 8 & 32 & 0 & 3 & 0 & 0 & 0 & 0 & 0 & 3.88
\end{tabular}


PROVINCE OF LGR

\section{PERCENTAGE OF VILLAGES GIVING FOLLOWING PRIORITY TO PROBLEM}

Rainfall

Crop Disease

Birds or Rats

Seed Availability

Fertilizer Availability

Crop Protection Chemicals

Credit

Irrigation Water Availability

Flooding

Labour Availabilty

Power for Land Preparation

Direct War Effects

Animal Diseases

Extension

Other Problems

\begin{tabular}{rrrrrrrrrrrrr}
1 & 2 & 3 & 4 & 5 & 6 & 7 & 8 & 9 & 10 & 11 & 12 & Heighted Priority \\
\hline 0 & 0 & 0 & 0 & 0 & 0 & 0 & 0 & 0 & 0 & 0 & 0 & 0.00 \\
14 & 19 & 0 & 3 & 0 & 0 & 0 & 0 & 0 & 0 & 0 & 0 & 22.54 \\
5 & 8 & 8 & 5 & 0 & 3 & 0 & 0 & 0 & 0 & 0 & 0 & 13.31 \\
5 & 3 & 8 & 0 & 3 & 0 & 3 & 0 & 0 & 0 & 0 & 0 & 9.90 \\
0 & 0 & 0 & 0 & 0 & 0 & 0 & 0 & 0 & 0 & 0 & 0 & 0.00 \\
0 & 0 & 3 & 0 & 0 & 0 & 0 & 0 & 0 & 0 & 0 & 0 & 0.86 \\
0 & 0 & 0 & 0 & 0 & 0 & 0 & 0 & 0 & 0 & 0 & 0 & 0.00 \\
8 & 5 & 3 & 0 & 0 & 0 & 0 & 0 & 0 & 0 & 0 & 0 & 11.16 \\
5 & 3 & 0 & 3 & 0 & 0 & 0 & 0 & 0 & 0 & 0 & 0 & 7.08 \\
0 & 3 & 5 & 0 & 0 & 0 & 0 & 0 & 0 & 0 & 0 & 0 & 3.00 \\
0 & 0 & 0 & 3 & 0 & 0 & 0 & 0 & 0 & 0 & 0 & 0 & 0.64 \\
11 & 5 & 0 & 0 & 0 & 0 & 0 & 0 & 0 & 0 & 0 & 0 & 12.88 \\
5 & 8 & 3 & 0 & 5 & 0 & 0 & 0 & 0 & 0 & 0 & 0 & 10.90 \\
0 & 0 & 0 & 0 & 0 & 0 & 0 & 0 & 0 & 0 & 0 & 0 & 0.00 \\
3 & 3 & 8 & 5 & 0 & 0 & 0 & 0 & 0 & 0 & 0 & 0 & 7.73
\end{tabular}

\section{8}

\section{Rainfall}

Crop Disease

Birds or Rats

Seed Availability

Fertilizer Availability

Crop Protection Chemicals

Credit

Irrigation Water Availability

Flooding

Labour Availabilty

Power for Land Preparation

Direct War Effects

Animal Diseases

Extension

Other Problems

\begin{tabular}{rrrrrrrrrrrrr}
1 & 2 & 3 & 4 & 5 & 6 & 7 & 8 & 9 & 10 & 11 & 12 & Weighted Priority \\
\hline 0 & 0 & 0 & 0 & 0 & 0 & 0 & 0 & 0 & 0 & 0 & 0 & 0.00 \\
8 & 22 & 5 & 0 & 0 & 0 & 0 & 0 & 0 & 0 & 0 & 0 & 23.66 \\
3 & 5 & 19 & 5 & 0 & 0 & 0 & 0 & 0 & 0 & 0 & 0 & 14.92 \\
3 & 0 & 3 & 5 & 0 & 0 & 0 & 0 & 0 & 0 & 0 & 0 & 5.66 \\
0 & 0 & 0 & 0 & 0 & 0 & 0 & 0 & 0 & 0 & 0 & 0 & 0.00 \\
3 & 0 & 3 & 0 & 3 & 0 & 0 & 0 & 0 & 0 & 0 & 0 & 4.73 \\
0 & 0 & 0 & 0 & 0 & 0 & 0 & 0 & 0 & 0 & 0 & 0 & 0.00 \\
0 & 0 & 0 & 0 & 0 & 0 & 0 & 0 & 0 & 0 & 0 & 0 & 0.00 \\
11 & 5 & 3 & 3 & 0 & 0 & 0 & 0 & 0 & 0 & 0 & 0 & 17.23 \\
0 & 0 & 0 & 0 & 0 & 0 & 0 & 0 & 0 & 0 & 0 & 0 & 0.00 \\
0 & 0 & 0 & 0 & 0 & 0 & 0 & 0 & 0 & 0 & 0 & 0 & 0.00 \\
0 & 0 & 0 & 0 & 0 & 0 & 0 & 0 & 0 & 0 & 0 & 0 & 0.00 \\
14 & 14 & 5 & 3 & 0 & 0 & 0 & 0 & 0 & 0 & 0 & 0 & 25.98 \\
0 & 0 & 0 & 0 & 3 & 0 & 0 & 0 & 0 & 0 & 0 & 0 & 0.62 \\
5 & 0 & 3 & 0 & 0 & 0 & 0 & 0 & 0 & 0 & 0 & 0 & 7.20
\end{tabular}




\section{LAGHMAN}




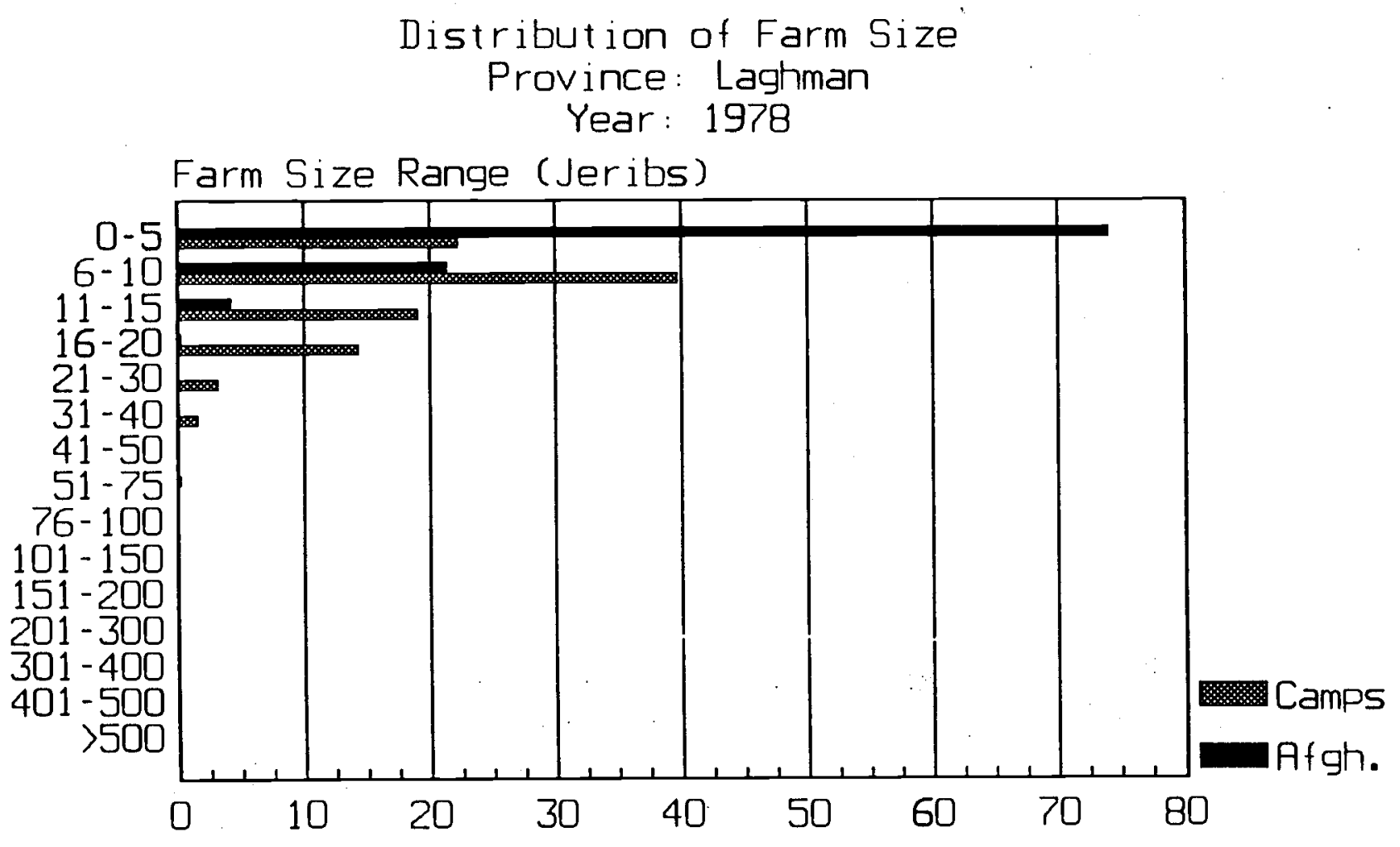

Percentage of Farmers

Farmers in Afghanistan

Total Number of Farms

Average Area

Average Area Irrigated

Average Dryland Area

Farmers who left in 1987

Total Number of Farms

Average Area

Average Area Irrigated

Average Dryland Area

\section{Average of both Groups}

Provincial Average Area

Provincial Average Irrigated Area

Provincial Average Dryland Area $\underline{1978}$

404

4.48

4.45

2.50

63

10.63

10.42

2.17 $\underline{1987}$

404

2.33

2.31

2.56

(All areas in Jeribs. 5 Jeribs $=1$ Hectare)

2.33

2.31

5.26

2.35 

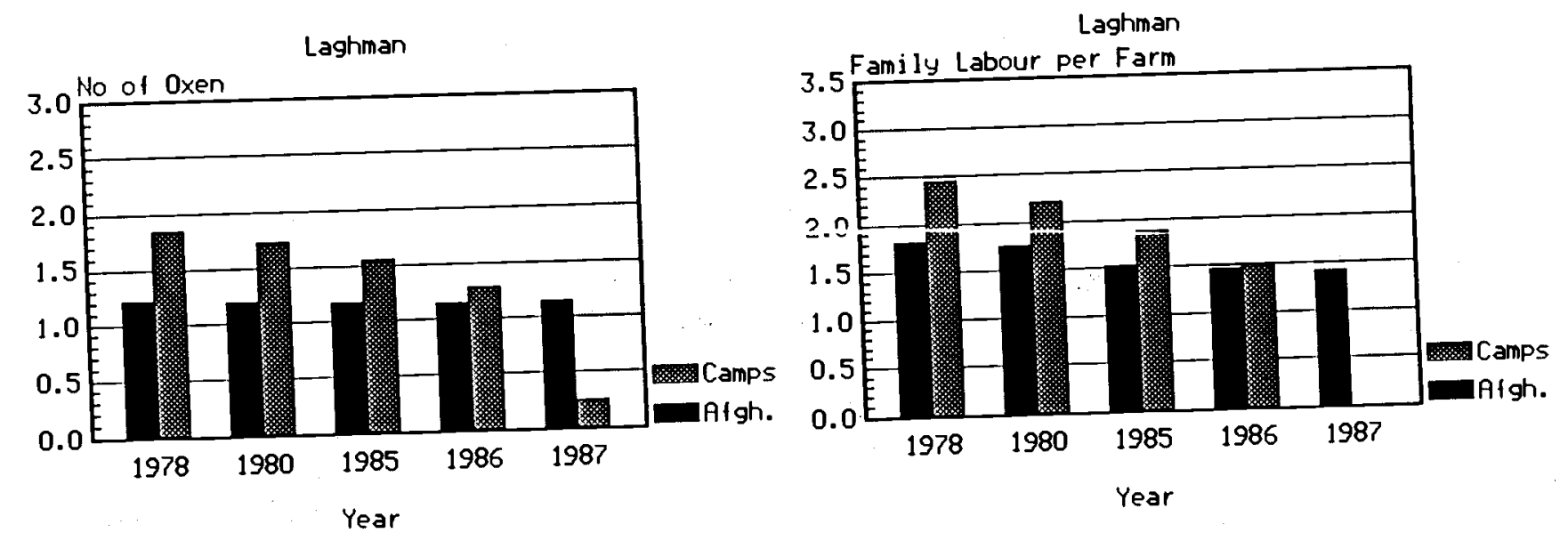

Laghman

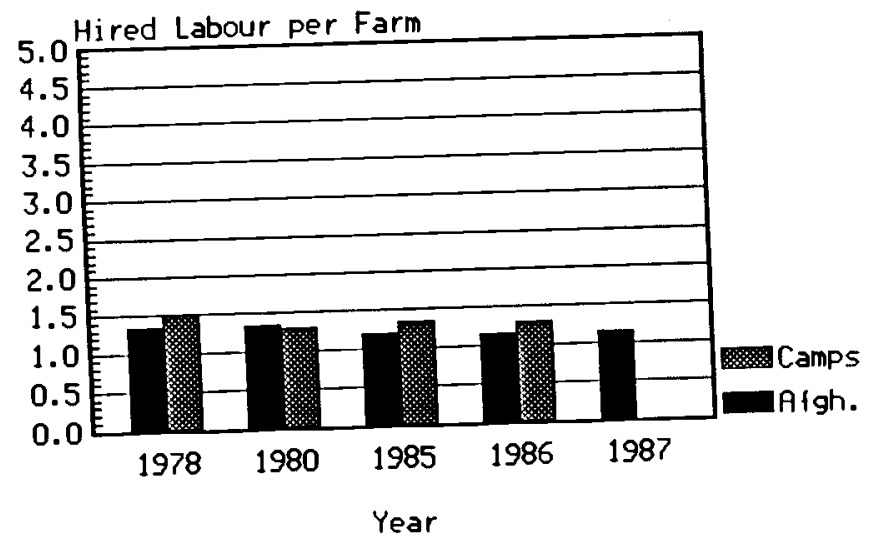

Laghman

Percentage of Farms using

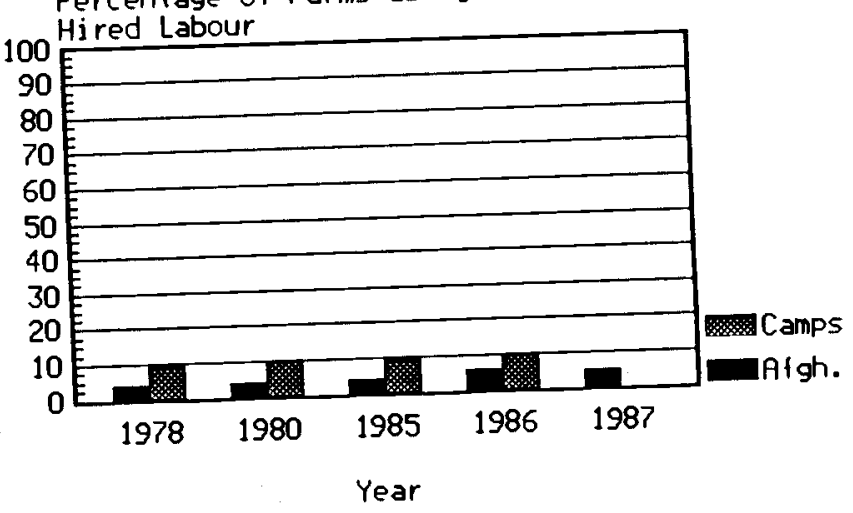


PROVINCE OF LMN

YEAR

1978 Number of Families $\%$ of Families

1980 Number of Families

x of Families

1985 Number of Families

\% of Families

1986 Number of Families

$x$ of Families

1987 Number of Families

$\%$ of Families

Average Number of Oxen per Family:

Number of Farmers Questioned 395

$\begin{array}{ll}1978 & 1.21 \\ 1980 & 1.19 \\ 1985 & 1.16 \\ 1986 & 1.14 \\ 1987 & 1.14\end{array}$

$\begin{array}{lcccc}\text { NUMBER OF } & \text { TRAINED } & \text { OXEN } & \text { PER FAMILY } \\ 0 & 1 & 2 & 3 & 4\end{array}$

$\begin{array}{llllll}74 & 165 & 156 & 0 & 0 & 0\end{array}$

$\begin{array}{llllll}18.73 & 41.77 & 39.49 & 0.00 & 0.00 & 0.00\end{array}$

$\begin{array}{llllll}77 & 164 & 154 & 0 & 0 & 0\end{array}$

$\begin{array}{llllll}19.49 & 41.52 & 38.99 & 0.00 & 0.00 & 0.00\end{array}$

$\begin{array}{llllll}81 & 170 & 144 & 0 & 0 & 0\end{array}$

$\begin{array}{llllll}20.51 & 43.04 & 36.46 & 0.00 & 0.00 & 0.00\end{array}$

$\begin{array}{llllll}82 & 177 & 135 & 1 & 0 & 0\end{array}$

$\begin{array}{llllll}20.76 & 44.81 & 34.18 & 0.25 & 0.00 & 0.00\end{array}$

$\begin{array}{llllll}82 & 178 & 134 & 1 & 0 & 0\end{array}$

$\begin{array}{llllll}20.76 & 45.06 & 33.92 & 0.25 & 0.00 & 0.00\end{array}$

\section{IABLE 7B - AVERAGE NUMBER OF TRAINED OXEN FOR FARMERS IN THE CAMPS}

PROVINCE OF LMN

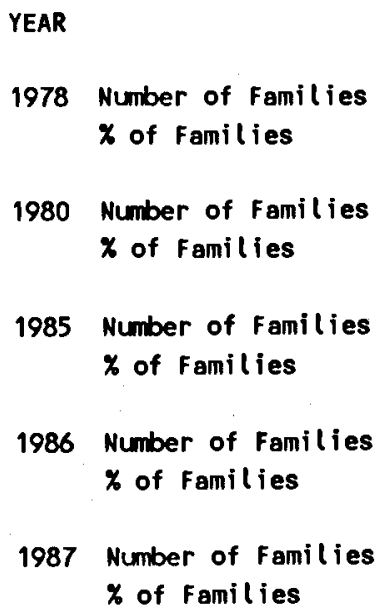

Number of Farmers Questioned 67 
PROVINCE OF LMN

\begin{tabular}{|c|c|c|c|c|c|}
\hline & $19 / 8$ & iצ̄ồu & 19Ẽ & 1980 & 1987 \\
\hline Average Family Labour per Farm & 1.81 & 1.76 & 1.52 & 1.46 & 1.42 \\
\hline $\begin{array}{l}\text { Average No. of Hired Labour per Farm } \\
\text { (for farms using hired labour) }\end{array}$ & 1.33 & 1.33 & 1.18 & 1.14 & 1.15 \\
\hline Number of Farms using Hired Labour & 15 & 15 & 17 & 22 & 20 \\
\hline
\end{tabular}

TABLE $8(B)$ - AVERAGE LABOUR (FAMILY and HIRED) FOR FARMERS IN CAMPS

PROVINCE OF LMN

$\begin{array}{lrrrrr}\text { Average Family Labour per Farm } & 2.45 & 2.21 & 1.88 & 1.51 & 0.31 \\ \begin{array}{l}\text { Average No. of Hired Labour per Farm } \\ \text { (for farms using hired labour) }\end{array} & 1.50 & 1.29 & 1.33 & 1.29 * \star \star \star * \\ \text { Number of Farms using Hired Labour } & 6 & 7 & 6 & 7 & 0 \\ \text { Number of Farmers Questioned } 67 & & & & & \end{array}$

$\begin{array}{lllll}1978 & 1980 & 1985 & 1986 & 1987\end{array}$

$2.45 \quad 2.21 \quad 1.88 \quad 1.51 \quad 0.31$ 
TABLE 9A - DIRECT EFFECTS OF THE WAR - FARMERS IN AFGANISTAN

PROVINCE OF LMN

Average Number of Livestock shot

Number of Formers reporting

Livestock Shot

Average Number of Livestock Killed

by Mines

Number of Farmers reporting Livestock killed by Mines

Number of Farmers Questioned 395

$\begin{array}{rrrrrr}1978 & 1980 & 1985 & 1986 & 1987 \\ 3.00 & 2.14 & 7.50 & 4.55 & 1.50 \\ 1 & 21 & 108 & 49 & 6 \\ * \star \star \star * & 1.80 & 3.09 & 3.18 & 1.00\end{array}$

$\begin{array}{lllll}0 & 5 & 23 & 11 & 4\end{array}$

\section{TABLE 9B - DIRECT EFFECTS OF THE WAR - FARMERS IN CAMPS}

PROVINCE OF LMN

Average Number of Livestock shot

Number of Farmers reporting

Livestock Shot

Average Number of Livestock Killed by Mines

Number of Farmers reporting Livestock killed by Mines

Number of Farmers Questioned 67

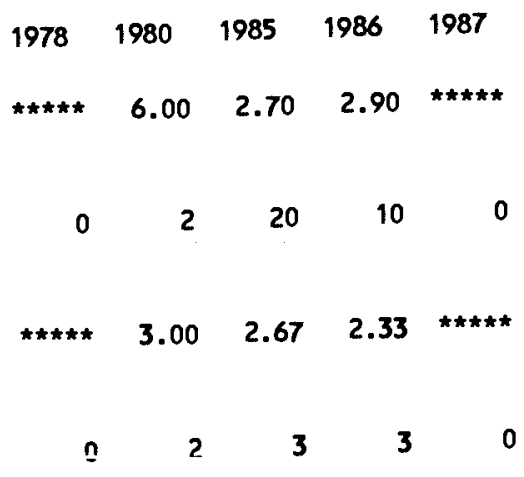




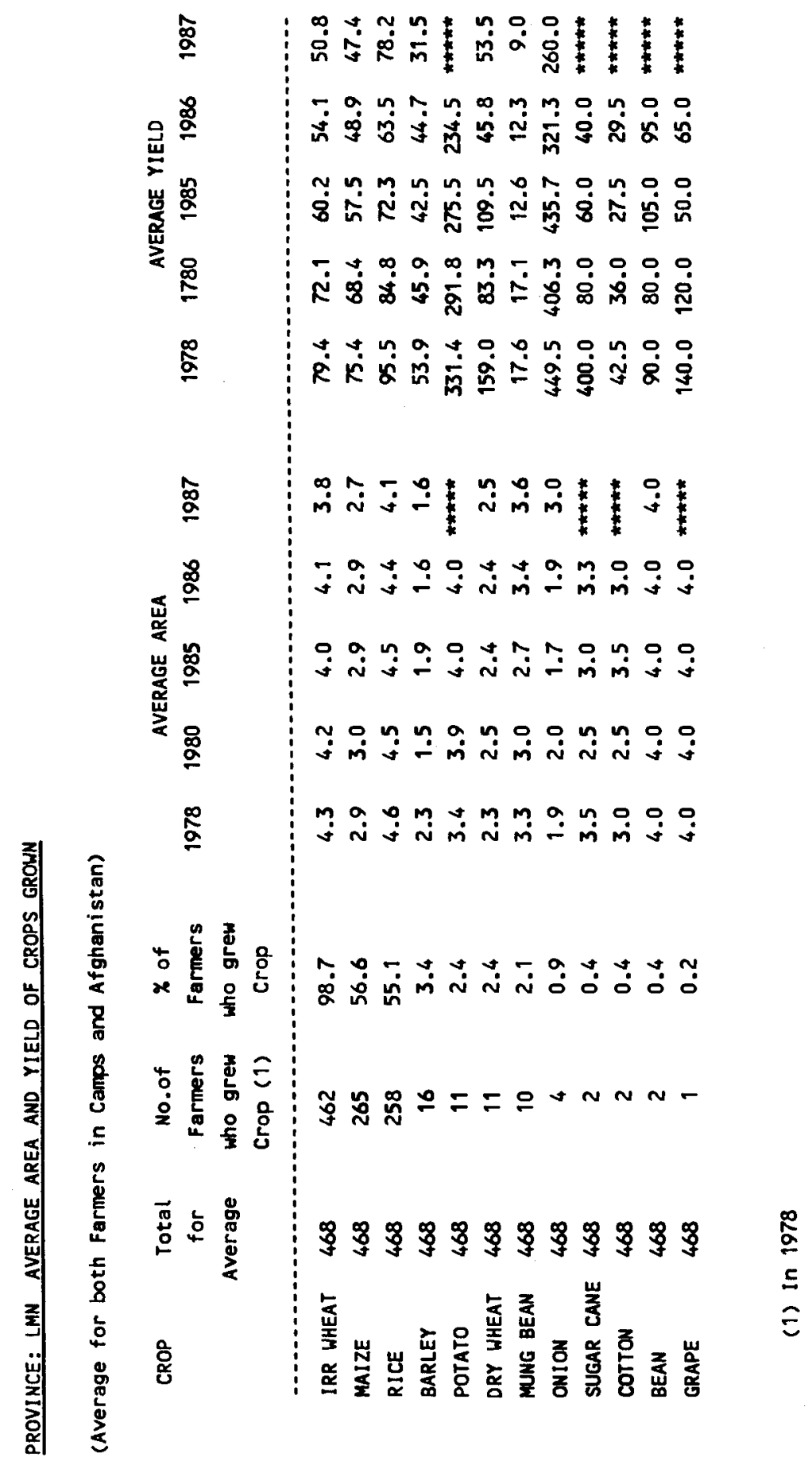


PROVINCE OF LMN

Rainfall

Crop Disease

Birds or Rats

Seed Availability

Fertilizer Availability

Crop Protection Chemicals

Credit

Irrigation Water Availability

Flooding

Labour Availabilty

Power for Land Preperation

Direct War Effects

Animal Diseases

Extension

Other Problems

$\begin{array}{rrrrrrrrrrrrr}1 & 2 & 3 & 4 & 5 & 6 & 7 & 8 & 9 & 10 & 11 & 12 & \text { Weighted Priority } \\ 0 & 0 & 0 & 0 & 0 & 0 & 0 & 0 & 0 & 0 & 0 & 0 & 0.00 \\ 0 & 20 & 0 & 40 & 20 & 0 & 0 & 0 & 0 & 0 & 0 & 0 & 10.68 \\ 0 & 0 & 0 & 0 & 0 & 0 & 20 & 0 & 0 & 0 & 0 & 0 & 1.27 \\ 20 & 0 & 0 & 0 & 0 & 0 & 0 & 0 & 0 & 0 & 0 & 0 & 8.90 \\ 0 & 0 & 40 & 20 & 0 & 0 & 0 & 0 & 0 & 0 & 0 & 0 & 8.16 \\ 0 & 0 & 20 & 0 & 0 & 0 & 0 & 0 & 0 & 0 & 0 & 0 & 2.97 \\ 0 & 0 & 0 & 0 & 40 & 0 & 0 & 20 & 0 & 0 & 0 & 0 & 4.67 \\ 20 & 0 & 0 & 0 & 0 & 0 & 0 & 0 & 0 & 0 & 0 & 0 & 8.90 \\ 0 & 20 & 0 & 0 & 0 & 0 & 0 & 0 & 0 & 0 & 0 & 0 & 4.45 \\ 0 & 0 & 0 & 0 & 0 & 0 & 0 & 0 & 0 & 0 & 0 & 0 & 0.00 \\ 40 & 20 & 0 & 0 & 0 & 0 & 0 & 0 & 0 & 0 & 0 & 0 & 22.25 \\ 0 & 40 & 20 & 20 & 0 & 0 & 0 & 0 & 0 & 0 & 0 & 0 & 14.09 \\ 20 & 0 & 0 & 0 & 20 & 20 & 0 & 0 & 0 & 0 & 0 & 0 & 12.16 \\ 0 & 0 & 0 & 0 & 0 & 0 & 0 & 0 & 0 & 0 & 0 & 0 & 0.00 \\ 0 & 0 & 0 & 0 & 0 & 20 & 0 & 0 & 0 & 0 & 0 & 0 & 1.48\end{array}$

1986

Rainfall

Crop Disease

Birds or Rats

Seed Availability

Fertilizer Availability

Crop Protection Chemicals

Credit

Irrigation Hater Availability

Flooding

Labour Availabilty

Power for Land Preparation

Direct Har Effects

Animal Diseases

Extension

Other Problems

1985

\section{Rainfall}

Crop Disease

Birds or Rats

Seed Availability

Fertilizer Availability

Crop Protection Chemicals

Credit

Irrigation Water Availability

Flooding

Labour Availabilty

Power for Land Preparation

Direct War Effects

Animal Diseases

Extension

other Problems

\begin{tabular}{rrrrrrrrrrrrr}
1 & 2 & 3 & 4 & 5 & 6 & 7 & 8 & 9 & 10 & 11 & 12 & Heighted Priority \\
\hline 0 & 0 & 0 & 0 & 0 & 0 & 0 & 0 & 0 & 0 & 0 & 0 & 0.00 \\
0 & 0 & 0 & 0 & 20 & 20 & 0 & 0 & 0 & 0 & 0 & 0 & 3.25 \\
0 & 0 & 0 & 0 & 0 & 0 & 0 & 0 & 0 & 0 & 0 & 0 & 0.00 \\
20 & 0 & 0 & 0 & 0 & 0 & 0 & 0 & 0 & 0 & 0 & 0 & 8.87 \\
0 & 40 & 20 & 0 & 0 & 0 & 0 & 0 & 0 & 0 & 0 & 0 & 11.82 \\
20 & 0 & 0 & 20 & 0 & 0 & 0 & 0 & 0 & 0 & 0 & 0 & 11.09 \\
0 & 0 & 0 & 0 & 40 & 0 & 20 & 0 & 0 & 0 & 0 & 0 & 4.81 \\
40 & 0 & 0 & 0 & 0 & 0 & 0 & 0 & 0 & 0 & 0 & 0 & 17.74 \\
0 & 20 & 20 & 0 & 0 & 0 & 0 & 0 & 0 & 0 & 0 & 0 & 7.39 \\
0 & 0 & 0 & 0 & 0 & 0 & 0 & 0 & 0 & 0 & 0 & 0 & 0.00 \\
0 & 0 & 0 & 40 & 0 & 0 & 0 & 0 & 0 & 0 & 0 & 0 & 4.43 \\
20 & 20 & 20 & 20 & 0 & 0 & 0 & 0 & 0 & 0 & 0 & 0 & 18.48 \\
0 & 20 & 20 & 0 & 0 & 20 & 0 & 0 & 0 & 0 & 0 & 0 & 8.87 \\
0 & 0 & 0 & 0 & 0 & 0 & 0 & 0 & 0 & 0 & 0 & 0 & 0.00 \\
0 & 0 & 0 & 0 & 20 & 20 & 0 & 0 & 0 & 0 & 0 & 0 & 3.25
\end{tabular}

$\begin{array}{rrrrrrrrrrrrr}1 & 2 & 3 & 4 & 5 & 6 & 7 & 8 & 9 & 10 & 11 & 12 & \text { Weighted Priority } \\ 0 & 0 & 0 & 0 & 0 & 0 & 0 & 0 & 0 & 0 & 0 & 0 & 0.00 \\ 0 & 0 & 0 & 0 & 0 & 0 & 0 & 0 & 0 & 0 & 0 & 0 & 0.00 \\ 20 & 0 & 0 & 40 & 0 & 0 & 0 & 0 & 0 & 0 & 0 & 0 & 19.07 \\ 0 & 0 & 0 & 0 & 0 & 0 & 0 & 0 & 0 & 0 & 0 & 0 & 0.00 \\ 0 & 40 & 0 & 0 & 0 & 0 & 0 & 0 & 0 & 0 & 0 & 0 & 12.71 \\ 0 & 0 & 0 & 0 & 0 & 0 & 0 & 0 & 0 & 0 & 0 & 0 & 0.00 \\ 0 & 20 & 20 & 0 & 0 & 20 & 0 & 0 & 0 & 0 & 0 & 0 & 12.71 \\ 0 & 0 & 0 & 0 & 0 & 0 & 0 & 0 & 0 & 0 & 0 & 0 & 0.00 \\ 0 & 0 & 0 & 0 & 0 & 0 & 0 & 0 & 0 & 0 & 0 & 0 & 0.00 \\ 0 & 0 & 0 & 0 & 0 & 0 & 0 & 0 & 0 & 0 & 0 & 0 & 0.00 \\ 0 & 0 & 0 & 0 & 0 & 0 & 0 & 0 & 0 & 0 & 0 & 0 & 0.00 \\ 60 & 20 & 0 & 0 & 0 & 0 & 0 & 0 & 0 & 0 & 0 & 0 & 44.49 \\ 0 & 0 & 20 & 0 & 0 & 0 & 0 & 0 & 0 & 0 & 0 & 0 & 4.24 \\ 0 & 0 & 0 & 0 & 0 & 0 & 0 & 0 & 0 & 0 & 0 & 0 & 0.00 \\ 0 & 0 & 20 & 0 & 20 & 0 & 0 & 0 & 0 & 0 & 0 & 0 & 6.78\end{array}$


PROVINCE OF LMN

Rainfall

\begin{tabular}{rrrrrrrrrrrrr}
1 & 2 & 3 & 4 & 5 & 6 & 7 & 8 & 9 & 10 & 11 & 12 & Weighted Priority \\
\hline 0 & 0 & 0 & 0 & 0 & 0 & 0 & 0 & 0 & 0 & 0 & 0 & 0.00 \\
0 & 0 & 0 & 0 & 0 & 0 & 0 & 0 & 0 & 0 & 0 & 0 & 0.00 \\
0 & 0 & 0 & 0 & 0 & 0 & 0 & 0 & 0 & 0 & 0 & 0 & 0.00 \\
0 & 0 & 0 & 0 & 0 & 0 & 0 & 0 & 0 & 0 & 0 & 0 & 0.00 \\
0 & 0 & 0 & 0 & 0 & 0 & 0 & 0 & 0 & 0 & 0 & 0 & 0.00 \\
0 & 20 & 0 & 0 & 0 & 0 & 0 & 0 & 0 & 0 & 0 & 0 & 17.65 \\
0 & 0 & 20 & 0 & 0 & 0 & 0 & 0 & 0 & 0 & 0 & 0 & 11.76 \\
0 & 0 & 0 & 0 & 0 & 0 & 0 & 0 & 0 & 0 & 0 & 0 & 0.00 \\
0 & 0 & 0 & 0 & 0 & 0 & 0 & 0 & 0 & 0 & 0 & 0 & 0.00 \\
0 & 0 & 0 & 0 & 0 & 0 & 0 & 0 & 0 & 0 & 0 & 0 & 0.00 \\
0 & 0 & 0 & 0 & 0 & 0 & 0 & 0 & 0 & 0 & 0 & 0 & 0.00 \\
40 & 0 & 0 & 0 & 0 & 0 & 0 & 0 & 0 & 0 & 0 & 0 & 70.59 \\
0 & 0 & 0 & 0 & 0 & 0 & 0 & 0 & 0 & 0 & 0 & 0 & 0.00 \\
0 & 0 & 0 & 0 & 0 & 0 & 0 & 0 & 0 & 0 & 0 & 0 & 0.00 \\
0 & 0 & 0 & 0 & 0 & 0 & 0 & 0 & 0 & 0 & 0 & 0 & 0.00
\end{tabular}

$\underline{1978}$

Rainfall

Crop Disease

Birds or Rats

Seed Availability

Fertilizer Availability

Crop Protection Chemicals

Credit

Irrigation Water Availability

Flooding

Labour Availabilty

Power for Land Preparation

Direct War Effects

Animal Diseases

Extension

other Problems

\begin{tabular}{|c|c|c|c|c|c|c|c|c|c|c|c|c|}
\hline 1 & 2 & 3 & 4 & 5 & 6 & 7 & 8 & 9 & 10 & 11 & 12 & Weighted Priority \\
\hline 0 & 0 & 0 & 0 & 0 & 0 & 0 & 0 & 0 & 0 & 0 & 0 & $\star \star \star \star \star \hbar$ \\
\hline 0 & 0 & 0 & 0 & 0 & 0 & 0 & 0 & 0 & 0 & 0 & 0 & $\star \star \star \star * *$ \\
\hline 0 & 0 & 0 & 0 & 0 & 0 & 0 & 0 & $\mathbf{0}$ & 0 & 0 & 0 & $\star \star \star \star \star \star$ \\
\hline 0 & 0 & 0 & 0 & 0 & 0 & 0 & 0 & 0 & 0 & 0 & 0 & 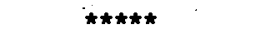 \\
\hline 0 & 0 & 0 & 0 & 0 & 0 & 0 & 0 & 0 & 0 & 0 & 0 & 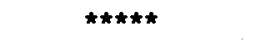 \\
\hline 0 & 0 & 0 & 0 & 0 & 0 & 0 & 0 & 0 & 0 & 0 & 0 & 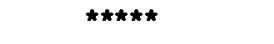 \\
\hline 0 & 0 & 0 & 0 & 0 & 0 & 0 & 0 & 0 & 0 & 0 & 0 & 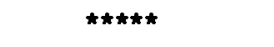 \\
\hline 0 & 0 & 0 & 0 & 0 & 0 & 0 & 0 & 0 & 0 & 0 & 0 & $\star \star \star \star \star \star$ \\
\hline 0 & 0 & 0 & 0 & 0 & 0 & 0 & 0 & 0 & 0 & 0 & 0 & 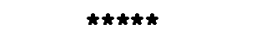 \\
\hline 0 & 0 & 0 & 0 & 0 & 0 & 0 & 0 & 0 & 0 & 0 & 0 & $\star \star \star \star \star \star$ \\
\hline 0 & 0 & 0 & 0 & 0 & 0 & 0 & 0 & 0 & 0 & 0 & 0 & 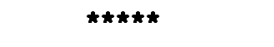 \\
\hline 0 & 0 & 0 & 0 & 0 & 0 & 0 & 0 & 0 & 0 & 0 & 0 & 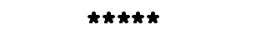 \\
\hline 0 & 0 & 0 & 0 & 0 & 0 & 0 & 0 & 0 & 0 & 0 & 0 & 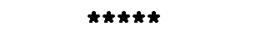 \\
\hline 0 & 0 & 0 & 0 & 0 & 0 & 0 & 0 & 0 & 0 & 0 & 0 & 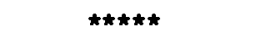 \\
\hline & 0 & 0 & 0 & 0 & 0 & 0 & 0 & 0 & 0 & 0 & 0 & $\star \star \star \star \star \star$ \\
\hline
\end{tabular}


NINGREHAR 


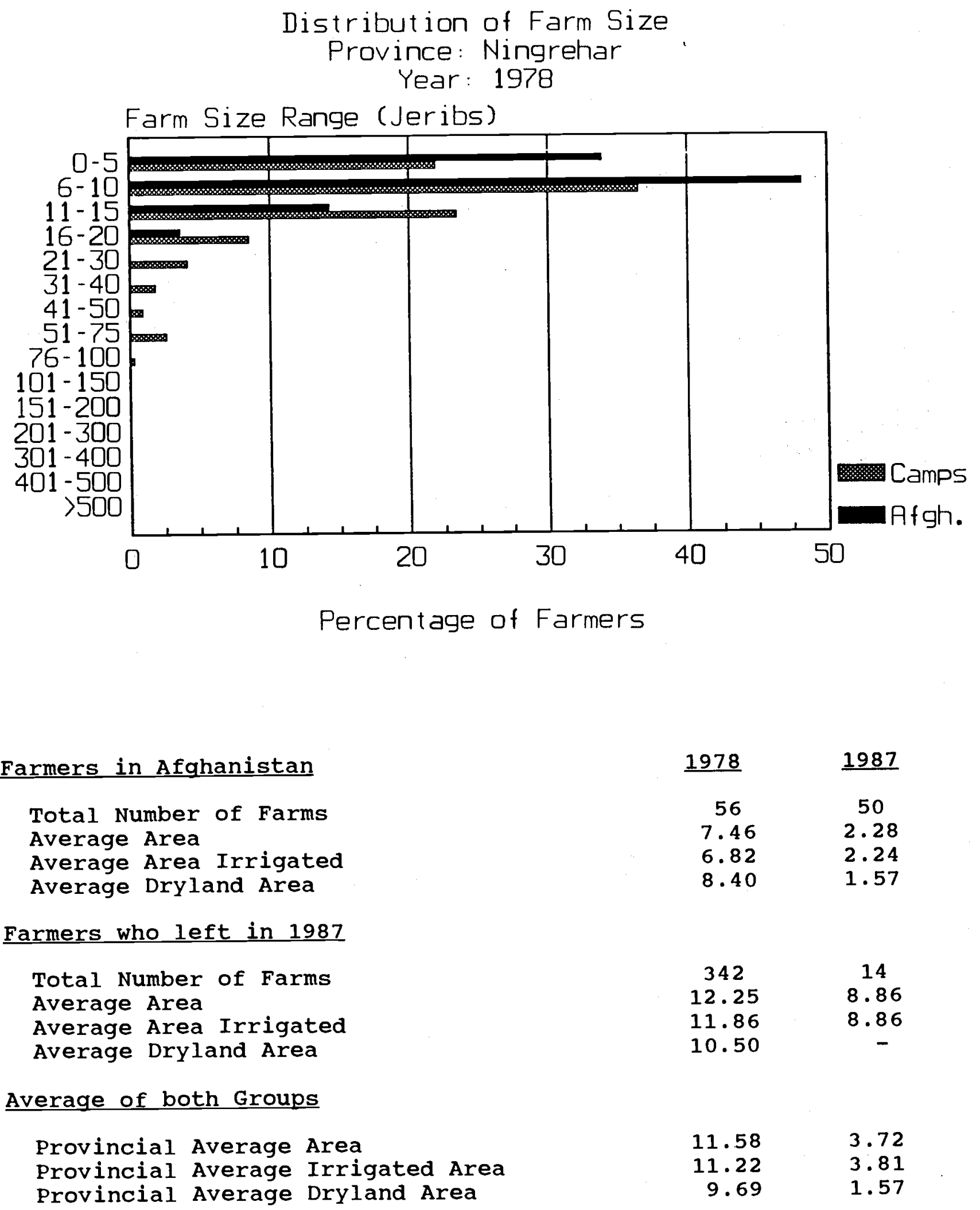

(All areas in Jeribs. 5 Jeribs $=1$ Hectare) 

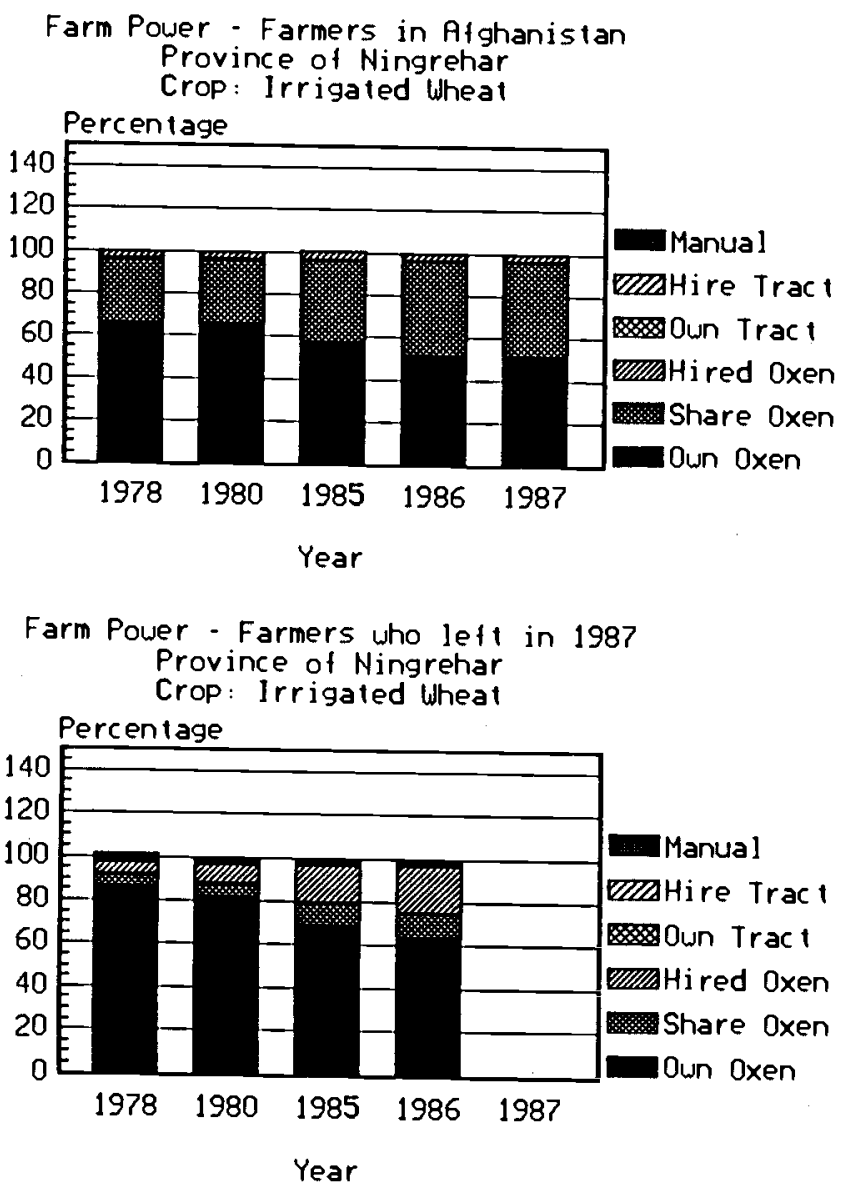

Base Figures for above graphs

Province: Ningrehar

Farmers in Afghanistan

Irr. Wheat Own oxen Shared oxen Hired oxen Own Tractor Hired Tractor Maize Manual Means Own Oxen Shared oxen Hired oxen Own Tractor Hired Tractor Manual Means

Farmers who left in 1987

Irr. Wheat Own oxen Shared oxen Hired oxen own Tractor Hired Tractor Maize Manual Means Own oxen Shared oxen Hired oxen Own Tractor Hired Tractor
Farm Power - Farmers in Atghanistan Province of Ningrehar Crop: Maize

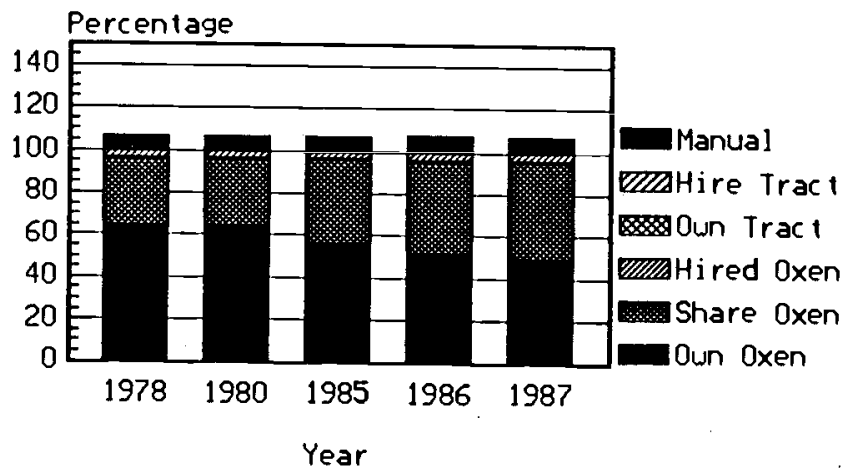

Farm Power - Farmers who left in 1987 Province of Ningrehar Crop: Maize

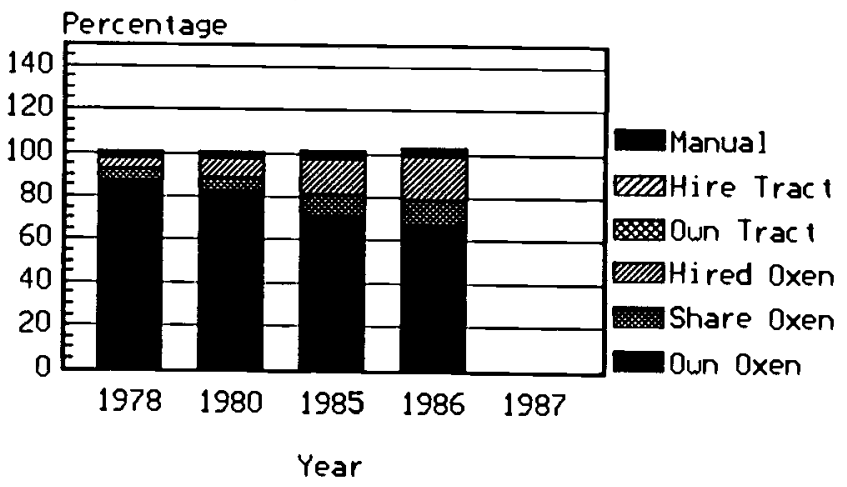

\begin{tabular}{|c|c|c|c|c|}
\hline 1978 & $\begin{array}{r}\text { Percen } \\
1980\end{array}$ & $\frac{\text { age of }}{1985}$ & $\frac{\text { Farmer }}{1986}$ & 1987 \\
\hline $\begin{array}{r}65.31 \\
30.61 \\
4.08\end{array}$ & $\begin{array}{r}65.31 \\
30.61 \\
4.08\end{array}$ & $\begin{array}{r}57.45 \\
38.30 \\
4.26\end{array}$ & $\begin{array}{r}51.11 \\
44.44 \\
4.44\end{array}$ & $\begin{array}{r}51.11 \\
44.44 \\
4.44\end{array}$ \\
\hline- & - & - & $\overline{-}$ & - \\
\hline - & - & - & - & - \\
\hline $\begin{array}{c}63.83 \\
31.91 \\
4.26 \\
-\end{array}$ & $\begin{array}{c}63.83 \\
31.91 \\
4.26 \\
-\end{array}$ & $\begin{array}{r}55.56 \\
40.00 \\
4.44\end{array}$ & $\begin{array}{c}51.16 \\
44.19 \\
4.65 \\
-\end{array}$ & $\begin{array}{c}48.84 \\
46.51 \\
4.65 \\
-\end{array}$ \\
\hline 6.38 & 6.38 & 6.67 & 6.98 & 6.98 \\
\hline
\end{tabular}

$\begin{array}{rrccc}85.94 & 81.45 & 68.28 & 63.53 & 58.33 \\ 5.63 & 6.29 & 11.00 & 10.98 & 25.00 \\ 5.94 & 8.49 & 17.15 & 21.96 & 16.67 \\ .31 & - & - & - & - \\ .63 & .94 & .32 & .78 & - \\ 2.50 & 2.52 & 2.27 & 2.75 & - \\ 86.67 & 82.16 & 70.70 & 66.67 & - \\ 5.19 & 6.32 & 10.16 & 11.59 & - \\ 5.56 & 8.55 & 16.80 & 20.29 & - \\ - & - & - & - & - \\ .37 & .74 & - & - & -\end{array}$


PROVINCE OF NGR

YEAR

1978 Number of Families

$x$ of Families

1980 Number of Families

$x$ of Families

1985 Number of Families

X of Families

1986 Number of Families

$x$ of Fanilies

1987 Number of Fanilies

$x$ of Fanilies

Average Number of Oxen per Family:

Number of Farmers Questioned 56
NUMBER OF TRAINED OXEN PER FAMILY

$\begin{array}{llllll}0 & 1 & 2 & 3 & 4\end{array}$

$\begin{array}{cccccc}3 & 21 & 32 & 0 & 0 & 0 \\ 5.36 & 37.50 & 57.14 & 0.00 & 0.00 & 0.00\end{array}$

$\begin{array}{llllll}3 & 20 & 33 & 0 & 0 & 0\end{array}$

$\begin{array}{llllll}5.36 & 35.71 & 58.93 & 0.00 & 0.00 & 0.00\end{array}$

$\begin{array}{llllll}7 & 22 & 27 & 0 & 0 & 0\end{array}$

$\begin{array}{llllll}12.50 & 39.29 & 48.21 & 0.00 & 0.00 & 0.00\end{array}$

$\begin{array}{llllll}9 & 24 & 23 & 0 & 0 & 0\end{array}$

$\begin{array}{llllll}16.07 & 42.86 & 41.07 & 0.00 & 0.00 & 0.00\end{array}$

$\begin{array}{llllll}9 & 24 & 23 & 0 & 0 & 0\end{array}$

$\begin{array}{llllll}16.07 & 42.86 & 41.07 & 0.00 & 0.00 & 0.00\end{array}$

$\begin{array}{ll}1978 & 1.52 \\ 1980 & 1.54 \\ 1985 & 1.36 \\ 1986 & 1.25 \\ 1987 & 1.25\end{array}$

IABLE 7B - AVERAGE NUMBER OF TRAINED OXEN FOR FARMERS IN THE CAMPS

PROVINCE OF NGR

\begin{tabular}{|c|c|c|c|c|c|c|c|}
\hline YEAR & & 0 & 1 & 2 & 3 & 4 & 5 \\
\hline 1978 & $\begin{array}{l}\text { Number of Families } \\
x \text { of Families }\end{array}$ & $\begin{array}{l}23 \\
7.26\end{array}$ & $\begin{array}{l}25 \\
7.89\end{array}$ & $\begin{array}{l}248 \\
78.23\end{array}$ & $\begin{array}{c}9 \\
2.84\end{array}$ & $\begin{array}{l}12 \\
3.79\end{array}$ & $\begin{array}{c}0 \\
0.00\end{array}$ \\
\hline 1980 & $\begin{array}{l}\text { Number of Families } \\
x \text { of Families }\end{array}$ & $\begin{array}{c}35 \\
11.04\end{array}$ & $\begin{array}{l}27 \\
8.52\end{array}$ & $\begin{array}{l}238 \\
75.08\end{array}$ & $\begin{array}{l}7 \\
2.21\end{array}$ & $\begin{array}{l}10 \\
3.15\end{array}$ & $\begin{array}{c}0 \\
0.00\end{array}$ \\
\hline 1985 & $\begin{array}{l}\text { Number of Families } \\
x \text { of Families }\end{array}$ & $\begin{array}{c}69 \\
21.43\end{array}$ & $\begin{array}{c}49 \\
15.22\end{array}$ & $\begin{array}{l}194 \\
60.25\end{array}$ & $1^{4}$ & $\begin{array}{c}6 \\
1.86\end{array}$ & $\begin{array}{l}0 \\
0.00\end{array}$ \\
\hline 1986 & $\begin{array}{l}\text { Number of Families } \\
x \text { of Families }\end{array}$ & $\begin{array}{l}131 \\
40.56\end{array}$ & $\begin{array}{c}45 \\
13.93\end{array}$ & $\begin{array}{l}142 \\
43.96\end{array}$ & $\begin{array}{c}1 \\
0.31\end{array}$ & ${ }^{4} .24$ & $\begin{array}{c}0 \\
0.00\end{array}$ \\
\hline 1987 & $\begin{array}{l}\text { Number of Families } \\
x \text { of Families }\end{array}$ & $\begin{array}{l}318 \\
98.45\end{array}$ & $\begin{array}{c}1 \\
0.31\end{array}$ & $\begin{array}{l}4 \\
1.24\end{array}$ & $\begin{array}{c}0 \\
0.00\end{array}$ & $\begin{array}{c}0 \\
0.00\end{array}$ & $\begin{array}{c}0 \\
0.00\end{array}$ \\
\hline Avera & ge Number of Oxen per Family: & $\begin{array}{l}1978 \\
1980 \\
1985 \\
1986 \\
1987\end{array}$ & & $\begin{array}{l}1.88 \\
1.78 \\
1.47 \\
1.08 \\
.03\end{array}$ & & & \\
\hline
\end{tabular}

Number of Farmers Questioned 323 
PROVINCE OF NGR

\begin{tabular}{|c|c|c|c|c|c|}
\hline & 1978 & 1980 & 1985 & 1986 & 1987 \\
\hline Average Family Labour per Farm & 2.04 & 2.02 & 1.71 & 1.38 & 1.27 \\
\hline $\begin{array}{l}\text { Average No. of Hired Labour per Farm } \\
\text { (for farms using hired (abour) }\end{array}$ & 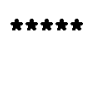 & 1.00 & 1.00 & $\star \star \star \star * *$ & $\star \star \star \star \star \star$ \\
\hline Number of Farms using Hired Labour & 0 & 1 & 1 & 0 & 0 \\
\hline Number of Farmers Questioned 56 & & & & & \\
\hline
\end{tabular}

IABLE 8(B) - AVERAGE LABOUR (FAMILY and HIRED) FOR FARMERS IN CAMPS

PROVINCE OF NGR

$\begin{array}{lllll}1978 & 1980 & 1985 & 1986 & 1987\end{array}$

Average Family Labour per Farm

$\begin{array}{lllll}2.85 & 2.54 & 1.85 & 1.34 & 0.01\end{array}$

Average No. of Hired Labour per Farm

$\begin{array}{lllll}1.95 & 1.74 & 1.21 & 1.15 & 1.00\end{array}$

(for farms using hired (abour)

Number of Farms using Hired Labour

$\begin{array}{lllll}44 & 44 & 52 & 40 & 1\end{array}$

Number of Farmers Questioned 323 
PROVINCE OF NGR

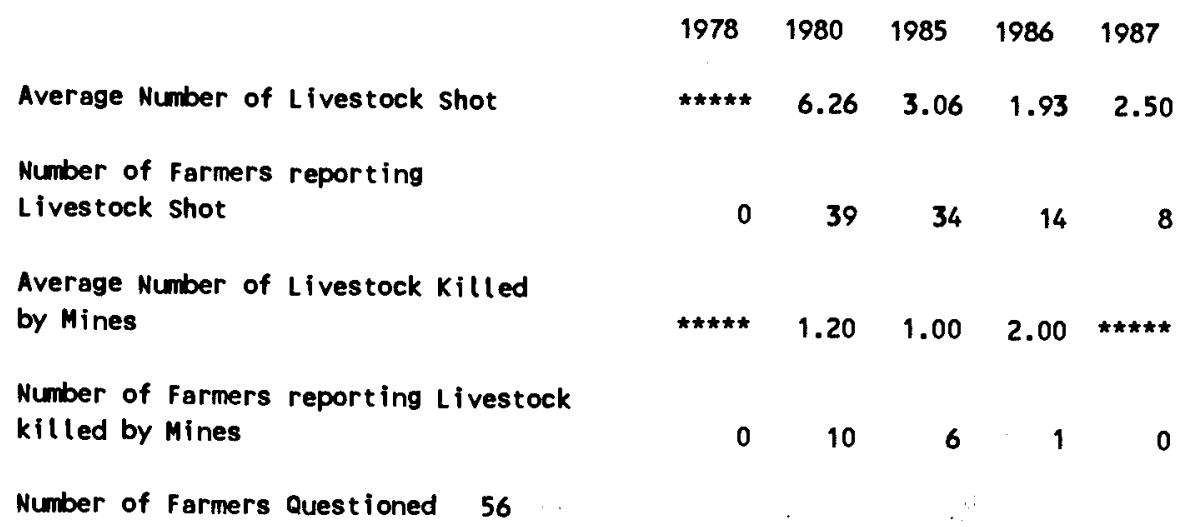

\section{IABLE 9B - DIRECT EFFECTS OF THE WAR - FARMERS IN CAMPS}

PROVINCE OF NGR

Average Number of Livestock Shot

Number of Farmers reporting

Livestock shot

Average Number of Livestock Killed

by Mines

Number of Farmers reporting Livestock killed by Mines

$\begin{array}{rrrrr}1978 & 1980 & 1985 & 1986 & 1987 \\ \star \star \star \star \star & 6.65 & 5.91 & 3.28 & \star \star \star \star \star \\ 0 & 51 & 102 & 36 & 0\end{array}$

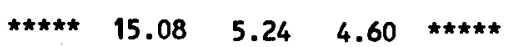

$\begin{array}{lllll}0 & 13 & 29 & 15 & 0\end{array}$

Number of Farmers Questioned 323 


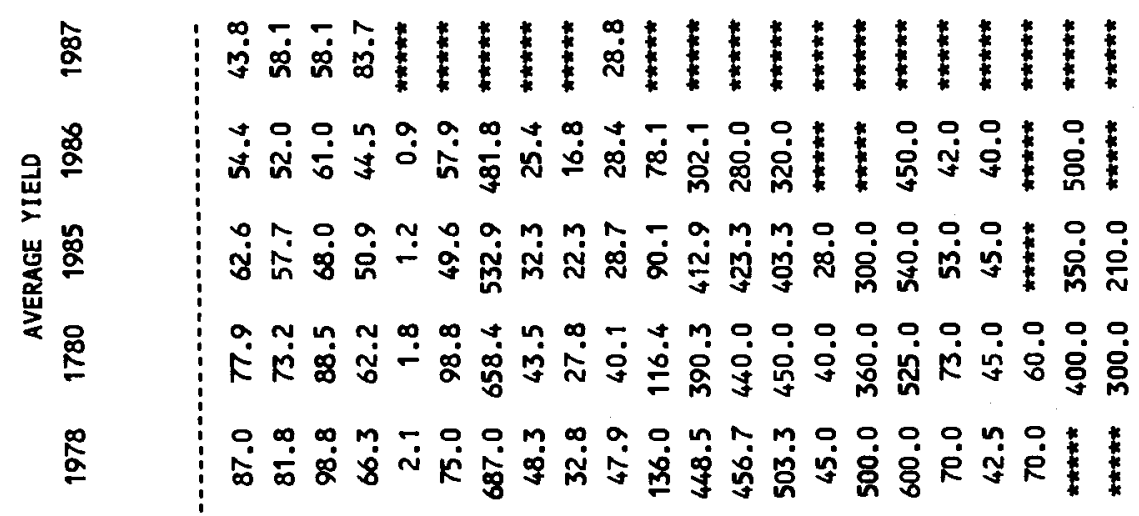

产

จ்ุ

䢓

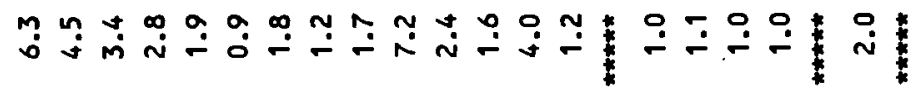

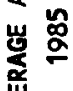

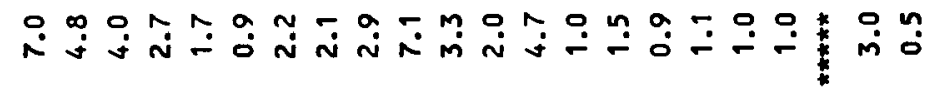

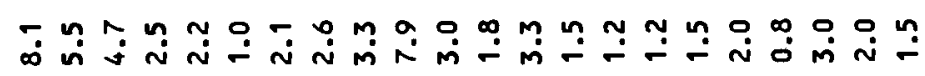

品

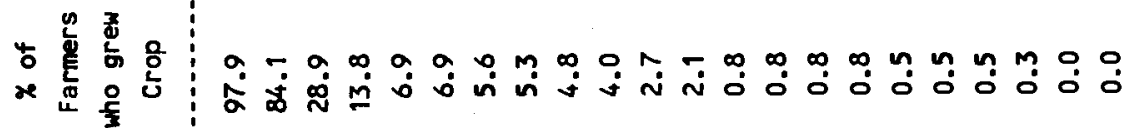

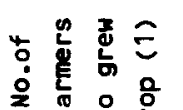

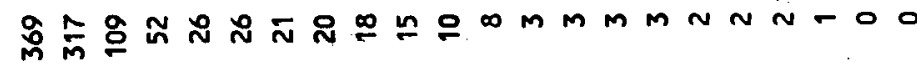

孪

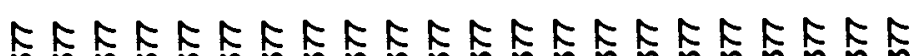

号

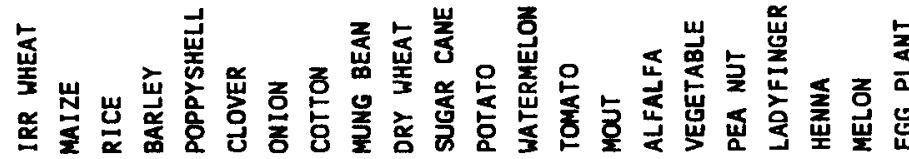


PROVINCE OF NGR

PERCENTAGE OF VILLAGES GIVING FOLLOWING PRIORITY TO PROBLEM

Rainfall

Crop Disease

Birds or Rats

Seed Availability

Fertilizer Availability

Crop Protection Chemicals

Credit

Irrigation Water Availability

Flooding

Labour Availabilty

Power for Land Preparation

Direct Her Effects

Animal Diseases

Extension

Other Problems

\begin{tabular}{rrrrrrrrrrrrr}
1 & 2 & 3 & 4 & 5 & 6 & 7 & 8 & 9 & 10 & 11 & 12 & Weighted Priority \\
\hline 0 & 0 & 0 & 0 & 0 & 0 & 0 & 0 & 0 & 0 & 0 & 0 & 0.00 \\
0 & 0 & 0 & 0 & 0 & 100 & 0 & 0 & 0 & 0 & 0 & 0 & 6.80 \\
0 & 0 & 0 & 100 & 0 & 0 & 0 & 0 & 0 & 0 & 0 & 0 & 10.20 \\
0 & 0 & 0 & 0 & 0 & 0 & 0 & 0 & 0 & 0 & 0 & 0 & 0.00 \\
0 & 0 & 0 & 0 & 0 & 0 & 0 & 0 & 0 & 0 & 0 & 0 & 0.00 \\
0 & 0 & 50 & 0 & 0 & 0 & 0 & 0 & 0 & 0 & 0 & 0 & 6.80 \\
0 & 0 & 0 & 0 & 0 & 0 & 0 & 0 & 0 & 0 & 0 & 0 & 0.00 \\
0 & 0 & 0 & 0 & 0 & 0 & 0 & 0 & 0 & 0 & 0 & 0 & 0.00 \\
0 & 0 & 0 & 0 & 50 & 0 & 0 & 0 & 0 & 0 & 0 & 0 & 4.08 \\
0 & 0 & 50 & 0 & 50 & 0 & 0 & 0 & 0 & 0 & 0 & 0 & 10.88 \\
0 & 0 & 0 & 0 & 0 & 0 & 0 & 0 & 0 & 0 & 0 & 0 & 0.00 \\
0 & 100 & 0 & 0 & 0 & 0 & 0 & 0 & 0 & 0 & 0 & 0 & 20.41 \\
0 & 0 & 0 & 0 & 0 & 0 & 0 & 0 & 0 & 0 & 0 & 0 & 0.00 \\
100 & 0 & 0 & 0 & 0 & 0 & 0 & 0 & 0 & 0 & 0 & 0 & 40.82 \\
0 & 0 & 0 & 0 & 0 & 0 & 0 & 0 & 0 & 0 & 0 & 0 & 0.00
\end{tabular}

1986

Rainfall

Crop Disease

Birds or Rats

Seed Avai lability

Fertilizer Availability

Crop Protection Chemicals

Credit

Irrigation Hater Availability

Flooding

Labour Avail labilty

Power for Land Preparation

Direct War Effects

Animal Diseases

Extension

other Problems

$\begin{array}{rrrrrrrrrrrrr}1 & 2 & 3 & 4 & 5 & 6 & 7 & 8 & 9 & 10 & 11 & 12 & \text { Heighted Priority } \\ 0 & 0 & 0 & 0 & 0 & 0 & 0 & 0 & 0 & 0 & 0 & 0 & 0.00 \\ 0 & 0 & 0 & 100 & 0 & 0 & 0 & 0 & 0 & 0 & 0 & 0 & 10.56 \\ 0 & 0 & 0 & 0 & 100 & 0 & 0 & 0 & 0 & 0 & 0 & 0 & 8.45 \\ 0 & 0 & 0 & 0 & 0 & 0 & 0 & 0 & 0 & 0 & 0 & 0 & 0.00 \\ 0 & 0 & 0 & 0 & 0 & 0 & 0 & 0 & 0 & 0 & 0 & 0 & 0.00 \\ 50 & 0 & 0 & 0 & 0 & 0 & 0 & 0 & 0 & 0 & 0 & 0 & 21.13 \\ 0 & 0 & 0 & 0 & 0 & 0 & 0 & 0 & 0 & 0 & 0 & 0 & 0.00 \\ 0 & 0 & 0 & 0 & 0 & 0 & 0 & 0 & 0 & 0 & 0 & 0 & 0.00 \\ 0 & 0 & 50 & 0 & 0 & 0 & 0 & 0 & 0 & 0 & 0 & 0 & 7.04 \\ 50 & 0 & 50 & 0 & 0 & 0 & 0 & 0 & 0 & 0 & 0 & 0 & 28.17 \\ 0 & 0 & 0 & 0 & 0 & 0 & 0 & 0 & 0 & 0 & 0 & 0 & 0.00 \\ 0 & 100 & 0 & 0 & 0 & 0 & 0 & 0 & 0 & 0 & 0 & 0 & 21.13 \\ 0 & 0 & 0 & 0 & 0 & 0 & 0 & 0 & 0 & 0 & 0 & 0 & 0.00 \\ 0 & 0 & 0 & 0 & 0 & 50 & 0 & 0 & 0 & 0 & 0 & 0 & 3.52 \\ 0 & 0 & 0 & 0 & 0 & 0 & 0 & 0 & 0 & 0 & 0 & 0 & 0.00\end{array}$

1985

Rainfall

Crop Disease

Birds or Rats

Seed Availability

Fertilizer Avai lability

Crop Protection chemicals

Credit

Irrigation Water Availability

Flooding

Labour Availabilty

Power for Land Preparation

Direct War Effects

Animal Diseases

Extension

Other Problems

\begin{tabular}{rrrrrrrrrrrrr}
1 & 2 & 3 & 4 & 5 & 6 & 7 & 8 & 9 & 10 & 11 & 12 & Heighted Priority \\
\hline 0 & 0 & 0 & 0 & 0 & 0 & 0 & 0 & 0 & 0 & 0 & 0 & 0.00 \\
100 & 0 & 0 & 0 & 0 & 0 & 0 & 0 & 0 & 0 & 0 & 0 & 40.82 \\
0 & 0 & 0 & 0 & 0 & 100 & 0 & 0 & 0 & 0 & 0 & 0 & 6.80 \\
0 & 0 & 0 & 0 & 0 & 0 & 0 & 0 & 0 & 0 & 0 & 0 & 0.00 \\
0 & 0 & 0 & 0 & 0 & 0 & 0 & 0 & 0 & 0 & 0 & 0 & 0.00 \\
0 & 0 & 50 & 0 & 0 & 0 & 0 & 0 & 0 & 0 & 0 & 0 & 6.80 \\
0 & 0 & 0 & 0 & 0 & 0 & 0 & 0 & 0 & 0 & 0 & 0 & 0.00 \\
0 & 0 & 0 & 0 & 0 & 0 & 0 & 0 & 0 & 0 & 0 & 0 & 0.00 \\
0 & 0 & 0 & 50 & 0 & 0 & 0 & 0 & 0 & 0 & 0 & 0 & 5.10 \\
0 & 0 & 50 & 50 & 0 & 0 & 0 & 0 & 0 & 0 & 0 & 0 & 11.90 \\
0 & 0 & 0 & 0 & 0 & 0 & 0 & 0 & 0 & 0 & 0 & 0 & 0.00 \\
0 & 100 & 0 & 0 & 0 & 0 & 0 & 0 & 0 & 0 & 0 & 0 & 20.41 \\
0 & 0 & 0 & 0 & 0 & 0 & 0 & 0 & 0 & 0 & 0 & 0 & 0.00 \\
0 & 0 & 0 & 0 & 100 & 0 & 0 & 0 & 0 & 0 & 0 & 0 & 8.16 \\
0 & 0 & 0 & 0 & 0 & 0 & 0 & 0 & 0 & 0 & 0 & 0 & 0.00
\end{tabular}


IABLE 1-5(contd) - ANALYSIS OF GREATEST FARMING PROBLEMS - COMMUNITY SURVEY

PROVINCE OF NGR

Rainfall

Crop Disease

Birds or Rats

Seed Availability

Fertilizer Availability

Crop Protection Chemicals

Credit

Irrigation Water Availability

Flooding

Labour Availabilty

Power for Land Preparation

Direct War Effects

Animal Diseases

Extension

Other Problems

\begin{tabular}{rrrrrrrrrrrrr}
1 & 2 & 3 & 4 & 5 & 6 & 7 & 8 & 9 & 10 & 11 & 12 & Weighted Priority \\
\hline 0 & 0 & 0 & 0 & 0 & 0 & 0 & 0 & 0 & 0 & 0 & 0 & 0.00 \\
0 & 0 & 100 & 0 & 0 & 0 & 0 & 0 & 0 & 0 & 0 & 0 & 13.61 \\
0 & 0 & 0 & 0 & 0 & 100 & 0 & 0 & 0 & 0 & 0 & 0 & 6.80 \\
0 & 0 & 0 & 0 & 0 & 0 & 0 & 0 & 0 & 0 & 0 & 0 & 0.00 \\
0 & 0 & 0 & 0 & 0 & 0 & 0 & 0 & 0 & 0 & 0 & 0 & 0.00 \\
0 & 50 & 0 & 0 & 0 & 0 & 0 & 0 & 0 & 0 & 0 & 0 & 10.20 \\
0 & 0 & 0 & 0 & 0 & 0 & 0 & 0 & 0 & 0 & 0 & 0 & 0.00 \\
0 & 0 & 0 & 0 & 0 & 0 & 0 & 0 & 0 & 0 & 0 & 0 & 0.00 \\
0 & 0 & 0 & 0 & 50 & 0 & 0 & 0 & 0 & 0 & 0 & 0 & 4.08 \\
0 & 50 & 0 & 0 & 50 & 0 & 0 & 0 & 0 & 0 & 0 & 0 & 14.29 \\
0 & 0 & 0 & 0 & 0 & 0 & 0 & 0 & 0 & 0 & 0 & 0 & 0.00 \\
100 & 0 & 0 & 0 & 0 & 0 & 0 & 0 & 0 & 0 & 0 & 0 & 40.82 \\
0 & 0 & 0 & 0 & 0 & 0 & 0 & 0 & 0 & 0 & 0 & 0 & 0.00 \\
0 & 0 & 0 & 100 & 0 & 0 & 0 & 0 & 0 & 0 & 0 & 0 & 10.20 \\
0 & 0 & 0 & 0 & 0 & 0 & 0 & 0 & 0 & 0 & 0 & 0 & 0.00
\end{tabular}

1978

Rainfall

Crop Disease

Birds or Rats

Seed Availability

Fertilizer Availability

Crop Protection Chemicals

Credit

Irrigation Water Availability

flooding

Labour Availabilty

Power for Land Preparation

Direct War Effects

Animal Diseases

Extension

Other Problems

\begin{tabular}{lllllllllllll}
1 & 2 & 3 & 4 & 5 & 6 & 7 & 8 & 9 & 10 & 11 & 12 & Weighted Priority \\
\hline 0 & 0 & 0 & 0 & 0 & 0 & 0 & 0 & 0 & 0 & 0 & 0 & $* * * *$ \\
0 & 0 & 0 & 0 & 0 & 0 & 0 & 0 & 0 & 0 & 0 & 0 & $* * * * *$ \\
0 & 0 & 0 & 0 & 0 & 0 & 0 & 0 & 0 & 0 & 0 & 0 & $* * * * *$ \\
0 & 0 & 0 & 0 & 0 & 0 & 0 & 0 & 0 & 0 & 0 & 0 & $* * * * *$ \\
0 & 0 & 0 & 0 & 0 & 0 & 0 & 0 & 0 & 0 & 0 & 0 & $* * * * *$ \\
0 & 0 & 0 & 0 & 0 & 0 & 0 & 0 & 0 & 0 & 0 & 0 & $* * * * *$ \\
0 & 0 & 0 & 0 & 0 & 0 & 0 & 0 & 0 & 0 & 0 & 0 & $* * * * *$ \\
0 & 0 & 0 & 0 & 0 & 0 & 0 & 0 & 0 & 0 & 0 & 0 & $* * * * *$ \\
0 & 0 & 0 & 0 & 0 & 0 & 0 & 0 & 0 & 0 & 0 & 0 & $* * * * *$ \\
0 & 0 & 0 & 0 & 0 & 0 & 0 & 0 & 0 & 0 & 0 & 0 & $* * * * *$ \\
0 & 0 & 0 & 0 & 0 & 0 & 0 & 0 & 0 & 0 & 0 & 0 & $* * * *$ \\
0 & 0 & 0 & 0 & 0 & 0 & 0 & 0 & 0 & 0 & 0 & 0 & $* * * * *$ \\
0 & 0 & 0 & 0 & 0 & 0 & 0 & 0 & 0 & 0 & 0 & 0 & $* * * * *$ \\
0 & 0 & 0 & 0 & 0 & 0 & 0 & 0 & 0 & 0 & 0 & 0 & $* * * * *$ \\
0 & 0 & 0 & 0 & 0 & 0 & 0 & 0 & 0 & 0 & 0 & 0 & $* * * * *$
\end{tabular}




\section{NIMRUZ}




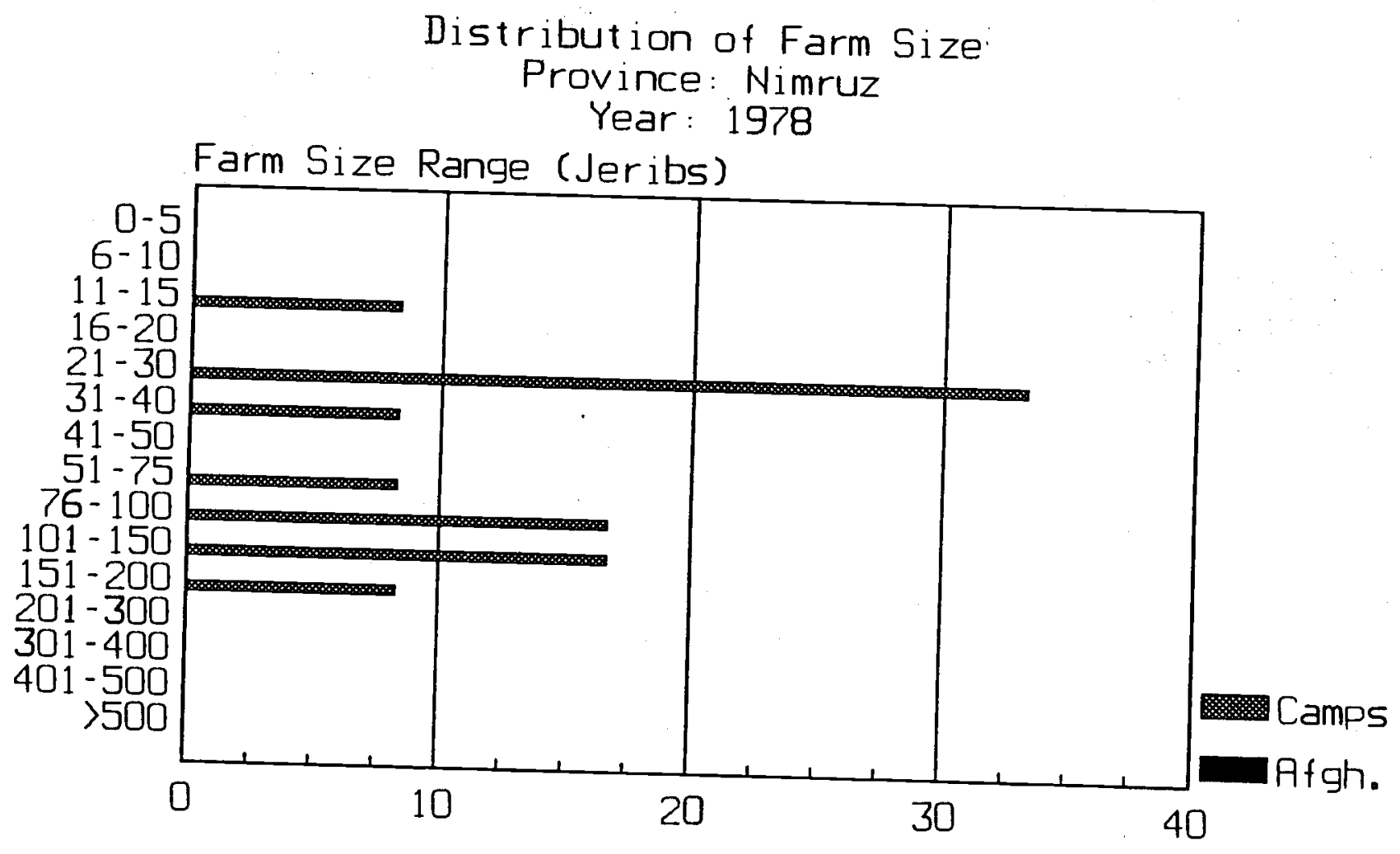

Percentage of Farmers

Farmers in Afghanistans

Total Number of Farms

Average Area

Average Area Irrigated

Average Dryland Area

Farmers who left in 1987

Total Number of Farms

Average Area

Average Area Irrigated

Average Dryland Area

Average of both Groups

\begin{abstract}
Provincial Average Area
Provincial Average Irrigated Area

Provincial Average Dryland Area
\end{abstract}

$\underline{1978}$

$\underline{1987}$

$\begin{array}{ll}0 & 0 \\ - & -\end{array}$

$\begin{array}{cc}12 & 0 \\ 68.90 & 0 \\ - & -\end{array}$

68.92

68.92

(All areas in Jeribs. 5 Jeribs $=1$ Hectare) 
Farm Power - Farmers in Alghanistan

Province of Nimruz

Crop: Irrigated wheat

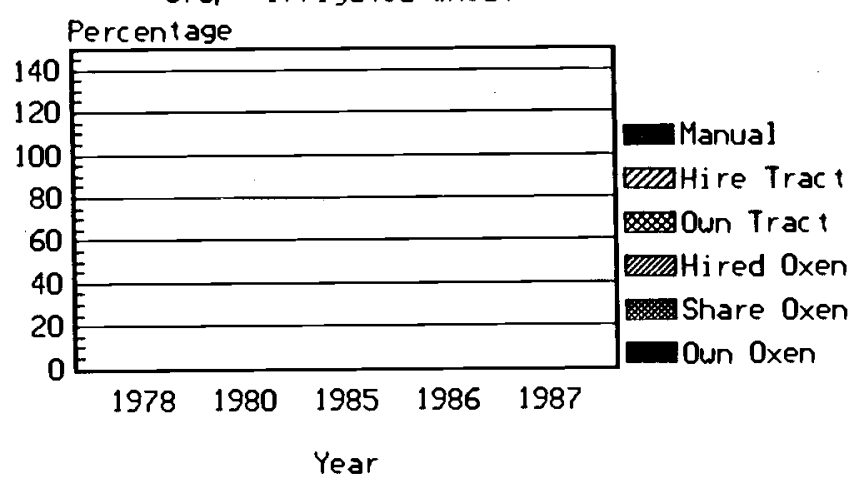

Farm Power - Farmers who lett in 1987

Province of Nimruz

Crop: Irrigated wheat

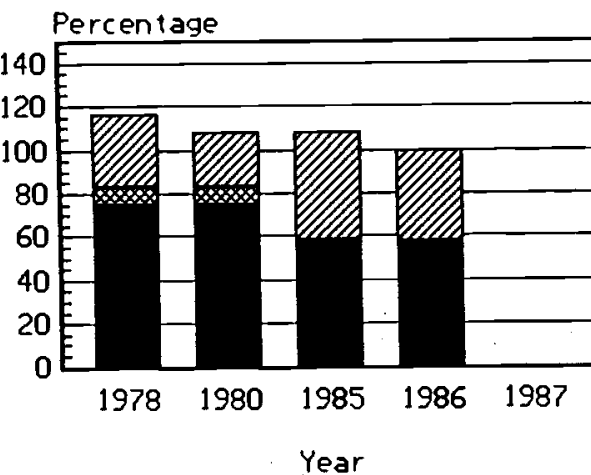

Base Figures for above graphs

Province: Nimruz

Farmers in Afghanistan

Irr. Wheat Own oxen

Shared oxen

Hired oxen

Own Tractor

Hired Tractor

Maize

Manual Means

own oxen

Shared oxen

Hired oxen

own Tractor

Hired Tractor

Manual Means

Farmers who left in 1987

Irr. Wheat own oxen

Shared oxen

Hired Oxen

Own Tractor

Hired Tractor

Maize

Manual Means

own oxen

Shared oxen

Hired oxen

nwn Tractor
Farm Power - Farmers in Atghanistan

Province of Nimruz

Crop: Maize

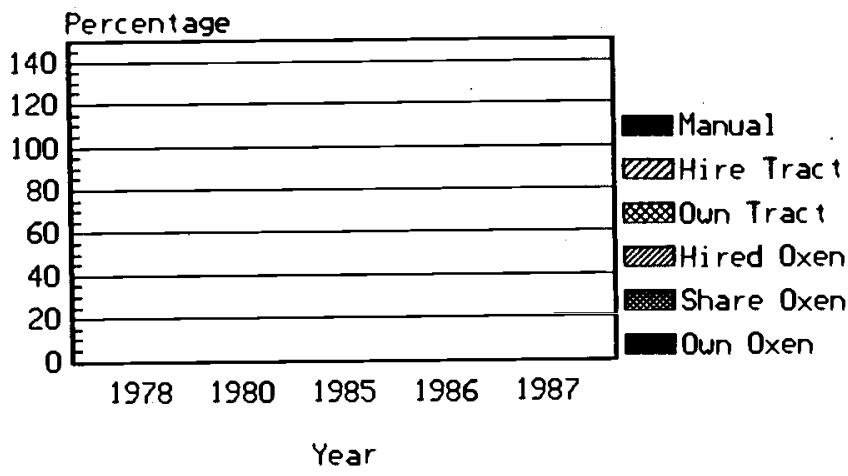

Farm Power - Farmers who left in 1987

Province of Nimruz

Crop: Maize

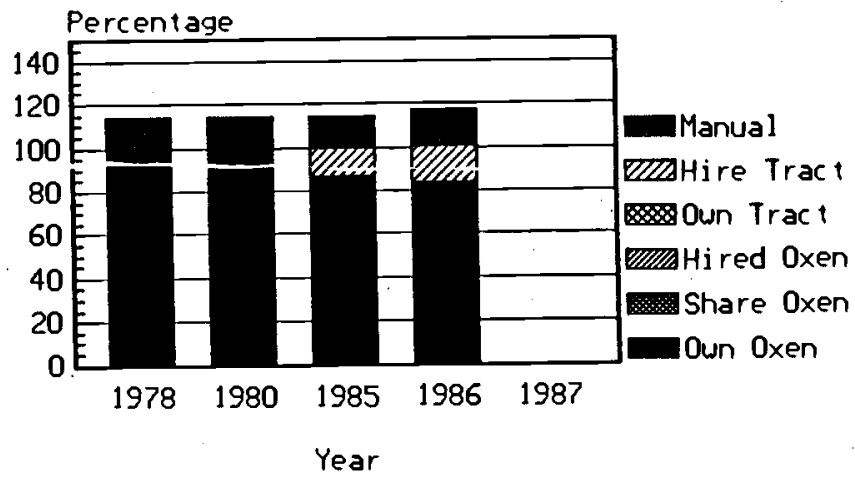

$\begin{array}{lllll}- & - & - & - & - \\ - & - & - & - & - \\ - & - & - & - & - \\ - & - & - & - & - \\ - & - & - & - & - \\ - & - & - & - & - \\ - & - & - & - & - \\ - & - & - & - & - \\ - & - & - & - & -\end{array}$

$\begin{array}{ccccc}75.00 & 75.00 & 58.33 & 57.14 & - \\ - & - & - & - & - \\ - & - & - & - & - \\ 8.33 & 8.33 & - & - & - \\ 33.33 & 25.00 & 50.00 & 42.86 & - \\ - & - & - & - & - \\ 100.00 & 100.00 & 85.71 & 83.33 & - \\ - & - & - & - & - \\ - & - & - & - & - \\ - & - & - & - & -\end{array}$



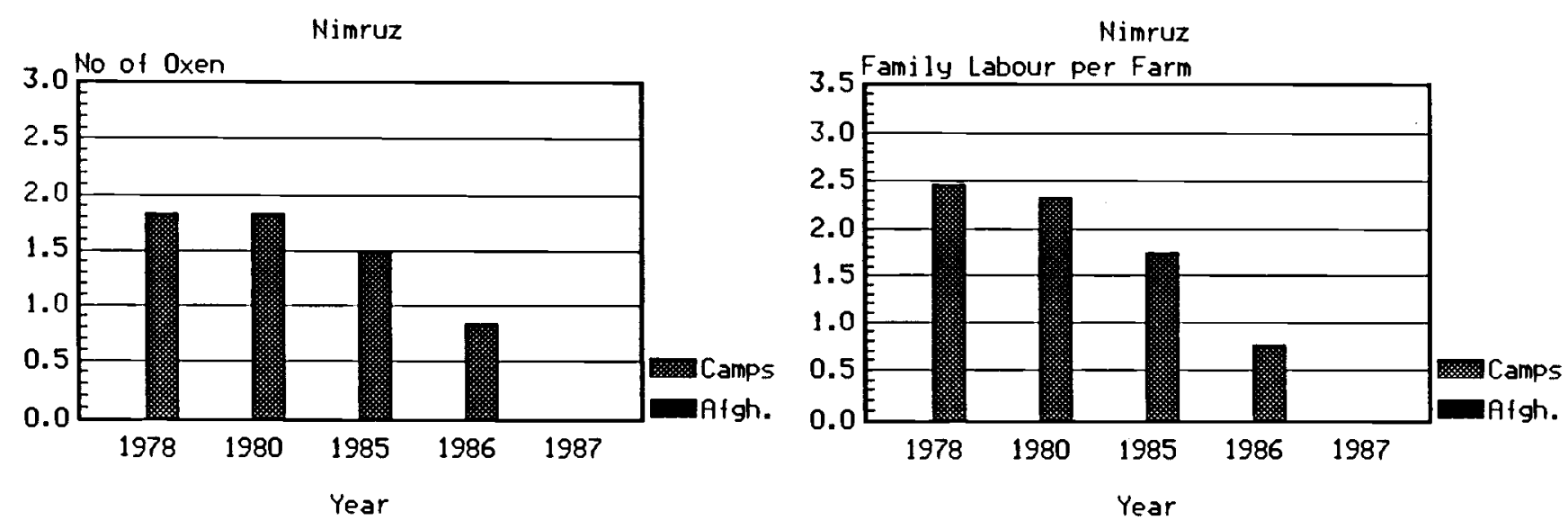

Nimruz

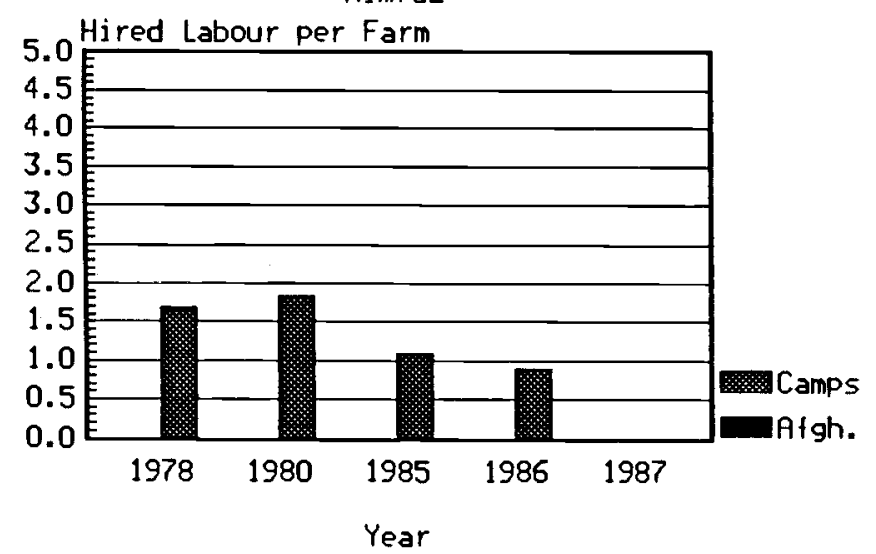

Nimroz

Percentage of Farms using

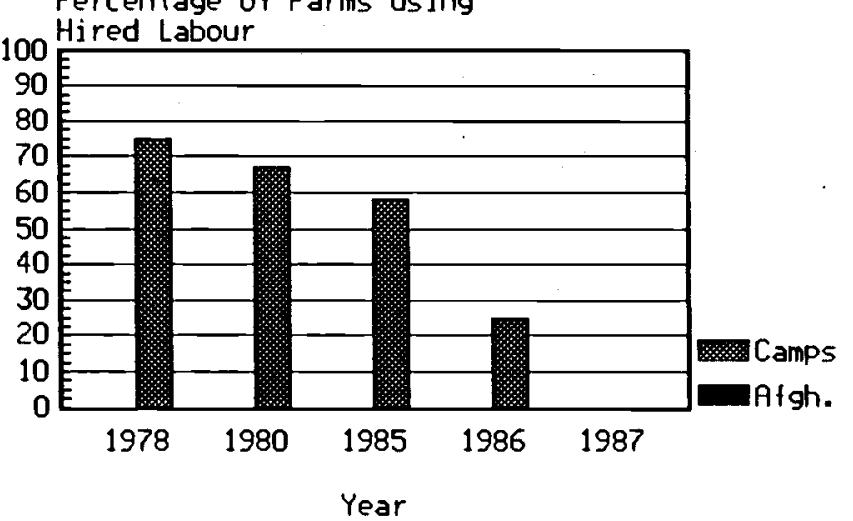


PROVINCE OF NRZ

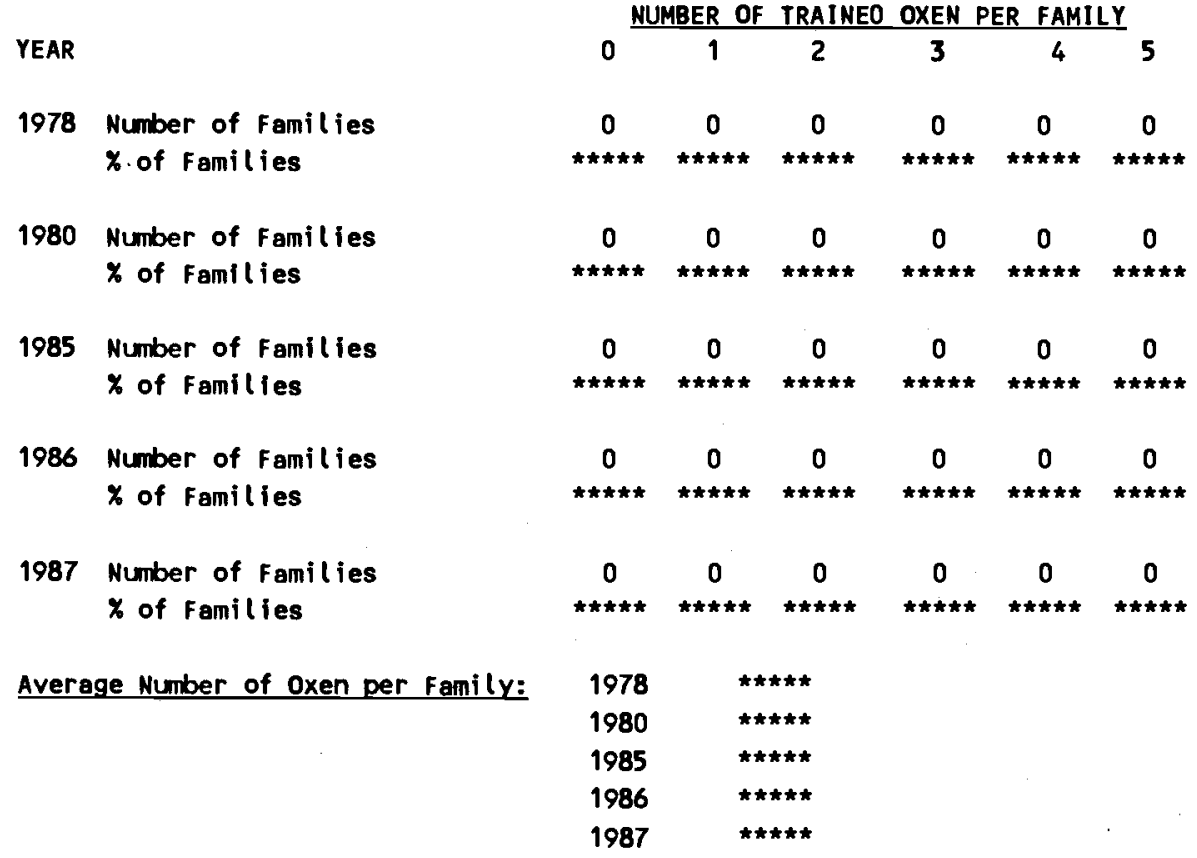

Number of Farmers Questioned 0

\section{IABLE 7B - AVERAGE NUMBER OF TRAINED OXEN FOR FARMERS IN THE CAMPS}

PROVINCE OF NRZ

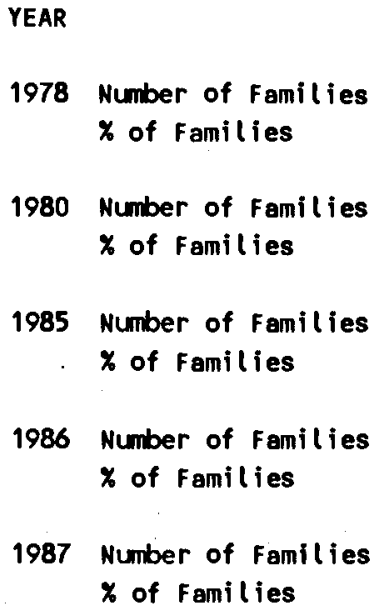

$\begin{array}{lll}\text { Average Number of Oxen per Family: } & 1978 & 1.83 \\ & 1980 & 1.83 \\ 1985 & 1.50 \\ & 1986 & 0.83 \\ & 1987 & 0.00\end{array}$

Number of Farmers Questioned 12 
IABLE $8(A)$ - AVERAGE LABOUR (FAMILY and HIREO) FOR FARMERS IN AFGANISTAN

PROVINCE OF NRZ

Average Family Labour per Farm

$\begin{array}{lllll}1978 & 1980 & 1985 & 1986 & 1987\end{array}$

Average No. of Hired Labour per Farm

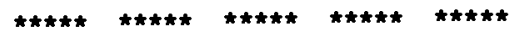

(for farms using hired labour)

Number of Farms using Hired Labour

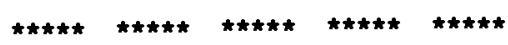

Number of Farmers Questioned 0

IABLE $8(B)$ - AVERAGE LABOUR. (FAMILY and HIREO) FOR FARMERS IN CAMPS

PROVIHCE OF NRZ

Average Family Labour per Farm

Average No. of Hired Labour per Farm

(for farms using hired labour)

Number of Farms using Hired Labour

Number of Farmers Questioned 12

$\begin{array}{lllll}1978 & 1980 & 1985 & 1986 & 1987 \\ 2.46 & 2.33 & 1.75 & 0.75 & 0.00 \\ 1.68 & 1.83 & 1.07 & 0.89 & \star \star \star \star \star\end{array}$

$\begin{array}{lllll}9 & 8 & 7 & 3 & 0\end{array}$ 
PROVINCE OF NRZ

Average Number of Livestock Shot

$\begin{array}{lllll}1978 & 1980 & 1985 & 1986 & 1987 \\ \star \star \star \star \star & \star \star \star \star \star & \star \star \star \star \star & \star \star \star \star \star & \star \star \star \star \star\end{array}$

Number of Farmers reporting

Livestock Shot

$\begin{array}{lllll}0 & 0 & 0 & 0 & 0\end{array}$

Average Number of Livestock Killed by Mines

Number of Farmers reporting Livestock killed by Mines

$\begin{array}{lllll}0 & 0 & 0 & 0 & 0\end{array}$

Number of Farmers Questioned 0

IABLE 9B - DIRECT EFFECTS OF THE WAR - FARMERS IN CAMPS

PROVINCE OF NRZ

Average Number of Livestock Shot

Number of Farmers reporting

Livestock Shot

Average Number of Livestock Killed by Mines

Number of Farmers reporting Livestock killed by Mines

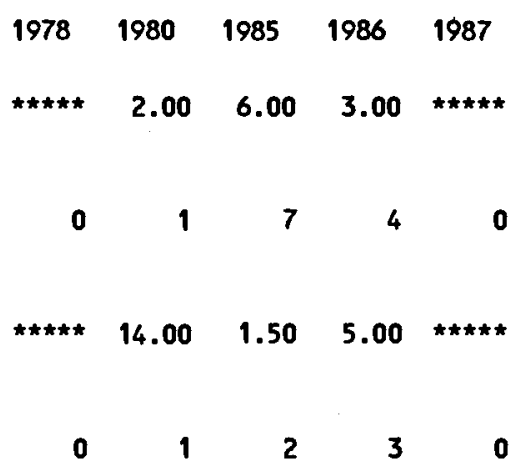

Number of Farmers Questioned 12 


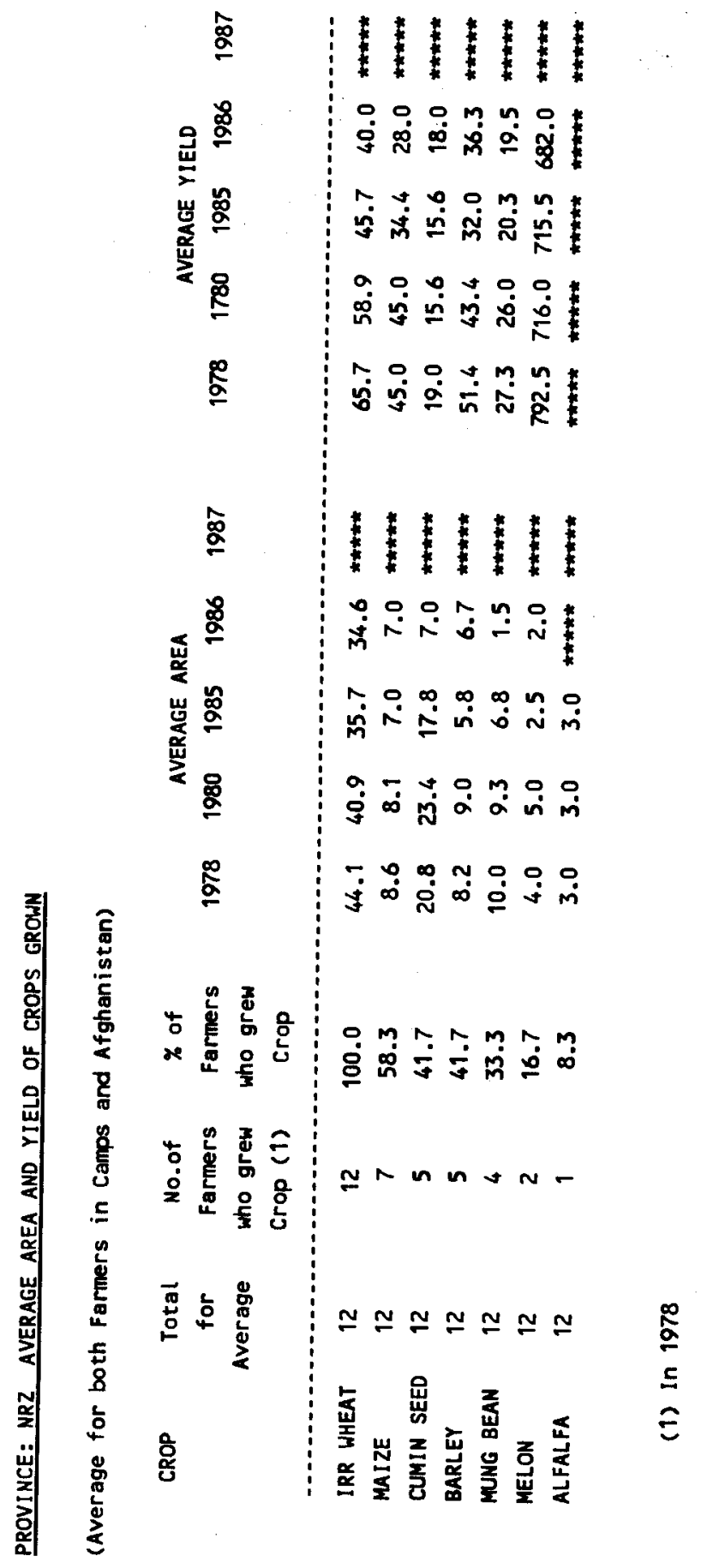


PROVINCE OF NRZ

\section{PERCENTAGE OF VILLAGES GIVING FOLLONING PRIORITY TO PROBLEM}

Rainfall

Crop Disease

Birds or Rats

Seed Availability

Fertilizer Availability

Crop Protection Chemicals Credit

Irrigation Water Availability

Flooding

Labour Availabilty

Power for Land Preparation

Direct War Effects

Animal Diseases

Extension

Other Problems

\section{6}

Rainfall

Crop Disease

Birds or Rats

Seed Avail lability

Fertilizer Availability

Crop Protection Chemicals

Credit

Irrigation Water Availability

Flooding

Labour Availabilty

Power for Land Preparation

Direct War Effects

Animal Diseases

Extension

Other Problems

1985

Rainfall

Crop Disease

Birds or Rats

Seed Availability

Fertilizer Availability

Crop Protection Chemicals

Credit

Irrigation Water Availability

Flooding

Labour Availabilty

Power for Land Preparation

Direct War Effects

Animal Diseases

Extension

other Problems

\begin{tabular}{|c|c|c|c|c|c|c|c|c|c|c|c|c|}
\hline & 2 & 3 & 4 & 5 & 6 & 7 & 8 & 9 & 10 & 11 & 12 & Weighted $\mathbf{P}$ \\
\hline & & $* *$ & 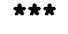 & 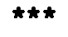 & $\star \star \star \star$ & $\star \star \star$ & $\star \star \star$ & $\star \star \star$ & $\star \star \star$ & $\star \star \star \star$ & $\star \star \star \star$ & 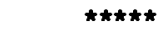 \\
\hline & $\star \star \star \star$ & $\star \star \star$ & $\star \star \star$ & $\star \star \star \star$ & $\star \star \star \star$ & $\star \star \star$ & $\star \star \star \star$ & $\star \star \star$ & $\star \star \star \star$ & $\star \star \star \star$ & $\star \star \star$ & 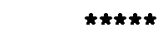 \\
\hline & 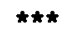 & $\star \star \star \star$ & $\star \star \star \star$ & $\star \star \star \star$ & $\star \star \star$ & $\star \star \star \star$ & $\star \star \star$ & $\star \star \star \star$ & $\star \star \star$ & $\star \star \star \star$ & $\star \star \star$ & $\star \star \star \star \star \star$ \\
\hline & $\star \star * *$ & $\star \star \star \star$ & $\star \star \star$ & $\star \star \star \star$ & $\star \star \star$ & $\star \star \star$ & $\star \star \star$ & $\star \star \star \star$ & $\star \star \star \star$ & $\star \star \star$ & $\star \star \star$ & $\star \star \star \star \star \star$ \\
\hline & $\star \star \star \star$ & $\star \star \star \star$ & $\star \star \star$ & $\star \star \star \star$ & $\star \star \star$ & $\star \star \star \star$ & $\star \star \star$ & $\star \star \star$ & $\star \star \star$ & $\star \star \star \star$ & 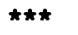 & $\star \star \star \star \star \star$ \\
\hline & XI & $\star \star \star \star$ & $\star \star \star$ & $\star \star \star \star$ & $\star \star \star \star$ & $\star \star \star \star$ & $\star \star \star \star$ & $\star \star \star \star$ & $\star \star \star \star$ & $\star \star \star \star$ & $\star \star \star \star *$ & $\star \star \star \star \star \star$ \\
\hline & $\star \star \star \star$ & $\star \star \star$ & $\star \star \star \star$ & $\star \star \star \star$ & $\star \star \star \star$ & 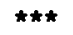 & 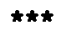 & 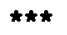 & $\star \star \star \star$ & $\star \star \star \star$ & $\star \star \star \star$ & $\star \star \star \star \star \star$ \\
\hline & 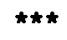 & $\star \star \star$ & $\star \star \star$ & $\star \star \star$ & $\star \star \star$ & $\star \star \star \star$ & $\star \star \star$ & $\star \star \star$ & 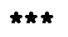 & 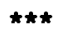 & $\star \star \star *$ & 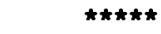 \\
\hline & & $\star \star \star$ & $\star \star \star \star$ & $\star \star \star$ & $\star \star \star$ & $\star \star \star \star$ & $\star \star \star$ & $\star \star \star$ & $\star \star \star$ & $\star \star \star$ & $\star \star \star$ & 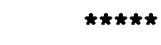 \\
\hline & $\star \star \star$ & $\star \star \star \star$ & $\star \star \star$ & $\star \star \star \star$ & $\star \star \star \star$ & 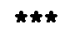 & $\star \star \star$ & $\star \star \star$ & $\star \star \star \star$ & $\star \star \star$ & 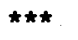 & 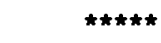 \\
\hline & $\star \star \star$ & $\star \star \star$ & $\star \star \star$ & $\star \star \star$ & $\star \star \star$ & $\star \star \star$ & $\star \star \star$ & $\star \star \star$ & $\star \star \star$ & $\star \star \star$ & $\star \star \star$ & $\star \star \star \star \star \star$ \\
\hline & $\star \star \star$ & $\star \star \star \star$ & $\star \star \star \star$ & $\star \star \star$ & $\star \star \star$ & $\star \star \star \star$ & $\star \star \star \star$ & $\star \star \star \star$ & $\star \star \star$ & $\star \star \star$ & $\star \star \star \star$ & 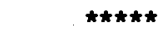 \\
\hline & $\star \star \star$ & $\star \star \star \star$ & $\star \star \star \star$ & $\star \star \star \star$ & $\star \star \star \star$ & $\star \star \star \star$ & $\star \star \star \star$ & $\star \star \star \star$ & $\star \star \star \star$ & $\star \star \star *$ & $\star \star \star \star$ & 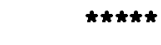 \\
\hline & 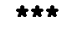 & $\star \star \star \star$ & $\star \star \star \star$ & $\star \star \star \star$ & $\star \star \star \star$ & 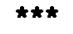 & $\star \star \star \star$ & $\star \star \star \star$ & $\star \star \star \star$ & $\star \star \star$ & $\star \star \star$ & $\star \star \star \star \star \star$ \\
\hline & 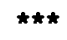 & $\star \star \star$ & $\star \star \star \star$ & $\star \star \star$ & $\star \star \star$ & $\star \star \star$ & $\star \star \star$ & $\star \star \star$ & $\star \star \star \star$ & $\star \star \star \star$ & $\star \star \star *$ & $\star \star \star \star \star \star$ \\
\hline
\end{tabular}

$\begin{array}{lllllllllllll}1 & 2 & 3 & 4 & 5 & 6 & 7 & 8 & 9 & 10 & 11 & 12 & \text { Heighted Priority }\end{array}$

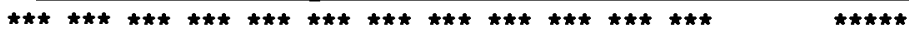

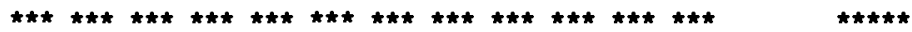

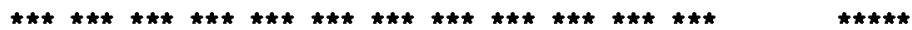

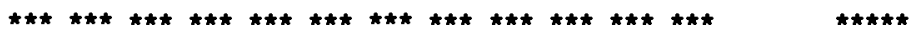

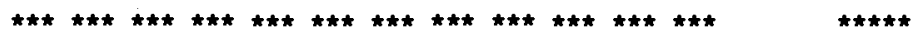

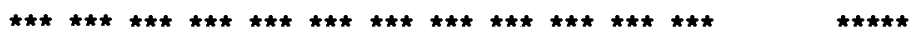

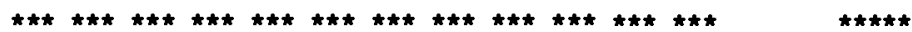

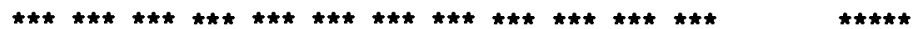

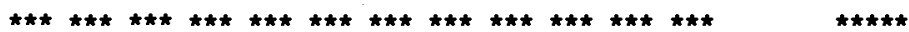

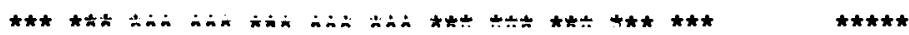

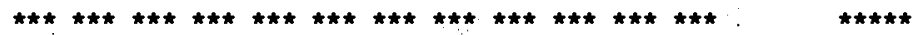

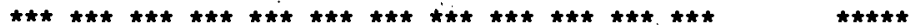

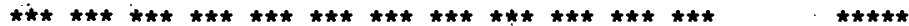

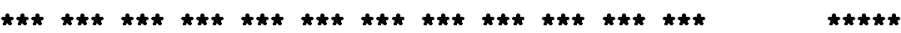

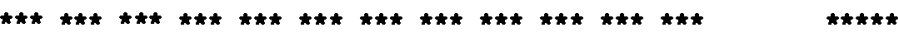

$\begin{array}{lllllllllllll}1 & 2 & 3 & 4 & 5 & 6 & 7 & 8 & 9 & 10 & 11 & 12 & \text { Weighted Priority }\end{array}$

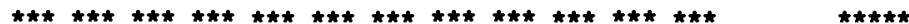

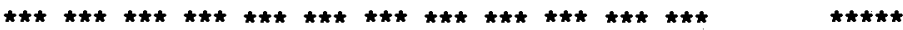

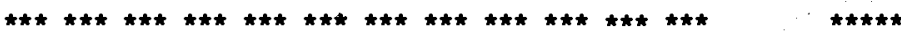

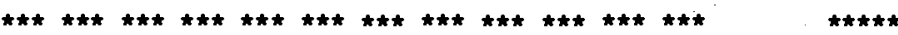

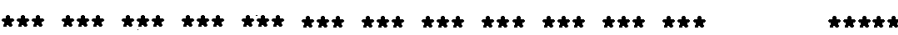

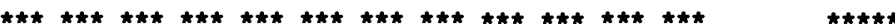

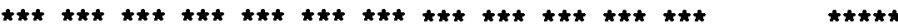

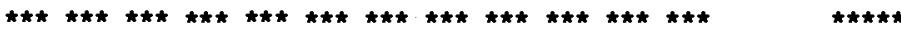

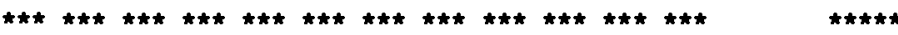

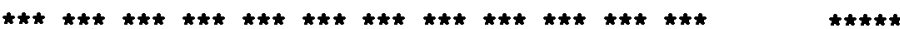

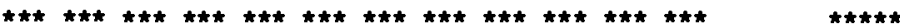

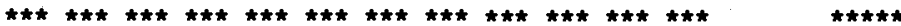

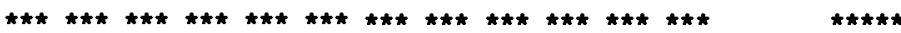

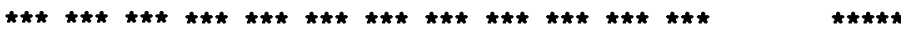

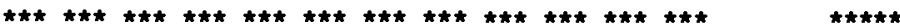


PROVINCE OF NRZ

\section{PERCENTAGE OF VILLAGES GIVING FOLLOWING PRIORITY TO PROBLEM}

Rainfall

Crop Disease

Birds or Rats

Seed Availability

Fertilizer Availability

Crop Protection Chemicals

Credit

Irrigation Water Availability

flooding

Labour Avail abilty

Power for Land Preparation

Direct War Effects

Animal Diseases

Extension

other Problems

1978

\section{Rainfall}

Crop Disease

Birds or Rats

Seed Availability

Fertilizer Availability

Crop Protection Chemicals

Credit

Irrigation Water Availability

Flooding

Labour Availabilty

Power for Land Preparation

Dírect War Effects

Animal Diseases

Extension

other Problems

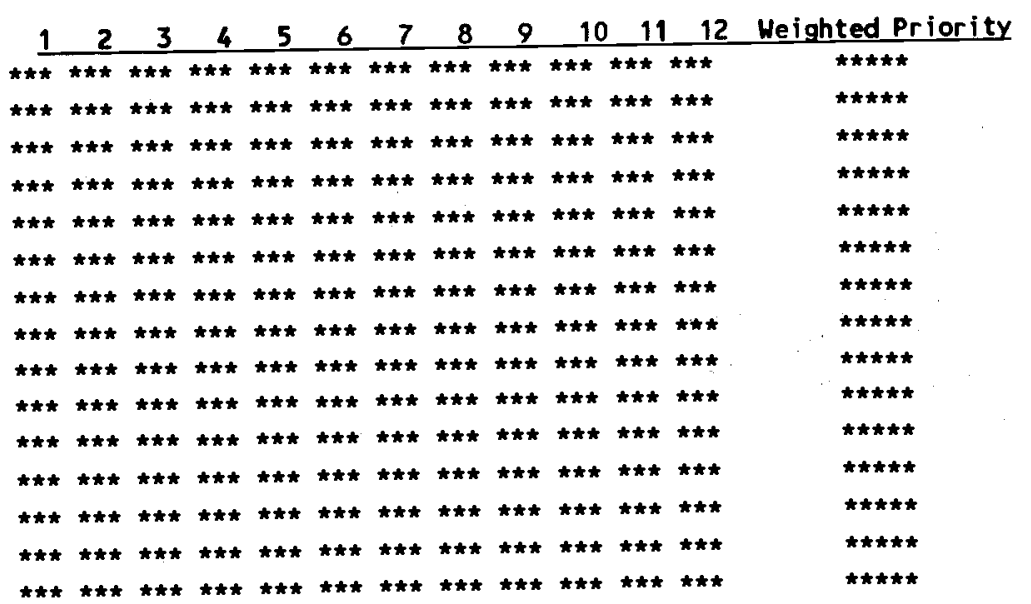

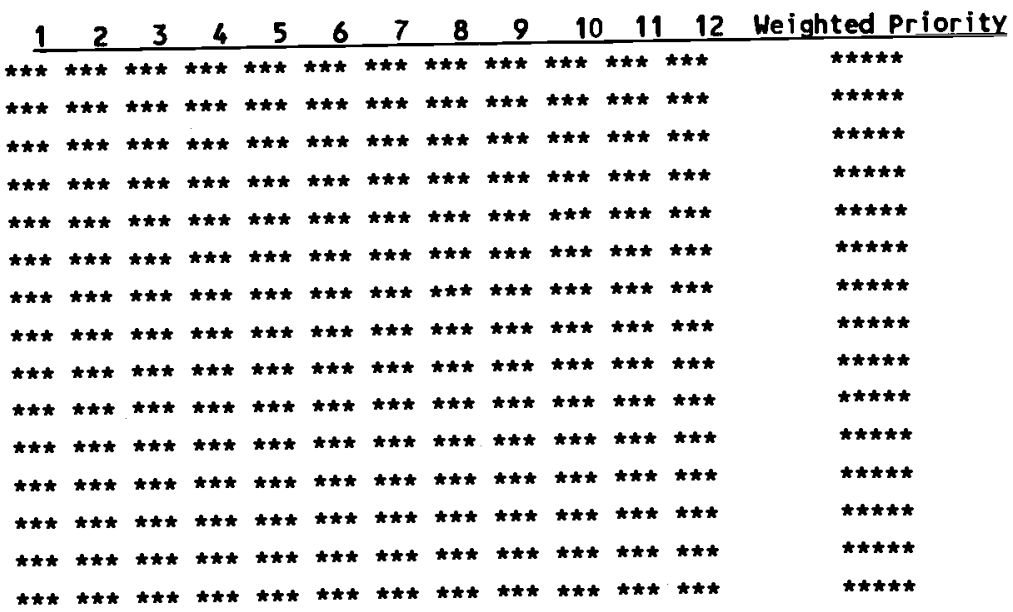


PAKTIA 


\section{Distribution of Farm Size \\ Province: Paktia \\ Year: 1978}

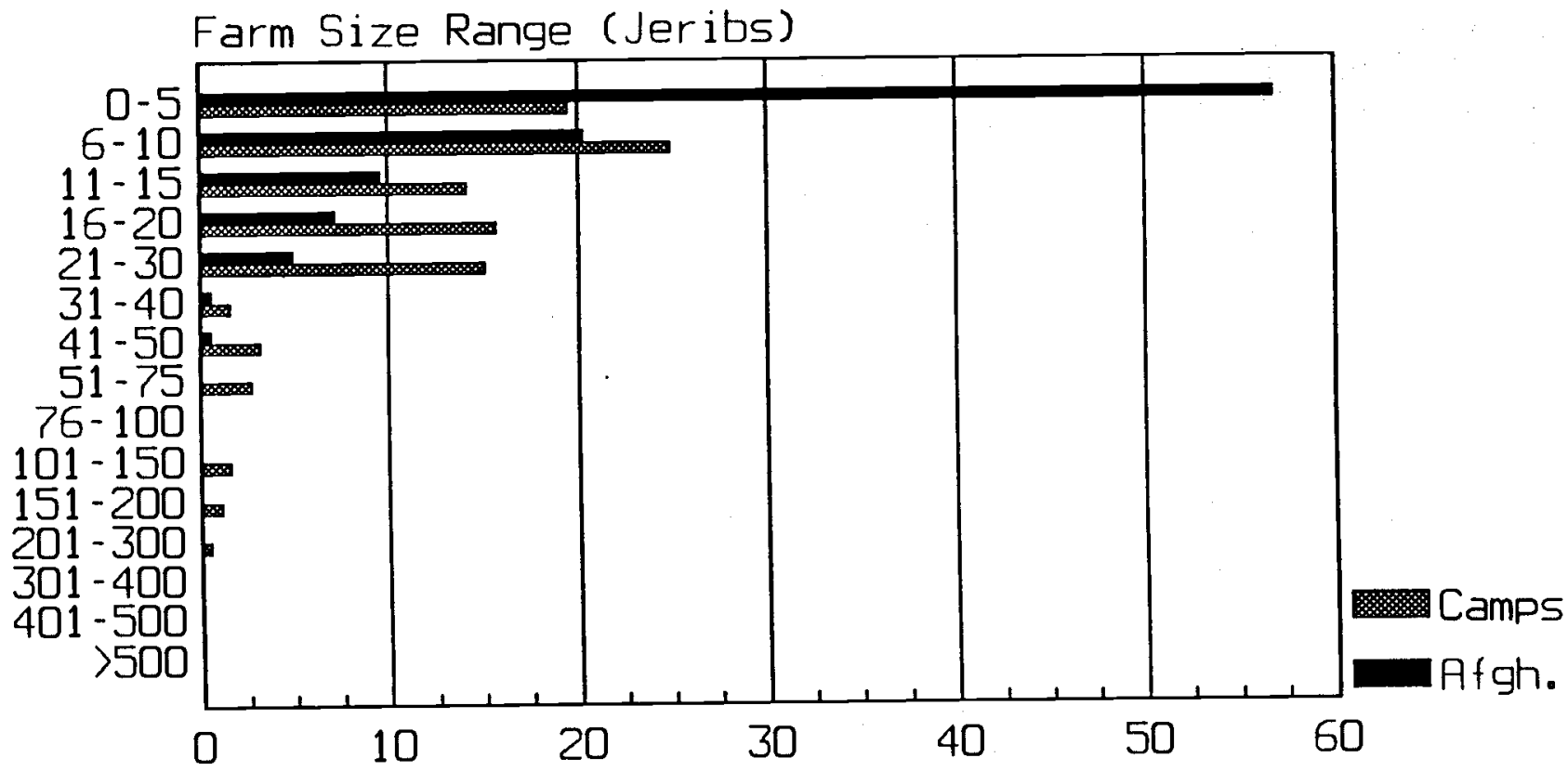

Percentage of Farmers

Farmers in Afghanistans

1978

345

7.60

7.17

7.86
Total Number of Farms Average Area Average Area Irrigated Average Dryland Area
185

Total Number of Farms

Average Area

Average Area Irrigated

Average Dryland Area

Average of both Groups

Provincial Average Area

Provincial Average Irrigated Area

Provincial Average Dryland Area
12.34

10.45

16.75 $\underline{1987}$

348

4.19

4.18

2.00

(All areas in Jeribs. 5 Jeribs $=1$ Hectare) 
Farm Power - Farmers in Atghanistan Province of Paktia

Crop: Irrigated wheat

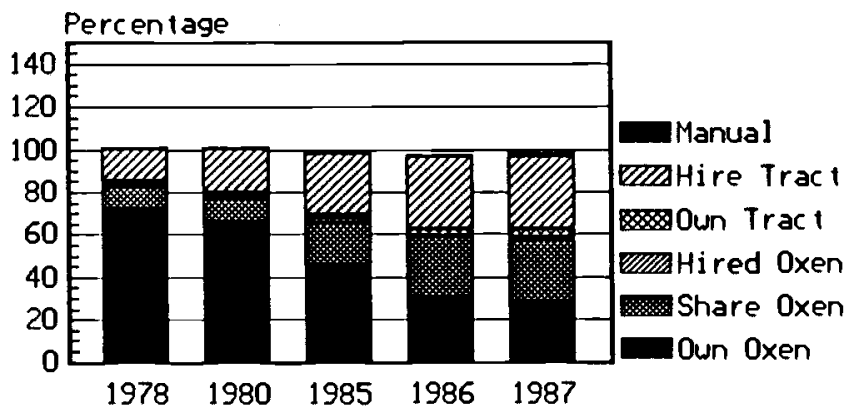

Year

Farm Power - Farmers who lett in 1987 Province of Paktia

Crop: Irrigated theat

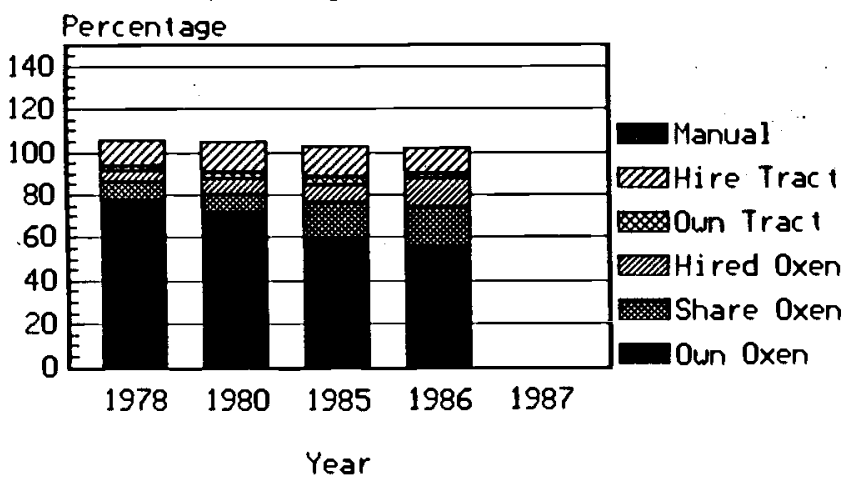

Base Figures for above graphs

Province: Paktia

Farmers in Afghanistan

Irr. Wheat own oxen

Shared Oxen

Hired oxen

Own Tractor

Hired Tractor

Manual Means

Maize Own oxen

Shared oxen

Hired oxen

Own Tractor

Hired Tractor

Manual Means

Farmers who left in 1987

Irr. Wheat Own oxen

Shared Oxen

Hired oxen

Own Tractor

Hired Tractor

Maize

Manual Means

Own Oxen

Shared oxen

Hired oxen

Own Tractor

Hired Tractor
Farm Power - Farmers in Atghanistan

Province of Paktia Crop: Maize

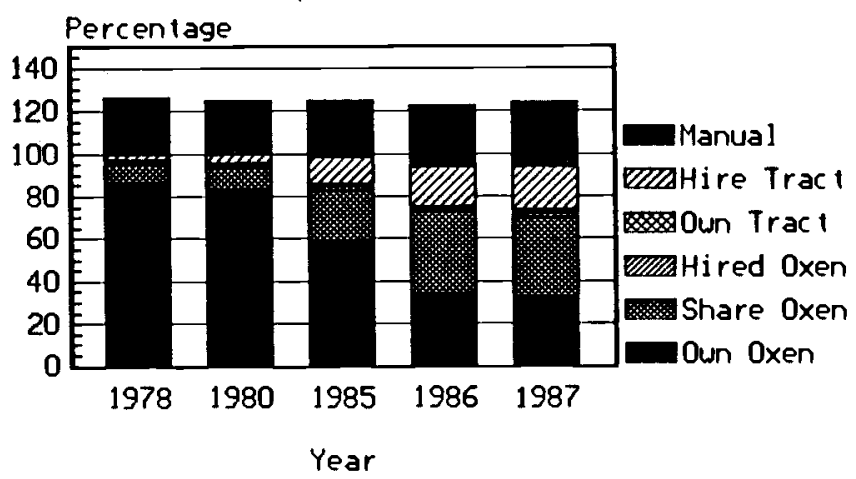
Farm Power - Farmers who lett in 1987 Province of Pakija Crop: Maize

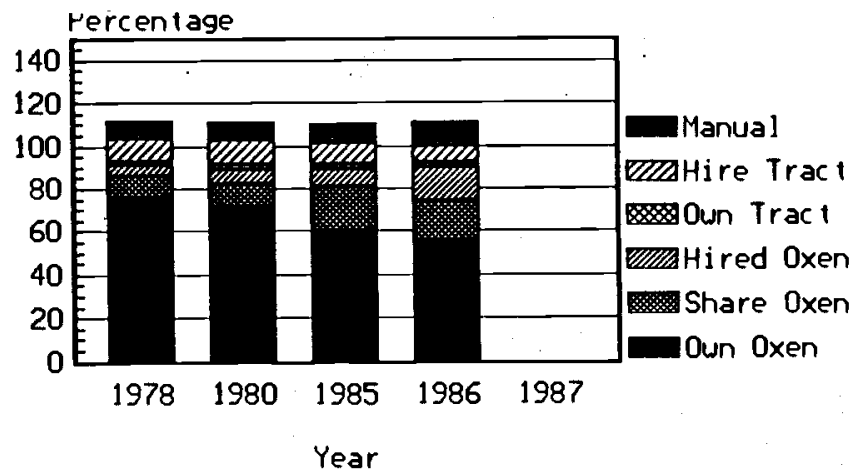

Percentage of Farmers

$1978 \quad \underline{1980} \quad \underline{1985} \quad \underline{1986} \quad \underline{1987}$

$\begin{array}{rrrrr}72.06 & 65.99 & 46.18 & 30.45 & 28.05 \\ 10.12 & 10.53 & 19.68 & 28.40 & 28.86 \\ 1.21 & 1.62 & 1.61 & .82 & 2.03 \\ 1.62 & 1.62 & 2.01 & 2.47 & 3.25 \\ 15.38 & 20.24 & 28.92 & 34.16 & 34.55 \\ .81 & 1.21 & 1.20 & 1.23 & 1.22 \\ 86.19 & 83.18 & 58.64 & 33.63 & 32.55 \\ 8.57 & 10.28 & 23.64 & 38.57 & 36.79 \\ .95 & .93 & 2.27 & 1.35 & 1.89 \\ .95 & .93 & .91 & 1.35 & 1.89 \\ 3.33 & 4.67 & 12.27 & 18.39 & 20.28 \\ 26.19 & 24.77 & 26.82 & 29.15 & 30.19\end{array}$

77.38

71.34

59.64

55.38

8.33

8.54

16.87

18.46

5.36

7.32

7.83

13.85

2.38

3.05

3.61

2.31

11.90

14.63

14.46

76.39

71.33

60.43

11.54

55.56

20.14

18.52

4.86

11.19

8.63

15.74

2.08

6.29

2.16

1.85

10.42 .

10.07

8.33 
Paktia

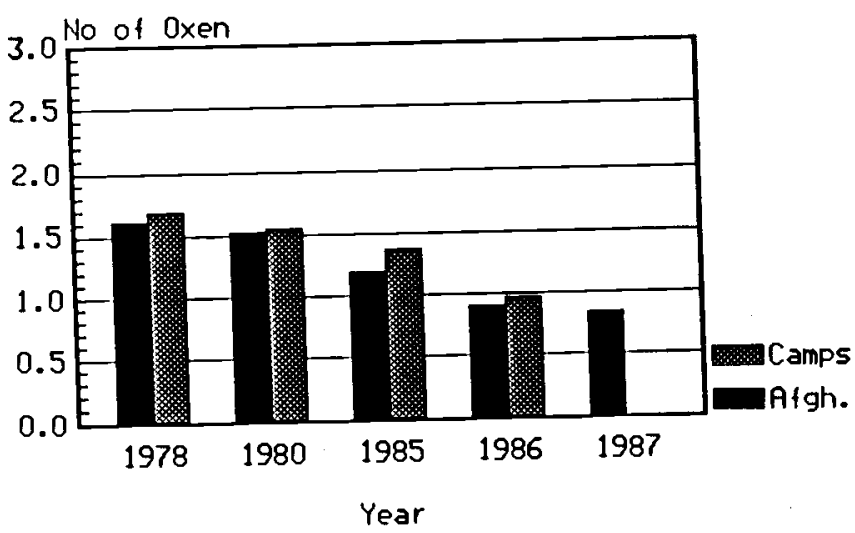

Paktia

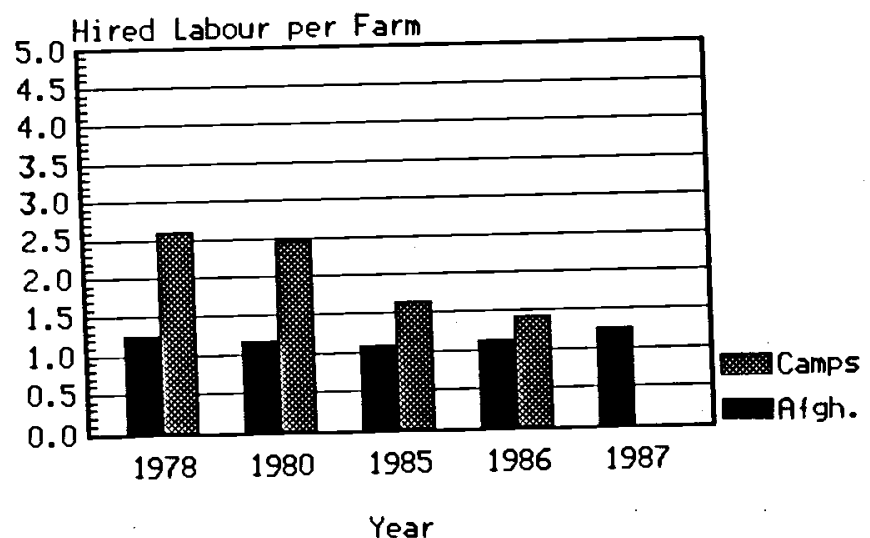

Paktia

3.5 Family Labour per Farm

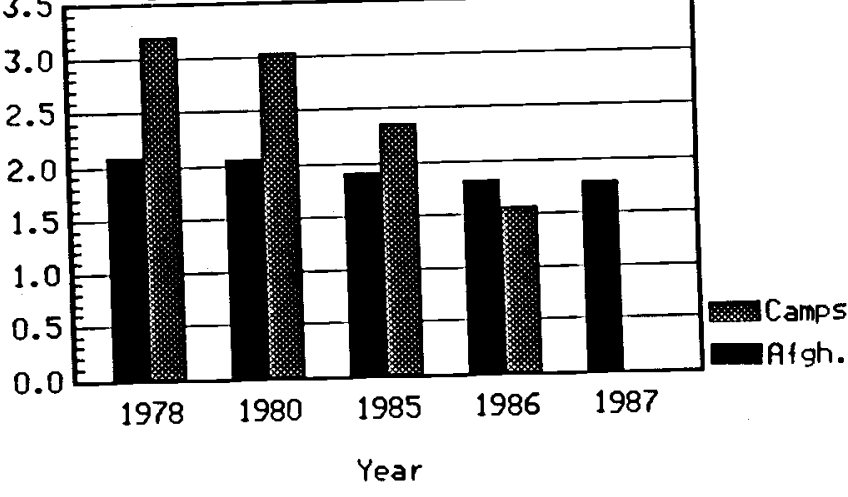

Paktia

Percentage of Farms using Hired Labour

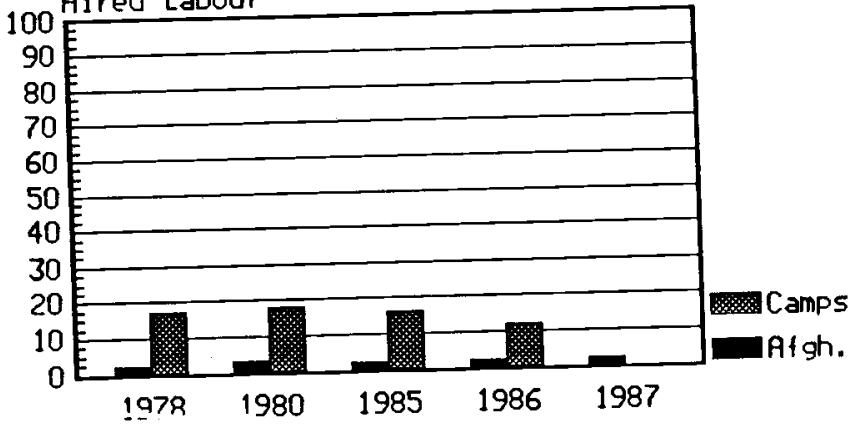


PROVINCE OF PKY

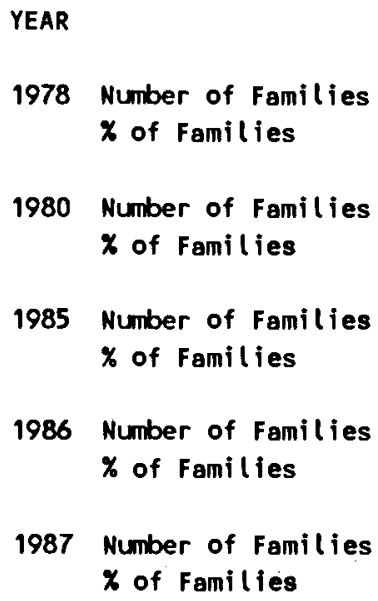


PROVINCE OF PKY

\begin{tabular}{|c|c|c|c|c|c|}
\hline & 1978 & 1980 & 1985 & 1986 & 1987 \\
\hline Average Family Labour per Farm & 2.09 & 2.06 & 1.91 & 1.82 & 1.79 \\
\hline $\begin{array}{l}\text { Average No. of Hired Labour per Farm } \\
\text { (for farms using hired labour) }\end{array}$ & 1.25 & 1.17 & 1.08 & 1.13 & 1.25 \\
\hline Number of Farms using Hired Labour & 8 & 9 & 6 & 8 & 8 \\
\hline \multicolumn{6}{|l|}{ Number of Farmers Questioned 348} \\
\hline \multicolumn{6}{|c|}{ IABLE $8(B)$ - AVERAGE LABOUR (FAMILY and HIRED) FOR FARMERS IN CAMPS } \\
\hline \multicolumn{6}{|l|}{ PROVINCE OF PKY } \\
\hline & 1978 & 1980 & 1985 & 1986 & 1987 \\
\hline Average Family Labour per Farm & 3.20 & 3.03 & 2.35 & 1.56 & 0.01 \\
\hline $\begin{array}{l}\text { Average No. of Hired Labour per Farm } \\
\text { (for farms using hired (abour) }\end{array}$ & 2.60 & 2.44 & 1.63 & 1.43 & $\star \star \star \star \star \star$ \\
\hline Number of Farms using Hired Labour & 31 & 34 & 30 & 23 & 0 \\
\hline Umber of Farmers Questioned 186 & & & & & \\
\hline
\end{tabular}


PROVINCE OF PKY

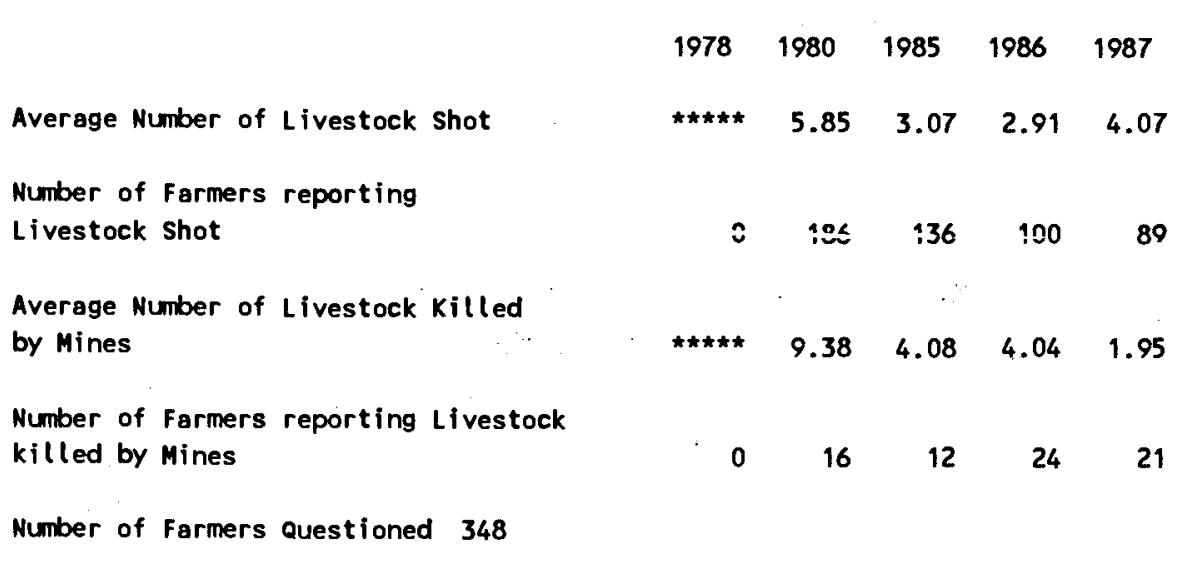

\section{TABLE 9B - DIRECT EFFECTS OF THE WAR - FARMERS IN CAMPS}

PROVINCE OF PKY

$\begin{array}{lrrrrr} & 1978 & 1980 & 1985 & 1986 & 1987 \\ \begin{array}{l}\text { Average Number of Livestock Shot } \\ \text { Number of Farmers reporting }\end{array} & \star \star \star \star \star & 10.38 & 8.17 & 11.84 & \star \star \star \star \star \\ \text { Livestock Shot } & 0 & 16 & 46 & 25 & 0 \\ \begin{array}{l}\text { Average Number of Livestock Killed } \\ \text { by Mines }\end{array} & \star \star \star \star \star & 2.75 & 7.90 & 8.10 & \\ \begin{array}{l}\text { Number of Farmers reporting Livestock } \\ \text { killed by Mines }\end{array} & & & & & \\ \end{array}$




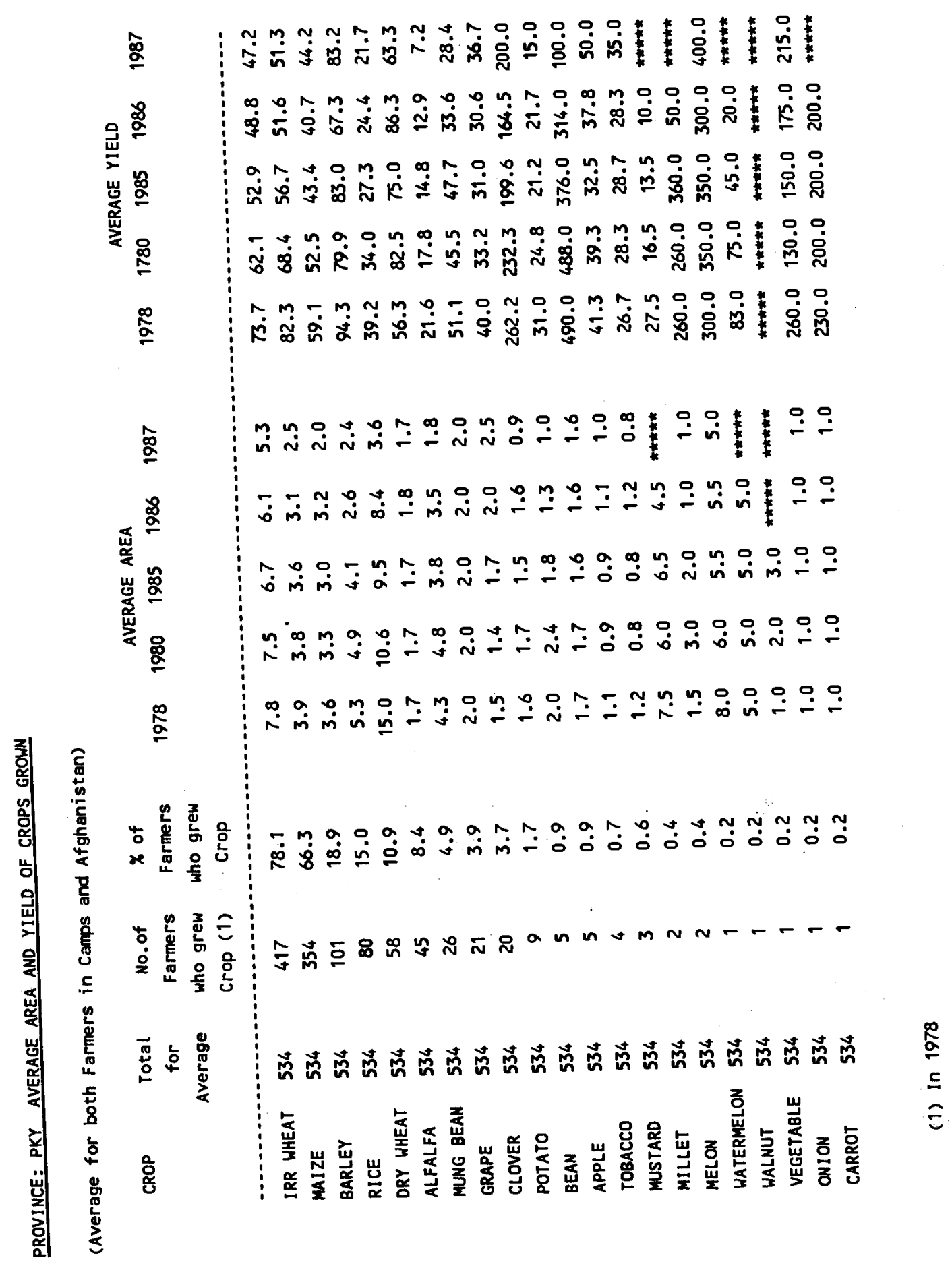


PROVINCE OF PKY

Rainfall

Crop 0 isease

Birds or Rats

Seed Availability

Fertilizer Availability

Crop Protection Chemicals

Credit

Irrigation Water Availability

Flooding

Labour Availabilty

Power for Land Preparation

Direct War Effects

Animal Diseases

Extension

Other Problems

\begin{tabular}{rrrrrrrrrrrrr}
1 & 2 & 3 & 4 & 5 & 6 & 7 & 8 & 9 & 10 & 11 & 12 & Weighted Priority \\
\hline 0 & 0 & 0 & 0 & 0 & 0 & 0 & 0 & 0 & 0 & 0 & 0 & 0.00 \\
0 & 0 & 0 & 3 & 7 & 12 & 14 & 14 & 5 & 3 & 0 & 0 & 3.07 \\
0 & 0 & 0 & 0 & 2 & 7 & 3 & 6 & 14 & 16 & 0 & 0 & 2.10 \\
0 & 0 & 0 & 0 & 0 & 0 & 2 & 2 & 0 & 0 & 0 & 0 & 0.20 \\
46 & 37 & 16 & 1 & 0 & 0 & 0 & 0 & 0 & 0 & 0 & 0 & 24.65 \\
0 & 0 & 0 & 0 & 1 & 5 & 56 & 26 & 4 & 1 & 1 & 0 & 4.57 \\
0 & 0 & 0 & 0 & 0 & 1 & 1 & 0 & 0 & 0 & 0 & 0 & 0.11 \\
43 & 28 & 22 & 1 & 0 & 0 & 0 & 0 & 0 & 0 & 0 & 0 & 22.80 \\
0 & 0 & 0 & 0 & 1 & 2 & 1 & 0 & 0 & 1 & 0 & 0 & 0.29 \\
0 & 0 & 0 & 2 & 3 & 5 & 3 & 24 & 16 & 5 & 2 & 0 & 2.80 \\
4 & 4 & 4 & 69 & 11 & 6 & 0 & 1 & 0 & 0 & 0 & 0 & 9.95 \\
7 & 31 & 56 & 6 & 0 & 0 & 0 & 0 & 0 & 0 & 0 & 0 & 15.02 \\
0 & 0 & 0 & 6 & 39 & 28 & 9 & 4 & 1 & 1 & 0 & 0 & 5.69 \\
0 & 0 & 0 & 0 & 0 & 1 & 3 & 9 & 40 & 13 & 5 & 0 & 2.81 \\
0 & 0 & 1 & 9 & 33 & 31 & 6 & 4 & 5 & 5 & 1 & 0 & 5.95
\end{tabular}

$\underline{1986}$

Rainfall

Crop 0 isease

Birds or Rats

Seed Availability

Fertilizer Availability

Crop Protection Chemicals

Credit

Irrigation Water Availability

Flooding

Labour Availabilty

Power for Land Preparation

Direct War Effects

Animal 0 iseases

Extension

other Problems

\begin{tabular}{rrrrrrrrrrrrr}
1 & 2 & 3 & 4 & 5 & 6 & 7 & 8 & 9 & 10 & 11 & 12 & Weighted Priority \\
\hline 0 & 0 & 0 & 0 & 0 & 0 & 0 & 0 & 0 & 0 & 0 & 0 & 0.00 \\
0 & 0 & 3 & 6 & 12 & 7 & 6 & 4 & 4 & 0 & 0 & 0 & 2.83 \\
0 & 0 & 1 & 0 & 3 & 8 & 4 & 6 & 16 & 15 & 1 & 0 & 2.49 \\
0 & 0 & 0 & 0 & 0 & 0 & 0 & 0 & 0 & 0 & 0 & 0 & 0.00 \\
49 & 36 & 14 & 1 & 0 & 0 & 0 & 0 & 0 & 0 & 0 & 0 & 25.30 \\
0 & 0 & 0 & 0 & 1 & 6 & 20 & 49 & 16 & 1 & 1 & 0 & 4.30 \\
0 & 0 & 0 & 0 & 0 & 0 & 0 & 2 & 0 & 0 & 0 & 0 & 0.09 \\
0 & 8 & 24 & 34 & 15 & 8 & 3 & 1 & 0 & 0 & 0 & 0 & 8.98 \\
0 & 4 & 35 & 21 & 7 & 5 & 2 & 5 & 1 & 1 & 0 & 0 & 7.88 \\
0 & 0 & 0 & 2 & 2 & 2 & 6 & 3 & 14 & 5 & 1 & 0 & 1.66 \\
5 & 2 & 3 & 16 & 49 & 18 & 5 & 1 & 0 & 0 & 0 & 0 & 8.79 \\
45 & 47 & 6 & 0 & 0 & 1 & 0 & 0 & 0 & 0 & 0 & 0 & 24.96 \\
0 & 0 & 3 & 13 & 6 & 25 & 17 & 5 & 5 & 0 & 0 & 0 & 4.67 \\
0 & 1 & 0 & 0 & 1 & 0 & 5 & 9 & 20 & 17 & 3 & 0 & 2.41 \\
0 & 0 & 12 & 7 & 3 & 19 & 25 & 13 & 7 & 5 & 1 & 0 & 5.65
\end{tabular}

1985

Rainfall

Crop Disease

Birds or Rats

Seed Avail ability

Fertilizer Availability

Crop Protection Chemicals

Credit

Irrigation Water Availability

Flooding

Labour Availabilty

Power for Land Preparation

Direct War Effects

Animal Diseases

Extension

other Problems

\begin{tabular}{rrrrrrrrrrrrr}
1 & 2 & 3 & 4 & 5 & 6 & 7 & 8 & 9 & 10 & 11 & 12 & Weighted Priority \\
\hline 0 & 0 & 0 & 0 & 0 & 0 & 0 & 0 & 0 & 0 & 0 & 0 & 0.00 \\
0 & 1 & 1 & 6 & 12 & 8 & 9 & 3 & 2 & 0 & 0 & 0 & 2.89 \\
0 & 0 & 0 & 4 & 4 & 4 & 9 & 9 & 7 & 4 & 1 & 0 & 2.29 \\
0 & 0 & 0 & 0 & 0 & 1 & 1 & 0 & 1 & 1 & 0 & 0 & 0.19 \\
8 & 46 & 40 & 5 & 0 & 0 & 0 & 0 & 0 & 0 & 0 & 0 & 16.40 \\
0 & 0 & 0 & 1 & 2 & 8 & 20 & 32 & 20 & 1 & 0 & 0 & 3.98 \\
0 & 0 & 0 & 0 & 0 & 0 & 2 & 0 & 0 & 0 & 0 & 0 & 0.11 \\
3 & 37 & 38 & 15 & 5 & 0 & 0 & 1 & 1 & 0 & 0 & 0 & 13.90 \\
0 & 1 & 8 & 15 & 17 & 7 & 6 & 5 & 1 & 2 & 0 & 0 & 4.79 \\
0 & 0 & 1 & 0 & 3 & 1 & 4 & 5 & 14 & 6 & 1 & 0 & 1.65 \\
1 & 4 & 3 & 3 & 16 & 49 & 17 & 5 & 0 & 0 & 0 & 0 & 6.91 \\
87 & 12 & 1 & 0 & 0 & 0 & 0 & 0 & 0 & 0 & 0 & 0 & 33.17 \\
0 & 0 & 1 & 12 & 29 & 9 & 16 & 7 & 1 & 0 & 0 & 0 & 4.97 \\
0 & 0 & 0 & 1 & 2 & 2 & 2 & 9 & 19 & 7 & 0 & 1 & 1.93 \\
0 & 0 & 6 & 36 & 12 & 9 & 14 & 9 & 4 & 5 & 1 & 0 & 6.80
\end{tabular}


PROVINCE OF PKY

PERCENTAGE OF VILLAGES GIVING FOLLONING PRIORITY TO PROBLEM

Rainfall

Crop 0 isease

Birds or Rats

Seed Availability

Fertilizer Availability

Crop Protection Chemicals

Credit

Irrigation Water Availability

Flooding

Labour Availabilty

Power for Land Preparation

0 irect War Effects

Animal Oiseases

Extension

other Problems

$\begin{array}{ccccccccccccc}1 & 2 & 3 & 4 & 5 & 6 & 7 & 8 & 9 & 10 & 11 & 12 & \text { Weighted Priority } \\ 0 & 0 & 0 & 0 & 0 & 0 & 0 & 0 & 0 & 0 & 0 & 0 & 0.00 \\ 0 & 0 & 1 & 5 & 8 & 8 & 5 & 1 & 2 & 2 & 0 & 0 & \end{array}$

$\begin{array}{lllllllllllll}0 & 0 & 1 & 5 & 8 & 8 & 5 & 1 & 2 & 2 & 0 & 0 & 2.26\end{array}$

$\begin{array}{lllllllllllll}0 & 0 & 0 & 0 & 3 & 1 & 3 & 5 & 3 & 2 & 0 & 0 & 0.92\end{array}$

$\begin{array}{lllllllllllll}0 & 0 & 0 & 0 & 0 & 0 & 0 & 0 & 0 & 0 & 0 & 0 & 0.00\end{array}$

$\begin{array}{lllllllllllll}0 & 59 & 29 & 5 & 2 & 0 & 0 & 0 & 0 & 0 & 0 & 0 & 15.28\end{array}$

$\begin{array}{lllllllllllll}0 & 0 & 0 & 0 & 2 & 16 & 17 & 31 & 9 & 2 & 0 & 0 & 3.92\end{array}$

$\begin{array}{lllllllllllll}0 & 0 & 0 & 0 & 1 & 0 & 0 & 0 & 0 & 0 & 0 & 0 & 0.08\end{array}$

$\begin{array}{lllllllllllll}0 & 28 & 33 & 12 & 9 & 4 & 2 & 4 & 0 & 0 & 0 & 0 & 11.69\end{array}$

$\begin{array}{lllllllllllll}0 & 4 & 15 & 24 & 13 & 9 & 5 & 1 & 0 & 0 & 0 & 0 & 6.72\end{array}$

$\begin{array}{lllllllllllll}0 & 1 & 2 & 5 & 1 & 3 & 5 & 3 & 3 & 0 & 0 & 0 & 1.78\end{array}$

$\begin{array}{lllllllllllll}0 & 1 & 2 & 2 & 1 & 19 & 39 & 17 & 7 & 0 & 0 & 0 & 5.07\end{array}$

\begin{tabular}{lllllllllllll}
99 & 0 & 0 & 0 & 0 & 0 & 0 & 0 & 0 & 0 & 0 & 0 & \\
\hline
\end{tabular}

$\begin{array}{lllllllllllll}0 & 2 & 7 & 18 & 23 & 22 & 4 & 2 & 0 & 0 & 0 & 0 & 6.39\end{array}$

$\begin{array}{lllllllllllll}0 & 0 & 0 & 0 & 2 & 3 & 3 & 11 & 14 & 4 & 0 & 0 & 1.73\end{array}$

$\begin{array}{lllllllllllll}0 & 1 & 7 & 24 & 33 & 9 & 8 & 5 & 0 & 5 & 1 & 0 & \\ \end{array}$

1978

Rainfall

Crop 0 isease

Birds or Rats

Seed Availability

Fertilizer Availability

Crop Protection Chemicals

Credit

Irrigation Water Availability

Flooding

Labour Availabilty

Power for Land Preparation

Oirect War Effects

Animal 0 iseases

Extension

Other Problems

\begin{tabular}{rrrrrrrrrrrrr}
1 & 2 & 3 & 4 & 5 & 6 & 7 & 8 & 9 & 10 & 11 & 12 & Weighted Priority \\
\hline 0 & 0 & 0 & 0 & 0 & 0 & 0 & 0 & 0 & 0 & 0 & 0 & 0.00 \\
0 & 0 & 0 & 0 & 0 & 0 & 0 & 0 & 0 & 0 & 0 & 0 & 0.00 \\
1 & 0 & 0 & 0 & 0 & 0 & 0 & 0 & 0 & 0 & 0 & 0 & 6.21 \\
0 & 0 & 0 & 0 & 0 & 0 & 0 & 0 & 0 & 0 & 0 & 0 & 0.00 \\
0 & 0 & 1 & 0 & 0 & 0 & 0 & 0 & 0 & 0 & 0 & 0 & 2.07 \\
0 & 0 & 0 & 0 & 0 & 0 & 0 & 0 & 1 & 0 & 0 & 0 & 0.69 \\
0 & 0 & 0 & 0 & 0 & 0 & 0 & 0 & 0 & 0 & 0 & 0 & 0.00 \\
0 & 0 & 0 & 0 & 0 & 0 & 0 & 0 & 0 & 0 & 0 & 0 & 0.00 \\
1 & 0 & 0 & 0 & 0 & 0 & 0 & 0 & 0 & 0 & 0 & 0 & 6.21 \\
0 & 0 & 0 & 0 & 0 & 0 & 0 & 0 & 0 & 0 & 0 & 0 & 0.00 \\
0 & 1 & 1 & 0 & 0 & 0 & 0 & 0 & 0 & 0 & 0 & 0 & 5.17 \\
0 & 0 & 0 & 0 & 0 & 0 & 0 & 0 & 0 & 0 & 0 & 0 & 0.00 \\
0 & 0 & 1 & 0 & 0 & 0 & 0 & 0 & 0 & 0 & 0 & 0 & 2.07 \\
13 & 0 & 0 & 0 & 0 & 0 & 0 & 0 & 0 & 0 & 0 & 0 & 74.48 \\
0 & 1 & 0 & 0 & 0 & 0 & 0 & 0 & 0 & 0 & 0 & 0 & 3.10
\end{tabular}


PARWAN 


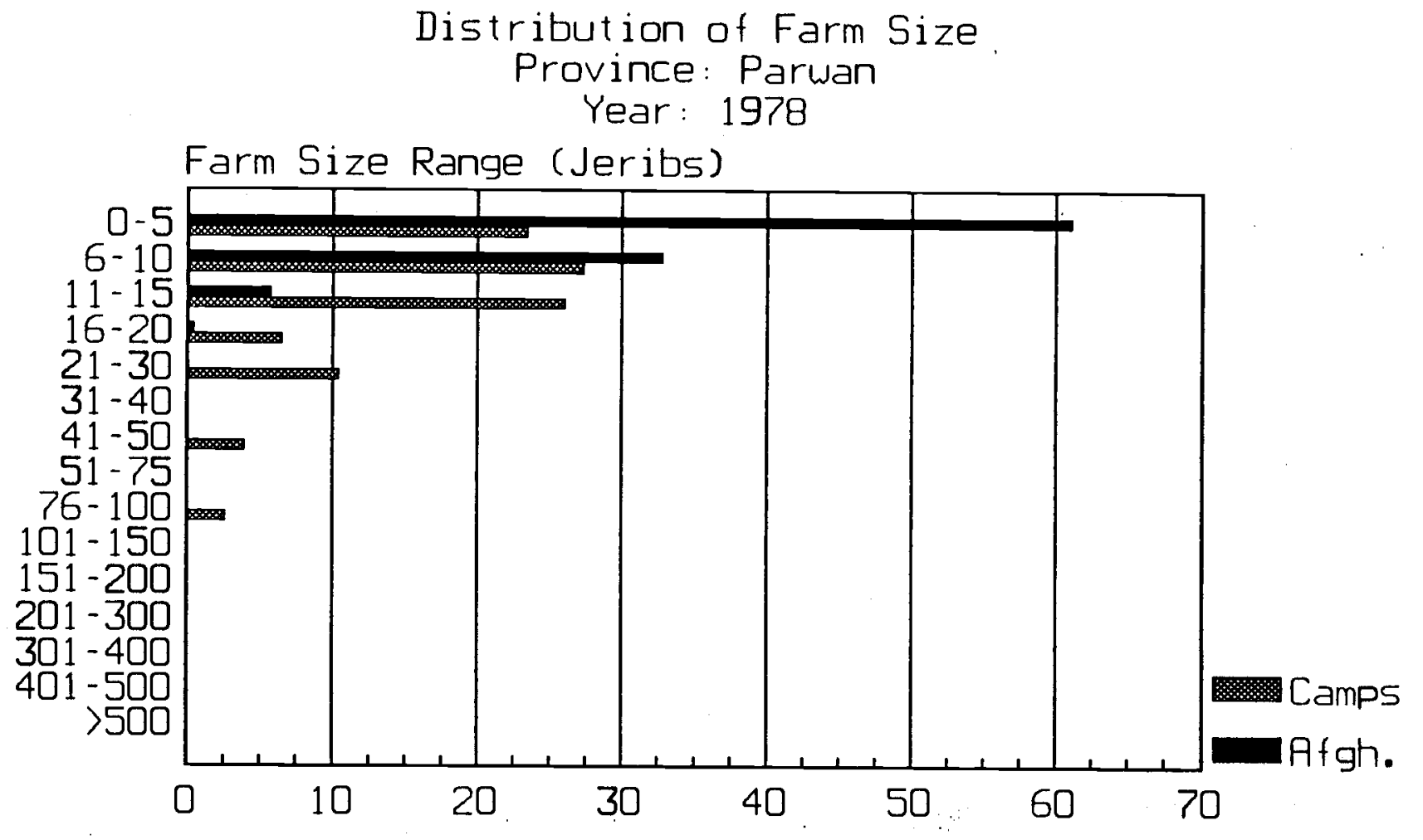

Percentage of Farmers

Farmers in Afghanistans

Total Number of Farms

Average Area

Average Area Irrigated

Average Dryland Area

Farmers who left in 1987

Total Number of Farms

Average Area

Average Area Irrigated

Average Dryland Area

Average of both Groups

Provincial Average Area

Provincial Average Irrigated Area

Provincial Average Dryland Area $\underline{1978}$

244

5.29

5.29

$-$

77

14.39

9.60

15.70

7.48

6.33

15.70
1987

245

2.16

2.16

(All areas in Jeribs. 5 Jeribs $=1$ Hectare) 


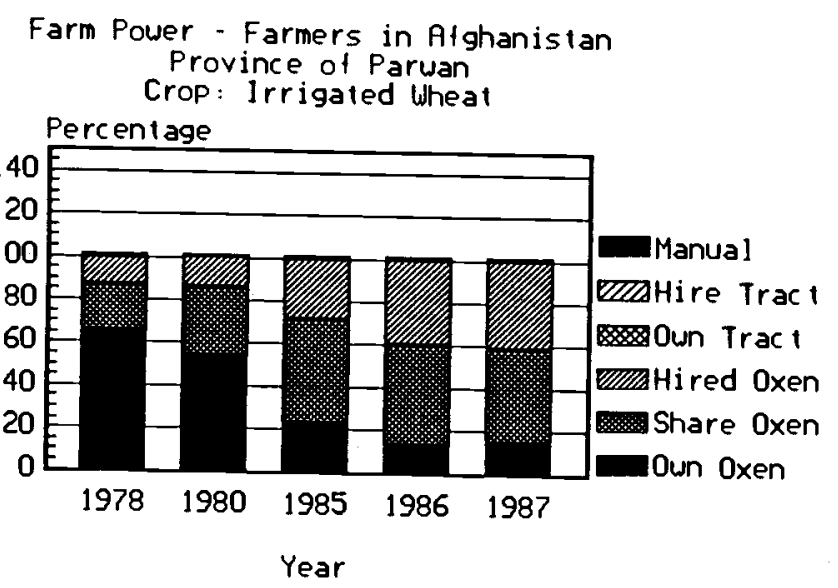

Farm Power - Farmers who lett in 1987

Province of Paruan

Crop: Irrigated wheat

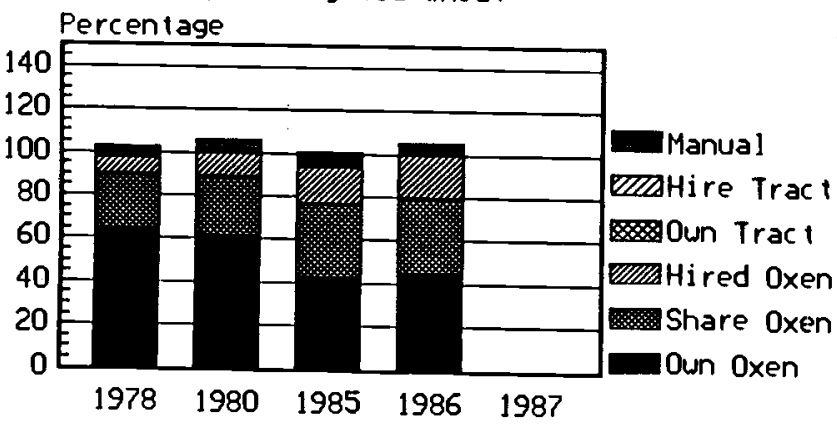

Year

Base Figures for above graphs

\section{Province: Parwan}

\section{Farmers in Afghanistan}

Irr. Wheat Own Oxen

Shared oxen

Hired oxen

Own Tractor

Hired Tractor

Maize

Manual Means

Own Oxen

Shared oxen

Hired oxen

Own Tractor

Hired Tractor

Manual Means

Farmers who left in 1987

$\begin{array}{ll}\text { Irr. Wheat } & \text { Own Oxen } \\ & \text { Shared Oxen } \\ & \text { Hired oxen } \\ \text { Own Tractor } & \text { Hired Tractor } \\ & \text { Manual Means } \\ \text { Maize } & \text { Own oxen } \\ & \text { Shared Oxen } \\ & \text { Hired oxen } \\ & \text { Own Tractor } \\ & \text { Hired Tractor }\end{array}$

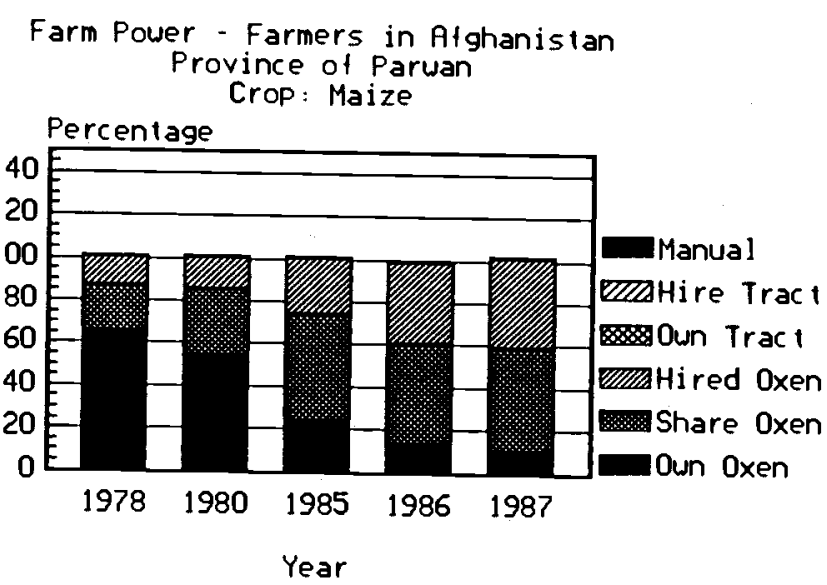

Farm Power - Farmers who lett in 1987 Province of Parwan Crop: Maize

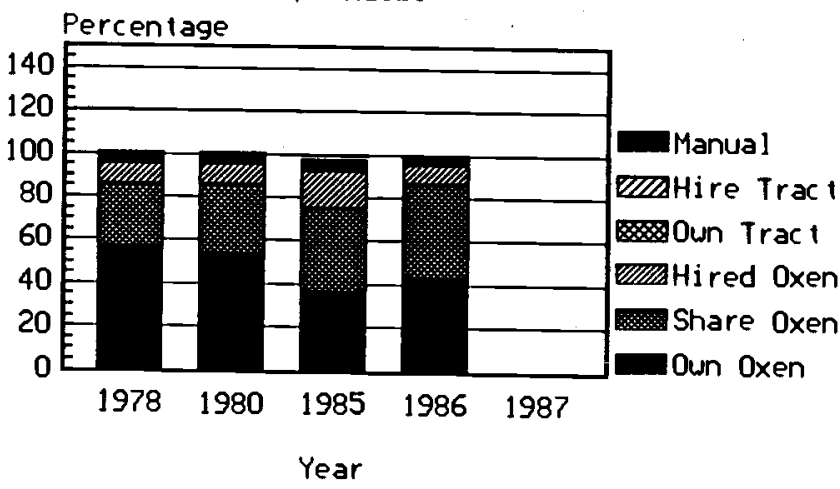

\begin{tabular}{cccccc} 
& \multicolumn{2}{l}{ Percentage of } & Farmers & \\
\cline { 5 - 6 } & & $\underline{1980}$ & $\underline{1985}$ & $\underline{1986}$ & 1987 \\
65.19 & 54.40 & 23.16 & 13.56 & 14.61 \\
21.55 & 31.87 & 48.59 & 46.89 & 44.38 \\
13.26 & 13.74 & 27.68 & 38.98 & 40.45 \\
- & - & - & - & - \\
- & - & - & - & - \\
1.10 & 1.10 & 1.69 & 1.69 & 1.69 \\
65.90 & 54.29 & 23.78 & 13.50 & 10.39 \\
20.81 & 30.86 & 50.00 & 46.63 & 48.70 \\
13.87 & 14.86 & 26.83 & 38.65 & 42.21 \\
- & - & - & - & - \\
- & - & - & - & - \\
.58 & .57 & .61 & .61 & .65
\end{tabular}

.

$\begin{array}{ccccc}64.38 & 61.19 & 41.94 & 44.19 & - \\ 24.66 & 26.87 & 33.87 & 34.88 & - \\ 8.22 & 11.94 & 17.74 & 20.93 & - \\ - & - & - & - & - \\ - & - & - & - & - \\ 5.48 & 5.97 & 6.45 & 4.65 & - \\ 56.10 & 53.66 & 36.11 & 42.86 & - \\ 29.27 & 31.71 & 38.89 & 42.86 & - \\ 9.76 & 9.76 & 16.67 & 9.52 & - \\ - & - & - & - & - \\ - & - & - & - & \end{array}$



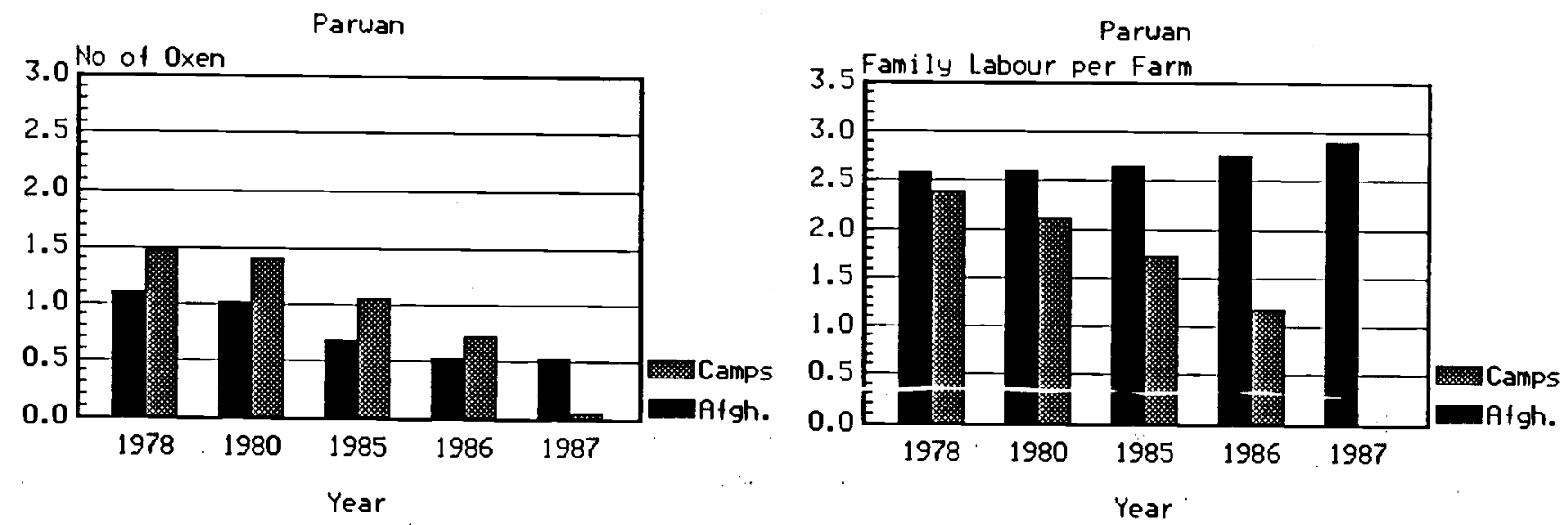

Parwan

5.0 Hired Labour per Farm

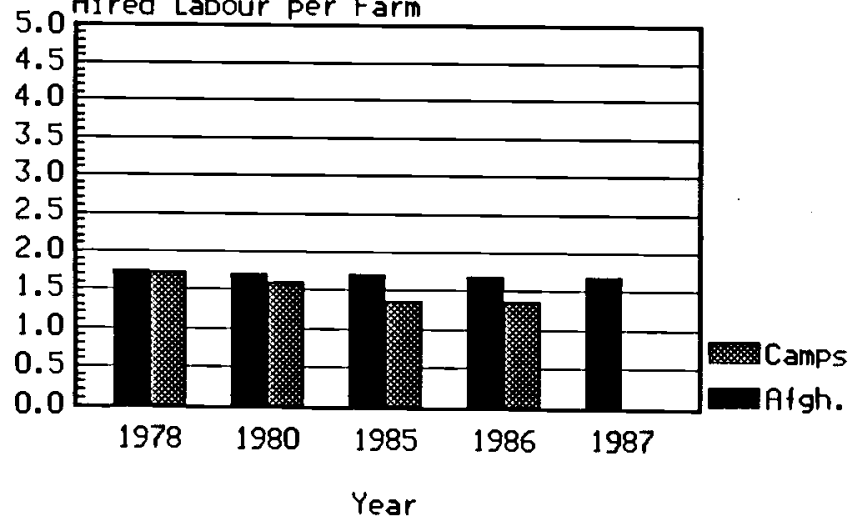

Parwan

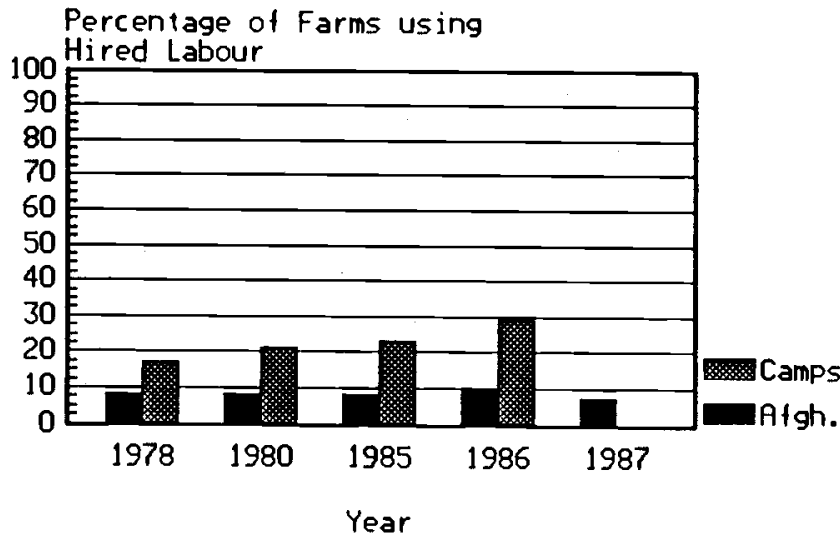


PROVINCE OF PRW

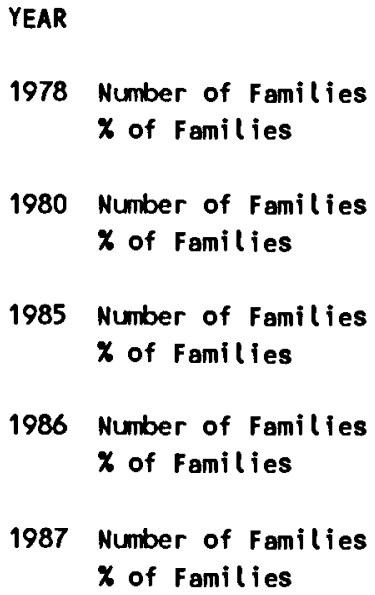

Number of Farmers Questioned 243

\section{IABLE 7B - AVERAGE NUMBER OF TRAINED OXEN FOR FARMERS IN THE CAMPS}

PROVINCE OF PRW

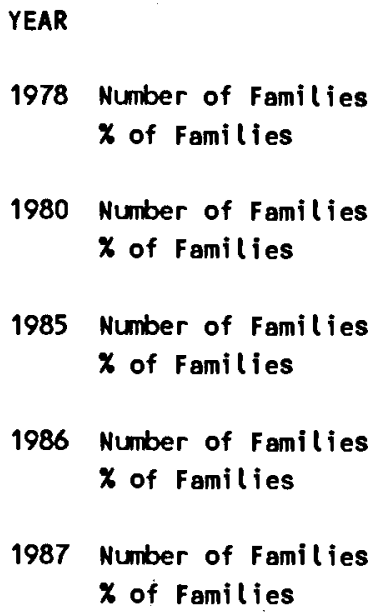

Average Number of Oxen per Family:

$\begin{array}{ll}1978 & 1.47 \\ 1980 & 1.40 \\ 1985 & 1.05 \\ 1986 & 0.71 \\ 1987 & 0.04\end{array}$

NUMBER OF TRAINED OXEN PER FAMILY

$\begin{array}{llllll}13 & 17 & 45 & 2 & 0 & 0\end{array}$

$\begin{array}{llllll}16.88 & 22.08 & 58.44 & 2.60 & 0.00 & 0.00\end{array}$

$\begin{array}{llllll}16 & 16 & 43 & 2 & 0 & 0\end{array}$

$\begin{array}{llllll}20.78 & 20.78 & 55.84 & 2.60 & 0.00 & 0.00\end{array}$

$\begin{array}{llllll}26 & 21 & 30 & 0 & 0 & 0\end{array}$

$\begin{array}{llllll}33.77 & 27.27 & 38.96 & 0.00 & 0.00 & 0.00\end{array}$

$\begin{array}{llllll}42 & 15 & 20 & 0 & 0 & 0\end{array}$

$\begin{array}{llllll}54.55 & 19.48 & 25.97 & 0.00 & 0.00 & 0.00\end{array}$

$\begin{array}{cccccc}75 & 1 & 1 & 0 & 0 & 0 \\ 97.40 & 1.30 & 1.30 & 0.00 & 0.00 & 0.00\end{array}$


PROVINCE OF PRW

\begin{tabular}{|c|c|c|c|c|c|}
\hline & 1978 & 1980 & 1985 & 1986 & 1987 \\
\hline Average Family Labour per Farm & 2.58 & 2.60 & 2.64 & 2.76 & 2.90 \\
\hline $\begin{array}{l}\text { Average No. of Hired Labour per Farm } \\
\text { (for farms using hired labour) }\end{array}$ & 1.74 & 1.70 & 1.70 & 1.67 & 1.67 \\
\hline Number of Farms using Hired Labour & 19 & 20 & 20 & 21 & 18 \\
\hline \multirow[t]{3}{*}{$\begin{array}{l}\text { Number of Farmers Questioned } 243 \\
\text { TABLE } 8(B) \text { - AVERAGE LABOUR (FAMILY and }\end{array}$} & HIRED ) & FOR FAI & RMERS IN & N CAMPS & \\
\hline & & & & & \\
\hline & 1978 & 1980 & 1985 & 1986 & 1987 \\
\hline Average Family Labour per Farm & 2.39 & 2.12 & 1.73 & 1.18 & 0.08 \\
\hline $\begin{array}{l}\text { Average No. of Hired Labour per Farm } \\
\text { (for farms using hired (abour) }\end{array}$ & 1.73 & 1.59 & 1.36 & 1.36 & $\star \star * \star * \star$ \\
\hline Number of Farms using Hired Labour & 13 & 16 & 18 & 22 & 0 \\
\hline Number of Farmers Questioned 77 & & & & & \\
\hline
\end{tabular}


PROVINCE OF PRW

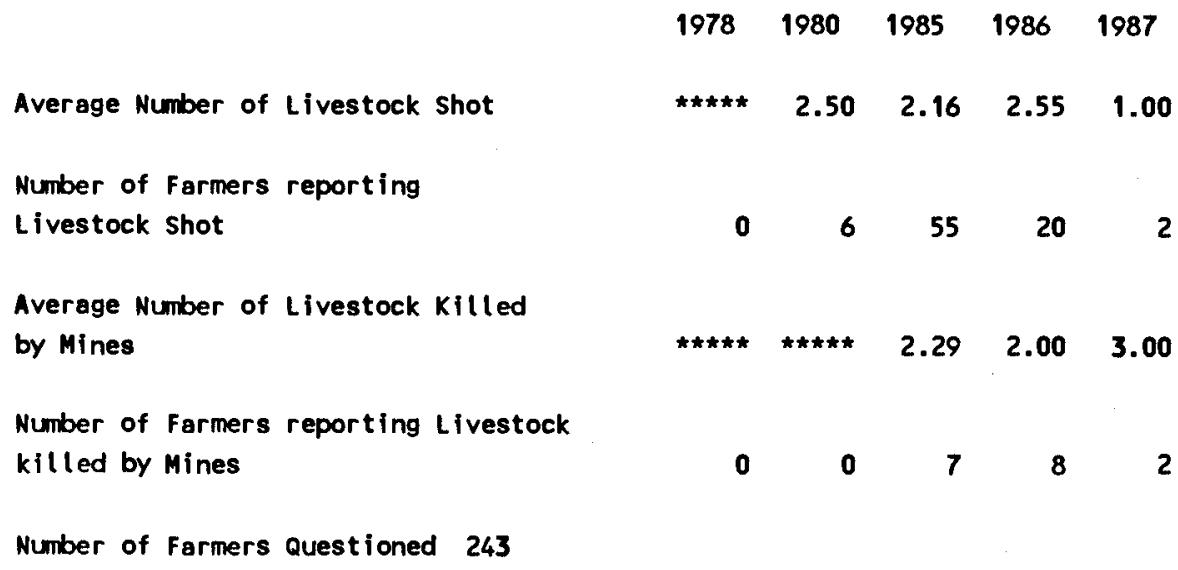

IABLE 9B - DIRECT EFFECTS OF THE WAR - FARMERS IN CAMPS

PROVINCE OF PRW

Average Number of Livestock Shot

Number of Farmers reporting

Livestock shot

Average Number of Livestock Killed by Mines

Number of Farmers reporting Livestock killed by Mines

Number of Farmers Questioned

77 $\begin{array}{lllll}1978 & 1980 & 1985 & 1986 & 1987\end{array}$

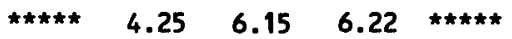

$\begin{array}{lllll}0 & 4 & 34 & 9 & 0\end{array}$

$\begin{array}{lllll}\star \star \star \star \star & 2.00 & 3.56 & 1.83 & \star \star \star \star \star\end{array}$

$\begin{array}{lllll}\dot{u} & i & \bar{y} & \dot{0} & 0\end{array}$ 
ฐ

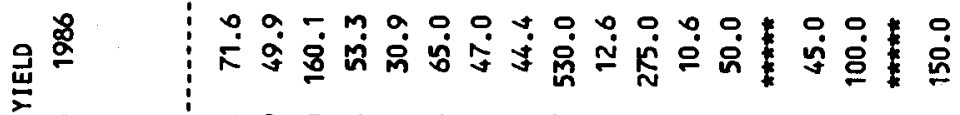
岁哭 8

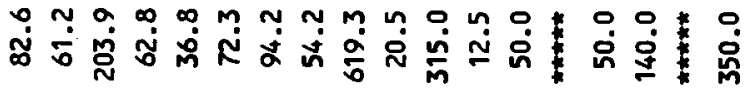

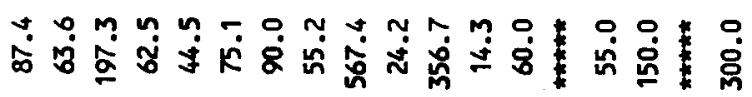
テัง m $\infty$ m $\because a m a$ ․․ $m$ m $m$ m mَّ

Rُ

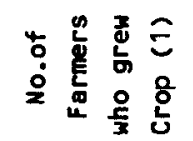

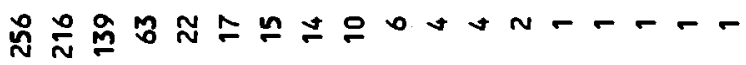
莒高兽 $\bar{N} \bar{N} \bar{N} \bar{N} \bar{N} \bar{N} \bar{N} \bar{N} \bar{N} \bar{N} \bar{N} \bar{N} \bar{N} \bar{N} \bar{N} \bar{N} \bar{N} \bar{N}$

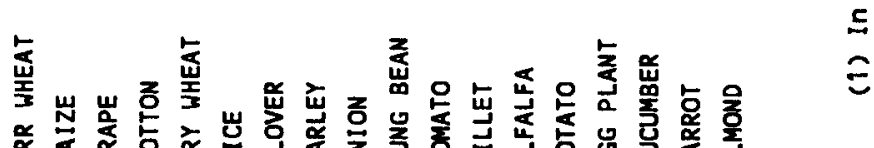
용

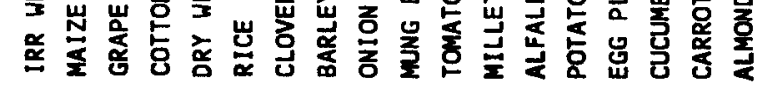


PROVINCE OF PRW

Rainfall

Crop Disease

Birds or Rats

Seed Availability

Crop Protection Chemicals

Credit

Irrigation Water Avai lability

Flooding

Labour Availabilty

Power for Land Preparation

Animal Diseases

Extension

Other Problems
Fertilizer Availability

Direct War Effects

$\begin{array}{rrrrrrrrrrrrr}1 & 2 & 3 & 4 & 5 & 6 & 7 & 8 & 9 & 10 & 11 & 12 & \text { Weighted Priority } \\ 0 & 0 & 0 & 0 & 0 & 0 & 0 & 0 & 0 & 0 & 0 & 0 & 0.00 \\ 3 & 47 & 18 & 9 & 8 & 2 & 0 & 0 & 0 & 0 & 0 & 0 & 15.47 \\ 0 & 1 & 7 & 9 & 6 & 6 & 1 & 2 & 0 & 0 & 0 & 0 & 3.20 \\ 0 & 2 & 7 & 0 & 0 & 2 & 1 & 1 & 1 & 1 & 1 & 0 & 1.85 \\ 0 & 7 & 7 & 2 & 5 & 5 & 6 & 3 & 2 & 0 & 0 & 0 & 3.93 \\ 0 & 2 & 13 & 23 & 11 & 9 & 11 & 3 & 0 & 1 & 0 & 0 & 7.08 \\ 0 & 0 & 1 & 0 & 0 & 0 & 0 & 0 & 0 & 0 & 0 & 0 & 0.16 \\ 0 & 16 & 0 & 0 & 0 & 0 & 0 & 0 & 0 & 0 & 0 & 0 & 3.33 \\ 0 & 0 & 2 & 2 & 5 & 6 & 3 & 0 & 2 & 0 & 0 & 0 & 1.64 \\ 0 & 0 & 3 & 6 & 3 & 1 & 2 & 3 & 0 & 0 & 0 & 0 & 1.75 \\ 0 & 15 & 9 & 8 & 9 & 11 & 8 & 9 & 2 & 0 & 0 & 0 & 7.79 \\ 94 & 3 & 0 & 0 & 0 & 0 & 0 & 0 & 0 & 0 & 0 & 0 & 40.14 \\ 0 & 2 & 5 & 8 & 6 & 3 & 1 & 0 & 1 & 0 & 0 & 0 & 2.77 \\ 0 & 0 & 3 & 9 & 10 & 3 & 3 & 2 & 0 & 0 & 0 & 0 & 2.84 \\ 0 & 3 & 22 & 18 & 14 & 10 & 7 & 2 & 0 & 1 & 0 & 0 & 8.05\end{array}$

1986

Rainfall

Crop Disease

Birds or Rats

Seed Availability

Fertilizer Availability

Crop Protection Chemicals

Credit

Irrigation Water Availability

Flooding

Labour Availabilty

Power for Land Preparation

Direct War Effects

Animal Diseases

Extension

Other Problems

$\begin{array}{rrrrrrrrrrrrr}1 & 2 & 3 & 4 & 5 & 6 & 7 & 8 & 9 & 10 & 11 & 12 & \text { Weighted Priority } \\ 0 & 1 & 0 & 0 & 0 & 0 & 0 & 0 & 0 & 0 & 0 & 0 & 0.24 \\ 0 & 17 & 16 & 5 & 13 & 2 & 3 & 0 & 0 & 0 & 0 & 0 & 7.65 \\ 1 & 0 & 11 & 14 & 2 & 11 & 9 & 1 & 1 & 0 & 0 & 0 & 5.11 \\ 0 & 5 & 2 & 3 & 0 & 1 & 2 & 0 & 0 & 0 & 0 & 0 & 1.83 \\ 0 & 0 & 7 & 0 & 0 & 0 & 0 & 0 & 0 & 0 & 0 & 0 & 0.95 \\ 0 & 15 & 18 & 11 & 17 & 9 & 1 & 1 & 1 & 0 & 1 & 0 & 9.07 \\ 0 & 0 & 1 & 0 & 1 & 0 & 0 & 0 & 0 & 0 & 0 & 0 & 0.25 \\ 0 & 14 & 0 & 2 & 0 & 0 & 0 & 0 & 0 & 0 & 0 & 0 & 3.08 \\ 0 & 1 & 7 & 1 & 5 & 1 & 3 & 0 & 0 & 0 & 0 & 0 & 1.97 \\ 0 & 0 & 8 & 14 & 10 & 6 & 5 & 1 & 0 & 1 & 0 & 0 & 4.16 \\ 0 & 24 & 5 & 6 & 10 & 11 & 8 & 9 & 2 & 0 & 0 & 0 & 8.90 \\ 98 & 2 & 0 & 0 & 0 & 0 & 0 & 0 & 0 & 0 & 0 & 0 & 41.25 \\ 0 & 11 & 5 & 1 & 2 & 2 & 0 & 0 & 0 & 0 & 0 & 0 & 3.47 \\ 0 & 3 & 3 & 13 & 11 & 1 & 1 & 0 & 0 & 0 & 0 & 0 & 3.58 \\ 1 & 7 & 15 & 25 & 10 & 9 & 5 & 2 & 0 & 1 & 0 & 0 & 8.48\end{array}$

$\underline{1985}$

Rainfall

Crop Disease

Birds or Rats

Seed Avai lability

Fertilizer Availability

Crop Protection Chemicals

Credit

Irrigation Water Availability

Flooding

Labour Availabilty

Power for Land Preparation

Direct War Effects

Animal Diseases

Extension

Other Problems

$\begin{array}{rrrrrrrrrrrrr}1 & 2 & 3 & 4 & 5 & 6 & 7 & 8 & 9 & 10 & 11 & 12 & \text { Weighted Priority } \\ 0 & 0 & 0 & 0 & 0 & 0 & 0 & 0 & 0 & 0 & 0 & 0 & 0.00 \\ 0 & 10 & 22 & 7 & 17 & 0 & 0 & 0 & 1 & 0 & 0 & 0 & 7.36 \\ 0 & 1 & 6 & 11 & 9 & 11 & 2 & 1 & 0 & 0 & 0 & 0 & 3.98 \\ 0 & 3 & 5 & 3 & 0 & 5 & 2 & 1 & 0 & 0 & 0 & 0 & 2.22 \\ 0 & 2 & 3 & 0 & 0 & 0 & 0 & 0 & 0 & 0 & 0 & 0 & 0.95 \\ 0 & 14 & 16 & 14 & 16 & 11 & 6 & 2 & 0 & 0 & 0 & 0 & 9.10 \\ 0 & 0 & 1 & 0 & 0 & 0 & 0 & 0 & 0 & 0 & 0 & 0 & 0.16 \\ 5 & 22 & 2 & 1 & 0 & 0 & 0 & 0 & 0 & 0 & 0 & 0 & 6.87 \\ 0 & 0 & 1 & 7 & 5 & 1 & 1 & 0 & 0 & 0 & 0 & 0 & 1.40 \\ 0 & 0 & 6 & 8 & 5 & 2 & 3 & 1 & 0 & 0 & 0 & 0 & 2.43 \\ 0 & 23 & 8 & 8 & 9 & 6 & 19 & 6 & 1 & 0 & 0 & 0 & 9.38 \\ 95 & 3 & 0 & 1 & 0 & 0 & 0 & 0 & 0 & 0 & 0 & 0 & 40.86 \\ 0 & 1 & 5 & 10 & 1 & 5 & 0 & 0 & 0 & 0 & 0 & 0 & 2.36 \\ 0 & 7 & 10 & 5 & 11 & 1 & 1 & 0 & 0 & 0 & 0 & 0 & 4.44 \\ 0 & 13 & 11 & 19 & 10 & 15 & 3 & 1 & 1 & 1 & 0 & 0 & 8.49\end{array}$


IABLE 1-5(contd) - ANALYSIS OF GREATEST FARMING PROBLEMS - COMMUNITY SURVEY

PROVINCE OF PRW

PERCENTAGE OF VILLAGES GIVING FOLLOWING PRIORITY TO PROBLEM

Rainfall

Crop Disease

Birds or Rats

Seed Availability

Fertilizer Availability

Crop Protection Chemicals

Credit

Irrigation Water Availability

Flooding

Labour Availabilty

Power for Land Preparation

Direct War Effects

Animal Diseases

Extension

other Problems

\begin{tabular}{rrrrrrrrrrrrr}
1 & 2 & 3 & 4 & 5 & 6 & 7 & 8 & 9 & 10 & 11 & 12 & Weighted Priority \\
\hline 0 & 0 & 0 & 0 & 0 & 0 & 0 & 0 & 0 & 0 & 0 & 0 & 0.00 \\
3 & 5 & 1 & 0 & 1 & 0 & 0 & 0 & 0 & 0 & 0 & 0 & 14.93 \\
2 & 1 & 2 & 0 & 0 & 1 & 0 & 0 & 0 & 0 & 0 & 0 & 9.00 \\
0 & 1 & 0 & 0 & 0 & 0 & 0 & 0 & 0 & 0 & 0 & 0 & 1.35 \\
0 & 0 & 0 & 0 & 0 & 0 & 0 & 0 & 0 & 0 & 0 & 0 & 0.00 \\
0 & 1 & 1 & 1 & 0 & 0 & 0 & 0 & 0 & 0 & 0 & 0 & 2.92 \\
0 & 1 & 0 & 0 & 0 & 0 & 0 & 0 & 0 & 0 & 0 & 0 & 1.35 \\
1 & 0 & 0 & 0 & 0 & 0 & 0 & 0 & 0 & 0 & 0 & 0 & 2.70 \\
3 & 0 & 0 & 0 & 0 & 0 & 0 & 0 & 0 & 0 & 0 & 0 & 8.10 \\
0 & 0 & 0 & 0 & 0 & 0 & 0 & 0 & 0 & 0 & 0 & 0 & 0.00 \\
0 & 2 & 0 & 0 & 0 & 0 & 0 & 0 & 0 & 0 & 0 & 0 & 2.70 \\
16 & 0 & 1 & 0 & 0 & 0 & 0 & 0 & 0 & 0 & 0 & 0 & 38.69 \\
0 & 2 & 1 & 0 & 0 & 0 & 0 & 0 & 0 & 0 & 0 & 0 & 3.60 \\
0 & 1 & 3 & 0 & 0 & 0 & 0 & 0 & 0 & 0 & 0 & 0 & 4.05 \\
0 & 5 & 5 & 2 & 0 & 0 & 0 & 0 & 0 & 1 & 0 & 0 & 10.62
\end{tabular}

\section{8}

Rainfall

Crop Disease

Birds or Rats

Seed Availability

Fertilizer Availability

Crop Protection Chemicals

Credit

Irrigation Water Availability

Flooding

Labour Avail labilty

Power for Land Preparation

Direct War Effects

Animal Diseases

Extension

other Problems

\begin{tabular}{rrllllllllllc}
1 & 2 & 3 & 4 & 5 & 6 & 7 & 8 & 9 & 10 & 11 & 12 & Weighted Priority \\
\hline 0 & 0 & 0 & 0 & 0 & 0 & 0 & 0 & 0 & 0 & 0 & 0 & 0.00 \\
1 & 0 & 0 & 0 & 0 & 0 & 0 & 0 & 0 & 0 & 0 & 0 & 13.19 \\
3 & 0 & 1 & 0 & 0 & 0 & 0 & 0 & 0 & 0 & 0 & 0 & 43.96 \\
0 & 0 & 0 & 0 & 0 & 0 & 0 & 0 & 0 & 0 & 0 & 0 & 0.00 \\
0 & 0 & 0 & 0 & 0 & 0 & 0 & 0 & 0 & 0 & 0 & 0 & 0.00 \\
0 & 0 & 0 & 0 & 0 & 0 & 0 & 0 & 0 & 0 & 0 & 0 & 0.00 \\
0 & 0 & 0 & 0 & 0 & 0 & 0 & 0 & 0 & 0 & 0 & 0 & 0.00 \\
0 & 0 & 0 & 0 & 0 & 0 & 0 & 0 & 0 & 0 & 0 & 0 & 0.00 \\
0 & 0 & 0 & 0 & 0 & 0 & 0 & 0 & 0 & 0 & 0 & 0 & 0.00 \\
0 & 0 & 0 & 0 & 0 & 0 & 0 & 0 & 0 & 0 & 0 & 0 & 0.00 \\
0 & 0 & 0 & 0 & 0 & 0 & 0 & 0 & 0 & 0 & 0 & 0 & 0.00 \\
0 & 0 & 0 & 0 & 0 & 0 & 0 & 0 & 0 & 0 & 0 & 0 & 0.00 \\
0 & 1 & 0 & 0 & 0 & 0 & 0 & 0 & 0 & 0 & 0 & 0 & 6.59 \\
0 & 2 & 0 & 0 & 0 & 0 & 0 & 0 & 0 & 0 & 0 & 0 & 13.19 \\
1 & 1 & 0 & 1 & 0 & 0 & 0 & 0 & 0 & 0 & 0 & 0 & 23.08
\end{tabular}




\section{PAKTIKA}




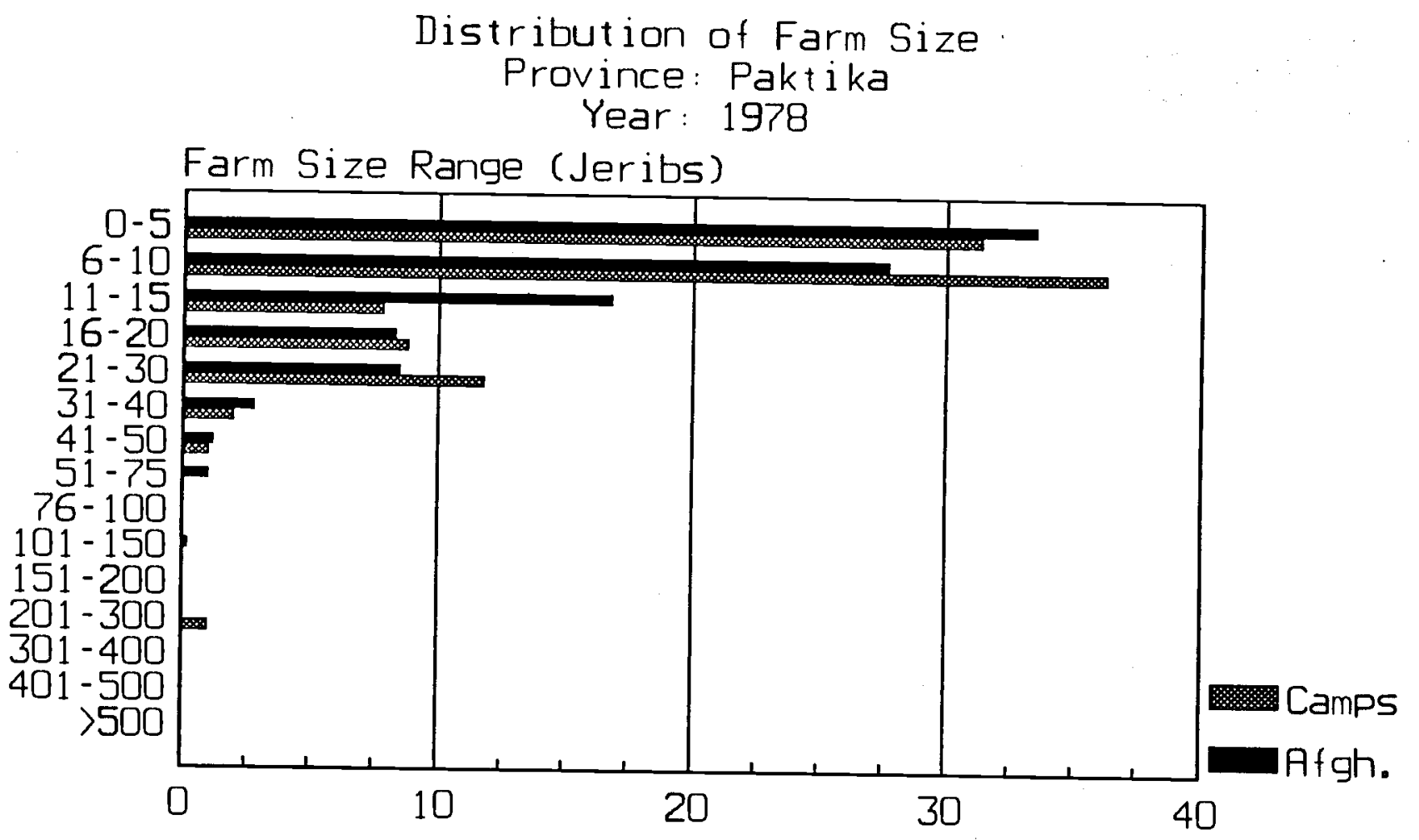

Percentage of Farmers

Farmers in Afghanistans

Total Number of Farms Average Area

Average Area Irrigated

Average Dryland Area

Farmers who left in 1987

Total Number of Farms

Average Area

Average Area Irrigated

Average Dryland Area

1978

495

11.66

10.80

7.20 $\underline{1987}$

487

2.67

2.66

2.56

Average of both Groups

Provincial Average Area

Provincial Average Irrigated Area

102

0

13.14

10.73

20.83

$-$

$-$

-

Provincial Average Dryland Area

11.91

2.67

10.79

2.66

9.51

2. 56

(All areas in Jeribs. 5 Jeribs $=1$ Hectare) 

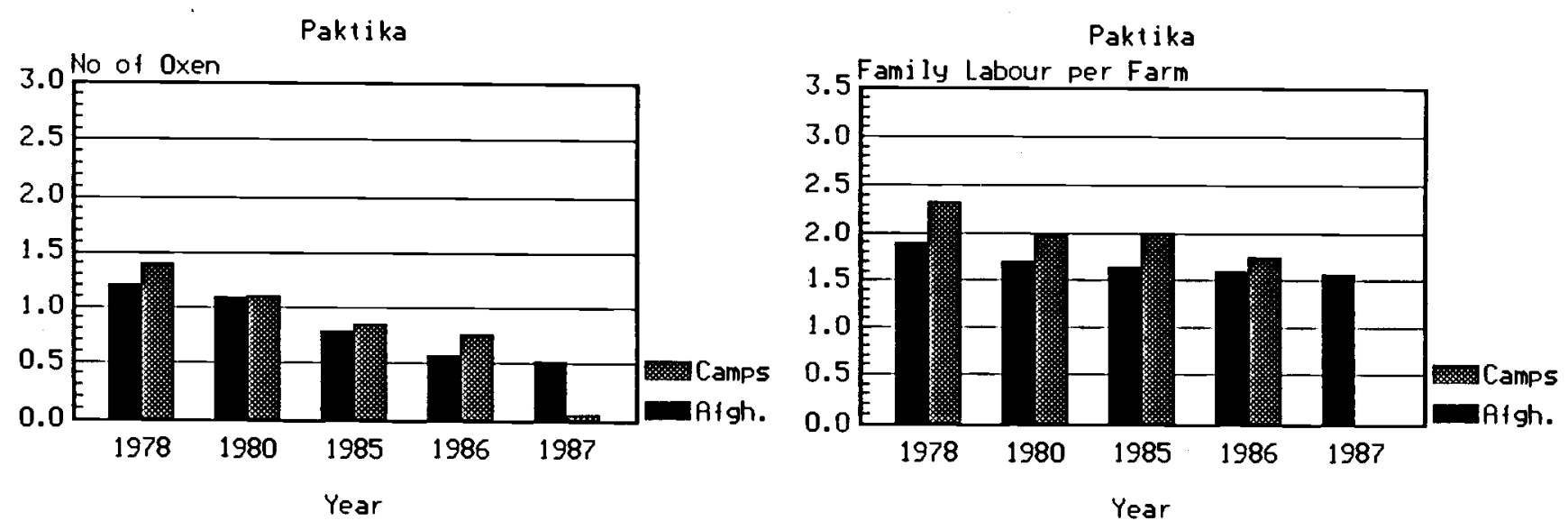

Paktika

Paktika
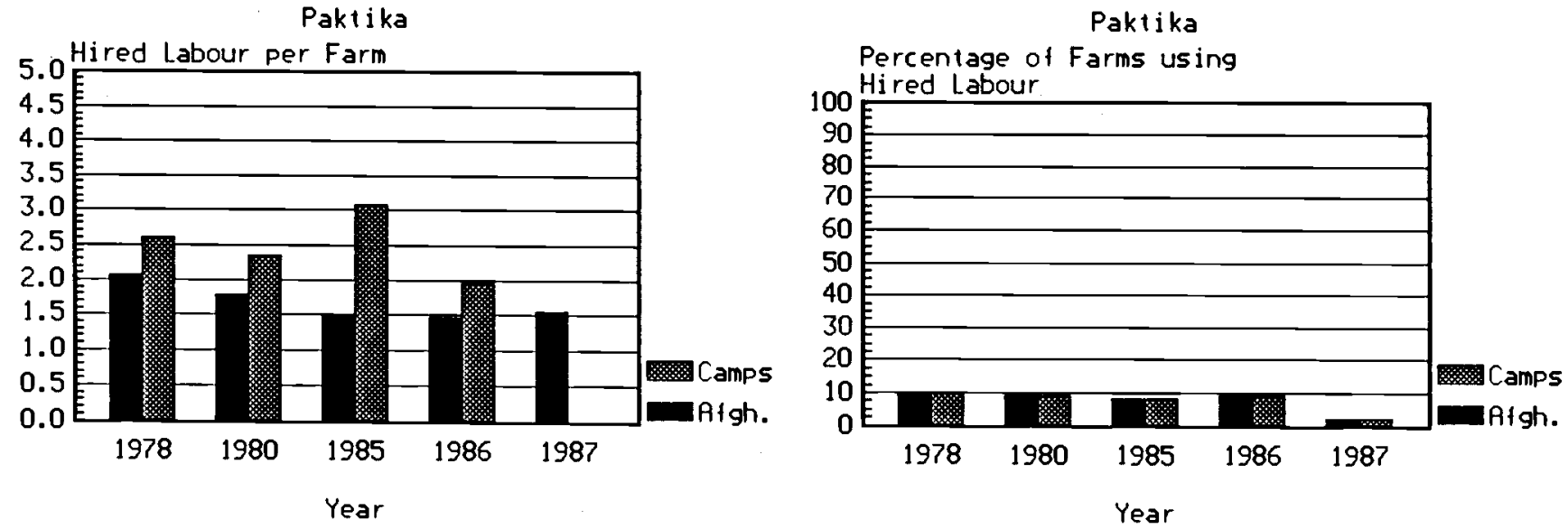
PROVINCE OF PTK

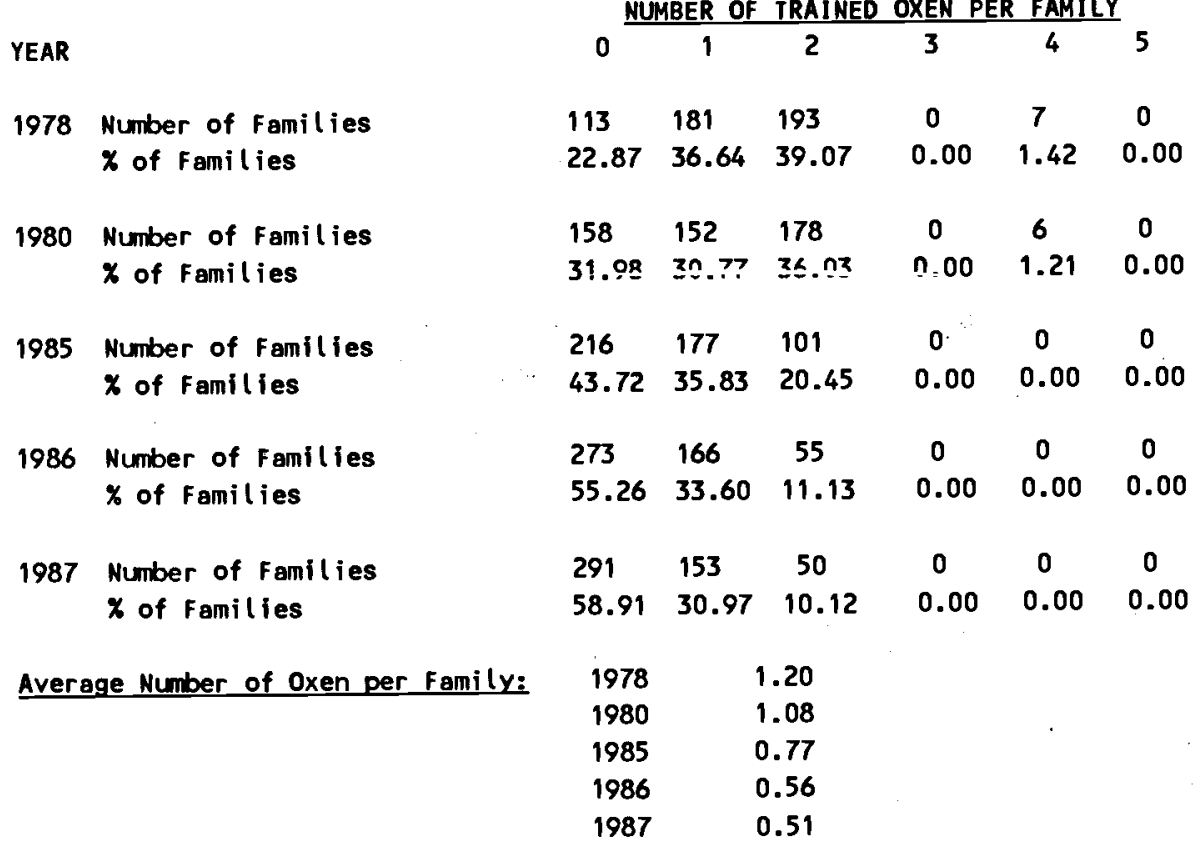

Number of Farmers Questioned 494

IABLE 7B - AVERAGE NUMBER OF TRAINED OXEN FOR FARMERS IN THE CAMPS

PROVINCE OF PTK

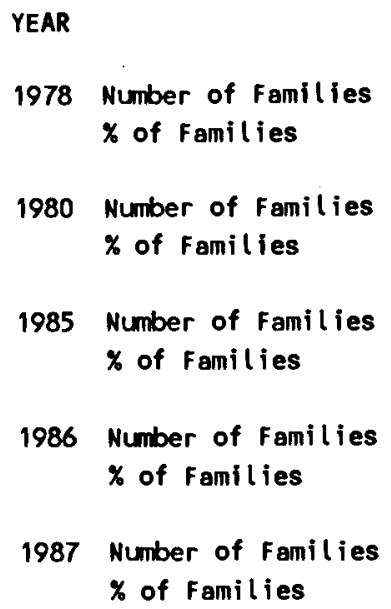

\begin{tabular}{|c|c|c|c|c|c|}
\hline 0 & 1 & 2 & 3 & 4 & 5 \\
\hline 19 & 35 & 45 & 3 & 3 & 0 \\
\hline 18.10 & 33.33 & 42.86 & 2.86 & 2.86 & 0.00 \\
\hline 35 & 33 & 34 & 1 & 3 & 0 \\
\hline 33.02 & 31.13 & 32.08 & 0.94 & 2.83 & 0.00 \\
\hline 48 & 30 & 26 & 1 & 1 & 0 \\
\hline 45.28 & 28.30 & 24.53 & 0.94 & 0.94 & 0.00 \\
\hline 53 & 27 & & 1 & 0 & 0 \\
\hline 50.00 & 25.47 & 8 & 0.94 & 0.00 & 0.00 \\
\hline 104 & 0 & & 0 & 0 & 0 \\
\hline 88.11 & 0.00 & 1.89 & 0.00 & 0.00 & 0.00 \\
\hline
\end{tabular}

$\begin{array}{ll}1978 & 1.39 \\ 1980 & 1.09 \\ 1985 & 0.84 \\ 1986 & 0.75 \\ 1987 & 0.04\end{array}$




\section{IABLE $8(A)$ - AVERAGE LABOUR (FAMILY and HIRED) FOR FARMERS IN AFGANISTAN}

PROVINCE OF PIK

\begin{tabular}{|c|c|c|c|c|c|}
\hline & 1978 & 1980 & 1985 & 1986 & 1987 \\
\hline Average Family Labour per Farm & 1.89 & 1.70 & 1.64 & 1.60 & 1.56 \\
\hline $\begin{array}{l}\text { Average No. of Hired Labour per Farm } \\
\text { (for farms using hired (abour) }\end{array}$ & 2.07 & 1.78 & 1.51 & 1.46 & 1.55 \\
\hline Number of Farms using Hired Labour & 43 & 51 & 82 & 94 & 86 \\
\hline
\end{tabular}

\section{IABLE $8(B)$ - AVERAGE LABOUR (FAMILY and HIRED) FOR FARMERS IN CAMPS}

PROVINCE OF PTK

Average Family Labour per Farm

Average No. of Hired Labour per Farm

(for farms using hired labour)

Number of Farms using Hired Labour

Number of Farmers Questioned 106

$\begin{array}{rrrrr}1978 & 1980 & 1985 & 1986 & 1987 \\ 2.32 & 1.98 & 1.99 & 1.75 & 0.08 \\ 2.59 & 2.35 & 3.06 & 1.95 & 3.50 \\ & & & & \\ 11 & 10 & 9 & 10 & 2\end{array}$


PROVINCE OF PTK

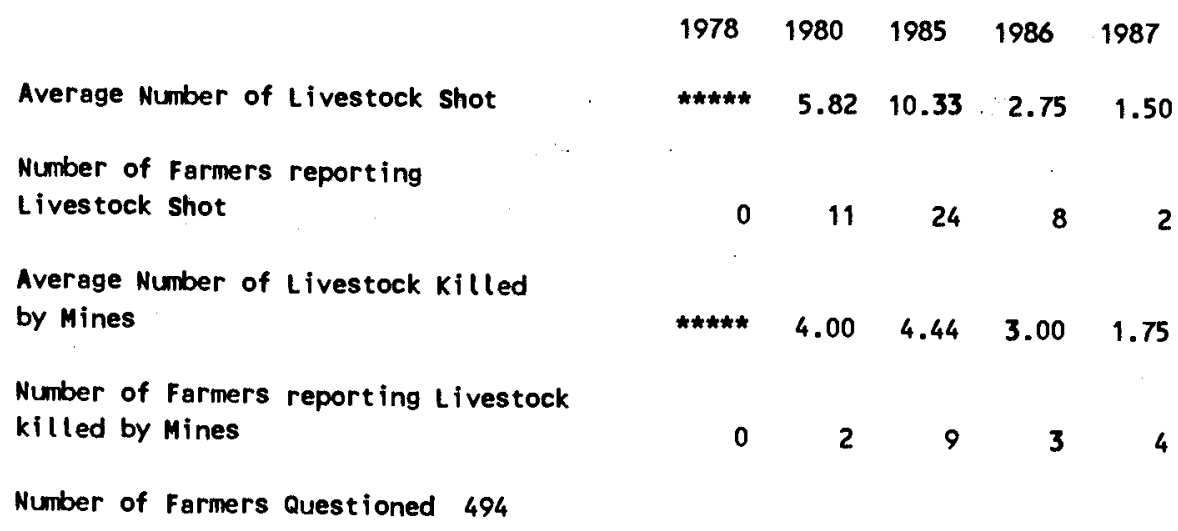

\section{IABLE 9B - DIRECT EFFECIS OF THE WAR - FARMERS IN CAMPS}

PROVINCE OF PTK

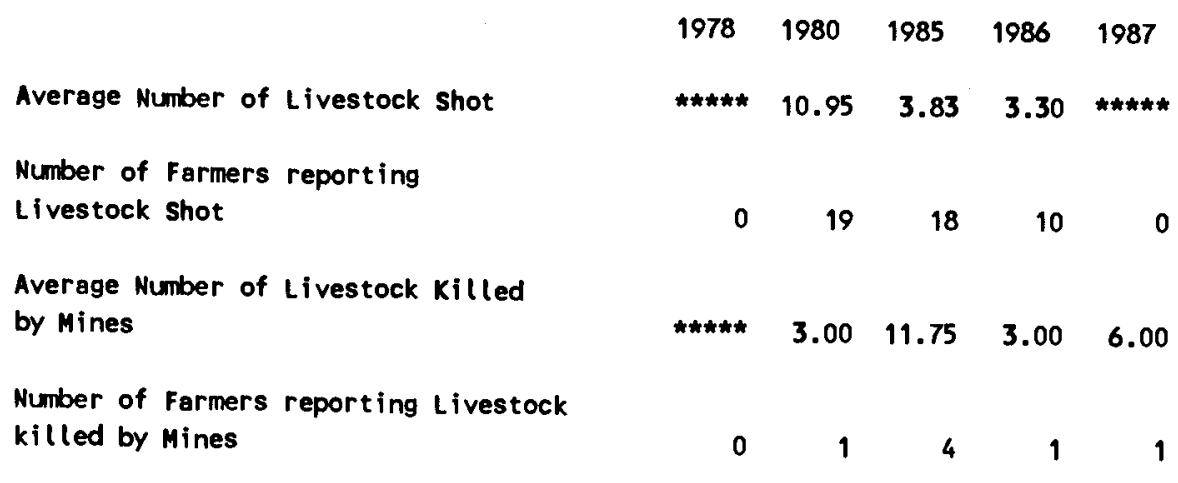

Number of Farmers Questioned 106 


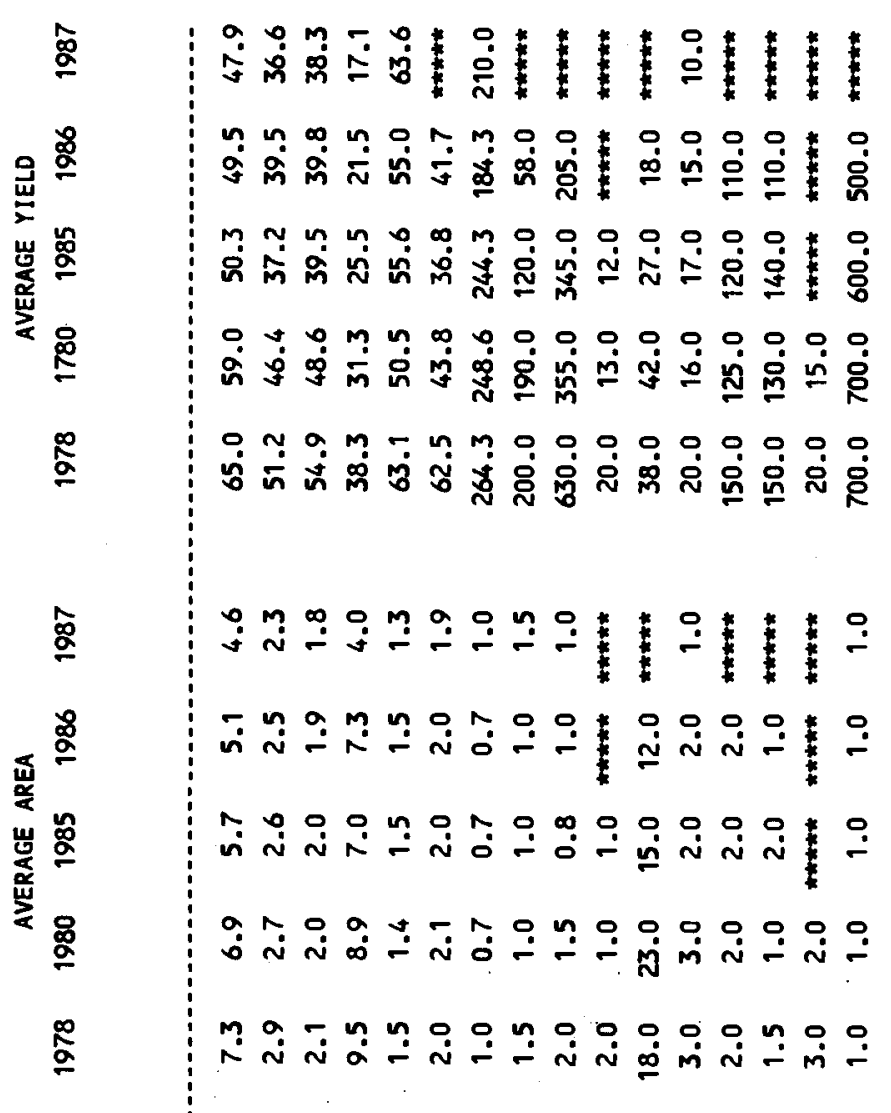

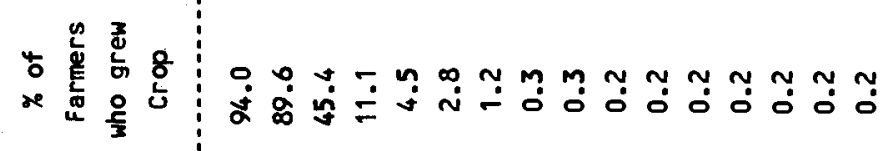

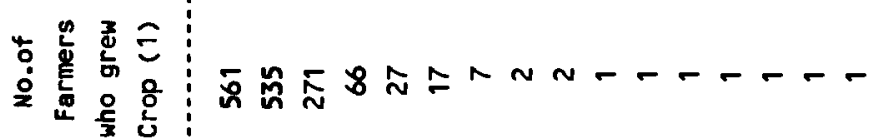

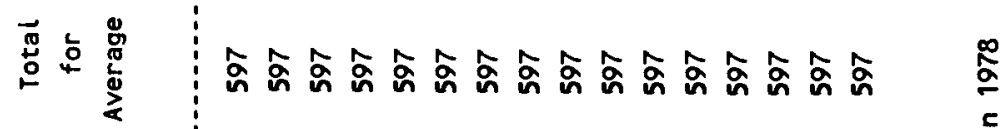

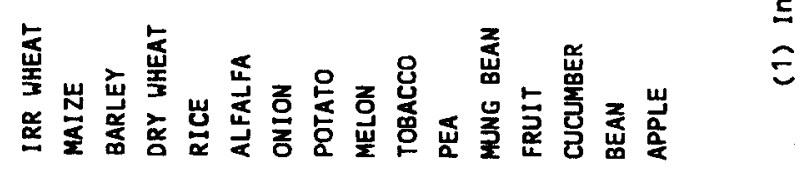
: 
PROVINCE OF PTK

\section{PERCENTAGE OF VILLAGES GIVING FOLLOWING PRIORITY TO PROBLEM}

Rainfall

Crop Disease

Birds or Rats

Seed Availability

Fertilizer Availability

Crop Protection Chemicals

Credit

Irrigation Water Availability

Flooding

Labour Avail labilty

Power for Land Preparation

Direct War Effects

Animal Diseases

Extension

Other Problems

$\begin{array}{rrrrrrrrrrrrr}1 & 2 & 3 & 4 & 5 & 6 & 7 & 8 & 9 & 10 & 11 & 12 & \text { Weighted Priority } \\ 1 & 0 & 0 & 0 & 0 & 0 & 0 & 0 & 0 & 0 & 0 & 0 & 0.33 \\ 8 & 6 & 6 & 5 & 7 & 6 & 4 & 0 & 0 & 0 & 0 & 0 & 8.31 \\ 1 & 1 & 6 & 6 & 6 & 1 & 1 & 1 & 0 & 0 & 0 & 0 & 3.11 \\ 1 & 2 & 7 & 3 & 3 & 1 & 2 & 0 & 0 & 0 & 0 & 0 & 2.86 \\ 22 & 43 & 16 & 3 & 1 & 1 & 0 & 0 & 0 & 0 & 0 & 0 & 23.55 \\ 0 & 1 & 1 & 5 & 5 & 6 & 1 & 1 & 0 & 0 & 0 & 0 & 1.89 \\ 1 & 0 & 1 & 1 & 0 & 0 & 0 & 0 & 0 & 0 & 0 & 0 & 0.86 \\ 29 & 5 & 1 & 9 & 2 & 3 & 1 & 1 & 0 & 0 & 0 & 0 & 16.53 \\ 2 & 0 & 2 & 1 & 2 & 1 & 0 & 0 & 0 & 0 & 0 & 0 & 1.66 \\ 0 & 1 & 0 & 0 & 1 & 0 & 0 & 0 & 0 & 0 & 0 & 0 & 0.40 \\ 16 & 21 & 39 & 4 & 0 & 1 & 0 & 0 & 0 & 0 & 0 & 0 & 19.31 \\ 13 & 8 & 1 & 3 & 7 & 5 & 11 & 2 & 0 & 0 & 0 & 0 & 10.51 \\ 6 & 3 & 6 & 17 & 10 & 4 & 1 & 0 & 1 & 0 & 0 & 0 & 7.69 \\ 0 & 0 & 2 & 6 & 6 & 7 & 1 & 3 & 1 & 1 & 0 & 0 & 2.51 \\ 0 & 1 & 0 & 1 & 0 & 0 & 0 & 0 & 0 & 0 & 0 & 0 & 0.50\end{array}$

1986

Rainfall

Crop Disease

Birds or Rats

Seed Availability

Fertilizer Availability

Crop Protection Chemicals

Credit

Irrigation Water Availability

Flooding

Labour Avai labilty

Power for Land Preparation

Direct War Effects

Animal Diseases

Extension

Other Problems

$\begin{array}{rrrrrrrrrrrrr}1 & 2 & 3 & 4 & 5 & 6 & 7 & 8 & 9 & 10 & 11 & 12 & \text { Heighted Priority } \\ 1 & 0 & 0 & 1 & 0 & 0 & 0 & 0 & 0 & 0 & 0 & 0 & 0.41 \\ 6 & 6 & 6 & 6 & 6 & 6 & 0 & 1 & 0 & 0 & 0 & 0 & 6.84 \\ 1 & 0 & 3 & 8 & 4 & 3 & 1 & 0 & 0 & 0 & 0 & 0 & 2.45 \\ 0 & 3 & 7 & 3 & 5 & 1 & 1 & 1 & 0 & 0 & 0 & 0 & 2.78 \\ 26 & 42 & 13 & 3 & 2 & 1 & 1 & 0 & 0 & 0 & 0 & 0 & 24.71 \\ 0 & 1 & 1 & 7 & 6 & 4 & 3 & 0 & 0 & 0 & 0 & 0 & 2.24 \\ 1 & 0 & 0 & 1 & 0 & 0 & 1 & 0 & 0 & 0 & 0 & 0 & 0.46 \\ 6 & 3 & 2 & 6 & 2 & 9 & 8 & 4 & 0 & 0 & 0 & 0 & 6.30 \\ 13 & 3 & 9 & 3 & 7 & 3 & 1 & 0 & 0 & 0 & 0 & 0 & 9.35 \\ 1 & 0 & 1 & 0 & 1 & 1 & 0 & 0 & 0 & 0 & 0 & 0 & 0.88 \\ 25 & 20 & 28 & 5 & 2 & 0 & 0 & 0 & 0 & 0 & 0 & 0 & 21.66 \\ 12 & 15 & 6 & 6 & 5 & 10 & 1 & 2 & 0 & 0 & 0 & 0 & 12.13 \\ 6 & 3 & 8 & 14 & 3 & 1 & 2 & 1 & 0 & 0 & 0 & 0 & 7.26 \\ 1 & 0 & 4 & 4 & 6 & 1 & 4 & 1 & 1 & 0 & 0 & 0 & 2.46 \\ 0 & 0 & 0 & 0 & 1 & 0 & 0 & 0 & 0 & 0 & 0 & 0 & 0.07\end{array}$

1985

Rainfall

Crop Disease

Birds or Rats

Seed Availability

Fertilizer Availability

Crop Protection Chemicals

Credit

Irrigation Water Availability

Flooding

Labour Availabilty

Power for Land Preparation

Direct War Effects

Animal Diseases

Extension

Other Problems

\begin{tabular}{rrrrrrrrrrrrr}
1 & 2 & 3 & 4 & 5 & 6 & 7 & 8 & 9 & 10 & 11 & 12 & Weighted Priority \\
\hline 0 & 0 & 0 & 0 & 1 & 0 & 0 & 0 & 0 & 0 & 0 & 0 & 0.07 \\
9 & 1 & 6 & 4 & 6 & 4 & 3 & 1 & 0 & 0 & 0 & 0 & 7.15 \\
0 & 1 & 1 & 4 & 8 & 5 & 2 & 0 & 0 & 0 & 0 & 0 & 2.01 \\
0 & 2 & 3 & 2 & 3 & 2 & 0 & 0 & 1 & 0 & 0 & 0 & 1.64 \\
13 & 43 & 20 & 6 & 1 & 0 & 1 & 0 & 0 & 0 & 0 & 0 & 20.36 \\
0 & 0 & 0 & 3 & 3 & 4 & 4 & 1 & 0 & 0 & 0 & 0 & 1.39 \\
0 & 1 & 0 & 0 & 1 & 1 & 0 & 0 & 0 & 0 & 0 & 0 & 0.57 \\
20 & 3 & 3 & 8 & 3 & 5 & 6 & 5 & 1 & 0 & 0 & 0 & 13.28 \\
10 & 2 & 6 & 16 & 5 & 7 & 0 & 1 & 0 & 0 & 0 & 0 & 9.38 \\
1 & 1 & 1 & 1 & 1 & 0 & 0 & 0 & 0 & 0 & 0 & 0 & 1.24 \\
13 & 17 & 33 & 5 & 3 & 3 & 1 & 0 & 0 & 0 & 0 & 0 & 16.26 \\
31 & 18 & 8 & 3 & 3 & 1 & 0 & 0 & 0 & 0 & 0 & 0 & 20.64 \\
0 & 0 & 6 & 12 & 10 & 1 & 1 & 0 & 0 & 0 & 0 & 0 & 3.46 \\
0 & 0 & 2 & 3 & 4 & 3 & 10 & 3 & 1 & 0 & 0 & 0 & 2.26 \\
0 & 1 & 0 & 0 & 1 & 0 & 0 & 0 & 0 & 0 & 0 & 0 & 0.30
\end{tabular}


PROVINCE OF PTK

Rainfall

Crop Disease

Birds or Rats

Seed Avail lability

Fertilizer Avallability

Crop Protection Chemicals

Credit

Irrigation Water Availability

Flooding

Labour Availabilty

Power for Land Preparation

Direct War Effects

Animal Diseases

Extens ion

other Problems

\begin{tabular}{rrrrrrrrrrrrr}
1 & 2 & 3 & 4 & 5 & 6 & 7 & 8 & 9 & 10 & 11 & 12 & Weighted Priority \\
\hline 0 & 1 & 0 & 0 & 0 & 0 & 0 & 0 & 0 & 0 & 0 & 0 & 0.17 \\
2 & 2 & 2 & 5 & 6 & 3 & 3 & 0 & 0 & 0 & 0 & 0 & 3.52 \\
0 & 1 & 3 & 6 & 5 & 5 & 1 & 2 & 0 & 0 & 0 & 0 & 2.75 \\
6 & 5 & 5 & 3 & 2 & 8 & 1 & 1 & 0 & 0 & 0 & 0 & 6.00 \\
8 & 47 & 17 & 1 & 1 & 0 & 0 & 0 & 0 & 0 & 0 & 0 & 17.95 \\
0 & 1 & 4 & 6 & 13 & 4 & 3 & 1 & 0 & 0 & 0 & 0 & 3.31 \\
1 & 1 & 1 & 1 & 1 & 0 & 1 & 0 & 0 & 0 & 0 & 0 & 0.99 \\
1 & 6 & 3 & 8 & 1 & 3 & 3 & 3 & 1 & 1 & 0 & 0 & 4.02 \\
8 & 3 & 7 & 8 & 3 & 5 & 1 & 1 & 0 & 0 & 0 & 0 & 7.43 \\
0 & 1 & 1 & 1 & 0 & 0 & 0 & 0 & 0 & 0 & 0 & 0 & 0.73 \\
3 & 8 & 26 & 8 & 3 & 1 & 2 & 1 & 1 & 0 & 0 & 0 & 9.03 \\
70 & 15 & 1 & 1 & 0 & 0 & 0 & 0 & 0 & 0 & 0 & 0 & 37.85 \\
2 & 2 & 4 & 4 & 3 & 3 & 1 & 0 & 0 & 0 & 0 & 0 & 3.36 \\
0 & 0 & 3 & 6 & 5 & 6 & 6 & 1 & 1 & 0 & 0 & 0 & 2.85 \\
0 & 0 & 0 & 0 & 0 & 0 & 1 & 0 & 0 & 0 & 0 & 0 & 0.05
\end{tabular}

\section{8}

Rainfall

Crop Disease

Birds or Rats

Seed Availability

Fertilizer Availability

Crop Protection Chemicals

Credit

Irrigation Water Availability

Flooding

Labour Availabilty

Power for Land Preparation

Direct War Effects

Animal Diseases

Extension

Other Problems

\begin{tabular}{rrrrrrrrrrrrc}
1 & 2 & 3 & 4 & 5 & 6 & 7 & 8 & 9 & 10 & 11 & 12 & Weighted Priority \\
\hline 0 & $n$ & $n$ & 2 & 2 & $n$ & 0 & $n$ & $n$ & $n$ & 0 & 0 & 0.00 \\
2 & 0 & 3 & 5 & 3 & 1 & 1 & 0 & 0 & 0 & 0 & 0 & 3.65 \\
1 & 1 & 1 & 7 & 6 & 6 & 1 & 1 & 0 & 0 & 0 & 0 & 4.63 \\
18 & 21 & 15 & 6 & 1 & 1 & 0 & 0 & 0 & 0 & 0 & 0 & 24.87 \\
44 & 1 & 0 & 0 & 0 & 0 & 0 & 0 & 0 & 0 & 0 & 0 & 31.60 \\
0 & 8 & 19 & 9 & 1 & 1 & 0 & 0 & 0 & 0 & 0 & 0 & 8.95 \\
1 & 2 & 0 & 0 & 0 & 0 & 0 & 0 & 0 & 0 & 0 & 0 & 1.73 \\
1 & 0 & 0 & 0 & 0 & 0 & 0 & 0 & 0 & 0 & 0 & 0 & 0.99 \\
2 & 0 & 1 & 2 & 3 & 1 & 1 & 0 & 0 & 0 & 0 & 0 & 2.73 \\
0 & 1 & 1 & 0 & 0 & 0 & 0 & 0 & 0 & 0 & 0 & 0 & 0.41 \\
1 & 11 & 1 & 0 & 1 & 3 & 1 & 1 & 0 & 0 & 0 & 0 & 5.89 \\
0 & 0 & 0 & 0 & 0 & 0 & 0 & 0 & 1 & 0 & 0 & 0 & 0.05 \\
6 & 8 & 0 & 4 & 2 & 2 & 1 & 0 & 0 & 0 & 0 & 0 & 8.51 \\
1 & 1 & 3 & 5 & 15 & 7 & 5 & 0 & 0 & 0 & 0 & 0 & 5.90 \\
0 & 0 & 0 & 0 & 0 & 1 & 0 & 0 & 0 & 0 & 0 & 0 & 0.08
\end{tabular}


SAMANGAN 


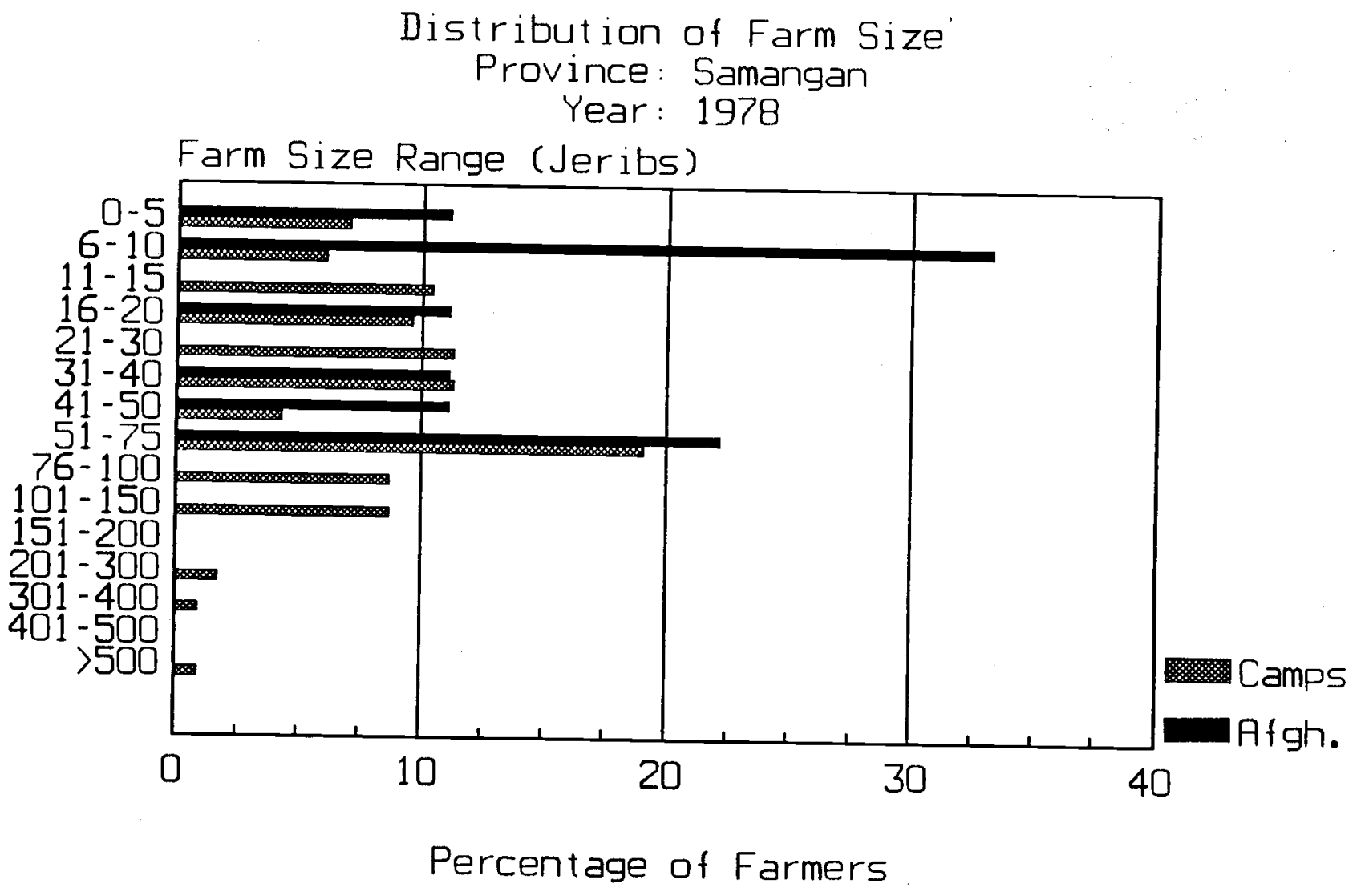

Farmers in Afghanistans

Total Number of Farms Average Area

Average Area Irrigated

Average Dryland Area

1978

9

30.78

18.75

28.86

115

55.69

24.14

64.81

Average Dryland Area

Average of both Groups

Provincial Average Area

Provincial Average Irrigated Area

Provincial Average Dryland Area
53.88

23.88

61.50 $\underline{1987}$

24.67

18.75

23.14

(All areas in Jeribs. 5 Jeribs $=1$ Hectare) 
Farm Pouer - Farmers in Atghanistan Province of Samangan

Crop: Irrigated wheat

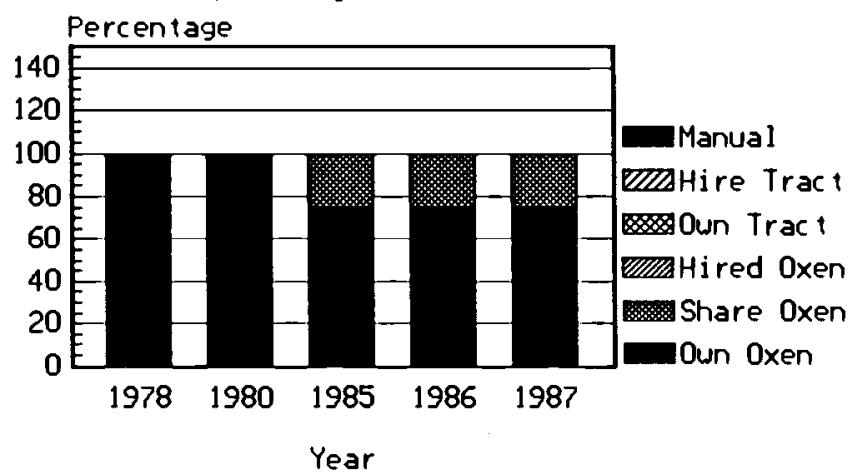

Farm Power - Farmers who lett in 1987 Province of Samangan Crop: Irrigated wheat

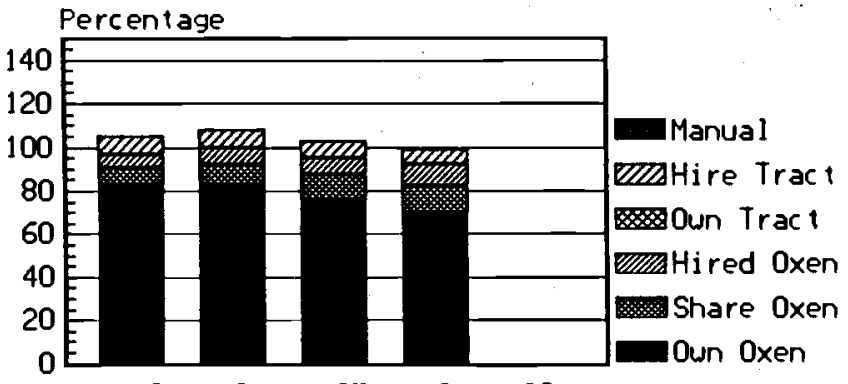

$\begin{array}{lllll}1978 & 1980 & 1985 & 1986 & 1987\end{array}$

Year
Farm Power - Farmers in Atghanistan Province of Samangan Crop: Barley

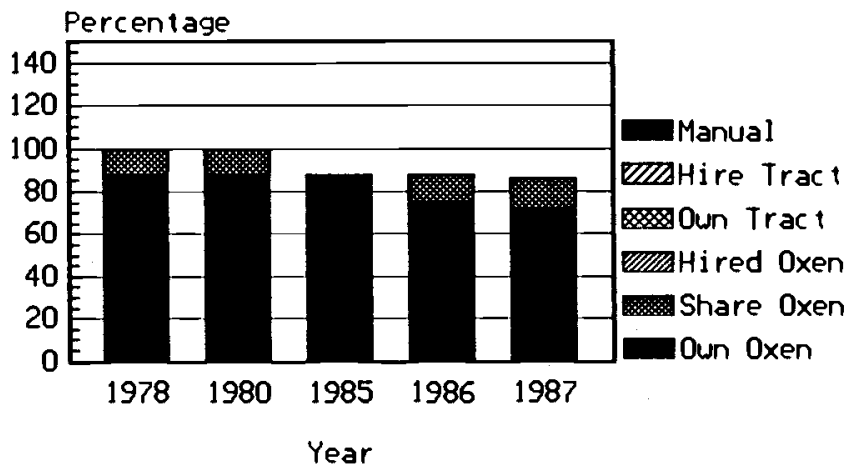

Farm Power - Farmers who lett in 1987 Province of Samangan Crop: Barley

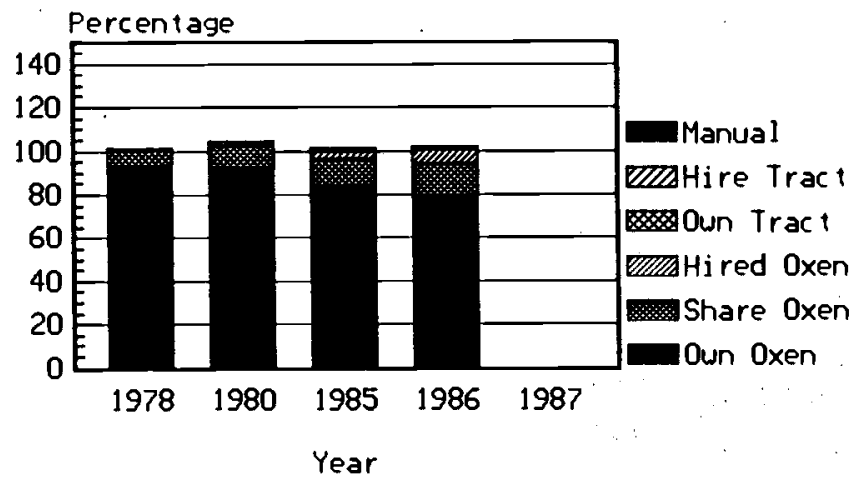

Base Fiqures for above graphs

Province: Samangan

Farmers in Afqhanistan

Irr. Wheat own Oxen

Shared oxen

Hired oxen

Own Tractor

Hired Tractor

Barley

Manual Means

Own Oxen

Shared oxen

Hired oxen

Own Tractor

Hired Tractor

Manual Means

Farmers who left in 1987

Irr. Wheat own oxen

Shared oxen

Hired oxen

Own Tractor

Hired Tractor

Barley

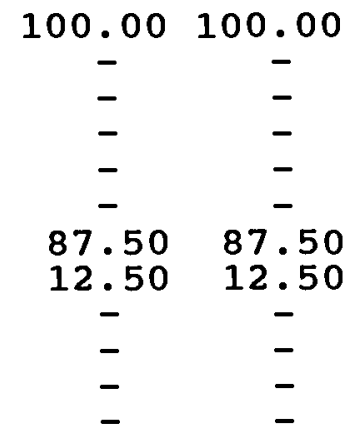

Percentage of Farmers $1978 \quad \underline{1980} \quad \underline{1985} \quad \underline{1986}$

$\underline{1987}$

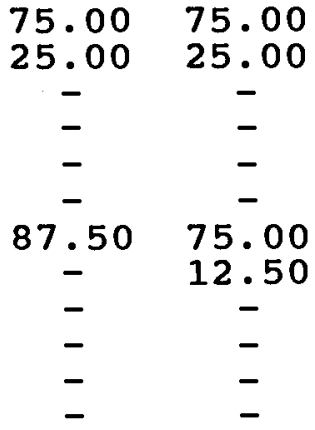

75.00

25.00

$-$

$-$

$-$

71.43

14.29

$-$

$-$

$-$
Manual Means

Own oxen

Shared oxen

Hired oxen

Own Tractor

$\begin{array}{ccccc}82.93 & 83.08 & 75.95 & 69.86 & 100.00 \\ 7.32 & 9.23 & 11.39 & 12.33 & - \\ 6.10 & 7.69 & 7.59 & 9.59 & - \\ - & - & - & - & - \\ 8.54 & 7.69 & 7.59 & 6.85 & - \\ - & - & - & 1.37 & - \\ 92.54 & 92.00 & 83.82 & 79.37 & 100.00 \\ 7.46 & 10.00 & 11.76 & 14.29 & - \\ - & - & 4.41 & 6.35 & - \\ - & - & - & - & - \\ 1.19 & - & 1.47 & 1.59 & -\end{array}$


Samangan

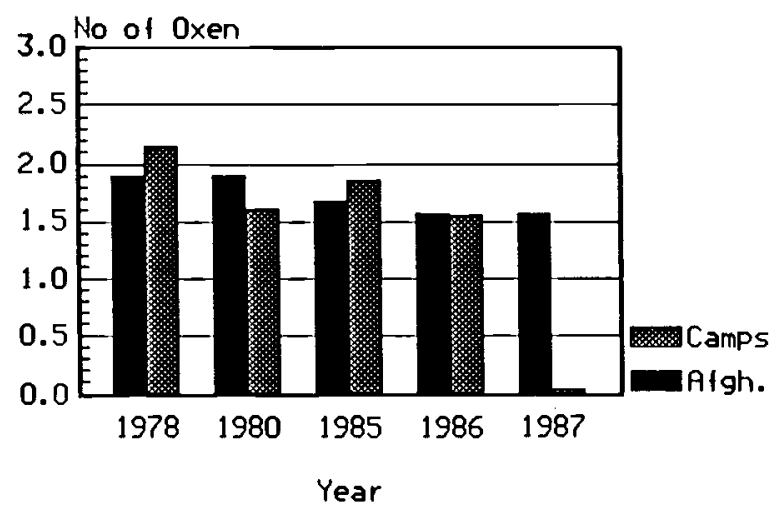

Samangan

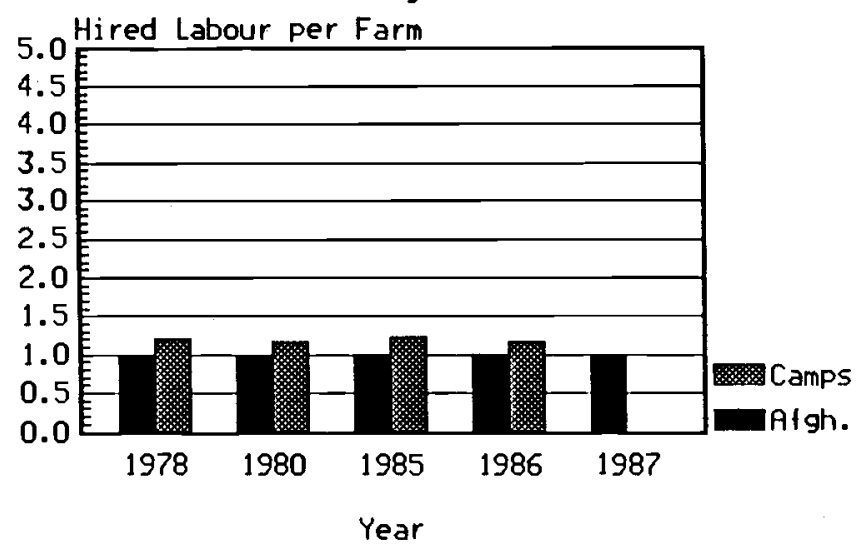

Samangan

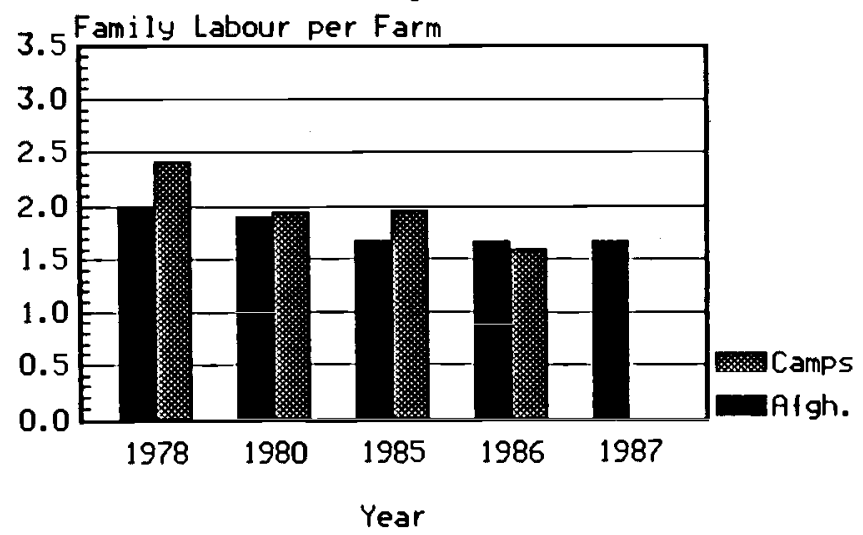

Samangan

Percentage of Farms using

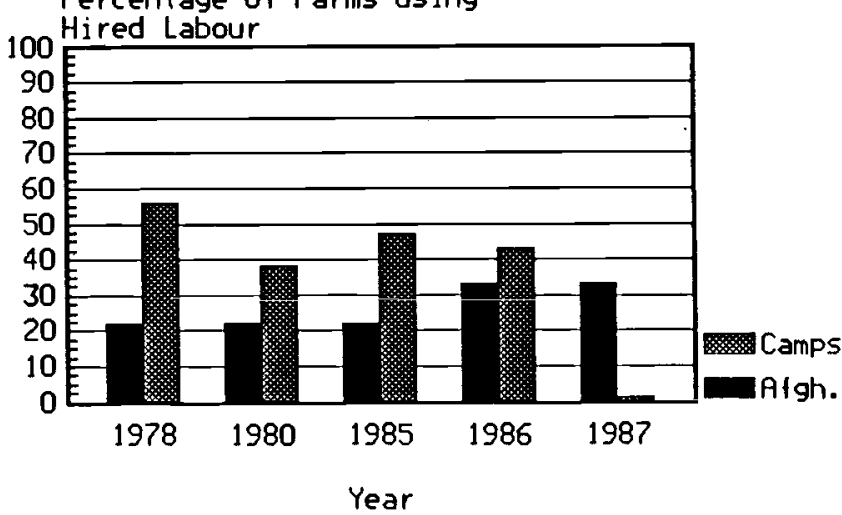


PROVINCE OF SMG

YEAR

1978 Number of Families

$X$ of Families

1980 Number of Families

$x$ of Families

1985 Number of Families

X of Families

1986 Number of Families

X of Families

1987 Number of Families

$x$ of Fanilies

Average Number of Oxen per Family:

1978

1980

1985

1986

1987

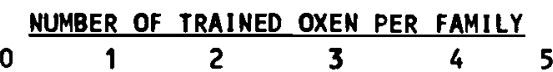

$\begin{array}{cccccc}0 & 1 & 8 & 0 & 0 & 0 \\ 0.00 & 11.11 & 88.89 & 0.00 & 0.00 & 0.00\end{array}$

$\begin{array}{llllll}0 & 1 & 8 & 0 & 0 & 0\end{array}$

$\begin{array}{llllll}0.00 & 11.11 & 88.89 & 0.00 & 0.00 & 0.00\end{array}$

$\begin{array}{cccccc}1 & 1 & 7 & 0 & 0 & 0 \\ 11.11 & 11.11 & 77.78 & 0.00 & 0.00 & 0.00\end{array}$

$\begin{array}{llllll}1 & 2 & 6 & 0 & 0 & 0\end{array}$

$\begin{array}{llllll}11.11 & 22.22 & 66.67 & 0.00 & 0.00 & 0.00\end{array}$

$\begin{array}{llllll}1 & 2 & 6 & 0 & 0 & 0\end{array}$

$\begin{array}{llllll}11.11 & 22.22 & 66.67 & 0.00 & 0.00 & 0.00\end{array}$

$\begin{array}{ll}978 & 1.89 \\ 980 & 1.89 \\ 985 & 1.67 \\ 986 & 1.56 \\ 987 & 1.56\end{array}$

Number of Farmers Questioned 9

IABLE 7B - AVERAGE NUMBER OF TRAINED OXEN FOR FARMERS IN THE CAMPS

PROVINCE OF SMG

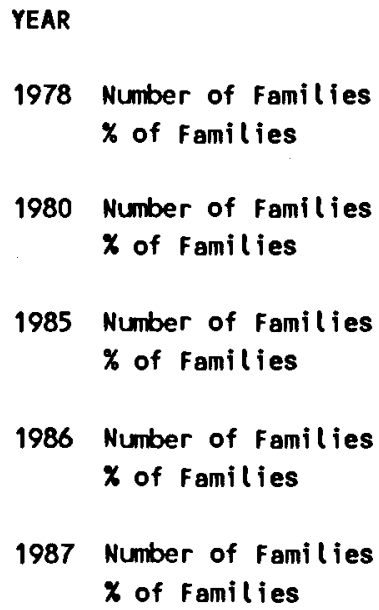

$\begin{array}{ll}1978 & 2.14 \\ 1980 & 1.61 \\ 1985 & 1.85 \\ 1986 & 1.55 \\ 1987 & 0.03\end{array}$

Number of Farmers Questioned 114 
PROVINCE OF SMG

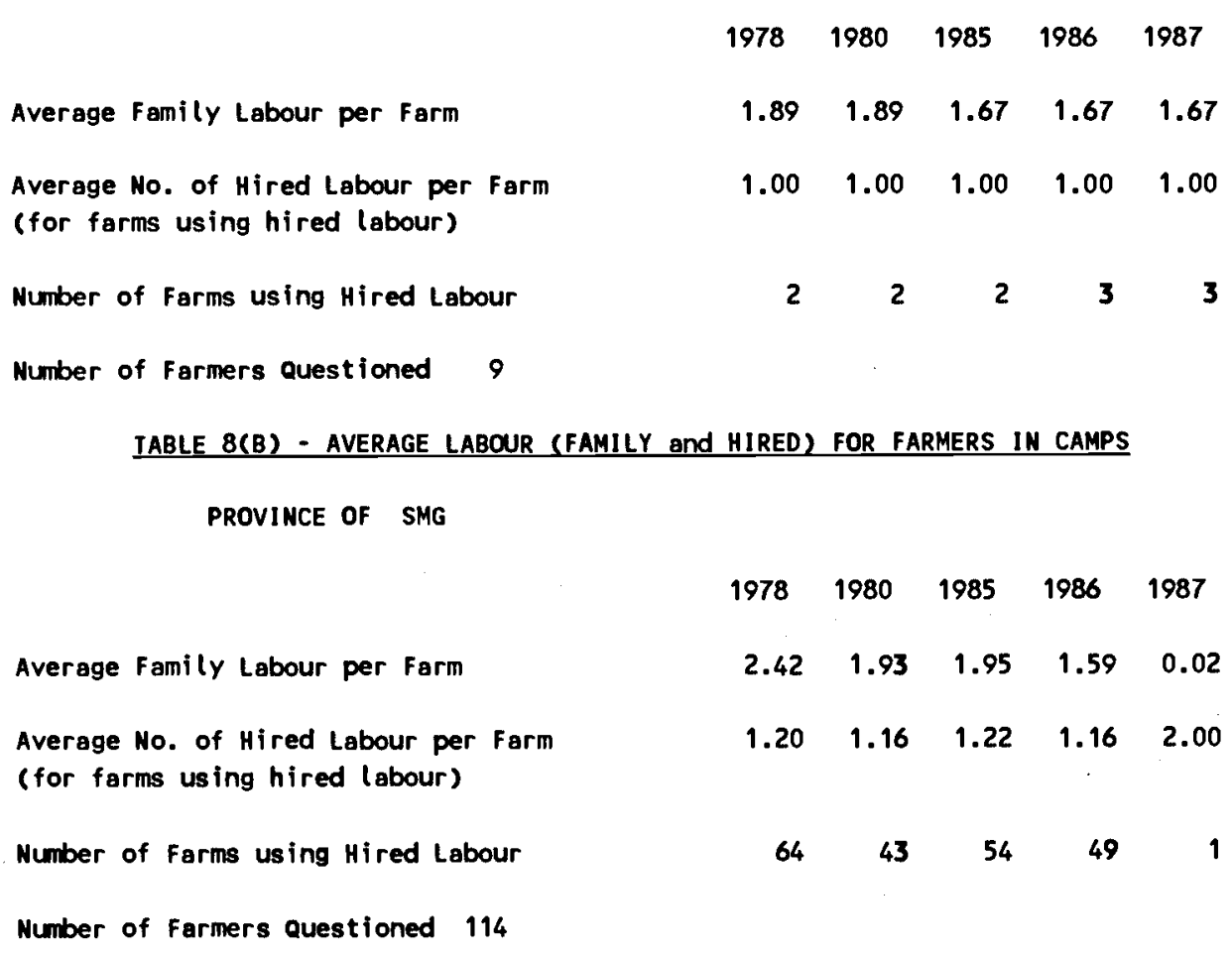


PROVINCE OF SMG

Average Number of Livestock Shot

$\begin{array}{lllll}1978 & 1980 & 1985 & 1986 & 1987 \\ \star \star \star \star \star \star & \star \star \star \star \star & 7.25 & 2.00 & 2.00\end{array}$

Number of Farmers reporting

Livestock Shot

$\begin{array}{lllll}0 & 0 & 4 & 2 & 1\end{array}$

Average Number of Livestock Killed by Mines

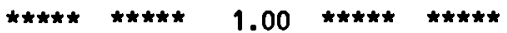

Number of Farmers reporting Livestock

killed by Mines

$\begin{array}{lllll}0 & 0 & 1 & 0 & 0\end{array}$

Number of Farmers Questioned 9

\section{IABLE 9B - DIRECT EFFECTS OF THE HAR - FARMERS IN CAMPS}

PROVINCE OF SMG

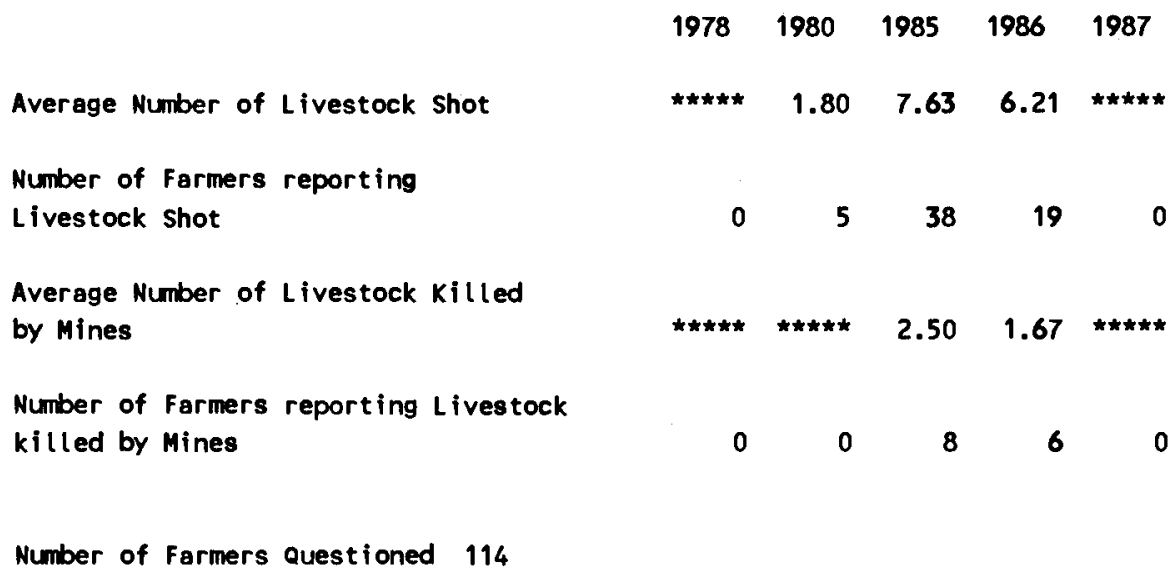



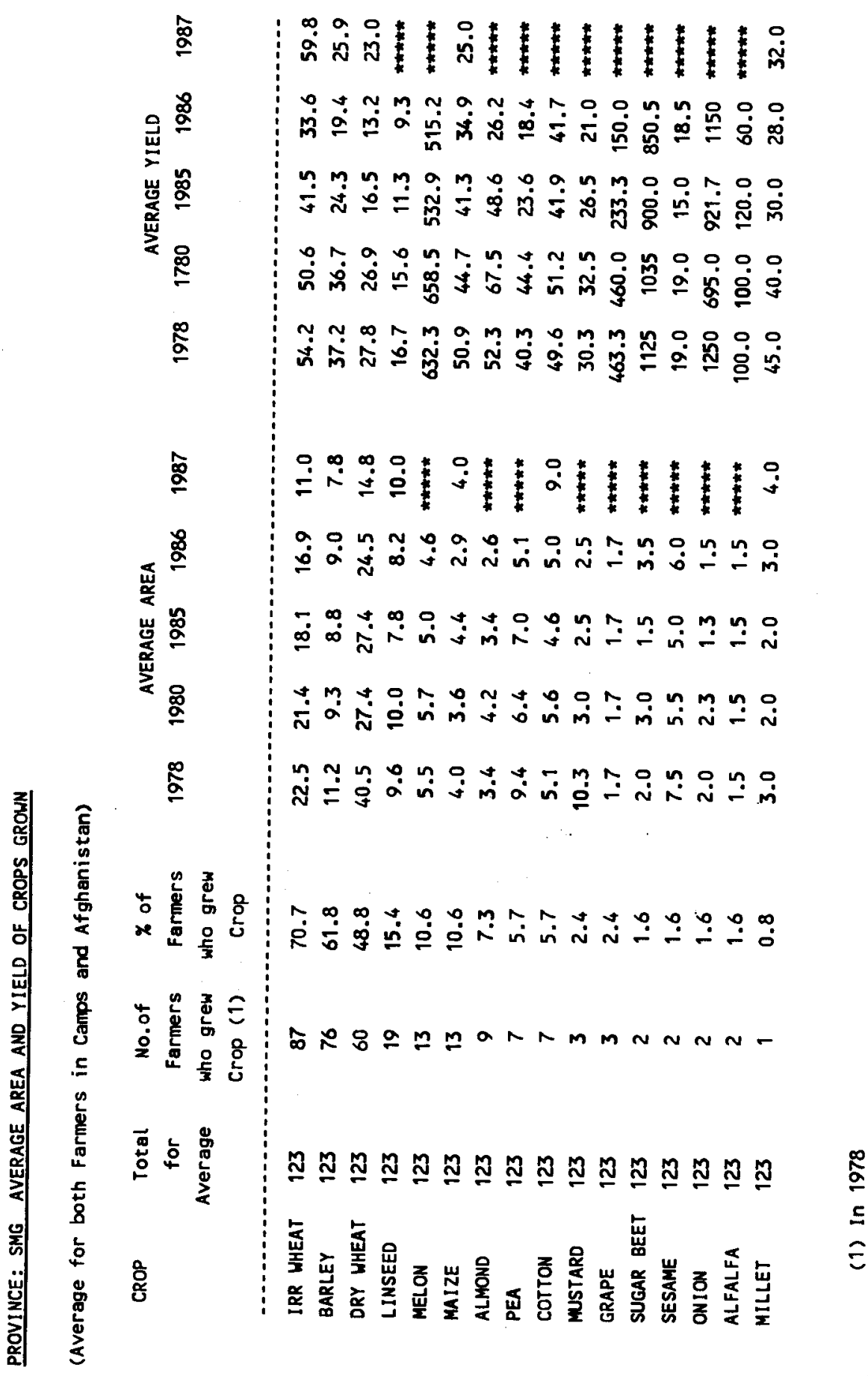
PROVINCE OF SMG

\section{PERCENTAGE OF VILLAGES GIVING FOLLOWING PRIORITY TO PROBLEM}

Rainfall

Crop Disease

Birds or Rats

Seed Avail lability

Fertilizer Availability

Crop Protection Chemicals

Credit

Irrigation Hater Availability

flooding

Labour Avai labilty

Power for Land Preparation

Direct War Effects

Animal Diseases

Extension

Other Problems

\section{Rainfall}

Crop Disease

Birds or Rats

Seed Availability

Fertilizer Avai lability

Crop Protection Chemicals

Credit

Irrigation Water Availability

Flooding

Labour Availabilty

Power for Land Preparation

Direct War Effects

Animal $D$ iseases

Extension

Other Problems $\begin{array}{rrrrrrrrrrrrr}1 & 2 & 3 & 4 & 5 & 6 & 7 & 8 & 9 & 10 & 11 & 12 & \text { Heighted Priority }\end{array}$

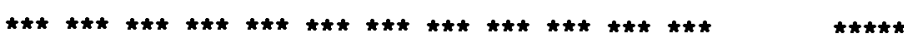

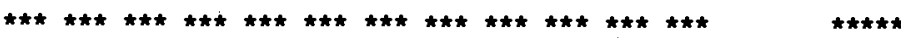

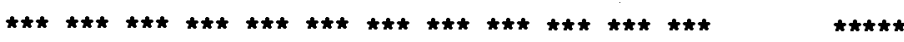

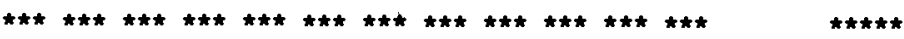

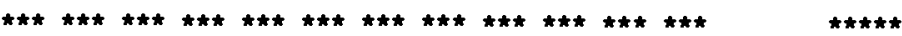

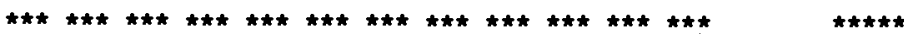

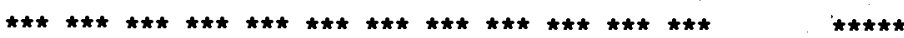

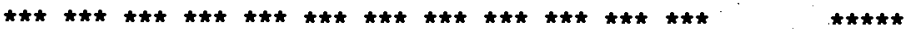

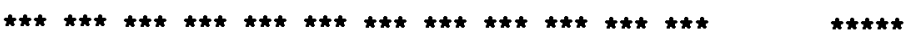

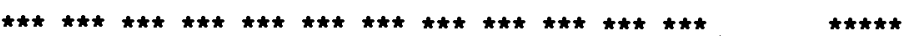

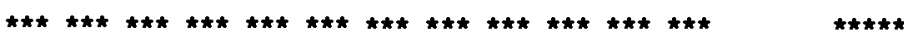

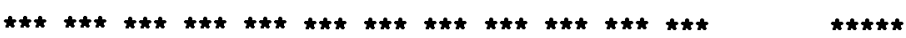

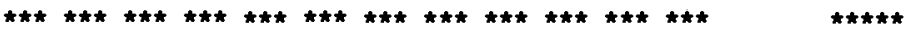

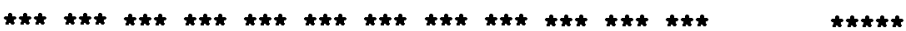

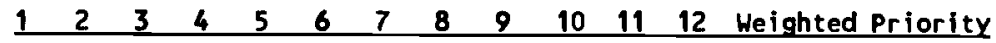

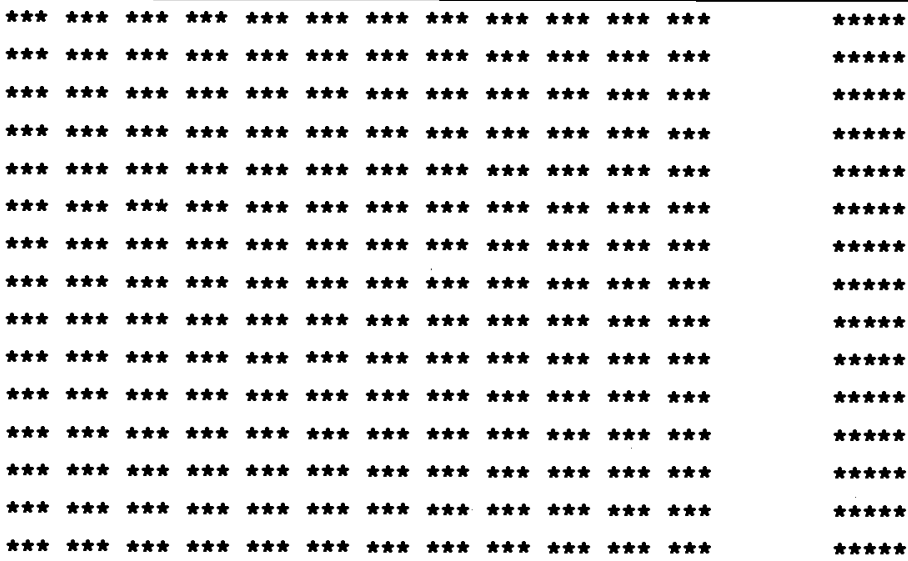

1985

Rainfall

Crop Disease

Birds or Rats

Seed Availability

Fertilizer Availability

Crop Protection Chemicals

Credit

Irrigation Water Availability

Flooding

Labour Availabilty

Power for Land Preparation

Direct War Effects

Animal Diseases

Extension

Other Problems \begin{tabular}{llllllllllll}
1 & 2 & 3 & 4 & 5 & 6 & 7 & 8 & 9 & 10 & 11 & 12 \\
\hline
\end{tabular}

\begin{tabular}{|c|c|c|c|c|c|c|c|c|c|c|c|}
\hline $\begin{array}{l}\star \star \star \\
\star \star \star\end{array}$ & $\begin{array}{l}\star \star \star \\
\star \star \star\end{array}$ & $\begin{array}{l}\star \star \star \\
\star \star \star\end{array}$ & $\begin{array}{l}\star \star \star \\
\star \star \star\end{array}$ & $\begin{array}{l}\star \star \star \\
\star \star \star\end{array}$ & $\begin{array}{l}\star \star \star \\
\star \star \star\end{array}$ & $\begin{array}{l}\star \star \star \\
\star \star \star\end{array}$ & $\begin{array}{l}\star \star \star \\
\star \star \star\end{array}$ & $\begin{array}{l}\star \star \star \\
\star \star \star\end{array}$ & $\begin{array}{l}\star \star \star * \\
\star \star \star *\end{array}$ & $\begin{array}{l}\star \star \star \\
\star \star \star\end{array}$ & $\begin{array}{l}\star \star * \\
\star \star \star\end{array}$ \\
\hline 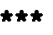 & $\star \star \star \star$ & $\star \star \star *$ & $\star \star \star \star *$ & $\star * *$ & $* \star *$ & $\star \star \star *$ & $\star \star \star \star$ & $\star \star \star \star$ & $\star * *$ & $\star \star \star \star$ & $\star \star \star \star *$ \\
\hline 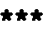 & 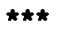 & 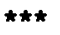 & 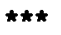 & $\star \star \star \star$ & $\star \star \star \star$ & $\star \star \star$ & $\star \star \star \star$ & $\star \star \star \star$ & $* * *$ & $\star * *$ & $\star \star * *$ \\
\hline$\star * *$ & $\star \star \star \star *$ & $\star \star * *$ & $\star \star \star \star$ & $\star \star \star \star$ & $\star \star \star \star$ & $\star \star \star \star$ & $\star \star \star$ & $\star \star \star \star$ & $\star \star \star \star$ & $\star \star \star \star$ & 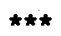 \\
\hline$\star \star \star \star$ & $\star \star \star \star$ & $\star * *$ & $\star \star * *$ & $* * *$ & $\star \star \star \star$ & $\star \star \star$ & $\star \star \star \star$ & $\star \star \star$ & $\star \star \star \star$ & $\star \star \star \star$ & 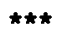 \\
\hline 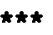 & $\star \star \star \star$ & $\star \star \star \star *$ & 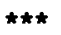 & 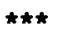 & $\star \star \star \star$ & $\star \star \star \star$ & $\star \star \star \star$ & $\star * *$ & $* * *$ & $\star * *$ & $\star \star \star \star *$ \\
\hline$\star \star \star \star t$ & $\star \star \star \star$ & $\star \star \star \star$ & $\hbar \star \star \star$ & 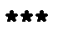 & $\hbar \star \star$ & 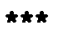 & $\star \star \star *$ & 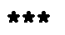 & $\star \star \star \star$ & $\star \star \star \star$ & $\star \star \star \star *$ \\
\hline 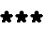 & $\star \star \star *$ & $\star \star \star *$ & $t * *$ & $t * *$ & $\star \star \star \star *$ & $\star \star \star \star$ & $\star \star \star$ & $\star \star \star \star$ & $\star \star \star \star$ & $\star \star \star \star$ & 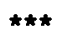 \\
\hline$\star \star \star$ & $\star \star \star$ & $\star \star \star \star$ & 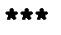 & $\star \star \star \star$ & $\star \star \star$ & $\star \star \star \star$ & $\star \star \star \star$ & $\star \star \star *$ & $\star \star * *$ & $\star \star \star \star$ & $\star \star * *$ \\
\hline$\star \star \star \star$ & $\star * *$ & $\star \star * *$ & $\star \star \star *$ & $\star \star \star *$ & $\star \star \star \star$ & $\star \star \star$ & $\star \star \star$ & 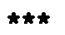 & $\hbar \star \star$ & $\star \star \star \star \star$ & 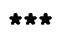 \\
\hline 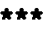 & $\star \star \star \star$ & $\star \star \star \star *$ & $\star \star \star \star$ & $\star * *$ & $\star * \star *$ & $\star \star * *$ & $\star \star * *$ & $\star \star \star \star *$ & $\star \star * *$ & $\star \star \star \star$ & 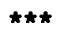 \\
\hline$\star \star$ & $\star \star \star \star$ & $\star \star \star$ & 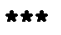 & 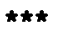 & $\star \star \star \star$ & $\star \star \star \star *$ & $\star \star \star *$ & $\star \star \star \star$ & $\star \star \star *$ & $\star \star \star \star *$ & $\star \star * *$ \\
\hline$\star$ & $* * *$ & $\star \star *$ & & & & & $\star t$ & 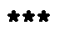 & 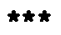 & $\star \star \star \star$ & 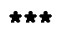 \\
\hline 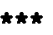 & $\star \star * *$ & $\star \star \star *$ & $\star * *$ & $* *$ & $\star * *$ & $\star \star \star \star$ & $\star \star * *$ & $\star \star * *$ & $\star \star \star \star$ & 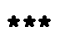 & *** \\
\hline
\end{tabular}


IABLE 1-5(contd) - ANALYSIS OF GREATEST FARMING PROBLEMS - COMMUNITY SURVEY

PROVINCE OF SMG

1980 PERCENTAGE OF VILLAGES GIVING FOLLOWING PRIORITY TO PROBLEM

Rainfall

Crop Disease

Birds or Rats

Seed Availability

Fertilizer Availability

Crop Protection Chemicals

Credit

Irrigation Water Availability

flooding

Labour Availabilty

Power for Land Preparation

Direct War Effects

Animal Diseases

Extension

other Problems

\begin{tabular}{|c|c|c|c|c|c|c|c|c|c|c|c|c|}
\hline 1 & 2 & 3 & 4 & 5 & 6 & 7 & 8 & 9 & 10 & 11 & 12. & Weighted Priority \\
\hline ** * & $\star \star \star \star$ & $\star \star \star *$ & $\star \star \star *$ & $\star \star$ & $\star \star$ & $\star$ & & * & $\star \star * *$ & $\star \star \star *$ & $\star * *$ & $\star \star \star \star \star \star$ \\
\hline$\star * *$ & $\star \star * *$ & $\star \star \star *$ & $\star \star \star$ & $\star \star \star$ & $\star \star \star \star$ & $\star \star \star$ & $\star \star \star$ & $\star \star *$ & $\star \star * *$ & $\star \star \star \star$ & $\star \star \star$ & 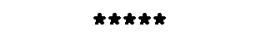 \\
\hline$\star \star \star \star$ & $\star \star \star$ & $\star \star \star \star$ & 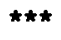 & $\star \star \star \star$ & $\star * *$ & $\star \star * *$ & $\star \star \star *$ & $\star \star \star \star$ & $\star \star \star$ & 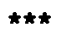 & 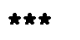 & $\star \star \star \star \star \star *$ \\
\hline$\star \star \star *$ & $\star \star \star *$ & $\star \star * *$ & $\star \star \star$ & $\star \star \star$ & $\star \star \star$ & $\star \star \star$ & $\star \star \star$ & $\star \star \star$ & $\star \star \star$ & $\star * *$ & $\star \star \star *$ & $\star \star \star \star \star$ \\
\hline$\star \star \star$ & $\star \star \star$ & $\star \star \star$ & $\star \star \star *$ & $\star \star \star *$ & $\star \star * *$ & $\star \star \star$ & $\star \star \star \star$ & $\star \star \star \star$ & $\star \star \star$ & $\star \star \star$ & $\star \star \star *$ & $\star * * * *$ \\
\hline$\star \star \star$ & $\star \star *$ & $\star * *$ & $\star * \star$ & $\star \star \star$ & $\star \star \star$ & $\star \star \star \star$ & $\star \star \star$ & $\star \star \star \star$ & $\star \star \star$ & $\star \star \star *$ & $\star \star \star *$ & $\star \star \star \star \star$ \\
\hline$\star \star \star \star$ & $\star \star \star$ & $\star \star \star *$ & $\star \star \star$ & $\star \star \star$ & 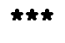 & $\star * *$ & $\star \star \star *$ & 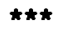 & $t \star \star t$ & 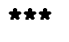 & $\star \star \star \star$ & $\star * \star * * *$ \\
\hline$\star \star \star *$ & $\star * *$ & $\star \star \star \star *$ & $\star \star \star \star *$ & $\star \star \star$ & $\star \star \star \star$ & $\star \star \star *$ & $\star \star \star *$ & 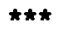 & $\star \star *$ & $\star \star \star *$ & $\star \star \star *$ & $\star \star \star \star \star \star$ \\
\hline$\star \star \star$ & $\star \star \star \star$ & $\star \star \star \star$ & $\star \star \star \star$ & $\star \star \star$ & $\star \star \star \star$ & $\star \star \star$ & $\star \star \star \star$ & $\star \star \star$ & $\star \star \star$ & $\star \star \star$ & $\star \star \star \star$ & $\star \star \star \star \star \star$ \\
\hline$\star \star \star$ & 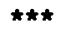 & 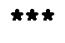 & 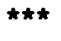 & $\star \star \star$ & 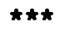 & $\star \star \star \star$ & $\star \star \star$ & 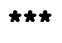 & $\star \star \star \star$ & $\star \star \star$ & $\star \star \star *$ & $\star \star \star \star \star \star$ \\
\hline$\star \star \star$ & $* * *$ & $\star \star \star *$ & $\star \star \star *$ & $\star \star \star \star$ & $\star \star \star$ & 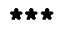 & 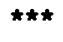 & $\star \star \star *$ & $\star \star \star \star$ & $\star \star \star *$ & $\star \star \star \star *$ & 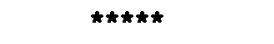 \\
\hline$\star \star \star$ & 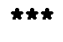 & $\star \star \star \star$ & $* * *$ & $\star \star *$ & $\star \star \star \star *$ & $\star \star \star \star *$ & $\star \star \star$ & 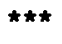 & $\star \star \star \star$ & 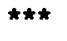 & $\star \star \star *$ & $\star \star \star \star \star \star$ \\
\hline$\star \star \star *$ & $\star \star \star \star$ & $\star \star \star$ & 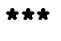 & $\star \star \star$ & 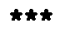 & 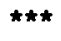 & 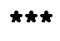 & $\star \star *$ & $\star \star \star \star$ & 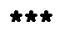 & $\star \star \star \star$ & 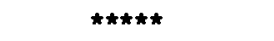 \\
\hline$\star \star \star \star$ & $\star \star \star$ & $\star \star \star *$ & $\star \star \star \star$ & $\star \star \star \star$ & $\star \star \star$ & $\star \star \star \star$ & 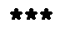 & 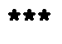 & $\star \star \star$ & $\star \star \star \star$ & $* * *$ & 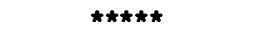 \\
\hline$\star$ & $\star \star \star$ & $\star \star \star \star$ & $\star \star \star$ & $\star \star \star$ & $\star \star \star \star$ & 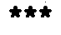 & $\star \star \star \star$ & $\star \star \star \star$ & $\star \star \star$ & $\star \star \star$ & $\star \star \star$ & $\star \star \star \star \star \star$ \\
\hline
\end{tabular}

$\underline{1978}$

Rainfall

Crop Disease

Birds or Rats

Seed Availability

Fertilizer Availability

Crop Protection Chemicals

Credit

Irrigation Water Availability flooding

Labour Availabilty

Power for Land Preparation

Direct War Effects

Animal Diseases

Extension

Other Problems

\begin{tabular}{|c|c|c|c|c|c|c|c|c|c|c|c|c|}
\hline 1 & 2 & 3 & 4 & 5 & 6 & 7 & 8 & 9 & 10 & 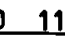 & 12 & Weighted \\
\hline$*$ & $\star \star \star \star$ & $\star \star \star$ & 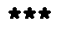 & $\star \star \star \star$ & $\star$ & $\star *$ & $* * *$ & $* * *$ & $\star \star \star \star *$ & $\star \star \star \star$ & $\star \star$ & $\star \star \star \star \star$ \\
\hline 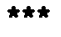 & $\star \star \star *$ & $\star * *$ & $* * *$ & $\star * *$ & $\star \star \star \star$ & $\star \star \star$ & $\star \star \star$ & $\star \star \star$ & $\star \star \star \star$ & $\star \star \star *$ & $* * *$ & 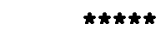 \\
\hline$t *$ & $\star \star \star \star$ & $\star \star \star \star$ & 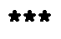 & $\star \star \star \star$ & $\star \star \star *$ & $\star \star \star *$ & $\star * *$ & $\star \star \star \star$ & $\star \star \star \star$ & $\star \star \star \star$ & 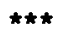 & 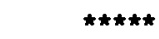 \\
\hline$* * *$ & $* * *$ & $\star * * *$ & 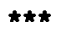 & $\star \star \star \star$ & $\star \star \star$ & $\star \star \star$ & $\star \star \star *$ & $\star \star \star$ & $\star * *$ & $\star * *$ & $\star \star * \star$ & $\star \star \star \star \star \star$ \\
\hline 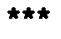 & $\star \star \star \star$ & $\star \star * *$ & $\star \star \star *$ & $* * *$ & $\star \star \star \star$ & $\star \star \star \star *$ & $\star \star \star \star$ & $\star \star \star$ & $\star \star \star$ & $\star \star \star \star$ & $\star \star * *$ & $\star \star * \star * *$ \\
\hline$* \star *$ & $\star \star \star \star$ & $\star \star \star *$ & $\star \star \star \star$ & $\star \star \star$ & $\star \star \star$ & $\star \star \star$ & $\star \star \star \star$ & $\star \star \star \star *$ & $\star \star \star \star$ & $\star \star \star \star$ & $\star \star \star$ & 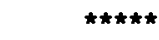 \\
\hline 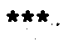 & $\star \star \star$ & $\star \star \star$ & 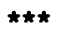 & $\star \star \star$ & $\star * *$ & $\star \star * *$ & $\star \star \star \star$ & $\star \star \star$ & $\star \star \star$ & $\star \star \star$ & $\star \star * *$ & $\star \star \star * \star *$ \\
\hline$k * *$ & $\star \star \star \star$ & $\star \star \star$ & $\star \star \star$ & $\star \star \star$ & $\star \star \star$ & $\star \star \star \star$ & $\star \star \star \star$ & $\star \star \star \star$ & $\star \star \star \star$ & $\star \star \star$ & $\star \star \star$ & 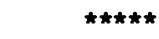 \\
\hline$\star \star \star \star$ & $\star \star \star \star$ & $\star \star \star *$ & $\star \star \star \star$ & $\star \star \star \star$ & $\star \star \star$ & $\star \star \star \star$ & 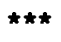 & $\star \star \star$ & $\star \star \star *$ & $\star \star \star$ & $* * *$ & $\star \star \star \star \star$ \\
\hline$* \star \star *$ & $\star \star \star \star$ & $\star \star \star \star$ & $\star \star \star$ & $\star \star \star$ & $\star \star \star$ & $\star \star \star \star$ & $\star \star \star \star$ & $\star \star \star \star$ & $\star \star \star$ & $\star \star \star$ & $\star \star \star \star$ & 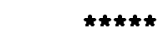 \\
\hline$\star \star \star \star$ & 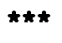 & $\star \star \star *$ & $\star \star \star \star$ & $\star \star \star \star$ & $\star \star \star$ & $\star \star \star \star$ & $\star \star \star \star$ & $\star \star \star \star$ & $\star \star \star *$ & $\star \star \star \star$ & $\star \star \star *$ & $\star \star \star \star \star \star$ \\
\hline$\star \star \star$ & $\star \star \star \star$ & $\star \star \star \star$ & 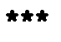 & $\star \star \star \star$ & $\star \star \star *$ & $\star \star \star \star$ & $\star \star \star \star$ & $\star \star \star$ & $\star \star \star$ & $\star \star \star$ & $\star \star \star$ & $\star \star \star \star \star \star *$ \\
\hline$* *$ & $\star \star \star *$ & $\star \star \star \star$ & $\star \star \star$ & $\star \star \star$ & $\star \star \star$ & $\star \star \star$ & $\star \star \star$ & $\star \star \star *$ & $\star \star \star *$ & $\star \star * *$ & $\star \star \star *$ & 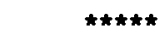 \\
\hline$\star$ & 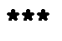 & 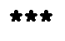 & $\star \star \star$ & 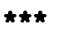 & $\star \star \star \star$ & $\star \star \star *$ & 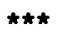 & $\star \star \star$ & $\star \star \star$ & $\star \star \star$ & $\star \star \star$ & 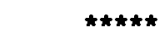 \\
\hline$\star \star$ & $\star * *$ & $\star \star \star \star$ & $\star \star \star$ & $\star \star \star$ & $\star \star \star$ & $\star \star \star \star$ & $\star \star \star \star$ & $\star \star \star$ & $\star \star \star$ & $\star \star \star$ & $\star \star \star \star$ & 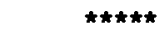 \\
\hline
\end{tabular}




\section{TAKHAR}




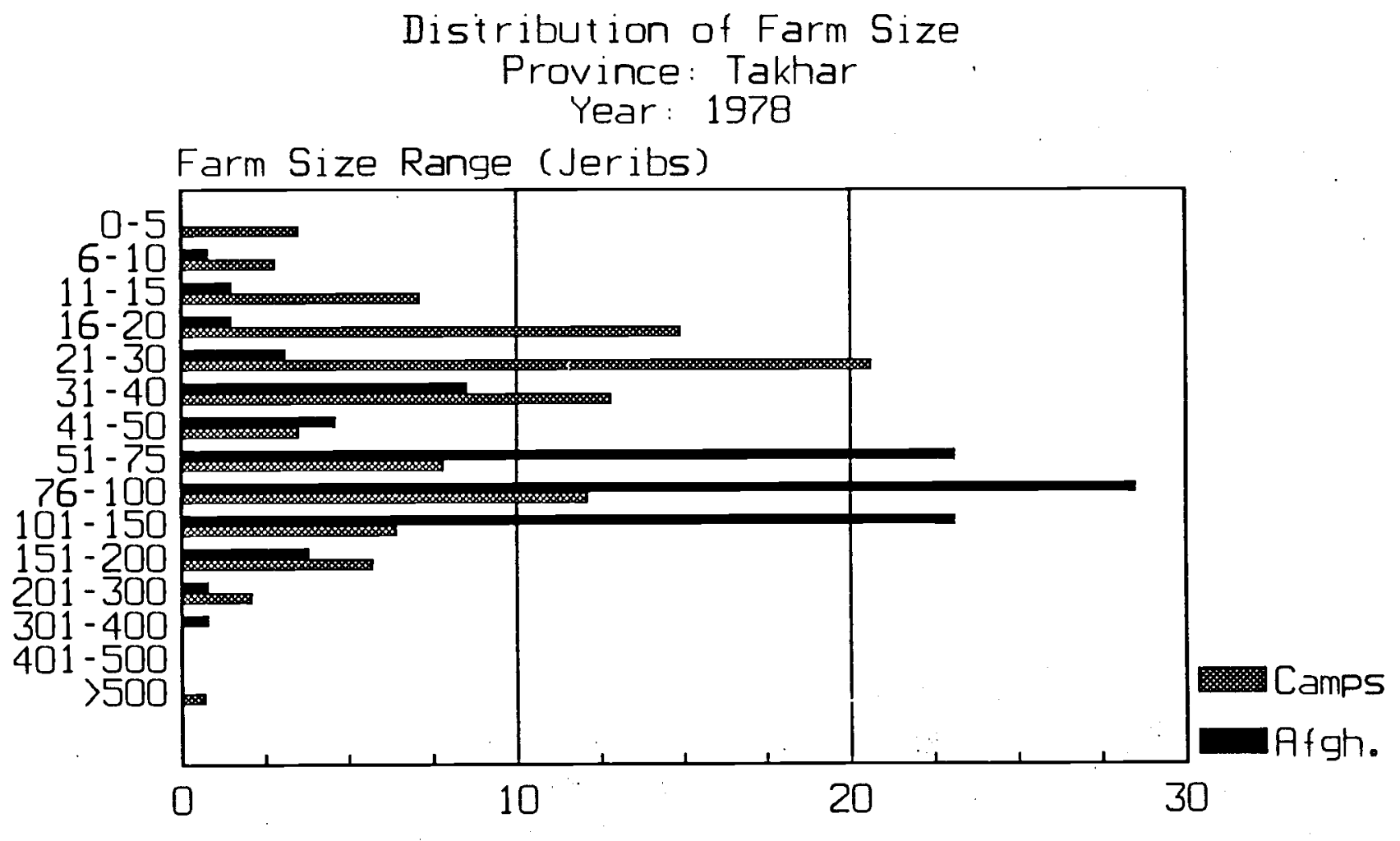

Percentage of Farmers

Farmers in Afghanistans

$\underline{1978}$

1987

Total Number of Farms

130

130

Average Area

87.39

80.58

Average Area Irrigated

16.88

12.44

Average Dryland Area

79.48

72.91

Farmers who left in 1987

Total Number of Farms

Average Area

Average Area Irrigated

141

Average Dryland Area

60.04

26.00

87.75

Average of both Groups

Provincial Average Area

Provincial Average Irrigated Area

73.16

80.58

Provincial Average Dryland Area

21.69

12.44

82.19

72.91

(All areas in Jeribs. 5 Jeribs $=1$ Hectare) 


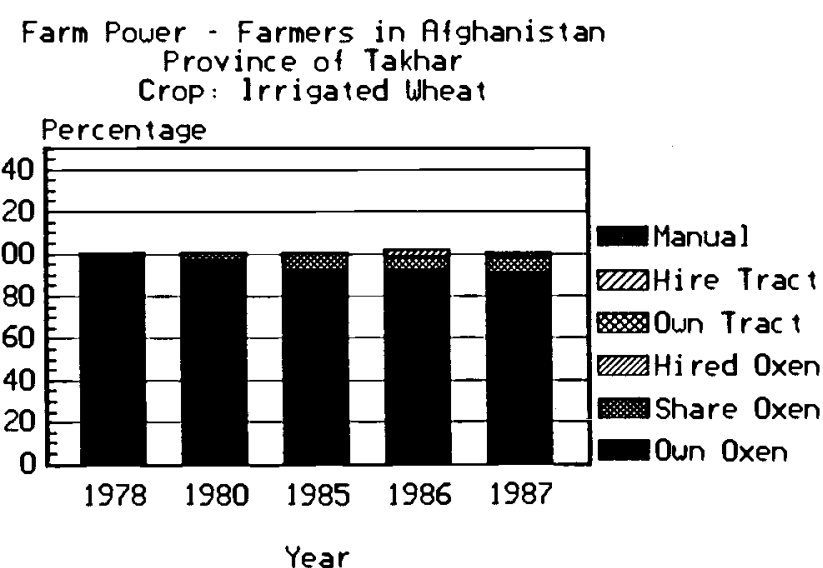

Farm Power - Farmers who lett in 1987 Province of Takhar

Crop: Irrigated theat

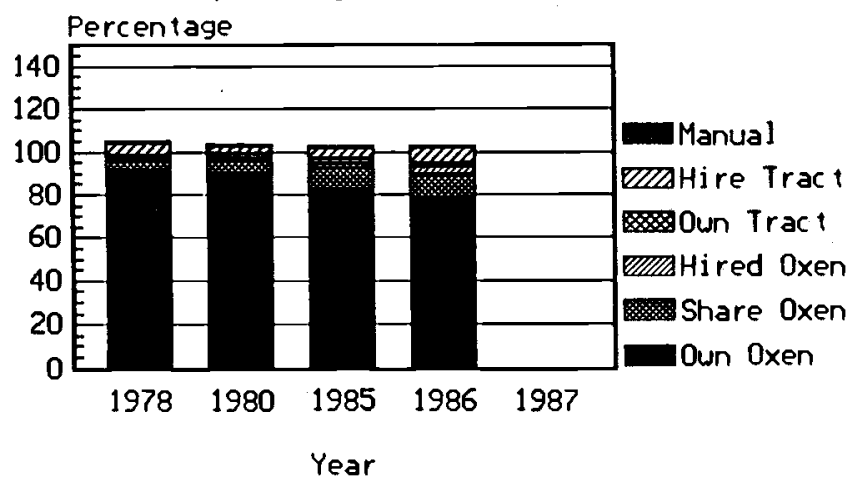

Base Figures for above graphs

Province: Takhar

Farmers in Afghanistan

Irr. Wheat Own oxen

Shared oxen

Hired oxen

Own Tractor

Hired Tractor

Barley

Manual Means

Own Oxen

Shared Oxen

Hired oxen

Own Tractor

Hired Tractor

Manual Means

Farmers who left in 1987

Irr. Wheat Own oxen

Shared oxen

Hired oxen

Own Tractor

Hired Tractor

Manual Means

Barley Own oxen

Shared Oxen

Hired oxen

Own Tractor

Hired Tractor

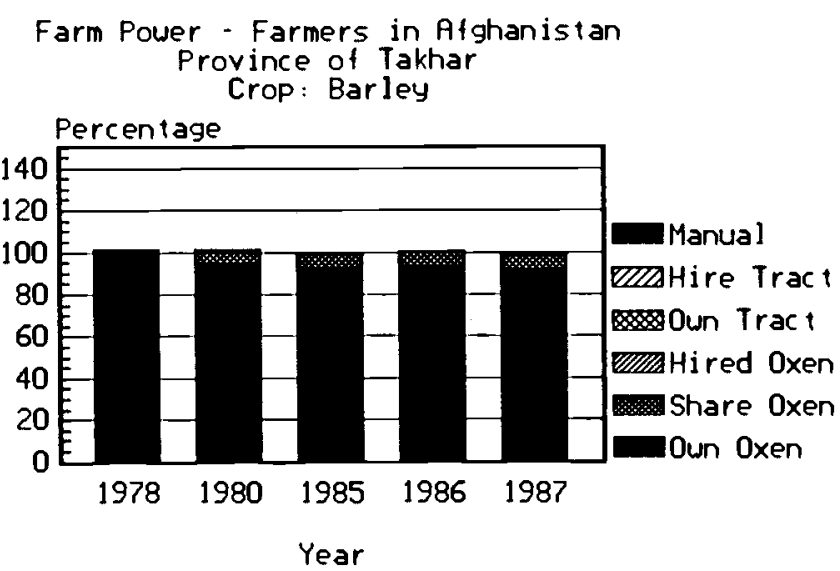

Farm Power - Farmers who left in 1987 Province of Takhar Crop: Barley

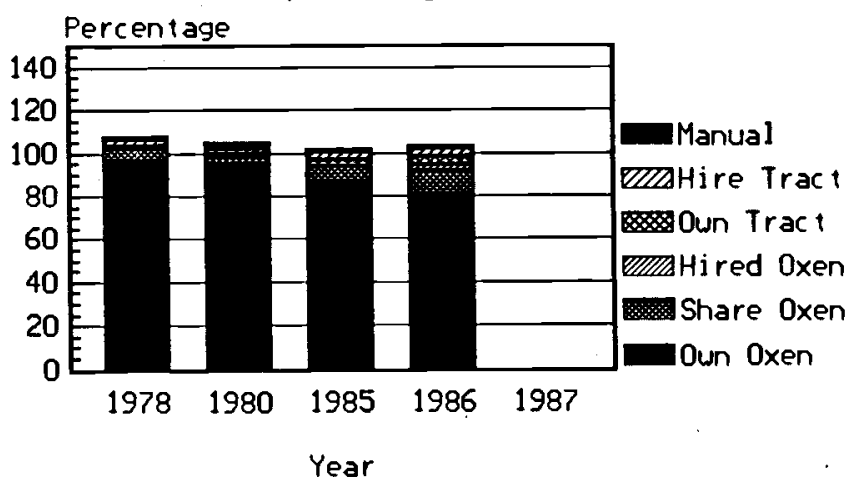

Percentage of Farmers $1978 \quad \underline{1980} \quad \underline{1985} \quad \underline{1986} \quad 1987$

$\begin{array}{ccccc}98.44 & 96.88 & 92.19 & 92.19 & 90.63 \\ 1.56 & 3.13 & 7.81 & 6.25 & 7.81 \\ - & - & - & 3.13 & 1.56 \\ - & - & - & - & - \\ - & - & - & - & - \\ - & - & - & - & - \\ 98.20 & 94.59 & 91.82 & 92.73 & 90.99 \\ 1.80 & 5.41 & 7.27 & 7.27 & 7.21 \\ - & - & - & - & .90 \\ - & - & - & - & - \\ .90 & .90 & .91 & - & .90 \\ - & - & - & - & -\end{array}$

$\begin{array}{rrrr}91.53 & 89.66 & 82.05 & 78.30 \\ 4.24 & 6.03 & 10.26 & 10.38 \\ .85 & .86 & 1.71 & 3.77 \\ 1.69 & 2.59 & 2.56 & 1.89 \\ 5.93 & 3.45 & 5.13 & 7.55 \\ .85 & .86 & .85 & .94 \\ 96.77 & 94.92 & 86.44 & 80.36 \\ 4.84 & 3.39 & 6.78 & 10.71 \\ - & 1.69 & - & 3.57 \\ 1.61 & 1.69 & 3.39 & 3.57 \\ 3.23 & 1.69 & 3.39 & 3.57\end{array}$



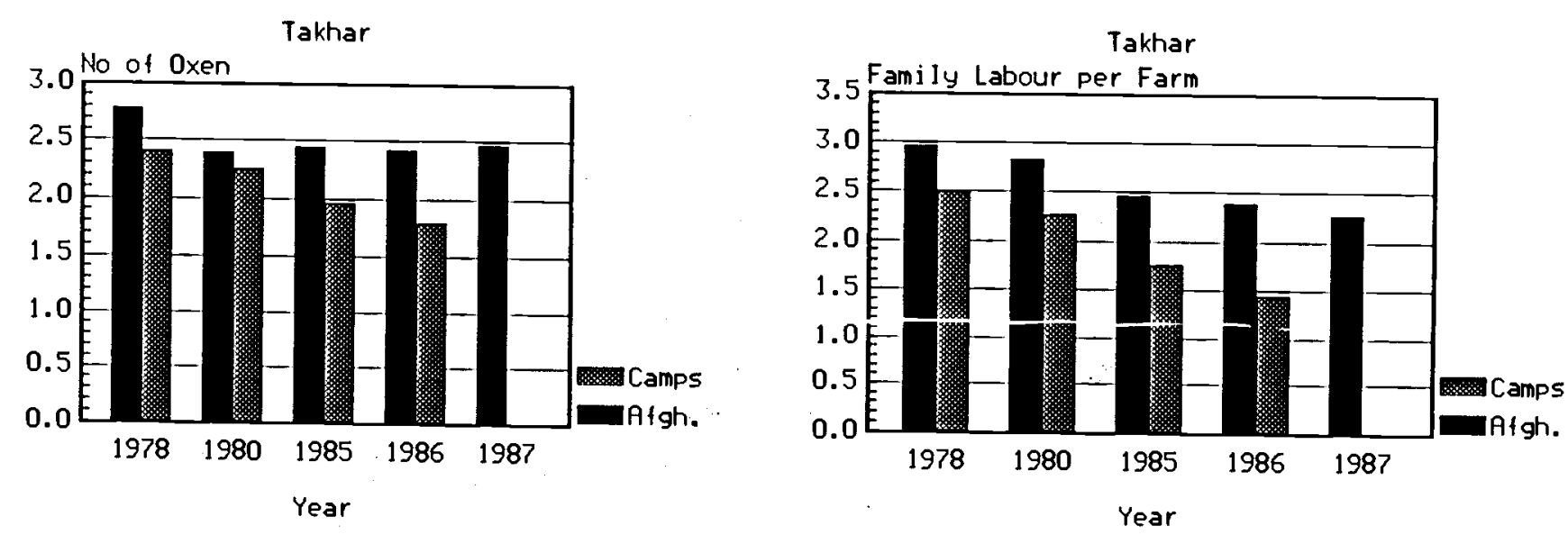

Takhar

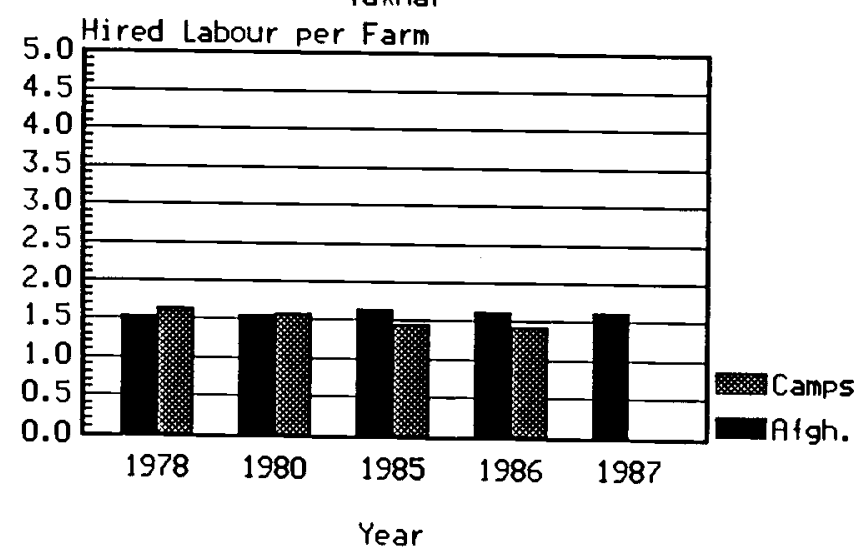

Takhar

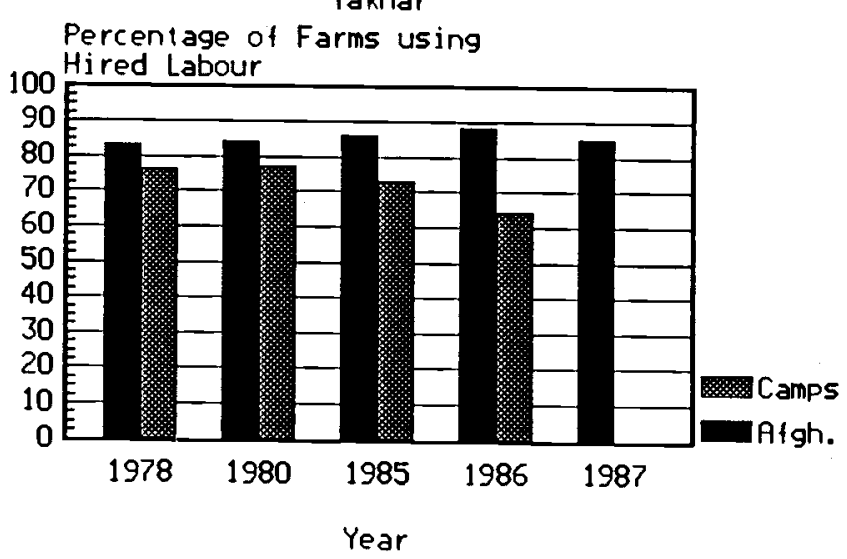


PROVINCE OF TKR

YEAR

1978 Number of Families

$x$ of Families

1980 Number of Families

$x$ of Families

1985 Number of Families

$x$ of Families

1986 Number of Families

$X$ of Families

1987 Number of Families

$X$ of Families

Average Number of Oxen per Family: $\begin{array}{ccccc}\text { NUMBER } & \text { OF } & \text { TRAINED } & \text { OXEN } & \text { PER FAMILY } \\ 0 & 1 & 2 & 3 & 4\end{array}$

$\begin{array}{llllll}0 & 2 & 62 & 30 & 20 & 9\end{array}$

$\begin{array}{llllll}0.00 & 1.63 & 50.41 & 24.39 & 16.26 & 7.32\end{array}$

$\begin{array}{llllll}0 & 6 & 76 & 15 & 23 & 5\end{array}$

$\begin{array}{llllll}0.00 & 4.80 & 60.80 & 12.00 & 18.40 & 4.00\end{array}$

$\begin{array}{llllll}1 & 9 & 76 & 14 & 25 & 1\end{array}$

$\begin{array}{llllll}0.79 & 7.14 & 60.32 & 11.11 & 19.84 & 0.79\end{array}$

$\begin{array}{llllll}2 & 8 & 76 & 15 & 25 & 0\end{array}$

$\begin{array}{llllll}1.59 & 6.35 & 60.32 & 11.90 & 19.84 & 0.00\end{array}$

$\begin{array}{llllll}2 & 8 & 72 & 18 & 25 & 1\end{array}$

$\begin{array}{llllll}1.59 & 6.35 & 57.14 & 14.29 & 19.84 & 0.79\end{array}$

$\begin{array}{ll}1978 & 2.77 \\ 1980 & 2.56 \\ 1985 & 2.44 \\ 1986 & 2.42 \\ 1987 & 2.47\end{array}$

Number of Farmers Questioned 130

IABLE 7B - AVERAGE NUMBER OF TRAINED OXEN FOR FARMERS IN THE CAMPS

PROVINCE OF TKR

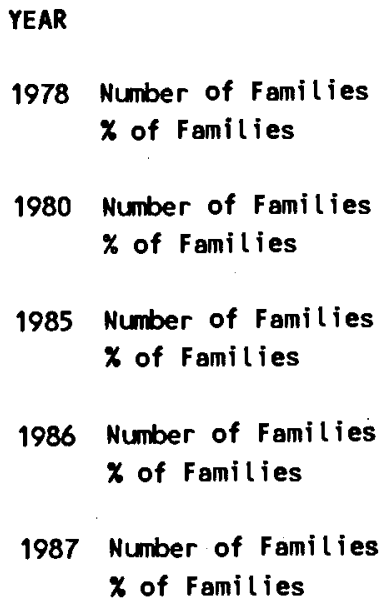

$\begin{array}{llllll}21 & 13 & 72 & 11 & 17 & 1\end{array}$

$\begin{array}{llllll}15.56 & 9.63 & 53.33 & 8.15 & 12.59 & 0.74\end{array}$

$\begin{array}{cccccc}32 & 12 & 61 & 12 & 18 & 0 \\ 23.70 & 8.89 & 45.19 & 8.89 & 13.33 & 0.00\end{array}$

$\begin{array}{llllll}135 & 0 & 0 & 0 & 0 & 0\end{array}$

$\begin{array}{llllll}100.0 & 0.00 & 0.00 & 0.00 & 0.00 & 0.00\end{array}$


PROVINCE OF TKR

\begin{tabular}{|c|c|c|c|c|c|}
\hline & 1978 & 1980 & 1985 & 1986 & 1987 \\
\hline Average Family Labour per Farm & 2.96 & 2.82 & 2.46 & 2.38 & 2.27 \\
\hline $\begin{array}{l}\text { Average No. of Hired Labour per Farm } \\
\text { (for farms using hired (abour) }\end{array}$ & 1.52 & 1.55 & 1.63 & 1.62 & 1.61 \\
\hline Number of Farms using Hired Labour & 108 & 109 & 112 & 114 & 111 \\
\hline Number of Farmers Questioned 130 & & & & & \\
\hline
\end{tabular}

PROVINCE OF TKR

\begin{tabular}{|c|c|c|c|c|c|}
\hline & 1978 & 1980 & 1985 & 1986 & 1987 \\
\hline Average Family Labour per Farm & 2.49 & 2.27 & 1.76 & 1.44 & 0.00 \\
\hline $\begin{array}{l}\text { Average No. of Hired Labour per Farm } \\
\text { (for farms using hired (abour) }\end{array}$ & 1.64 & 1.56 & 1.45 & 1.42 & $\star \star \star \star \star *$ \\
\hline Number of Farms using Hired Labour & 102 & 104 & 99 & 86 & 0 \\
\hline
\end{tabular}

Number of Farmers Questioned 135 


\section{TABLE 9A - DIRECT EFFECTS OF THE WAR - FARMERS IN AFGANISTAN}

PROVINCE OF TKR

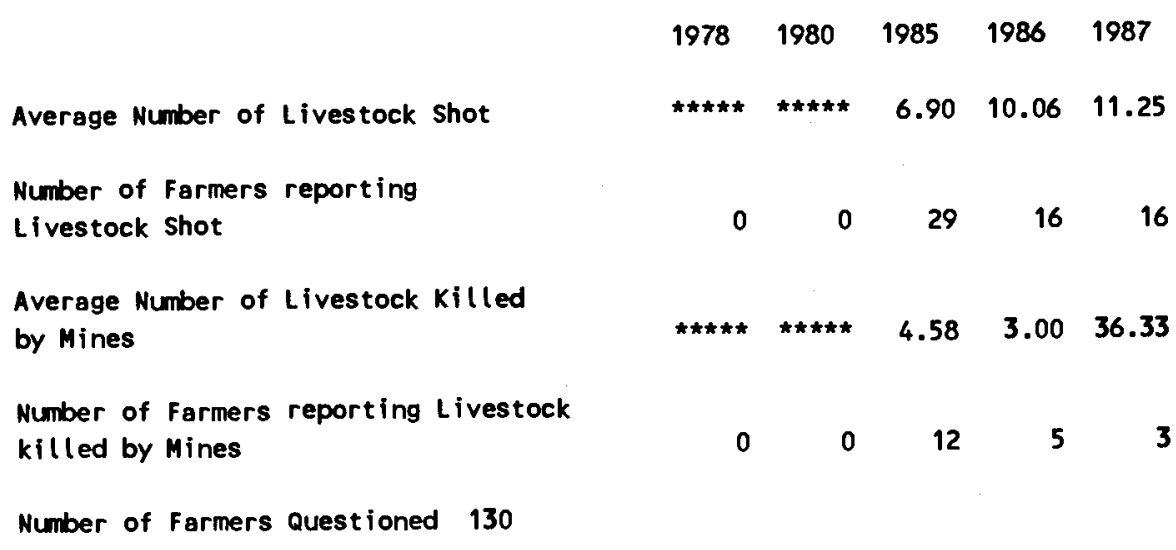

IABLE 9B - DIRECT EFFECTS OF THE WAR - FARMERS IN CAMPS

PROVINCE OF TKR

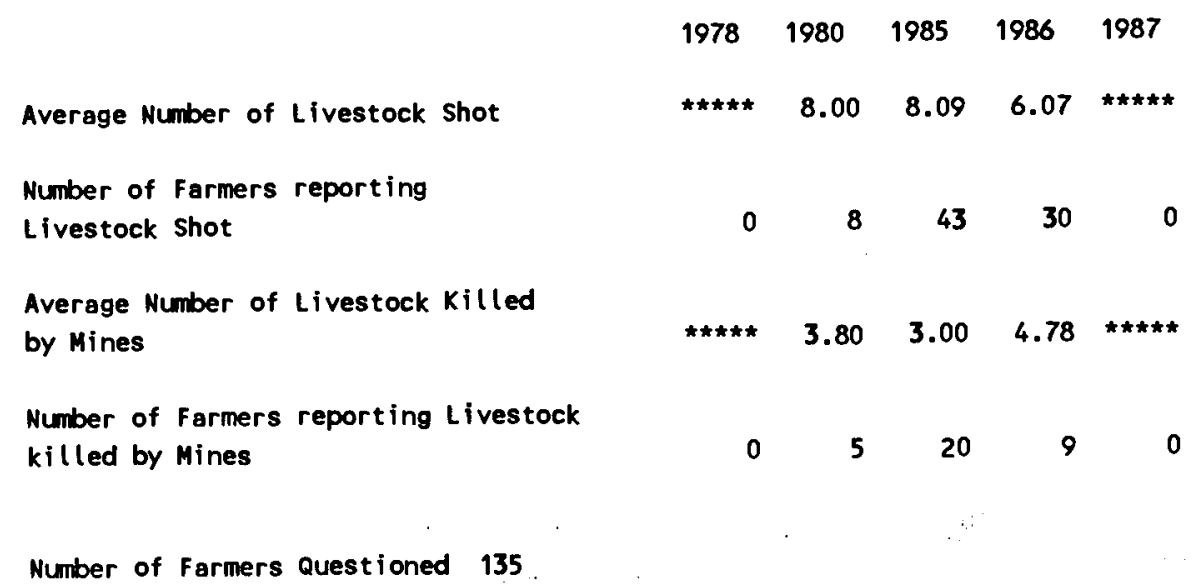




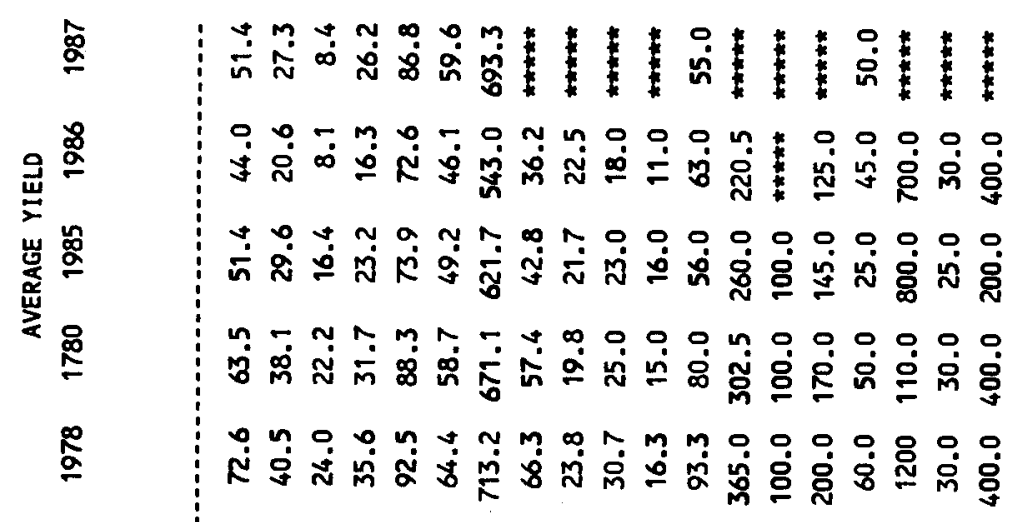

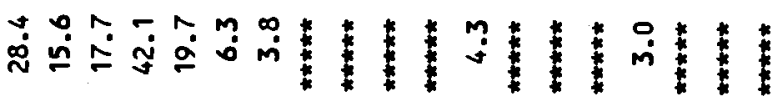

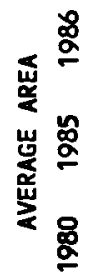

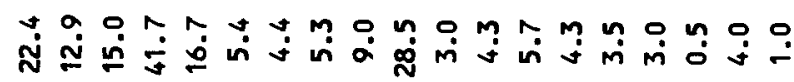

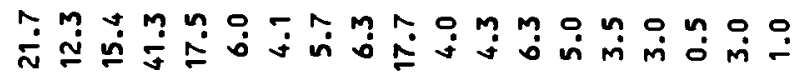

ஸ்

ஸ்

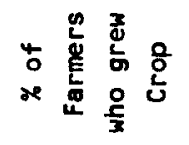

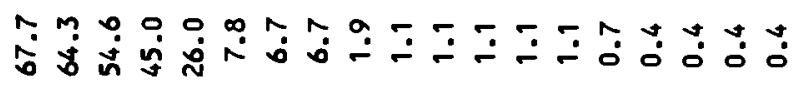

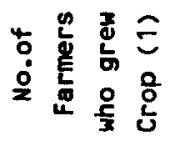

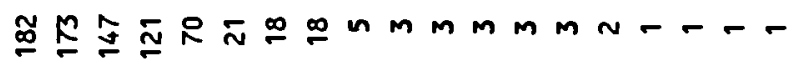

嵒 亯

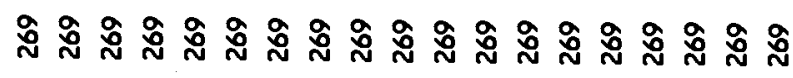

$\stackrel{\infty}{2}$

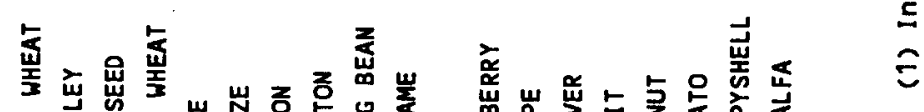


PROVINCE OF TKR

\section{PERCENTAGE OF VILLAGES GIVING FOLLOWING PRIORITY TO PROBLEM}

Rainfall

Crop Disease

Birds or Rats

Seed Availability

Fertilizer Availability

Crop Protection Chemicals

Credit

Irrigation Water Availability

Flooding

Labour Avai labilty

Power for Land Preparation

Direct War Effects

Animal Diseases

Extension

Other Problems

\begin{tabular}{rrrrrrrrrrrrr}
1 & 2 & 3 & 4 & 5 & 6 & 7 & 8 & 9 & 10 & 11 & 12 & Weighted Priority \\
\hline 0 & 0 & 0 & 0 & 0 & 0 & 0 & 0 & 0 & 0 & 0 & 0 & 0.00 \\
9 & 20 & 26 & 6 & 0 & 3 & 0 & 0 & 0 & 0 & 0 & 0 & 14.33 \\
0 & 0 & 3 & 9 & 0 & 0 & 0 & 0 & 0 & 0 & 0 & 0 & 1.53 \\
0 & 11 & 14 & 14 & 0 & 0 & 0 & 0 & 0 & 0 & 0 & 0 & 6.93 \\
0 & 9 & 3 & 3 & 3 & 0 & 0 & 0 & 0 & 0 & 0 & 0 & 3.22 \\
0 & 0 & 0 & 0 & 3 & 0 & 0 & 0 & 0 & 0 & 0 & 0 & 0.28 \\
0 & 0 & 3 & 3 & 3 & 3 & 0 & 0 & 0 & 0 & 0 & 0 & 1.34 \\
3 & 0 & 0 & 0 & 3 & 0 & 0 & 0 & 0 & 0 & 0 & 0 & 1.69 \\
0 & 0 & 3 & 3 & 3 & 0 & 0 & 0 & 0 & 0 & 0 & 0 & 1.10 \\
9 & 43 & 26 & 6 & 6 & 3 & 0 & 0 & 0 & 0 & 0 & 0 & 20.54 \\
3 & 6 & 6 & 6 & 0 & 0 & 0 & 0 & 0 & 0 & 0 & 0 & 4.46 \\
77 & 11 & 11 & 0 & 0 & 0 & 0 & 0 & 0 & 0 & 0 & 0 & 42.76 \\
0 & 0 & 0 & 3 & 3 & 0 & 0 & 0 & 0 & 0 & 0 & 0 & 0.63 \\
0 & 0 & 3 & 6 & 0 & 0 & 0 & 0 & 0 & 0 & 0 & 0 & 1.17 \\
0 & 0 & 0 & 0 & 0 & 0 & 0 & 0 & 0 & 0 & 0 & 0 & 0.00
\end{tabular}

1986

Rainfall

Crop Disease

Birds or Rats

Seed Avail ability

Fertilizer Availability

Crop Protection Chemicals

Credit

Irrigation Water Availability

Flooding

Labour Availabilty

Power for Land Preparation

Direct War Effects

Animal Diseases

Extension

Other Problems

\begin{tabular}{rrrrrrrrrrrrr}
1 & 2 & 3 & 4 & 5 & 6 & 7 & 8 & 9 & 10 & 11 & 12 & Weighted Priority \\
\hline 6 & 14 & 0 & 14 & 20 & 3 & 0 & 0 & 0 & 0 & 0 & 0 & 9.51 \\
20 & 11 & 29 & 11 & 6 & 3 & 0 & 0 & 0 & 0 & 0 & 0 & 18.07 \\
0 & 0 & 0 & 14 & 9 & 0 & 0 & 0 & 0 & 0 & 0 & 0 & 2.40 \\
3 & 6 & 20 & 11 & 3 & 0 & 3 & 0 & 0 & 0 & 0 & 0 & 7.38 \\
0 & 0 & 11 & 3 & 0 & 0 & 0 & 0 & 0 & 0 & 0 & 0 & 2.06 \\
0 & 0 & 0 & 0 & 0 & 0 & 0 & 0 & 0 & 0 & 0 & 0 & 0.00 \\
0 & 0 & 0 & 6 & 6 & 0 & 0 & 0 & 0 & 0 & 0 & 0 & 1.17 \\
3 & 0 & 0 & 0 & 3 & 0 & 0 & 0 & 0 & 0 & 0 & 0 & 1.56 \\
0 & 0 & 0 & 3 & 0 & 0 & 0 & 0 & 0 & 0 & 0 & 0 & 0.32 \\
20 & 26 & 26 & 11 & 3 & 0 & 0 & 0 & 0 & 0 & 0 & 0 & 20.40 \\
0 & 3 & 3 & 9 & 3 & 0 & 0 & 0 & 0 & 0 & 0 & 0 & 2.32 \\
49 & 43 & 6 & 0 & 0 & 0 & 0 & 0 & 0 & 0 & 0 & 0 & 32.71 \\
0 & 3 & 3 & 3 & 3 & 3 & 0 & 0 & 0 & 0 & 0 & 0 & 1.88 \\
0 & 0 & 0 & 0 & 0 & 3 & 0 & 0 & 0 & 0 & 0 & 0 & 0.22 \\
0 & 0 & 0 & 0 & 0 & 0 & 0 & 0 & 0 & 0 & 0 & 0 & 0.00
\end{tabular}

1985

Rainfall

Crop Disease

Birds or Rats

Seed Avail lability

Fertilizer Availability

Crop Protection Chemicals

Credit

Irrigation Water Availability

Flooding

Labour Aveilabilty

Power for Land Preparation

Direct War Effects

Animal Diseases

Extension

Other Problems

$\begin{array}{rrrrrrrrrrrrc}1 & 2 & 3 & 4 & 5 & 6 & 7 & 8 & 9 & 10 & 11 & 12 & \text { Weighted Priority } \\ 17 & 11 & 14 & 20 & 9 & 0 & 0 & 0 & 0 & 0 & 0 & 0 & 16.45 \\ 11 & 17 & 23 & 9 & 3 & 0 & 0 & 0 & 0 & 0 & 0 & 0 & 14.53 \\ 0 & 0 & 0 & 14 & 3 & 0 & 0 & 0 & 0 & 0 & 0 & 0 & 1.98 \\ 0 & 6 & 14 & 17 & 0 & 0 & 0 & 0 & 0 & 0 & 0 & 0 & 5.70 \\ 0 & 0 & 3 & 6 & 6 & 0 & 0 & 0 & 0 & 0 & 0 & 0 & 1.69 \\ 0 & 0 & 0 & 0 & 0 & 0 & 0 & 0 & 0 & 0 & 0 & 0 & 0.00 \\ 0 & 0 & 3 & 3 & 0 & 0 & 0 & 0 & 0 & 0 & 0 & 0 & 0.80 \\ 11 & 0 & 3 & 6 & 0 & 0 & 0 & 0 & 0 & 0 & 0 & 0 & 6.61 \\ 3 & 0 & 0 & 0 & 0 & 0 & 0 & 0 & 0 & 0 & 0 & 0 & 1.37 \\ 9 & 20 & 23 & 9 & 6 & 3 & 0 & 0 & 0 & 0 & 0 & 0 & 14.35 \\ 0 & 6 & 6 & 0 & 6 & 3 & 0 & 0 & 0 & 0 & 0 & 0 & 3.06 \\ 46 & 40 & 9 & 0 & 0 & 0 & 0 & 0 & 0 & 0 & 0 & 0 & 32.85 \\ 0 & 0 & 0 & 3 & 0 & 0 & 0 & 0 & 0 & 0 & 0 & 0 & 0.34 \\ 0 & 0 & 0 & 0 & 3 & 0 & 0 & 0 & 0 & 0 & 0 & 0 & 0.27 \\ 0 & 0 & 0 & 0 & 0 & 0 & 0 & 0 & 0 & 0 & 0 & 0 & 0.00\end{array}$


IABLE $1-5$ (contd) - ANALYSIS OF GREATEST FARMING PROBLEMS - COMMUNITY SURVEY

PROVINCE OF TKR

PERCENTAGE OF VILLAGES GIVING FOLLOWING PRIORITY TO PROBLEM

Rainfall

Crop Disease

Birds or Rats

Seed Avail ability

Fertilizer Availability

Crop Protection Chemicals

Credit

Irrigation Water Avallability

Flooding

Labour Availabilty

Power for Lend Preparation

Direct War Effects

Animal Diseases

Extension

Other Problems

$\begin{array}{rrrrrrrrrrrrc}1 & 2 & 3 & 4 & 5 & 6 & 7 & 8 & 9 & 10 & 11 & 12 & \text { Neighted Priority } \\ 3 & 9 & 0 & 0 & 0 & 0 & 0 & 0 & 0 & 0 & 0 & 0 & 5.38 \\ 17 & 3 & 3 & 6 & 0 & 0 & 0 & 0 & 0 & 0 & 0 & 0 & 15.78 \\ 3 & 0 & 0 & 0 & 0 & 0 & 0 & 0 & 0 & 0 & 0 & 0 & 2.15 \\ 0 & 6 & 6 & 0 & 0 & 3 & 0 & 0 & 0 & 0 & 0 & 0 & 3.95 \\ 0 & 6 & 3 & 3 & 0 & 0 & 0 & 0 & 0 & 0 & 0 & 0 & 3.41 \\ 0 & 0 & 0 & 0 & 0 & 0 & 0 & 0 & 0 & 0 & 0 & 0 & 0.00 \\ 6 & 0 & 0 & 3 & 0 & 0 & 0 & 0 & 0 & 0 & 0 & 0 & 4.84 \\ 0 & 6 & 0 & 0 & 0 & 0 & 0 & 0 & 0 & 0 & 0 & 0 & 2.15 \\ 6 & 0 & 0 & 0 & 0 & 0 & 0 & 0 & 0 & 0 & 0 & 0 & 4.30 \\ 3 & 3 & 0 & 3 & 3 & 0 & 0 & 0 & 0 & 0 & 0 & 0 & 4.20 \\ 6 & 9 & 0 & 0 & 3 & 0 & 0 & 0 & 0 & 0 & 0 & 0 & 7.96 \\ 40 & 17 & 9 & 0 & 0 & 0 & 0 & 0 & 0 & 0 & 0 & 0 & 38.74 \\ 3 & 0 & 3 & 3 & 6 & 0 & 0 & 0 & 0 & 0 & 0 & 0 & 4.27 \\ 0 & 6 & 3 & 0 & 0 & 0 & 0 & 0 & 0 & 0 & 0 & 0 & 2.87 \\ 0 & 0 & 0 & 0 & 0 & 0 & 0 & 0 & 0 & 0 & 0 & 0 & 0.00\end{array}$

1978

Rainfall

Crop Disease

Birds or Rats

Seed Availability

Fertilizer Availability

Crop Protection Chemicals

Credit

Irrigation Water Availability

Flooding

Labour Availabilty

Power for Land Preparation

Oirect War Effects

Animal Oiseases

Extension

Other Problems

\begin{tabular}{llllllllllllc}
1 & 2 & 3 & 4 & 5 & 6 & 7 & 8 & 9 & 10 & 11 & 12 & Weighted Priority \\
\hline 3 & 0 & 0 & 0 & 0 & 0 & 0 & 0 & 0 & 0 & 0 & 0 & 8.21 \\
0 & 3 & 0 & 3 & 0 & 3 & 0 & 0 & 0 & 0 & 0 & 0 & 7.52 \\
3 & 0 & 0 & 0 & 0 & 0 & 0 & 0 & 0 & 0 & 0 & 0 & 8.21 \\
0 & 3 & 0 & 3 & 0 & 0 & 0 & 0 & 0 & 0 & 0 & 0 & 6.16 \\
0 & 3 & 6 & 0 & 0 & 0 & 0 & 0 & 0 & 0 & 0 & 0 & 9.58 \\
0 & 0 & 0 & 0 & 3 & 0 & 0 & 0 & 0 & 0 & 0 & 0 & 1.64 \\
6 & 3 & 0 & 3 & 0 & 0 & 0 & 0 & 0 & 0 & 0 & 0 & 22.57 \\
0 & 0 & 0 & 0 & 0 & 0 & 0 & 0 & 0 & 0 & 0 & 0 & 0.00 \\
0 & 0 & 0 & 0 & 0 & 0 & 0 & 0 & 0 & 0 & 0 & 0 & 0.00 \\
0 & 3 & 0 & 0 & 0 & 0 & 0 & 0 & 0 & 0 & 0 & 0 & 4.10 \\
6 & 0 & 3 & 0 & 0 & 0 & 0 & 0 & 0 & 0 & 0 & 0 & 19.15 \\
0 & 0 & 0 & 0 & 0 & 0 & 0 & 0 & 0 & 0 & 0 & 0 & 0.00 \\
0 & 0 & 3 & 0 & 6 & 0 & 0 & 0 & 0 & 0 & 0 & 0 & 10.12 \\
0 & 0 & 3 & 0 & 0 & 0 & 0 & 0 & 0 & 0 & 0 & 0 & 2.74 \\
0 & 0 & 0 & 0 & 0 & 0 & 0 & 0 & 0 & 0 & 0 & 0 & 0.00
\end{tabular}




\section{URZGHAN}




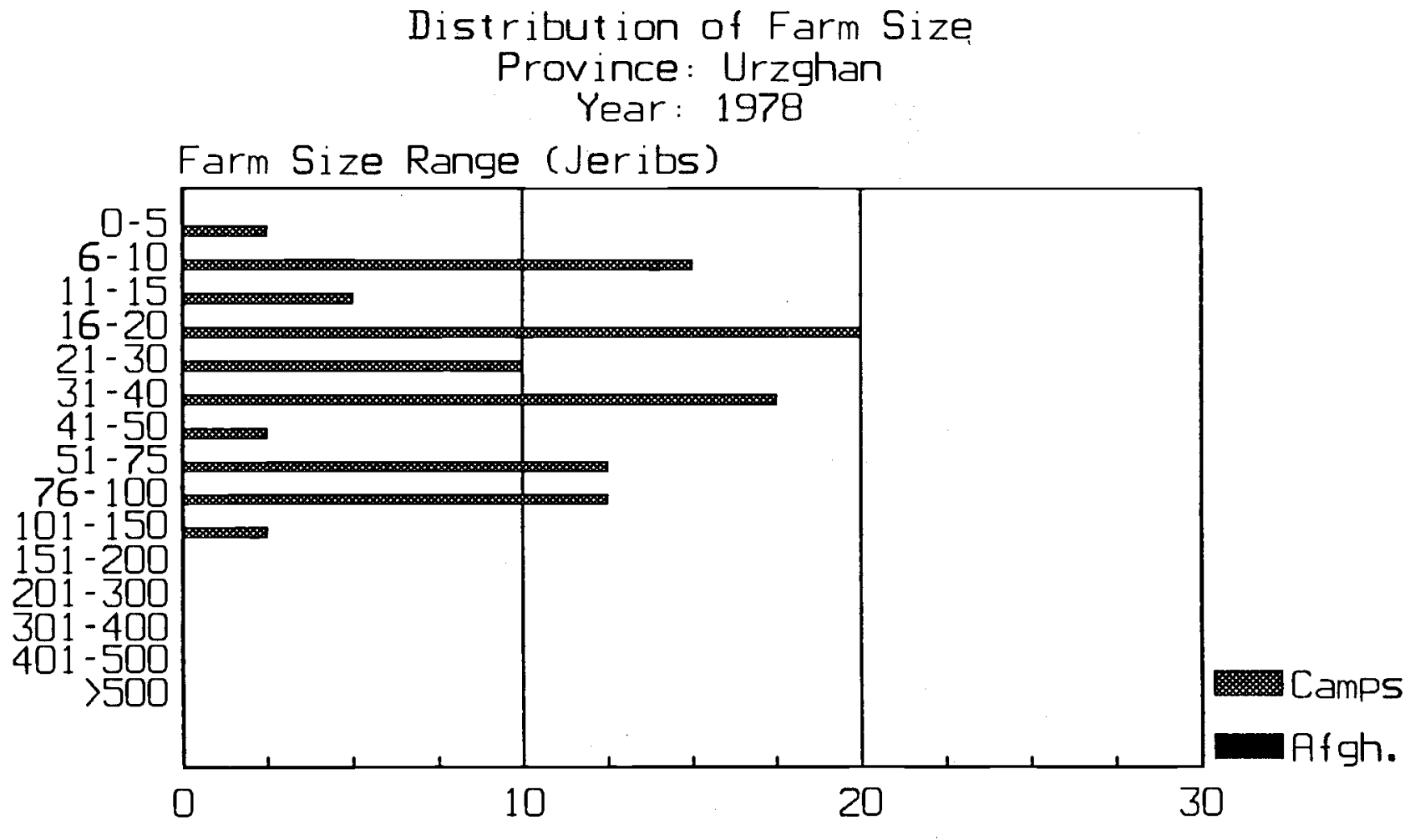

Percentage of Farmers

Farmers in Afghanistans

Total Number of Farms

Average Area

Average Area Irrigated

Average Dryland Area

Farmers who left in 1987

Total Number of Farms

Average Area

Average Area Irrigated

Average Dryland Area

$\begin{array}{ll}0 & 0 \\ - & -\end{array}$

\section{Average of both Groups}

Provincial Average Area

Provincial Average Irrigated Area

37.78

Provincial Average Dryland Area

40
-
32.55
34.83

(All areas in Jeribs. 5 Jeribs $=1$ Hectare) 
Farm Power - Farmers in Atghanistan Province of Urzgan

Crop: Irrigated wheat

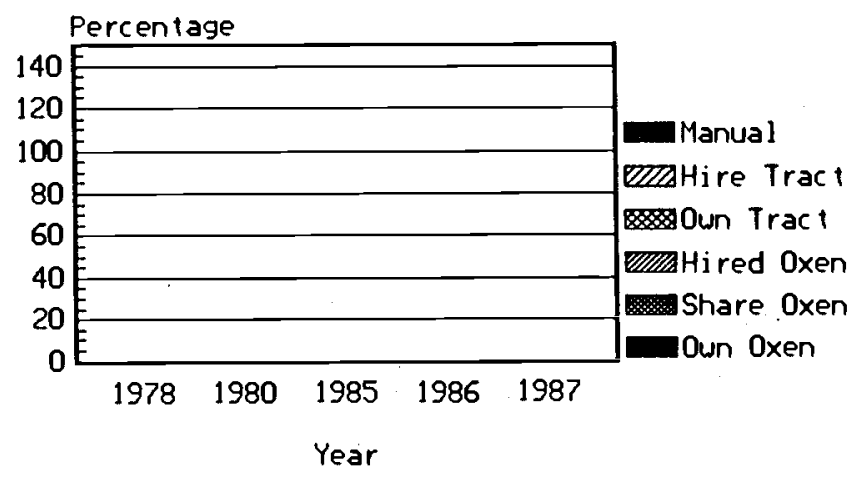

Farm Power - Farmers who lett in 1987 Province of Urzgan Crop: Irrigated wheat

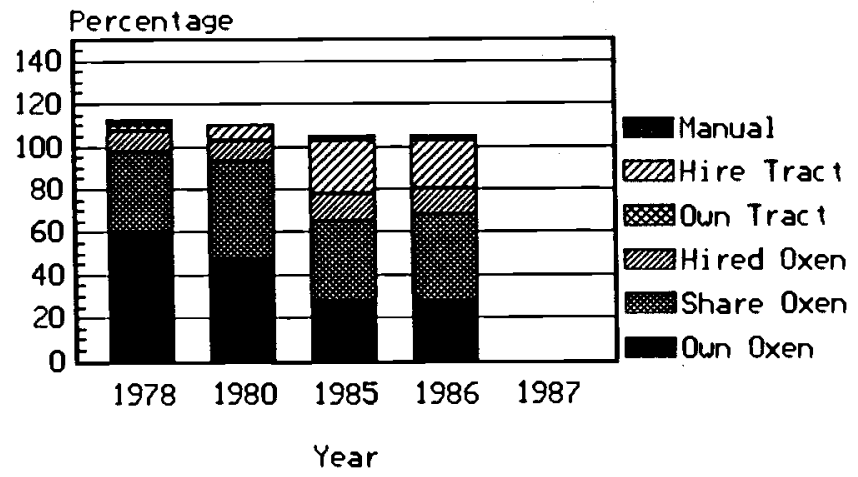

Base Fiqures for above graphs

Province: Urzgan

Farmers in Afghanistan

Irr. Wheat own Oxen Shared Oxen Hired oxen Own Tractor Hired Tractor

Maize Manual Means Own Oxen Shared oxen Hired oxen Own Tractor Hired Tractor Manual Means

Farmers who left in 1987

Irr. Wheat own Oxen

Shared oxen

Hired oxen

Own Tractor

Hired Tractor

Manual Means

Maize
Own Oxen

Shared oxen

Hired oxen

Own Tractor
Farm Power - Farmers in Atghanistan Province of Urzgan Crop: Maize

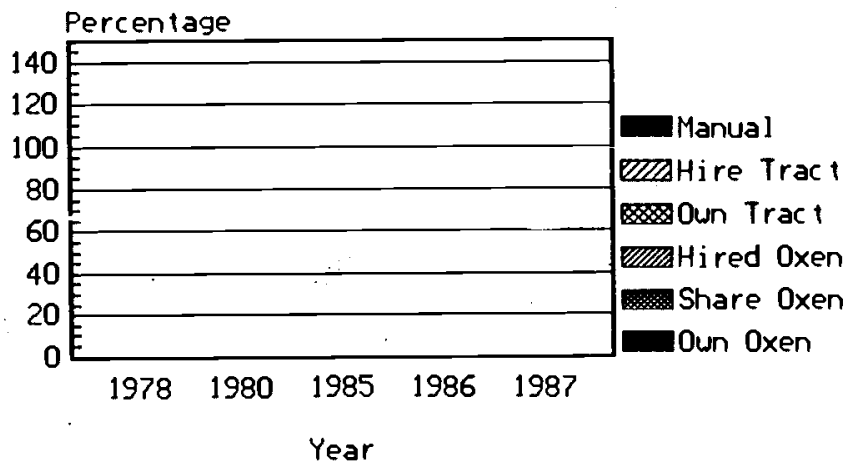

Farm Power - Farmers who lett in 1987 Province of Urzgan Crop: Maize

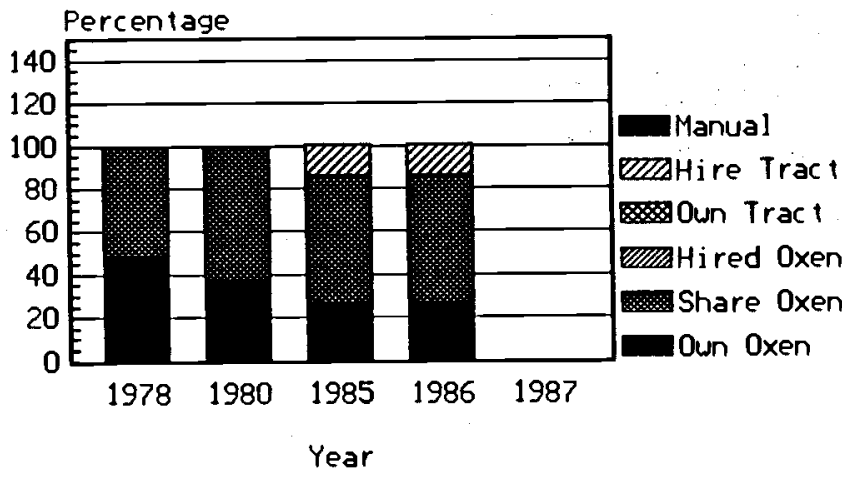

$1978 \stackrel{\text { Percentage of Farmers }}{1980 \quad \underline{1985} \quad \underline{1986}} \quad 1987$

$\begin{array}{lllll}- & - & - & - & - \\ - & - & - & - & - \\ - & - & - & - & - \\ - & - & - & - & - \\ - & - & - & - & - \\ - & - & - & - & - \\ - & - & - & - & - \\ - & - & - & - & - \\ - & - & - & - & - \\ - & - & - & - & -\end{array}$

60.00

37.50

47.50

27.50

27.50

10.00

45.00

37.50

40-

2.50

10.00

12.50

12.50

2. 50

7.50

25.00

22.50

$\overline{8.15}$

51.85

$-$

2.50

2.50

37.04

25.93

25.93

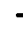

62.96

59.26

59.26 

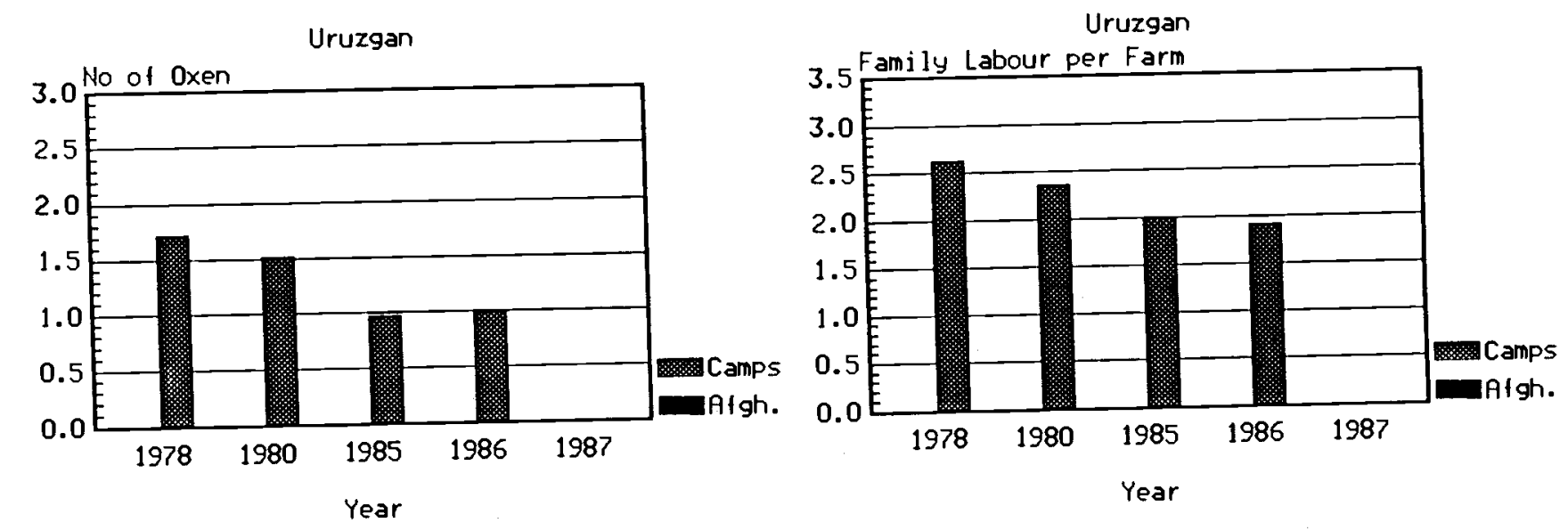

Uruzgan

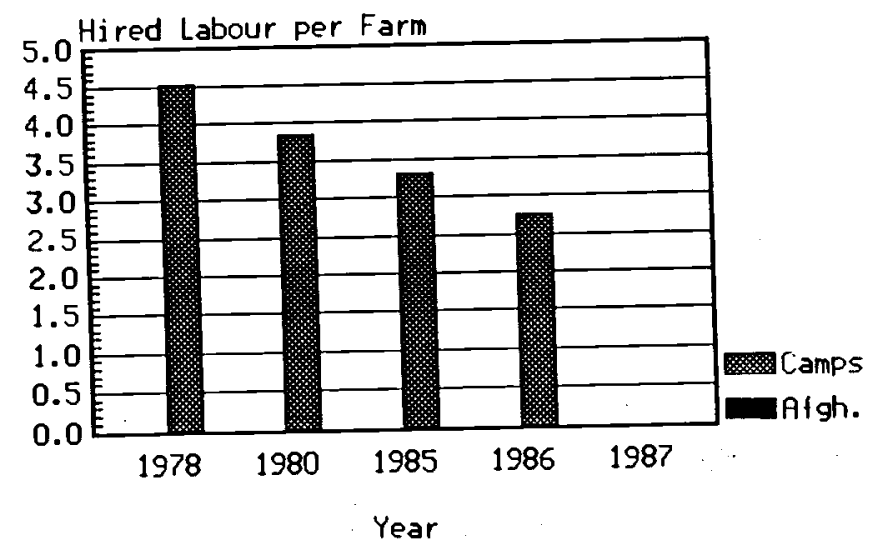

Uruzgan

Percentage of Farms using

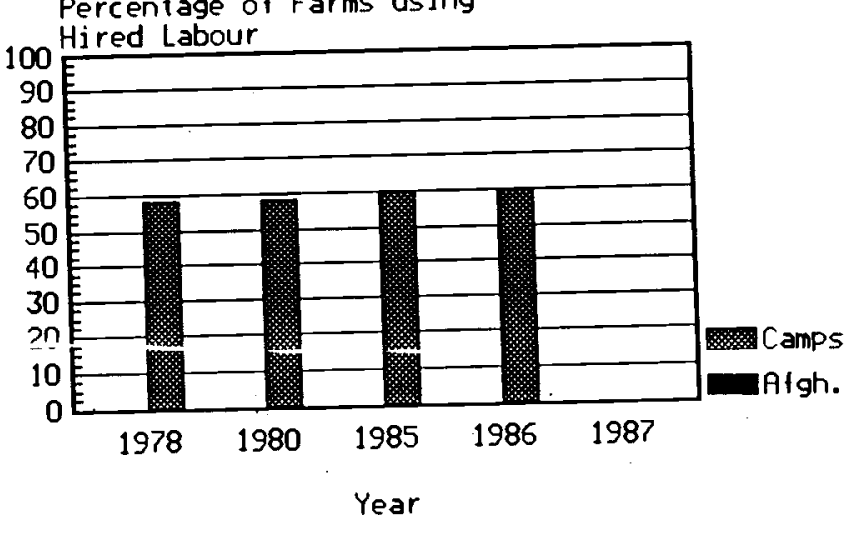


PROVINCE OF URG

YEAR

1978 Number of Families

$\boldsymbol{X}$ of Families

1980 Number of Families

\% of Families

1985 Number of Families

$\boldsymbol{X}$ of Families

1986 Number of Families

$x$ of Families

1987 Number of Families

X of Families

Average Number of Oxen per Family:

IABLE 7B - AVERAGE NUMBER OF TRAINED OXEN FOR FARMERS IN THE CAMPS

PROVINCE OF URG

YEAR

1978 Number of Families

\% of Families

1980 Number of Families

$x$ of Families

1985 Number of Families

$X$ of Families

1986 Number of Families

$x$ of Families

1987 Number of Families

$x$ of Families

Average Number of Oxen per Family:
1978

1980

1985

1986

1987

NUMBER OF TRAINED OXEN PER FAMILY

$\begin{array}{lllll}0 & 1 & 2 & 3 & 4\end{array}$

$\begin{array}{llllll}1 & 15 & 15 & 1 & 3 & 0\end{array}$

$\begin{array}{llllll}2.86 & 42.86 & 42.86 & 2.86 & 8.57 & 0.00\end{array}$

$\begin{array}{llllll}5 & 17 & 10 & 1 & 4 & 0\end{array}$

$\begin{array}{llllll}13.51 & 45.95 & 27.03 & 2.70 & 10.81 & 0.00\end{array}$

$\begin{array}{llllll}16 & 14 & 8 & 0 & 2 & 0\end{array}$

$\begin{array}{llllll}40.00 & 35.00 & 20.00 & 0.00 & 5.00 & 0.00\end{array}$

$\begin{array}{llllll}15 & 15 & 8 & 0 & 2 & 0\end{array}$

$\begin{array}{llllll}37.50 & 37.50 & 20.00 & 0.00 & 5.00 & 0.00\end{array}$

$\begin{array}{llllll}40 & 0 & 0 & 0 & 0 & 0\end{array}$

$\begin{array}{llllll}100.0 & 0.00 & 0.00 & 0.00 & 0.00 & 0.00\end{array}$

$\begin{array}{ll}978 & 1.71 \\ 1980 & 1.51 \\ 985 & 0.95 \\ 986 & 0.98 \\ 987 & 0.00\end{array}$

Number of Farmers Questioned 40 
PROVINCE OF URG

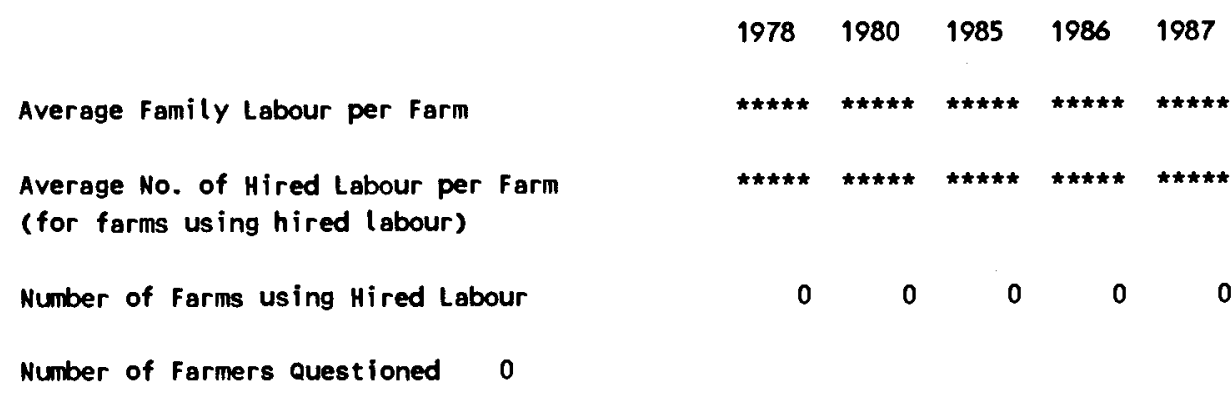

TABLE 8(B) - AVERAGE LABOUR (FAMILY and HIRED) FOR FARMERS IN CAMPS

PROVINCE OF URG

Average Family Labour per Farm

Average No. of Hired Labour per Farm

(for farms using hired labour)

Number of Farms using Hired Labour

Number of Farmers Questioned 40

$\begin{array}{rrrrr}1978 & 1980 & 1985 & 1986 & 1987 \\ 2.63 & 2.35 & 2.00 & 1.90 & 0.00 \\ 4.52 & 3.83 & 3.31 & 2.75 & \star \star \star \star \star \\ 23 & 23 & 24 & 24 & 0\end{array}$


PROVINCE OF URG

Average Number of Livestock Shot

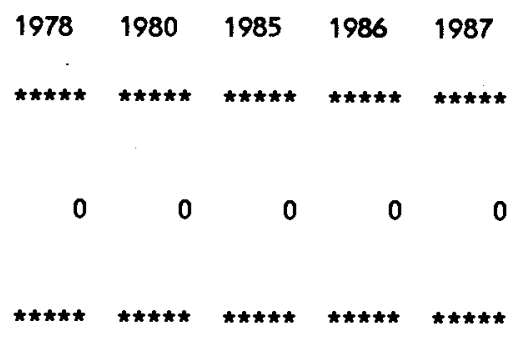

Average Number of Livestock Killed by Mines

$\begin{array}{lllll}0 & 0 & 0 & 0 & 0\end{array}$

Number of Farmers reporting Livestock

killed by Mines

Number of Farmers Questioned 0

\section{TABLE 9B - DIRECT EFFECTS OF THE HAR - FARMERS IN CAMPS}

PROVINCE OF URG

Average Number of Livestock Shot

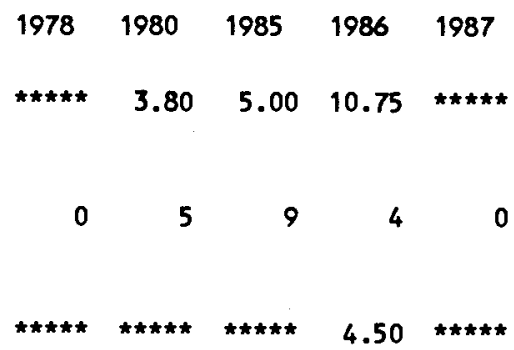

Average Number of Livestock Killed by Mines

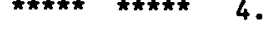

Number of Farmers reporting Livestock

killed by Mines

$\begin{array}{lllll}0 & 0 & 0 & 2 & 0\end{array}$

Number of Farmers Questioned 40 


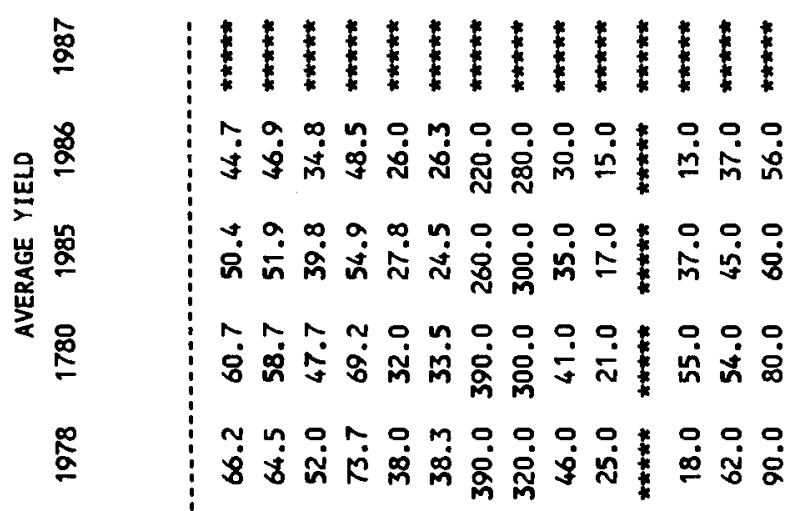

ڤิ

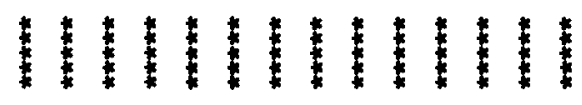

崖

岁哭

\&్

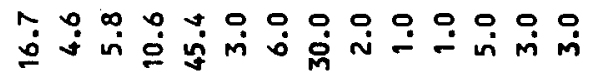

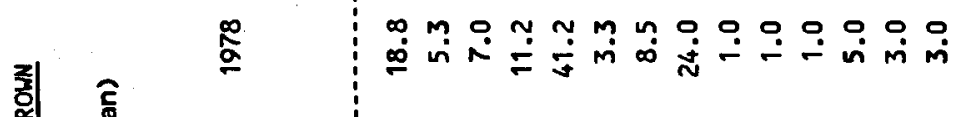

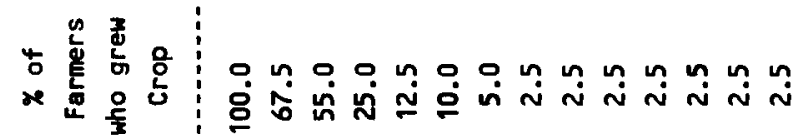

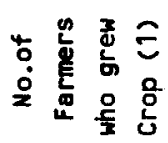

芯 就

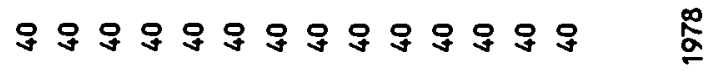

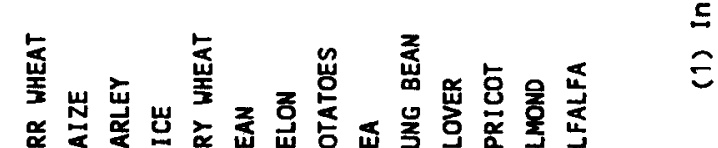

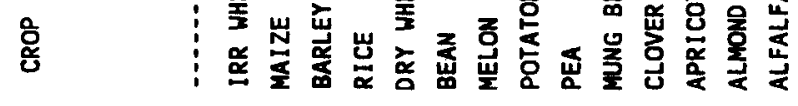


PROVINCE OF URG

\section{PERCENTAGE OF VILLAGES GIVING FOLLONING PRIORITY TO PROBLEM}

Rainfall

Crop Disease

Birds or Rats

Seed Availability

Fertilizer Availability

Crop Protection Chemicals

Credit

Irrigation Water Availability

flooding

Labour Availabilty

Power for Land Preparation

Direct War Effects

Animal Diseases

Extension

Other Problems

\section{6}

Rainfall

Crop Disease

Birds or Rats

Seed Availability

Fertilizer Availability

Crop Protection Chemicals

Credit

Irrigation Water Availability

Flooding

Labour Availabilty

Power for Land Preparation

Direct War Effects

Animal Diseases

Extension

other Problems

$\underline{1985}$

\section{Rainfall}

Crop Disease

Birds or Rats

Seed Availability

Fertilizer Availability

Crop Protection Chemicals

Credit

Irrigation Water Availability

Flooding

Labour Availabilty

Power for Land Preparation

Direct War Effects

Animal Diseases

Extension

other Problems

\begin{tabular}{|c|c|c|c|c|c|c|c|c|c|c|c|c|}
\hline 1 & 2 & 3 & 4 & 5 & 6 & 7 & 8 & 9 & 10 & 11 & 12 & Weighted \\
\hline$\star \star \star$ & 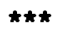 & $\star \star \star \star$ & $\star \star \star *$ & 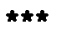 & 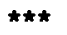 & $\star \star \star$ & $\star \star \star$ & $\star \star \star \star$ & $\star \star \star *$ & $\star \star \star \star$ & $\star \star \star$ & 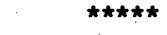 \\
\hline$\star \star \star$ & $\star \star \star \star$ & $\hbar k \star$ & $\star \star \star \star$ & $\star \star \star \star$ & $\star \star \star \star$ & $\star \star \star \star$ & $\star \star \star \star$ & $\star \star \star$ & $\star \star \star$ & $\star \star \star$ & 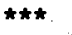 & 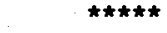 \\
\hline$\star \star \star *$ & $\star \star * *$ & $\star \star \star \star$ & $\star \star \star \star$ & $\star \star \star \star$ & $\star \star \star$ & $\star \star \star$ & $\star \star \star \star$ & $\star \star \star \star$ & $\star \star \star \star$ & $\star \star \star$ & $\star \star \star$ & $\star \star \star \star \star \star$ \\
\hline$\star \star \star \star$ & $\star \star \star \star$ & $\hbar \star \hbar$ & $\star \star \star \star$ & $\star \star \star \star$ & $\star \star \star \star$ & $\star \star \star \star$ & $\star \star \star \star$ & $\star \star \star$ & $\star \star \star$ & $\star \star \star *$ & $\star \star \star$ & 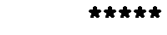 \\
\hline$\star \star \star *$ & $\star \star * *$ & $\star \star * *$ & $\star \star \star \star$ & 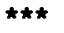 & $\star \star \star$ & $\star \star \star$ & $\star \star \star \star$ & $\star \star \star$ & $\star \star \star$ & $\star \star \star$ & $\hbar \star \star$ & 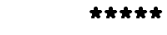 \\
\hline$\star \star \star$ & $\star \star \star$ & $\star \star \star$ & $\star \star \star \star$ & $\star * *$ & $\star \star \star \star$ & $\star \star \star \star$ & $\star \star \star \star$ & $\star \star \star \star$ & $\star \star \star \star$ & $\star \star \star \star$ & $\star \star \star \star$ & $\star \star \star \star \star \star$ \\
\hline$\star \star \star$ & $\star \star \star$ & $\star \star \star \star$ & $\star \star \star$ & $\star \star \star$ & $\star \star \star$ & $\star \star \star \star$ & $\star \star \star \star$ & $\star \star \star *$ & $\star \star \star \star$ & $\star \star \star$ & $\star \star \star$ & $\star \star \star \star \star \star *$ \\
\hline$\star \star \star$ & $\star \star \star *$ & $\star \star \star$ & $\star \star \star$ & $\star \star \star$ & $\star \star \star$ & $\star \star \star$ & $\star \star \star$ & $\star \star \star$ & $\star \star \star \star$ & $\star \star \star$ & $\star \star \star$ & 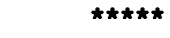 \\
\hline 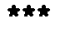 & $\star \star \star$ & $\star \star \star$ & $\star \star \star$ & $\star \star \star \star$ & $\star \star \star$ & $\star \star \star \star$ & $\star \star \star$ & $\star \star \star$ & $\star \star \star$ & $\star \star \star$ & $\star \star \star$ & 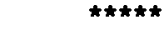 \\
\hline$* * *$ & $\star \star \star$ & $\star \star \star$ & $\star \star \star \star$ & $\star \star \star \star$ & $\star \star \star$ & $\star \star \star$ & 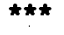 & $\star \star \star$ & $\star \star \star \star$ & $\star \star \star$ & $\star \star \star$ & 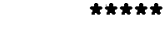 \\
\hline$\star \star \star \star$ & $\star \star \star \star$ & $\star \star \star *$ & $\star \star \star *$ & $\star \star \star \star$ & $\star \star \star$ & $\star \star \star$ & $\star \star \star \star$ & $\star \star \star \star$ & $\star \star \star \star$ & $\star \star \star \star$ & 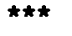 & 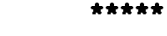 \\
\hline$\star \star \star *$ & $\star \star \star \star$ & $\star \star \star$ & $\star \star \star$ & $\star \star \star *$ & 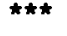 & $\star \star \star$ & $\star \star \star \star$ & $\star \star \star$ & $\star \star \star \star$ & $\star \star \star \star$ & $\star \star \star \star$ & 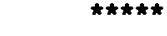 \\
\hline$\star \star \star$ & $\star \star \star$ & $\star \star \star$ & $\star \star \star \star$ & $\star \star \star$ & $\star \star \star *$ & $\star \star \star \star$ & $\star \star \star \star$ & 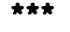 & $\star \star \star \star *$ & $\star \star \star \star$ & $\star \star \star *$ & 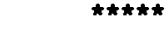 \\
\hline 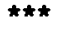 & $\star \star \star$ & $\star \star \star$ & $\star \star \star$ & $\star \star \star$ & $\star \star \star$ & $\star \star \star \star *$ & $\star \star \star \star$ & $\star \star \star$ & $\star \star \star \star$ & $\star \star \star \star$ & $\star \star \star$ & 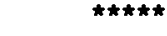 \\
\hline 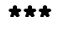 & $\star \star \star \star$ & $\star \star \star$ & $\star \star \star$ & $\star \star \star$ & $\star \star \star$ & $\star \star \star$ & $\star * *$ & $\star \star \star *$ & $\star \star \star$ & $\star \star \star \star$ & $\star \star \star$ & 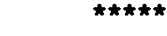 \\
\hline
\end{tabular}

$\begin{array}{llllllllllll}1 & 2 & 3 & 4 & 5 & 6 & 7 & 8 & 9 & 10 & 11 & 12\end{array}$ Weighted Priority

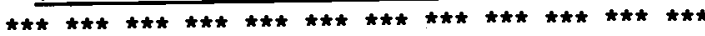

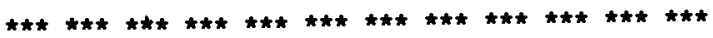

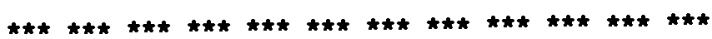

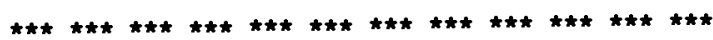

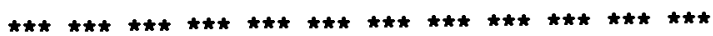

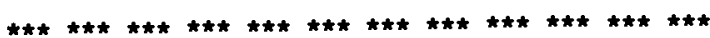

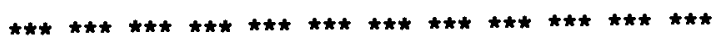

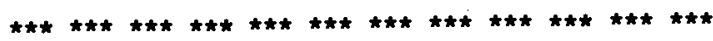

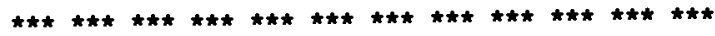

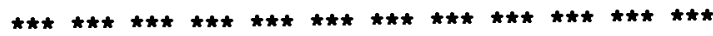

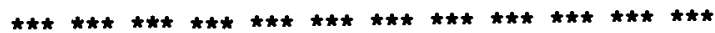

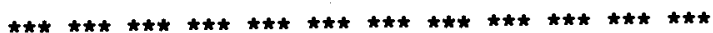

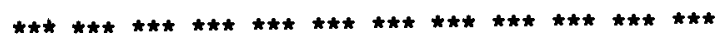

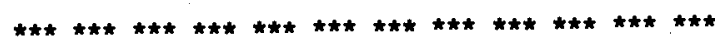

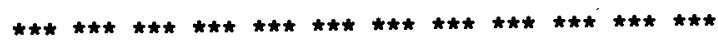

$\star \star \star \star \star$
$\star \star \star \star \star$
$\star \star \star \star \star$
$\star \star \star \star \star$
$\star \star \star \star \star$
$\star \star \star \star \star$
$\star \star \star \star \star$
$\star \star \star \star \star$
$\star \star \star \star \star$
$\star \star \star \star \star$
$\star \star \star \star \star$
$\star \star \star \star \star$
$\star \star \star \star \star$
$\star \star \star \star \star$
$\star \star \star \star \star$

\begin{tabular}{|c|c|c|c|c|c|c|c|c|c|c|c|c|}
\hline 1 & 2 & 3 & 4 & 5 & 6 & 7 & 8 & 9 & 10 & 11 & 12 & Weighted Priority \\
\hline$\star \star \star *$ & $\star \star \star *$ & $\star \star \star$ & $\star \star \star$ & $\star \star \star \star$ & $\star * *$ & $\star \star \star \star$ & $\star \star \star \star$ & $\star \star \star$ & $\star \star \star$ & $\star \star \star \star *$ & $\star * *$ & 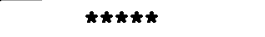 \\
\hline$* \star *$ & $\star \star \star$ & $\star \star \star$ & $\star \star \star \star$ & $\star \star *$ & 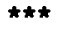 & $\star \star \star$ & $\star \star \star$ & $\star \star \star \star$ & $\star \star \star *$ & $\star \star \star$ & $\star \star *$ & $\star \star \star \star \star \star * *$ \\
\hline$\star * *$ & $\star \star \star \star$ & $\star \star \star *$ & $\star \star \star \star$ & $\star \star \star \star$ & $\star \star \star \star$ & $\star \star \star$ & $\star \star \star \star$ & $\star \star \star \star$ & $\star \star \star$ & $\star \star \star$ & $\star \star \star \star$ & $\star \star \star \star \star \star *$ \\
\hline$\star \star \star \star$ & $\star \star \star \star$ & $\star \star \star$ & $\star \star \star$ & $\star \star \star$ & $\star \star \star$ & $\star \star \star *$ & $\star \star \star \star$ & $\star \star \star$ & $\star \star \star$ & $\star \star \star$ & $\star \star \star \star$ & 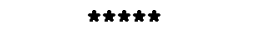 \\
\hline * * & $\star \star \star \star$ & $\star \star * *$ & $\star * *$ & $\star * *$ & $\star \star \star *$ & $\star \star \star *$ & $\star \star \star$ & $\star \star \star \star$ & $\star \star \star \star$ & $* * *$ & $\star \star \star \star$ & $\star \star \star \star \star \star$ \\
\hline$* \star * *$ & $\star \star \star *$ & $\star \star \star \star$ & $\star \star \star$ & $\star \star \star \star$ & $\star \star \star$ & $\star \star \star \star$ & $\star \star \star \star$ & $\star * *$ & $\star \star \star *$ & $\star \star \star \star$ & $\star \star \star \star$ & $\star \star \star \star \star \star *$ \\
\hline $\boldsymbol{t} \star \star \star$ & $\star \star \star$ & $\star \star \star \star$ & $\star \star \star$ & $\star \star \star \star *$ & $* * *$ & $\star \star \star *$ & $\star \star \star \star$ & 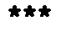 & $\star \star \star$ & $\star \star \star$ & $\star \star \star \star$ & $\star \star \star \star \star \star$ \\
\hline$t \star \star$ & $\star \star \star \star$ & $\star \star \star *$ & $\star \star \star \star$ & $\star \star \star$ & 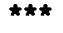 & $\star \star \star \star$ & $\star \star \star \star$ & $\star * *$ & $* * *$ & $\star * *$ & $\star \star * *$ & 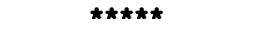 \\
\hline$\star \star \star \star$ & 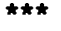 & $\star \star \star$ & 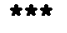 & $\star \star \star \star$ & $\star \star \star \star$ & 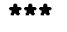 & $\star \star \star \star$ & $\star \star \star *$ & $\star \star \star$ & $\star \star \star \star$ & $\star \star \star$ & 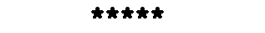 \\
\hline$\star *$ & $\star \star \star \star$ & $\star * *$ & $\star \star \star *$ & $* \star *$ & 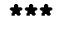 & 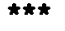 & $\star \star \star$ & $\star \star \star$ & $\star \star \star \star$ & $\star \star \star *$ & $\star \star \star$ & $\star \star \star \star \star$ \\
\hline$\star \star \star \star$ & $\star \star \star$ & $\star \star \star$ & $\star \star \star$ & $\star \star \star \star$ & $\star \star \star *$ & $\star \star \star \star$ & $* * *$ & $\star \star \star *$ & $\star \star \star$ & $\star \star \star$ & $\star \star \star \star$ & $\star \star \star \star \star \star$ \\
\hline 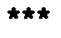 & $\star \star \star \star$ & $\star \star \star \star$ & $\star \star \star \star$ & $\star * *$ & $\star * \star$ & $\star \star \star$ & $\star \star \star$ & $\star \star \star$ & $\star \star \star \star$ & $\star \star \star \star$ & $\star \star \star \star$ & $\star \star \star \star \star \star$ \\
\hline$\star \star \star *$ & $\star * *$ & $\star \star \star *$ & $\star \star \star \star$ & $\star \star \star \star$ & $\star \star \star$ & $\star \star \star$ & $\star \star \star \star$ & $\star * *$ & $\star \star \star$ & $\star \star \star$ & $\star \star \star \star$ & $\star \star \star \star \star \star$ \\
\hline$\star \star \star \star$ & $\star \star \star \star$ & $\star \star \star \star$ & $\star \star \star *$ & $\star \star \star \star$ & $\star \star * *$ & $\star \star \star \star$ & $\star \star \star \star$ & $\star \star \star *$ & $\star \star \star$ & $\star \star \star \star$ & $\star \star \star \star$ & $\star \star \star \star \star \star$ \\
\hline & 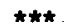 & $\star \star \star$ & 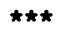 & $\star \star \star$ & 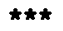 & $\star \star *$ & $\star \star \star *$ & 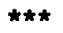 & $\star \star \star \star *$ & $\star \star \star \star$ & $\star \star \star *$ & $\star \star \star \star \star \star$ \\
\hline
\end{tabular}


PROVINCE OF URG

\section{PERCENTAGE OF VILLAGES GIVING FOLLOHING PRIORITY TO PROBLEM}

Rainfall

Crop Disease

Birds or Rats

Seed Availability

Fertilizer Availability

Crop Protection Chemicals

Credit

Irrigation Water Availability

Flooding

Labour Avail labilty

Power for Land Preparation

Direct War Effects

Animal Diseases

Extension

other Problems

\section{8}

Rainfall

Crop Disease

Birds or Rats

Seed Availability

Fertilizer Availability

Crop Protection Chemicals

credit

Irrigation Water Avai lability

Flooding

Labour Availabilty

Power for Land Preparation

Direct War Effects

Animal Diseases

Extension

other Problems

\begin{tabular}{|c|c|c|c|c|c|c|c|c|c|c|c|c|}
\hline 1 & 2 & 3 & 4 & 5 & 6 & 7 & 8 & 9 & 10 & 11 & 12 & Weighted Priority \\
\hline$\star * *$ & $\star * *$ & $\star \star \star$ & $\star \star \star$ & $\star \star \star$ & $\star \star \star$ & $\star \star \star$ & $\star \star \star \star$ & $\star \star \star$ & $\star \star \star$ & $\star \star \star$ & $\star \star$ & $\star \star \star \star \star \star$ \\
\hline$\star \star \star$ & $\star \star *$ & $\star \star \star *$ & $\star \star \star$ & $\star \star \star$ & $\star \star \star$ & $\star \star \star$ & $\star \star \star$ & $\star \star \star$ & $\star \star \star$ & $\star \star \star$ & $\star \star \star$ & 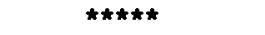 \\
\hline$\star \star \star$ & $\star \star \star *$ & $\star * *$ & $\star \star \star$ & $\star \star \star$ & $\star \star \star$ & $\star \star \star$ & $\star \star \star$ & $\star \star \star$ & $\star \star \star$ & $\star \star \star *$ & $\star \star \star$ & 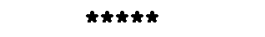 \\
\hline$\star \star \star$ & $\star \star \star$ & $\star \star \star \star$ & $\star \star \star \star$ & $\star \star \star \star$ & $\star \star \star$ & $\star \star \star$ & $\star \star \star$ & $\star \star \star$ & $\star \star \star$ & $\star \star \star$ & $\star \star \star$ & $\star \star \star \star \star$ \\
\hline$\star \star \star *$ & $\star \star \star *$ & $* * *$ & $\star \star \star *$ & $\star \star *$ & 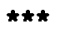 & $\star \star \star *$ & $\star \star \star *$ & 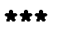 & $\star \star \star$ & $\star \star \star$ & $\star \star \star$ & 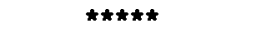 \\
\hline$\star \star \star *$ & $\star \star \star \star$ & $\star \star * *$ & $\star \star \star \star *$ & $\star \star \star \star$ & $\star \star \star \star$ & $\star \star \star \star$ & $* * *$ & $\star * *$ & $\star \star \star *$ & $* * *$ & $\star \star * *$ & $\star * * * *$ \\
\hline$\star \star \star$ & $\star \star \star \star$ & $\star \star \star \star$ & $\star \star *$ & 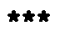 & $\star \star \star \star$ & $\star \star \star \star$ & $\star \star \star \star$ & $\star \star \star$ & $\star \star \star$ & $\star \star \star$ & $\star \star \star \star$ & $\star \star \star \star \star \star$ \\
\hline$\star * *$ & $\star * *$ & $\star \star \star \star$ & $\star * *$ & $\star \star \star *$ & $\star \star \star *$ & $\star * *$ & $* * *$ & $\star \star \star *$ & $\star \star * *$ & $\star * *$ & $\star \star \star$ & $\star \star \star \star \star \star *$ \\
\hline$\star \star \star$ & $\star \star \star \star$ & $\star \star \star$ & $\star \star \star$ & $\star \star \star \star$ & $\star \star \star \star$ & $\star \star \star \star$ & $\star \star \star$ & $\star \star \star$ & $\star \star \star \star$ & $\star \star \star *$ & $\star \star \star \star$ & $\star \star \star \star \star \star *$ \\
\hline$\star \star \star \star$ & $\star \star \star$ & $\star \star \star$ & $\star \star \star$ & $\star \star \star \star$ & $\star \star \star *$ & $\star \star \star *$ & 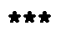 & 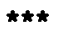 & $\star \star \star \star$ & $\star \star \star \star$ & $\star \star \star$ & $\star \star \star \star \star \star$ \\
\hline$\star \star \star$ & $\star \star \star$ & $\star \star \star \star$ & $\star \star \star \star *$ & $\star \star \star *$ & $\star \star \star \star *$ & $\star \star \star *$ & $\star \star \star \star$ & $\star \star \star *$ & $\star * *$ & $\star * *$ & $\star \star * *$ & 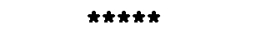 \\
\hline$\star \star \star \star$ & $\star \star \star \star$ & $\star \star \star \star$ & $\star \star \star \star$ & $\star \star \star \star$ & $\star * *$ & $\star \star *$ & $\star \star \star \star$ & $\star \star \star *$ & $\star \star \star *$ & $\star \star \star \star$ & $\star \star \star \star$ & 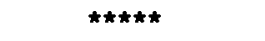 \\
\hline$\star \star \star$ & 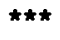 & $\star \star \star \star$ & $\star \star \star$ & $\star \star \star$ & $\star \star \star \star$ & 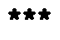 & $\star \star \star$ & $\star \star \star \star$ & $\star \star \star *$ & 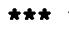 & $\star \star \star \star$ & $\star \star \star \star \star$ \\
\hline$\star \star \star$ & $\star \star \star \star$ & $\star \star \star$ & $\star \star \star$ & $\star \star \star$ & $\star \star * \star$ & $\star \star * *$ & $\star \star \star \star *$ & $\neq * *$ & $\star * *$ & $\star \star * *$ & $* * *$ & $\star * \star * \star *$ \\
\hline$\star \star \star *$ & $\star \star \star$ & $\star \star \star \star$ & $\star \star \star \star$ & $\star \star \star$ & 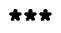 & $\star \star \star \star$ & 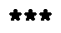 & 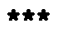 & $\star \star \star \star *$ & 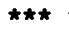 & *** & 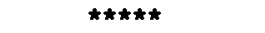 \\
\hline
\end{tabular}

$\begin{array}{lllllllllllll}1 & 2 & 3 & 4 & 5 & 6 & 7 & 8 & 9 & 10 & 11 & 12 & \text { Weighted Priority }\end{array}$

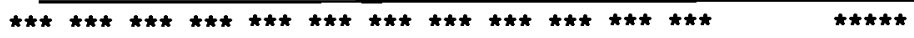

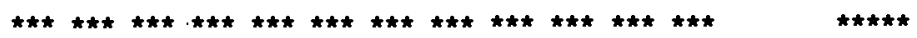

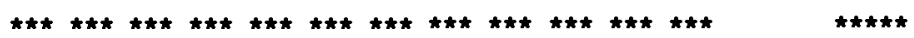

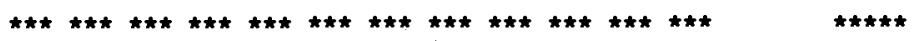

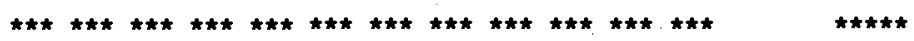

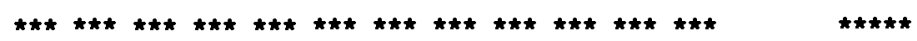

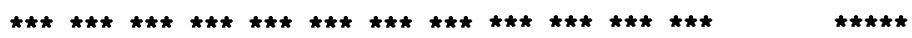

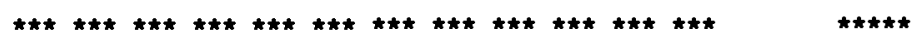

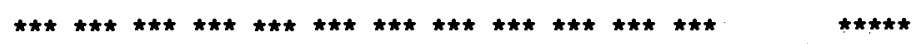

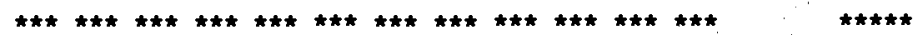

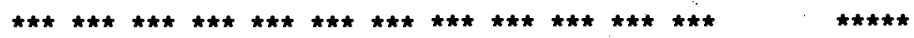

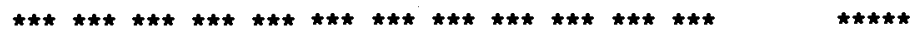

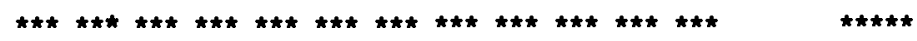

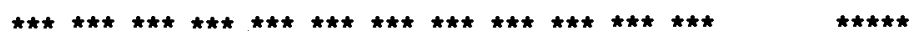

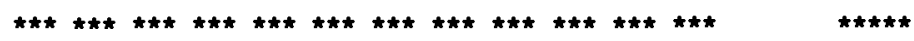


WARDAK 


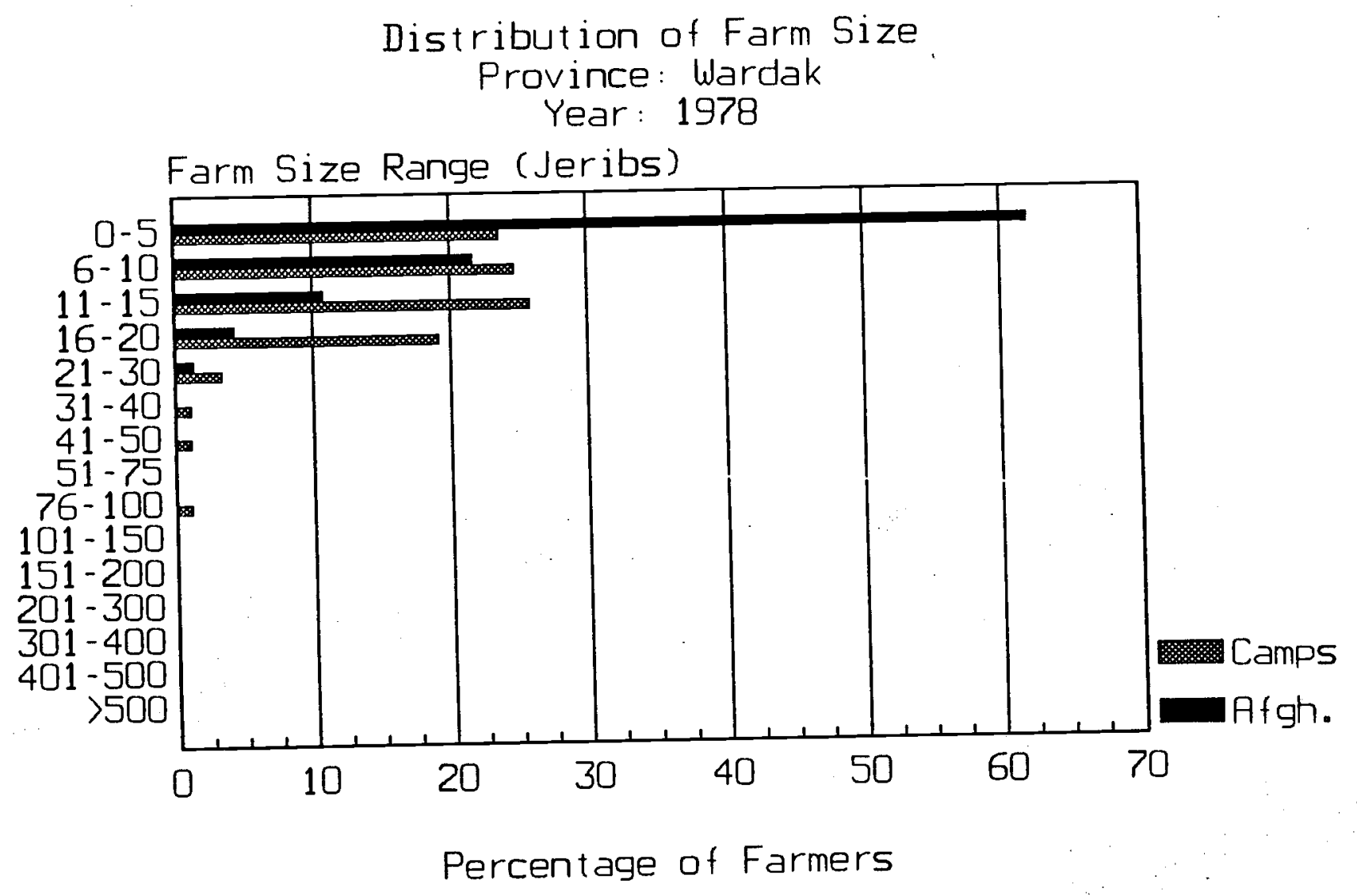

Farmers in Afghanistans

$\underline{1978}$

$\underline{1987}$

Total Number of Farms

Average Area

Average Area Irrigated

Average Dryland Area

6.28

3.12

6.15

3.12

6.33

2.00

Farmers who left in 1987

Total Number of Farms

Average Area

Average Area Irrigated

Average Dryland Area

\section{Average of both Groups}

Provincial Average Area

Provincial Average Irrigated Area

Provincial Average Dryland Area

8.71
8.50
7.00

3.12

3.12

2.00

(A11 areas in Jeribs. 5 Jeribs $=1$ Hectare) 


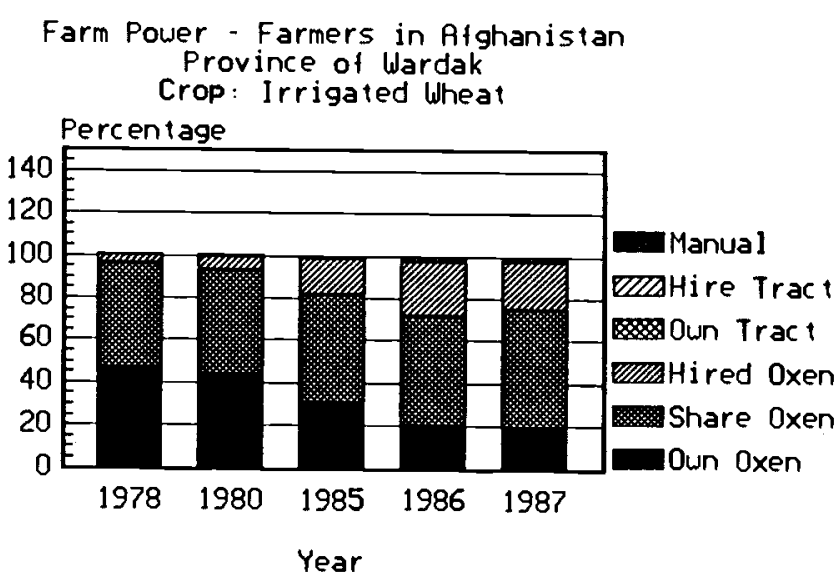

Farm Power - Farmers who left in 1987 Province of Wardak Crop: Irrigated wheat

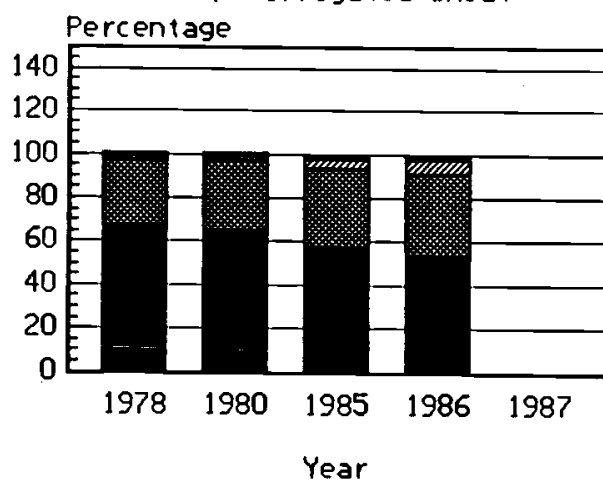

Base Figures for above graphs
Province: Wardak

\section{Farmers in Afghanistan}

Irr. Wheat own oxen Shared oxen Hired oxen Own Tractor Hired Tractor Rice Manual Means

Shared oxen

Hired oxen Own Tractor Hired Tractor Manual Means

Farmers who left in 1987

Irr. Wheat own oxen

Shared oxen

Hired oxen

Own Tractor

Hired Tractor

Rice Own oxen
Manual Means

Own Oxen

Shared oxen

Hired oxen

Own Tractor

Hired Tractor Manual Means

\section{Farm Power - Farmers in Atghanistan Province of Wardak Crop: Rice}

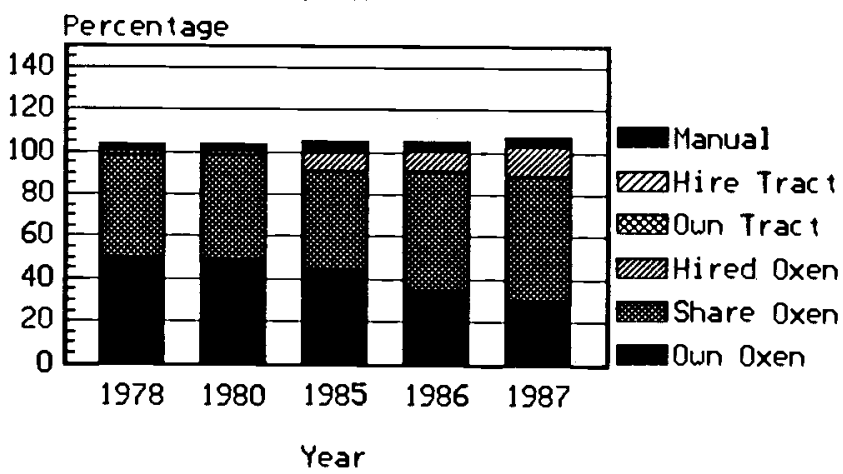

Farm Power - Farmers who left in 1987 Province of Wardak Crop: Rice

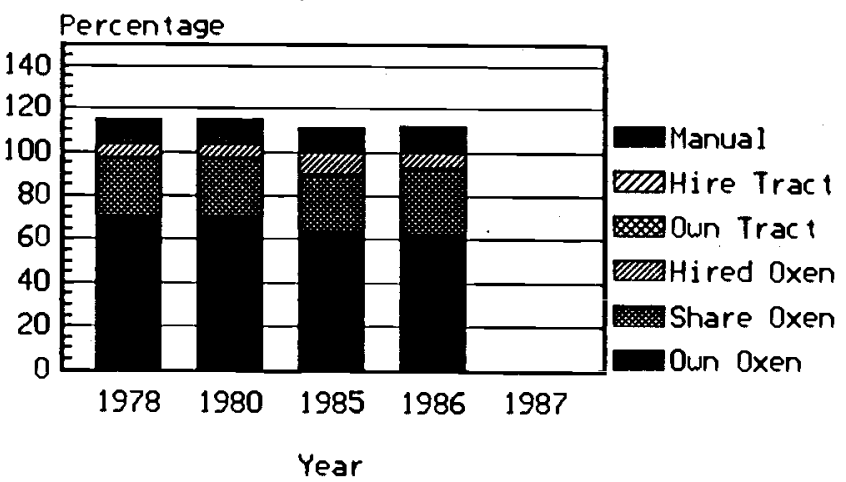

\begin{tabular}{ccccc}
1978 & \multicolumn{2}{c}{ Percentage of } & Farmers & \\
\cline { 5 - 5 } & $\underline{1980}$ & $\underline{1985}$ & $\underline{1986}$ & $\underline{1987}$ \\
46.38 & 43.48 & 30.43 & 20.44 & 19.57 \\
49.28 & 49.28 & 50.72 & 51.09 & 55.07 \\
4.35 & 6.52 & 17.39 & 25.55 & 22.46 \\
- & - & - & - & - \\
- & -72 & -72 & .73 & .72 \\
- & - & - & - & - \\
50.00 & 49.09 & 44.19 & 34.15 & 30.23 \\
48.15 & 49.09 & 46.51 & 56.10 & 58.14 \\
1.85 & 1.82 & 9.30 & 9.76 & 13.95 \\
- & - & - & - & - \\
- & - & - & - & - \\
3.70 & 3.64 & 4.65 & 4.88 & 4.65
\end{tabular}

67.05

29.55

2.27

64.77

57.47

53.57

31.82

35.63

2.27

4.60

36.90

$-$

1.14

-

$70 \cdot 37$

1. 14

70.37

25.93

25.93

-

1.15

$62 \cdot 96$

25.93

11.11

7.14

$-$

1. 19

$61 \cdot 54$

30.77

7.69

$-$
7.41 
PROVINCE OF WRD

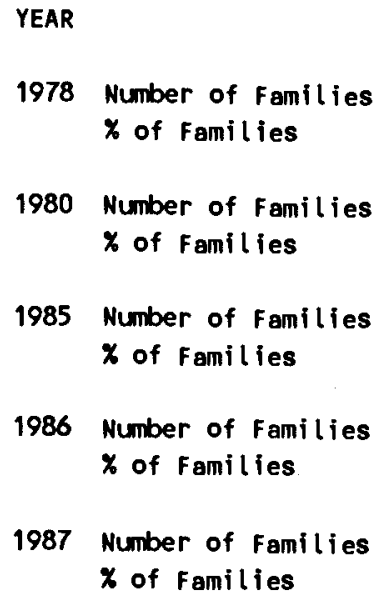

$\begin{array}{ll}1978 & 1.40 \\ 1980 & 1.35 \\ 1985 & 1.09 \\ 1986 & 0.92 \\ 1987 & 0.92\end{array}$

\begin{tabular}{|c|c|c|c|c|c|}
\hline \multicolumn{2}{|c|}{ NUMBER OF } & IRAINED & OXEN $P$ & \multicolumn{2}{|c|}{ FAMILY } \\
\hline 0 & 1 & 2 & 3 & 4 & 5 \\
\hline 8 & 72 & 58 & 2 & 1 & 0 \\
\hline 5.67 & 51.06 & 41.13 & 1.42 & 0.71 & 0.00 \\
\hline 12 & 72 & 54 & 2 & 1 & 0 \\
\hline 8.51 & 51.06 & 38.30 & 1.42 & 0.71 & 0.00 \\
\hline 30 & 71 & 39 & 0 & 1 & 0 \\
\hline 21.28 & 50.35 & 27.66 & 0.00 & 0.71 & 0.00 \\
\hline 40 & 74 & 26 & 0 & 1 & 0 \\
\hline 28.37 & 52.48 & 18.44 & 0.00 & 0.71 & 0.00 \\
\hline 37 & 78 & 26 & 0 & 0 & 0 \\
\hline 26.24 & 55.32 & 18.44 & 0.00 & 0.00 & 0.00 \\
\hline
\end{tabular}

Number of Farmers Questioned 141

\section{IABLE 7B - AVERAGE NUMBER OF TRAINED OXEN FOR FARMERS IN THE CAMPS}

PROVINCE OF WRD

YEAR

1978 Number of Families

$X$ of Families

1980 Number of Families

\% of Fanilies

1985 Number of Families

$\boldsymbol{x}$ of Families

1986 Number of Families

X of Families

1987 Number of Families

\% of Families

Average Number of Oxen per Family:

$$
\begin{aligned}
& 1980 \\
& 1985 \\
& 1986 \\
& 1987
\end{aligned}
$$

NUMBER OF TRAINED OXEN PER FAMILY

$\begin{array}{llllll}0 & 1 & 2 & 3 & 4\end{array}$

$\begin{array}{llllll}5 & 25 & 53 & 3 & 3 & 0\end{array}$

$\begin{array}{llllll}5.62 & 29.09 & 59.55 & 3.37 & 3.37 & 0.00\end{array}$

$\begin{array}{llllll}5 & 29 & 50 & 2 & 3 & 0\end{array}$

$\begin{array}{llllll}5.62 & 32.58 & 56.18 & 2.25 & 3.37 & 0.00\end{array}$

$\begin{array}{cccccc}6 & 33 & 48 & 1 & 1 & 0 \\ 6.74 & 37.08 & 53.93 & 1.12 & 1.12 & 0.00 \\ 11 & 34 & 42 & 0 & 1 & 0 \\ 12.50 & 38.64 & 47.73 & 0.00 & 1.14 & 0.00\end{array}$

$\begin{array}{llllll}89 & 0 & 0 & 0 & 0 & 0\end{array}$

$\begin{array}{llllll}100.0 & 0.00 & 0.00 & 0.00 & 0.00 & 0.00\end{array}$

$\begin{array}{ll}1978 & 1.71 \\ 1980 & 1.65 \\ 1985 & 1.53 \\ 1986 & 1.39 \\ 1987 & 0.00\end{array}$

Number of Farmers Questioned 89 
PROVINCE OF WRO

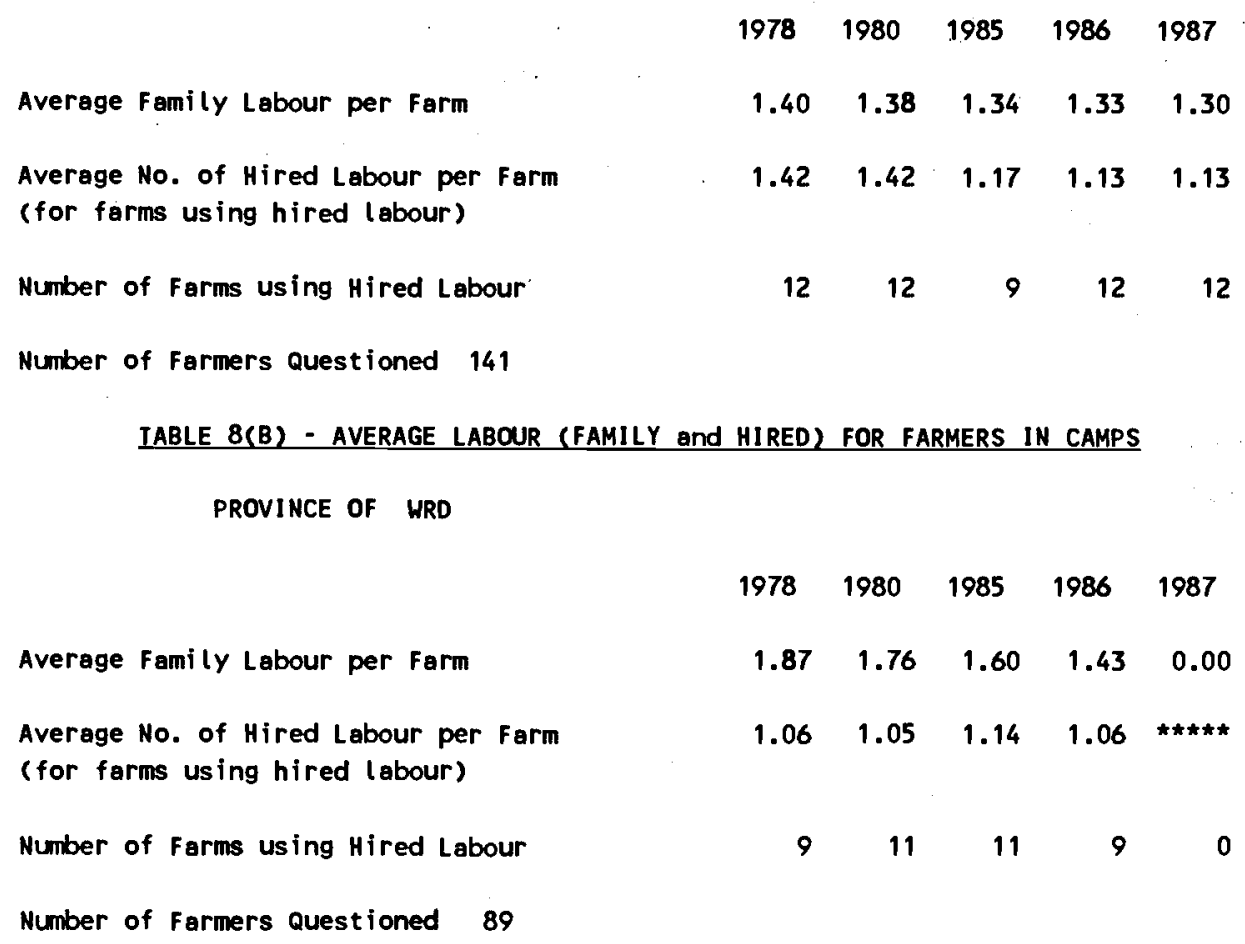


PROVINCE OF WRD

Average Number of Livestock Shot

$$
\begin{aligned}
& \begin{array}{lllll}
1978 & 1980 & 1985 & 1986 & 1987
\end{array} \\
& \begin{array}{lllll}
* * * * * & 6.04 & 2.76 & 1.43 & 1.60
\end{array}
\end{aligned}
$$

Number of Farmers reporting

Livestock shot

Average Number of Livestock Killed by Mines

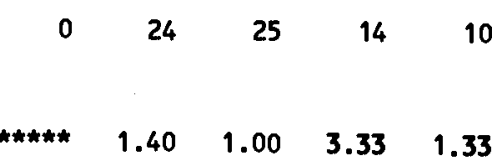

Number of Farmers reporting Livestock killed by Mines

$\begin{array}{lllll}0 & 5 & 3 & 6 & 3\end{array}$

Number of Farmers Questioned 141

\section{IABLE 9B - DIRECT EFFECTS OF THE WAR - FARMERS IN CAMPS}

PROVINCE OF WRD

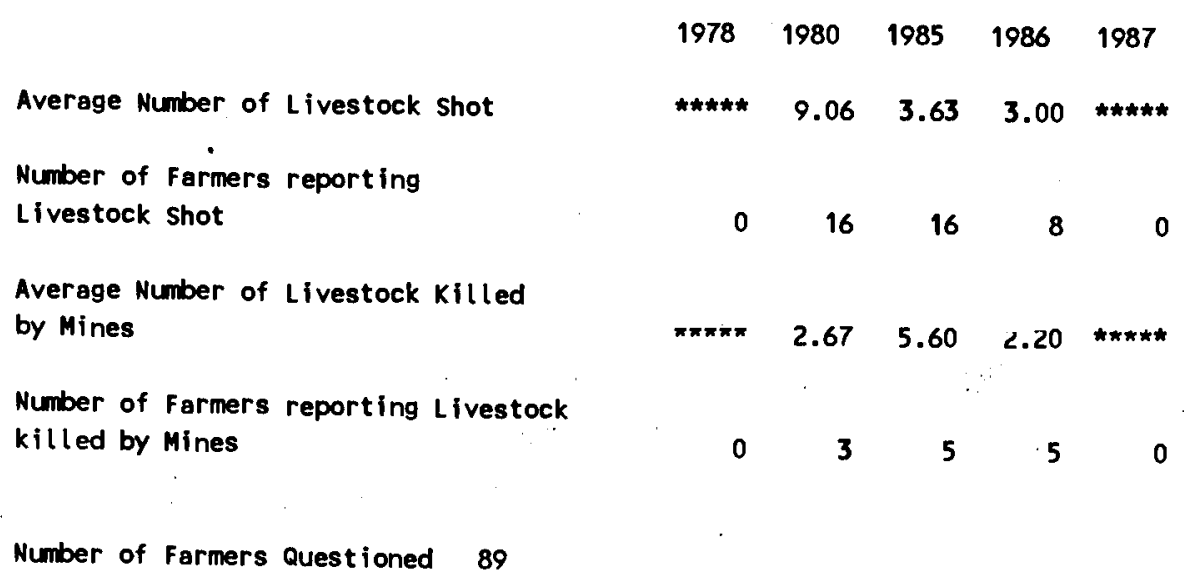




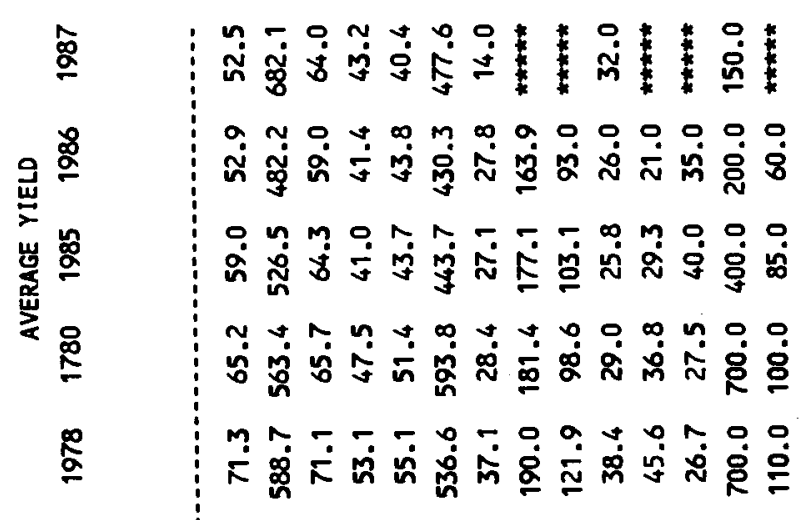

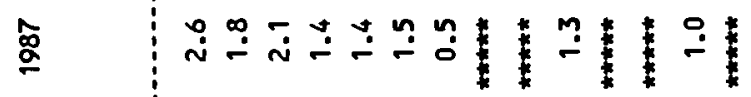

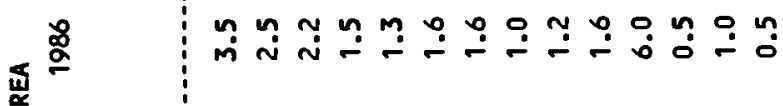

岁㚘

\& $\quad$ M̀

萦

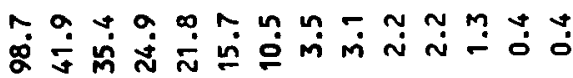

సัำ

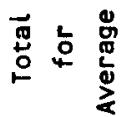

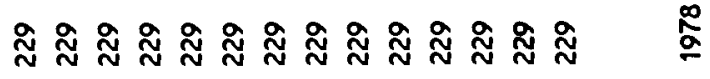

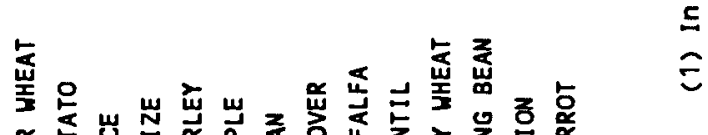

$\underset{8}{2}$

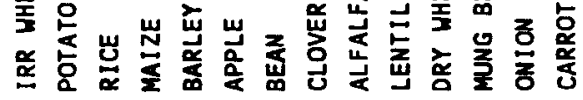


TABLE 1-5 - ANALYSIS OF GREATEST FARMING PROBLEMS - COMUNITY SURVEY

PROVINCE OF WRD

\section{PERCENTAGE OF VILLAGES GIVING FOLLOHING PRIORITY TO PROBLEM}

Rainfall

Crop Disease

Birds or Rats

Seed Availability

Fertilizer Availability

Crop Protection Chemicals

Credit

Irrigation Water Availability

Flooding

Labour Availabilty

Power for Land Preparation

Direct War Effects

Animal Diseases

Extension

Other Problems

\begin{tabular}{rrrrrrrrrrrrr}
1 & 2 & 3 & 4 & 5 & 6 & 7 & 8 & 9 & 10 & 11 & 12 & Weighted Priority \\
\hline 0 & 0 & 0 & 0 & 0 & 0 & 0 & 0 & 0 & 0 & 0 & 0 & 0.00 \\
0 & 7 & 4 & 6 & 6 & 6 & 7 & 0 & 0 & 0 & 0 & 0 & 3.71 \\
4 & 17 & 13 & 15 & 11 & 17 & 7 & 0 & 0 & 0 & 0 & 0 & 10.28 \\
0 & 6 & 11 & 35 & 22 & 11 & 2 & 0 & 0 & 0 & 0 & 0 & 8.60 \\
7 & 0 & 2 & 4 & 4 & 2 & 0 & 0 & 0 & 0 & 0 & 0 & 3.94 \\
2 & 4 & 0 & 6 & 11 & 24 & 9 & 6 & 0 & 0 & 0 & 0 & 5.25 \\
15 & 6 & 0 & 0 & 0 & 0 & 0 & 0 & 0 & 0 & 0 & 0 & 6.93 \\
50 & 2 & 4 & 4 & 0 & 0 & 0 & 0 & 0 & 0 & 0 & 0 & 20.90 \\
2 & 0 & 0 & 0 & 0 & 2 & 0 & 0 & 0 & 0 & 0 & 0 & 0.85 \\
0 & 2 & 0 & 0 & 2 & 0 & 0 & 0 & 0 & 0 & 0 & 0 & 0.51 \\
2 & 9 & 52 & 13 & 15 & 0 & 2 & 0 & 0 & 0 & 0 & 0 & 11.90 \\
13 & 24 & 2 & 2 & 4 & 0 & 0 & 0 & 0 & 0 & 0 & 0 & 10.56 \\
0 & 0 & 7 & 6 & 6 & 4 & 6 & 0 & 0 & 0 & 0 & 0 & 2.51 \\
0 & 0 & 2 & 0 & 6 & 9 & 17 & 11 & 0 & 0 & 0 & 0 & 2.77 \\
7 & 22 & 4 & 11 & 13 & 17 & 6 & 0 & 0 & 0 & 0 & 0 & 11.30
\end{tabular}

1986

Rainfall

Crop Disease

Birds or Rats

Seed Availability

Fertilizer Availability

Crop Protection Chemicals

Credit

Irrigation Water Availability

Flooding

Labour Availabilty

Power for Land Preparation

Direct Wor Effects

Animal Diseases

Extension

Other Problems

\begin{tabular}{rrrrrrrrrrrrr}
1 & 2 & 3 & 4 & 5 & 6 & 7 & 8 & 9 & 10 & 11 & 12 & Weighted Priority \\
\hline 0 & 0 & 0 & 0 & 0 & 0 & 0 & 0 & 0 & 0 & 0 & 0 & 0.00 \\
2 & 9 & 4 & 4 & 6 & 7 & 4 & 0 & 0 & 0 & 0 & 0 & 4.69 \\
3 & 20 & 9 & 9 & 17 & 9 & 4 & 0 & 0 & 0 & 0 & 0 & 13.82 \\
0 & 9 & 17 & 17 & 26 & 11 & 0 & 0 & 0 & 0 & 0 & 0 & 8.70 \\
2 & 2 & 2 & 0 & 4 & 0 & 0 & 0 & 0 & 0 & 0 & 0 & 1.68 \\
4 & 0 & 6 & 6 & 6 & 19 & 11 & 2 & 0 & 0 & 0 & 0 & 5.27 \\
3 & 6 & 2 & 0 & 0 & 0 & 0 & 0 & 0 & 0 & 0 & 0 & 6.66 \\
1 & 11 & 9 & 4 & 0 & 0 & 0 & 0 & 0 & 0 & 0 & 0 & 20.47 \\
2 & 2 & 0 & 0 & 2 & 0 & 0 & 0 & 0 & 0 & 0 & 0 & 1.28 \\
0 & 0 & 0 & 2 & 0 & 0 & 0 & 0 & 0 & 0 & 0 & 0 & 0.19 \\
0 & 4 & 26 & 43 & 9 & 4 & 0 & 0 & 0 & 0 & 0 & 0 & 9.61 \\
9 & 17 & 11 & 2 & 2 & 0 & 0 & 0 & 0 & 0 & 0 & 0 & 9.00 \\
0 & 4 & 4 & 2 & 4 & 0 & 7 & 2 & 0 & 0 & 0 & 0 & 2.27 \\
0 & 0 & 0 & 2 & 6 & 15 & 13 & 9 & 0 & 0 & 0 & 0 & 2.87 \\
3 & 19 & 9 & 13 & 13 & 7 & 6 & 0 & 0 & 0 & 0 & 0 & 13.50
\end{tabular}

1985

Rainfall

Crop Disease

Birds or Rats

Seed Availability

Fertilizer Availability

crop Protection Chemicals

Credit

Irrigation Water Availability

Flooding

Labour Availabilty

Power for Land Preparation

Direct War Effects

Animal Diseases

Extension

other Problems \begin{tabular}{ccccccccccccc}
1 & 2 & 3 & 4 & 5 & 6 & 7 & 8 & 9 & 10 & 11 & 12 & Weighted Priority \\
\hline 0 & 0 & 0 & 0 & 0 & 0 & 0 & 0 & 0 & 0 & 0 & 0 & 0.00
\end{tabular}

$\begin{array}{lllllllllllll}4 & 2 & 6 & 7 & 0 & 0 & 0 & 2 & 0 & 0 & 0 & 0 & 3.74\end{array}$

$\begin{array}{lllllllllllll}22 & 19 & 19 & 7 & 2 & 6 & 2 & 0 & 0 & 0 & 0 & 0 & 17.93\end{array}$

\begin{tabular}{lllllllllllll}
7 & 4 & 7 & 13 & 28 & 9 & 4 & 0 & 0 & 0 & 0 & 0 & \\
\hline & 0 & 0 & 0 & 0 & 0 & 0 & 0 & 0 & 0 & 0 & 0 & 9.87
\end{tabular}

$\begin{array}{lllllllllllll}0 & 6 & 6 & 6 & 7 & 4 & 4 & 0 & 0 & 0 & 0 & 0 & 3.78\end{array}$

$\begin{array}{lllllllllllll}0 & 0 & 0 & 0 & 0 & 0 & 0 & 0 & 0 & 0 & 0 & 0 & 0.00\end{array}$

$\begin{array}{lllllllllllll}4 & 30 & 19 & 2 & 4 & 2 & 0 & 0 & 0 & 0 & 0 & 0 & 11.44\end{array}$

$\begin{array}{lllllllllllll}2 & 0 & 2 & 2 & 0 & 0 & 2 & 0 & 0 & 0 & 0 & 0 & 1.40\end{array}$

$\begin{array}{lllllllllllll}0 & 0 & 0 & 2 & 0 & 0 & 0 & 0 & 0 & 0 & 0 & 0 & 0.20\end{array}$

$\begin{array}{lllllllllllll}4 & 2 & 15 & 26 & 11 & 2 & 0 & 0 & 0 & 0 & 0 & 0 & 8.11\end{array}$

$\begin{array}{lllllllllllll}33 & 22 & 11 & 6 & 2 & 2 & 0 & 0 & 0 & 0 & 0 & 0 & 21.93\end{array}$

$\begin{array}{lllllllllllll}4 & 2 & 2 & 2 & 2 & 4 & 0 & 0 & 0 & 0 & 0 & 0 & 2.92\end{array}$

$\begin{array}{lllllllllllll}0 & 0 & 2 & 2 & 11 & 22 & 4 & 0 & 0 & 0 & 0 & 0 & 3.29\end{array}$

$\begin{array}{lllllllllllll}20 & 13 & 4 & 13 & 17 & 2 & 2 & 0 & 0 & 0 & 0 & 0 & \end{array}$ $\begin{array}{lllllllllllll}0 & 0 & 0 & 0 & 0 & 0 & 0 & 0 & 0 & 0 & 0 & 0 & 0.00\end{array}$ 
PROVINCE OF WRD

\section{Percentage of VILLAges gIVING FOLLOWING PRIORITY TO PROBLEM}

Rainfall

Crop Disease

Birds or Rats

Seed Availability

Fertilizer Availability

Crop Protection Chemicals

Credit

Irrigation Hater Availability

Flooding

Labour Availabilty

Power for Land Preperation

Direct Har Effects

Animal Diseases

Extension

Other Problems

\begin{tabular}{rrrrrrrrrrrrr}
1 & 2 & 3 & 4 & 5 & 6 & 7 & 8 & 9 & 10 & 11 & 12 & Weighted Priority \\
\hline 0 & 0 & 0 & 0 & 0 & 0 & 0 & 0 & 0 & 0 & 0 & 0 & 0.00 \\
2 & 0 & 2 & 0 & 0 & 0 & 0 & 0 & 0 & 0 & 0 & 0 & 1.38 \\
33 & 26 & 11 & 6 & 0 & 0 & 0 & 0 & 0 & 0 & 0 & 0 & 28.68 \\
7 & 0 & 6 & 0 & 0 & 2 & 0 & 0 & 0 & 0 & 0 & 0 & 5.34 \\
0 & 0 & 0 & 0 & 0 & 0 & 0 & 0 & 0 & 0 & 0 & 0 & 0.00 \\
0 & 4 & 4 & 2 & 0 & 0 & 0 & 0 & 0 & 0 & 0 & 0 & 1.98 \\
0 & 0 & 0 & 0 & 0 & 0 & 0 & 0 & 0 & 0 & 0 & 0 & 0.00 \\
0 & 2 & 2 & 4 & 6 & 2 & 0 & 0 & 0 & 0 & 0 & 0 & 2.17 \\
0 & 6 & 0 & 6 & 2 & 0 & 0 & 0 & 0 & 0 & 0 & 0 & 2.53 \\
0 & 0 & 2 & 0 & 0 & 0 & 0 & 0 & 0 & 0 & 0 & 0 & 0.34 \\
0 & 0 & 2 & 9 & 4 & 0 & 0 & 0 & 0 & 0 & 0 & 0 & 2.05 \\
35 & 19 & 11 & 4 & 0 & 0 & 0 & 0 & 0 & 0 & 0 & 0 & 27.39 \\
2 & 2 & 2 & 4 & 2 & 0 & 0 & 0 & 0 & 0 & 0 & 0 & 2.62 \\
0 & 6 & 7 & 13 & 9 & 4 & 0 & 0 & 0 & 0 & 0 & 0 & 6.12 \\
15 & 19 & 26 & 4 & 6 & 0 & 0 & 0 & 0 & 0 & 0 & 0 & 19.40
\end{tabular}

1978

Rainfall

Crop Disease

Birds or Rats

Seed Availability

Fertilizer Availability

Crop Protection Chemicals

Credit

Irrigation Water Availability

Flooding

Labour Avai labilty

Power for Land Preparation

Direct War Effects

Animal Diseases

Extension

Other Problems

\begin{tabular}{rrrrrrrrrrrrr}
1 & 2 & 3 & 4 & 5 & 6 & 7 & 8 & 9 & 10 & 11 & 12 & Heighted Priority \\
\hline 0 & 0 & 0 & 0 & 0 & 0 & 0 & 0 & 0 & 0 & 0 & 0 & 0.00 \\
0 & 0 & 2 & 0 & 0 & 0 & 0 & 0 & 0 & 0 & 0 & 0 & 0.56 \\
33 & 26 & 0 & 0 & 0 & 0 & 0 & 0 & 0 & 0 & 0 & 0 & 41.96 \\
2 & 0 & 0 & 0 & 0 & 0 & 0 & 0 & 0 & 0 & 0 & 0 & 1.68 \\
0 & 0 & 0 & 0 & 0 & 0 & 0 & 0 & 0 & 0 & 0 & 0 & 0.00 \\
0 & 0 & 0 & 0 & 0 & 0 & 0 & 0 & 0 & 0 & 0 & 0 & 0.00 \\
0 & 0 & 0 & 0 & 0 & 0 & 0 & 0 & 0 & 0 & 0 & 0 & 0.00 \\
0 & 0 & 0 & 0 & 0 & 0 & 0 & 0 & 0 & 0 & 0 & 0 & 0.00 \\
0 & 6 & 2 & 0 & 0 & 0 & 0 & 0 & 0 & 0 & 0 & 0 & 3.08 \\
0 & 0 & 0 & 0 & 0 & 0 & 0 & 0 & 0 & 0 & 0 & 0 & 0.00 \\
0 & 0 & 0 & 0 & 0 & 0 & 0 & 0 & 0 & 0 & 0 & 0 & 0.00 \\
0 & 0 & 0 & 0 & 0 & 0 & 0 & 0 & 0 & 0 & 0 & 0 & 0.00 \\
2 & 2 & 2 & 0 & 0 & 0 & 0 & 0 & 0 & 0 & 0 & 0 & 3.08 \\
0 & 0 & 0 & 2 & 0 & 0 & 0 & 0 & 0 & 0 & 0 & 0 & 0.42 \\
46 & 15 & 2 & 0 & 0 & 0 & 0 & 0 & 0 & 0 & 0 & 0 & 49.23
\end{tabular}




\section{ZABUL}




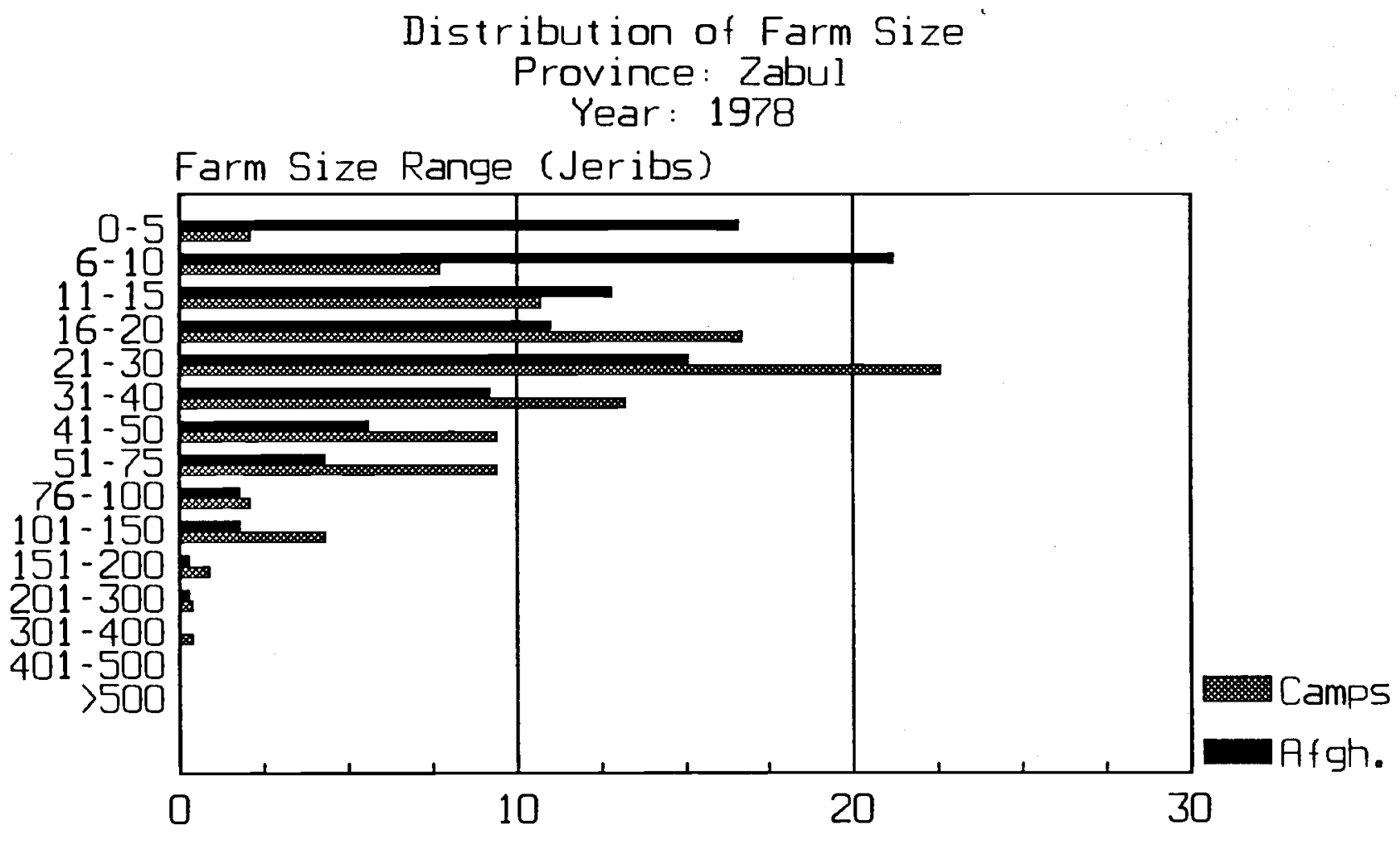

Percentage of Farmers

Farmers in Afghanistans

Total Number of Farms

Average Area

Average Area Irrigated

Average Dryland Area

Farmers who left in 1987

Total Number of Farms

Average Area

Average Area Irrigated

Average Dryland Area

Average of both Groups

Provincial Average Area

Provincial Average Irrigated Area

Provincial Average Dryland Area
1978

391

22.86

21.52

9.87
1987

19.3

5.13

4.36

3.54

0

-

-

-

27.99

5.13

25.88

4.36

14.27

3.54

(All areas in Jeribs. 5 Jeribs $=1$ Hectare) 
Farm Power - Farmers in Atghanistan Province of Zabul

Crop: Irrigated theat

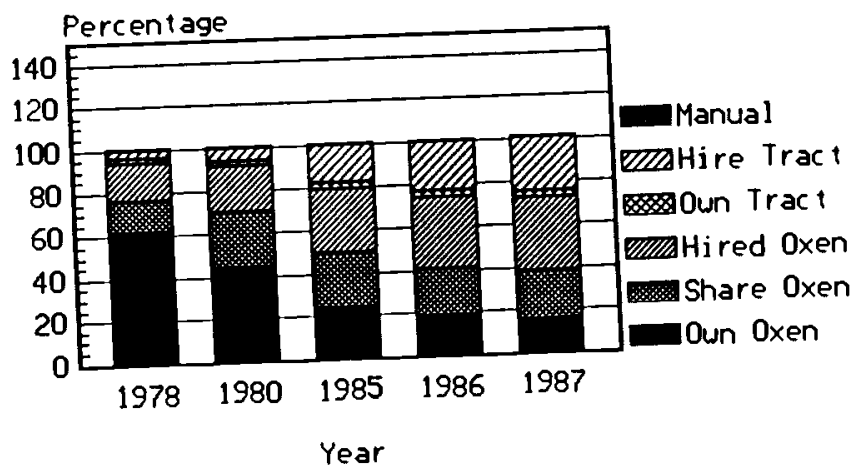

Farm Power - Farmers who lett in 1987 Province of Zabul

Crop: Irrigated wheat

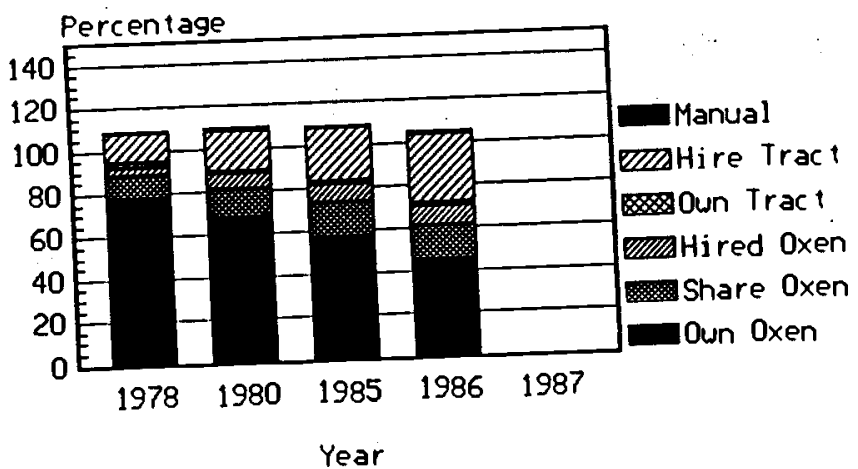

Base Figures for above graphs

Province: $\mathrm{Zabul}$

Farmers in Afghanistan

Irr. Wheat own oxen

Shared oxen

Hired oxen

own Tractor

Hired Tractor

Manual Means

Maize

Own Oxen

Shared oxen

Hired oxen

Own Tractor

Hired Tractor

Manual Means

Farmers who left in 1987

Irr. Wheat

Own oxen

shared oxen

Hired Oxen

own Tractor

Hired Tractor

Manual Means

Maize

shared oxen

Hired oxen

own Tractor

Farm Power - Farmers in Atghanistan Province of Zabul

Crop: Maize

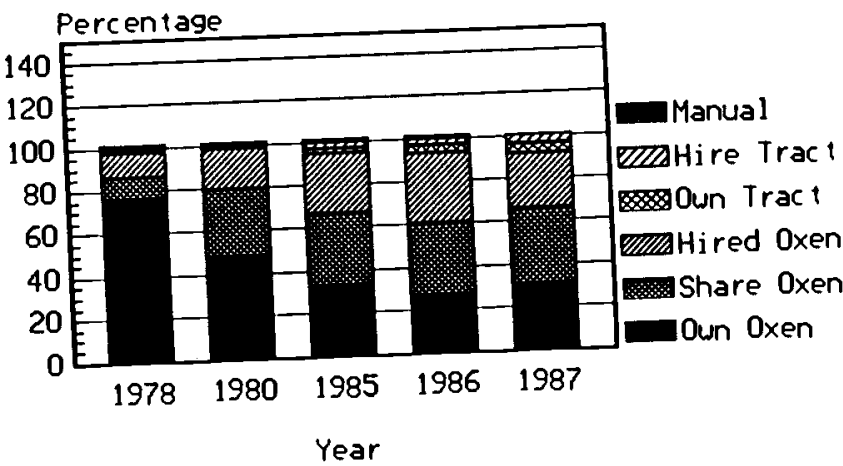

Farm Power - Farmers who lett in 1987 Province of Zabul Crop: ilaize

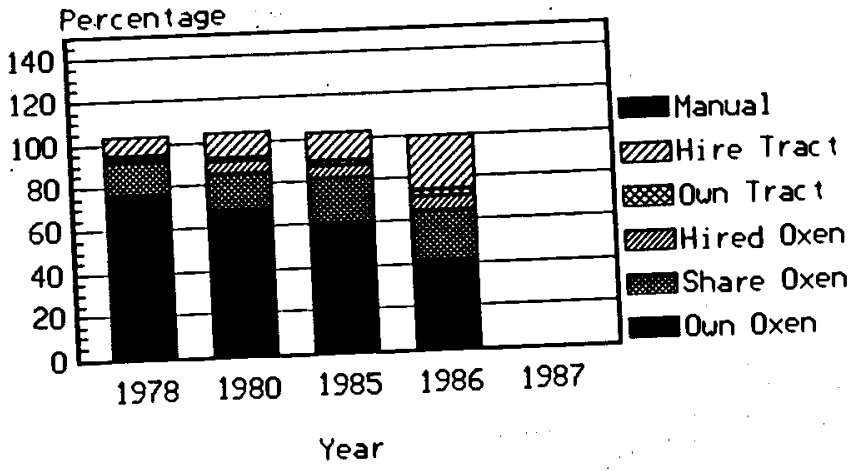

$1978 \stackrel{\text { Percentage of Farmers }}{1980 \quad \underline{1985} \quad \underline{1986}} \underline{1987}$

$\begin{array}{rrrrr}61.47 & 44.19 & 23.68 & 18.34 & 15.29 \\ 14.45 & 25.58 & 26.02 & 21.89 & 22.93 \\ 17.28 & 21.22 & 29.53 & 33.73 & 33.76 \\ 2.27 & 2.33 & 2.63 & 2.96 & 3.18 \\ 3.97 & 6.10 & 17.54 & 22.49 & 25.48 \\ .85 & .87 & .88 & .89 & .64 \\ 76.47 & 48.45 & 33.33 & 27.27 & 30.43 \\ 9.80 & 30.93 & 33.33 & 32.95 & 34.78 \\ 10.78 & 18.56 & 27.78 & 32.95 & 26.09 \\ 1.96 & 1.03 & 2.22 & 3.41 & 4.35 \\ .98 & 1.03 & 3.33 & 3.41 & 4.35 \\ 6.86 & 7.22 & 4.44 & 4.55 & -\end{array}$

77.49

67.70

57.21

45.04

10.39

12.83

16.22

4.33

7.52

1.73

1.33

13.85

18.58

1.30

1.33

76.11

67.62

15.04

17.14

1.77

5.71

1.77

1.90

8.11

1.35

24.77

1.35

60.00

21.00

6.00

2.00

16.03

9.16

1.53

31.30

1.53

41.38

22.41

6.90

8.85

11.43 
Zabul

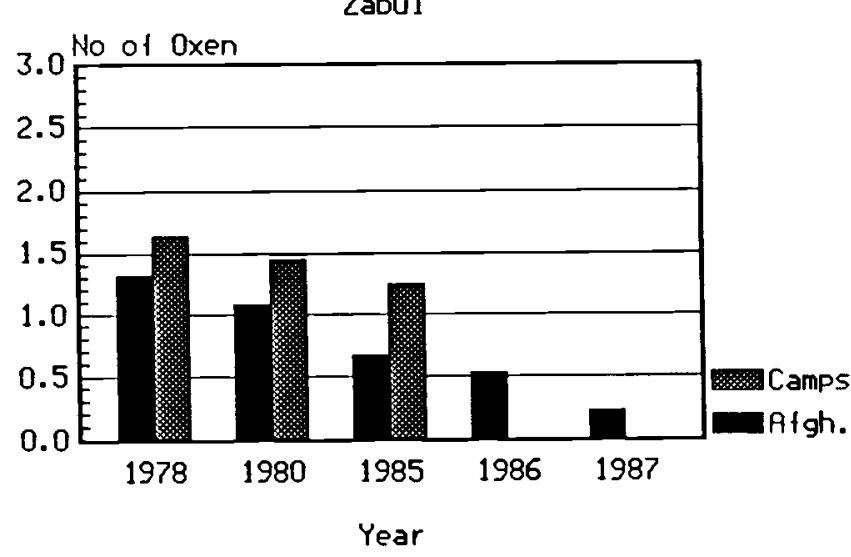

Zabul

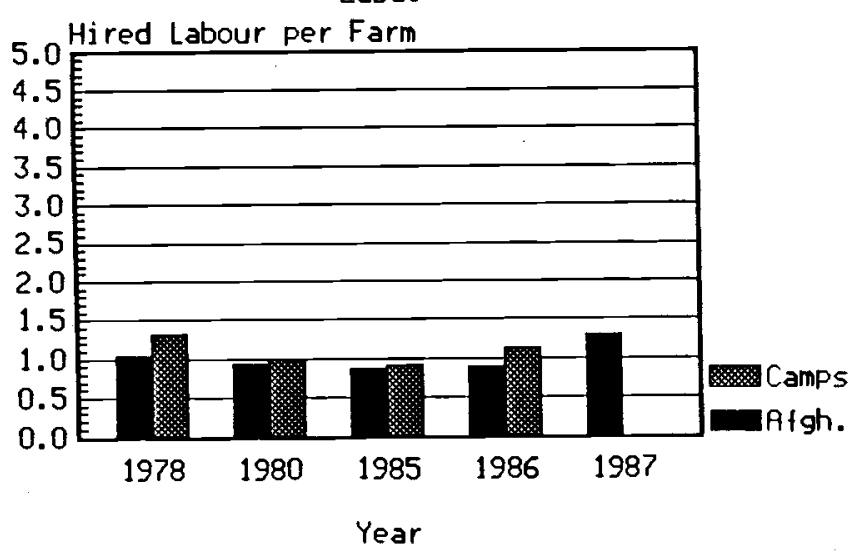

Zabul

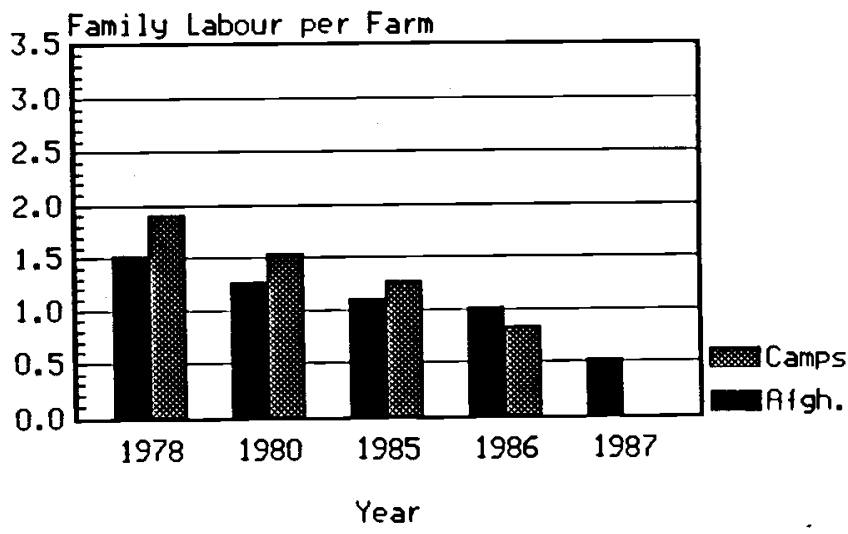

Zabul

Percentage of Farms using

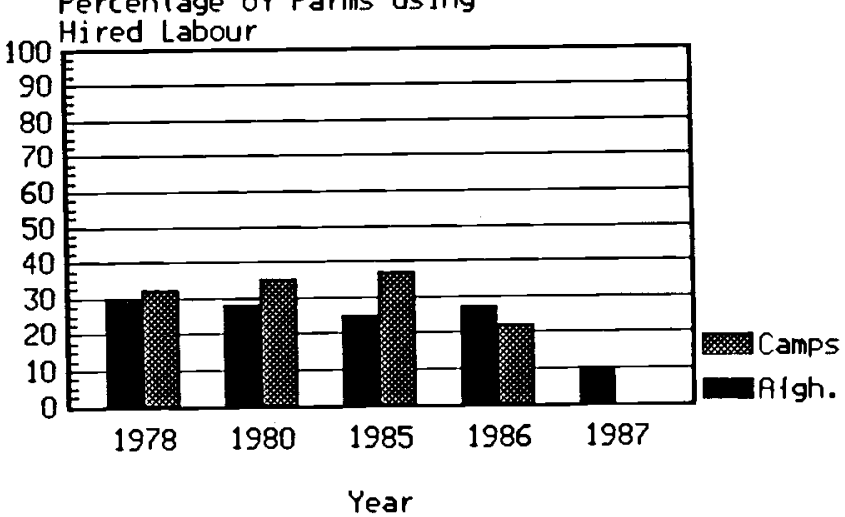


PROVINCE OF ZBL

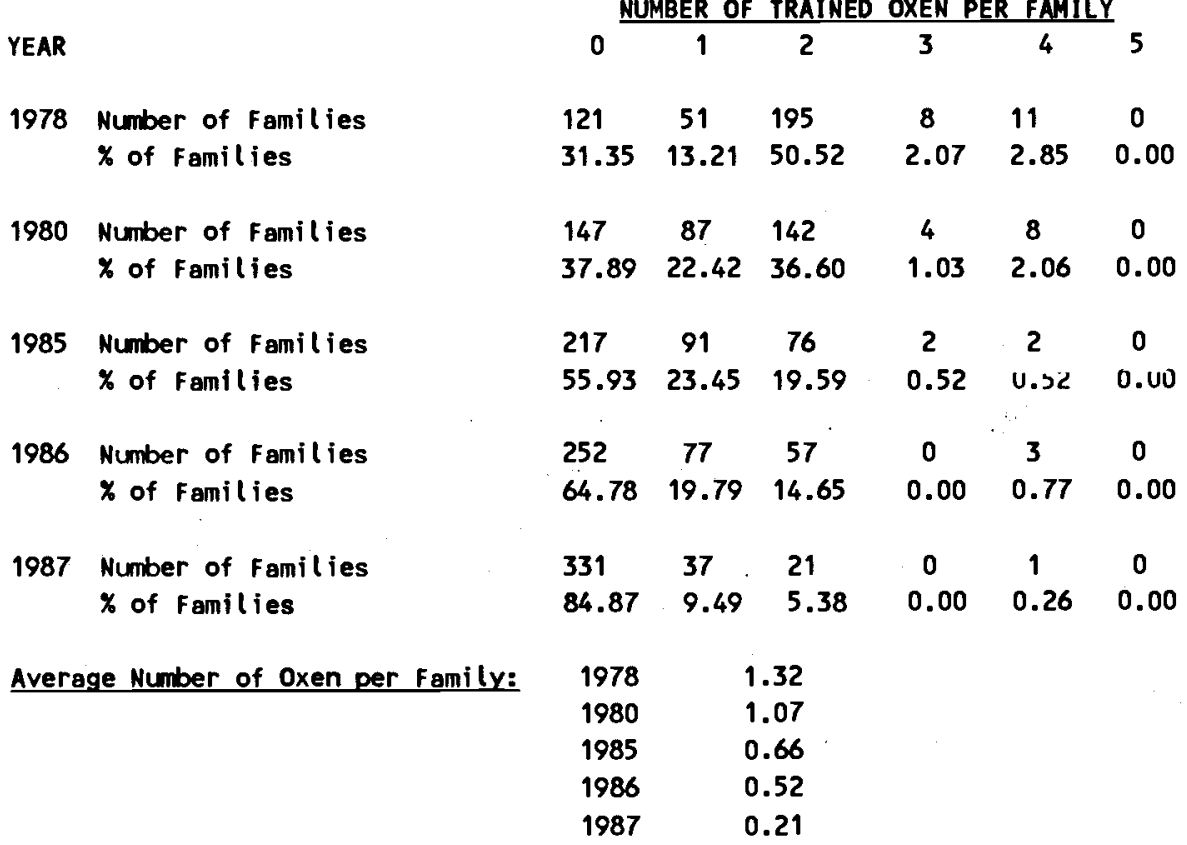

Number of Farmers Questioned 390

\section{IABLE 7B - AVERAGE NUMBER OF TRAINED OXEN FOR FARMERS IN THE CAMPS}

PROVINCE OF ZBL

\begin{tabular}{|c|c|c|c|c|c|c|c|}
\hline \multirow{2}{*}{ YEAR } & & & & & & & \\
\hline & & 0 & 1 & 2 & 3 & 4 & 5 \\
\hline \multirow[t]{2}{*}{1978} & Number of Families & 32 & 28 & 169 & 4 & 2 & 0 \\
\hline & $x$ of Families & 13.62 & 11.91 & 71.91 & 1.70 & 0.85 & 0.00 \\
\hline \multirow[t]{2}{*}{1980} & Number of Families & 54 & 28 & 152 & 0 & 2 & 0 \\
\hline & $x$ of Families & 22.88 & 11.86 & 64.41 & 0.00 & 0.85 & 0.00 \\
\hline \multirow[t]{2}{*}{1985} & Number of Families & 72 & 35 & 129 & 0 & 0 & 0 \\
\hline & $\%$ of Families & 30.51 & 14.83 & 54.66 & 0.00 & 0.00 & 0.00 \\
\hline \multirow[t]{2}{*}{1986} & Number of Families & 152 & 20 & 64 & 0 & 0 & 0 \\
\hline & $\%$ of Families & 64.41 & 8.47 & 27.12 & 0.00 & 0.00 & 0.00 \\
\hline \multirow[t]{2}{*}{1987} & Number of Families & 235 & 1 & 0 & 0 & 0 & 0 \\
\hline & \% of Families & 99.58 & 0.42 & 0.00 & 0.00 & 0.00 & 0.00 \\
\hline \multirow{5}{*}{\multicolumn{2}{|c|}{ Average Number of Oxen per Family: }} & 1978 & \multicolumn{2}{|c|}{1.64} & & & \\
\hline & & 1980 & \multicolumn{2}{|c|}{1.44} & & & \\
\hline & & 1985 & \multicolumn{2}{|c|}{1.24} & & & \\
\hline & & 1986 & \multicolumn{2}{|r|}{0.63} & & & \\
\hline & & 1987 & \multicolumn{2}{|c|}{0.00} & & & \\
\hline
\end{tabular}


PROVINCE OF ZBL

\begin{tabular}{|c|c|c|c|c|c|}
\hline & 1978 & 1980 & 1985 & 1986 & 1987 \\
\hline Average Family Labour per Farm & 1.52 & 1.27 & 1.10 & 1.01 & 0.51 \\
\hline $\begin{array}{l}\text { Average No. of Hired Labour per Farm } \\
\text { (for farms using hired (abour) }\end{array}$ & 1.04 & 0.93 & 0.86 & 0.89 & 1.29 \\
\hline Number of Farms using Hired Labour & 115 & 110 & 98 & 105 & 34 \\
\hline
\end{tabular}

IABLE 8(B) - AVERAGE LABOUR (FAMILY and HIRED) FOR FARMERS IN CAMPS

PROVINCE OF ZBL

\begin{tabular}{|c|c|c|c|c|c|}
\hline & 1978 & 1980 & 1985 & 1986 & 1987 \\
\hline Average Family Labour per Farm & 1.90 & 1.54 & 1.27 & 0.83 & 0.00 \\
\hline $\begin{array}{l}\text { Average No. of Hired Labour per Farm } \\
\text { (for farms using hired (abour) }\end{array}$ & 1.32 & 1.00 & 0.90 & 1.12 & 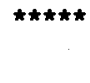 \\
\hline Number of Farms using Hired Labour & 75 & 83 & 88 & 51 & 0 \\
\hline
\end{tabular}


PROVINCE OF ZBL

\begin{tabular}{|c|c|c|c|c|c|}
\hline & 1978 & 1980 & 1985 & 1986 & 1987 \\
\hline Average Number of Livestock Shot & $\star \star \star \star \star$ & 10.38 & 5.45 & 6.44 & 2.50 \\
\hline $\begin{array}{l}\text { Number of Farmers reporting } \\
\text { Livestock Shot }\end{array}$ & 0 & 16 & 22 & 9 & 2 \\
\hline $\begin{array}{l}\text { Average Number of Livestock Killed } \\
\text { by Mines }\end{array}$ & 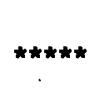 & $\star \star \star \star \star \star$ & 5.00 & 9.00 & 3.50 \\
\hline $\begin{array}{l}\text { Number of Farmers reporting Livestock } \\
\text { killed by Mines }\end{array}$ & 0 & 0 & 9 & 2 & 2 \\
\hline
\end{tabular}

IABLE 9B - OIRECT EFFECTS OF THE HAR - FARMERS IN CAMPS

PROVINCE OF ZBL

\begin{tabular}{|c|c|c|c|c|c|}
\hline & 1978 & 1980 & 1985 & 1986 & 1987 \\
\hline Average Number of Livestock Shot & 2.00 & 9.59 & 4.76 & 6.25 & 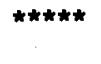 \\
\hline $\begin{array}{l}\text { Number of Farmers reporting } \\
\text { Livestock Shot }\end{array}$ & 1 & 37 & 55 & 4 & 0 \\
\hline $\begin{array}{l}\text { Average Number of Livestock Killed } \\
\text { by Mines }\end{array}$ & $\star \star \star \star \star$ & 4.75 & 4.11 & 2.57 & $\star \star \star \star \star *$ \\
\hline $\begin{array}{l}\text { Number of Farmers reporting Livestock } \\
\text { killed by Mines }\end{array}$ & 0 & 8 & 19 & 7 & \\
\hline
\end{tabular}




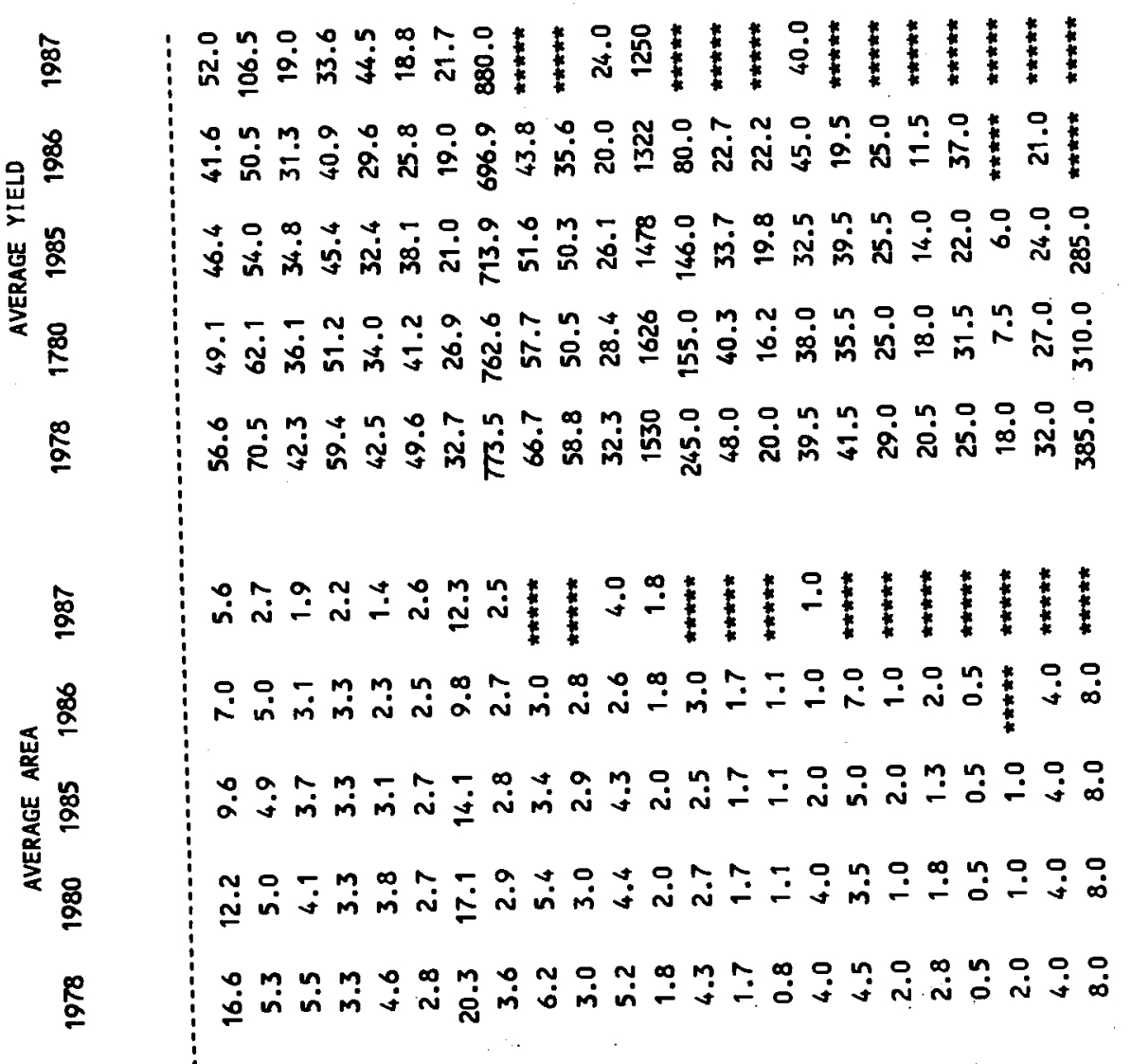


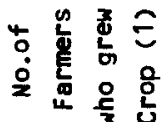

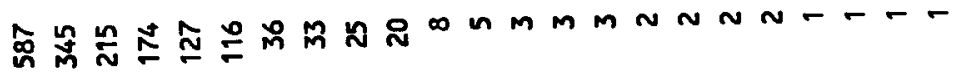

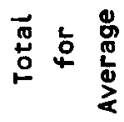

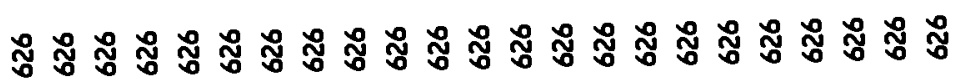

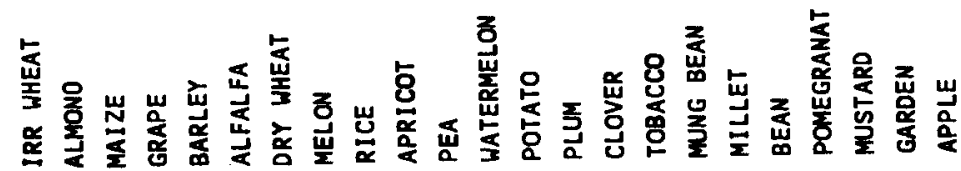


PROVINCE OF ZBL

\section{PERCENTAGE OF VILLAGES GIVING FOLLOWING PRIORITY TO PROBLEM}

Rainfall

Crop Disease

Birds or Rats

Seed Availability

Fertilizer Availability

Crop Protection Chemicals

Credit

Irrigation Water Availability

Flooding

Labour Availabilty

Power for Land Preparation

Direct Har Effects

Animal Diseases

Extension

other Problems
Rainfall

Crop Disease

Birds or Rats

Seed Availability

Fertilizer Availability

Crop Protection Chemicals

Credit

Irrigation Hater Availability

Flooding

Labour Availabilty

Power for Land Preparation

Direct War Effects

Animal Diseases

Extension

Other Problems

1985

Rainfall

Crop Disease

Birds or Rats

Seed Availability

Fertilizer Availability

Crop Protection Chemicals

Credit

Irrigation Water Availability

Flooding

Labour Availabilty

Power for Land Preparation

Direct War Effects

Animal Diseases

Extension

other Problems $\begin{array}{llllllllllll}1 & 2 & 3 & 4 & 5 & 6 & 7 & 8 & 9 & 10 & 11 & 12\end{array}$ Heighted Priority

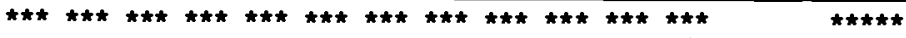

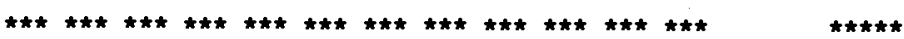

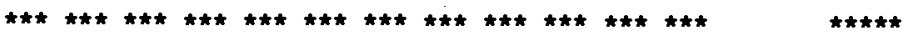

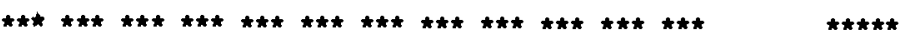

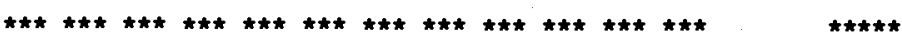

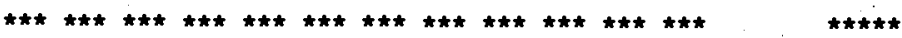

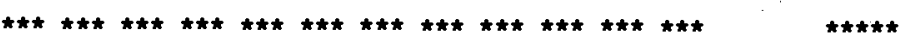

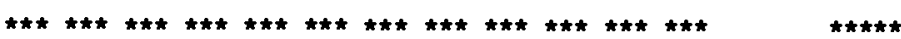

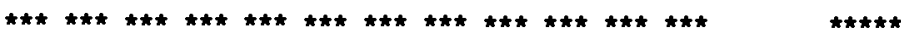

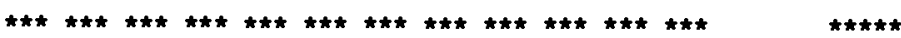

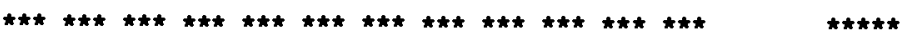

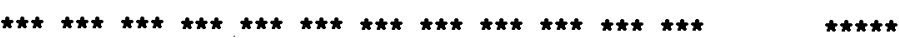

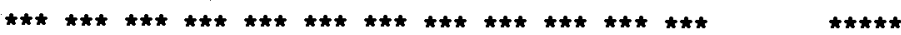

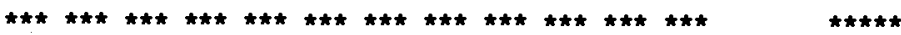

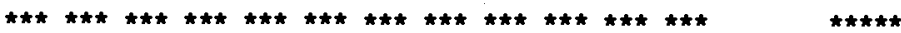

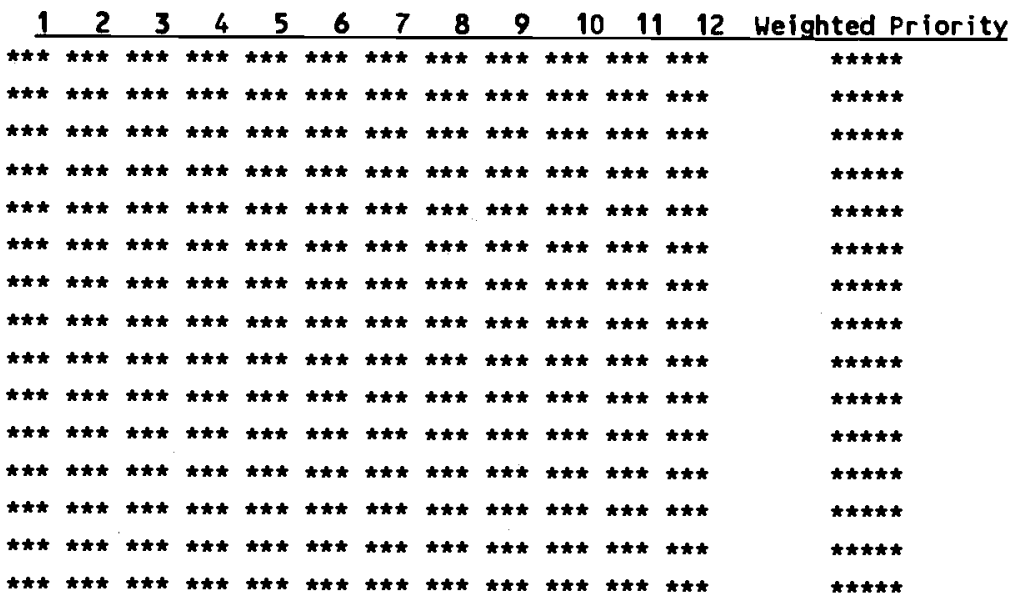

$\begin{array}{lllllllllllll}1 & 2 & 3 & 4 & 5 & 6 & 7 & 8 & 9 & 10 & 11 & 12 & \text { Weighted Priority }\end{array}$

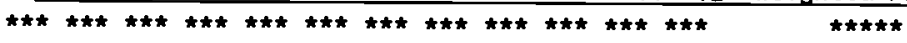

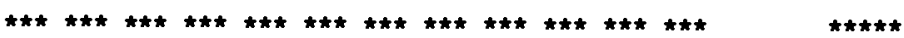

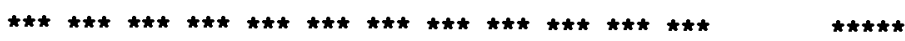

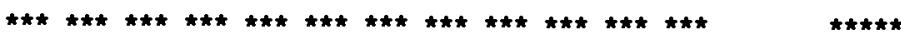

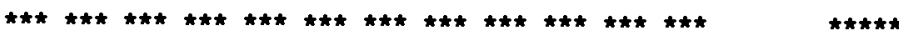

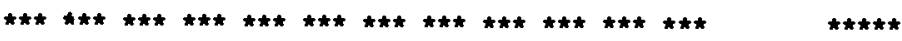

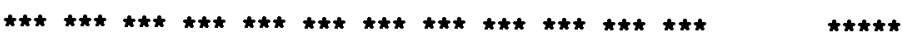

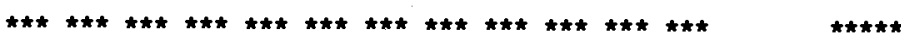

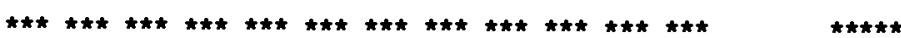

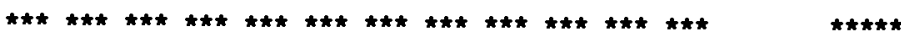

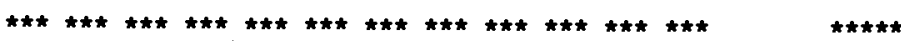

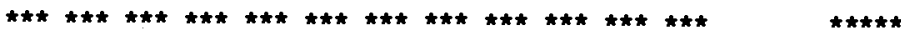

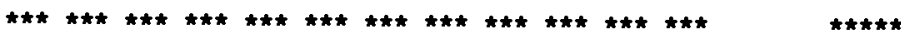

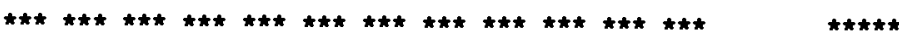

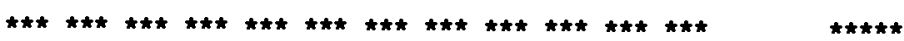


PROVINCE OF ZBL

Rainfall

Crop Disease

Birds or Rats

Seed Availability

Fertilizer Availability

Crop Protection Chemicals

Credit

Irrigation Water Availability

Flooding

Labour Availabilty

Power for Land Preparation

Direct War Effects

Animal Oiseases

Extension

other Problems

\begin{tabular}{|c|c|c|c|c|c|c|c|c|c|c|c|c|}
\hline 1 & 2 & 3 & 4 & 5 & 6 & 7 & 8 & 9 & 10 & 11 & 12 & Weighted Priority \\
\hline & $\star \star \star \star$ & 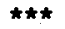 & $\star \star \star$ & $\star \star \star$ & 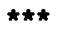 & $\star \star \star$ & $\star \star \star$ & $\star \star \star$ & $\star \star \star$ & 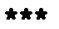 & 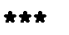 & 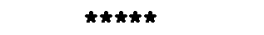 \\
\hline & $\star \star \star$ & $\star \star \star \star$ & *** & $\star \star \star$ & $\star \star \star \star$ & $\star \star \star$ & $\star \star \star$ & $\star \star \star \star$ & $\star \star \star \star$ & $\star \star \star$ & $\star \star \star$ & 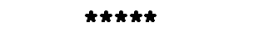 \\
\hline & $\star \star \star$ & $\star \star \star$ & $\star \star \star$ & $\star \star \star$ & $\star \star \star$ & $\star \star \star$ & $\star \star \star$ & $\star \star \star$ & $\star \star \star$ & $\star \star \star$ & $\star \star \star$ & 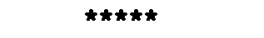 \\
\hline & $\star \star \star *$ & $\star \star \star \star$ & 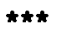 & $\star \star \star$ & $\star \star \star \star$ & 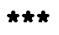 & 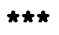 & 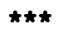 & $\star \star \star \star$ & $\star \star \star$ & $\star \star \star$ & 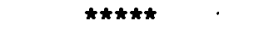 \\
\hline & $\star \star \star \star$ & $\star \star \star$ & $\star \star \star$ & $\star \star \star$ & $\star \star \star \star$ & $\star \star \star$ & $\star \star \star$ & 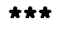 & $\star \star \star \star$ & $\star \star \star$ & $\star \star \star$ & 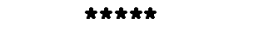 \\
\hline & $\star \star \star$ & $\star \star \star$ & $\star \star \star$ & $\star \star \star$ & $\star \star \star$ & $\star \star \star$ & $\star \star \star$ & $\star \star \star$ & $\star \star \star$ & $\star \star \star$ & $\star \star \star$ & 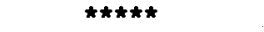 \\
\hline & $\star \star \star$ & $\star \star \star$ & $\star \star \star$ & $\star \star \star$ & $\star \star \star$ & $\star \star \star$ & $\star \star \star$ & $\star \star \star$ & $\star \star \star \star$ & $\star \star \star \star$ & $\star \star \star$ & 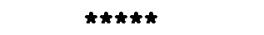 \\
\hline & $\star \star \star \star$ & $\star \star \star \star$ & $\star \star \star \star$ & $\star \star \star$ & $\star \star \star$ & 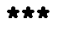 & $\star \star \star$ & $\star \star \star \star *$ & 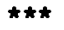 & 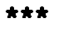 & 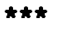 & 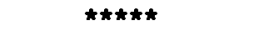 \\
\hline & $\star \star \star \star$ & $\star \star \star$ & $\star \star \star \star$ & 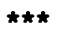 & $\star \star \star \star$ & $\star \star \star \star$ & $\star \star \star \star$ & $\star \star \star$ & $\star \star \star \star$ & $\star \star \star$ & $\star \star \star$ & 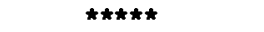 \\
\hline & $\star \star \star$ & $\star \star \star \star$ & $\star \star \star \star$ & $\star \star \star$ & $\star \star \star$ & $\star \star \star \star$ & $\star \star \star$ & $\star \star \star$ & $\star \star \star$ & $\star \star \star$ & $\star \star \star$ & 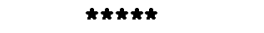 \\
\hline & $\star \star \star$ & $\star \star \star$ & 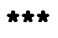 & $\star \star \star$ & $\star \star \star$ & 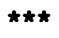 & 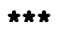 & $\star \star \star$ & $\star \star \star \star *$ & $\star \star \star *$ & 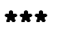 & 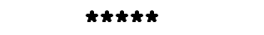 \\
\hline & $\star \star \star \star$ & $\star \star \star \star$ & $\star \star \star *$ & 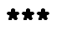 & 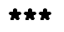 & $\star \star \star \star$ & $\star \star \star \star$ & $\star \star \star \star$ & 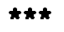 & $\star \star \star \star$ & $\star \star \star$ & 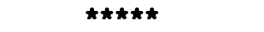 \\
\hline & $\star \star \star \star$ & $\star \star \star \star$ & $\star \star \star \star$ & 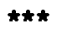 & $\star \star \star \star$ & 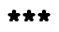 & $\star \star \star \star$ & $\star \star \star \star$ & $\star \star \star \star$ & $\hbar \star \star$ & $\star \star \star \star$ & 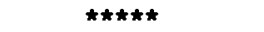 \\
\hline & $\star \star \star \star$ & $\star \star \star$ & $\star \star *$ & $\star \star \star *$ & $\star \star \star$ & $\star \star \star$ & $\star \star \star *$ & $\star \star \star$ & $\star \star \star \star$ & $\star \star \star *$ & $\star \star \star \star$ & 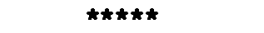 \\
\hline$\star \star \star \star$ & $\hbar \star \star \star$ & $\star \star \star \star$ & $\star \star \star$ & $\star \star \star *$ & $\hbar \star \star \star$ & $\star \star \star \star$ & $\star \star \star \star$ & $\hbar \star \star$ & $\hbar \star \star \star$ & $\hbar \star \star$ & $\hbar \star \star$ & 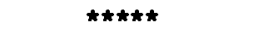 \\
\hline
\end{tabular}

1978

Rainfall

Crop Disease

Birds or Rats

Seed Availability

Fertilizer Availability

Crop Protection Chemicals

Credit

Irrigation Water Availability

flooding

Labour Availabilty

Power for Land Preparation

Direct War Effects

Animal Diseases

Extension

Other Problems

\begin{tabular}{|c|c|c|c|c|c|c|c|c|c|c|c|c|c|}
\hline 1 & 2 & 3 & 4 & 5 & 6 & 7 & 8 & 9 & 10 & 11 & 12 & Weighted P & Priorits \\
\hline$* *$ * & $\star \star \star *$ & $\hbar * \star$ & *** & $\star \star \star *$ & 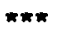 & $\approx * \hbar$ & $\star \star \star \star$ & $i=i$ & $\star \star \star$ & $\dot{i} * \dot{k}$ & $+*$ * & 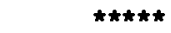 & \\
\hline$* * *$ & $* * *$ & $* * *$ & $\star * *$ & $* * *$ & $* * *$ & $* * *$ & $* * \hbar$. & $* * *$ & $\star \star * *$ & $\star \star *$ & $\star \star \star *$ & $\star \star \star \star \star * \star$ & \\
\hline$\star \star \star *$ & $\star \star \star$ & 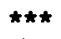 & $\star \star \star$ & $\star \star \star \dot{*}$ & $\star \star \star$ & 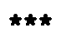 & $\star * *$ & 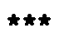 & $\star \star \star$ & 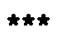 & $\star \star \star$ & $\star \star \star \star \star \star$ & \\
\hline 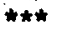 & $\star \star \star \star$ & 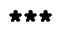 & $\star \star \star \star$ & $\star \star \star \star$ & $\star \star \star \star$ & $\star \star \star *$ & $\star * *$ & $\star \star * *$ & $\star \star \star *$ & $\star * *$ & $\star * *$ & $\star \star \star \star \star * \star$ & \\
\hline$\star \star \star \star$ & $\star \star \star \star$ & $\star \star \star \star$ & $\star \star \star \star$ & $\star \star \star \star$ & 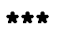 & $\star \star \star$ & 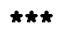 & $\star \star \star \star$ & 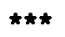 & $\star \star * *$ & 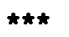 & 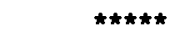 & \\
\hline$\star \star \star \star$ & $\star \star \star *$ & $\star \star \star \star$ & $\star \star \star \star *$ & $\star \star \star \star$ & $\star \star * *$ & 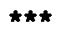 & $\star \star \star \star$ & $\star \star *$ & $\star \star \star \star$ & $\star \star \star *$ & $\star \star \star *$ & 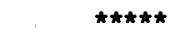 & \\
\hline$\star \star \star$ & $\star \star \star \star$ & $\star \star \star$ & $\star \star \star$ & $\star \star \star$ & $\star \star \star$ & $\star \star \star$ & $\star \star \star$ & $\star \star \star$ & $\star \star \star$ & $\star \star \star$ & $\star \star \star$ & $\star \star \star \star \star$ & \\
\hline$\star \star \star \star$ & $\star \star \star \star$ & $\star \star \star \star$ & $\star \star \star \star$ & $\star \star \star \star$ & $\star \star \star \star$ & $\star \star \star \star$ & 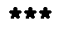 & $\star \star \star \star$ & 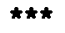 & $\star \star \star \star$ & $\star \star \star *$ & 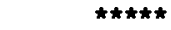 & \\
\hline$\star \star \star *$ & $\star \star \star \star$ & $\star \star \star \star$ & $\star \star \star \star$ & $\star \star \star \star$ & $\star \star \star$ & 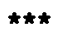 & $\star \star \star$ & $\star \star \star$ & $\star \star \star$ & 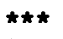 & $\star \star \star$ & 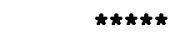 & \\
\hline$\star \star \star *$ & $\star \star \star$ & $\star \star \star$ & $\star \star \star$ & $\star \star \star$ & $\star \star \star$ & $\star \star \star$ & $\star \star \star$ & $\star \star \star$ & $\star \star \star$ & $\star \star \star$ & $\star \star \star$ & 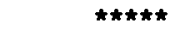 & \\
\hline$\star \star \star *$ & $\star \star \star \star$ & $\star \star \star \star$ & 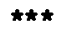 & $\star \star \star *$ & $\star \star \star$ & $\star \star \star *$ & $\star \star \star \star$ & $\star \star \star *$ & $\star \star \star$ & $\star \star \star *$ & $\star \star \star$ & 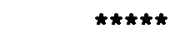 & * \\
\hline 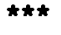 & $\star \star \star$ & $\star \star \star$ & $\star \star \star$ & $\star \star \star$ & $\star \star \star \star$ & $\star \star \star \star$ & $\star \star \star \star$ & $\star \star \star \star$ & $\star \star \star *$ & $\star \star \star$ & $\star \star \star *$ & 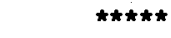 & \\
\hline$\star \star \star \star$ & $\star \star \star \star$ & $\star \star \star \star$ & $\star \star \star \star$ & * & $\star$ & $\star \star \star \star$ & $\star$ & $\star$ & $\star \star \star \star$ & $\star \star \star \star$ & $\star \star \star$ & 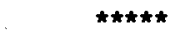 & * \\
\hline 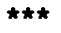 & $\star \star$ & $\star \star \star *$ & $\star \star \star \star$ & $\star \star \star \star$ & $\star \star \star$ & $\star \star \star *$ & $\star \star \star$ & $\star \star \star \star$ & $\star \star \star *$ & $\star \star \star$ & $\star \star \star$ & 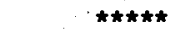 & \\
\hline 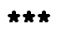 & $\star \star \star$ & 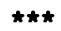 & $\star \star \star$ & $\star \star \star \star$ & $\star \star \star$ & $\star \star \star \star$ & $\star \star \star$ & $\star \star \star \star$ & $\star \star \star$ & $\star \star \star$ & $\star \star \star$ & 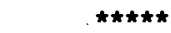 & \\
\hline
\end{tabular}


PROVINCE OF KBL

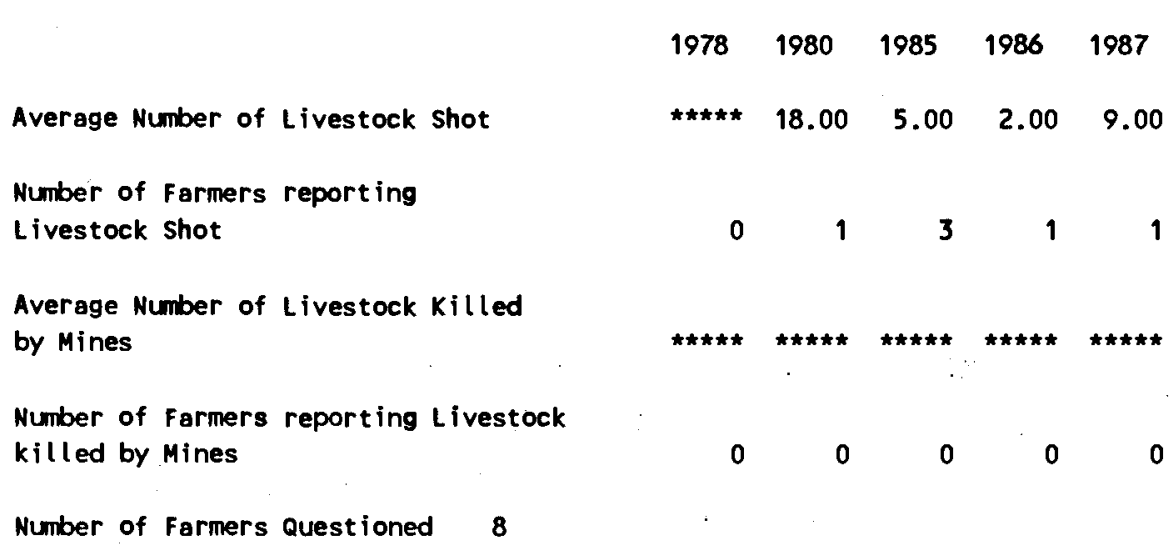

IABLE 9B - DIRECT EFFECTS OF THE WAR - FARMERS IN CAMPS

PROVINCE OF KBL

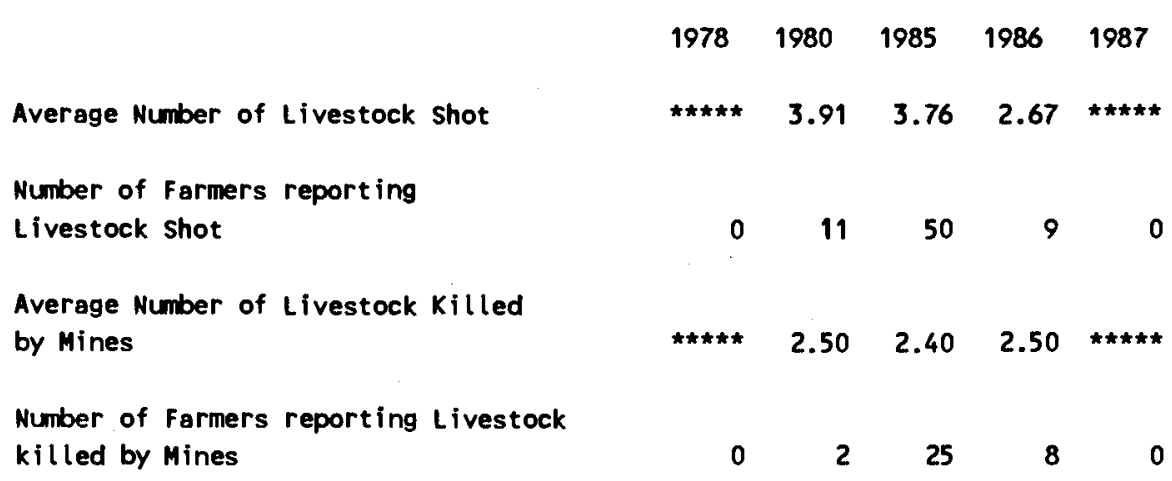

Number of Farmers Questioned 192 